Prepared for the U.S. Department of Energy under Contract DE-AC05-76RL01830

\title{
Geochemical Characterization of Sediments from UPR 200-E-81
}

Michael Lindberg

JULY 2008

Pacific Northwest

NATIONAL LABORATORY 
07/28/08 11:02

To: Fredrick Mann

From: Michael J. Lindberg

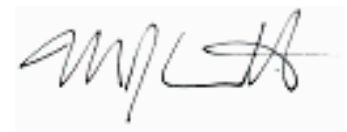

Environmental Sciences Laboratory

Energy and Environment Directorate, Pacific Northwest National Laboratory

Subject: Geochemical Study of Grab Samples Collected From UPR 200-E-81, Sample Delivery Group ESL080022, SAF Number V08-003

This letter contains the following information for sample delivery group ESL080022

- $\quad$ Cover Sheet

- $\quad$ Narrative

- Analytical Results

- Quality Control

- Geologic Logs

- Geologic Photos

- Chain of Custodies 


\section{Introduction}

Between May 15, 2008 and June 9, 2008 soil cores and grab samples were received from UPR 200-E-81 for geochemical studies.

\section{Analytical Results/Methodology}

The analyses for this project were performed at the 325 building located in the 300 Area of the Hanford Site. The analyses were performed according to Pacific Northwest National Laboratory (PNNL) approved procedures and/or nationally recognized test procedures. The data sets include the sample identification numbers, analytical results, estimated quantification limits (EQL), and quality control data.

\section{Quality Control}

The preparatory and analytical quality control requirements, calibration requirements, acceptance criteria, and failure actions are defined in the on-line QA plan “Conducting Analytical Work in Support of Regulatory Programs” (CAW). This QA plan implements the Hanford Analytical Services Quality Assurance Requirements Documents (HASQARD) for PNNL.

\section{Definitions}

Dup Duplicate

RPD Relative Percent Difference

\section{Sample Receipt}

Samples were received with a chain of custody (COC) and were analyzed according to the sample identification numbers supplied by the client. All Samples were refrigerated upon receipt until prepared for analysis.

All samples were received with custody seals intact unless noted in the Case Narrative.

\section{Holding Times}

Holding time is defined as the time from sample preparation to the time of analyses. The prescribed holding times were met for all analytes unless noted in the Case Narrative.

\section{Analytical Results}

All reported analytical results meet the requirements of the CAW or client specified SOW unless noted in the case narrative. 


\section{Case Narrative Report}

\section{Hold time:}

\section{Preparation Blank (PB):}

\section{Duplicate (DUP):}

Duplicate RPD for Barium (38.7\%) was above the acceptance limit (35) in 8F30003-DUP1 for ICP-OES Vadose-WE All other duplicates and QC associated with the batch were in limits. Duplicate failure may be due to sample heterogeneity. There should be no impact to sample data as reported.

Duplicate RPD for Calcium (40.6\%) was above the acceptance limit (35) in 8F30003-DUP1 for ICP-OES Vadose-WE All other duplicates and QC associated with the batch were in limits. Duplicate failure may be due to sample heterogeneity. There should be no impact to sample data as reported.

Duplicate RPD for Magnesium (36.6\%) was above the acceptance limit (35) in 8F30003-DUP1 for ICP-OES Vadose-WE All other duplicates and QC associated with the batch were in limits. Duplicate failure may be due to sample heterogeneity. There should be no impact to sample data as reported.

Duplicate RPD for Manganese (44.4\%) was above the acceptance limit (35) in 8F30003-DUP1 for ICP-OES Vadose-WE

All other duplicates and QC associated with the batch were in limits. Duplicate failure may be due to sample heterogeneity. There should be no impact to sample data as reported.

Duplicate RPD for Titanium (58.9\%) was above the acceptance limit (35) in 8F30003-DUP1 for ICP-OES Vadose-WE All other duplicates and QC associated with the batch were in limits. Duplicate failure may be due to sample heterogeneity. There should be no impact to sample data as reported.

\section{Laboratory control samples (LCS):}

The acid extraction laboratory control standard failed criteria for silver. It was determined that silver is not stable in the nitric acid digestion as performed. Silver is not reported for acid extracted samples.

Post spike (PS) and post spike duplicate (PSD):

\section{Matrix spike (MS) and matrix spike duplicate (MSD):}

Not Applicable.

\section{Other QC Criteria:}

Continuing Calibration Blank 3 analyzed with the water extracts showed a concentration of sodium, 258 ug/L, above the EQL, 223 ug/L. All other calibration blanks analyzed met QC criteria. There should be no impact to the sodium data as reported, 


\section{DISCLAIMER}

This report was prepared as an account of work sponsored by an agency of the United States Government. Neither the United States Government nor any agency thereof, nor Battelle Memorial Institute, nor any of their employees, makes any warranty, express or implied, or assumes any legal liability or responsibility for the accuracy, completeness, or usefulness of any information, apparatus, product, or process disclosed, or represents that its use would not infringe privately owned rights. Reference herein to any specific commercial product, process, or service by trade name, trademark, manufacturer, or otherwise does not necessarily constitute or imply its endorsement, recommendation, or favoring by the United States Government or any agency thereof, or Battelle Memorial Institute. The views and opinions of authors expressed herein do not necessarily state or reflect those of the United States Government or any agency thereof.

\section{SAMPLES INCLUDED IN THIS REPORT}

\section{C-Farm Direct Push C6394}

$\begin{array}{lll}\text { HEIS No. } & \text { Laboratory ID } & \text { Matrix } \\ \text { B1VJ54B } & 0805020-01 & \text { SOIL } \\ \text { B1VJ54C } & 0805020-02 & \text { SOIL } \\ \text { B1VJ55B } & 0805020-04 & \text { SOIL } \\ \text { B1VJ55C } & 0805020-05 & \text { SOIL } \\ \text { B1VJ56A } & 0805020-07 & \text { SOIL } \\ \text { B1VJ56B } & 0805020-08 & \text { SOIL } \\ \text { B1VJ56C } & 0805020-09 & \text { SOIL } \\ \text { B1VJ58C } & 0805020-10 & \text { SOIL } \\ \text { B1VJ58B } & 0805020-11 & \text { SOIL } \\ \text { B1VJ58A } & 0805020-12 & \text { SOIL } \\ \text { B1VJ59C } & 0805020-14 & \text { SOIL } \\ \text { B1VJ59B } & 0805020-15 & \text { SOIL } \\ \text { B1VJ59A } & 0805020-16 & \text { SOIL } \\ \text { B1VJ60C } & 0805020-18 & \text { SOIL } \\ \text { B1VJ60B } & 0805020-19 & \text { SOIL } \\ \text { B1VJ60A } & 0805020-20 & \text { SOIL } \\ \text { B1VJ61C } & 0805020-22 & \text { SOIL } \\ \text { B1VJ61B } & 0805020-23 & \text { SOIL } \\ \text { B1VJ61A } & 0805020-24 & \text { SOIL } \\ \text { B1VJ62C } & 0805020-26 & \text { SOIL } \\ \text { B1VJ62B } & 0805020-27 & \text { SOIL } \\ \text { B1VJ62A } & 0805020-28 & \text { SOIL } \\ \text { B1VJ64C } & 0805020-30 & \text { SOIL } \\ \text { B1VJ64B } & 0805020-31 & \text { SOIL } \\ \text { B1VJ64A } & 0805020-32 & \text { SOIL }\end{array}$

$\begin{array}{llll}\text { Date Collected } & \text { Date Received } \\ \text { 5/13/08 } & 10: 25 & 5 / 15 / 08 & 13: 20 \\ \text { 5/13/08 } & 10: 25 & 5 / 15 / 08 & 13: 20 \\ \text { 5/13/08 } & 11: 30 & 5 / 15 / 08 & 13: 20 \\ \text { 5/13/08 } & 11: 30 & 5 / 15 / 08 & 13: 20 \\ 5 / 14 / 08 & 12: 00 & 5 / 15 / 08 & 13: 20 \\ 5 / 14 / 08 & 12: 00 & 5 / 15 / 08 & 13: 20 \\ \text { 5/14/08 } & 12: 00 & 5 / 15 / 08 & 13: 20 \\ \text { 5/19/08 } & 09: 45 & 5 / 19 / 08 & 14: 00 \\ \text { 5/19/08 } & 09: 45 & 5 / 19 / 08 & 14: 00 \\ 5 / 19 / 08 & 09: 45 & 5 / 19 / 08 & 14: 00 \\ 5 / 22 / 08 & 11: 00 & 5 / 28 / 08 & 14: 45 \\ 5 / 22 / 08 & 11: 00 & 5 / 28 / 08 & 14: 45 \\ 5 / 22 / 08 & 11: 00 & 5 / 28 / 08 & 14: 45 \\ 5 / 23 / 08 & 10: 00 & 5 / 28 / 08 & 14: 45 \\ 5 / 23 / 08 & 10: 00 & 5 / 28 / 08 & 14: 45 \\ 5 / 23 / 08 & 10: 00 & 5 / 28 / 08 & 14: 45 \\ 5 / 27 / 08 & 13: 30 & 5 / 28 / 08 & 14: 45 \\ 5 / 27 / 08 & 13: 30 & 5 / 28 / 08 & 14: 45 \\ 5 / 27 / 08 & 13: 30 & 5 / 28 / 08 & 14: 45 \\ 5 / 28 / 08 & 10: 10 & 5 / 29 / 08 & 13: 40 \\ 5 / 28 / 08 & 10: 10 & 5 / 29 / 08 & 13: 40 \\ 5 / 28 / 08 & 10: 10 & 5 / 29 / 08 & 13: 40 \\ \text { 6/9/08 } & 10: 10 & 6 / 9 / 08 & 13: 40 \\ \text { 6/9/08 } & 10: 10 & 6 / 9 / 08 & 13: 40 \\ \text { 6/9/08 } & 10: 10 & 6 / 9 / 08 & 13: 40\end{array}$


The following analyses were performed on the following samples included in this report:

Metals 1:1 DI Water Extract by ICPMS

Metals Acid Extract by ICPMS

1:1 DI Water Extract

Actinide Acid Extract by ICPMS

AGG-TOC-001

Alkalinity, Titrimetic ( $\mathrm{pH} 4.5)$

Anions By Ion Chromatography

Carbon, Total, Combustion or Oxidation

GEA No Preparation

geological description

Inorganic Carbon, Total, Combustion or Oxidation

Metals 1:1 Water Extract by ICPOES

Metals Acid Extract by ICPOES

Moisture Content

Nitric Acid Digestion

Percent Solids

$\mathrm{pH}$ of Waters By Electrode

Specific Conductance

Tc_U Acid Extract by ICPMS

Tc_U 1:1 DI Water Extract by ICPMS

Total Alpha Total Beta 1:1 DI Water Extract By LSC

Total Alpha Total Beta Acid Extract By LSC 


$\begin{array}{lll}\text { HEIS No. } & \text { Laboratory ID } & \text { Matrix } \\ \text { B1VJ54B } & 0805020-01 & \text { SOIL } \\ \text { B1VJ54C } & 0805020-02 & \text { SOIL } \\ \text { B1VJ55B } & 0805020-04 & \text { SOIL } \\ \text { B1VJ55C } & 0805020-05 & \text { SOIL } \\ \text { B1VJ56A } & 0805020-07 & \text { SOIL } \\ \text { B1VJ56B } & 0805020-08 & \text { SOIL } \\ \text { B1VJ56C } & 0805020-09 & \text { SOIL } \\ \text { B1VJ58C } & 0805020-10 & \text { SOIL } \\ \text { B1VJ58B } & 0805020-11 & \text { SOIL } \\ \text { B1VJ58A } & 0805020-12 & \text { SOIL } \\ \text { B1VJ59C } & 0805020-14 & \text { SOIL } \\ \text { B1VJ59B } & 0805020-15 & \text { SOIL } \\ \text { B1VJ59A } & 0805020-16 & \text { SOIL } \\ \text { B1VJ60C } & 0805020-18 & \text { SOIL } \\ \text { B1VJ60B } & 0805020-19 & \text { SOIL } \\ \text { B1VJ60A } & 0805020-20 & \text { SOIL } \\ \text { B1VJ61C } & 0805020-22 & \text { SOIL } \\ \text { B1VJ61B } & 0805020-23 & \text { SOIL } \\ \text { B1VJ61A } & 0805020-24 & \text { SOIL } \\ \text { B1VJ62C } & 0805020-26 & \text { SOIL } \\ \text { B1VJ62B } & 0805020-27 & \text { SOIL } \\ \text { B1VJ62A } & 0805020-28 & \text { SOIL } \\ \text { B1VJ64C } & 0805020-30 & \text { SOIL } \\ \text { B1VJ64B } & 0805020-31 & \text { SOIL } \\ \text { B1VJ64A } & 0805020-32 & \text { SOIL }\end{array}$

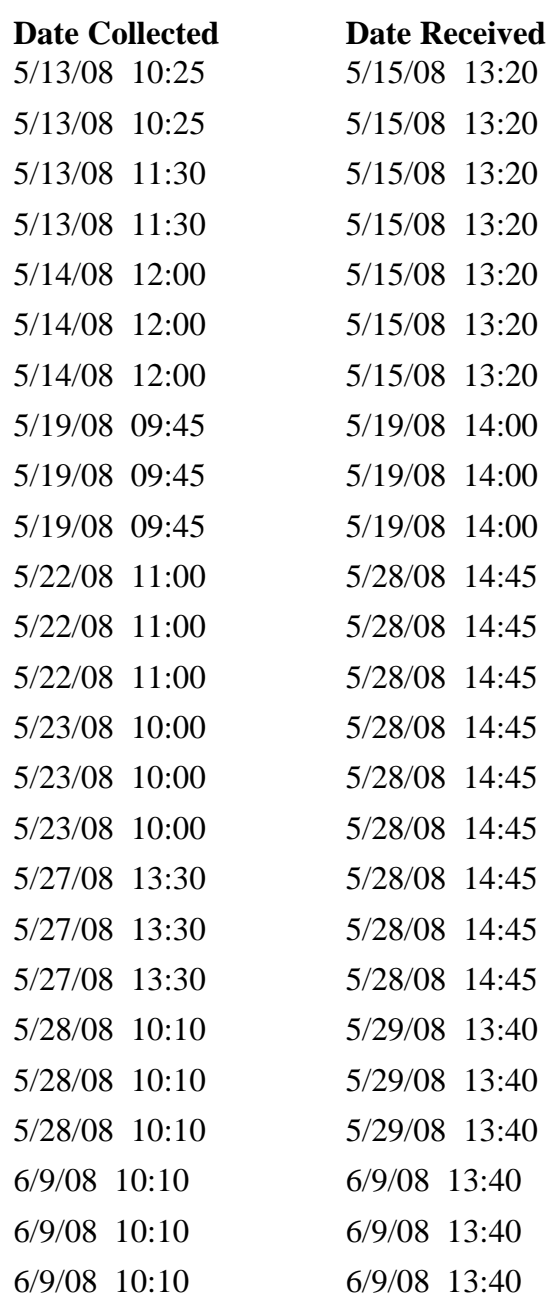




\section{Wet Chemistry}

\begin{tabular}{|c|c|c|c|c|c|}
\hline $\begin{array}{l}\text { Alkalinity } \\
\text { Lab ID }\end{array}$ & $\begin{array}{c}\text { CaCO3 (l } \\
\text { HEIS No. }\end{array}$ & $\begin{array}{l}\text { Methoc } \\
\text { Results }\end{array}$ & $\begin{array}{l}\text { 20B } \\
\text { EQL }\end{array}$ & Analyzed & Batch \\
\hline 0805020-01 & B1VJ54B & $9.26 \mathrm{E} 1$ & 2.39E1 & 6/30/08 & 8F30007 \\
\hline 0805020-05 & B1VJ55C & 1.17E2 & 2.35E1 & 6/30/08 & 8 F30007 \\
\hline 0805020-07 & B1VJ56A & 4.93E2 & 2.52E1 & 6/30/08 & 8F30007 \\
\hline 0805020-08 & B1VJ56B & $5.26 \mathrm{E} 2$ & 2.35E1 & 6/30/08 & $8 \mathrm{~F} 30007$ \\
\hline 0805020-09 & B1VJ56C & 4.04E2 & 2.35E1 & 6/30/08 & 8 F30007 \\
\hline 0805020-10 & B1VJ58C & 8.32E2 & 2.35E1 & 6/30/08 & 8 F30007 \\
\hline 0805020-11 & B1VJ58B & $9.25 \mathrm{E} 2$ & 2.36E1 & 6/30/08 & $8 \mathrm{~F} 30007$ \\
\hline 0805020-12 & B1VJ58A & 8.61E2 & $2.35 \mathrm{E} 1$ & 6/30/08 & $8 \mathrm{~F} 30007$ \\
\hline 0805020-14 & B1VJ59C & 8.51E1 & 2.35E1 & 6/30/08 & 8F30007 \\
\hline 0805020-15 & B1VJ59B & 4.56E1 & 2.35E1 & 6/30/08 & 8 F30007 \\
\hline 0805020-16 & B1VJ59A & 4.52E1 & 2.37E1 & 6/30/08 & 8F30007 \\
\hline 0805020-18 & B1VJ60C & 8.05E1 & 2.35E1 & 6/30/08 & 8F30007 \\
\hline 0805020-19 & B1VJ60B & 4.18E1 & 2.35E1 & 6/30/08 & 8 F30007 \\
\hline 0805020-20 & B1VJ60A & 4.10E1 & 2.35E1 & 6/30/08 & $8 \mathrm{~F} 30007$ \\
\hline 0805020-22 & B1VJ61C & 4.79E1 & 2.35E1 & 6/30/08 & $8 \mathrm{~F} 30007$ \\
\hline 0805020-23 & B1VJ61B & 4.15E1 & 2.37E1 & 6/30/08 & 8F30007 \\
\hline 0805020-24 & B1VJ61A & 3.94E1 & $2.44 \mathrm{E} 1$ & 6/30/08 & 8 F30007 \\
\hline 0805020-26 & B1VJ62C & $5.85 \mathrm{E} 1$ & 2.35E1 & 6/30/08 & 8 F30007 \\
\hline 0805020-27 & B1VJ62B & 3.96E1 & 2.35E1 & 6/30/08 & $8 \mathrm{~F} 30008$ \\
\hline 0805020-28 & B1VJ62A & 3.88E1 & 2.35E1 & 6/30/08 & 8F30008 \\
\hline 0805020-30 & B1VJ64C & 5.17E1 & 2.35E1 & 6/30/08 & 8F30008 \\
\hline 0805020-31 & B1VJ64B & 4.10E1 & 2.35E1 & 6/30/08 & 8F30008 \\
\hline 0805020-32 & B1VJ64A & 4.04E1 & 2.23E1 & 6/30/08 & 8F30008 \\
\hline
\end{tabular}




\section{Wet Chemistry}

\begin{tabular}{llcccc}
\multicolumn{2}{l}{$\begin{array}{l}\text { Specific Conductance (EC) (mS/cm) by EPA 120.1 } \\
\text { Lab ID }\end{array}$} & Results & EQL & Analyzed & Batch \\
\hline $0805020-01$ & B1VJ54B & $1.76 \mathrm{E}-1$ & $1.00 \mathrm{E}-2$ & $6 / 26 / 08$ & $8 \mathrm{~F} 26007$ \\
$0805020-05$ & B1VJ55C & $2.52 \mathrm{E}-1$ & $1.00 \mathrm{E}-2$ & $6 / 26 / 08$ & $8 \mathrm{~F} 26007$ \\
$0805020-07$ & B1VJ56A & $8.23 \mathrm{E}-1$ & $1.00 \mathrm{E}-2$ & $6 / 26 / 08$ & $8 \mathrm{~F} 26007$ \\
$0805020-08$ & B1VJ56B & $9.68 \mathrm{E}-1$ & $1.00 \mathrm{E}-2$ & $6 / 26 / 08$ & $8 \mathrm{~F} 26007$ \\
$0805020-09$ & B1VJ56C & $7.69 \mathrm{E}-1$ & $1.00 \mathrm{E}-2$ & $6 / 26 / 08$ & $8 \mathrm{~F} 26007$ \\
$0805020-10$ & B1VJ58C & $1.84 \mathrm{E} 0$ & $1.00 \mathrm{E}-2$ & $6 / 26 / 08$ & $8 \mathrm{~F} 26007$ \\
$0805020-11$ & B1VJ58B & $2.01 \mathrm{E} 0$ & $1.00 \mathrm{E}-2$ & $6 / 26 / 08$ & $8 \mathrm{~F} 26007$ \\
$0805020-12$ & B1VJ58A & $1.91 \mathrm{E} 0$ & $1.00 \mathrm{E}-2$ & $6 / 26 / 08$ & $8 \mathrm{~F} 26007$ \\
$0805020-14$ & B1VJ59C & $2.34 \mathrm{E}-1$ & $1.00 \mathrm{E}-2$ & $6 / 26 / 08$ & $8 \mathrm{~F} 26007$ \\
$0805020-15$ & B1VJ59B & $1.32 \mathrm{E}-1$ & $1.00 \mathrm{E}-2$ & $6 / 26 / 08$ & $8 \mathrm{~F} 26007$ \\
$0805020-16$ & B1VJ59A & $1.20 \mathrm{E}-1$ & $1.00 \mathrm{E}-2$ & $6 / 26 / 08$ & $8 \mathrm{~F} 26007$ \\
$0805020-18$ & B1VJ60C & $3.30 \mathrm{E}-1$ & $1.00 \mathrm{E}-2$ & $6 / 26 / 08$ & $8 \mathrm{~F} 26007$ \\
$0805020-19$ & B1VJ60B & $3.19 \mathrm{E}-1$ & $1.00 \mathrm{E}-2$ & $6 / 26 / 08$ & $8 \mathrm{~F} 26007$ \\
$0805020-20$ & B1VJ60A & $2.18 \mathrm{E}-1$ & $1.00 \mathrm{E}-2$ & $6 / 26 / 08$ & $8 \mathrm{~F} 26007$ \\
$0805020-22$ & B1VJ61C & $2.66 \mathrm{E}-1$ & $1.00 \mathrm{E}-2$ & $6 / 26 / 08$ & $8 \mathrm{~F} 26007$ \\
$0805020-23$ & B1VJ61B & $2.24 \mathrm{E}-1$ & $1.00 \mathrm{E}-2$ & $6 / 26 / 08$ & $8 \mathrm{~F} 26007$ \\
$0805020-24$ & B1VJ61A & $2.18 \mathrm{E}-1$ & $1.00 \mathrm{E}-2$ & $6 / 26 / 08$ & $8 \mathrm{~F} 26007$ \\
$0805020-26$ & B1VJ62C & $6.71 \mathrm{E}-1$ & $1.00 \mathrm{E}-2$ & $6 / 26 / 08$ & $8 \mathrm{~F} 26007$ \\
$0805020-27$ & B1VJ62B & $4.52 \mathrm{E}-1$ & $1.00 \mathrm{E}-2$ & $6 / 26 / 08$ & $8 \mathrm{~F} 26008$ \\
$0805020-28$ & B1VJ62A & $4.66 \mathrm{E}-1$ & $1.00 \mathrm{E}-2$ & $6 / 26 / 08$ & $8 \mathrm{~F} 26008$ \\
$0805020-30$ & B1VJ64C & $4.22 \mathrm{E}-1$ & $1.00 \mathrm{E}-2$ & $6 / 26 / 08$ & $8 \mathrm{~F} 26008$ \\
$0805020-31$ & B1VJ64B & $2.75 \mathrm{E}-1$ & $1.00 \mathrm{E}-2$ & 60808 \\
$0805020-32$ & B1VJ64A & $2.66 \mathrm{E}-1$ & $1.00 \mathrm{E}-2$ & \\
\hline
\end{tabular}




\section{Wet Chemistry}

\begin{tabular}{|c|c|c|c|c|c|}
\hline $\begin{array}{l}\text { Moisture } \\
\text { Lab ID }\end{array}$ & $\begin{array}{c}\text { ntent ( } \% \text { b } \\
\text { HEIS No. }\end{array}$ & $\begin{array}{l}\text { C-001 } \\
\text { Results }\end{array}$ & EQL & Analyzed & Batch \\
\hline 0805020-01 & B1VJ54B & $9.86 \mathrm{E} 0$ & N/A & 6/20/08 & $8 \mathrm{~F} 11008$ \\
\hline 0805020-02 & B1VJ54C & $9.52 \mathrm{E} 0$ & N/A & $6 / 20 / 08$ & $8 \mathrm{~F} 11008$ \\
\hline 0805020-04 & B1VJ55B & $8.90 \mathrm{E} 0$ & N/A & 6/20/08 & $8 F 11008$ \\
\hline 0805020-05 & B1VJ55C & $9.84 \mathrm{E} 0$ & N/A & $6 / 20 / 08$ & 8F11008 \\
\hline 0805020-07 & B1VJ56A & $8.00 \mathrm{E} 0$ & N/A & 6/20/08 & $8 \mathrm{~F} 11008$ \\
\hline 0805020-08 & B1VJ56B & 8.07E0 & N/A & $6 / 20 / 08$ & $8 F 11008$ \\
\hline 0805020-09 & B1VJ56C & 5.61E0 & N/A & 6/20/08 & $8 F 11008$ \\
\hline 0805020-10 & B1VJ58C & $1.25 \mathrm{E} 1$ & N/A & 6/20/08 & $8 F 11008$ \\
\hline 0805020-11 & B1VJ58B & $1.36 \mathrm{E} 1$ & N/A & $6 / 20 / 08$ & $8 \mathrm{~F} 11008$ \\
\hline 0805020-12 & B1VJ58A & $1.25 \mathrm{E} 1$ & N/A & $6 / 20 / 08$ & 8F11008 \\
\hline 0805020-14 & B1VJ59C & $6.71 \mathrm{E} 0$ & N/A & 6/20/08 & $8 F 11008$ \\
\hline 0805020-15 & B1VJ59B & 3.87E0 & N/A & 6/20/08 & $8 F 11008$ \\
\hline 0805020-16 & B1VJ59A & 3.04E0 & N/A & $6 / 20 / 08$ & $8 F 11008$ \\
\hline 0805020-18 & B1VJ60C & 4.07E0 & N/A & 6/20/08 & $8 F 11008$ \\
\hline 0805020-19 & B1VJ60B & $5.30 \mathrm{E} 0$ & N/A & 6/20/08 & $8 \mathrm{~F} 11008$ \\
\hline 0805020-20 & B1VJ60A & $2.66 \mathrm{E} 0$ & N/A & 6/20/08 & $8 \mathrm{~F} 11008$ \\
\hline 0805020-22 & B1VJ61C & 2.89E0 & N/A & $6 / 20 / 08$ & 8F11008 \\
\hline 0805020-23 & B1VJ61B & 2.49E0 & N/A & $6 / 20 / 08$ & 8F11008 \\
\hline 0805020-24 & B1VJ61A & 2.47E0 & N/A & 6/20/08 & $8 F 11008$ \\
\hline 0805020-26 & B1VJ62C & $8.29 \mathrm{E} 0$ & N/A & $6 / 20 / 08$ & $8 F 11008$ \\
\hline $0805020-27$ & B1VJ62B & $6.85 \mathrm{E} 0$ & N/A & 6/20/08 & $8 \mathrm{~F} 11008$ \\
\hline 0805020-28 & B1VJ62A & $6.90 \mathrm{E} 0$ & N/A & 6/20/08 & $8 F 11008$ \\
\hline 0805020-30 & B1VJ64C & $6.08 \mathrm{E} 0$ & N/A & 6/20/08 & $8 F 11008$ \\
\hline 0805020-31 & B1VJ64B & $3.48 \mathrm{E} 0$ & N/A & $6 / 20 / 08$ & 8F11008 \\
\hline 0805020-32 & B1VJ64A & $3.10 \mathrm{E} 0$ & N/A & $6 / 20 / 08$ & 8F11008 \\
\hline
\end{tabular}




\section{Wet Chemistry}

\begin{tabular}{|c|c|c|c|c|c|}
\hline Lab ID & HEIS No. & Results & EQL & Analyzed & Batch \\
\hline 0805020-01 & B1VJ54B & 8.67E0 & N/A & $6 / 26 / 08$ & $8 F 26009$ \\
\hline 0805020-05 & B1VJ55C & $8.80 \mathrm{E} 0$ & N/A & 6/26/08 & 8 F26009 \\
\hline 0805020-07 & B1VJ56A & $1.01 \mathrm{E} 1$ & N/A & 6/26/08 & $8 F 26009$ \\
\hline 0805020-08 & B1VJ56B & $1.00 \mathrm{E} 1$ & N/A & 6/26/08 & 8F26009 \\
\hline 0805020-09 & B1VJ56C & $9.85 \mathrm{E} 0$ & N/A & 6/26/08 & $8 F 26009$ \\
\hline 0805020-10 & B1VJ58C & $9.66 \mathrm{E} 0$ & N/A & $6 / 26 / 08$ & 8F26009 \\
\hline 0805020-11 & B1VJ58B & $9.64 \mathrm{E} 0$ & N/A & $6 / 26 / 08$ & 8F26009 \\
\hline 0805020-12 & B1VJ58A & $9.71 \mathrm{E} 0$ & N/A & 6/26/08 & 8F26009 \\
\hline 0805020-14 & B1VJ59C & 8.29E0 & N/A & 6/26/08 & 8F26009 \\
\hline 0805020-15 & B1VJ59B & 8.19E0 & N/A & 6/26/08 & $8 F 26009$ \\
\hline 0805020-16 & B1VJ59A & 8.07E0 & N/A & $6 / 26 / 08$ & 8F26009 \\
\hline 0805020-18 & B1VJ60C & $8.16 \mathrm{E} 0$ & N/A & $6 / 26 / 08$ & 8F26009 \\
\hline 0805020-19 & B1VJ60B & 7.98E0 & N/A & 6/26/08 & $8 F 26009$ \\
\hline 0805020-20 & B1VJ60A & 7.98E0 & N/A & 6/26/08 & 8F26009 \\
\hline 0805020-22 & B1VJ61C & 8.06E0 & N/A & $6 / 26 / 08$ & 8F26009 \\
\hline 0805020-23 & B1VJ61B & 7.99E0 & N/A & $6 / 26 / 08$ & 8F26009 \\
\hline 0805020-24 & B1VJ61A & 7.94E0 & N/A & 6/26/08 & 8F26009 \\
\hline 0805020-26 & B1VJ62C & $8.11 \mathrm{E} 0$ & N/A & $6 / 26 / 08$ & 8F26009 \\
\hline 0805020-27 & B1VJ62B & 7.79E0 & N/A & $6 / 26 / 08$ & 8F26010 \\
\hline 0805020-28 & B1VJ62A & $7.80 \mathrm{E} 0$ & N/A & 6/26/08 & 8 F26010 \\
\hline 0805020-30 & B1VJ64C & 7.94E0 & N/A & 6/26/08 & 8F26010 \\
\hline 0805020-31 & B1VJ64B & 7.79E0 & N/A & 6/26/08 & 8 F26010 \\
\hline 0805020-32 & B1VJ64A & 7.75E0 & N/A & 6/26/08 & $8 F 26010$ \\
\hline
\end{tabular}




\section{Anions by Ion Chromatography}

\begin{tabular}{|c|c|c|c|c|c|c|c|}
\hline CAS \# & Analyte & Results & Units & EQL & Analyzed & Batch & Method \\
\hline HEIS No. & B1VJ54B & \multicolumn{3}{|c|}{ Lab ID: $\quad$ 0805020-01 } & & & \\
\hline $16984-48-8$ & Fluoride & 1.17E0 & ug/g dry & 2.03E-1 & 6/26/08 & 8F26011 & AGG-IC-001 \\
\hline 16887-00-6 & Chloride & $<5.08 \mathrm{E}-1$ & ug/g dry & $5.08 \mathrm{E}-1$ & 6/26/08 & 8F26011 & AGG-IC-001 \\
\hline 14797-65-0 & Nitrite & $<1.02 \mathrm{E} 0$ & ug/g dry & $1.02 \mathrm{E} 0$ & 6/26/08 & 8F26011 & AGG-IC-001 \\
\hline 24959-67-9 & Bromide & $<1.02 \mathrm{E} 0$ & ug/g dry & $1.02 \mathrm{E} 0$ & $6 / 26 / 08$ & $8 \mathrm{~F} 26011$ & AGG-IC-001 \\
\hline $14797-55-8$ & Nitrate & 8.20E0 & ug/g dry & $1.02 \mathrm{E} 0$ & 6/26/08 & $8 \mathrm{~F} 26011$ & AGG-IC-001 \\
\hline 14808-79-8 & Sulfate & 4.74E0 & ug/g dry & $1.52 \mathrm{E} 0$ & 6/26/08 & 8 F26011 & AGG-IC-001 \\
\hline $14265-44-2$ & Phosphate & $<1.52 \mathrm{E} 0$ & ug/g dry & $1.52 \mathrm{E} 0$ & 6/26/08 & 8F26011 & AGG-IC-001 \\
\hline HEIS No. & B1VJ55C & \multicolumn{3}{|c|}{ Lab ID: $\quad$ 0805020-05 } & & & \\
\hline 16984-48-8 & Fluoride & $1.25 \mathrm{E} 0$ & ug/g dry & $2.00 \mathrm{E}-1$ & $6 / 26 / 08$ & 8 F26011 & AGG-IC-001 \\
\hline $16887-00-6$ & Chloride & $3.20 \mathrm{E} 0$ & ug/g dry & $5.00 \mathrm{E}-1$ & 6/26/08 & 8F26011 & AGG-IC-001 \\
\hline 14797-65-0 & Nitrite & $<1.00 \mathrm{E} 0$ & ug/g dry & $1.00 \mathrm{E} 0$ & 6/26/08 & $8 \mathrm{~F} 26011$ & AGG-IC-001 \\
\hline 24959-67-9 & Bromide & $<1.00 \mathrm{E} 0$ & ug/g dry & $1.00 \mathrm{E} 0$ & $6 / 26 / 08$ & $8 \mathrm{~F} 26011$ & AGG-IC-001 \\
\hline $14797-55-8$ & Nitrate & 1.66E1 & ug/g dry & $1.00 \mathrm{E} 0$ & 6/26/08 & $8 \mathrm{~F} 26011$ & AGG-IC-001 \\
\hline 14808-79-8 & Sulfate & $1.54 \mathrm{E} 1$ & ug/g dry & $1.50 \mathrm{E} 0$ & 6/26/08 & $8 F 26011$ & AGG-IC-001 \\
\hline $14265-44-2$ & Phosphate & $<1.50 \mathrm{E} 0$ & ug/g dry & $1.50 \mathrm{E} 0$ & 6/26/08 & $8 F 26011$ & AGG-IC-001 \\
\hline HEIS No. & B1VJ56A & \multicolumn{3}{|c|}{ Lab ID: $\quad$ 0805020-07 } & & & \\
\hline 16984-48-8 & Fluoride & 1.67E0 & ug/g dry & $2.14 \mathrm{E}-1$ & 6/30/08 & 8 F26011 & AGG-IC-001 \\
\hline 16887-00-6 & Chloride & 7.29E-1 & ug/g dry & $5.36 \mathrm{E}-1$ & 6/30/08 & 8F26011 & AGG-IC-001 \\
\hline $14797-65-0$ & Nitrite & $<1.07 \mathrm{E} 0$ & ug/g dry & $1.07 \mathrm{E} 0$ & 6/30/08 & $8 \mathrm{~F} 26011$ & AGG-IC-001 \\
\hline 24959-67-9 & Bromide & $<1.07 \mathrm{E} 0$ & ug/g dry & 1.07E0 & 6/30/08 & 8F26011 & AGG-IC-001 \\
\hline 14797-55-8 & Nitrate & 7.77E0 & ug/g dry & 1.07E0 & 6/30/08 & $8 \mathrm{~F} 26011$ & AGG-IC-001 \\
\hline 14808-79-8 & Sulfate & 8.63E0 & ug/g dry & $1.61 \mathrm{E} 0$ & 6/30/08 & $8 \mathrm{~F} 26011$ & AGG-IC-001 \\
\hline $14265-44-2$ & Phosphate & 2.77E0 & ug/g dry & $1.61 \mathrm{E} 0$ & 6/30/08 & $8 F 26011$ & AGG-IC-001 \\
\hline HEIS No. & B1VJ56B & \multicolumn{3}{|c|}{ Lab ID: $\quad$ 0805020-08 } & & & \\
\hline 16984-48-8 & Fluoride & 2.33E0 & ug/g dry & $2.00 \mathrm{E}-1$ & 6/30/08 & $8 \mathrm{~F} 26011$ & AGG-IC-001 \\
\hline $16887-00-6$ & Chloride & $9.62 \mathrm{E}-1$ & ug/g dry & $5.01 \mathrm{E}-1$ & 6/30/08 & 8F26011 & AGG-IC-001 \\
\hline $14797-65-0$ & Nitrite & $<1.00 \mathrm{E} 0$ & ug/g dry & $1.00 \mathrm{E} 0$ & 6/30/08 & 8F26011 & AGG-IC-001 \\
\hline 24959-67-9 & Bromide & $<1.00 \mathrm{E} 0$ & ug/g dry & $1.00 \mathrm{E} 0$ & 6/30/08 & 8F26011 & AGG-IC-001 \\
\hline $14797-55-8$ & Nitrate & $9.24 \mathrm{E} 0$ & ug/g dry & $1.00 \mathrm{E} 0$ & 6/30/08 & $8 \mathrm{~F} 26011$ & AGG-IC-001 \\
\hline 14808-79-8 & Sulfate & $9.90 \mathrm{E} 0$ & ug/g dry & $1.50 \mathrm{E} 0$ & 6/30/08 & $8 \mathrm{~F} 26011$ & AGG-IC-001 \\
\hline $14265-44-2$ & Phosphate & 3.19E0 & ug/g dry & $1.50 \mathrm{E} 0$ & 6/30/08 & 8F26011 & AGG-IC-001 \\
\hline HEIS No. & B1VJ56C & \multicolumn{3}{|c|}{ Lab ID: $\quad$ 0805020-09 } & & & \\
\hline $16984-48-8$ & Fluoride & $1.47 \mathrm{E} 0$ & ug/g dry & 2.00E-1 & 6/30/08 & 8F26011 & AGG-IC-001 \\
\hline 16887-00-6 & Chloride & 1.62E0 & ug/g dry & $5.00 \mathrm{E}-1$ & 6/30/08 & $8 \mathrm{~F} 26011$ & AGG-IC-001 \\
\hline $14797-65-0$ & Nitrite & $<1.00 \mathrm{E} 0$ & ug/g dry & $1.00 \mathrm{E} 0$ & 6/30/08 & $8 \mathrm{~F} 26011$ & AGG-IC-001 \\
\hline 24959-67-9 & Bromide & $<1.00 \mathrm{E} 0$ & ug/g dry & $1.00 \mathrm{E} 0$ & 6/30/08 & $8 \mathrm{~F} 26011$ & AGG-IC-001 \\
\hline $14797-55-8$ & Nitrate & $1.80 \mathrm{E} 0$ & ug/g dry & $1.00 \mathrm{E} 0$ & 6/30/08 & $8 \mathrm{~F} 26011$ & AGG-IC-001 \\
\hline 14808-79-8 & Sulfate & 8.57E0 & ug/g dry & $1.50 \mathrm{E} 0$ & 6/30/08 & $8 \mathrm{~F} 26011$ & AGG-IC-001 \\
\hline $14265-44-2$ & Phosphate & $<1.50 \mathrm{E} 0$ & ug/g dry & $1.50 \mathrm{E} 0$ & 6/30/08 & $8 F 26011$ & AGG-IC-001 \\
\hline HEIS No. & B1VJ58C & \multicolumn{3}{|c|}{ Lab ID: $\quad$ 0805020-10 } & & & \\
\hline 16984-48-8 & Fluoride & $1.79 \mathrm{E} 1$ & ug/g dry & $2.00 \mathrm{E} 0$ & 6/30/08 & $8 \mathrm{~F} 26011$ & AGG-IC-001 \\
\hline $16887-00-6$ & Chloride & $<5.00 \mathrm{E} 0$ & ug/g dry & $5.00 \mathrm{E} 0$ & 6/30/08 & $8 \mathrm{~F} 26011$ & AGG-IC-001 \\
\hline $14797-65-0$ & Nitrite & $<1.00 \mathrm{E} 1$ & ug/g dry & $1.00 \mathrm{E} 1$ & 6/30/08 & $8 F 26011$ & AGG-IC-001 \\
\hline 24959-67-9 & Bromide & $<1.00 \mathrm{E} 1$ & ug/g dry & $1.00 \mathrm{E} 1$ & 6/30/08 & 8F26011 & AGG-IC-001 \\
\hline 14797-55-8 & Nitrate & $1.68 \mathrm{E} 2$ & ug/g dry & $1.00 \mathrm{E} 1$ & 6/30/08 & 8F26011 & AGG-IC-001 \\
\hline 14808-79-8 & Sulfate & $1.90 \mathrm{E} 1$ & ug/g dry & $1.50 \mathrm{E} 1$ & 6/30/08 & $8 \mathrm{~F} 26011$ & AGG-IC-001 \\
\hline 14265-44-2 & Phosphate & $<1.50 \mathrm{E} 1$ & ug/g dry & $1.50 \mathrm{E} 1$ & 6/30/08 & $8 F 26011$ & AGG-IC-001 \\
\hline HEIS No. & B1VJ58B & \multicolumn{2}{|c|}{ Lab ID: } & 5020-11 & & & \\
\hline
\end{tabular}




\section{Anions by Ion Chromatography}

\begin{tabular}{|c|c|c|c|c|c|c|c|}
\hline CAS \# & Analyte & Results & Units & EQL & Analyzed & Batch & Method \\
\hline HEIS No. & B1VJ58B & \multicolumn{3}{|c|}{ Lab ID: $\quad$ 0805020-11 } & & & \\
\hline 16984-48-8 & Fluoride & $2.00 \mathrm{E} 1$ & ug/g dry & $2.01 \mathrm{E} 0$ & 6/30/08 & $8 F 26011$ & AGG-IC-001 \\
\hline $16887-00-6$ & Chloride & $<5.03 \mathrm{E} 0$ & ug/g dry & 5.03E0 & 6/30/08 & $8 F 26011$ & AGG-IC-001 \\
\hline $14797-65-0$ & Nitrite & $<1.01 \mathrm{E} 1$ & ug/g dry & $1.01 \mathrm{E} 1$ & 6/30/08 & $8 F 26011$ & AGG-IC-001 \\
\hline 24959-67-9 & Bromide & $<1.01 \mathrm{E} 1$ & ug/g dry & $1.01 \mathrm{E} 1$ & 6/30/08 & $8 F 26011$ & AGG-IC-001 \\
\hline $14797-55-8$ & Nitrate & $1.99 \mathrm{E} 2$ & ug/g dry & $1.01 \mathrm{E} 1$ & 6/30/08 & $8 F 26011$ & AGG-IC-001 \\
\hline $14808-79-8$ & Sulfate & $1.83 \mathrm{E} 1$ & ug/g dry & $1.51 \mathrm{E} 1$ & 6/30/08 & $8 \mathrm{~F} 26011$ & AGG-IC-001 \\
\hline $14265-44-2$ & Phosphate & $<1.51 \mathrm{E} 1$ & ug/g dry & $1.51 \mathrm{E} 1$ & 6/30/08 & 8F26011 & AGG-IC-001 \\
\hline HEIS No. & B1VJ58A & \multicolumn{3}{|c|}{ Lab ID: $\quad 0805020-12$} & & & \\
\hline $16984-48-8$ & Fluoride & $1.78 \mathrm{E} 1$ & ug/g dry & $2.00 \mathrm{E} 0$ & 6/30/08 & $8 F 26011$ & AGG-IC-001 \\
\hline $16887-00-6$ & Chloride & $<5.00 \mathrm{E} 0$ & ug/g dry & $5.00 \mathrm{E} 0$ & 6/30/08 & $8 F 26011$ & AGG-IC-001 \\
\hline $14797-65-0$ & Nitrite & $<9.99 \mathrm{E} 0$ & ug/g dry & 9.99E0 & 6/30/08 & $8 F 26011$ & AGG-IC-001 \\
\hline 24959-67-9 & Bromide & $<9.99 \mathrm{E} 0$ & ug/g dry & $9.99 \mathrm{E} 0$ & 6/30/08 & 8F26011 & AGG-IC-001 \\
\hline 14797-55-8 & Nitrate & $1.85 \mathrm{E} 2$ & ug/g dry & 9.99E0 & 6/30/08 & $8 F 26011$ & AGG-IC-001 \\
\hline $14808-79-8$ & Sulfate & $1.78 \mathrm{E} 1$ & ug/g dry & $1.50 \mathrm{E} 1$ & 6/30/08 & $8 F 26011$ & AGG-IC-001 \\
\hline $14265-44-2$ & Phosphate & $1.60 \mathrm{E} 1$ & ug/g dry & $1.50 \mathrm{E} 1$ & 6/30/08 & $8 F 26011$ & AGG-IC-001 \\
\hline HEIS No. & B1VJ59C & \multicolumn{3}{|c|}{ Lab ID: $\quad$ 0805020-14 } & & & \\
\hline $16984-48-8$ & Fluoride & $7.52 \mathrm{E}-1$ & ug/g dry & $2.00 \mathrm{E}-1$ & $6 / 26 / 08$ & $8 F 26011$ & AGG-IC-001 \\
\hline $16887-00-6$ & Chloride & 2.77E0 & ug/g dry & $5.00 \mathrm{E}-1$ & 6/26/08 & 8F26011 & AGG-IC-001 \\
\hline $14797-65-0$ & Nitrite & $<1.00 \mathrm{E} 0$ & ug/g dry & $1.00 \mathrm{E} 0$ & $6 / 26 / 08$ & $8 F 26011$ & AGG-IC-001 \\
\hline 24959-67-9 & Bromide & $<1.00 \mathrm{E} 0$ & ug/g dry & $1.00 \mathrm{E} 0$ & 6/26/08 & $8 F 26011$ & AGG-IC-001 \\
\hline $14797-55-8$ & Nitrate & $<1.00 \mathrm{E} 0$ & ug/g dry & $1.00 \mathrm{E} 0$ & 6/26/08 & $8 F 26011$ & AGG-IC-001 \\
\hline $14808-79-8$ & Sulfate & 2.85E1 & ug/g dry & $1.50 \mathrm{E} 0$ & $6 / 26 / 08$ & $8 F 26011$ & AGG-IC-001 \\
\hline $14265-44-2$ & Phosphate & $<1.50 \mathrm{E} 0$ & ug/g dry & $1.50 \mathrm{E} 0$ & 6/26/08 & $8 F 26011$ & AGG-IC-001 \\
\hline HEIS No. & B1VJ59B & \multicolumn{3}{|c|}{ Lab ID: $\quad$ 0805020-15 } & & & \\
\hline $16984-48-8$ & Fluoride & 4.53E-1 & ug/g dry & $2.00 \mathrm{E}-1$ & 6/26/08 & $8 F 26011$ & AGG-IC-001 \\
\hline $16887-00-6$ & Chloride & 1.23E0 & ug/g dry & $5.00 \mathrm{E}-1$ & 6/26/08 & $8 F 26011$ & AGG-IC-001 \\
\hline $14797-65-0$ & Nitrite & $<1.00 \mathrm{E} 0$ & ug/g dry & $1.00 \mathrm{E} 0$ & 6/26/08 & $8 F 26011$ & AGG-IC-001 \\
\hline 24959-67-9 & Bromide & $<1.00 \mathrm{E} 0$ & ug/g dry & $1.00 \mathrm{E} 0$ & 6/26/08 & $8 F 26011$ & AGG-IC-001 \\
\hline $14797-55-8$ & Nitrate & 2.93E0 & ug/g dry & $1.00 \mathrm{E} 0$ & 6/26/08 & $8 F 26011$ & AGG-IC-001 \\
\hline $14808-79-8$ & Sulfate & $1.78 \mathrm{E} 1$ & ug/g dry & $1.50 \mathrm{E} 0$ & 6/26/08 & $8 F 26011$ & AGG-IC-001 \\
\hline 14265-44-2 & Phosphate & $<1.50 \mathrm{E} 0$ & ug/g dry & $1.50 \mathrm{E} 0$ & 6/26/08 & $8 F 26011$ & AGG-IC-001 \\
\hline HEIS No. & B1VJ59A & \multicolumn{3}{|c|}{ Lab ID: $\quad$ 0805020-16 } & & & \\
\hline $16984-48-8$ & Fluoride & $4.18 \mathrm{E}-1$ & ug/g dry & $2.02 \mathrm{E}-1$ & 6/26/08 & $8 F 26011$ & AGG-IC-001 \\
\hline $16887-00-6$ & Chloride & $9.93 \mathrm{E}-1$ & ug/g dry & $5.04 \mathrm{E}-1$ & 6/26/08 & $8 F 26011$ & AGG-IC-001 \\
\hline $14797-65-0$ & Nitrite & $<1.01 \mathrm{E} 0$ & ug/g dry & $1.01 \mathrm{E} 0$ & 6/26/08 & $8 F 26011$ & AGG-IC-001 \\
\hline 24959-67-9 & Bromide & $<1.01 \mathrm{E} 0$ & ug/g dry & $1.01 \mathrm{E} 0$ & 6/26/08 & $8 F 26011$ & AGG-IC-001 \\
\hline $14797-55-8$ & Nitrate & $2.41 \mathrm{E} 0$ & ug/g dry & $1.01 \mathrm{E} 0$ & 6/26/08 & $8 F 26011$ & AGG-IC-001 \\
\hline 14808-79-8 & Sulfate & $1.50 \mathrm{E} 1$ & ug/g dry & $1.51 \mathrm{E} 0$ & 6/26/08 & $8 F 26011$ & AGG-IC-001 \\
\hline $14265-44-2$ & Phosphate & $<1.51 \mathrm{E} 0$ & ug/g dry & $1.51 \mathrm{E} 0$ & 6/26/08 & $8 F 26011$ & AGG-IC-001 \\
\hline HEIS No. & B1VJ60C & \multicolumn{3}{|c|}{ Lab ID: $\quad 0805020-18$} & & & \\
\hline $16984-48-8$ & Fluoride & $1.42 \mathrm{E} 0$ & ug/g dry & $2.00 \mathrm{E}-1$ & 6/26/08 & $8 F 26011$ & AGG-IC-001 \\
\hline $16887-00-6$ & Chloride & $3.04 \mathrm{E} 0$ & ug/g dry & $5.00 \mathrm{E}-1$ & 6/26/08 & $8 F 26011$ & AGG-IC-001 \\
\hline $14797-65-0$ & Nitrite & $<1.00 \mathrm{E} 0$ & ug/g dry & $1.00 \mathrm{E} 0$ & 6/26/08 & $8 F 26011$ & AGG-IC-001 \\
\hline 24959-67-9 & Bromide & $<1.00 \mathrm{E} 0$ & ug/g dry & $1.00 \mathrm{E} 0$ & 6/26/08 & $8 F 26011$ & AGG-IC-001 \\
\hline $14797-55-8$ & Nitrate & $1.54 \mathrm{E} 0$ & ug/g dry & $1.00 \mathrm{E} 0$ & 6/26/08 & $8 F 26011$ & AGG-IC-001 \\
\hline $14808-79-8$ & Sulfate & 7.30E1 & ug/g dry & $1.50 \mathrm{E} 0$ & 6/26/08 & $8 F 26011$ & AGG-IC-001 \\
\hline $14265-44-2$ & Phosphate & $<1.50 \mathrm{E} 0$ & ug/g dry & $1.50 \mathrm{E} 0$ & 6/26/08 & $8 F 26011$ & AGG-IC-001 \\
\hline HEIS No. & B1VJ60B & $\mathbf{L a}$ & b ID: & $5020-19$ & & & \\
\hline
\end{tabular}




\section{Anions by Ion Chromatography}

\begin{tabular}{|c|c|c|c|c|c|c|c|}
\hline CAS \# & Analyte & Results & Units & EQL & Analyzed & Batch & Method \\
\hline HEIS No. & B1VJ60B & \multicolumn{3}{|c|}{ Lab ID: $\quad$ 0805020-19 } & & & \\
\hline 16984-48-8 & Fluoride & $5.35 \mathrm{E}-1$ & ug/g dry & $2.00 \mathrm{E}-1$ & 6/27/08 & $8 F 26011$ & AGG-IC-001 \\
\hline $16887-00-6$ & Chloride & 1.63E0 & ug/g dry & $5.00 \mathrm{E}-1$ & $6 / 27 / 08$ & $8 F 26011$ & AGG-IC-001 \\
\hline $14797-65-0$ & Nitrite & $<1.00 \mathrm{E} 0$ & ug/g dry & $1.00 \mathrm{E} 0$ & $6 / 27 / 08$ & $8 F 26011$ & AGG-IC-001 \\
\hline 24959-67-9 & Bromide & $<1.00 \mathrm{E} 0$ & ug/g dry & $1.00 \mathrm{E} 0$ & $6 / 27 / 08$ & $8 F 26011$ & AGG-IC-001 \\
\hline $14797-55-8$ & Nitrate & 7.33E0 & ug/g dry & $1.00 \mathrm{E} 0$ & 6/27/08 & $8 F 26011$ & AGG-IC-001 \\
\hline $14808-79-8$ & Sulfate & $1.02 \mathrm{E} 2$ & ug/g dry & $1.50 \mathrm{E} 0$ & $6 / 27 / 08$ & $8 \mathrm{~F} 26011$ & AGG-IC-001 \\
\hline $14265-44-2$ & Phosphate & $<1.50 \mathrm{E} 0$ & ug/g dry & $1.50 \mathrm{E} 0$ & $6 / 27 / 08$ & 8F26011 & AGG-IC-001 \\
\hline HEIS No. & B1VJ60A & \multicolumn{3}{|c|}{$\begin{array}{ll}\text { Lab ID: } & 0805020-20\end{array}$} & & & \\
\hline $16984-48-8$ & Fluoride & 5.23E-1 & ug/g dry & $2.00 \mathrm{E}-1$ & 6/27/08 & $8 F 26011$ & AGG-IC-001 \\
\hline $16887-00-6$ & Chloride & $1.10 \mathrm{E} 0$ & ug/g dry & $5.00 \mathrm{E}-1$ & $6 / 27 / 08$ & $8 F 26011$ & AGG-IC-001 \\
\hline $14797-65-0$ & Nitrite & $<1.00 \mathrm{E} 0$ & ug/g dry & $1.00 \mathrm{E} 0$ & $6 / 27 / 08$ & $8 F 26011$ & AGG-IC-001 \\
\hline 24959-67-9 & Bromide & $<1.00 \mathrm{E} 0$ & ug/g dry & $1.00 \mathrm{E} 0$ & 6/27/08 & 8F26011 & AGG-IC-001 \\
\hline 14797-55-8 & Nitrate & 4.24E0 & ug/g dry & $1.00 \mathrm{E} 0$ & 6/27/08 & $8 F 26011$ & AGG-IC-001 \\
\hline $14808-79-8$ & Sulfate & 5.78E1 & ug/g dry & $1.50 \mathrm{E} 0$ & 6/27/08 & $8 F 26011$ & AGG-IC-001 \\
\hline 14265-44-2 & Phosphate & $<1.50 \mathrm{E} 0$ & ug/g dry & $1.50 \mathrm{E} 0$ & $6 / 27 / 08$ & $8 F 26011$ & AGG-IC-001 \\
\hline HEIS No. & B1VJ61C & \multicolumn{3}{|c|}{ Lab ID: $\quad$ 0805020-22 } & & & \\
\hline $16984-48-8$ & Fluoride & 8.75E-1 & ug/g dry & $2.00 \mathrm{E}-1$ & $6 / 27 / 08$ & $8 F 26011$ & AGG-IC-001 \\
\hline $16887-00-6$ & Chloride & 4.53E0 & ug/g dry & $5.00 \mathrm{E}-1$ & 6/27/08 & $8 F 26011$ & AGG-IC-001 \\
\hline $14797-65-0$ & Nitrite & $<1.00 \mathrm{E} 0$ & ug/g dry & $1.00 \mathrm{E} 0$ & $6 / 27 / 08$ & $8 F 26011$ & AGG-IC-001 \\
\hline 24959-67-9 & Bromide & $<1.00 \mathrm{E} 0$ & ug/g dry & $1.00 \mathrm{E} 0$ & $6 / 27 / 08$ & $8 F 26011$ & AGG-IC-001 \\
\hline $14797-55-8$ & Nitrate & $8.41 \mathrm{E} 0$ & ug/g dry & $1.00 \mathrm{E} 0$ & 6/27/08 & $8 F 26011$ & AGG-IC-001 \\
\hline $14808-79-8$ & Sulfate & $6.21 \mathrm{E} 1$ & ug/g dry & $1.50 \mathrm{E} 0$ & $6 / 27 / 08$ & $8 F 26011$ & AGG-IC-001 \\
\hline $14265-44-2$ & Phosphate & $<1.50 \mathrm{E} 0$ & ug/g dry & $1.50 \mathrm{E} 0$ & $6 / 27 / 08$ & $8 F 26011$ & AGG-IC-001 \\
\hline HEIS No. & B1VJ61B & \multicolumn{3}{|c|}{ Lab ID: $\quad$ 0805020-23 } & & & \\
\hline $16984-48-8$ & Fluoride & $5.01 \mathrm{E}-1$ & ug/g dry & $2.02 \mathrm{E}-1$ & $6 / 27 / 08$ & $8 F 26011$ & AGG-IC-001 \\
\hline $16887-00-6$ & Chloride & $3.52 \mathrm{E} 0$ & ug/g dry & 5.05E-1 & 6/27/08 & $8 F 26011$ & AGG-IC-001 \\
\hline $14797-65-0$ & Nitrite & $<1.01 \mathrm{E} 0$ & ug/g dry & $1.01 \mathrm{E} 0$ & 6/27/08 & $8 F 26011$ & AGG-IC-001 \\
\hline 24959-67-9 & Bromide & $<1.01 \mathrm{E} 0$ & ug/g dry & $1.01 \mathrm{E} 0$ & 6/27/08 & $8 F 26011$ & AGG-IC-001 \\
\hline $14797-55-8$ & Nitrate & 7.04E0 & ug/g dry & $1.01 \mathrm{E} 0$ & 6/27/08 & $8 F 26011$ & AGG-IC-001 \\
\hline $14808-79-8$ & Sulfate & 5.53E1 & ug/g dry & $1.52 \mathrm{E} 0$ & 6/27/08 & $8 F 26011$ & AGG-IC-001 \\
\hline 14265-44-2 & Phosphate & $<1.52 \mathrm{E} 0$ & ug/g dry & $1.52 \mathrm{E} 0$ & $6 / 27 / 08$ & $8 F 26011$ & AGG-IC-001 \\
\hline HEIS No. & B1VJ61A & \multicolumn{3}{|c|}{ Lab ID: $\quad$ 0805020-24 } & & & \\
\hline $16984-48-8$ & Fluoride & $4.30 \mathrm{E}-1$ & ug/g dry & $2.08 \mathrm{E}-1$ & $6 / 27 / 08$ & $8 F 26011$ & AGG-IC-001 \\
\hline $16887-00-6$ & Chloride & $3.61 \mathrm{E} 0$ & ug/g dry & $5.19 \mathrm{E}-1$ & 6/27/08 & $8 F 26011$ & AGG-IC-001 \\
\hline $14797-65-0$ & Nitrite & $<1.04 \mathrm{E} 0$ & ug/g dry & $1.04 \mathrm{E} 0$ & 6/27/08 & $8 F 26011$ & AGG-IC-001 \\
\hline 24959-67-9 & Bromide & $<1.04 \mathrm{E} 0$ & ug/g dry & $1.04 \mathrm{E} 0$ & $6 / 27 / 08$ & $8 F 26011$ & AGG-IC-001 \\
\hline $14797-55-8$ & Nitrate & $7.00 \mathrm{E} 0$ & ug/g dry & $1.04 \mathrm{E} 0$ & 6/27/08 & $8 F 26011$ & AGG-IC-001 \\
\hline 14808-79-8 & Sulfate & $5.84 \mathrm{E} 1$ & ug/g dry & $1.56 \mathrm{E} 0$ & 6/27/08 & $8 F 26011$ & AGG-IC-001 \\
\hline $14265-44-2$ & Phosphate & $<1.56 \mathrm{E} 0$ & ug/g dry & $1.56 \mathrm{E} 0$ & $6 / 27 / 08$ & $8 F 26011$ & AGG-IC-001 \\
\hline HEIS No. & B1VJ62C & \multicolumn{3}{|c|}{ Lab ID: $\quad 0805020-26$} & & & \\
\hline $16984-48-8$ & Fluoride & $<2.00 \mathrm{E} 0$ & ug/g dry & $2.00 \mathrm{E} 0$ & $6 / 27 / 08$ & $8 F 26011$ & AGG-IC-001 \\
\hline $16887-00-6$ & Chloride & $7.21 \mathrm{E} 1$ & ug/g dry & $5.00 \mathrm{E} 0$ & $6 / 27 / 08$ & $8 F 26011$ & AGG-IC-001 \\
\hline $14797-65-0$ & Nitrite & $<1.00 \mathrm{E} 1$ & ug/g dry & $1.00 \mathrm{E} 1$ & 6/27/08 & $8 F 26011$ & AGG-IC-001 \\
\hline 24959-67-9 & Bromide & $<1.00 \mathrm{E} 1$ & ug/g dry & $1.00 \mathrm{E} 1$ & $6 / 27 / 08$ & $8 F 26011$ & AGG-IC-001 \\
\hline $14797-55-8$ & Nitrate & $<1.00 \mathrm{E} 1$ & ug/g dry & $1.00 \mathrm{E} 1$ & 6/27/08 & $8 F 26011$ & AGG-IC-001 \\
\hline $14808-79-8$ & Sulfate & $1.59 \mathrm{E} 2$ & ug/g dry & $1.50 \mathrm{E} 1$ & 6/27/08 & $8 F 26011$ & AGG-IC-001 \\
\hline $14265-44-2$ & Phosphate & $<1.50 \mathrm{E} 1$ & ug/g dry & $1.50 \mathrm{E} 1$ & 6/27/08 & $8 F 26011$ & AGG-IC-001 \\
\hline HEIS No. & B1VJ62B & \multicolumn{2}{|c|}{ Lab ID: } & $5020-27$ & & & \\
\hline
\end{tabular}




\section{Anions by Ion Chromatography}

\begin{tabular}{|c|c|c|c|c|c|c|c|}
\hline CAS \# & Analyte & Results & Units & EQL & Analyzed & Batch & Method \\
\hline HEIS No. & B1VJ62B & \multicolumn{3}{|c|}{ Lab ID: $\quad 0805020-27$} & & & \\
\hline 16984-48-8 & Fluoride & $<2.00 \mathrm{E} 0$ & ug/g dry & $2.00 \mathrm{E} 0$ & 6/27/08 & 8F26016 & AGG-IC-001 \\
\hline $16887-00-6$ & Chloride & 4.10E1 & ug/g dry & $5.00 \mathrm{E} 0$ & $6 / 27 / 08$ & $8 F 26016$ & AGG-IC-001 \\
\hline $14797-65-0$ & Nitrite & $<1.00 \mathrm{E} 1$ & ug/g dry & $1.00 \mathrm{E} 1$ & 6/27/08 & $8 \mathrm{~F} 26016$ & AGG-IC-001 \\
\hline 24959-67-9 & Bromide & $<1.00 \mathrm{E} 1$ & ug/g dry & $1.00 \mathrm{E} 1$ & 6/27/08 & $8 F 26016$ & AGG-IC-001 \\
\hline 14797-55-8 & Nitrate & $1.02 \mathrm{E} 1$ & ug/g dry & $1.00 \mathrm{E} 1$ & $6 / 27 / 08$ & 8F26016 & AGG-IC-001 \\
\hline 14808-79-8 & Sulfate & 1.15E2 & ug/g dry & $1.50 \mathrm{E} 1$ & 6/27/08 & $8 \mathrm{~F} 26016$ & AGG-IC-001 \\
\hline $14265-44-2$ & Phosphate & $<1.50 \mathrm{E} 1$ & ug/g dry & $1.50 \mathrm{E} 1$ & $6 / 27 / 08$ & 8F26016 & AGG-IC-001 \\
\hline HEIS No. & B1VJ62A & \multicolumn{3}{|c|}{ Lab ID: $\quad$ 0805020-28 } & & & \\
\hline $16984-48-8$ & Fluoride & 4.73E-1 & ug/g dry & $2.00 \mathrm{E}-1$ & 6/27/08 & 8F26016 & AGG-IC-001 \\
\hline $16887-00-6$ & Chloride & $2.21 \mathrm{E} 1$ & ug/g dry & $5.01 \mathrm{E}-1$ & $6 / 27 / 08$ & $8 \mathrm{~F} 26016$ & AGG-IC-001 \\
\hline $14797-65-0$ & Nitrite & $<1.00 \mathrm{E} 0$ & ug/g dry & $1.00 \mathrm{E} 0$ & $6 / 27 / 08$ & 8F26016 & AGG-IC-001 \\
\hline 24959-67-9 & Bromide & $<1.00 \mathrm{E} 0$ & ug/g dry & $1.00 \mathrm{E} 0$ & $6 / 27 / 08$ & $8 F 26016$ & AGG-IC-001 \\
\hline $14797-55-8$ & Nitrate & $1.14 \mathrm{E} 1$ & ug/g dry & $1.00 \mathrm{E} 0$ & 6/27/08 & $8 F 26016$ & AGG-IC-001 \\
\hline 14808-79-8 & Sulfate & 1.49E2 & ug/g dry & $1.50 \mathrm{E} 0$ & $6 / 27 / 08$ & 8F26016 & AGG-IC-001 \\
\hline $14265-44-2$ & Phosphate & $<1.50 \mathrm{E} 0$ & ug/g dry & $1.50 \mathrm{E} 0$ & $6 / 27 / 08$ & $8 \mathrm{~F} 26016$ & AGG-IC-001 \\
\hline HEIS No. & B1VJ64C & \multicolumn{3}{|c|}{ Lab ID: $\quad$ 0805020-30 } & & & \\
\hline $16984-48-8$ & Fluoride & $9.97 \mathrm{E}-1$ & ug/g dry & $2.00 \mathrm{E}-1$ & 6/27/08 & $8 F 26016$ & AGG-IC-001 \\
\hline 16887-00-6 & Chloride & $4.94 \mathrm{E} 0$ & ug/g dry & $5.00 \mathrm{E}-1$ & $6 / 27 / 08$ & $8 F 26016$ & AGG-IC-001 \\
\hline $14797-65-0$ & Nitrite & $<1.00 \mathrm{E} 0$ & ug/g dry & $1.00 \mathrm{E} 0$ & $6 / 27 / 08$ & $8 \mathrm{~F} 26016$ & AGG-IC-001 \\
\hline 24959-67-9 & Bromide & $<1.00 \mathrm{E} 0$ & ug/g dry & $1.00 \mathrm{E} 0$ & $6 / 27 / 08$ & $8 \mathrm{~F} 26016$ & AGG-IC-001 \\
\hline 14797-55-8 & Nitrate & 9.48E0 & ug/g dry & $1.00 \mathrm{E} 0$ & 6/27/08 & $8 F 26016$ & AGG-IC-001 \\
\hline $14808-79-8$ & Sulfate & $1.32 \mathrm{E} 2$ & ug/g dry & $1.50 \mathrm{E} 0$ & $6 / 27 / 08$ & $8 \mathrm{~F} 26016$ & AGG-IC-001 \\
\hline $14265-44-2$ & Phosphate & $<1.50 \mathrm{E} 0$ & ug/g dry & $1.50 \mathrm{E} 0$ & $6 / 27 / 08$ & $8 \mathrm{~F} 26016$ & AGG-IC-001 \\
\hline HEIS No. & B1VJ64B & \multicolumn{3}{|c|}{ Lab ID: $\quad$ 0805020-31 } & & & \\
\hline $16984-48-8$ & Fluoride & $5.97 \mathrm{E}-1$ & ug/g dry & $2.00 \mathrm{E}-1$ & $6 / 27 / 08$ & $8 F 26016$ & AGG-IC-001 \\
\hline $16887-00-6$ & Chloride & $2.58 \mathrm{E} 0$ & ug/g dry & $5.00 \mathrm{E}-1$ & 6/27/08 & $8 F 26016$ & AGG-IC-001 \\
\hline 14797-65-0 & Nitrite & $<1.00 \mathrm{E} 0$ & ug/g dry & $1.00 \mathrm{E} 0$ & $6 / 27 / 08$ & $8 F 26016$ & AGG-IC-001 \\
\hline 24959-67-9 & Bromide & $<1.00 \mathrm{E} 0$ & ug/g dry & $1.00 \mathrm{E} 0$ & $6 / 27 / 08$ & $8 F 26016$ & AGG-IC-001 \\
\hline $14797-55-8$ & Nitrate & 7.12E0 & ug/g dry & $1.00 \mathrm{E} 0$ & $6 / 27 / 08$ & $8 F 26016$ & AGG-IC-001 \\
\hline 14808-79-8 & Sulfate & 8.07E1 & ug/g dry & $1.50 \mathrm{E} 0$ & 6/27/08 & 8F26016 & AGG-IC-001 \\
\hline $14265-44-2$ & Phosphate & $<1.50 \mathrm{E} 0$ & ug/g dry & $1.50 \mathrm{E} 0$ & $6 / 27 / 08$ & $8 \mathrm{~F} 26016$ & AGG-IC-001 \\
\hline HEIS No. & B1VJ64A & \multicolumn{3}{|c|}{ Lab ID: $\quad$ 0805020-32 } & & & \\
\hline $16984-48-8$ & Fluoride & $5.61 \mathrm{E}-1$ & ug/g dry & $1.90 \mathrm{E}-1$ & $6 / 27 / 08$ & $8 F 26016$ & AGG-IC-001 \\
\hline 16887-00-6 & Chloride & $2.28 \mathrm{E} 0$ & ug/g dry & $4.74 \mathrm{E}-1$ & 6/27/08 & $8 F 26016$ & AGG-IC-001 \\
\hline $14797-65-0$ & Nitrite & $<9.49 \mathrm{E}-1$ & ug/g dry & $9.49 \mathrm{E}-1$ & 6/27/08 & $8 F 26016$ & AGG-IC-001 \\
\hline 24959-67-9 & Bromide & $<9.49 \mathrm{E}-1$ & ug/g dry & $9.49 \mathrm{E}-1$ & $6 / 27 / 08$ & 8F26016 & AGG-IC-001 \\
\hline $14797-55-8$ & Nitrate & $1.08 \mathrm{E} 1$ & ug/g dry & $9.49 \mathrm{E}-1$ & $6 / 27 / 08$ & $8 F 26016$ & AGG-IC-001 \\
\hline $14808-79-8$ & Sulfate & $6.89 \mathrm{E} 1$ & ug/g dry & $1.42 \mathrm{E} 0$ & 6/27/08 & $8 F 26016$ & AGG-IC-001 \\
\hline 14265-44-2 & Phosphate & $<1.42 \mathrm{E} 0$ & ug/g dry & 1.42E0 & $6 / 27 / 08$ & $8 F 26016$ & AGG-IC-001 \\
\hline
\end{tabular}


Total Metals by PNNL-AGG-ICP-AES/Water Extract

\begin{tabular}{|c|c|c|c|c|c|c|c|}
\hline CAS \# & Analyte & Results & Units & EQL & Analyzed & Batch & Method \\
\hline HEIS No. & B1VJ54B & \multicolumn{3}{|c|}{ Lab ID: $\quad 0805020-01$} & & & \\
\hline 7429-90-5 & Aluminum & 8.61E-1 & ug/g dry & $8.71 \mathrm{E}-2$ & 6/30/08 & 8F30003 & PNNL-AGG-ICP-AES \\
\hline $7440-38-2$ & Arsenic & $<3.66 \mathrm{E}-1$ & ug/g dry & $3.66 \mathrm{E}-1$ & 6/30/08 & $8 F 30003$ & PNNL-AGG-ICP-AES \\
\hline $7440-42-8$ & Boron & $<1.97 \mathrm{E} 0$ & ug/g dry & 1.97E0 & 6/30/08 & 8F30003 & PNNL-AGG-ICP-AES \\
\hline $7440-39-3$ & Barium & $1.82 \mathrm{E}-2$ & ug/g dry & 8.93E-3 & 6/30/08 & 8F30003 & PNNL-AGG-ICP-AES \\
\hline $7440-41-7$ & Beryllium & $<2.89 \mathrm{E}-2$ & ug/g dry & $2.89 \mathrm{E}-2$ & 6/30/08 & $8 F 30003$ & PNNL-AGG-ICP-AES \\
\hline $7440-69-9$ & Bismuth & $<1.91 \mathrm{E}-1$ & ug/g dry & $1.91 \mathrm{E}-1$ & 6/30/08 & $8 F 30003$ & PNNL-AGG-ICP-AES \\
\hline $7440-70-2$ & Calcium & 6.80E0 & ug/g dry & 3.93E-1 & 6/30/08 & 8F30003 & PNNL-AGG-ICP-AES \\
\hline $7440-43-9$ & Cadmium & $<2.73 E-2$ & ug/g dry & 2.73E-2 & 6/30/08 & 8F30003 & PNNL-AGG-ICP-AES \\
\hline $7440-48-4$ & Cobalt & $<9.75 \mathrm{E}-2$ & ug/g dry & $9.75 \mathrm{E}-2$ & 6/30/08 & 8F30003 & PNNL-AGG-ICP-AES \\
\hline $7440-47-3$ & Chromium & $<3.38 \mathrm{E}-2$ & ug/g dry & $3.38 \mathrm{E}-2$ & 6/30/08 & 8F30003 & PNNL-AGG-ICP-AES \\
\hline $7440-50-8$ & Copper & $<8.17 \mathrm{E}-2$ & ug/g dry & 8.17E-2 & 6/30/08 & $8 F 30003$ & PNNL-AGG-ICP-AES \\
\hline 7439-89-6 & Iron & 1.17E0 & ug/g dry & $1.44 \mathrm{E}-1$ & 6/30/08 & 8F30003 & PNNL-AGG-ICP-AES \\
\hline 7440-09-7 & Potassium & 3.95E0 & ug/g dry & 2.36E0 & 6/30/08 & 8F30003 & PNNL-AGG-ICP-AES \\
\hline 7439-93-2 & Lithium & $<5.48 \mathrm{E}-1$ & ug/g dry & $5.48 \mathrm{E}-1$ & 6/30/08 & $8 F 30003$ & PNNL-AGG-ICP-AES \\
\hline $7439-95-4$ & Magnesium & 1.18E0 & ug/g dry & $8.47 \mathrm{E}-2$ & 6/30/08 & 8F30003 & PNNL-AGG-ICP-AES \\
\hline 7439-96-5 & Manganese & $<1.74 \mathrm{E}-2$ & ug/g dry & $1.74 \mathrm{E}-2$ & 6/30/08 & 8F30003 & PNNL-AGG-ICP-AES \\
\hline 7439-98-7 & Molybdenum & $<1.41 \mathrm{E}-1$ & ug/g dry & $1.41 \mathrm{E}-1$ & 6/30/08 & $8 F 30003$ & PNNL-AGG-ICP-AES \\
\hline $7440-02-0$ & Nickel & $<9.48 \mathrm{E}-2$ & ug/g dry & $9.48 \mathrm{E}-2$ & 6/30/08 & $8 F 30003$ & PNNL-AGG-ICP-AES \\
\hline $7723-14-0$ & Phosphorus & $<1.05 \mathrm{E} 0$ & ug/g dry & $1.05 \mathrm{E} 0$ & 6/30/08 & $8 F 30003$ & PNNL-AGG-ICP-AES \\
\hline $7439-92-1$ & Lead & $<4.39 \mathrm{E}-1$ & ug/g dry & $4.39 \mathrm{E}-1$ & 6/30/08 & 8F30003 & PNNL-AGG-ICP-AES \\
\hline $7782-49-2$ & Selenium & $<1.98 \mathrm{E} 0$ & ug/g dry & $1.98 \mathrm{E} 0$ & 6/30/08 & 8F30003 & PNNL-AGG-ICP-AES \\
\hline $7440-24-6$ & Strontium & $<5.30 \mathrm{E}-2$ & ug/g dry & $5.30 \mathrm{E}-2$ & 6/30/08 & $8 F 30003$ & PNNL-AGG-ICP-AES \\
\hline $7440-28-0$ & Thallium & $<1.04 \mathrm{E} 0$ & ug/g dry & $1.04 \mathrm{E} 0$ & 6/30/08 & 8F30003 & PNNL-AGG-ICP-AES \\
\hline $7440-62-2$ & Vanadium & $9.82 \mathrm{E}-2$ & ug/g dry & $4.60 \mathrm{E}-2$ & 6/30/08 & 8F30003 & PNNL-AGG-ICP-AES \\
\hline $7440-66-6$ & Zinc & $<9.42 \mathrm{E}-2$ & ug/g dry & $9.42 \mathrm{E}-2$ & 6/30/08 & 8F30003 & PNNL-AGG-ICP-AES \\
\hline $7440-23-5$ & Sodium & 3.44E1 & ug/g dry & $6.80 \mathrm{E}-1$ & 6/30/08 & 8F30003 & PNNL-AGG-ICP-AES \\
\hline $7440-21-3$ & Silicon & $1.72 \mathrm{E} 1$ & ug/g dry & $1.52 \mathrm{E} 0$ & 6/30/08 & 8F30003 & PNNL-AGG-ICP-AES \\
\hline 7704-34-9 & Sulfur & $<3.12 \mathrm{E} 0$ & ug/g dry & $3.12 \mathrm{E} 0$ & 6/30/08 & 8F30003 & PNNL-AGG-ICP-AES \\
\hline $7440-32-6$ & Titanium & 5.32E-2 & ug/g dry & $8.90 \mathrm{E}-3$ & 6/30/08 & 8F30003 & PNNL-AGG-ICP-AES \\
\hline $7440-67-7$ & Zirconium & $<1.52 \mathrm{E}-1$ & ug/g dry & $1.52 \mathrm{E}-1$ & 6/30/08 & $8 F 30003$ & PNNL-AGG-ICP-AES \\
\hline $7440-22-4$ & Silver & $<7.83 \mathrm{E}-2$ & ug/g dry & 7.83E-2 & 6/30/08 & 8F30003 & PNNL-AGG-ICP-AES \\
\hline $7440-15-5$ & Rhenium & $<1.54 \mathrm{E}-1$ & ug/g dry & $1.54 \mathrm{E}-1$ & 6/30/08 & 8F30003 & PNNL-AGG-ICP-AES \\
\hline $7440-36-0$ & Antimony & $<6.43 \mathrm{E}-1$ & ug/g dry & $6.43 \mathrm{E}-1$ & 6/30/08 & $8 F 30003$ & PNNL-AGG-ICP-AES \\
\hline HEIS No. & B1VJ55C & & b ID: & 5020-05 & & & \\
\hline $7429-90-5$ & Aluminum & $7.28 \mathrm{E}-1$ & ug/g dry & $8.58 \mathrm{E}-2$ & 6/30/08 & $8 F 30003$ & PNNL-AGG-ICP-AES \\
\hline $7440-38-2$ & Arsenic & $<3.60 \mathrm{E}-1$ & ug/g dry & $3.60 \mathrm{E}-1$ & 6/30/08 & 8F30003 & PNNL-AGG-ICP-AES \\
\hline $7440-42-8$ & Boron & $<1.94 \mathrm{E} 0$ & ug/g dry & $1.94 \mathrm{E} 0$ & 6/30/08 & $8 F 30003$ & PNNL-AGG-ICP-AES \\
\hline $7440-39-3$ & Barium & 2.11E-2 & ug/g dry & $8.79 \mathrm{E}-3$ & 6/30/08 & 8F30003 & PNNL-AGG-ICP-AES \\
\hline $7440-41-7$ & Beryllium & $<2.84 \mathrm{E}-2$ & ug/g dry & $2.84 \mathrm{E}-2$ & 6/30/08 & 8F30003 & PNNL-AGG-ICP-AES \\
\hline 7440-69-9 & Bismuth & $<1.88 \mathrm{E}-1$ & ug/g dry & $1.88 \mathrm{E}-1$ & 6/30/08 & 8F30003 & PNNL-AGG-ICP-AES \\
\hline $7440-70-2$ & Calcium & 9.13E0 & ug/g dry & $3.87 \mathrm{E}-1$ & 6/30/08 & 8F30003 & PNNL-AGG-ICP-AES \\
\hline $7440-43-9$ & Cadmium & $<2.69 \mathrm{E}-2$ & ug/g dry & 2.69E-2 & 6/30/08 & $8 F 30003$ & PNNL-AGG-ICP-AES \\
\hline $7440-48-4$ & Cobalt & $<9.60 \mathrm{E}-2$ & ug/g dry & $9.60 \mathrm{E}-2$ & 6/30/08 & 8F30003 & PNNL-AGG-ICP-AES \\
\hline $7440-47-3$ & Chromium & $<3.33 \mathrm{E}-2$ & ug/g dry & 3.33E-2 & 6/30/08 & 8F30003 & PNNL-AGG-ICP-AES \\
\hline $7440-50-8$ & Copper & $<8.04 \mathrm{E}-2$ & ug/g dry & 8.04E-2 & 6/30/08 & 8F30003 & PNNL-AGG-ICP-AES \\
\hline 7439-89-6 & Iron & 1.07E0 & ug/g dry & $1.42 \mathrm{E}-1$ & 6/30/08 & 8F30003 & PNNL-AGG-ICP-AES \\
\hline 7440-09-7 & Potassium & 3.40E0 & ug/g dry & 2.33E0 & 6/30/08 & 8F30003 & PNNL-AGG-ICP-AES \\
\hline 7439-93-2 & Lithium & $<5.40 \mathrm{E}-1$ & ug/g dry & $5.40 \mathrm{E}-1$ & 6/30/08 & $8 F 30003$ & PNNL-AGG-ICP-AES \\
\hline
\end{tabular}


Total Metals by PNNL-AGG-ICP-AES/Water Extract

\begin{tabular}{|c|c|c|c|c|c|c|c|}
\hline CAS \# & Analyte & Results & Units & EQL & Analyzed & Batch & Method \\
\hline HEIS No. & B1VJ55C & \multicolumn{3}{|c|}{ Lab ID: $\quad$ 0805020-05 } & & & \\
\hline 7439-95-4 & Magnesium & $1.27 \mathrm{E} 0$ & ug/g dry & 8.34E-2 & 6/30/08 & 8F30003 & PNNL-AGG-ICP-AES \\
\hline 7439-96-5 & Manganese & $2.15 \mathrm{E}-2$ & ug/g dry & $1.71 \mathrm{E}-2$ & 6/30/08 & $8 F 30003$ & PNNL-AGG-ICP-AES \\
\hline 7439-98-7 & Molybdenum & $<1.39 \mathrm{E}-1$ & ug/g dry & $1.39 \mathrm{E}-1$ & 6/30/08 & 8F30003 & PNNL-AGG-ICP-AES \\
\hline $7440-02-0$ & Nickel & $<9.33 \mathrm{E}-2$ & ug/g dry & 9.33E-2 & 6/30/08 & 8F30003 & PNNL-AGG-ICP-AES \\
\hline $7723-14-0$ & Phosphorus & $<1.03 \mathrm{E} 0$ & ug/g dry & 1.03E0 & 6/30/08 & $8 F 30003$ & PNNL-AGG-ICP-AES \\
\hline 7439-92-1 & Lead & $<4.32 \mathrm{E}-1$ & ug/g dry & $4.32 \mathrm{E}-1$ & 6/30/08 & $8 F 30003$ & PNNL-AGG-ICP-AES \\
\hline $7782-49-2$ & Selenium & $<1.95 \mathrm{E} 0$ & ug/g dry & $1.95 \mathrm{E} 0$ & 6/30/08 & 8F30003 & PNNL-AGG-ICP-AES \\
\hline $7440-24-6$ & Strontium & $<5.22 \mathrm{E}-2$ & ug/g dry & $5.22 \mathrm{E}-2$ & 6/30/08 & 8F30003 & PNNL-AGG-ICP-AES \\
\hline $7440-28-0$ & Thallium & $<1.03 \mathrm{E} 0$ & ug/g dry & $1.03 \mathrm{E} 0$ & 6/30/08 & 8F30003 & PNNL-AGG-ICP-AES \\
\hline $7440-62-2$ & Vanadium & $1.44 \mathrm{E}-1$ & ug/g dry & $4.53 \mathrm{E}-2$ & 6/30/08 & 8F30003 & PNNL-AGG-ICP-AES \\
\hline $7440-66-6$ & Zinc & $<9.27 \mathrm{E}-2$ & ug/g dry & $9.27 \mathrm{E}-2$ & 6/30/08 & $8 F 30003$ & PNNL-AGG-ICP-AES \\
\hline $7440-23-5$ & Sodium & $5.10 \mathrm{E} 1$ & ug/g dry & $6.69 \mathrm{E}-1$ & 6/30/08 & 8F30003 & PNNL-AGG-ICP-AES \\
\hline $7440-21-3$ & Silicon & $1.76 \mathrm{E} 1$ & ug/g dry & $1.50 \mathrm{E} 0$ & 6/30/08 & 8F30003 & PNNL-AGG-ICP-AES \\
\hline $7704-34-9$ & Sulfur & $5.54 \mathrm{E} 0$ & ug/g dry & 3.07E0 & 6/30/08 & 8F30003 & PNNL-AGG-ICP-AES \\
\hline $7440-32-6$ & Titanium & 3.97E-2 & ug/g dry & 8.76E-3 & 6/30/08 & $8 F 30003$ & PNNL-AGG-ICP-AES \\
\hline $7440-67-7$ & Zirconium & $<1.50 \mathrm{E}-1$ & ug/g dry & $1.50 \mathrm{E}-1$ & 6/30/08 & 8F30003 & PNNL-AGG-ICP-AES \\
\hline $7440-22-4$ & Silver & $<7.71 \mathrm{E}-2$ & ug/g dry & $7.71 \mathrm{E}-2$ & 6/30/08 & $8 F 30003$ & PNNL-AGG-ICP-AES \\
\hline $7440-15-5$ & Rhenium & $<1.52 \mathrm{E}-1$ & ug/g dry & $1.52 \mathrm{E}-1$ & 6/30/08 & 8F30003 & PNNL-AGG-ICP-AES \\
\hline $7440-36-0$ & Antimony & $<6.33 \mathrm{E}-1$ & ug/g dry & $6.33 \mathrm{E}-1$ & 6/30/08 & $8 F 30003$ & PNNL-AGG-ICP-AES \\
\hline HEIS No. & B1VJ56A & \multicolumn{3}{|c|}{ Lab ID: $\quad$ 0805020-07 } & & & \\
\hline 7429-90-5 & Aluminum & 6.95E0 & ug/g dry & $9.20 \mathrm{E}-2$ & 6/30/08 & 8F30003 & PNNL-AGG-ICP-AES \\
\hline $7440-38-2$ & Arsenic & $<3.86 \mathrm{E}-1$ & ug/g dry & $3.86 \mathrm{E}-1$ & 6/30/08 & $8 F 30003$ & PNNL-AGG-ICP-AES \\
\hline $7440-42-8$ & Boron & $<2.08 \mathrm{E} 0$ & ug/g dry & $2.08 \mathrm{E} 0$ & 6/30/08 & $8 F 30003$ & PNNL-AGG-ICP-AES \\
\hline $7440-39-3$ & Barium & $6.11 \mathrm{E}-2$ & ug/g dry & $9.42 \mathrm{E}-3$ & 6/30/08 & 8F30003 & PNNL-AGG-ICP-AES \\
\hline $7440-41-7$ & Beryllium & $<3.04 \mathrm{E}-2$ & ug/g dry & $3.04 \mathrm{E}-2$ & 6/30/08 & 8F30003 & PNNL-AGG-ICP-AES \\
\hline $7440-69-9$ & Bismuth & $<2.02 \mathrm{E}-1$ & ug/g dry & $2.02 \mathrm{E}-1$ & 6/30/08 & $8 F 30003$ & PNNL-AGG-ICP-AES \\
\hline $7440-70-2$ & Calcium & 2.69E0 & ug/g dry & 4.15E-1 & 6/30/08 & 8F30003 & PNNL-AGG-ICP-AES \\
\hline $7440-43-9$ & Cadmium & $<2.88 \mathrm{E}-2$ & ug/g dry & $2.88 \mathrm{E}-2$ & 6/30/08 & 8F30003 & PNNL-AGG-ICP-AES \\
\hline $7440-48-4$ & Cobalt & $<1.03 \mathrm{E}-1$ & ug/g dry & $1.03 \mathrm{E}-1$ & 6/30/08 & 8F30003 & PNNL-AGG-ICP-AES \\
\hline $7440-47-3$ & Chromium & $<3.57 \mathrm{E}-2$ & ug/g dry & $3.57 \mathrm{E}-2$ & 6/30/08 & 8F30003 & PNNL-AGG-ICP-AES \\
\hline $7440-50-8$ & Copper & $<8.62 \mathrm{E}-2$ & ug/g dry & 8.62E-2 & 6/30/08 & 8F30003 & PNNL-AGG-ICP-AES \\
\hline 7439-89-6 & Iron & $1.05 \mathrm{E} 1$ & ug/g dry & $1.52 \mathrm{E}-1$ & 6/30/08 & 8F30003 & PNNL-AGG-ICP-AES \\
\hline 7440-09-7 & Potassium & 3.62E0 & ug/g dry & $2.50 \mathrm{E} 0$ & 6/30/08 & 8F30003 & PNNL-AGG-ICP-AES \\
\hline 7439-93-2 & Lithium & $<5.79 \mathrm{E}-1$ & ug/g dry & 5.79E-1 & 6/30/08 & $8 F 30003$ & PNNL-AGG-ICP-AES \\
\hline $7439-95-4$ & Magnesium & $1.87 \mathrm{E} 0$ & ug/g dry & 8.94E-2 & 6/30/08 & 8F30003 & PNNL-AGG-ICP-AES \\
\hline 7439-96-5 & Manganese & 1.43E-1 & ug/g dry & $1.84 \mathrm{E}-2$ & 6/30/08 & 8F30003 & PNNL-AGG-ICP-AES \\
\hline 7439-98-7 & Molybdenum & $<1.49 \mathrm{E}-1$ & ug/g dry & $1.49 \mathrm{E}-1$ & 6/30/08 & $8 F 30003$ & PNNL-AGG-ICP-AES \\
\hline $7440-02-0$ & Nickel & $<1.00 \mathrm{E}-1$ & ug/g dry & $1.00 \mathrm{E}-1$ & 6/30/08 & 8F30003 & PNNL-AGG-ICP-AES \\
\hline 7723-14-0 & Phosphorus & 1.21E0 & ug/g dry & $1.11 \mathrm{E} 0$ & 6/30/08 & 8F30003 & PNNL-AGG-ICP-AES \\
\hline 7439-92-1 & Lead & $<4.63 \mathrm{E}-1$ & ug/g dry & 4.63E-1 & 6/30/08 & 8F30003 & PNNL-AGG-ICP-AES \\
\hline $7782-49-2$ & Selenium & $<2.09 \mathrm{E} 0$ & ug/g dry & 2.09E0 & 6/30/08 & 8F30003 & PNNL-AGG-ICP-AES \\
\hline $7440-24-6$ & Strontium & $<5.59 \mathrm{E}-2$ & ug/g dry & $5.59 \mathrm{E}-2$ & 6/30/08 & $8 F 30003$ & PNNL-AGG-ICP-AES \\
\hline $7440-28-0$ & Thallium & $<1.10 \mathrm{E} 0$ & ug/g dry & $1.10 \mathrm{E} 0$ & 6/30/08 & 8F30003 & PNNL-AGG-ICP-AES \\
\hline $7440-62-2$ & Vanadium & 7.53E-1 & ug/g dry & 4.86E-2 & 6/30/08 & 8F30003 & PNNL-AGG-ICP-AES \\
\hline $7440-66-6$ & Zinc & $<9.94 \mathrm{E}-2$ & ug/g dry & $9.94 \mathrm{E}-2$ & 6/30/08 & 8F30003 & PNNL-AGG-ICP-AES \\
\hline $7440-23-5$ & Sodium & 2.08E2 & ug/g dry & 7.17E-1 & 6/30/08 & 8F30003 & PNNL-AGG-ICP-AES \\
\hline $7440-21-3$ & Silicon & $3.94 \mathrm{E} 1$ & ug/g dry & $1.61 \mathrm{E} 0$ & 6/30/08 & 8F30003 & PNNL-AGG-ICP-AES \\
\hline 7704-34-9 & Sulfur & 3.50E0 & ug/g dry & 3.30E0 & 6/30/08 & $8 F 30003$ & PNNL-AGG-ICP-AES \\
\hline
\end{tabular}


Total Metals by PNNL-AGG-ICP-AES/Water Extract

\begin{tabular}{|c|c|c|c|c|c|c|c|}
\hline CAS \# & Analyte & Results & Units & EQL & Analyzed & Batch & Method \\
\hline HEIS No. & B1VJ56A & \multicolumn{3}{|c|}{ Lab ID: $\quad$ 0805020-07 } & & & \\
\hline $7440-32-6$ & Titanium & $4.98 \mathrm{E}-1$ & ug/g dry & $9.39 \mathrm{E}-3$ & 6/30/08 & 8F30003 & PNNL-AGG-ICP-AES \\
\hline $7440-67-7$ & Zirconium & $<1.61 \mathrm{E}-1$ & ug/g dry & $1.61 \mathrm{E}-1$ & 6/30/08 & $8 F 30003$ & PNNL-AGG-ICP-AES \\
\hline $7440-22-4$ & Silver & $<8.26 \mathrm{E}-2$ & ug/g dry & $8.26 \mathrm{E}-2$ & 6/30/08 & 8 F30003 & PNNL-AGG-ICP-AES \\
\hline $7440-15-5$ & Rhenium & $<1.63 \mathrm{E}-1$ & ug/g dry & 1.63E-1 & 6/30/08 & $8 F 30003$ & PNNL-AGG-ICP-AES \\
\hline $7440-36-0$ & Antimony & $<6.78 \mathrm{E}-1$ & ug/g dry & $6.78 \mathrm{E}-1$ & 6/30/08 & $8 F 30003$ & PNNL-AGG-ICP-AES \\
\hline HEIS No. & B1VJ56B & \multicolumn{3}{|c|}{ Lab ID: $\quad$ 0805020-08 } & & & \\
\hline $7429-90-5$ & Aluminum & 2.29E0 & ug/g dry & 8.59E-2 & 6/30/08 & 8F30003 & PNNL-AGG-ICP-AES \\
\hline $7440-38-2$ & Arsenic & $<3.61 \mathrm{E}-1$ & ug/g dry & $3.61 \mathrm{E}-1$ & 6/30/08 & $8 F 30003$ & PNNL-AGG-ICP-AES \\
\hline $7440-42-8$ & Boron & $<1.94 \mathrm{E} 0$ & ug/g dry & $1.94 \mathrm{E} 0$ & 6/30/08 & 8F30003 & PNNL-AGG-ICP-AES \\
\hline $7440-39-3$ & Barium & $3.40 \mathrm{E}-2$ & ug/g dry & $8.80 \mathrm{E}-3$ & 6/30/08 & $8 F 30003$ & PNNL-AGG-ICP-AES \\
\hline $7440-41-7$ & Beryllium & $<2.85 \mathrm{E}-2$ & ug/g dry & $2.85 \mathrm{E}-2$ & 6/30/08 & $8 F 30003$ & PNNL-AGG-ICP-AES \\
\hline 7440-69-9 & Bismuth & $<1.88 \mathrm{E}-1$ & ug/g dry & $1.88 \mathrm{E}-1$ & 6/30/08 & $8 F 30003$ & PNNL-AGG-ICP-AES \\
\hline $7440-70-2$ & Calcium & $1.76 \mathrm{E} 0$ & ug/g dry & $3.88 \mathrm{E}-1$ & 6/30/08 & 8F30003 & PNNL-AGG-ICP-AES \\
\hline $7440-43-9$ & Cadmium & $<2.69 \mathrm{E}-2$ & ug/g dry & $2.69 \mathrm{E}-2$ & 6/30/08 & 8F30003 & PNNL-AGG-ICP-AES \\
\hline $7440-48-4$ & Cobalt & $<9.61 \mathrm{E}-2$ & ug/g dry & $9.61 \mathrm{E}-2$ & 6/30/08 & $8 F 30003$ & PNNL-AGG-ICP-AES \\
\hline $7440-47-3$ & Chromium & $<3.34 \mathrm{E}-2$ & ug/g dry & $3.34 \mathrm{E}-2$ & 6/30/08 & 8F30003 & PNNL-AGG-ICP-AES \\
\hline $7440-50-8$ & Copper & $<8.05 \mathrm{E}-2$ & ug/g dry & 8.05E-2 & 6/30/08 & 8F30003 & PNNL-AGG-ICP-AES \\
\hline 7439-89-6 & Iron & 4.76E0 & ug/g dry & $1.42 \mathrm{E}-1$ & 6/30/08 & $8 F 30003$ & PNNL-AGG-ICP-AES \\
\hline $7440-09-7$ & Potassium & $2.71 \mathrm{E} 0$ & ug/g dry & 2.33Е0 & 6/30/08 & 8F30003 & PNNL-AGG-ICP-AES \\
\hline 7439-93-2 & Lithium & $<5.41 \mathrm{E}-1$ & ug/g dry & $5.41 \mathrm{E}-1$ & 6/30/08 & 8F30003 & PNNL-AGG-ICP-AES \\
\hline $7439-95-4$ & Magnesium & 1.03E0 & ug/g dry & 8.35E-2 & 6/30/08 & 8F30003 & PNNL-AGG-ICP-AES \\
\hline $7439-96-5$ & Manganese & $9.74 \mathrm{E}-2$ & ug/g dry & $1.72 \mathrm{E}-2$ & 6/30/08 & 8F30003 & PNNL-AGG-ICP-AES \\
\hline 7439-98-7 & Molybdenum & $1.71 \mathrm{E}-1$ & ug/g dry & $1.39 \mathrm{E}-1$ & 6/30/08 & $8 F 30003$ & PNNL-AGG-ICP-AES \\
\hline $7440-02-0$ & Nickel & $<9.34 \mathrm{E}-2$ & ug/g dry & $9.34 \mathrm{E}-2$ & 6/30/08 & 8F30003 & PNNL-AGG-ICP-AES \\
\hline 7723-14-0 & Phosphorus & 1.29E0 & ug/g dry & 1.03E0 & 6/30/08 & 8F30003 & PNNL-AGG-ICP-AES \\
\hline 7439-92-1 & Lead & $<4.33 \mathrm{E}-1$ & ug/g dry & 4.33E-1 & 6/30/08 & $8 F 30003$ & PNNL-AGG-ICP-AES \\
\hline $7782-49-2$ & Selenium & $<1.95 \mathrm{E} 0$ & ug/g dry & $1.95 \mathrm{E} 0$ & 6/30/08 & 8F30003 & PNNL-AGG-ICP-AES \\
\hline $7440-24-6$ & Strontium & $<5.23 \mathrm{E}-2$ & ug/g dry & $5.23 \mathrm{E}-2$ & 6/30/08 & 8F30003 & PNNL-AGG-ICP-AES \\
\hline $7440-28-0$ & Thallium & $<1.03 \mathrm{E} 0$ & ug/g dry & $1.03 \mathrm{E} 0$ & 6/30/08 & 8F30003 & PNNL-AGG-ICP-AES \\
\hline $7440-62-2$ & Vanadium & $9.43 \mathrm{E}-1$ & ug/g dry & $4.54 \mathrm{E}-2$ & 6/30/08 & $8 F 30003$ & PNNL-AGG-ICP-AES \\
\hline $7440-66-6$ & Zinc & $<9.28 \mathrm{E}-2$ & ug/g dry & $9.28 \mathrm{E}-2$ & 6/30/08 & 8F30003 & PNNL-AGG-ICP-AES \\
\hline $7440-23-5$ & Sodium & 2.28E2 & ug/g dry & $6.70 \mathrm{E}-1$ & 6/30/08 & 8F30003 & PNNL-AGG-ICP-AES \\
\hline $7440-21-3$ & Silicon & $2.25 \mathrm{E} 1$ & ug/g dry & $1.50 \mathrm{E} 0$ & 6/30/08 & 8F30003 & PNNL-AGG-ICP-AES \\
\hline $7704-34-9$ & Sulfur & $4.21 \mathrm{E} 0$ & ug/g dry & $3.08 \mathrm{E} 0$ & 6/30/08 & $8 F 30003$ & PNNL-AGG-ICP-AES \\
\hline $7440-32-6$ & Titanium & $1.56 \mathrm{E}-1$ & ug/g dry & 8.77E-3 & 6/30/08 & 8F30003 & PNNL-AGG-ICP-AES \\
\hline $7440-67-7$ & Zirconium & $<1.50 \mathrm{E}-1$ & ug/g dry & $1.50 \mathrm{E}-1$ & 6/30/08 & $8 F 30003$ & PNNL-AGG-ICP-AES \\
\hline $7440-22-4$ & Silver & $<7.72 \mathrm{E}-2$ & ug/g dry & $7.72 \mathrm{E}-2$ & 6/30/08 & $8 F 30003$ & PNNL-AGG-ICP-AES \\
\hline $7440-15-5$ & Rhenium & $<1.52 \mathrm{E}-1$ & ug/g dry & $1.52 \mathrm{E}-1$ & 6/30/08 & $8 F 30003$ & PNNL-AGG-ICP-AES \\
\hline $7440-36-0$ & Antimony & $<6.34 \mathrm{E}-1$ & ug/g dry & 6.34E-1 & 6/30/08 & 8F30003 & PNNL-AGG-ICP-AES \\
\hline HEIS No. & B1VJ56C & & b ID: & 5020-09 & & & \\
\hline $7429-90-5$ & Aluminum & $2.24 \mathrm{E} 0$ & ug/g dry & $8.58 \mathrm{E}-2$ & 6/30/08 & 8F30003 & PNNL-AGG-ICP-AES \\
\hline $7440-38-2$ & Arsenic & $<3.60 \mathrm{E}-1$ & ug/g dry & $3.60 \mathrm{E}-1$ & 6/30/08 & $8 F 30003$ & PNNL-AGG-ICP-AES \\
\hline $7440-42-8$ & Boron & $<1.94 \mathrm{E} 0$ & ug/g dry & $1.94 \mathrm{E} 0$ & 6/30/08 & 8F30003 & PNNL-AGG-ICP-AES \\
\hline $7440-39-3$ & Barium & $2.92 \mathrm{E}-2$ & ug/g dry & 8.79E-3 & 6/30/08 & 8F30003 & PNNL-AGG-ICP-AES \\
\hline $7440-41-7$ & Beryllium & $<2.84 \mathrm{E}-2$ & ug/g dry & $2.84 \mathrm{E}-2$ & 6/30/08 & $8 F 30003$ & PNNL-AGG-ICP-AES \\
\hline 7440-69-9 & Bismuth & $<1.88 \mathrm{E}-1$ & ug/g dry & $1.88 \mathrm{E}-1$ & 6/30/08 & $8 F 30003$ & PNNL-AGG-ICP-AES \\
\hline $7440-70-2$ & Calcium & 1.82E0 & ug/g dry & 3.87E-1 & 6/30/08 & 8F30003 & PNNL-AGG-ICP-AES \\
\hline $7440-43-9$ & Cadmium & $<2.69 \mathrm{E}-2$ & ug/g dry & $2.69 \mathrm{E}-2$ & 6/30/08 & 8F30003 & PNNL-AGG-ICP-AES \\
\hline
\end{tabular}


Total Metals by PNNL-AGG-ICP-AES/Water Extract

\begin{tabular}{|c|c|c|c|c|c|c|c|}
\hline CAS \# & Analyte & Results & Units & EQL & Analyzed & Batch & Method \\
\hline HEIS No. & B1VJ56C & \multicolumn{3}{|c|}{ Lab ID: $\quad$ 0805020-09 } & & & \\
\hline $7440-48-4$ & Cobalt & $<9.60 \mathrm{E}-2$ & ug/g dry & $9.60 \mathrm{E}-2$ & 6/30/08 & 8 F30003 & PNNL-AGG-ICP-AES \\
\hline $7440-47-3$ & Chromium & $<3.33 \mathrm{E}-2$ & ug/g dry & 3.33E-2 & 6/30/08 & 8F30003 & PNNL-AGG-ICP-AES \\
\hline $7440-50-8$ & Copper & $<8.04 \mathrm{E}-2$ & ug/g dry & 8.04E-2 & 6/30/08 & 8F30003 & PNNL-AGG-ICP-AES \\
\hline 7439-89-6 & Iron & 3.99E0 & ug/g dry & $1.42 \mathrm{E}-1$ & 6/30/08 & 8F30003 & PNNL-AGG-ICP-AES \\
\hline 7440-09-7 & Potassium & $2.53 \mathrm{E} 0$ & ug/g dry & 2.33Е0 & 6/30/08 & $8 F 30003$ & PNNL-AGG-ICP-AES \\
\hline 7439-93-2 & Lithium & $<5.40 \mathrm{E}-1$ & ug/g dry & $5.40 \mathrm{E}-1$ & 6/30/08 & $8 F 30003$ & PNNL-AGG-ICP-AES \\
\hline $7439-95-4$ & Magnesium & 9.12E-1 & ug/g dry & 8.34E-2 & 6/30/08 & 8F30003 & PNNL-AGG-ICP-AES \\
\hline 7439-96-5 & Manganese & $6.85 \mathrm{E}-2$ & ug/g dry & $1.71 \mathrm{E}-2$ & 6/30/08 & $8 F 30003$ & PNNL-AGG-ICP-AES \\
\hline 7439-98-7 & Molybdenum & $<1.39 \mathrm{E}-1$ & ug/g dry & $1.39 \mathrm{E}-1$ & 6/30/08 & 8F30003 & PNNL-AGG-ICP-AES \\
\hline $7440-02-0$ & Nickel & $<9.33 \mathrm{E}-2$ & ug/g dry & $9.33 \mathrm{E}-2$ & 6/30/08 & 8F30003 & PNNL-AGG-ICP-AES \\
\hline $7723-14-0$ & Phosphorus & $<1.03 \mathrm{E} 0$ & ug/g dry & 1.03E0 & 6/30/08 & 8F30003 & PNNL-AGG-ICP-AES \\
\hline 7439-92-1 & Lead & $<4.32 \mathrm{E}-1$ & ug/g dry & $4.32 \mathrm{E}-1$ & 6/30/08 & $8 F 30003$ & PNNL-AGG-ICP-AES \\
\hline $7782-49-2$ & Selenium & $<1.95 \mathrm{E} 0$ & ug/g dry & $1.95 \mathrm{E} 0$ & 6/30/08 & $8 F 30003$ & PNNL-AGG-ICP-AES \\
\hline $7440-24-6$ & Strontium & $<5.22 \mathrm{E}-2$ & ug/g dry & $5.22 \mathrm{E}-2$ & 6/30/08 & 8F30003 & PNNL-AGG-ICP-AES \\
\hline $7440-28-0$ & Thallium & $<1.03 \mathrm{E} 0$ & ug/g dry & $1.03 \mathrm{E} 0$ & 6/30/08 & 8F30003 & PNNL-AGG-ICP-AES \\
\hline $7440-62-2$ & Vanadium & $3.42 \mathrm{E}-1$ & ug/g dry & 4.53E-2 & 6/30/08 & $8 F 30003$ & PNNL-AGG-ICP-AES \\
\hline $7440-66-6$ & Zinc & $<9.27 \mathrm{E}-2$ & ug/g dry & $9.27 \mathrm{E}-2$ & 6/30/08 & 8F30003 & PNNL-AGG-ICP-AES \\
\hline $7440-23-5$ & Sodium & $1.86 \mathrm{E} 2$ & ug/g dry & $6.69 \mathrm{E}-1$ & 6/30/08 & 8F30003 & PNNL-AGG-ICP-AES \\
\hline $7440-21-3$ & Silicon & $1.71 \mathrm{E} 1$ & ug/g dry & $1.50 \mathrm{E} 0$ & 6/30/08 & 8F30003 & PNNL-AGG-ICP-AES \\
\hline $7704-34-9$ & Sulfur & $3.51 \mathrm{E} 0$ & ug/g dry & $3.08 \mathrm{E} 0$ & 6/30/08 & 8F30003 & PNNL-AGG-ICP-AES \\
\hline $7440-32-6$ & Titanium & $1.28 \mathrm{E}-1$ & ug/g dry & $8.76 \mathrm{E}-3$ & 6/30/08 & $8 F 30003$ & PNNL-AGG-ICP-AES \\
\hline $7440-67-7$ & Zirconium & $<1.50 \mathrm{E}-1$ & ug/g dry & $1.50 \mathrm{E}-1$ & 6/30/08 & 8F30003 & PNNL-AGG-ICP-AES \\
\hline $7440-22-4$ & Silver & $<7.71 \mathrm{E}-2$ & ug/g dry & $7.71 \mathrm{E}-2$ & 6/30/08 & $8 F 30003$ & PNNL-AGG-ICP-AES \\
\hline $7440-15-5$ & Rhenium & $<1.52 \mathrm{E}-1$ & ug/g dry & $1.52 \mathrm{E}-1$ & 6/30/08 & 8F30003 & PNNL-AGG-ICP-AES \\
\hline $7440-36-0$ & Antimony & $<6.33 \mathrm{E}-1$ & ug/g dry & $6.33 \mathrm{E}-1$ & 6/30/08 & 8F30003 & PNNL-AGG-ICP-AES \\
\hline HEIS No. & B1VJ58C & \multicolumn{3}{|c|}{ Lab ID: $\quad$ 0805020-10 } & & & \\
\hline $7429-90-5$ & Aluminum & $1.49 \mathrm{E} 0$ & ug/g dry & $8.59 \mathrm{E}-2$ & 6/30/08 & 8F30003 & PNNL-AGG-ICP-AES \\
\hline $7440-38-2$ & Arsenic & $4.90 \mathrm{E}-1$ & ug/g dry & $3.60 \mathrm{E}-1$ & 6/30/08 & $8 F 30003$ & PNNL-AGG-ICP-AES \\
\hline $7440-42-8$ & Boron & $<1.94 \mathrm{E} 0$ & ug/g dry & $1.94 \mathrm{E} 0$ & 6/30/08 & 8F30003 & PNNL-AGG-ICP-AES \\
\hline $7440-39-3$ & Barium & 5.67E-2 & ug/g dry & $8.80 \mathrm{E}-3$ & 6/30/08 & 8F30003 & PNNL-AGG-ICP-AES \\
\hline $7440-41-7$ & Beryllium & $<2.84 \mathrm{E}-2$ & ug/g dry & $2.84 \mathrm{E}-2$ & 6/30/08 & $8 F 30003$ & PNNL-AGG-ICP-AES \\
\hline $7440-69-9$ & Bismuth & $<1.88 \mathrm{E}-1$ & ug/g dry & $1.88 \mathrm{E}-1$ & 6/30/08 & $8 F 30003$ & PNNL-AGG-ICP-AES \\
\hline $7440-70-2$ & Calcium & 8.41E0 & ug/g dry & $3.87 \mathrm{E}-1$ & 6/30/08 & $8 F 30003$ & PNNL-AGG-ICP-AES \\
\hline $7440-43-9$ & Cadmium & $<2.69 \mathrm{E}-2$ & ug/g dry & $2.69 \mathrm{E}-2$ & 6/30/08 & 8F30003 & PNNL-AGG-ICP-AES \\
\hline $7440-48-4$ & Cobalt & $<9.61 \mathrm{E}-2$ & ug/g dry & $9.61 \mathrm{E}-2$ & 6/30/08 & $8 F 30003$ & PNNL-AGG-ICP-AES \\
\hline $7440-47-3$ & Chromium & $<3.33 \mathrm{E}-2$ & ug/g dry & 3.33E-2 & 6/30/08 & $8 F 30003$ & PNNL-AGG-ICP-AES \\
\hline $7440-50-8$ & Copper & 1.36E-1 & ug/g dry & 8.05E-2 & 6/30/08 & $8 F 30003$ & PNNL-AGG-ICP-AES \\
\hline 7439-89-6 & Iron & $2.55 \mathrm{E} 0$ & ug/g dry & 1.42E-1 & 6/30/08 & 8F30003 & PNNL-AGG-ICP-AES \\
\hline 7440-09-7 & Potassium & 4.45E0 & ug/g dry & 2.33Е0 & 6/30/08 & 8F30003 & PNNL-AGG-ICP-AES \\
\hline 7439-93-2 & Lithium & $<5.41 \mathrm{E}-1$ & ug/g dry & $5.41 \mathrm{E}-1$ & 6/30/08 & 8F30003 & PNNL-AGG-ICP-AES \\
\hline 7439-95-4 & Magnesium & 8.27E-1 & ug/g dry & 8.35E-2 & 6/30/08 & $8 F 30003$ & PNNL-AGG-ICP-AES \\
\hline 7439-96-5 & Manganese & 8.02E-2 & ug/g dry & $1.71 \mathrm{E}-2$ & 6/30/08 & 8F30003 & PNNL-AGG-ICP-AES \\
\hline 7439-98-7 & Molybdenum & $1.77 \mathrm{E}-1$ & ug/g dry & $1.39 \mathrm{E}-1$ & 6/30/08 & 8F30003 & PNNL-AGG-ICP-AES \\
\hline $7440-02-0$ & Nickel & $<9.34 \mathrm{E}-2$ & ug/g dry & $9.34 \mathrm{E}-2$ & 6/30/08 & $8 F 30003$ & PNNL-AGG-ICP-AES \\
\hline 7723-14-0 & Phosphorus & 3.77E0 & ug/g dry & 1.03E0 & 6/30/08 & $8 F 30003$ & PNNL-AGG-ICP-AES \\
\hline $7439-92-1$ & Lead & $<4.32 \mathrm{E}-1$ & ug/g dry & $4.32 \mathrm{E}-1$ & 6/30/08 & 8F30003 & PNNL-AGG-ICP-AES \\
\hline $7782-49-2$ & Selenium & $<1.95 \mathrm{E} 0$ & ug/g dry & $1.95 \mathrm{E} 0$ & 6/30/08 & 8F30003 & PNNL-AGG-ICP-AES \\
\hline $7440-24-6$ & Strontium & $<5.22 \mathrm{E}-2$ & ug/g dry & $5.22 \mathrm{E}-2$ & 6/30/08 & 8F30003 & PNNL-AGG-ICP-AES \\
\hline
\end{tabular}


Total Metals by PNNL-AGG-ICP-AES/Water Extract

\begin{tabular}{|c|c|c|c|c|c|c|c|}
\hline CAS \# & Analyte & Results & Units & EQL & Analyzed & Batch & Method \\
\hline HEIS No. & B1VJ58C & \multicolumn{3}{|c|}{ Lab ID: $\quad$ 0805020-10 } & & & \\
\hline $7440-28-0$ & Thallium & $<1.03 \mathrm{E} 0$ & ug/g dry & 1.03E0 & 6/30/08 & 8F30003 & PNNL-AGG-ICP-AES \\
\hline $7440-62-2$ & Vanadium & $2.46 \mathrm{E}-1$ & ug/g dry & 4.53E-2 & 6/30/08 & $8 F 30003$ & PNNL-AGG-ICP-AES \\
\hline $7440-66-6$ & Zinc & $<9.28 \mathrm{E}-2$ & ug/g dry & $9.28 \mathrm{E}-2$ & 6/30/08 & $8 F 30003$ & PNNL-AGG-ICP-AES \\
\hline $7440-23-5$ & Sodium & 4.50E2 & ug/g dry & $6.70 \mathrm{E}-1$ & 6/30/08 & $8 F 30003$ & PNNL-AGG-ICP-AES \\
\hline $7440-21-3$ & Silicon & $1.52 \mathrm{E} 1$ & ug/g dry & $1.50 \mathrm{E} 0$ & 6/30/08 & $8 F 30003$ & PNNL-AGG-ICP-AES \\
\hline 7704-34-9 & Sulfur & 7.45E0 & ug/g dry & $3.08 \mathrm{E} 0$ & 6/30/08 & 8 F30003 & PNNL-AGG-ICP-AES \\
\hline $7440-32-6$ & Titanium & 8.38E-2 & ug/g dry & 8.77E-3 & 6/30/08 & $8 F 30003$ & PNNL-AGG-ICP-AES \\
\hline $7440-67-7$ & Zirconium & $<1.50 \mathrm{E}-1$ & ug/g dry & $1.50 \mathrm{E}-1$ & 6/30/08 & 8F30003 & PNNL-AGG-ICP-AES \\
\hline $7440-22-4$ & Silver & $<7.72 \mathrm{E}-2$ & ug/g dry & 7.72E-2 & 6/30/08 & $8 F 30003$ & PNNL-AGG-ICP-AES \\
\hline $7440-15-5$ & Rhenium & $<1.52 \mathrm{E}-1$ & ug/g dry & $1.52 \mathrm{E}-1$ & 6/30/08 & 8F30003 & PNNL-AGG-ICP-AES \\
\hline $7440-36-0$ & Antimony & $<6.34 \mathrm{E}-1$ & ug/g dry & $6.34 \mathrm{E}-1$ & 6/30/08 & $8 F 30003$ & PNNL-AGG-ICP-AES \\
\hline HEIS No. & B1VJ58B & \multicolumn{3}{|c|}{ Lab ID: $\quad$ 0805020-11 } & & & \\
\hline $7429-90-5$ & Aluminum & 2.57E0 & ug/g dry & 8.63E-2 & $6 / 30 / 08$ & 8F30003 & PNNL-AGG-ICP-AES \\
\hline $7440-38-2$ & Arsenic & 7.70E-1 & ug/g dry & $3.62 \mathrm{E}-1$ & 6/30/08 & 8F30003 & PNNL-AGG-ICP-AES \\
\hline $7440-42-8$ & Boron & $<1.95 \mathrm{E} 0$ & ug/g dry & $1.95 \mathrm{E} 0$ & 6/30/08 & $8 F 30003$ & PNNL-AGG-ICP-AES \\
\hline $7440-39-3$ & Barium & 1.07E-1 & ug/g dry & 8.85E-3 & $7 / 07 / 08$ & 8F30003 & PNNL-AGG-ICP-AES \\
\hline $7440-41-7$ & Beryllium & $<2.86 \mathrm{E}-2$ & ug/g dry & $2.86 \mathrm{E}-2$ & 6/30/08 & 8F30003 & PNNL-AGG-ICP-AES \\
\hline 7440-69-9 & Bismuth & $<1.89 \mathrm{E}-1$ & ug/g dry & $1.89 \mathrm{E}-1$ & 6/30/08 & 8F30003 & PNNL-AGG-ICP-AES \\
\hline $7440-70-2$ & Calcium & 1.77E1 & ug/g dry & 3.89E-1 & $7 / 07 / 08$ & 8F30003 & PNNL-AGG-ICP-AES \\
\hline $7440-43-9$ & Cadmium & $<2.70 \mathrm{E}-2$ & ug/g dry & $2.70 \mathrm{E}-2$ & 6/30/08 & 8F30003 & PNNL-AGG-ICP-AES \\
\hline $7440-48-4$ & Cobalt & $<9.66 \mathrm{E}-2$ & ug/g dry & $9.66 \mathrm{E}-2$ & 6/30/08 & 8F30003 & PNNL-AGG-ICP-AES \\
\hline $7440-47-3$ & Chromium & $<3.35 \mathrm{E}-2$ & ug/g dry & $3.35 \mathrm{E}-2$ & 6/30/08 & $8 F 30003$ & PNNL-AGG-ICP-AES \\
\hline $7440-50-8$ & Copper & $1.24 \mathrm{E}-1$ & ug/g dry & 8.09E-2 & 6/30/08 & $8 F 30003$ & PNNL-AGG-ICP-AES \\
\hline 7439-89-6 & Iron & $3.90 \mathrm{E} 0$ & ug/g dry & 1.43E-1 & 6/30/08 & 8F30003 & PNNL-AGG-ICP-AES \\
\hline $7440-09-7$ & Potassium & 4.88E0 & ug/g dry & $2.34 \mathrm{E} 0$ & 6/30/08 & 8F30003 & PNNL-AGG-ICP-AES \\
\hline 7439-93-2 & Lithium & $<5.43 \mathrm{E}-1$ & ug/g dry & $5.43 \mathrm{E}-1$ & 6/30/08 & 8F30003 & PNNL-AGG-ICP-AES \\
\hline $7439-95-4$ & Magnesium & 1.58E0 & ug/g dry & 8.39E-2 & $7 / 07 / 08$ & 8F30003 & PNNL-AGG-ICP-AES \\
\hline 7439-96-5 & Manganese & $1.65 \mathrm{E}-1$ & ug/g dry & $1.72 \mathrm{E}-2$ & $7 / 07 / 08$ & $8 F 30003$ & PNNL-AGG-ICP-AES \\
\hline 7439-98-7 & Molybdenum & $<1.40 \mathrm{E}-1$ & ug/g dry & $1.40 \mathrm{E}-1$ & 6/30/08 & 8F30003 & PNNL-AGG-ICP-AES \\
\hline $7440-02-0$ & Nickel & $<9.39 \mathrm{E}-2$ & ug/g dry & $9.39 \mathrm{E}-2$ & 6/30/08 & 8F30003 & PNNL-AGG-ICP-AES \\
\hline $7723-14-0$ & Phosphorus & 5.11E0 & ug/g dry & $1.04 \mathrm{E} 0$ & 6/30/08 & 8F30003 & PNNL-AGG-ICP-AES \\
\hline $7439-92-1$ & Lead & $<4.35 \mathrm{E}-1$ & ug/g dry & $4.35 \mathrm{E}-1$ & 6/30/08 & 8F30003 & PNNL-AGG-ICP-AES \\
\hline $7782-49-2$ & Selenium & $<1.96 \mathrm{E} 0$ & ug/g dry & $1.96 \mathrm{E} 0$ & 6/30/08 & 8F30003 & PNNL-AGG-ICP-AES \\
\hline $7440-24-6$ & Strontium & $<5.25 \mathrm{E}-2$ & ug/g dry & $5.25 \mathrm{E}-2$ & 6/30/08 & $8 F 30003$ & PNNL-AGG-ICP-AES \\
\hline $7440-28-0$ & Thallium & $<1.03 \mathrm{E} 0$ & ug/g dry & 1.03E0 & 6/30/08 & 8F30003 & PNNL-AGG-ICP-AES \\
\hline $7440-62-2$ & Vanadium & 3.58E-1 & ug/g dry & $4.56 \mathrm{E}-2$ & $7 / 07 / 08$ & 8F30003 & PNNL-AGG-ICP-AES \\
\hline $7440-66-6$ & Zinc & $<9.33 \mathrm{E}-2$ & ug/g dry & $9.33 \mathrm{E}-2$ & 6/30/08 & $8 F 30003$ & PNNL-AGG-ICP-AES \\
\hline $7440-23-5$ & Sodium & 4.95E2 & ug/g dry & $6.73 \mathrm{E}-1$ & 6/30/08 & 8F30003 & PNNL-AGG-ICP-AES \\
\hline $7440-21-3$ & Silicon & 2.03E1 & ug/g dry & $1.51 \mathrm{E} 0$ & 6/30/08 & 8F30003 & PNNL-AGG-ICP-AES \\
\hline $7704-34-9$ & Sulfur & 7.02E0 & ug/g dry & 3.09E0 & 6/30/08 & $8 F 30003$ & PNNL-AGG-ICP-AES \\
\hline $7440-32-6$ & Titanium & 3.05E-1 & ug/g dry & $8.81 \mathrm{E}-3$ & $7 / 07 / 08$ & $8 F 30003$ & PNNL-AGG-ICP-AES \\
\hline $7440-67-7$ & Zirconium & $<1.51 \mathrm{E}-1$ & ug/g dry & $1.51 \mathrm{E}-1$ & 6/30/08 & $8 F 30003$ & PNNL-AGG-ICP-AES \\
\hline $7440-22-4$ & Silver & $<7.76 \mathrm{E}-2$ & ug/g dry & 7.76E-2 & 6/30/08 & 8F30003 & PNNL-AGG-ICP-AES \\
\hline $7440-15-5$ & Rhenium & $<1.53 \mathrm{E}-1$ & ug/g dry & $1.53 \mathrm{E}-1$ & 6/30/08 & 8F30003 & PNNL-AGG-ICP-AES \\
\hline $7440-36-0$ & Antimony & $<6.37 \mathrm{E}-1$ & ug/g dry & 6.37E-1 & 6/30/08 & 8F30003 & PNNL-AGG-ICP-AES \\
\hline HEIS No. & B1VJ58A & & b ID: & $05020-12$ & & & \\
\hline $7429-90-5$ & Aluminum & $2.16 \mathrm{E} 0$ & ug/g dry & $8.58 \mathrm{E}-2$ & 6/30/08 & 8F30003 & PNNL-AGG-ICP-AES \\
\hline $7440-38-2$ & Arsenic & $8.88 \mathrm{E}-1$ & ug/g dry & $3.60 \mathrm{E}-1$ & 6/30/08 & $8 F 30003$ & PNNL-AGG-ICP-AES \\
\hline
\end{tabular}


Total Metals by PNNL-AGG-ICP-AES/Water Extract

\begin{tabular}{|c|c|c|c|c|c|c|c|}
\hline CAS \# & Analyte & Results & Units & EQL & Analyzed & Batch & Method \\
\hline HEIS No. & B1VJ58A & & b ID: & 5020-12 & & & \\
\hline $7440-42-8$ & Boron & $<1.94 \mathrm{E} 0$ & ug/g dry & $1.94 \mathrm{E} 0$ & 6/30/08 & 8 F30003 & PNNL-AGG-ICP-AES \\
\hline $7440-39-3$ & Barium & $5.95 \mathrm{E}-2$ & ug/g dry & $8.78 \mathrm{E}-3$ & 6/30/08 & 8F30003 & PNNL-AGG-ICP-AES \\
\hline $7440-41-7$ & Beryllium & $<2.84 \mathrm{E}-2$ & ug/g dry & $2.84 \mathrm{E}-2$ & 6/30/08 & $8 F 30003$ & PNNL-AGG-ICP-AES \\
\hline 7440-69-9 & Bismuth & $<1.88 \mathrm{E}-1$ & ug/g dry & $1.88 \mathrm{E}-1$ & 6/30/08 & 8F30003 & PNNL-AGG-ICP-AES \\
\hline $7440-70-2$ & Calcium & 7.34E0 & ug/g dry & $3.87 \mathrm{E}-1$ & 6/30/08 & $8 F 30003$ & PNNL-AGG-ICP-AES \\
\hline $7440-43-9$ & Cadmium & $<2.69 \mathrm{E}-2$ & ug/g dry & 2.69E-2 & 6/30/08 & $8 F 30003$ & PNNL-AGG-ICP-AES \\
\hline $7440-48-4$ & Cobalt & $<9.59 \mathrm{E}-2$ & ug/g dry & $9.59 \mathrm{E}-2$ & 6/30/08 & 8F30003 & PNNL-AGG-ICP-AES \\
\hline $7440-47-3$ & Chromium & $<3.33 \mathrm{E}-2$ & ug/g dry & 3.33E-2 & 6/30/08 & $8 F 30003$ & PNNL-AGG-ICP-AES \\
\hline $7440-50-8$ & Copper & $1.42 \mathrm{E}-1$ & ug/g dry & $8.04 \mathrm{E}-2$ & 6/30/08 & 8F30003 & PNNL-AGG-ICP-AES \\
\hline 7439-89-6 & Iron & 3.59E0 & ug/g dry & 1.42E-1 & 6/30/08 & 8F30003 & PNNL-AGG-ICP-AES \\
\hline 7440-09-7 & Potassium & $4.56 \mathrm{E} 0$ & ug/g dry & 2.33Е0 & 6/30/08 & 8F30003 & PNNL-AGG-ICP-AES \\
\hline $7439-93-2$ & Lithium & $<5.40 \mathrm{E}-1$ & ug/g dry & $5.40 \mathrm{E}-1$ & 6/30/08 & $8 F 30003$ & PNNL-AGG-ICP-AES \\
\hline $7439-95-4$ & Magnesium & $9.08 \mathrm{E}-1$ & ug/g dry & 8.34E-2 & 6/30/08 & $8 F 30003$ & PNNL-AGG-ICP-AES \\
\hline 7439-96-5 & Manganese & $9.41 \mathrm{E}-2$ & ug/g dry & $1.71 \mathrm{E}-2$ & 6/30/08 & $8 F 30003$ & PNNL-AGG-ICP-AES \\
\hline 7439-98-7 & Molybdenum & $<1.39 \mathrm{E}-1$ & ug/g dry & $1.39 \mathrm{E}-1$ & 6/30/08 & 8F30003 & PNNL-AGG-ICP-AES \\
\hline $7440-02-0$ & Nickel & $<9.32 \mathrm{E}-2$ & ug/g dry & $9.32 \mathrm{E}-2$ & 6/30/08 & 8F30003 & PNNL-AGG-ICP-AES \\
\hline 7723-14-0 & Phosphorus & 5.74E0 & ug/g dry & 1.03E0 & 6/30/08 & 8F30003 & PNNL-AGG-ICP-AES \\
\hline $7439-92-1$ & Lead & $<4.32 \mathrm{E}-1$ & ug/g dry & 4.32E-1 & 6/30/08 & 8F30003 & PNNL-AGG-ICP-AES \\
\hline $7782-49-2$ & Selenium & $<1.95 \mathrm{E} 0$ & ug/g dry & $1.95 \mathrm{E} 0$ & 6/30/08 & 8F30003 & PNNL-AGG-ICP-AES \\
\hline $7440-24-6$ & Strontium & $<5.22 \mathrm{E}-2$ & ug/g dry & $5.22 \mathrm{E}-2$ & 6/30/08 & 8F30003 & PNNL-AGG-ICP-AES \\
\hline $7440-28-0$ & Thallium & $<1.03 \mathrm{E} 0$ & ug/g dry & $1.03 \mathrm{E} 0$ & 6/30/08 & $8 F 30003$ & PNNL-AGG-ICP-AES \\
\hline $7440-62-2$ & Vanadium & 3.98E-1 & ug/g dry & 4.53E-2 & 6/30/08 & 8F30003 & PNNL-AGG-ICP-AES \\
\hline $7440-66-6$ & Zinc & $<9.26 \mathrm{E}-2$ & ug/g dry & $9.26 \mathrm{E}-2$ & 6/30/08 & $8 F 30003$ & PNNL-AGG-ICP-AES \\
\hline $7440-23-5$ & Sodium & 4.62E2 & ug/g dry & $6.69 \mathrm{E}-1$ & 6/30/08 & $8 F 30003$ & PNNL-AGG-ICP-AES \\
\hline $7440-21-3$ & Silicon & 2.03E1 & ug/g dry & $1.50 \mathrm{E} 0$ & 6/30/08 & $8 F 30003$ & PNNL-AGG-ICP-AES \\
\hline 7704-34-9 & Sulfur & 7.09E0 & ug/g dry & 3.07E0 & 6/30/08 & 8F30003 & PNNL-AGG-ICP-AES \\
\hline $7440-32-6$ & Titanium & $1.41 \mathrm{E}-1$ & ug/g dry & $8.75 \mathrm{E}-3$ & 6/30/08 & 8F30003 & PNNL-AGG-ICP-AES \\
\hline $7440-67-7$ & Zirconium & $<1.50 \mathrm{E}-1$ & ug/g dry & $1.50 \mathrm{E}-1$ & 6/30/08 & 8F30003 & PNNL-AGG-ICP-AES \\
\hline $7440-22-4$ & Silver & $<7.71 \mathrm{E}-2$ & ug/g dry & $7.71 \mathrm{E}-2$ & 6/30/08 & 8F30003 & PNNL-AGG-ICP-AES \\
\hline $7440-15-5$ & Rhenium & $<1.52 \mathrm{E}-1$ & ug/g dry & $1.52 \mathrm{E}-1$ & 6/30/08 & $8 F 30003$ & PNNL-AGG-ICP-AES \\
\hline $7440-36-0$ & Antimony & $<6.33 \mathrm{E}-1$ & ug/g dry & 6.33E-1 & 6/30/08 & 8F30003 & PNNL-AGG-ICP-AES \\
\hline HEIS No. & B1VJ59C & & b ID: & 5020-14 & & & \\
\hline $7429-90-5$ & Aluminum & 2.61E-1 & ug/g dry & $8.58 \mathrm{E}-2$ & 6/30/08 & 8F30003 & PNNL-AGG-ICP-AES \\
\hline $7440-38-2$ & Arsenic & $<3.60 \mathrm{E}-1$ & ug/g dry & $3.60 \mathrm{E}-1$ & 6/30/08 & $8 F 30003$ & PNNL-AGG-ICP-AES \\
\hline $7440-42-8$ & Boron & $<1.94 \mathrm{E} 0$ & ug/g dry & $1.94 \mathrm{E} 0$ & 6/30/08 & $8 F 30003$ & PNNL-AGG-ICP-AES \\
\hline $7440-39-3$ & Barium & 2.63E-2 & ug/g dry & 8.79E-3 & 6/30/08 & 8F30003 & PNNL-AGG-ICP-AES \\
\hline $7440-41-7$ & Beryllium & $<2.84 \mathrm{E}-2$ & ug/g dry & $2.84 \mathrm{E}-2$ & 6/30/08 & $8 F 30003$ & PNNL-AGG-ICP-AES \\
\hline $7440-69-9$ & Bismuth & $<1.88 \mathrm{E}-1$ & ug/g dry & $1.88 \mathrm{E}-1$ & 6/30/08 & 8F30003 & PNNL-AGG-ICP-AES \\
\hline $7440-70-2$ & Calcium & 1.66E1 & ug/g dry & 3.87E-1 & 6/30/08 & 8F30003 & PNNL-AGG-ICP-AES \\
\hline $7440-43-9$ & Cadmium & $<2.69 \mathrm{E}-2$ & ug/g dry & $2.69 \mathrm{E}-2$ & 6/30/08 & 8F30003 & PNNL-AGG-ICP-AES \\
\hline $7440-48-4$ & Cobalt & $<9.60 \mathrm{E}-2$ & ug/g dry & $9.60 \mathrm{E}-2$ & 6/30/08 & $8 F 30003$ & PNNL-AGG-ICP-AES \\
\hline $7440-47-3$ & Chromium & $<3.33 \mathrm{E}-2$ & ug/g dry & 3.33E-2 & 6/30/08 & 8F30003 & PNNL-AGG-ICP-AES \\
\hline $7440-50-8$ & Copper & $<8.04 \mathrm{E}-2$ & ug/g dry & 8.04E-2 & 6/30/08 & 8F30003 & PNNL-AGG-ICP-AES \\
\hline 7439-89-6 & Iron & $<1.42 \mathrm{E}-1$ & ug/g dry & $1.42 \mathrm{E}-1$ & 6/30/08 & 8F30003 & PNNL-AGG-ICP-AES \\
\hline 7440-09-7 & Potassium & 7.47E0 & ug/g dry & 2.33Е0 & 6/30/08 & $8 F 30003$ & PNNL-AGG-ICP-AES \\
\hline $7439-93-2$ & Lithium & $<5.40 \mathrm{E}-1$ & ug/g dry & $5.40 \mathrm{E}-1$ & 6/30/08 & 8F30003 & PNNL-AGG-ICP-AES \\
\hline $7439-95-4$ & Magnesium & $5.60 \mathrm{E} 0$ & ug/g dry & 8.34E-2 & 6/30/08 & 8F30003 & PNNL-AGG-ICP-AES \\
\hline 7439-96-5 & Manganese & $<1.71 \mathrm{E}-2$ & ug/g dry & $1.71 \mathrm{E}-2$ & 6/30/08 & 8F30003 & PNNL-AGG-ICP-AES \\
\hline
\end{tabular}


Total Metals by PNNL-AGG-ICP-AES/Water Extract

\begin{tabular}{|c|c|c|c|c|c|c|c|}
\hline CAS \# & Analyte & Results & Units & EQL & Analyzed & Batch & Method \\
\hline HEIS No. & B1VJ59C & \multicolumn{3}{|c|}{ Lab ID: $\quad 0805020-14$} & & & \\
\hline 7439-98-7 & Molybdenum & $<1.39 \mathrm{E}-1$ & ug/g dry & $1.39 \mathrm{E}-1$ & 6/30/08 & 8F30003 & PNNL-AGG-ICP-AES \\
\hline $7440-02-0$ & Nickel & $<9.33 \mathrm{E}-2$ & ug/g dry & 9.33E-2 & 6/30/08 & $8 F 30003$ & PNNL-AGG-ICP-AES \\
\hline 7723-14-0 & Phosphorus & $<1.03 \mathrm{E} 0$ & ug/g dry & 1.03E0 & 6/30/08 & 8F30003 & PNNL-AGG-ICP-AES \\
\hline 7439-92-1 & Lead & $<4.32 \mathrm{E}-1$ & ug/g dry & $4.32 \mathrm{E}-1$ & 6/30/08 & 8F30003 & PNNL-AGG-ICP-AES \\
\hline $7782-49-2$ & Selenium & $<1.95 \mathrm{E} 0$ & ug/g dry & $1.95 \mathrm{E} 0$ & 6/30/08 & 8F30003 & PNNL-AGG-ICP-AES \\
\hline $7440-24-6$ & Strontium & $8.64 \mathrm{E}-2$ & ug/g dry & $5.22 \mathrm{E}-2$ & 6/30/08 & 8F30003 & PNNL-AGG-ICP-AES \\
\hline $7440-28-0$ & Thallium & $<1.03 \mathrm{E} 0$ & ug/g dry & $1.03 \mathrm{E} 0$ & 6/30/08 & 8F30003 & PNNL-AGG-ICP-AES \\
\hline $7440-62-2$ & Vanadium & $<4.53 \mathrm{E}-2$ & ug/g dry & 4.53E-2 & 6/30/08 & 8F30003 & PNNL-AGG-ICP-AES \\
\hline $7440-66-6$ & Zinc & $<9.27 \mathrm{E}-2$ & ug/g dry & $9.27 \mathrm{E}-2$ & 6/30/08 & $8 F 30003$ & PNNL-AGG-ICP-AES \\
\hline $7440-23-5$ & Sodium & 2.03E1 & ug/g dry & $6.69 \mathrm{E}-1$ & 6/30/08 & 8F30003 & PNNL-AGG-ICP-AES \\
\hline $7440-21-3$ & Silicon & 8.01E0 & ug/g dry & $1.50 \mathrm{E} 0$ & 6/30/08 & $8 F 30003$ & PNNL-AGG-ICP-AES \\
\hline 7704-34-9 & Sulfur & $1.01 \mathrm{E} 1$ & ug/g dry & 3.08E0 & 6/30/08 & 8F30003 & PNNL-AGG-ICP-AES \\
\hline $7440-32-6$ & Titanium & $<8.76 \mathrm{E}-3$ & ug/g dry & 8.76E-3 & 6/30/08 & 8F30003 & PNNL-AGG-ICP-AES \\
\hline $7440-67-7$ & Zirconium & $<1.50 \mathrm{E}-1$ & ug/g dry & $1.50 \mathrm{E}-1$ & 6/30/08 & 8F30003 & PNNL-AGG-ICP-AES \\
\hline $7440-22-4$ & Silver & $<7.71 \mathrm{E}-2$ & ug/g dry & $7.71 \mathrm{E}-2$ & 6/30/08 & 8F30003 & PNNL-AGG-ICP-AES \\
\hline $7440-15-5$ & Rhenium & $<1.52 \mathrm{E}-1$ & ug/g dry & $1.52 \mathrm{E}-1$ & 6/30/08 & 8F30003 & PNNL-AGG-ICP-AES \\
\hline $7440-36-0$ & Antimony & $<6.33 \mathrm{E}-1$ & ug/g dry & 6.33E-1 & 6/30/08 & 8F30003 & PNNL-AGG-ICP-AES \\
\hline HEIS No. & B1VJ59B & \multicolumn{3}{|c|}{$\begin{array}{ll}\text { Lab ID: } & \text { 0805020-15 }\end{array}$} & & & \\
\hline $7429-90-5$ & Aluminum & 2.12E-1 & ug/g dry & $8.59 \mathrm{E}-2$ & 6/30/08 & 8F30003 & PNNL-AGG-ICP-AES \\
\hline $7440-38-2$ & Arsenic & $<3.60 \mathrm{E}-1$ & ug/g dry & $3.60 \mathrm{E}-1$ & 6/30/08 & 8F30003 & PNNL-AGG-ICP-AES \\
\hline $7440-42-8$ & Boron & $<1.94 \mathrm{E} 0$ & ug/g dry & $1.94 \mathrm{E} 0$ & 6/30/08 & 8F30003 & PNNL-AGG-ICP-AES \\
\hline $7440-39-3$ & Barium & $1.16 \mathrm{E}-2$ & ug/g dry & $8.80 \mathrm{E}-3$ & 6/30/08 & 8F30003 & PNNL-AGG-ICP-AES \\
\hline $7440-41-7$ & Beryllium & $<2.84 \mathrm{E}-2$ & ug/g dry & $2.84 \mathrm{E}-2$ & 6/30/08 & $8 F 30003$ & PNNL-AGG-ICP-AES \\
\hline $7440-69-9$ & Bismuth & $<1.88 \mathrm{E}-1$ & ug/g dry & $1.88 \mathrm{E}-1$ & 6/30/08 & 8F30003 & PNNL-AGG-ICP-AES \\
\hline $7440-70-2$ & Calcium & 6.98E0 & ug/g dry & 3.87E-1 & 6/30/08 & $8 F 30003$ & PNNL-AGG-ICP-AES \\
\hline $7440-43-9$ & Cadmium & $<2.69 \mathrm{E}-2$ & ug/g dry & 2.69E-2 & 6/30/08 & $8 F 30003$ & PNNL-AGG-ICP-AES \\
\hline $7440-48-4$ & Cobalt & $<9.61 \mathrm{E}-2$ & ug/g dry & $9.61 \mathrm{E}-2$ & 6/30/08 & 8F30003 & PNNL-AGG-ICP-AES \\
\hline $7440-47-3$ & Chromium & $<3.33 \mathrm{E}-2$ & ug/g dry & 3.33E-2 & 6/30/08 & 8F30003 & PNNL-AGG-ICP-AES \\
\hline $7440-50-8$ & Copper & $<8.05 \mathrm{E}-2$ & ug/g dry & 8.05E-2 & 6/30/08 & 8F30003 & PNNL-AGG-ICP-AES \\
\hline 7439-89-6 & Iron & 1.67E-1 & ug/g dry & 1.42E-1 & 6/30/08 & 8F30003 & PNNL-AGG-ICP-AES \\
\hline 7440-09-7 & Potassium & 4.66E0 & ug/g dry & 2.33E0 & 6/30/08 & 8F30003 & PNNL-AGG-ICP-AES \\
\hline 7439-93-2 & Lithium & $<5.40 \mathrm{E}-1$ & ug/g dry & $5.40 \mathrm{E}-1$ & 6/30/08 & 8F30003 & PNNL-AGG-ICP-AES \\
\hline 7439-95-4 & Magnesium & 2.13E0 & ug/g dry & 8.35E-2 & 6/30/08 & 8F30003 & PNNL-AGG-ICP-AES \\
\hline $7439-96-5$ & Manganese & $<1.71 \mathrm{E}-2$ & ug/g dry & $1.71 \mathrm{E}-2$ & 6/30/08 & $8 F 30003$ & PNNL-AGG-ICP-AES \\
\hline 7439-98-7 & Molybdenum & $<1.39 \mathrm{E}-1$ & ug/g dry & $1.39 \mathrm{E}-1$ & 6/30/08 & 8F30003 & PNNL-AGG-ICP-AES \\
\hline $7440-02-0$ & Nickel & $<9.34 \mathrm{E}-2$ & ug/g dry & $9.34 \mathrm{E}-2$ & 6/30/08 & 8F30003 & PNNL-AGG-ICP-AES \\
\hline $7723-14-0$ & Phosphorus & $<1.03 \mathrm{E} 0$ & ug/g dry & 1.03Е0 & 6/30/08 & $8 F 30003$ & PNNL-AGG-ICP-AES \\
\hline $7439-92-1$ & Lead & $<4.32 \mathrm{E}-1$ & ug/g dry & $4.32 \mathrm{E}-1$ & 6/30/08 & 8F30003 & PNNL-AGG-ICP-AES \\
\hline $7782-49-2$ & Selenium & $<1.95 \mathrm{E} 0$ & ug/g dry & $1.95 \mathrm{E} 0$ & 6/30/08 & 8F30003 & PNNL-AGG-ICP-AES \\
\hline $7440-24-6$ & Strontium & $<5.22 \mathrm{E}-2$ & ug/g dry & $5.22 \mathrm{E}-2$ & 6/30/08 & 8F30003 & PNNL-AGG-ICP-AES \\
\hline $7440-28-0$ & Thallium & $<1.03 \mathrm{E} 0$ & ug/g dry & $1.03 \mathrm{E} 0$ & 6/30/08 & 8F30003 & PNNL-AGG-ICP-AES \\
\hline $7440-62-2$ & Vanadium & $<4.53 \mathrm{E}-2$ & ug/g dry & 4.53E-2 & 6/30/08 & $8 F 30003$ & PNNL-AGG-ICP-AES \\
\hline $7440-66-6$ & Zinc & $<9.28 \mathrm{E}-2$ & ug/g dry & $9.28 \mathrm{E}-2$ & 6/30/08 & 8F30003 & PNNL-AGG-ICP-AES \\
\hline $7440-23-5$ & Sodium & $1.31 \mathrm{E} 1$ & ug/g dry & $6.69 \mathrm{E}-1$ & 6/30/08 & 8F30003 & PNNL-AGG-ICP-AES \\
\hline $7440-21-3$ & Silicon & 8.40E0 & ug/g dry & $1.50 \mathrm{E} 0$ & 6/30/08 & 8F30003 & PNNL-AGG-ICP-AES \\
\hline $7704-34-9$ & Sulfur & 6.19E0 & ug/g dry & 3.08E0 & 6/30/08 & 8F30003 & PNNL-AGG-ICP-AES \\
\hline $7440-32-6$ & Titanium & $<8.77 \mathrm{E}-3$ & ug/g dry & 8.77E-3 & 6/30/08 & 8F30003 & PNNL-AGG-ICP-AES \\
\hline $7440-67-7$ & Zirconium & $<1.50 \mathrm{E}-1$ & ug/g dry & $1.50 \mathrm{E}-1$ & 6/30/08 & 8F30003 & PNNL-AGG-ICP-AES \\
\hline
\end{tabular}


Total Metals by PNNL-AGG-ICP-AES/Water Extract

\begin{tabular}{|c|c|c|c|c|c|c|c|}
\hline CAS \# & Analyte & Results & Units & EQL & Analyzed & Batch & Method \\
\hline HEIS No. & B1VJ59B & \multicolumn{3}{|c|}{ Lab ID: $\quad$ 0805020-15 } & & & \\
\hline $7440-22-4$ & Silver & $<7.72 \mathrm{E}-2$ & ug/g dry & $7.72 \mathrm{E}-2$ & 6/30/08 & 8F30003 & PNNL-AGG-ICP-AES \\
\hline $7440-15-5$ & Rhenium & $<1.52 \mathrm{E}-1$ & ug/g dry & $1.52 \mathrm{E}-1$ & 6/30/08 & $8 F 30003$ & PNNL-AGG-ICP-AES \\
\hline $7440-36-0$ & Antimony & $<6.33 \mathrm{E}-1$ & ug/g dry & $6.33 \mathrm{E}-1$ & 6/30/08 & 8 F30003 & PNNL-AGG-ICP-AES \\
\hline HEIS No. & B1VJ59A & \multicolumn{3}{|c|}{ Lab ID: $\quad 0805020-16$} & & & \\
\hline $7429-90-5$ & Aluminum & $2.02 \mathrm{E}-1$ & ug/g dry & 8.65E-2 & 6/30/08 & 8F30003 & PNNL-AGG-ICP-AES \\
\hline $7440-38-2$ & Arsenic & $<3.63 \mathrm{E}-1$ & ug/g dry & 3.63E-1 & 6/30/08 & $8 F 30003$ & PNNL-AGG-ICP-AES \\
\hline $7440-42-8$ & Boron & $<1.96 \mathrm{E} 0$ & ug/g dry & 1.96E0 & 6/30/08 & 8F30003 & PNNL-AGG-ICP-AES \\
\hline $7440-39-3$ & Barium & $1.28 \mathrm{E}-2$ & ug/g dry & $8.86 \mathrm{E}-3$ & 6/30/08 & 8F30003 & PNNL-AGG-ICP-AES \\
\hline $7440-41-7$ & Beryllium & $<2.86 \mathrm{E}-2$ & ug/g dry & 2.86E-2 & 6/30/08 & 8F30003 & PNNL-AGG-ICP-AES \\
\hline $7440-69-9$ & Bismuth & $<1.90 \mathrm{E}-1$ & ug/g dry & $1.90 \mathrm{E}-1$ & 6/30/08 & 8F30003 & PNNL-AGG-ICP-AES \\
\hline $7440-70-2$ & Calcium & 6.74E0 & ug/g dry & $3.90 \mathrm{E}-1$ & 6/30/08 & 8F30003 & PNNL-AGG-ICP-AES \\
\hline $7440-43-9$ & Cadmium & $<2.71 \mathrm{E}-2$ & ug/g dry & $2.71 \mathrm{E}-2$ & 6/30/08 & 8F30003 & PNNL-AGG-ICP-AES \\
\hline $7440-48-4$ & Cobalt & $<9.67 \mathrm{E}-2$ & ug/g dry & $9.67 \mathrm{E}-2$ & 6/30/08 & 8F30003 & PNNL-AGG-ICP-AES \\
\hline $7440-47-3$ & Chromium & $<3.36 \mathrm{E}-2$ & ug/g dry & 3.36E-2 & 6/30/08 & $8 F 30003$ & PNNL-AGG-ICP-AES \\
\hline $7440-50-8$ & Copper & $<8.10 \mathrm{E}-2$ & ug/g dry & $8.10 \mathrm{E}-2$ & 6/30/08 & 8 F30003 & PNNL-AGG-ICP-AES \\
\hline 7439-89-6 & Iron & $1.45 \mathrm{E}-1$ & ug/g dry & 1.43E-1 & 6/30/08 & 8F30003 & PNNL-AGG-ICP-AES \\
\hline 7440-09-7 & Potassium & 4.74E0 & ug/g dry & 2.35E0 & 6/30/08 & $8 F 30003$ & PNNL-AGG-ICP-AES \\
\hline 7439-93-2 & Lithium & $<5.44 \mathrm{E}-1$ & ug/g dry & $5.44 \mathrm{E}-1$ & 6/30/08 & 8F30003 & PNNL-AGG-ICP-AES \\
\hline 7439-95-4 & Magnesium & 2.01E0 & ug/g dry & $8.40 \mathrm{E}-2$ & 6/30/08 & $8 F 30003$ & PNNL-AGG-ICP-AES \\
\hline 7439-96-5 & Manganese & $<1.73 \mathrm{E}-2$ & ug/g dry & 1.73E-2 & 6/30/08 & 8F30003 & PNNL-AGG-ICP-AES \\
\hline 7439-98-7 & Molybdenum & $<1.40 \mathrm{E}-1$ & ug/g dry & $1.40 \mathrm{E}-1$ & 6/30/08 & 8F30003 & PNNL-AGG-ICP-AES \\
\hline $7440-02-0$ & Nickel & $<9.40 \mathrm{E}-2$ & ug/g dry & $9.40 \mathrm{E}-2$ & 6/30/08 & 8F30003 & PNNL-AGG-ICP-AES \\
\hline 7723-14-0 & Phosphorus & $<1.04 \mathrm{E} 0$ & ug/g dry & $1.04 \mathrm{E} 0$ & 6/30/08 & 8F30003 & PNNL-AGG-ICP-AES \\
\hline 7439-92-1 & Lead & $<4.35 \mathrm{E}-1$ & ug/g dry & 4.35E-1 & 6/30/08 & 8F30003 & PNNL-AGG-ICP-AES \\
\hline $7782-49-2$ & Selenium & $<1.96 \mathrm{E} 0$ & ug/g dry & $1.96 \mathrm{E} 0$ & 6/30/08 & 8F30003 & PNNL-AGG-ICP-AES \\
\hline $7440-24-6$ & Strontium & $<5.26 \mathrm{E}-2$ & ug/g dry & $5.26 \mathrm{E}-2$ & 6/30/08 & 8F30003 & PNNL-AGG-ICP-AES \\
\hline $7440-28-0$ & Thallium & $<1.03 \mathrm{E} 0$ & ug/g dry & 1.03E0 & 6/30/08 & $8 F 30003$ & PNNL-AGG-ICP-AES \\
\hline $7440-62-2$ & Vanadium & $<4.56 \mathrm{E}-2$ & ug/g dry & $4.56 \mathrm{E}-2$ & 6/30/08 & 8F30003 & PNNL-AGG-ICP-AES \\
\hline $7440-66-6$ & Zinc & $<9.34 \mathrm{E}-2$ & ug/g dry & $9.34 \mathrm{E}-2$ & 6/30/08 & 8F30003 & PNNL-AGG-ICP-AES \\
\hline $7440-23-5$ & Sodium & 1.18E1 & ug/g dry & $6.74 \mathrm{E}-1$ & 6/30/08 & $8 F 30003$ & PNNL-AGG-ICP-AES \\
\hline $7440-21-3$ & Silicon & 7.83E0 & ug/g dry & $1.51 \mathrm{E} 0$ & 6/30/08 & 8F30003 & PNNL-AGG-ICP-AES \\
\hline $7704-34-9$ & Sulfur & 5.20E0 & ug/g dry & 3.10E0 & 6/30/08 & 8F30003 & PNNL-AGG-ICP-AES \\
\hline $7440-32-6$ & Titanium & $<8.83 \mathrm{E}-3$ & ug/g dry & 8.83E-3 & 6/30/08 & 8F30003 & PNNL-AGG-ICP-AES \\
\hline $7440-67-7$ & Zirconium & $<1.51 \mathrm{E}-1$ & ug/g dry & $1.51 \mathrm{E}-1$ & 6/30/08 & $8 F 30003$ & PNNL-AGG-ICP-AES \\
\hline $7440-22-4$ & Silver & $<7.77 \mathrm{E}-2$ & ug/g dry & 7.77E-2 & 6/30/08 & 8F30003 & PNNL-AGG-ICP-AES \\
\hline $7440-15-5$ & Rhenium & $<1.53 \mathrm{E}-1$ & ug/g dry & $1.53 \mathrm{E}-1$ & 6/30/08 & 8F30003 & PNNL-AGG-ICP-AES \\
\hline $7440-36-0$ & Antimony & $<6.38 \mathrm{E}-1$ & ug/g dry & $6.38 \mathrm{E}-1$ & 6/30/08 & $8 F 30003$ & PNNL-AGG-ICP-AES \\
\hline HEIS No. & B1VJ60C & & b ID: & $5020-18$ & & & \\
\hline $7429-90-5$ & Aluminum & $1.10 \mathrm{E}-1$ & ug/g dry & $8.58 \mathrm{E}-2$ & 6/30/08 & 8F30003 & PNNL-AGG-ICP-AES \\
\hline $7440-38-2$ & Arsenic & $<3.60 \mathrm{E}-1$ & ug/g dry & $3.60 \mathrm{E}-1$ & 6/30/08 & 8F30003 & PNNL-AGG-ICP-AES \\
\hline $7440-42-8$ & Boron & $<1.94 \mathrm{E} 0$ & ug/g dry & $1.94 \mathrm{E} 0$ & 6/30/08 & 8F30003 & PNNL-AGG-ICP-AES \\
\hline $7440-39-3$ & Barium & 2.67E-2 & ug/g dry & 8.79E-3 & 6/30/08 & $8 F 30003$ & PNNL-AGG-ICP-AES \\
\hline $7440-41-7$ & Beryllium & $<2.84 \mathrm{E}-2$ & ug/g dry & $2.84 \mathrm{E}-2$ & 6/30/08 & 8F30003 & PNNL-AGG-ICP-AES \\
\hline $7440-69-9$ & Bismuth & $<1.88 \mathrm{E}-1$ & ug/g dry & $1.88 \mathrm{E}-1$ & 6/30/08 & 8F30003 & PNNL-AGG-ICP-AES \\
\hline $7440-70-2$ & Calcium & 2.13E1 & ug/g dry & 3.87E-1 & 6/30/08 & 8F30003 & PNNL-AGG-ICP-AES \\
\hline $7440-43-9$ & Cadmium & $<2.69 \mathrm{E}-2$ & ug/g dry & $2.69 \mathrm{E}-2$ & 6/30/08 & $8 F 30003$ & PNNL-AGG-ICP-AES \\
\hline $7440-48-4$ & Cobalt & $<9.60 \mathrm{E}-2$ & ug/g dry & $9.60 \mathrm{E}-2$ & 6/30/08 & 8F30003 & PNNL-AGG-ICP-AES \\
\hline $7440-47-3$ & Chromium & $<3.33 \mathrm{E}-2$ & ug/g dry & 3.33E-2 & 6/30/08 & $8 F 30003$ & PNNL-AGG-ICP-AES \\
\hline
\end{tabular}


Total Metals by PNNL-AGG-ICP-AES/Water Extract

\begin{tabular}{|c|c|c|c|c|c|c|c|}
\hline CAS \# & Analyte & Results & Units & EQL & Analyzed & Batch & Method \\
\hline HEIS No. & B1VJ60C & \multicolumn{3}{|c|}{ Lab ID: $\quad 0805020-18$} & & & \\
\hline $7440-50-8$ & Copper & $<8.04 \mathrm{E}-2$ & ug/g dry & $8.04 \mathrm{E}-2$ & 6/30/08 & 8 F30003 & PNNL-AGG-ICP-AES \\
\hline 7439-89-6 & Iron & $<1.42 \mathrm{E}-1$ & ug/g dry & $1.42 \mathrm{E}-1$ & 6/30/08 & 8F30003 & PNNL-AGG-ICP-AES \\
\hline 7440-09-7 & Potassium & 1.34E1 & ug/g dry & 2.33Е0 & 6/30/08 & $8 F 30003$ & PNNL-AGG-ICP-AES \\
\hline 7439-93-2 & Lithium & $<5.40 \mathrm{E}-1$ & ug/g dry & $5.40 \mathrm{E}-1$ & 6/30/08 & 8F30003 & PNNL-AGG-ICP-AES \\
\hline 7439-95-4 & Magnesium & 8.45E0 & ug/g dry & $8.34 \mathrm{E}-2$ & 6/30/08 & $8 F 30003$ & PNNL-AGG-ICP-AES \\
\hline 7439-96-5 & Manganese & $2.85 \mathrm{E}-2$ & ug/g dry & $1.71 \mathrm{E}-2$ & 6/30/08 & $8 F 30003$ & PNNL-AGG-ICP-AES \\
\hline 7439-98-7 & Molybdenum & 2.32E-1 & ug/g dry & $1.39 \mathrm{E}-1$ & 6/30/08 & 8F30003 & PNNL-AGG-ICP-AES \\
\hline $7440-02-0$ & Nickel & $<9.33 \mathrm{E}-2$ & ug/g dry & $9.33 \mathrm{E}-2$ & 6/30/08 & $8 F 30003$ & PNNL-AGG-ICP-AES \\
\hline 7723-14-0 & Phosphorus & $<1.03 \mathrm{E} 0$ & ug/g dry & 1.03E0 & 6/30/08 & 8F30003 & PNNL-AGG-ICP-AES \\
\hline $7439-92-1$ & Lead & $<4.32 \mathrm{E}-1$ & ug/g dry & $4.32 \mathrm{E}-1$ & 6/30/08 & 8F30003 & PNNL-AGG-ICP-AES \\
\hline $7782-49-2$ & Selenium & $<1.95 \mathrm{E} 0$ & ug/g dry & $1.95 \mathrm{E} 0$ & 6/30/08 & 8F30003 & PNNL-AGG-ICP-AES \\
\hline $7440-24-6$ & Strontium & $1.15 \mathrm{E}-1$ & ug/g dry & $5.22 \mathrm{E}-2$ & 6/30/08 & $8 F 30003$ & PNNL-AGG-ICP-AES \\
\hline $7440-28-0$ & Thallium & $<1.03 \mathrm{E} 0$ & ug/g dry & $1.03 \mathrm{E} 0$ & 6/30/08 & $8 F 30003$ & PNNL-AGG-ICP-AES \\
\hline $7440-62-2$ & Vanadium & $<4.53 \mathrm{E}-2$ & ug/g dry & $4.53 \mathrm{E}-2$ & 6/30/08 & 8F30003 & PNNL-AGG-ICP-AES \\
\hline $7440-66-6$ & Zinc & $<9.27 \mathrm{E}-2$ & ug/g dry & $9.27 \mathrm{E}-2$ & 6/30/08 & $8 F 30003$ & PNNL-AGG-ICP-AES \\
\hline $7440-23-5$ & Sodium & 2.83E1 & ug/g dry & $6.69 \mathrm{E}-1$ & 6/30/08 & $8 F 30003$ & PNNL-AGG-ICP-AES \\
\hline $7440-21-3$ & Silicon & $5.50 \mathrm{E} 0$ & ug/g dry & $1.50 \mathrm{E} 0$ & 6/30/08 & 8F30003 & PNNL-AGG-ICP-AES \\
\hline $7704-34-9$ & Sulfur & $2.54 \mathrm{E} 1$ & ug/g dry & 3.07E0 & 6/30/08 & 8F30003 & PNNL-AGG-ICP-AES \\
\hline $7440-32-6$ & Titanium & $<8.76 \mathrm{E}-3$ & ug/g dry & $8.76 \mathrm{E}-3$ & 6/30/08 & 8F30003 & PNNL-AGG-ICP-AES \\
\hline $7440-67-7$ & Zirconium & $<1.50 \mathrm{E}-1$ & ug/g dry & $1.50 \mathrm{E}-1$ & 6/30/08 & 8F30003 & PNNL-AGG-ICP-AES \\
\hline $7440-22-4$ & Silver & $<7.71 \mathrm{E}-2$ & ug/g dry & $7.71 \mathrm{E}-2$ & 6/30/08 & $8 F 30003$ & PNNL-AGG-ICP-AES \\
\hline $7440-15-5$ & Rhenium & $<1.52 \mathrm{E}-1$ & ug/g dry & $1.52 \mathrm{E}-1$ & 6/30/08 & 8F30003 & PNNL-AGG-ICP-AES \\
\hline $7440-36-0$ & Antimony & $<6.33 \mathrm{E}-1$ & ug/g dry & $6.33 \mathrm{E}-1$ & 6/30/08 & 8F30003 & PNNL-AGG-ICP-AES \\
\hline HEIS No. & B1VJ60B & \multicolumn{3}{|c|}{$\begin{array}{ll}\text { Lab ID: } & \text { 0805020-19 }\end{array}$} & & & \\
\hline $7429-90-5$ & Aluminum & $<8.58 \mathrm{E}-2$ & ug/g dry & $8.58 \mathrm{E}-2$ & 6/30/08 & 8F30003 & PNNL-AGG-ICP-AES \\
\hline $7440-38-2$ & Arsenic & $<3.60 \mathrm{E}-1$ & ug/g dry & $3.60 \mathrm{E}-1$ & $6 / 30 / 08$ & 8F30003 & PNNL-AGG-ICP-AES \\
\hline $7440-42-8$ & Boron & $<1.94 \mathrm{E} 0$ & ug/g dry & $1.94 \mathrm{E} 0$ & 6/30/08 & $8 F 30003$ & PNNL-AGG-ICP-AES \\
\hline $7440-39-3$ & Barium & $2.10 \mathrm{E}-2$ & ug/g dry & $8.80 \mathrm{E}-3$ & 6/30/08 & $8 F 30003$ & PNNL-AGG-ICP-AES \\
\hline $7440-41-7$ & Beryllium & $<2.84 \mathrm{E}-2$ & ug/g dry & $2.84 \mathrm{E}-2$ & 6/30/08 & 8F30003 & PNNL-AGG-ICP-AES \\
\hline $7440-69-9$ & Bismuth & $<1.88 \mathrm{E}-1$ & ug/g dry & $1.88 \mathrm{E}-1$ & $6 / 30 / 08$ & 8F30003 & PNNL-AGG-ICP-AES \\
\hline $7440-70-2$ & Calcium & 2.21E1 & ug/g dry & 3.87E-1 & 6/30/08 & $8 F 30003$ & PNNL-AGG-ICP-AES \\
\hline $7440-43-9$ & Cadmium & $<2.69 \mathrm{E}-2$ & ug/g dry & $2.69 \mathrm{E}-2$ & 6/30/08 & $8 F 30003$ & PNNL-AGG-ICP-AES \\
\hline $7440-48-4$ & Cobalt & $<9.61 \mathrm{E}-2$ & ug/g dry & $9.61 \mathrm{E}-2$ & 6/30/08 & $8 F 30003$ & PNNL-AGG-ICP-AES \\
\hline $7440-47-3$ & Chromium & $<3.33 \mathrm{E}-2$ & ug/g dry & 3.33E-2 & 6/30/08 & 8F30003 & PNNL-AGG-ICP-AES \\
\hline $7440-50-8$ & Copper & $<8.04 \mathrm{E}-2$ & ug/g dry & $8.04 \mathrm{E}-2$ & 6/30/08 & $8 F 30003$ & PNNL-AGG-ICP-AES \\
\hline 7439-89-6 & Iron & $<1.42 \mathrm{E}-1$ & ug/g dry & $1.42 \mathrm{E}-1$ & 6/30/08 & $8 F 30003$ & PNNL-AGG-ICP-AES \\
\hline 7440-09-7 & Potassium & 1.07E1 & ug/g dry & 2.33Е0 & 6/30/08 & $8 F 30003$ & PNNL-AGG-ICP-AES \\
\hline $7439-93-2$ & Lithium & $<5.40 \mathrm{E}-1$ & ug/g dry & $5.40 \mathrm{E}-1$ & 6/30/08 & 8F30003 & PNNL-AGG-ICP-AES \\
\hline $7439-95-4$ & Magnesium & 9.22E0 & ug/g dry & $8.34 \mathrm{E}-2$ & 6/30/08 & 8F30003 & PNNL-AGG-ICP-AES \\
\hline $7439-96-5$ & Manganese & $<1.71 \mathrm{E}-2$ & ug/g dry & $1.71 \mathrm{E}-2$ & 6/30/08 & 8F30003 & PNNL-AGG-ICP-AES \\
\hline 7439-98-7 & Molybdenum & $<1.39 \mathrm{E}-1$ & ug/g dry & $1.39 \mathrm{E}-1$ & 6/30/08 & $8 F 30003$ & PNNL-AGG-ICP-AES \\
\hline $7440-02-0$ & Nickel & $<9.34 \mathrm{E}-2$ & ug/g dry & $9.34 \mathrm{E}-2$ & 6/30/08 & 8F30003 & PNNL-AGG-ICP-AES \\
\hline $7723-14-0$ & Phosphorus & $<1.03 \mathrm{E} 0$ & ug/g dry & $1.03 \mathrm{E} 0$ & 6/30/08 & 8F30003 & PNNL-AGG-ICP-AES \\
\hline $7439-92-1$ & Lead & $<4.32 \mathrm{E}-1$ & ug/g dry & $4.32 \mathrm{E}-1$ & 6/30/08 & 8F30003 & PNNL-AGG-ICP-AES \\
\hline 7782-49-2 & Selenium & $<1.95 \mathrm{E} 0$ & ug/g dry & $1.95 \mathrm{E} 0$ & 6/30/08 & $8 F 30003$ & PNNL-AGG-ICP-AES \\
\hline $7440-24-6$ & Strontium & $1.20 \mathrm{E}-1$ & ug/g dry & $5.22 \mathrm{E}-2$ & 6/30/08 & 8F30003 & PNNL-AGG-ICP-AES \\
\hline $7440-28-0$ & Thallium & $<1.03 \mathrm{E} 0$ & ug/g dry & $1.03 \mathrm{E} 0$ & 6/30/08 & 8F30003 & PNNL-AGG-ICP-AES \\
\hline $7440-62-2$ & Vanadium & $<4.53 \mathrm{E}-2$ & ug/g dry & 4.53E-2 & 6/30/08 & 8F30003 & PNNL-AGG-ICP-AES \\
\hline
\end{tabular}


Total Metals by PNNL-AGG-ICP-AES/Water Extract

\begin{tabular}{|c|c|c|c|c|c|c|c|}
\hline CAS \# & Analyte & Results & Units & EQL & Analyzed & Batch & Method \\
\hline HEIS No. & B1VJ60B & \multicolumn{3}{|c|}{ Lab ID: $\quad$ 0805020-19 } & & & \\
\hline $7440-66-6$ & Zinc & $<9.28 \mathrm{E}-2$ & ug/g dry & $9.28 \mathrm{E}-2$ & 6/30/08 & 8F30003 & PNNL-AGG-ICP-AES \\
\hline $7440-23-5$ & Sodium & 1.96E1 & ug/g dry & $6.69 \mathrm{E}-1$ & 6/30/08 & $8 F 30003$ & PNNL-AGG-ICP-AES \\
\hline $7440-21-3$ & Silicon & 7.95E0 & ug/g dry & $1.50 \mathrm{E} 0$ & 6/30/08 & 8 F30003 & PNNL-AGG-ICP-AES \\
\hline 7704-34-9 & Sulfur & 3.34E1 & ug/g dry & 3.08E0 & 6/30/08 & $8 F 30003$ & PNNL-AGG-ICP-AES \\
\hline $7440-32-6$ & Titanium & $<8.77 \mathrm{E}-3$ & ug/g dry & 8.77E-3 & 6/30/08 & $8 F 30003$ & PNNL-AGG-ICP-AES \\
\hline $7440-67-7$ & Zirconium & $<1.50 \mathrm{E}-1$ & ug/g dry & $1.50 \mathrm{E}-1$ & 6/30/08 & $8 F 30003$ & PNNL-AGG-ICP-AES \\
\hline $7440-22-4$ & Silver & $<7.71 \mathrm{E}-2$ & ug/g dry & $7.71 \mathrm{E}-2$ & 6/30/08 & $8 F 30003$ & PNNL-AGG-ICP-AES \\
\hline $7440-15-5$ & Rhenium & $<1.52 \mathrm{E}-1$ & ug/g dry & $1.52 \mathrm{E}-1$ & 6/30/08 & $8 F 30003$ & PNNL-AGG-ICP-AES \\
\hline $7440-36-0$ & Antimony & $<6.33 \mathrm{E}-1$ & ug/g dry & $6.33 \mathrm{E}-1$ & 6/30/08 & $8 F 30003$ & PNNL-AGG-ICP-AES \\
\hline HEIS No. & B1VJ60A & \multicolumn{3}{|c|}{ Lab ID: $\quad$ 0805020-20 } & & & \\
\hline $7429-90-5$ & Aluminum & 2.63E-1 & ug/g dry & $8.58 \mathrm{E}-2$ & 6/30/08 & 8F30003 & PNNL-AGG-ICP-AES \\
\hline $7440-38-2$ & Arsenic & $<3.60 \mathrm{E}-1$ & ug/g dry & $3.60 \mathrm{E}-1$ & 6/30/08 & 8F30003 & PNNL-AGG-ICP-AES \\
\hline $7440-42-8$ & Boron & $<1.94 \mathrm{E} 0$ & ug/g dry & $1.94 \mathrm{E} 0$ & 6/30/08 & 8F30003 & PNNL-AGG-ICP-AES \\
\hline $7440-39-3$ & Barium & $1.47 \mathrm{E}-2$ & ug/g dry & $8.79 \mathrm{E}-3$ & 6/30/08 & $8 F 30003$ & PNNL-AGG-ICP-AES \\
\hline $7440-41-7$ & Beryllium & $<2.84 \mathrm{E}-2$ & ug/g dry & $2.84 \mathrm{E}-2$ & 6/30/08 & $8 F 30003$ & PNNL-AGG-ICP-AES \\
\hline 7440-69-9 & Bismuth & $<1.88 \mathrm{E}-1$ & ug/g dry & $1.88 \mathrm{E}-1$ & 6/30/08 & 8F30003 & PNNL-AGG-ICP-AES \\
\hline $7440-70-2$ & Calcium & 1.27E1 & ug/g dry & 3.87E-1 & 6/30/08 & $8 F 30003$ & PNNL-AGG-ICP-AES \\
\hline 7440-43-9 & Cadmium & $<2.69 \mathrm{E}-2$ & ug/g dry & 2.69E-2 & 6/30/08 & 8F30003 & PNNL-AGG-ICP-AES \\
\hline $7440-48-4$ & Cobalt & $<9.60 \mathrm{E}-2$ & ug/g dry & $9.60 \mathrm{E}-2$ & 6/30/08 & 8F30003 & PNNL-AGG-ICP-AES \\
\hline $7440-47-3$ & Chromium & $<3.33 \mathrm{E}-2$ & ug/g dry & 3.33E-2 & 6/30/08 & 8F30003 & PNNL-AGG-ICP-AES \\
\hline $7440-50-8$ & Copper & $<8.04 \mathrm{E}-2$ & ug/g dry & 8.04E-2 & 6/30/08 & 8F30003 & PNNL-AGG-ICP-AES \\
\hline 7439-89-6 & Iron & $<1.42 \mathrm{E}-1$ & ug/g dry & $1.42 \mathrm{E}-1$ & 6/30/08 & 8F30003 & PNNL-AGG-ICP-AES \\
\hline 7440-09-7 & Potassium & 8.44E0 & ug/g dry & 2.33E0 & 6/30/08 & 8F30003 & PNNL-AGG-ICP-AES \\
\hline 7439-93-2 & Lithium & $<5.40 \mathrm{E}-1$ & ug/g dry & $5.40 \mathrm{E}-1$ & 6/30/08 & 8F30003 & PNNL-AGG-ICP-AES \\
\hline 7439-95-4 & Magnesium & 5.33E0 & ug/g dry & 8.34E-2 & 6/30/08 & 8F30003 & PNNL-AGG-ICP-AES \\
\hline 7439-96-5 & Manganese & $<1.71 \mathrm{E}-2$ & ug/g dry & $1.71 \mathrm{E}-2$ & 6/30/08 & 8F30003 & PNNL-AGG-ICP-AES \\
\hline 7439-98-7 & Molybdenum & $<1.39 \mathrm{E}-1$ & ug/g dry & $1.39 \mathrm{E}-1$ & 6/30/08 & $8 F 30003$ & PNNL-AGG-ICP-AES \\
\hline $7440-02-0$ & Nickel & $<9.33 \mathrm{E}-2$ & ug/g dry & 9.33E-2 & 6/30/08 & 8F30003 & PNNL-AGG-ICP-AES \\
\hline $7723-14-0$ & Phosphorus & $<1.03 \mathrm{E} 0$ & ug/g dry & $1.03 \mathrm{E} 0$ & 6/30/08 & 8F30003 & PNNL-AGG-ICP-AES \\
\hline 7439-92-1 & Lead & $<4.32 \mathrm{E}-1$ & ug/g dry & $4.32 \mathrm{E}-1$ & 6/30/08 & $8 F 30003$ & PNNL-AGG-ICP-AES \\
\hline $7782-49-2$ & Selenium & $<1.95 \mathrm{E} 0$ & ug/g dry & $1.95 \mathrm{E} 0$ & 6/30/08 & 8F30003 & PNNL-AGG-ICP-AES \\
\hline $7440-24-6$ & Strontium & 7.19E-2 & ug/g dry & $5.22 \mathrm{E}-2$ & 6/30/08 & 8F30003 & PNNL-AGG-ICP-AES \\
\hline $7440-28-0$ & Thallium & $<1.03 \mathrm{E} 0$ & ug/g dry & $1.03 \mathrm{E} 0$ & 6/30/08 & 8F30003 & PNNL-AGG-ICP-AES \\
\hline $7440-62-2$ & Vanadium & $<4.53 \mathrm{E}-2$ & ug/g dry & 4.53E-2 & 6/30/08 & $8 F 30003$ & PNNL-AGG-ICP-AES \\
\hline $7440-66-6$ & Zinc & $<9.27 \mathrm{E}-2$ & ug/g dry & $9.27 \mathrm{E}-2$ & 6/30/08 & 8F30003 & PNNL-AGG-ICP-AES \\
\hline $7440-23-5$ & Sodium & 1.59E1 & ug/g dry & $6.69 \mathrm{E}-1$ & 6/30/08 & 8F30003 & PNNL-AGG-ICP-AES \\
\hline $7440-21-3$ & Silicon & 7.25E0 & ug/g dry & $1.50 \mathrm{E} 0$ & 6/30/08 & 8F30003 & PNNL-AGG-ICP-AES \\
\hline $7704-34-9$ & Sulfur & $1.92 \mathrm{E} 1$ & ug/g dry & 3.08E0 & 6/30/08 & 8F30003 & PNNL-AGG-ICP-AES \\
\hline $7440-32-6$ & Titanium & $<8.76 \mathrm{E}-3$ & ug/g dry & 8.76E-3 & 6/30/08 & 8F30003 & PNNL-AGG-ICP-AES \\
\hline $7440-67-7$ & Zirconium & $<1.50 \mathrm{E}-1$ & ug/g dry & $1.50 \mathrm{E}-1$ & 6/30/08 & 8F30003 & PNNL-AGG-ICP-AES \\
\hline $7440-22-4$ & Silver & $<7.71 \mathrm{E}-2$ & ug/g dry & $7.71 \mathrm{E}-2$ & 6/30/08 & $8 F 30003$ & PNNL-AGG-ICP-AES \\
\hline $7440-15-5$ & Rhenium & $<1.52 \mathrm{E}-1$ & ug/g dry & $1.52 \mathrm{E}-1$ & 6/30/08 & $8 F 30003$ & PNNL-AGG-ICP-AES \\
\hline $7440-36-0$ & Antimony & $<6.33 \mathrm{E}-1$ & ug/g dry & 6.33E-1 & 6/30/08 & 8F30003 & PNNL-AGG-ICP-AES \\
\hline HEIS No. & B1VJ61C & & b ID: & $5020-22$ & & & \\
\hline $7429-90-5$ & Aluminum & 1.72E-1 & ug/g dry & $8.58 \mathrm{E}-2$ & 6/30/08 & $8 F 30003$ & PNNL-AGG-ICP-AES \\
\hline $7440-38-2$ & Arsenic & $<3.60 \mathrm{E}-1$ & ug/g dry & $3.60 \mathrm{E}-1$ & 6/30/08 & $8 F 30003$ & PNNL-AGG-ICP-AES \\
\hline $7440-42-8$ & Boron & $<1.94 \mathrm{E} 0$ & ug/g dry & $1.94 \mathrm{E} 0$ & 6/30/08 & 8F30003 & PNNL-AGG-ICP-AES \\
\hline 7440-39-3 & Barium & $1.85 \mathrm{E}-2$ & ug/g dry & $8.79 \mathrm{E}-3$ & 6/30/08 & $8 F 30003$ & PNNL-AGG-ICP-AES \\
\hline
\end{tabular}


Total Metals by PNNL-AGG-ICP-AES/Water Extract

\begin{tabular}{|c|c|c|c|c|c|c|c|}
\hline CAS \# & Analyte & Results & Units & EQL & Analyzed & Batch & Method \\
\hline HEIS No. & B1VJ61C & \multicolumn{3}{|c|}{ Lab ID: $\quad 0805020-22$} & & & \\
\hline $7440-41-7$ & Beryllium & $<2.84 \mathrm{E}-2$ & ug/g dry & $2.84 \mathrm{E}-2$ & 6/30/08 & 8 F30003 & PNNL-AGG-ICP-AES \\
\hline 7440-69-9 & Bismuth & $<1.88 \mathrm{E}-1$ & ug/g dry & $1.88 \mathrm{E}-1$ & 6/30/08 & 8F30003 & PNNL-AGG-ICP-AES \\
\hline $7440-70-2$ & Calcium & 1.62E1 & ug/g dry & 3.87E-1 & 6/30/08 & $8 F 30003$ & PNNL-AGG-ICP-AES \\
\hline $7440-43-9$ & Cadmium & $<2.69 \mathrm{E}-2$ & ug/g dry & $2.69 \mathrm{E}-2$ & 6/30/08 & 8F30003 & PNNL-AGG-ICP-AES \\
\hline $7440-48-4$ & Cobalt & $<9.60 \mathrm{E}-2$ & ug/g dry & $9.60 \mathrm{E}-2$ & 6/30/08 & $8 F 30003$ & PNNL-AGG-ICP-AES \\
\hline $7440-47-3$ & Chromium & $<3.33 \mathrm{E}-2$ & ug/g dry & 3.33E-2 & 6/30/08 & $8 F 30003$ & PNNL-AGG-ICP-AES \\
\hline $7440-50-8$ & Copper & $<8.04 \mathrm{E}-2$ & ug/g dry & 8.04E-2 & 6/30/08 & 8F30003 & PNNL-AGG-ICP-AES \\
\hline 7439-89-6 & Iron & $<1.42 \mathrm{E}-1$ & ug/g dry & $1.42 \mathrm{E}-1$ & 6/30/08 & $8 F 30003$ & PNNL-AGG-ICP-AES \\
\hline 7440-09-7 & Potassium & $9.76 \mathrm{E} 0$ & ug/g dry & 2.33Е0 & 6/30/08 & 8F30003 & PNNL-AGG-ICP-AES \\
\hline $7439-93-2$ & Lithium & $<5.40 \mathrm{E}-1$ & ug/g dry & $5.40 \mathrm{E}-1$ & 6/30/08 & 8F30003 & PNNL-AGG-ICP-AES \\
\hline 7439-95-4 & Magnesium & 7.32E0 & ug/g dry & 8.34E-2 & 6/30/08 & 8F30003 & PNNL-AGG-ICP-AES \\
\hline 7439-96-5 & Manganese & $<1.71 \mathrm{E}-2$ & ug/g dry & $1.71 \mathrm{E}-2$ & 6/30/08 & $8 F 30003$ & PNNL-AGG-ICP-AES \\
\hline 7439-98-7 & Molybdenum & $<1.39 \mathrm{E}-1$ & ug/g dry & $1.39 \mathrm{E}-1$ & 6/30/08 & $8 F 30003$ & PNNL-AGG-ICP-AES \\
\hline $7440-02-0$ & Nickel & $<9.33 \mathrm{E}-2$ & ug/g dry & $9.33 \mathrm{E}-2$ & 6/30/08 & 8F30003 & PNNL-AGG-ICP-AES \\
\hline 7723-14-0 & Phosphorus & $<1.03 \mathrm{E} 0$ & ug/g dry & $1.03 \mathrm{E} 0$ & 6/30/08 & $8 F 30003$ & PNNL-AGG-ICP-AES \\
\hline 7439-92-1 & Lead & $<4.32 \mathrm{E}-1$ & ug/g dry & $4.32 \mathrm{E}-1$ & 6/30/08 & $8 F 30003$ & PNNL-AGG-ICP-AES \\
\hline $7782-49-2$ & Selenium & $<1.95 \mathrm{E} 0$ & ug/g dry & $1.95 \mathrm{E} 0$ & 6/30/08 & 8F30003 & PNNL-AGG-ICP-AES \\
\hline $7440-24-6$ & Strontium & $9.80 \mathrm{E}-2$ & ug/g dry & $5.22 \mathrm{E}-2$ & 6/30/08 & 8F30003 & PNNL-AGG-ICP-AES \\
\hline $7440-28-0$ & Thallium & $<1.03 \mathrm{E} 0$ & ug/g dry & $1.03 \mathrm{E} 0$ & 6/30/08 & 8F30003 & PNNL-AGG-ICP-AES \\
\hline $7440-62-2$ & Vanadium & $<4.53 \mathrm{E}-2$ & ug/g dry & 4.53E-2 & 6/30/08 & 8F30003 & PNNL-AGG-ICP-AES \\
\hline $7440-66-6$ & Zinc & $<9.27 \mathrm{E}-2$ & ug/g dry & $9.27 \mathrm{E}-2$ & 6/30/08 & $8 F 30003$ & PNNL-AGG-ICP-AES \\
\hline $7440-23-5$ & Sodium & 1.94E1 & ug/g dry & $6.69 \mathrm{E}-1$ & 6/30/08 & 8F30003 & PNNL-AGG-ICP-AES \\
\hline $7440-21-3$ & Silicon & $6.98 \mathrm{E} 0$ & ug/g dry & $1.50 \mathrm{E} 0$ & 6/30/08 & $8 F 30003$ & PNNL-AGG-ICP-AES \\
\hline 7704-34-9 & Sulfur & $2.12 \mathrm{E} 1$ & ug/g dry & 3.07E0 & 6/30/08 & 8F30003 & PNNL-AGG-ICP-AES \\
\hline $7440-32-6$ & Titanium & $<8.76 \mathrm{E}-3$ & ug/g dry & 8.76E-3 & 6/30/08 & 8F30003 & PNNL-AGG-ICP-AES \\
\hline $7440-67-7$ & Zirconium & $<1.50 \mathrm{E}-1$ & ug/g dry & $1.50 \mathrm{E}-1$ & 6/30/08 & 8F30003 & PNNL-AGG-ICP-AES \\
\hline $7440-22-4$ & Silver & $<7.71 \mathrm{E}-2$ & ug/g dry & $7.71 \mathrm{E}-2$ & 6/30/08 & 8F30003 & PNNL-AGG-ICP-AES \\
\hline $7440-15-5$ & Rhenium & $<1.52 \mathrm{E}-1$ & ug/g dry & $1.52 \mathrm{E}-1$ & 6/30/08 & 8F30003 & PNNL-AGG-ICP-AES \\
\hline $7440-36-0$ & Antimony & $<6.33 \mathrm{E}-1$ & ug/g dry & $6.33 \mathrm{E}-1$ & 6/30/08 & 8F30003 & PNNL-AGG-ICP-AES \\
\hline HEIS No. & B1VJ61B & & b ID: & $55020-23$ & & & \\
\hline $7429-90-5$ & Aluminum & $1.96 \mathrm{E}-1$ & ug/g dry & $8.67 \mathrm{E}-2$ & 6/30/08 & 8F30003 & PNNL-AGG-ICP-AES \\
\hline $7440-38-2$ & Arsenic & $<3.64 \mathrm{E}-1$ & ug/g dry & $3.64 \mathrm{E}-1$ & 6/30/08 & $8 F 30003$ & PNNL-AGG-ICP-AES \\
\hline $7440-42-8$ & Boron & $<1.96 \mathrm{E} 0$ & ug/g dry & $1.96 \mathrm{E} 0$ & 6/30/08 & $8 F 30003$ & PNNL-AGG-ICP-AES \\
\hline $7440-39-3$ & Barium & 2.37E-2 & ug/g dry & $8.88 \mathrm{E}-3$ & 6/30/08 & 8F30003 & PNNL-AGG-ICP-AES \\
\hline $7440-41-7$ & Beryllium & $<2.87 \mathrm{E}-2$ & ug/g dry & $2.87 \mathrm{E}-2$ & 6/30/08 & $8 F 30003$ & PNNL-AGG-ICP-AES \\
\hline 7440-69-9 & Bismuth & $<1.90 \mathrm{E}-1$ & ug/g dry & $1.90 \mathrm{E}-1$ & 6/30/08 & $8 F 30003$ & PNNL-AGG-ICP-AES \\
\hline $7440-70-2$ & Calcium & 1.40E1 & ug/g dry & 3.91E-1 & 6/30/08 & $8 F 30003$ & PNNL-AGG-ICP-AES \\
\hline $7440-43-9$ & Cadmium & $<2.72 \mathrm{E}-2$ & ug/g dry & $2.72 \mathrm{E}-2$ & 6/30/08 & 8F30003 & PNNL-AGG-ICP-AES \\
\hline $7440-48-4$ & Cobalt & $<9.70 \mathrm{E}-2$ & ug/g dry & $9.70 \mathrm{E}-2$ & 6/30/08 & 8F30003 & PNNL-AGG-ICP-AES \\
\hline $7440-47-3$ & Chromium & $<3.36 \mathrm{E}-2$ & ug/g dry & $3.36 \mathrm{E}-2$ & 6/30/08 & 8F30003 & PNNL-AGG-ICP-AES \\
\hline $7440-50-8$ & Copper & $<8.12 \mathrm{E}-2$ & ug/g dry & $8.12 \mathrm{E}-2$ & 6/30/08 & $8 F 30003$ & PNNL-AGG-ICP-AES \\
\hline 7439-89-6 & Iron & $<1.44 \mathrm{E}-1$ & ug/g dry & $1.44 \mathrm{E}-1$ & 6/30/08 & $8 F 30003$ & PNNL-AGG-ICP-AES \\
\hline 7440-09-7 & Potassium & $8.46 \mathrm{E} 0$ & ug/g dry & $2.35 \mathrm{E} 0$ & 6/30/08 & 8F30003 & PNNL-AGG-ICP-AES \\
\hline $7439-93-2$ & Lithium & $<5.45 \mathrm{E}-1$ & ug/g dry & $5.45 \mathrm{E}-1$ & 6/30/08 & 8F30003 & PNNL-AGG-ICP-AES \\
\hline 7439-95-4 & Magnesium & 6.20E0 & ug/g dry & $8.42 \mathrm{E}-2$ & 6/30/08 & $8 F 30003$ & PNNL-AGG-ICP-AES \\
\hline $7439-96-5$ & Manganese & $<1.73 \mathrm{E}-2$ & ug/g dry & $1.73 \mathrm{E}-2$ & 6/30/08 & 8F30003 & PNNL-AGG-ICP-AES \\
\hline 7439-98-7 & Molybdenum & $<1.41 \mathrm{E}-1$ & ug/g dry & $1.41 \mathrm{E}-1$ & 6/30/08 & 8F30003 & PNNL-AGG-ICP-AES \\
\hline 7440-02-0 & Nickel & $<9.42 \mathrm{E}-2$ & ug/g dry & $9.42 \mathrm{E}-2$ & 6/30/08 & 8F30003 & PNNL-AGG-ICP-AES \\
\hline
\end{tabular}


Total Metals by PNNL-AGG-ICP-AES/Water Extract

\begin{tabular}{|c|c|c|c|c|c|c|c|}
\hline CAS \# & Analyte & Results & Units & EQL & Analyzed & Batch & Method \\
\hline HEIS No. & B1VJ61B & \multicolumn{3}{|c|}{ Lab ID: $\quad 0805020-23$} & & & \\
\hline 7723-14-0 & Phosphorus & $<1.04 \mathrm{E} 0$ & ug/g dry & $1.04 \mathrm{E} 0$ & 6/30/08 & 8 F30003 & PNNL-AGG-ICP-AES \\
\hline 7439-92-1 & Lead & $<4.36 \mathrm{E}-1$ & ug/g dry & $4.36 \mathrm{E}-1$ & 6/30/08 & 8F30003 & PNNL-AGG-ICP-AES \\
\hline $7782-49-2$ & Selenium & $<1.97 \mathrm{E} 0$ & ug/g dry & 1.97E0 & 6/30/08 & 8F30003 & PNNL-AGG-ICP-AES \\
\hline $7440-24-6$ & Strontium & $8.66 \mathrm{E}-2$ & ug/g dry & $5.27 \mathrm{E}-2$ & 6/30/08 & 8F30003 & PNNL-AGG-ICP-AES \\
\hline $7440-28-0$ & Thallium & $<1.04 \mathrm{E} 0$ & ug/g dry & $1.04 \mathrm{E} 0$ & 6/30/08 & $8 F 30003$ & PNNL-AGG-ICP-AES \\
\hline $7440-62-2$ & Vanadium & $<4.58 \mathrm{E}-2$ & ug/g dry & $4.58 \mathrm{E}-2$ & 6/30/08 & $8 F 30003$ & PNNL-AGG-ICP-AES \\
\hline $7440-66-6$ & Zinc & $<9.36 \mathrm{E}-2$ & ug/g dry & $9.36 \mathrm{E}-2$ & $6 / 30 / 08$ & 8F30003 & PNNL-AGG-ICP-AES \\
\hline $7440-23-5$ & Sodium & 1.57E1 & ug/g dry & $6.76 \mathrm{E}-1$ & 6/30/08 & $8 F 30003$ & PNNL-AGG-ICP-AES \\
\hline $7440-21-3$ & Silicon & 7.20E0 & ug/g dry & $1.52 \mathrm{E} 0$ & 6/30/08 & 8F30003 & PNNL-AGG-ICP-AES \\
\hline $7704-34-9$ & Sulfur & $1.87 \mathrm{E} 1$ & ug/g dry & 3.11E0 & 6/30/08 & 8F30003 & PNNL-AGG-ICP-AES \\
\hline $7440-32-6$ & Titanium & $<8.85 \mathrm{E}-3$ & ug/g dry & 8.85E-3 & 6/30/08 & 8F30003 & PNNL-AGG-ICP-AES \\
\hline $7440-67-7$ & Zirconium & $<1.52 \mathrm{E}-1$ & ug/g dry & $1.52 \mathrm{E}-1$ & 6/30/08 & $8 F 30003$ & PNNL-AGG-ICP-AES \\
\hline $7440-22-4$ & Silver & $<7.79 \mathrm{E}-2$ & ug/g dry & 7.79E-2 & 6/30/08 & $8 F 30003$ & PNNL-AGG-ICP-AES \\
\hline $7440-15-5$ & Rhenium & $<1.53 \mathrm{E}-1$ & ug/g dry & $1.53 \mathrm{E}-1$ & 6/30/08 & 8F30003 & PNNL-AGG-ICP-AES \\
\hline $7440-36-0$ & Antimony & $<6.39 \mathrm{E}-1$ & ug/g dry & $6.39 \mathrm{E}-1$ & 6/30/08 & 8F30003 & PNNL-AGG-ICP-AES \\
\hline HEIS No. & B1VJ61A & \multicolumn{3}{|c|}{ Lab ID: $\quad$ 0805020-24 } & & & \\
\hline $7429-90-5$ & Aluminum & $2.04 \mathrm{E}-1$ & ug/g dry & $8.91 \mathrm{E}-2$ & 6/30/08 & 8F30003 & PNNL-AGG-ICP-AES \\
\hline $7440-38-2$ & Arsenic & $<3.74 \mathrm{E}-1$ & ug/g dry & $3.74 \mathrm{E}-1$ & 6/30/08 & 8F30003 & PNNL-AGG-ICP-AES \\
\hline $7440-42-8$ & Boron & $<2.01 \mathrm{E} 0$ & ug/g dry & $2.01 \mathrm{E} 0$ & $6 / 30 / 08$ & 8F30003 & PNNL-AGG-ICP-AES \\
\hline $7440-39-3$ & Barium & $1.95 \mathrm{E}-2$ & ug/g dry & $9.12 \mathrm{E}-3$ & 6/30/08 & 8F30003 & PNNL-AGG-ICP-AES \\
\hline $7440-41-7$ & Beryllium & $<2.95 \mathrm{E}-2$ & ug/g dry & $2.95 \mathrm{E}-2$ & 6/30/08 & $8 F 30003$ & PNNL-AGG-ICP-AES \\
\hline $7440-69-9$ & Bismuth & $<1.95 \mathrm{E}-1$ & ug/g dry & $1.95 \mathrm{E}-1$ & 6/30/08 & $8 F 30003$ & PNNL-AGG-ICP-AES \\
\hline $7440-70-2$ & Calcium & 1.39E1 & ug/g dry & 4.02E-1 & 6/30/08 & $8 F 30003$ & PNNL-AGG-ICP-AES \\
\hline $7440-43-9$ & Cadmium & $<2.79 \mathrm{E}-2$ & ug/g dry & $2.79 \mathrm{E}-2$ & 6/30/08 & $8 F 30003$ & PNNL-AGG-ICP-AES \\
\hline $7440-48-4$ & Cobalt & $<9.97 \mathrm{E}-2$ & ug/g dry & $9.97 \mathrm{E}-2$ & 6/30/08 & 8F30003 & PNNL-AGG-ICP-AES \\
\hline $7440-47-3$ & Chromium & $<3.46 \mathrm{E}-2$ & ug/g dry & 3.46E-2 & 6/30/08 & $8 F 30003$ & PNNL-AGG-ICP-AES \\
\hline $7440-50-8$ & Copper & $<8.35 \mathrm{E}-2$ & ug/g dry & 8.35E-2 & 6/30/08 & $8 F 30003$ & PNNL-AGG-ICP-AES \\
\hline 7439-89-6 & Iron & $<1.48 \mathrm{E}-1$ & ug/g dry & $1.48 \mathrm{E}-1$ & 6/30/08 & 8F30003 & PNNL-AGG-ICP-AES \\
\hline 7440-09-7 & Potassium & 7.57E0 & ug/g dry & $2.42 \mathrm{E} 0$ & 6/30/08 & 8F30003 & PNNL-AGG-ICP-AES \\
\hline $7439-93-2$ & Lithium & $<5.61 \mathrm{E}-1$ & ug/g dry & $5.61 \mathrm{E}-1$ & 6/30/08 & 8F30003 & PNNL-AGG-ICP-AES \\
\hline 7439-95-4 & Magnesium & 6.31E0 & ug/g dry & $8.66 \mathrm{E}-2$ & 6/30/08 & $8 F 30003$ & PNNL-AGG-ICP-AES \\
\hline $7439-96-5$ & Manganese & $<1.78 \mathrm{E}-2$ & ug/g dry & $1.78 \mathrm{E}-2$ & 6/30/08 & $8 F 30003$ & PNNL-AGG-ICP-AES \\
\hline 7439-98-7 & Molybdenum & $<1.44 \mathrm{E}-1$ & ug/g dry & $1.44 \mathrm{E}-1$ & 6/30/08 & $8 F 30003$ & PNNL-AGG-ICP-AES \\
\hline $7440-02-0$ & Nickel & $<9.68 \mathrm{E}-2$ & ug/g dry & $9.68 \mathrm{E}-2$ & 6/30/08 & 8F30003 & PNNL-AGG-ICP-AES \\
\hline $7723-14-0$ & Phosphorus & $<1.07 \mathrm{E} 0$ & ug/g dry & 1.07E0 & 6/30/08 & 8F30003 & PNNL-AGG-ICP-AES \\
\hline 7439-92-1 & Lead & $<4.48 \mathrm{E}-1$ & ug/g dry & $4.48 \mathrm{E}-1$ & 6/30/08 & 8F30003 & PNNL-AGG-ICP-AES \\
\hline 7782-49-2 & Selenium & $<2.02 \mathrm{E} 0$ & ug/g dry & $2.02 \mathrm{E} 0$ & 6/30/08 & $8 F 30003$ & PNNL-AGG-ICP-AES \\
\hline $7440-24-6$ & Strontium & $8.52 \mathrm{E}-2$ & ug/g dry & $5.42 \mathrm{E}-2$ & 6/30/08 & 8F30003 & PNNL-AGG-ICP-AES \\
\hline $7440-28-0$ & Thallium & $<1.07 \mathrm{E} 0$ & ug/g dry & 1.07E0 & 6/30/08 & 8F30003 & PNNL-AGG-ICP-AES \\
\hline $7440-62-2$ & Vanadium & $<4.70 \mathrm{E}-2$ & ug/g dry & $4.70 \mathrm{E}-2$ & 6/30/08 & 8F30003 & PNNL-AGG-ICP-AES \\
\hline $7440-66-6$ & Zinc & $<9.62 \mathrm{E}-2$ & ug/g dry & $9.62 \mathrm{E}-2$ & 6/30/08 & $8 F 30003$ & PNNL-AGG-ICP-AES \\
\hline $7440-23-5$ & Sodium & 1.46E1 & ug/g dry & $6.94 \mathrm{E}-1$ & 6/30/08 & $8 F 30003$ & PNNL-AGG-ICP-AES \\
\hline $7440-21-3$ & Silicon & 7.73E0 & ug/g dry & $1.56 \mathrm{E} 0$ & 6/30/08 & 8F30003 & PNNL-AGG-ICP-AES \\
\hline 7704-34-9 & Sulfur & $1.96 \mathrm{E} 1$ & ug/g dry & 3.19E0 & 6/30/08 & 8F30003 & PNNL-AGG-ICP-AES \\
\hline $7440-32-6$ & Titanium & $<9.09 \mathrm{E}-3$ & ug/g dry & $9.09 \mathrm{E}-3$ & 6/30/08 & $8 F 30003$ & PNNL-AGG-ICP-AES \\
\hline $7440-67-7$ & Zirconium & $<1.56 \mathrm{E}-1$ & ug/g dry & $1.56 \mathrm{E}-1$ & 6/30/08 & 8F30003 & PNNL-AGG-ICP-AES \\
\hline $7440-22-4$ & Silver & $<8.00 \mathrm{E}-2$ & ug/g dry & $8.00 \mathrm{E}-2$ & 6/30/08 & 8F30003 & PNNL-AGG-ICP-AES \\
\hline $7440-15-5$ & Rhenium & $<1.58 \mathrm{E}-1$ & ug/g dry & $1.58 \mathrm{E}-1$ & 6/30/08 & 8F30003 & PNNL-AGG-ICP-AES \\
\hline
\end{tabular}


Total Metals by PNNL-AGG-ICP-AES/Water Extract

\begin{tabular}{|c|c|c|c|c|c|c|c|}
\hline CAS \# & Analyte & Results & Units & EQL & Analyzed & Batch & Method \\
\hline HEIS No. & B1VJ61A & \multicolumn{3}{|c|}{ Lab ID: $\quad$ 0805020-24 } & & & \\
\hline $7440-36-0$ & Antimony & $<6.57 \mathrm{E}-1$ & ug/g dry & $6.57 \mathrm{E}-1$ & 6/30/08 & $8 F 30003$ & PNNL-AGG-ICP-AES \\
\hline HEIS No. & B1VJ62C & \multicolumn{3}{|c|}{ Lab ID: $\quad$ 0805020-26 } & & & \\
\hline $7429-90-5$ & Aluminum & $<8.58 \mathrm{E}-2$ & ug/g dry & $8.58 \mathrm{E}-2$ & 6/30/08 & 8F30003 & PNNL-AGG-ICP-AES \\
\hline $7440-38-2$ & Arsenic & $<3.60 \mathrm{E}-1$ & ug/g dry & $3.60 \mathrm{E}-1$ & 6/30/08 & 8F30003 & PNNL-AGG-ICP-AES \\
\hline $7440-42-8$ & Boron & $<1.94 \mathrm{E} 0$ & ug/g dry & $1.94 \mathrm{E} 0$ & 6/30/08 & 8F30003 & PNNL-AGG-ICP-AES \\
\hline 7440-39-3 & Barium & $3.01 \mathrm{E}-2$ & ug/g dry & 8.79E-3 & 6/30/08 & 8F30003 & PNNL-AGG-ICP-AES \\
\hline $7440-41-7$ & Beryllium & $<2.84 \mathrm{E}-2$ & ug/g dry & $2.84 \mathrm{E}-2$ & 6/30/08 & 8F30003 & PNNL-AGG-ICP-AES \\
\hline $7440-69-9$ & Bismuth & $<1.88 \mathrm{E}-1$ & ug/g dry & $1.88 \mathrm{E}-1$ & 6/30/08 & 8F30003 & PNNL-AGG-ICP-AES \\
\hline $7440-70-2$ & Calcium & 5.98E1 & ug/g dry & 3.87E-1 & 6/30/08 & 8F30003 & PNNL-AGG-ICP-AES \\
\hline $7440-43-9$ & Cadmium & $<2.69 \mathrm{E}-2$ & ug/g dry & $2.69 \mathrm{E}-2$ & 6/30/08 & 8F30003 & PNNL-AGG-ICP-AES \\
\hline $7440-48-4$ & Cobalt & $<9.60 \mathrm{E}-2$ & ug/g dry & $9.60 \mathrm{E}-2$ & 6/30/08 & 8F30003 & PNNL-AGG-ICP-AES \\
\hline $7440-47-3$ & Chromium & $<3.33 \mathrm{E}-2$ & ug/g dry & 3.33E-2 & 6/30/08 & 8F30003 & PNNL-AGG-ICP-AES \\
\hline $7440-50-8$ & Copper & $<8.04 \mathrm{E}-2$ & ug/g dry & 8.04E-2 & 6/30/08 & 8F30003 & PNNL-AGG-ICP-AES \\
\hline 7439-89-6 & Iron & $<1.42 \mathrm{E}-1$ & ug/g dry & 1.42E-1 & 6/30/08 & $8 F 30003$ & PNNL-AGG-ICP-AES \\
\hline 7440-09-7 & Potassium & 1.79E1 & ug/g dry & 2.33E0 & 6/30/08 & 8 F30003 & PNNL-AGG-ICP-AES \\
\hline 7439-93-2 & Lithium & $<5.40 \mathrm{E}-1$ & ug/g dry & $5.40 \mathrm{E}-1$ & 6/30/08 & 8F30003 & PNNL-AGG-ICP-AES \\
\hline 7439-95-4 & Magnesium & 2.34E1 & ug/g dry & 8.34E-2 & 6/30/08 & $8 F 30003$ & PNNL-AGG-ICP-AES \\
\hline 7439-96-5 & Manganese & 8.01E-2 & ug/g dry & $1.71 \mathrm{E}-2$ & 6/30/08 & 8F30003 & PNNL-AGG-ICP-AES \\
\hline 7439-98-7 & Molybdenum & $1.82 \mathrm{E}-1$ & ug/g dry & $1.39 \mathrm{E}-1$ & 6/30/08 & 8F30003 & PNNL-AGG-ICP-AES \\
\hline $7440-02-0$ & Nickel & $<9.33 \mathrm{E}-2$ & ug/g dry & $9.33 \mathrm{E}-2$ & 6/30/08 & 8F30003 & PNNL-AGG-ICP-AES \\
\hline $7723-14-0$ & Phosphorus & $<1.03 \mathrm{E} 0$ & ug/g dry & $1.03 \mathrm{E} 0$ & 6/30/08 & $8 F 30003$ & PNNL-AGG-ICP-AES \\
\hline 7439-92-1 & Lead & $<4.32 \mathrm{E}-1$ & ug/g dry & 4.32E-1 & 6/30/08 & 8F30003 & PNNL-AGG-ICP-AES \\
\hline $7782-49-2$ & Selenium & $<1.95 \mathrm{E} 0$ & ug/g dry & $1.95 \mathrm{E} 0$ & 6/30/08 & 8F30003 & PNNL-AGG-ICP-AES \\
\hline $7440-24-6$ & Strontium & $2.95 \mathrm{E}-1$ & ug/g dry & $5.22 \mathrm{E}-2$ & 6/30/08 & 8F30003 & PNNL-AGG-ICP-AES \\
\hline $7440-28-0$ & Thallium & $<1.03 \mathrm{E} 0$ & ug/g dry & 1.03E0 & 6/30/08 & 8F30003 & PNNL-AGG-ICP-AES \\
\hline $7440-62-2$ & Vanadium & $<4.53 \mathrm{E}-2$ & ug/g dry & 4.53E-2 & 6/30/08 & 8F30003 & PNNL-AGG-ICP-AES \\
\hline $7440-66-6$ & Zinc & $<9.27 \mathrm{E}-2$ & ug/g dry & $9.27 \mathrm{E}-2$ & 6/30/08 & 8F30003 & PNNL-AGG-ICP-AES \\
\hline $7440-23-5$ & Sodium & 3.19E1 & ug/g dry & $6.69 \mathrm{E}-1$ & 6/30/08 & 8F30003 & PNNL-AGG-ICP-AES \\
\hline $7440-21-3$ & Silicon & 7.31E0 & ug/g dry & $1.50 \mathrm{E} 0$ & 6/30/08 & 8F30003 & PNNL-AGG-ICP-AES \\
\hline 7704-34-9 & Sulfur & $5.45 \mathrm{E} 1$ & ug/g dry & 3.07E0 & 6/30/08 & 8F30003 & PNNL-AGG-ICP-AES \\
\hline $7440-32-6$ & Titanium & $<8.76 \mathrm{E}-3$ & ug/g dry & $8.76 \mathrm{E}-3$ & 6/30/08 & 8F30003 & PNNL-AGG-ICP-AES \\
\hline $7440-67-7$ & Zirconium & $<1.50 \mathrm{E}-1$ & ug/g dry & $1.50 \mathrm{E}-1$ & 6/30/08 & $8 F 30003$ & PNNL-AGG-ICP-AES \\
\hline $7440-22-4$ & Silver & $<7.71 \mathrm{E}-2$ & ug/g dry & $7.71 \mathrm{E}-2$ & 6/30/08 & $8 F 30003$ & PNNL-AGG-ICP-AES \\
\hline $7440-15-5$ & Rhenium & $<1.52 \mathrm{E}-1$ & ug/g dry & $1.52 \mathrm{E}-1$ & 6/30/08 & 8F30003 & PNNL-AGG-ICP-AES \\
\hline 7440-36-0 & Antimony & $<6.33 \mathrm{E}-1$ & ug/g dry & 6.33E-1 & 6/30/08 & 8F30003 & PNNL-AGG-ICP-AES \\
\hline HEIS No. & B1VJ62B & & b ID: & 5020-27 & & & \\
\hline $7429-90-5$ & Aluminum & $<8.59 \mathrm{E}-2$ & ug/g dry & $8.59 \mathrm{E}-2$ & 6/30/08 & 8F30003 & PNNL-AGG-ICP-AES \\
\hline $7440-38-2$ & Arsenic & $<3.60 \mathrm{E}-1$ & ug/g dry & $3.60 \mathrm{E}-1$ & 6/30/08 & $8 F 30003$ & PNNL-AGG-ICP-AES \\
\hline $7440-42-8$ & Boron & $<1.94 \mathrm{E} 0$ & ug/g dry & $1.94 \mathrm{E} 0$ & 6/30/08 & 8F30003 & PNNL-AGG-ICP-AES \\
\hline $7440-39-3$ & Barium & $3.50 \mathrm{E}-2$ & ug/g dry & $8.80 \mathrm{E}-3$ & 7/07/08 & 8F30003 & PNNL-AGG-ICP-AES \\
\hline $7440-41-7$ & Beryllium & $<2.84 \mathrm{E}-2$ & ug/g dry & $2.84 \mathrm{E}-2$ & 6/30/08 & 8F30003 & PNNL-AGG-ICP-AES \\
\hline 7440-69-9 & Bismuth & $<1.88 \mathrm{E}-1$ & ug/g dry & $1.88 \mathrm{E}-1$ & 6/30/08 & $8 F 30003$ & PNNL-AGG-ICP-AES \\
\hline $7440-70-2$ & Calcium & $4.11 \mathrm{E} 1$ & ug/g dry & 3.87E-1 & 7/07/08 & 8F30003 & PNNL-AGG-ICP-AES \\
\hline $7440-43-9$ & Cadmium & $<2.69 \mathrm{E}-2$ & ug/g dry & $2.69 \mathrm{E}-2$ & 6/30/08 & $8 F 30003$ & PNNL-AGG-ICP-AES \\
\hline $7440-48-4$ & Cobalt & $<9.61 \mathrm{E}-2$ & ug/g dry & $9.61 \mathrm{E}-2$ & 6/30/08 & 8F30003 & PNNL-AGG-ICP-AES \\
\hline $7440-47-3$ & Chromium & $<3.33 \mathrm{E}-2$ & ug/g dry & 3.33E-2 & 6/30/08 & 8F30003 & PNNL-AGG-ICP-AES \\
\hline $7440-50-8$ & Copper & $<8.05 \mathrm{E}-2$ & ug/g dry & 8.05E-2 & 6/30/08 & 8F30003 & PNNL-AGG-ICP-AES \\
\hline 7439-89-6 & Iron & $<1.42 \mathrm{E}-1$ & ug/g dry & $1.42 \mathrm{E}-1$ & 6/30/08 & 8F30003 & PNNL-AGG-ICP-AES \\
\hline
\end{tabular}


Total Metals by PNNL-AGG-ICP-AES/Water Extract

\begin{tabular}{|c|c|c|c|c|c|c|c|}
\hline CAS \# & Analyte & Results & Units & EQL & Analyzed & Batch & Method \\
\hline HEIS No. & B1VJ62B & \multicolumn{3}{|c|}{ Lab ID: $\quad 0805020-27$} & & & \\
\hline 7440-09-7 & Potassium & $1.23 \mathrm{E} 1$ & ug/g dry & $2.33 \mathrm{E} 0$ & 7/07/08 & 8 F30003 & PNNL-AGG-ICP-AES \\
\hline 7439-93-2 & Lithium & $<5.40 \mathrm{E}-1$ & ug/g dry & $5.40 \mathrm{E}-1$ & 6/30/08 & 8F30003 & PNNL-AGG-ICP-AES \\
\hline 7439-95-4 & Magnesium & 1.59E1 & ug/g dry & 8.35E-2 & $7 / 07 / 08$ & $8 F 30003$ & PNNL-AGG-ICP-AES \\
\hline 7439-96-5 & Manganese & $<1.71 \mathrm{E}-2$ & ug/g dry & $1.71 \mathrm{E}-2$ & 6/30/08 & 8F30003 & PNNL-AGG-ICP-AES \\
\hline 7439-98-7 & Molybdenum & $<1.39 \mathrm{E}-1$ & ug/g dry & $1.39 \mathrm{E}-1$ & 6/30/08 & $8 F 30003$ & PNNL-AGG-ICP-AES \\
\hline 7440-02-0 & Nickel & $<9.34 \mathrm{E}-2$ & ug/g dry & $9.34 \mathrm{E}-2$ & 6/30/08 & $8 F 30003$ & PNNL-AGG-ICP-AES \\
\hline $7723-14-0$ & Phosphorus & $<1.03 \mathrm{E} 0$ & ug/g dry & 1.03E0 & 6/30/08 & 8F30003 & PNNL-AGG-ICP-AES \\
\hline $7439-92-1$ & Lead & $<4.32 \mathrm{E}-1$ & ug/g dry & $4.32 \mathrm{E}-1$ & 6/30/08 & 8F30003 & PNNL-AGG-ICP-AES \\
\hline $7782-49-2$ & Selenium & $<1.95 \mathrm{E} 0$ & ug/g dry & $1.95 \mathrm{E} 0$ & 6/30/08 & 8F30003 & PNNL-AGG-ICP-AES \\
\hline $7440-24-6$ & Strontium & $1.93 \mathrm{E}-1$ & ug/g dry & $5.22 \mathrm{E}-2$ & 6/30/08 & 8F30003 & PNNL-AGG-ICP-AES \\
\hline $7440-28-0$ & Thallium & $<1.03 \mathrm{E} 0$ & ug/g dry & 1.03E0 & 6/30/08 & 8F30003 & PNNL-AGG-ICP-AES \\
\hline $7440-62-2$ & Vanadium & $<4.53 \mathrm{E}-2$ & ug/g dry & 4.53E-2 & 6/30/08 & $8 F 30003$ & PNNL-AGG-ICP-AES \\
\hline $7440-66-6$ & Zinc & $<9.28 \mathrm{E}-2$ & ug/g dry & $9.28 \mathrm{E}-2$ & 6/30/08 & $8 F 30003$ & PNNL-AGG-ICP-AES \\
\hline $7440-23-5$ & Sodium & 1.99E1 & ug/g dry & $6.70 \mathrm{E}-1$ & $7 / 07 / 08$ & 8F30003 & PNNL-AGG-ICP-AES \\
\hline $7440-21-3$ & Silicon & 8.60Е0 & ug/g dry & $1.50 \mathrm{E} 0$ & $7 / 07 / 08$ & 8F30003 & PNNL-AGG-ICP-AES \\
\hline $7704-34-9$ & Sulfur & 4.03E1 & ug/g dry & $3.08 \mathrm{E} 0$ & $7 / 07 / 08$ & $8 F 30003$ & PNNL-AGG-ICP-AES \\
\hline $7440-32-6$ & Titanium & $<8.77 \mathrm{E}-3$ & ug/g dry & 8.77E-3 & 6/30/08 & 8F30003 & PNNL-AGG-ICP-AES \\
\hline $7440-67-7$ & Zirconium & $<1.50 \mathrm{E}-1$ & ug/g dry & $1.50 \mathrm{E}-1$ & 6/30/08 & 8F30003 & PNNL-AGG-ICP-AES \\
\hline $7440-22-4$ & Silver & $<7.72 \mathrm{E}-2$ & ug/g dry & $7.72 \mathrm{E}-2$ & $7 / 07 / 08$ & 8F30003 & PNNL-AGG-ICP-AES \\
\hline $7440-15-5$ & Rhenium & $<1.52 \mathrm{E}-1$ & ug/g dry & $1.52 \mathrm{E}-1$ & 6/30/08 & 8F30003 & PNNL-AGG-ICP-AES \\
\hline $7440-36-0$ & Antimony & $<6.34 \mathrm{E}-1$ & ug/g dry & $6.34 \mathrm{E}-1$ & 6/30/08 & 8F30003 & PNNL-AGG-ICP-AES \\
\hline HEIS No. & B1VJ62A & \multicolumn{3}{|c|}{ Lab ID: $\quad 0805020-28$} & & & \\
\hline $7429-90-5$ & Aluminum & $<8.59 \mathrm{E}-2$ & ug/g dry & $8.59 \mathrm{E}-2$ & 6/30/08 & $8 F 30003$ & PNNL-AGG-ICP-AES \\
\hline $7440-38-2$ & Arsenic & $<3.61 \mathrm{E}-1$ & ug/g dry & $3.61 \mathrm{E}-1$ & 6/30/08 & $8 F 30003$ & PNNL-AGG-ICP-AES \\
\hline $7440-42-8$ & Boron & $<1.94 \mathrm{E} 0$ & ug/g dry & $1.94 \mathrm{E} 0$ & 6/30/08 & $8 F 30003$ & PNNL-AGG-ICP-AES \\
\hline $7440-39-3$ & Barium & $2.41 \mathrm{E}-2$ & ug/g dry & $8.80 \mathrm{E}-3$ & 6/30/08 & $8 F 30003$ & PNNL-AGG-ICP-AES \\
\hline $7440-41-7$ & Beryllium & $<2.85 \mathrm{E}-2$ & ug/g dry & $2.85 \mathrm{E}-2$ & 6/30/08 & 8F30003 & PNNL-AGG-ICP-AES \\
\hline $7440-69-9$ & Bismuth & $<1.88 \mathrm{E}-1$ & ug/g dry & $1.88 \mathrm{E}-1$ & 6/30/08 & 8F30003 & PNNL-AGG-ICP-AES \\
\hline $7440-70-2$ & Calcium & 4.16E1 & ug/g dry & $3.88 \mathrm{E}-1$ & 6/30/08 & 8F30003 & PNNL-AGG-ICP-AES \\
\hline $7440-43-9$ & Cadmium & $<2.69 \mathrm{E}-2$ & ug/g dry & $2.69 \mathrm{E}-2$ & 6/30/08 & 8F30003 & PNNL-AGG-ICP-AES \\
\hline $7440-48-4$ & Cobalt & $<9.62 \mathrm{E}-2$ & ug/g dry & $9.62 \mathrm{E}-2$ & 6/30/08 & $8 F 30003$ & PNNL-AGG-ICP-AES \\
\hline $7440-47-3$ & Chromium & $<3.34 \mathrm{E}-2$ & ug/g dry & $3.34 \mathrm{E}-2$ & 6/30/08 & $8 F 30003$ & PNNL-AGG-ICP-AES \\
\hline $7440-50-8$ & Copper & $<8.05 \mathrm{E}-2$ & ug/g dry & 8.05E-2 & 6/30/08 & $8 F 30003$ & PNNL-AGG-ICP-AES \\
\hline 7439-89-6 & Iron & $<1.42 \mathrm{E}-1$ & ug/g dry & $1.42 \mathrm{E}-1$ & 6/30/08 & 8F30003 & PNNL-AGG-ICP-AES \\
\hline 7440-09-7 & Potassium & $1.20 \mathrm{E} 1$ & ug/g dry & 2.33E0 & 6/30/08 & $8 F 30003$ & PNNL-AGG-ICP-AES \\
\hline $7439-93-2$ & Lithium & $<5.41 \mathrm{E}-1$ & ug/g dry & $5.41 \mathrm{E}-1$ & 6/30/08 & 8F30003 & PNNL-AGG-ICP-AES \\
\hline 7439-95-4 & Magnesium & 1.67E1 & ug/g dry & 8.35E-2 & 6/30/08 & $8 F 30003$ & PNNL-AGG-ICP-AES \\
\hline $7439-96-5$ & Manganese & $<1.72 \mathrm{E}-2$ & ug/g dry & $1.72 \mathrm{E}-2$ & 6/30/08 & 8F30003 & PNNL-AGG-ICP-AES \\
\hline 7439-98-7 & Molybdenum & $<1.39 \mathrm{E}-1$ & ug/g dry & $1.39 \mathrm{E}-1$ & 6/30/08 & 8F30003 & PNNL-AGG-ICP-AES \\
\hline $7440-02-0$ & Nickel & $<9.34 \mathrm{E}-2$ & ug/g dry & $9.34 \mathrm{E}-2$ & 6/30/08 & 8F30003 & PNNL-AGG-ICP-AES \\
\hline $7723-14-0$ & Phosphorus & $<1.03 \mathrm{E} 0$ & ug/g dry & 1.03E0 & 6/30/08 & $8 F 30003$ & PNNL-AGG-ICP-AES \\
\hline 7439-92-1 & Lead & $<4.33 \mathrm{E}-1$ & ug/g dry & 4.33E-1 & 6/30/08 & 8F30003 & PNNL-AGG-ICP-AES \\
\hline $7782-49-2$ & Selenium & $<1.95 \mathrm{E} 0$ & ug/g dry & $1.95 \mathrm{E} 0$ & 6/30/08 & 8F30003 & PNNL-AGG-ICP-AES \\
\hline $7440-24-6$ & Strontium & $2.05 \mathrm{E}-1$ & ug/g dry & $5.23 \mathrm{E}-2$ & 6/30/08 & $8 F 30003$ & PNNL-AGG-ICP-AES \\
\hline $7440-28-0$ & Thallium & $<1.03 \mathrm{E} 0$ & ug/g dry & 1.03E0 & 6/30/08 & $8 F 30003$ & PNNL-AGG-ICP-AES \\
\hline $7440-62-2$ & Vanadium & $<4.54 \mathrm{E}-2$ & ug/g dry & $4.54 \mathrm{E}-2$ & 6/30/08 & 8F30003 & PNNL-AGG-ICP-AES \\
\hline $7440-66-6$ & Zinc & $<9.28 \mathrm{E}-2$ & ug/g dry & $9.28 \mathrm{E}-2$ & 6/30/08 & 8F30003 & PNNL-AGG-ICP-AES \\
\hline $7440-23-5$ & Sodium & 1.82E1 & ug/g dry & $6.70 \mathrm{E}-1$ & 6/30/08 & 8F30003 & PNNL-AGG-ICP-AES \\
\hline
\end{tabular}


Total Metals by PNNL-AGG-ICP-AES/Water Extract

\begin{tabular}{|c|c|c|c|c|c|c|c|}
\hline CAS \# & Analyte & Results & Units & EQL & Analyzed & Batch & Method \\
\hline HEIS No. & B1VJ62A & \multicolumn{3}{|c|}{ Lab ID: $\quad 0805020-28$} & & & \\
\hline $7440-21-3$ & Silicon & $8.16 \mathrm{E} 0$ & ug/g dry & $1.50 \mathrm{E} 0$ & 6/30/08 & 8F30003 & PNNL-AGG-ICP-AES \\
\hline 7704-34-9 & Sulfur & $5.21 \mathrm{E} 1$ & ug/g dry & 3.08E0 & 6/30/08 & $8 F 30003$ & PNNL-AGG-ICP-AES \\
\hline $7440-32-6$ & Titanium & $<8.77 \mathrm{E}-3$ & ug/g dry & 8.77E-3 & 6/30/08 & 8 F30003 & PNNL-AGG-ICP-AES \\
\hline $7440-67-7$ & Zirconium & $<1.50 \mathrm{E}-1$ & ug/g dry & $1.50 \mathrm{E}-1$ & 6/30/08 & $8 F 30003$ & PNNL-AGG-ICP-AES \\
\hline $7440-22-4$ & Silver & $<7.72 \mathrm{E}-2$ & ug/g dry & $7.72 \mathrm{E}-2$ & 6/30/08 & $8 F 30003$ & PNNL-AGG-ICP-AES \\
\hline $7440-15-5$ & Rhenium & $<1.52 \mathrm{E}-1$ & ug/g dry & $1.52 \mathrm{E}-1$ & 6/30/08 & $8 F 30003$ & PNNL-AGG-ICP-AES \\
\hline $7440-36-0$ & Antimony & $<6.34 \mathrm{E}-1$ & ug/g dry & $6.34 \mathrm{E}-1$ & $6 / 30 / 08$ & 8F30003 & PNNL-AGG-ICP-AES \\
\hline HEIS No. & B1VJ64C & \multicolumn{3}{|c|}{ Lab ID: $\quad$ 0805020-30 } & & & \\
\hline $7429-90-5$ & Aluminum & $<8.58 \mathrm{E}-2$ & ug/g dry & $8.58 \mathrm{E}-2$ & 6/30/08 & 8F30003 & PNNL-AGG-ICP-AES \\
\hline $7440-38-2$ & Arsenic & $<3.60 \mathrm{E}-1$ & ug/g dry & 3.60E-1 & 6/30/08 & $8 F 30003$ & PNNL-AGG-ICP-AES \\
\hline $7440-42-8$ & Boron & $<1.94 \mathrm{E} 0$ & ug/g dry & $1.94 \mathrm{E} 0$ & 6/30/08 & $8 F 30003$ & PNNL-AGG-ICP-AES \\
\hline $7440-39-3$ & Barium & $2.20 \mathrm{E}-2$ & ug/g dry & 8.79E-3 & 6/30/08 & $8 F 30003$ & PNNL-AGG-ICP-AES \\
\hline $7440-41-7$ & Beryllium & $<2.84 \mathrm{E}-2$ & ug/g dry & $2.84 \mathrm{E}-2$ & 6/30/08 & 8F30003 & PNNL-AGG-ICP-AES \\
\hline $7440-69-9$ & Bismuth & $<1.88 \mathrm{E}-1$ & ug/g dry & $1.88 \mathrm{E}-1$ & 6/30/08 & $8 F 30003$ & PNNL-AGG-ICP-AES \\
\hline $7440-70-2$ & Calcium & 2.63E1 & ug/g dry & 3.87E-1 & 6/30/08 & $8 F 30003$ & PNNL-AGG-ICP-AES \\
\hline $7440-43-9$ & Cadmium & $<2.69 \mathrm{E}-2$ & ug/g dry & $2.69 \mathrm{E}-2$ & 6/30/08 & 8F30003 & PNNL-AGG-ICP-AES \\
\hline $7440-48-4$ & Cobalt & $<9.60 \mathrm{E}-2$ & ug/g dry & $9.60 \mathrm{E}-2$ & 6/30/08 & $8 F 30003$ & PNNL-AGG-ICP-AES \\
\hline $7440-47-3$ & Chromium & $<3.33 \mathrm{E}-2$ & ug/g dry & $3.33 \mathrm{E}-2$ & 6/30/08 & $8 F 30003$ & PNNL-AGG-ICP-AES \\
\hline $7440-50-8$ & Copper & $<8.04 \mathrm{E}-2$ & ug/g dry & $8.04 \mathrm{E}-2$ & 6/30/08 & $8 F 30003$ & PNNL-AGG-ICP-AES \\
\hline 7439-89-6 & Iron & $<1.42 \mathrm{E}-1$ & ug/g dry & $1.42 \mathrm{E}-1$ & 6/30/08 & 8F30003 & PNNL-AGG-ICP-AES \\
\hline $7440-09-7$ & Potassium & $1.78 \mathrm{E} 1$ & ug/g dry & 2.33E0 & 6/30/08 & 8F30003 & PNNL-AGG-ICP-AES \\
\hline 7439-93-2 & Lithium & $<5.40 \mathrm{E}-1$ & ug/g dry & $5.40 \mathrm{E}-1$ & 6/30/08 & $8 F 30003$ & PNNL-AGG-ICP-AES \\
\hline 7439-95-4 & Magnesium & 9.58E0 & ug/g dry & 8.34E-2 & 6/30/08 & $8 F 30003$ & PNNL-AGG-ICP-AES \\
\hline $7439-96-5$ & Manganese & $<1.71 \mathrm{E}-2$ & ug/g dry & $1.71 \mathrm{E}-2$ & 6/30/08 & 8F30003 & PNNL-AGG-ICP-AES \\
\hline 7439-98-7 & Molybdenum & $<1.39 \mathrm{E}-1$ & ug/g dry & $1.39 \mathrm{E}-1$ & 6/30/08 & 8F30003 & PNNL-AGG-ICP-AES \\
\hline $7440-02-0$ & Nickel & $<9.33 \mathrm{E}-2$ & ug/g dry & $9.33 \mathrm{E}-2$ & 6/30/08 & $8 F 30003$ & PNNL-AGG-ICP-AES \\
\hline $7723-14-0$ & Phosphorus & $<1.03 \mathrm{E} 0$ & ug/g dry & 1.03E0 & 6/30/08 & 8F30003 & PNNL-AGG-ICP-AES \\
\hline $7439-92-1$ & Lead & $<4.32 \mathrm{E}-1$ & ug/g dry & $4.32 \mathrm{E}-1$ & 6/30/08 & 8F30003 & PNNL-AGG-ICP-AES \\
\hline $7782-49-2$ & Selenium & $<1.95 \mathrm{E} 0$ & ug/g dry & $1.95 \mathrm{E} 0$ & 6/30/08 & 8F30003 & PNNL-AGG-ICP-AES \\
\hline $7440-24-6$ & Strontium & $1.44 \mathrm{E}-1$ & ug/g dry & $5.22 \mathrm{E}-2$ & 6/30/08 & $8 F 30003$ & PNNL-AGG-ICP-AES \\
\hline $7440-28-0$ & Thallium & $<1.03 \mathrm{E} 0$ & ug/g dry & 1.03E0 & 6/30/08 & 8F30003 & PNNL-AGG-ICP-AES \\
\hline $7440-62-2$ & Vanadium & $<4.53 \mathrm{E}-2$ & ug/g dry & 4.53E-2 & 6/30/08 & 8F30003 & PNNL-AGG-ICP-AES \\
\hline $7440-66-6$ & Zinc & $<9.27 \mathrm{E}-2$ & ug/g dry & $9.27 \mathrm{E}-2$ & 6/30/08 & 8F30003 & PNNL-AGG-ICP-AES \\
\hline $7440-23-5$ & Sodium & 3.62E1 & ug/g dry & $6.69 \mathrm{E}-1$ & 6/30/08 & $8 F 30003$ & PNNL-AGG-ICP-AES \\
\hline $7440-21-3$ & Silicon & $6.84 \mathrm{E} 0$ & ug/g dry & $1.50 \mathrm{E} 0$ & 6/30/08 & 8F30003 & PNNL-AGG-ICP-AES \\
\hline 7704-34-9 & Sulfur & 4.56E1 & ug/g dry & 3.08E0 & 6/30/08 & $8 F 30003$ & PNNL-AGG-ICP-AES \\
\hline $7440-32-6$ & Titanium & $<8.76 \mathrm{E}-3$ & ug/g dry & $8.76 \mathrm{E}-3$ & 6/30/08 & $8 F 30003$ & PNNL-AGG-ICP-AES \\
\hline $7440-67-7$ & Zirconium & $<1.50 \mathrm{E}-1$ & ug/g dry & $1.50 \mathrm{E}-1$ & 6/30/08 & $8 F 30003$ & PNNL-AGG-ICP-AES \\
\hline $7440-22-4$ & Silver & $<7.71 \mathrm{E}-2$ & ug/g dry & $7.71 \mathrm{E}-2$ & 6/30/08 & 8F30003 & PNNL-AGG-ICP-AES \\
\hline $7440-15-5$ & Rhenium & $<1.52 \mathrm{E}-1$ & ug/g dry & $1.52 \mathrm{E}-1$ & 6/30/08 & 8F30003 & PNNL-AGG-ICP-AES \\
\hline $7440-36-0$ & Antimony & $<6.33 \mathrm{E}-1$ & ug/g dry & $6.33 \mathrm{E}-1$ & 6/30/08 & 8F30003 & PNNL-AGG-ICP-AES \\
\hline HEIS No. & B1VJ64B & & b ID: & $5020-31$ & & & \\
\hline $7429-90-5$ & Aluminum & $1.40 \mathrm{E}-1$ & ug/g dry & $8.58 \mathrm{E}-2$ & 6/30/08 & 8F30003 & PNNL-AGG-ICP-AES \\
\hline $7440-38-2$ & Arsenic & $<3.60 \mathrm{E}-1$ & ug/g dry & $3.60 \mathrm{E}-1$ & 6/30/08 & 8F30003 & PNNL-AGG-ICP-AES \\
\hline $7440-42-8$ & Boron & $<1.94 \mathrm{E} 0$ & ug/g dry & $1.94 \mathrm{E} 0$ & 6/30/08 & $8 F 30003$ & PNNL-AGG-ICP-AES \\
\hline $7440-39-3$ & Barium & $1.38 \mathrm{E}-2$ & ug/g dry & 8.79E-3 & 6/30/08 & $8 F 30003$ & PNNL-AGG-ICP-AES \\
\hline $7440-41-7$ & Beryllium & $<2.84 \mathrm{E}-2$ & ug/g dry & $2.84 \mathrm{E}-2$ & 6/30/08 & 8F30003 & PNNL-AGG-ICP-AES \\
\hline 7440-69-9 & Bismuth & $<1.88 \mathrm{E}-1$ & ug/g dry & $1.88 \mathrm{E}-1$ & 6/30/08 & $8 F 30003$ & PNNL-AGG-ICP-AES \\
\hline
\end{tabular}


Total Metals by PNNL-AGG-ICP-AES/Water Extract

\begin{tabular}{|c|c|c|c|c|c|c|c|}
\hline CAS \# & Analyte & Results & Units & EQL & Analyzed & Batch & Method \\
\hline HEIS No. & B1VJ64B & \multicolumn{3}{|c|}{ Lab ID: $\quad 0805020-31$} & & & \\
\hline $7440-70-2$ & Calcium & $1.65 \mathrm{E} 1$ & ug/g dry & $3.87 \mathrm{E}-1$ & 6/30/08 & 8 F30003 & PNNL-AGG-ICP-AES \\
\hline $7440-43-9$ & Cadmium & $<2.69 \mathrm{E}-2$ & ug/g dry & $2.69 \mathrm{E}-2$ & 6/30/08 & 8F30003 & PNNL-AGG-ICP-AES \\
\hline $7440-48-4$ & Cobalt & $<9.60 \mathrm{E}-2$ & ug/g dry & $9.60 \mathrm{E}-2$ & 6/30/08 & 8F30003 & PNNL-AGG-ICP-AES \\
\hline $7440-47-3$ & Chromium & $<3.33 \mathrm{E}-2$ & ug/g dry & 3.33E-2 & 6/30/08 & 8F30003 & PNNL-AGG-ICP-AES \\
\hline $7440-50-8$ & Copper & $<8.04 \mathrm{E}-2$ & ug/g dry & $8.04 \mathrm{E}-2$ & 6/30/08 & $8 F 30003$ & PNNL-AGG-ICP-AES \\
\hline 7439-89-6 & Iron & $<1.42 \mathrm{E}-1$ & ug/g dry & $1.42 \mathrm{E}-1$ & 6/30/08 & $8 F 30003$ & PNNL-AGG-ICP-AES \\
\hline $7440-09-7$ & Potassium & 1.07E1 & ug/g dry & 2.33Е0 & 6/30/08 & 8F30003 & PNNL-AGG-ICP-AES \\
\hline $7439-93-2$ & Lithium & $<5.40 \mathrm{E}-1$ & ug/g dry & $5.40 \mathrm{E}-1$ & 6/30/08 & $8 F 30003$ & PNNL-AGG-ICP-AES \\
\hline 7439-95-4 & Magnesium & 6.14E0 & ug/g dry & 8.34E-2 & 6/30/08 & 8F30003 & PNNL-AGG-ICP-AES \\
\hline $7439-96-5$ & Manganese & $<1.71 \mathrm{E}-2$ & ug/g dry & $1.71 \mathrm{E}-2$ & 6/30/08 & 8F30003 & PNNL-AGG-ICP-AES \\
\hline 7439-98-7 & Molybdenum & $<1.39 \mathrm{E}-1$ & ug/g dry & $1.39 \mathrm{E}-1$ & 6/30/08 & 8F30003 & PNNL-AGG-ICP-AES \\
\hline $7440-02-0$ & Nickel & $<9.33 \mathrm{E}-2$ & ug/g dry & $9.33 \mathrm{E}-2$ & 6/30/08 & $8 F 30003$ & PNNL-AGG-ICP-AES \\
\hline $7723-14-0$ & Phosphorus & $<1.03 \mathrm{E} 0$ & ug/g dry & 1.03E0 & 6/30/08 & $8 F 30003$ & PNNL-AGG-ICP-AES \\
\hline 7439-92-1 & Lead & $<4.32 \mathrm{E}-1$ & ug/g dry & 4.32E-1 & 6/30/08 & $8 F 30003$ & PNNL-AGG-ICP-AES \\
\hline $7782-49-2$ & Selenium & $<1.95 \mathrm{E} 0$ & ug/g dry & $1.95 \mathrm{E} 0$ & 6/30/08 & 8F30003 & PNNL-AGG-ICP-AES \\
\hline $7440-24-6$ & Strontium & $9.01 \mathrm{E}-2$ & ug/g dry & $5.22 \mathrm{E}-2$ & 6/30/08 & $8 F 30003$ & PNNL-AGG-ICP-AES \\
\hline $7440-28-0$ & Thallium & $<1.03 \mathrm{E} 0$ & ug/g dry & 1.03E0 & 6/30/08 & 8F30003 & PNNL-AGG-ICP-AES \\
\hline $7440-62-2$ & Vanadium & $<4.53 \mathrm{E}-2$ & ug/g dry & 4.53E-2 & 6/30/08 & 8F30003 & PNNL-AGG-ICP-AES \\
\hline $7440-66-6$ & Zinc & $<9.27 \mathrm{E}-2$ & ug/g dry & $9.27 \mathrm{E}-2$ & 6/30/08 & 8F30003 & PNNL-AGG-ICP-AES \\
\hline $7440-23-5$ & Sodium & 2.14E1 & ug/g dry & $6.69 \mathrm{E}-1$ & 6/30/08 & 8F30003 & PNNL-AGG-ICP-AES \\
\hline $7440-21-3$ & Silicon & 7.22E0 & ug/g dry & $1.50 \mathrm{E} 0$ & 6/30/08 & $8 F 30003$ & PNNL-AGG-ICP-AES \\
\hline 7704-34-9 & Sulfur & 2.75E1 & ug/g dry & 3.07E0 & 6/30/08 & 8F30003 & PNNL-AGG-ICP-AES \\
\hline $7440-32-6$ & Titanium & $<8.76 \mathrm{E}-3$ & ug/g dry & $8.76 \mathrm{E}-3$ & 6/30/08 & $8 F 30003$ & PNNL-AGG-ICP-AES \\
\hline $7440-67-7$ & Zirconium & $<1.50 \mathrm{E}-1$ & ug/g dry & $1.50 \mathrm{E}-1$ & 6/30/08 & 8F30003 & PNNL-AGG-ICP-AES \\
\hline $7440-22-4$ & Silver & $<7.71 \mathrm{E}-2$ & ug/g dry & $7.71 \mathrm{E}-2$ & 6/30/08 & $8 F 30003$ & PNNL-AGG-ICP-AES \\
\hline $7440-15-5$ & Rhenium & $<1.52 \mathrm{E}-1$ & ug/g dry & $1.52 \mathrm{E}-1$ & 6/30/08 & 8F30003 & PNNL-AGG-ICP-AES \\
\hline $7440-36-0$ & Antimony & $<6.33 \mathrm{E}-1$ & ug/g dry & 6.33E-1 & 6/30/08 & 8F30003 & PNNL-AGG-ICP-AES \\
\hline HEIS No. & B1VJ64A & & b ID: & 5020-32 & & & \\
\hline 7429-90-5 & Aluminum & $1.66 \mathrm{E}-1$ & ug/g dry & $8.14 \mathrm{E}-2$ & 6/30/08 & 8F30003 & PNNL-AGG-ICP-AES \\
\hline $7440-38-2$ & Arsenic & $<3.42 \mathrm{E}-1$ & ug/g dry & 3.42E-1 & 6/30/08 & 8F30003 & PNNL-AGG-ICP-AES \\
\hline $7440-42-8$ & Boron & $<1.84 \mathrm{E} 0$ & ug/g dry & $1.84 \mathrm{E} 0$ & 6/30/08 & 8F30003 & PNNL-AGG-ICP-AES \\
\hline $7440-39-3$ & Barium & $1.31 \mathrm{E}-2$ & ug/g dry & 8.34E-3 & 6/30/08 & $8 F 30003$ & PNNL-AGG-ICP-AES \\
\hline $7440-41-7$ & Beryllium & $<2.70 \mathrm{E}-2$ & ug/g dry & $2.70 \mathrm{E}-2$ & 6/30/08 & $8 F 30003$ & PNNL-AGG-ICP-AES \\
\hline $7440-69-9$ & Bismuth & $<1.78 \mathrm{E}-1$ & ug/g dry & $1.78 \mathrm{E}-1$ & 6/30/08 & 8F30003 & PNNL-AGG-ICP-AES \\
\hline $7440-70-2$ & Calcium & $1.44 \mathrm{E} 1$ & ug/g dry & $3.67 \mathrm{E}-1$ & 6/30/08 & $8 F 30003$ & PNNL-AGG-ICP-AES \\
\hline $7440-43-9$ & Cadmium & $<2.55 \mathrm{E}-2$ & ug/g dry & $2.55 \mathrm{E}-2$ & 6/30/08 & $8 F 30003$ & PNNL-AGG-ICP-AES \\
\hline $7440-48-4$ & Cobalt & $<9.11 \mathrm{E}-2$ & ug/g dry & $9.11 \mathrm{E}-2$ & 6/30/08 & $8 F 30003$ & PNNL-AGG-ICP-AES \\
\hline $7440-47-3$ & Chromium & $<3.16 \mathrm{E}-2$ & ug/g dry & $3.16 \mathrm{E}-2$ & 6/30/08 & 8F30003 & PNNL-AGG-ICP-AES \\
\hline $7440-50-8$ & Copper & $<7.63 \mathrm{E}-2$ & ug/g dry & 7.63E-2 & 6/30/08 & 8F30003 & PNNL-AGG-ICP-AES \\
\hline 7439-89-6 & Iron & $<1.35 \mathrm{E}-1$ & ug/g dry & $1.35 \mathrm{E}-1$ & 6/30/08 & 8F30003 & PNNL-AGG-ICP-AES \\
\hline 7440-09-7 & Potassium & 9.30E0 & ug/g dry & $2.21 \mathrm{E} 0$ & 6/30/08 & $8 F 30003$ & PNNL-AGG-ICP-AES \\
\hline 7439-93-2 & Lithium & $<5.12 \mathrm{E}-1$ & ug/g dry & $5.12 \mathrm{E}-1$ & 6/30/08 & 8F30003 & PNNL-AGG-ICP-AES \\
\hline 7439-95-4 & Magnesium & $5.30 \mathrm{E} 0$ & ug/g dry & $7.91 \mathrm{E}-2$ & 6/30/08 & 8F30003 & PNNL-AGG-ICP-AES \\
\hline $7439-96-5$ & Manganese & $<1.63 \mathrm{E}-2$ & ug/g dry & $1.63 \mathrm{E}-2$ & 6/30/08 & 8F30003 & PNNL-AGG-ICP-AES \\
\hline 7439-98-7 & Molybdenum & $<1.32 \mathrm{E}-1$ & ug/g dry & $1.32 \mathrm{E}-1$ & 6/30/08 & $8 F 30003$ & PNNL-AGG-ICP-AES \\
\hline $7440-02-0$ & Nickel & $<8.85 \mathrm{E}-2$ & ug/g dry & 8.85E-2 & 6/30/08 & 8F30003 & PNNL-AGG-ICP-AES \\
\hline $7723-14-0$ & Phosphorus & $<9.79 \mathrm{E}-1$ & ug/g dry & $9.79 \mathrm{E}-1$ & 6/30/08 & 8F30003 & PNNL-AGG-ICP-AES \\
\hline 7439-92-1 & Lead & $<4.10 \mathrm{E}-1$ & ug/g dry & $4.10 \mathrm{E}-1$ & 6/30/08 & 8F30003 & PNNL-AGG-ICP-AES \\
\hline
\end{tabular}


Total Metals by PNNL-AGG-ICP-AES/Water Extract

\begin{tabular}{|c|c|c|c|c|c|c|c|}
\hline CAS \# & Analyte & Results & Units & EQL & Analyzed & Batch & Method \\
\hline HEIS No. & B1VJ64A & \multicolumn{3}{|c|}{ Lab ID: $\quad$ 0805020-32 } & & & \\
\hline 7782-49-2 & Selenium & $<1.85 \mathrm{E} 0$ & ug/g dry & $1.85 \mathrm{E} 0$ & 6/30/08 & 8F30003 & PNNL-AGG-ICP-AES \\
\hline $7440-24-6$ & Strontium & $7.74 \mathrm{E}-2$ & ug/g dry & 4.95E-2 & $6 / 30 / 08$ & 8F30003 & PNNL-AGG-ICP-AES \\
\hline $7440-28-0$ & Thallium & $<9.74 \mathrm{E}-1$ & ug/g dry & $9.74 \mathrm{E}-1$ & 6/30/08 & 8F30003 & PNNL-AGG-ICP-AES \\
\hline $7440-62-2$ & Vanadium & $<4.30 \mathrm{E}-2$ & ug/g dry & 4.30E-2 & $6 / 30 / 08$ & 8F30003 & PNNL-AGG-ICP-AES \\
\hline $7440-66-6$ & Zinc & $<8.80 \mathrm{E}-2$ & ug/g dry & 8.80E-2 & $6 / 30 / 08$ & 8F30003 & PNNL-AGG-ICP-AES \\
\hline $7440-23-5$ & Sodium & $2.00 \mathrm{E} 1$ & ug/g dry & 6.35E-1 & 6/30/08 & 8F30003 & PNNL-AGG-ICP-AES \\
\hline $7440-21-3$ & Silicon & 7.56E0 & ug/g dry & $1.42 \mathrm{E} 0$ & $6 / 30 / 08$ & 8F30003 & PNNL-AGG-ICP-AES \\
\hline 7704-34-9 & Sulfur & 2.39E1 & ug/g dry & 2.92E0 & 6/30/08 & 8F30003 & PNNL-AGG-ICP-AES \\
\hline $7440-32-6$ & Titanium & $<8.31 \mathrm{E}-3$ & ug/g dry & 8.31E-3 & 6/30/08 & 8F30003 & PNNL-AGG-ICP-AES \\
\hline $7440-67-7$ & Zirconium & $<1.42 \mathrm{E}-1$ & ug/g dry & $1.42 \mathrm{E}-1$ & 6/30/08 & 8F30003 & PNNL-AGG-ICP-AES \\
\hline $7440-22-4$ & Silver & $<7.32 \mathrm{E}-2$ & ug/g dry & 7.32E-2 & $6 / 30 / 08$ & 8F30003 & PNNL-AGG-ICP-AES \\
\hline $7440-15-5$ & Rhenium & $<1.44 \mathrm{E}-1$ & ug/g dry & $1.44 \mathrm{E}-1$ & $6 / 30 / 08$ & 8F30003 & PNNL-AGG-ICP-AES \\
\hline $7440-36-0$ & Antimony & $<6.01 \mathrm{E}-1$ & ug/g dry & 6.01E-1 & $6 / 30 / 08$ & 8F30003 & PNNL-AGG-ICP-AES \\
\hline
\end{tabular}


Total Metals by PNNL-AGG-ICP-AES/Acid Extract

\begin{tabular}{|c|c|c|c|c|c|c|c|}
\hline CAS \# & Analyte & Results & Units & EQL & Analyzed & Batch & Method \\
\hline HEIS No. & B1VJ54B & \multicolumn{3}{|c|}{ Lab ID: $\quad$ 0805020-01 } & & & \\
\hline $7429-90-5$ & Aluminum & $5.43 \mathrm{E} 3$ & ug/g dry & $3.43 \mathrm{E} 1$ & 7/01/08 & 8G01001 & PNNL-AGG-ICP-AES \\
\hline $7440-38-2$ & Arsenic & $<8.34 \mathrm{E} 1$ & ug/g dry & 8.34E1 & $7 / 01 / 08$ & 8G01001 & PNNL-AGG-ICP-AES \\
\hline $7440-42-8$ & Boron & $<7.26 \mathrm{E} 2$ & ug/g dry & $7.26 \mathrm{E} 2$ & 7/01/08 & 8G01001 & PNNL-AGG-ICP-AES \\
\hline $7440-39-3$ & Barium & 7.61E1 & ug/g dry & $3.27 \mathrm{E} 0$ & $7 / 01 / 08$ & 8G01001 & PNNL-AGG-ICP-AES \\
\hline $7440-41-7$ & Beryllium & $<1.30 \mathrm{E} 0$ & ug/g dry & $1.30 \mathrm{E} 0$ & $7 / 01 / 08$ & 8G01001 & PNNL-AGG-ICP-AES \\
\hline $7440-69-9$ & Bismuth & $<1.73 \mathrm{E} 1$ & ug/g dry & $1.73 \mathrm{E} 1$ & 7/01/08 & 8G01001 & PNNL-AGG-ICP-AES \\
\hline $7440-70-2$ & Calcium & 7.64E3 & ug/g dry & $1.27 \mathrm{E} 2$ & $7 / 01 / 08$ & 8G01001 & PNNL-AGG-ICP-AES \\
\hline $7440-43-9$ & Cadmium & $<1.95 \mathrm{E} 0$ & ug/g dry & $1.95 \mathrm{E} 0$ & 7/01/08 & 8G01001 & PNNL-AGG-ICP-AES \\
\hline $7440-48-4$ & Cobalt & 7.17E0 & ug/g dry & 7.12E0 & $7 / 01 / 08$ & 8G01001 & PNNL-AGG-ICP-AES \\
\hline $7440-47-3$ & Chromium & 4.61E0 & ug/g dry & $2.85 \mathrm{E} 0$ & $7 / 01 / 08$ & 8G01001 & PNNL-AGG-ICP-AES \\
\hline $7440-50-8$ & Copper & $<3.04 \mathrm{E} 1$ & ug/g dry & 3.04E1 & $7 / 01 / 08$ & 8G01001 & PNNL-AGG-ICP-AES \\
\hline 7439-89-6 & Iron & $1.33 \mathrm{E} 4$ & ug/g dry & $8.72 \mathrm{E} 1$ & 7/01/08 & 8G01001 & PNNL-AGG-ICP-AES \\
\hline 7440-09-7 & Potassium & 1.07E3 & ug/g dry & 8.09E1 & $7 / 01 / 08$ & 8G01001 & PNNL-AGG-ICP-AES \\
\hline 7439-93-2 & Lithium & $<1.24 \mathrm{E} 1$ & ug/g dry & $1.24 \mathrm{E} 1$ & $7 / 01 / 08$ & 8G01001 & PNNL-AGG-ICP-AES \\
\hline $7439-95-4$ & Magnesium & $4.00 \mathrm{E} 3$ & ug/g dry & $2.70 \mathrm{E} 1$ & 7/01/08 & 8G01001 & PNNL-AGG-ICP-AES \\
\hline 7439-96-5 & Manganese & 2.67E2 & ug/g dry & $1.02 \mathrm{E} 0$ & $7 / 01 / 08$ & 8G01001 & PNNL-AGG-ICP-AES \\
\hline 7439-98-7 & Molybdenum & $<1.93 \mathrm{E} 1$ & ug/g dry & $1.93 \mathrm{E} 1$ & $7 / 01 / 08$ & 8G01001 & PNNL-AGG-ICP-AES \\
\hline $7440-02-0$ & Nickel & 7.31E0 & ug/g dry & 7.02E0 & $7 / 01 / 08$ & 8G01001 & PNNL-AGG-ICP-AES \\
\hline $7723-14-0$ & Phosphorus & $7.20 \mathrm{E} 2$ & ug/g dry & 6.43E1 & 7/01/08 & 8G01001 & PNNL-AGG-ICP-AES \\
\hline $7439-92-1$ & Lead & $<2.71 \mathrm{E} 1$ & ug/g dry & $2.71 \mathrm{E} 1$ & 7/01/08 & 8G01001 & PNNL-AGG-ICP-AES \\
\hline $7440-24-6$ & Strontium & 2.72E1 & ug/g dry & $1.24 \mathrm{E} 0$ & $7 / 01 / 08$ & 8G01001 & PNNL-AGG-ICP-AES \\
\hline $7440-66-6$ & Zinc & $3.51 \mathrm{E} 1$ & ug/g dry & 5.35E0 & $7 / 01 / 08$ & 8G01001 & PNNL-AGG-ICP-AES \\
\hline $7440-23-5$ & Sodium & $<9.14 \mathrm{E} 2$ & ug/g dry & $9.14 \mathrm{E} 2$ & 7/01/08 & 8G01001 & PNNL-AGG-ICP-AES \\
\hline 7440-21-3 & Silicon & $<1.19 \mathrm{E} 3$ & ug/g dry & 1.19E3 & $7 / 01 / 08$ & 8G01001 & PNNL-AGG-ICP-AES \\
\hline $7704-34-9$ & Sulfur & $<2.02 \mathrm{E} 2$ & ug/g dry & $2.02 \mathrm{E} 2$ & $7 / 01 / 08$ & 8G01001 & PNNL-AGG-ICP-AES \\
\hline $7440-32-6$ & Titanium & 6.94E2 & ug/g dry & 2.69E1 & $7 / 01 / 08$ & 8G01001 & PNNL-AGG-ICP-AES \\
\hline $7440-67-7$ & Zirconium & $<1.74 \mathrm{E} 1$ & ug/g dry & $1.74 \mathrm{E} 1$ & 7/01/08 & 8G01001 & PNNL-AGG-ICP-AES \\
\hline $7440-15-5$ & Rhenium & $<1.50 \mathrm{E} 1$ & ug/g dry & $1.50 \mathrm{E} 1$ & 7/01/08 & 8G01001 & PNNL-AGG-ICP-AES \\
\hline $7440-36-0$ & Antimony & $<9.55 \mathrm{E} 1$ & ug/g dry & $9.55 \mathrm{E} 1$ & $7 / 01 / 08$ & 8G01001 & PNNL-AGG-ICP-AES \\
\hline HEIS No. & B1VJ55C & & b ID: & 5020-05 & & & \\
\hline $7429-90-5$ & Aluminum & 5.97E3 & ug/g dry & 4.01E1 & $7 / 01 / 08$ & 8G01001 & PNNL-AGG-ICP-AES \\
\hline $7440-38-2$ & Arsenic & $<9.73 \mathrm{E} 1$ & ug/g dry & $9.73 \mathrm{E} 1$ & 7/01/08 & 8G01001 & PNNL-AGG-ICP-AES \\
\hline $7440-42-8$ & Boron & $<8.48 \mathrm{E} 2$ & ug/g dry & $8.48 \mathrm{E} 2$ & $7 / 01 / 08$ & 8G01001 & PNNL-AGG-ICP-AES \\
\hline $7440-39-3$ & Barium & 7.67E1 & ug/g dry & 3.82E0 & $7 / 01 / 08$ & 8G01001 & PNNL-AGG-ICP-AES \\
\hline $7440-41-7$ & Beryllium & $<1.51 \mathrm{E} 0$ & ug/g dry & $1.51 \mathrm{E} 0$ & 7/01/08 & 8G01001 & PNNL-AGG-ICP-AES \\
\hline 7440-69-9 & Bismuth & $<2.02 \mathrm{E} 1$ & ug/g dry & $2.02 \mathrm{E} 1$ & 7/01/08 & 8G01001 & PNNL-AGG-ICP-AES \\
\hline $7440-70-2$ & Calcium & $1.10 \mathrm{E} 4$ & ug/g dry & 1.49E2 & $7 / 01 / 08$ & 8G01001 & PNNL-AGG-ICP-AES \\
\hline $7440-43-9$ & Cadmium & $<2.28 \mathrm{E} 0$ & ug/g dry & $2.28 \mathrm{E} 0$ & $7 / 01 / 08$ & 8G01001 & PNNL-AGG-ICP-AES \\
\hline $7440-48-4$ & Cobalt & $<8.32 \mathrm{E} 0$ & ug/g dry & 8.32E0 & 7/01/08 & 8G01001 & PNNL-AGG-ICP-AES \\
\hline $7440-47-3$ & Chromium & 4.53E0 & ug/g dry & 3.33Е0 & 7/01/08 & 8G01001 & PNNL-AGG-ICP-AES \\
\hline $7440-50-8$ & Copper & $<3.55 \mathrm{E} 1$ & ug/g dry & $3.55 \mathrm{E} 1$ & 7/01/08 & 8G01001 & PNNL-AGG-ICP-AES \\
\hline 7439-89-6 & Iron & $1.54 \mathrm{E} 4$ & ug/g dry & $1.02 \mathrm{E} 2$ & 7/01/08 & 8G01001 & PNNL-AGG-ICP-AES \\
\hline 7440-09-7 & Potassium & $1.18 \mathrm{E} 3$ & ug/g dry & $9.45 \mathrm{E} 1$ & 7/01/08 & 8G01001 & PNNL-AGG-ICP-AES \\
\hline 7439-93-2 & Lithium & $<1.44 \mathrm{E} 1$ & ug/g dry & $1.44 \mathrm{E} 1$ & $7 / 01 / 08$ & 8G01001 & PNNL-AGG-ICP-AES \\
\hline 7439-95-4 & Magnesium & $4.94 \mathrm{E} 3$ & ug/g dry & 3.15E1 & $7 / 01 / 08$ & 8G01001 & PNNL-AGG-ICP-AES \\
\hline 7439-96-5 & Manganese & 2.98E2 & ug/g dry & $1.19 \mathrm{E} 0$ & $7 / 01 / 08$ & 8G01001 & PNNL-AGG-ICP-AES \\
\hline 7439-98-7 & Molybdenum & $<2.26 \mathrm{E} 1$ & ug/g dry & 2.26E1 & 7/01/08 & 8G01001 & PNNL-AGG-ICP-AES \\
\hline $7440-02-0$ & Nickel & $<8.19 \mathrm{E} 0$ & ug/g dry & 8.19E0 & 7/01/08 & 8G01001 & PNNL-AGG-ICP-AES \\
\hline
\end{tabular}


Total Metals by PNNL-AGG-ICP-AES/Acid Extract

\begin{tabular}{|c|c|c|c|c|c|c|c|}
\hline CAS \# & Analyte & Results & Units & EQL & Analyzed & Batch & Method \\
\hline HEIS No. & B1VJ55C & \multicolumn{3}{|c|}{ Lab ID: $\quad$ 0805020-05 } & & & \\
\hline $7723-14-0$ & Phosphorus & 8.11E2 & ug/g dry & $7.50 \mathrm{E} 1$ & 7/01/08 & 8G01001 & PNNL-AGG-ICP-AES \\
\hline 7439-92-1 & Lead & $<3.17 \mathrm{E} 1$ & ug/g dry & 3.17E1 & $7 / 01 / 08$ & 8G01001 & PNNL-AGG-ICP-AES \\
\hline $7440-24-6$ & Strontium & 3.49E1 & ug/g dry & $1.44 \mathrm{E} 0$ & $7 / 01 / 08$ & 8G01001 & PNNL-AGG-ICP-AES \\
\hline $7440-66-6$ & Zinc & 3.90E1 & ug/g dry & $6.25 \mathrm{E} 0$ & $7 / 01 / 08$ & 8G01001 & PNNL-AGG-ICP-AES \\
\hline $7440-23-5$ & Sodium & $<1.07 \mathrm{E} 3$ & ug/g dry & $1.07 \mathrm{E} 3$ & 7/01/08 & 8G01001 & PNNL-AGG-ICP-AES \\
\hline $7440-21-3$ & Silicon & $<1.39 \mathrm{E} 3$ & ug/g dry & $1.39 \mathrm{E} 3$ & $7 / 01 / 08$ & 8G01001 & PNNL-AGG-ICP-AES \\
\hline $7704-34-9$ & Sulfur & $<2.36 \mathrm{E} 2$ & ug/g dry & 2.36E2 & $7 / 01 / 08$ & 8G01001 & PNNL-AGG-ICP-AES \\
\hline $7440-32-6$ & Titanium & 7.88E2 & ug/g dry & $3.14 \mathrm{E} 1$ & $7 / 01 / 08$ & 8G01001 & PNNL-AGG-ICP-AES \\
\hline $7440-67-7$ & Zirconium & $<2.03 \mathrm{E} 1$ & ug/g dry & 2.03E1 & 7/01/08 & 8G01001 & PNNL-AGG-ICP-AES \\
\hline $7440-15-5$ & Rhenium & $<1.76 \mathrm{E} 1$ & ug/g dry & $1.76 \mathrm{E} 1$ & $7 / 01 / 08$ & 8G01001 & PNNL-AGG-ICP-AES \\
\hline $7440-36-0$ & Antimony & $<1.12 \mathrm{E} 2$ & ug/g dry & $1.12 \mathrm{E} 2$ & 7/01/08 & 8G01001 & PNNL-AGG-ICP-AES \\
\hline HEIS No. & B1VJ56A & \multicolumn{3}{|c|}{ Lab ID: $\quad$ 0805020-07 } & & & \\
\hline $7429-90-5$ & Aluminum & 4.49E3 & ug/g dry & 3.34E1 & $7 / 01 / 08$ & 8G01001 & PNNL-AGG-ICP-AES \\
\hline $7440-38-2$ & Arsenic & $<8.11 \mathrm{E} 1$ & ug/g dry & $8.11 \mathrm{E} 1$ & $7 / 01 / 08$ & 8G01001 & PNNL-AGG-ICP-AES \\
\hline $7440-42-8$ & Boron & $<7.06 \mathrm{E} 2$ & ug/g dry & 7.06E2 & $7 / 01 / 08$ & 8G01001 & PNNL-AGG-ICP-AES \\
\hline $7440-39-3$ & Barium & 6.48E1 & ug/g dry & $3.18 \mathrm{E} 0$ & $7 / 01 / 08$ & 8G01001 & PNNL-AGG-ICP-AES \\
\hline $7440-41-7$ & Beryllium & $<1.26 \mathrm{E} 0$ & ug/g dry & $1.26 \mathrm{E} 0$ & $7 / 01 / 08$ & 8G01001 & PNNL-AGG-ICP-AES \\
\hline $7440-69-9$ & Bismuth & $<1.69 \mathrm{E} 1$ & ug/g dry & 1.69E1 & $7 / 01 / 08$ & 8G01001 & PNNL-AGG-ICP-AES \\
\hline $7440-70-2$ & Calcium & $6.08 \mathrm{E} 3$ & ug/g dry & $1.24 \mathrm{E} 2$ & $7 / 01 / 08$ & 8G01001 & PNNL-AGG-ICP-AES \\
\hline 7440-43-9 & Cadmium & $<1.89 \mathrm{E} 0$ & ug/g dry & $1.89 \mathrm{E} 0$ & $7 / 01 / 08$ & 8G01001 & PNNL-AGG-ICP-AES \\
\hline $7440-48-4$ & Cobalt & $<6.92 \mathrm{E} 0$ & ug/g dry & $6.92 \mathrm{E} 0$ & 7/01/08 & 8G01001 & PNNL-AGG-ICP-AES \\
\hline $7440-47-3$ & Chromium & 5.23E0 & ug/g dry & 2.77E0 & 7/01/08 & 8G01001 & PNNL-AGG-ICP-AES \\
\hline $7440-50-8$ & Copper & $<2.96 \mathrm{E} 1$ & ug/g dry & 2.96E1 & $7 / 01 / 08$ & 8G01001 & PNNL-AGG-ICP-AES \\
\hline 7439-89-6 & Iron & $1.26 \mathrm{E} 4$ & ug/g dry & $8.48 \mathrm{E} 1$ & $7 / 01 / 08$ & 8G01001 & PNNL-AGG-ICP-AES \\
\hline $7440-09-7$ & Potassium & 7.72E2 & ug/g dry & 7.87E1 & $7 / 01 / 08$ & 8G01001 & PNNL-AGG-ICP-AES \\
\hline 7439-93-2 & Lithium & $<1.20 \mathrm{E} 1$ & ug/g dry & $1.20 \mathrm{E} 1$ & $7 / 01 / 08$ & 8G01001 & PNNL-AGG-ICP-AES \\
\hline $7439-95-4$ & Magnesium & 3.09E3 & ug/g dry & $2.62 \mathrm{E} 1$ & $7 / 01 / 08$ & 8G01001 & PNNL-AGG-ICP-AES \\
\hline $7439-96-5$ & Manganese & 2.37E2 & ug/g dry & 9.93E-1 & 7/01/08 & 8G01001 & PNNL-AGG-ICP-AES \\
\hline 7439-98-7 & Molybdenum & $<1.88 \mathrm{E} 1$ & ug/g dry & $1.88 \mathrm{E} 1$ & 7/01/08 & 8G01001 & PNNL-AGG-ICP-AES \\
\hline $7440-02-0$ & Nickel & 7.90E0 & ug/g dry & 6.82E0 & $7 / 01 / 08$ & 8G01001 & PNNL-AGG-ICP-AES \\
\hline 7723-14-0 & Phosphorus & $7.22 \mathrm{E} 2$ & ug/g dry & $6.25 \mathrm{E} 1$ & $7 / 01 / 08$ & 8G01001 & PNNL-AGG-ICP-AES \\
\hline $7439-92-1$ & Lead & $<2.64 \mathrm{E} 1$ & ug/g dry & $2.64 \mathrm{E} 1$ & 7/01/08 & 8G01001 & PNNL-AGG-ICP-AES \\
\hline $7440-24-6$ & Strontium & $2.25 \mathrm{E} 1$ & ug/g dry & $1.20 \mathrm{E} 0$ & $7 / 01 / 08$ & 8G01001 & PNNL-AGG-ICP-AES \\
\hline $7440-66-6$ & Zinc & 2.82E1 & ug/g dry & $5.20 \mathrm{E} 0$ & $7 / 01 / 08$ & 8G01001 & PNNL-AGG-ICP-AES \\
\hline $7440-23-5$ & Sodium & 1.77E3 & ug/g dry & 8.89E2 & $7 / 01 / 08$ & 8G01001 & PNNL-AGG-ICP-AES \\
\hline 7440-21-3 & Silicon & $<1.16 \mathrm{E} 3$ & ug/g dry & $1.16 \mathrm{E} 3$ & 7/01/08 & 8G01001 & PNNL-AGG-ICP-AES \\
\hline $7704-34-9$ & Sulfur & $<1.97 \mathrm{E} 2$ & ug/g dry & $1.97 \mathrm{E} 2$ & 7/01/08 & 8G01001 & PNNL-AGG-ICP-AES \\
\hline $7440-32-6$ & Titanium & 7.66E2 & ug/g dry & 2.62E1 & $7 / 01 / 08$ & 8G01001 & PNNL-AGG-ICP-AES \\
\hline 7440-67-7 & Zirconium & $<1.69 \mathrm{E} 1$ & ug/g dry & 1.69E1 & $7 / 01 / 08$ & 8G01001 & PNNL-AGG-ICP-AES \\
\hline $7440-15-5$ & Rhenium & $<1.46 \mathrm{E} 1$ & ug/g dry & $1.46 \mathrm{E} 1$ & 7/01/08 & 8G01001 & PNNL-AGG-ICP-AES \\
\hline $7440-36-0$ & Antimony & $<9.29 \mathrm{E} 1$ & ug/g dry & $9.29 \mathrm{E} 1$ & $7 / 01 / 08$ & 8G01001 & PNNL-AGG-ICP-AES \\
\hline HEIS No. & B1VJ56B & & b ID: & $5020-08$ & & & \\
\hline $7429-90-5$ & Aluminum & 4.73E3 & ug/g dry & 3.58E1 & $7 / 01 / 08$ & 8G01001 & PNNL-AGG-ICP-AES \\
\hline $7440-38-2$ & Arsenic & $<8.69 \mathrm{E} 1$ & ug/g dry & 8.69E1 & 7/01/08 & 8G01001 & PNNL-AGG-ICP-AES \\
\hline $7440-42-8$ & Boron & $<7.56 \mathrm{E} 2$ & ug/g dry & $7.56 \mathrm{E} 2$ & 7/01/08 & 8G01001 & PNNL-AGG-ICP-AES \\
\hline 7440-39-3 & Barium & $6.85 \mathrm{E} 1$ & ug/g dry & $3.41 \mathrm{E} 0$ & $7 / 01 / 08$ & 8G01001 & PNNL-AGG-ICP-AES \\
\hline $7440-41-7$ & Beryllium & $<1.35 \mathrm{E} 0$ & ug/g dry & $1.35 \mathrm{E} 0$ & $7 / 01 / 08$ & 8G01001 & PNNL-AGG-ICP-AES \\
\hline $7440-69-9$ & Bismuth & $<1.81 \mathrm{E} 1$ & ug/g dry & $1.81 \mathrm{E} 1$ & 7/01/08 & 8G01001 & PNNL-AGG-ICP-AES \\
\hline
\end{tabular}


Total Metals by PNNL-AGG-ICP-AES/Acid Extract

\begin{tabular}{|c|c|c|c|c|c|c|c|}
\hline CAS \# & Analyte & Results & Units & EQL & Analyzed & Batch & Method \\
\hline HEIS No. & B1VJ56B & \multicolumn{3}{|c|}{ Lab ID: $\quad$ 0805020-08 } & & & \\
\hline $7440-70-2$ & Calcium & $5.84 \mathrm{E} 3$ & ug/g dry & $1.33 \mathrm{E} 2$ & 7/01/08 & 8G01001 & PNNL-AGG-ICP-AES \\
\hline $7440-43-9$ & Cadmium & $<2.03 \mathrm{E} 0$ & ug/g dry & 2.03E0 & $7 / 01 / 08$ & 8G01001 & PNNL-AGG-ICP-AES \\
\hline $7440-48-4$ & Cobalt & $<7.42 \mathrm{E} 0$ & ug/g dry & 7.42E0 & 7/01/08 & 8G01001 & PNNL-AGG-ICP-AES \\
\hline $7440-47-3$ & Chromium & 3.59E0 & ug/g dry & 2.97E0 & $7 / 01 / 08$ & 8G01001 & PNNL-AGG-ICP-AES \\
\hline $7440-50-8$ & Copper & $<3.17 \mathrm{E} 1$ & ug/g dry & 3.17E1 & $7 / 01 / 08$ & 8G01001 & PNNL-AGG-ICP-AES \\
\hline 7439-89-6 & Iron & $1.34 \mathrm{E} 4$ & ug/g dry & $9.08 \mathrm{E} 1$ & 7/01/08 & 8G01001 & PNNL-AGG-ICP-AES \\
\hline $7440-09-7$ & Potassium & 8.53E2 & ug/g dry & 8.43E1 & $7 / 01 / 08$ & 8G01001 & PNNL-AGG-ICP-AES \\
\hline 7439-93-2 & Lithium & $<1.29 \mathrm{E} 1$ & ug/g dry & $1.29 \mathrm{E} 1$ & 7/01/08 & 8G01001 & PNNL-AGG-ICP-AES \\
\hline 7439-95-4 & Magnesium & $3.21 \mathrm{E} 3$ & ug/g dry & $2.81 \mathrm{E} 1$ & $7 / 01 / 08$ & 8G01001 & PNNL-AGG-ICP-AES \\
\hline $7439-96-5$ & Manganese & $2.54 \mathrm{E} 2$ & ug/g dry & $1.06 \mathrm{E} 0$ & $7 / 01 / 08$ & 8G01001 & PNNL-AGG-ICP-AES \\
\hline 7439-98-7 & Molybdenum & $<2.02 \mathrm{E} 1$ & ug/g dry & 2.02E1 & $7 / 01 / 08$ & 8G01001 & PNNL-AGG-ICP-AES \\
\hline $7440-02-0$ & Nickel & 9.53E0 & ug/g dry & $7.31 \mathrm{E} 0$ & 7/01/08 & 8G01001 & PNNL-AGG-ICP-AES \\
\hline $7723-14-0$ & Phosphorus & $6.90 \mathrm{E} 2$ & ug/g dry & $6.70 \mathrm{E} 1$ & $7 / 01 / 08$ & 8G01001 & PNNL-AGG-ICP-AES \\
\hline $7439-92-1$ & Lead & $<2.83 \mathrm{E} 1$ & ug/g dry & 2.83E1 & $7 / 01 / 08$ & 8G01001 & PNNL-AGG-ICP-AES \\
\hline $7440-24-6$ & Strontium & 2.23E1 & ug/g dry & $1.29 \mathrm{E} 0$ & 7/01/08 & 8G01001 & PNNL-AGG-ICP-AES \\
\hline $7440-66-6$ & Zinc & $2.86 \mathrm{E} 1$ & ug/g dry & 5.57E0 & 7/01/08 & 8G01001 & PNNL-AGG-ICP-AES \\
\hline $7440-23-5$ & Sodium & $2.01 \mathrm{E} 3$ & ug/g dry & $9.53 \mathrm{E} 2$ & 7/01/08 & 8G01001 & PNNL-AGG-ICP-AES \\
\hline $7440-21-3$ & Silicon & $<1.24 \mathrm{E} 3$ & ug/g dry & $1.24 \mathrm{E} 3$ & $7 / 01 / 08$ & 8G01001 & PNNL-AGG-ICP-AES \\
\hline 7704-34-9 & Sulfur & $<2.11 \mathrm{E} 2$ & ug/g dry & $2.11 \mathrm{E} 2$ & 7/01/08 & 8G01001 & PNNL-AGG-ICP-AES \\
\hline $7440-32-6$ & Titanium & $8.40 \mathrm{E} 2$ & ug/g dry & $2.81 \mathrm{E} 1$ & 7/01/08 & 8G01001 & PNNL-AGG-ICP-AES \\
\hline $7440-67-7$ & Zirconium & $<1.81 \mathrm{E} 1$ & ug/g dry & $1.81 \mathrm{E} 1$ & $7 / 01 / 08$ & 8G01001 & PNNL-AGG-ICP-AES \\
\hline $7440-15-5$ & Rhenium & $<1.57 \mathrm{E} 1$ & ug/g dry & $1.57 \mathrm{E} 1$ & $7 / 01 / 08$ & 8G01001 & PNNL-AGG-ICP-AES \\
\hline $7440-36-0$ & Antimony & $<9.95 \mathrm{E} 1$ & ug/g dry & 9.95E1 & 7/01/08 & 8G01001 & PNNL-AGG-ICP-AES \\
\hline HEIS No. & B1VJ56C & \multicolumn{3}{|c|}{ Lab ID: $\quad 0805020-09$} & & & \\
\hline $7429-90-5$ & Aluminum & $5.02 \mathrm{E} 3$ & ug/g dry & $3.46 \mathrm{E} 1$ & $7 / 01 / 08$ & 8G01001 & PNNL-AGG-ICP-AES \\
\hline $7440-38-2$ & Arsenic & $<8.40 \mathrm{E} 1$ & ug/g dry & $8.40 \mathrm{E} 1$ & $7 / 01 / 08$ & 8G01001 & PNNL-AGG-ICP-AES \\
\hline $7440-42-8$ & Boron & $<7.32 \mathrm{E} 2$ & ug/g dry & 7.32E2 & $7 / 01 / 08$ & 8G01001 & PNNL-AGG-ICP-AES \\
\hline $7440-39-3$ & Barium & 5.86E1 & ug/g dry & $3.29 \mathrm{E} 0$ & 7/01/08 & 8G01001 & PNNL-AGG-ICP-AES \\
\hline $7440-41-7$ & Beryllium & $<1.31 \mathrm{E} 0$ & ug/g dry & $1.31 \mathrm{E} 0$ & 7/01/08 & 8G01001 & PNNL-AGG-ICP-AES \\
\hline $7440-69-9$ & Bismuth & $<1.75 \mathrm{E} 1$ & ug/g dry & $1.75 \mathrm{E} 1$ & $7 / 01 / 08$ & 8G01001 & PNNL-AGG-ICP-AES \\
\hline $7440-70-2$ & Calcium & 6.00E3 & ug/g dry & $1.29 \mathrm{E} 2$ & $7 / 01 / 08$ & 8G01001 & PNNL-AGG-ICP-AES \\
\hline $7440-43-9$ & Cadmium & $<1.96 \mathrm{E} 0$ & ug/g dry & $1.96 \mathrm{E} 0$ & 7/01/08 & 8G01001 & PNNL-AGG-ICP-AES \\
\hline $7440-48-4$ & Cobalt & $<7.18 \mathrm{E} 0$ & ug/g dry & $7.18 \mathrm{E} 0$ & $7 / 01 / 08$ & 8G01001 & PNNL-AGG-ICP-AES \\
\hline $7440-47-3$ & Chromium & 8.96E0 & ug/g dry & 2.87E0 & $7 / 01 / 08$ & 8G01001 & PNNL-AGG-ICP-AES \\
\hline $7440-50-8$ & Copper & $<3.06 \mathrm{E} 1$ & ug/g dry & 3.06E1 & 7/01/08 & 8G01001 & PNNL-AGG-ICP-AES \\
\hline 7439-89-6 & Iron & $1.26 \mathrm{E} 4$ & ug/g dry & 8.79E1 & 7/01/08 & 8G01001 & PNNL-AGG-ICP-AES \\
\hline $7440-09-7$ & Potassium & 8.87E2 & ug/g dry & 8.16E1 & $7 / 01 / 08$ & 8G01001 & PNNL-AGG-ICP-AES \\
\hline 7439-93-2 & Lithium & $<1.25 \mathrm{E} 1$ & ug/g dry & $1.25 \mathrm{E} 1$ & $7 / 01 / 08$ & 8G01001 & PNNL-AGG-ICP-AES \\
\hline 7439-95-4 & Magnesium & 3.37E3 & ug/g dry & 2.72E1 & 7/01/08 & 8G01001 & PNNL-AGG-ICP-AES \\
\hline 7439-96-5 & Manganese & $2.25 \mathrm{E} 2$ & ug/g dry & $1.03 \mathrm{E} 0$ & 7/01/08 & 8G01001 & PNNL-AGG-ICP-AES \\
\hline 7439-98-7 & Molybdenum & $<1.95 \mathrm{E} 1$ & ug/g dry & $1.95 \mathrm{E} 1$ & 7/01/08 & 8G01001 & PNNL-AGG-ICP-AES \\
\hline $7440-02-0$ & Nickel & 9.77E0 & ug/g dry & 7.07E0 & 7/01/08 & 8G01001 & PNNL-AGG-ICP-AES \\
\hline $7723-14-0$ & Phosphorus & 5.63E2 & ug/g dry & $6.48 \mathrm{E} 1$ & 7/01/08 & 8G01001 & PNNL-AGG-ICP-AES \\
\hline 7439-92-1 & Lead & $<2.73 \mathrm{E} 1$ & ug/g dry & 2.73E1 & $7 / 01 / 08$ & 8G01001 & PNNL-AGG-ICP-AES \\
\hline $7440-24-6$ & Strontium & $2.44 \mathrm{E} 1$ & ug/g dry & $1.25 \mathrm{E} 0$ & $7 / 01 / 08$ & 8G01001 & PNNL-AGG-ICP-AES \\
\hline $7440-66-6$ & Zinc & 2.93E1 & ug/g dry & 5.39E0 & $7 / 01 / 08$ & 8G01001 & PNNL-AGG-ICP-AES \\
\hline $7440-23-5$ & Sodium & $1.45 \mathrm{E} 3$ & ug/g dry & $9.22 \mathrm{E} 2$ & 7/01/08 & 8G01001 & PNNL-AGG-ICP-AES \\
\hline $7440-21-3$ & Silicon & $<1.20 \mathrm{E} 3$ & ug/g dry & $1.20 \mathrm{E} 3$ & 7/01/08 & 8G01001 & PNNL-AGG-ICP-AES \\
\hline
\end{tabular}


Total Metals by PNNL-AGG-ICP-AES/Acid Extract

\begin{tabular}{|c|c|c|c|c|c|c|c|}
\hline CAS \# & Analyte & Results & Units & EQL & Analyzed & Batch & Method \\
\hline HEIS No. & B1VJ56C & \multicolumn{3}{|c|}{ Lab ID: $\quad 0805020-09$} & & & \\
\hline 7704-34-9 & Sulfur & $<2.04 \mathrm{E} 2$ & ug/g dry & 2.04E2 & $7 / 01 / 08$ & 8G01001 & PNNL-AGG-ICP-AES \\
\hline $7440-32-6$ & Titanium & $6.82 \mathrm{E} 2$ & ug/g dry & 2.71E1 & $7 / 01 / 08$ & 8G01001 & PNNL-AGG-ICP-AES \\
\hline 7440-67-7 & Zirconium & $<1.75 \mathrm{E} 1$ & ug/g dry & $1.75 \mathrm{E} 1$ & $7 / 01 / 08$ & 8G01001 & PNNL-AGG-ICP-AES \\
\hline $7440-15-5$ & Rhenium & $<1.52 \mathrm{E} 1$ & ug/g dry & $1.52 \mathrm{E} 1$ & $7 / 01 / 08$ & 8G01001 & PNNL-AGG-ICP-AES \\
\hline $7440-36-0$ & Antimony & $<9.63 \mathrm{E} 1$ & ug/g dry & 9.63E1 & $7 / 01 / 08$ & 8G01001 & PNNL-AGG-ICP-AES \\
\hline HEIS No. & B1VJ58C & \multicolumn{3}{|c|}{ Lab ID: $\quad$ 0805020-10 } & & & \\
\hline 7429-90-5 & Aluminum & $6.47 \mathrm{E} 3$ & ug/g dry & 3.69E1 & $7 / 01 / 08$ & 8G01001 & PNNL-AGG-ICP-AES \\
\hline $7440-38-2$ & Arsenic & $<8.97 \mathrm{E} 1$ & ug/g dry & 8.97E1 & $7 / 01 / 08$ & 8G01001 & PNNL-AGG-ICP-AES \\
\hline $7440-42-8$ & Boron & $<7.81 \mathrm{E} 2$ & ug/g dry & $7.81 \mathrm{E} 2$ & $7 / 01 / 08$ & 8G01001 & PNNL-AGG-ICP-AES \\
\hline $7440-39-3$ & Barium & 8.26E1 & ug/g dry & 3.52E0 & $7 / 01 / 08$ & 8G01001 & PNNL-AGG-ICP-AES \\
\hline $7440-41-7$ & Beryllium & $<1.39 \mathrm{E} 0$ & ug/g dry & 1.39E0 & $7 / 01 / 08$ & 8G01001 & PNNL-AGG-ICP-AES \\
\hline 7440-69-9 & Bismuth & $<1.86 \mathrm{E} 1$ & ug/g dry & $1.86 \mathrm{E} 1$ & $7 / 01 / 08$ & 8G01001 & PNNL-AGG-ICP-AES \\
\hline $7440-70-2$ & Calcium & 8.79E3 & ug/g dry & $1.37 \mathrm{E} 2$ & 7/01/08 & 8G01001 & PNNL-AGG-ICP-AES \\
\hline $7440-43-9$ & Cadmium & $<2.10 \mathrm{E} 0$ & ug/g dry & $2.10 \mathrm{E} 0$ & 7/01/08 & 8G01001 & PNNL-AGG-ICP-AES \\
\hline $7440-48-4$ & Cobalt & $<7.66 \mathrm{E} 0$ & ug/g dry & 7.66E0 & $7 / 01 / 08$ & 8G01001 & PNNL-AGG-ICP-AES \\
\hline $7440-47-3$ & Chromium & 7.38E0 & ug/g dry & 3.07E0 & $7 / 01 / 08$ & 8G01001 & PNNL-AGG-ICP-AES \\
\hline $7440-50-8$ & Copper & $<3.27 \mathrm{E} 1$ & ug/g dry & 3.27E1 & $7 / 01 / 08$ & 8G01001 & PNNL-AGG-ICP-AES \\
\hline 7439-89-6 & Iron & $1.41 \mathrm{E} 4$ & ug/g dry & $9.38 \mathrm{E} 1$ & 7/01/08 & 8G01001 & PNNL-AGG-ICP-AES \\
\hline $7440-09-7$ & Potassium & $1.40 \mathrm{E} 3$ & ug/g dry & 8.71E1 & $7 / 01 / 08$ & 8G01001 & PNNL-AGG-ICP-AES \\
\hline 7439-93-2 & Lithium & $<1.33 \mathrm{E} 1$ & ug/g dry & 1.33E1 & $7 / 01 / 08$ & 8G01001 & PNNL-AGG-ICP-AES \\
\hline $7439-95-4$ & Magnesium & $4.25 \mathrm{E} 3$ & ug/g dry & $2.90 \mathrm{E} 1$ & 7/01/08 & 8G01001 & PNNL-AGG-ICP-AES \\
\hline 7439-96-5 & Manganese & $2.83 \mathrm{E} 2$ & ug/g dry & $1.10 \mathrm{E} 0$ & 7/01/08 & 8G01001 & PNNL-AGG-ICP-AES \\
\hline 7439-98-7 & Molybdenum & $<2.08 \mathrm{E} 1$ & ug/g dry & $2.08 \mathrm{E} 1$ & $7 / 01 / 08$ & 8G01001 & PNNL-AGG-ICP-AES \\
\hline $7440-02-0$ & Nickel & 8.96Е0 & ug/g dry & $7.55 \mathrm{E} 0$ & $7 / 01 / 08$ & 8G01001 & PNNL-AGG-ICP-AES \\
\hline 7723-14-0 & Phosphorus & 7.43E2 & ug/g dry & 6.91E1 & $7 / 01 / 08$ & 8G01001 & PNNL-AGG-ICP-AES \\
\hline 7439-92-1 & Lead & $<2.92 \mathrm{E} 1$ & ug/g dry & 2.92E1 & $7 / 01 / 08$ & 8G01001 & PNNL-AGG-ICP-AES \\
\hline $7440-24-6$ & Strontium & 2.92E1 & ug/g dry & $1.33 \mathrm{E} 0$ & $7 / 01 / 08$ & 8G01001 & PNNL-AGG-ICP-AES \\
\hline $7440-66-6$ & Zinc & 3.67E1 & ug/g dry & $5.75 \mathrm{E} 0$ & 7/01/08 & 8G01001 & PNNL-AGG-ICP-AES \\
\hline $7440-23-5$ & Sodium & $2.09 \mathrm{E} 3$ & ug/g dry & $9.84 \mathrm{E} 2$ & 7/01/08 & 8G01001 & PNNL-AGG-ICP-AES \\
\hline $7440-21-3$ & Silicon & $<1.28 \mathrm{E} 3$ & ug/g dry & $1.28 \mathrm{E} 3$ & $7 / 01 / 08$ & 8G01001 & PNNL-AGG-ICP-AES \\
\hline 7704-34-9 & Sulfur & $<2.17 \mathrm{E} 2$ & ug/g dry & 2.17E2 & $7 / 01 / 08$ & 8G01001 & PNNL-AGG-ICP-AES \\
\hline $7440-32-6$ & Titanium & $6.94 \mathrm{E} 2$ & ug/g dry & $2.90 \mathrm{E} 1$ & 7/01/08 & 8G01001 & PNNL-AGG-ICP-AES \\
\hline $7440-67-7$ & Zirconium & $<1.87 \mathrm{E} 1$ & ug/g dry & $1.87 \mathrm{E} 1$ & $7 / 01 / 08$ & 8G01001 & PNNL-AGG-ICP-AES \\
\hline $7440-15-5$ & Rhenium & $<1.62 \mathrm{E} 1$ & ug/g dry & $1.62 \mathrm{E} 1$ & $7 / 01 / 08$ & 8G01001 & PNNL-AGG-ICP-AES \\
\hline $7440-36-0$ & Antimony & $<1.03 \mathrm{E} 2$ & ug/g dry & $1.03 \mathrm{E} 2$ & $7 / 01 / 08$ & 8G01001 & PNNL-AGG-ICP-AES \\
\hline HEIS No. & B1VJ58B & \multicolumn{3}{|c|}{$\begin{array}{ll}\text { Lab ID: } & 0805020-11\end{array}$} & & & \\
\hline $7429-90-5$ & Aluminum & $6.11 \mathrm{E} 3$ & ug/g dry & 3.93E1 & $7 / 01 / 08$ & 8G01001 & PNNL-AGG-ICP-AES \\
\hline $7440-38-2$ & Arsenic & $<9.55 \mathrm{E} 1$ & ug/g dry & $9.55 \mathrm{E} 1$ & $7 / 01 / 08$ & 8G01001 & PNNL-AGG-ICP-AES \\
\hline $7440-42-8$ & Boron & $<8.32 \mathrm{E} 2$ & ug/g dry & 8.32E2 & $7 / 01 / 08$ & 8G01001 & PNNL-AGG-ICP-AES \\
\hline 7440-39-3 & Barium & 8.02E1 & ug/g dry & 3.74E0 & $7 / 01 / 08$ & 8G01001 & PNNL-AGG-ICP-AES \\
\hline $7440-41-7$ & Beryllium & $<1.48 \mathrm{E} 0$ & ug/g dry & $1.48 \mathrm{E} 0$ & 7/01/08 & 8G01001 & PNNL-AGG-ICP-AES \\
\hline $7440-69-9$ & Bismuth & $<1.99 \mathrm{E} 1$ & ug/g dry & $1.99 \mathrm{E} 1$ & $7 / 01 / 08$ & 8G01001 & PNNL-AGG-ICP-AES \\
\hline $7440-70-2$ & Calcium & 8.77E3 & ug/g dry & $1.46 \mathrm{E} 2$ & $7 / 01 / 08$ & 8G01001 & PNNL-AGG-ICP-AES \\
\hline $7440-43-9$ & Cadmium & $<2.23 \mathrm{E} 0$ & ug/g dry & 2.23E0 & $7 / 01 / 08$ & 8G01001 & PNNL-AGG-ICP-AES \\
\hline $7440-48-4$ & Cobalt & $<8.16 \mathrm{E} 0$ & ug/g dry & $8.16 \mathrm{E} 0$ & $7 / 01 / 08$ & 8G01001 & PNNL-AGG-ICP-AES \\
\hline $7440-47-3$ & Chromium & 4.96E0 & ug/g dry & 3.27E0 & $7 / 01 / 08$ & 8G01001 & PNNL-AGG-ICP-AES \\
\hline $7440-50-8$ & Copper & $<3.48 \mathrm{E} 1$ & ug/g dry & $3.48 \mathrm{E} 1$ & $7 / 01 / 08$ & 8G01001 & PNNL-AGG-ICP-AES \\
\hline 7439-89-6 & Iron & $1.33 \mathrm{E} 4$ & ug/g dry & 9.99E1 & 7/01/08 & 8G01001 & PNNL-AGG-ICP-AES \\
\hline
\end{tabular}


Total Metals by PNNL-AGG-ICP-AES/Acid Extract

\begin{tabular}{|c|c|c|c|c|c|c|c|}
\hline CAS \# & Analyte & Results & Units & EQL & Analyzed & Batch & Method \\
\hline HEIS No. & B1VJ58B & \multicolumn{3}{|c|}{ Lab ID: $\quad$ 0805020-11 } & & & \\
\hline $7440-09-7$ & Potassium & $1.27 \mathrm{E} 3$ & ug/g dry & $9.27 \mathrm{E} 1$ & 7/01/08 & 8G01001 & PNNL-AGG-ICP-AES \\
\hline 7439-93-2 & Lithium & $<1.42 \mathrm{E} 1$ & ug/g dry & 1.42E1 & $7 / 01 / 08$ & 8G01001 & PNNL-AGG-ICP-AES \\
\hline 7439-95-4 & Magnesium & 4.14E3 & ug/g dry & 3.09E1 & $7 / 01 / 08$ & 8G01001 & PNNL-AGG-ICP-AES \\
\hline 7439-96-5 & Manganese & $2.82 \mathrm{E} 2$ & ug/g dry & 1.17E0 & 7/01/08 & 8G01001 & PNNL-AGG-ICP-AES \\
\hline 7439-98-7 & Molybdenum & $<2.22 \mathrm{E} 1$ & ug/g dry & 2.22E1 & 7/01/08 & 8G01001 & PNNL-AGG-ICP-AES \\
\hline $7440-02-0$ & Nickel & $<8.04 \mathrm{E} 0$ & ug/g dry & 8.04E0 & $7 / 01 / 08$ & 8G01001 & PNNL-AGG-ICP-AES \\
\hline 7723-14-0 & Phosphorus & 6.73E2 & ug/g dry & 7.36E1 & $7 / 01 / 08$ & 8G01001 & PNNL-AGG-ICP-AES \\
\hline 7439-92-1 & Lead & $<3.11 \mathrm{E} 1$ & ug/g dry & $3.11 \mathrm{E} 1$ & $7 / 01 / 08$ & 8G01001 & PNNL-AGG-ICP-AES \\
\hline $7440-24-6$ & Strontium & $2.91 \mathrm{E} 1$ & ug/g dry & $1.42 \mathrm{E} 0$ & 7/01/08 & 8G01001 & PNNL-AGG-ICP-AES \\
\hline $7440-66-6$ & Zinc & $3.44 \mathrm{E} 1$ & ug/g dry & 6.13E0 & $7 / 01 / 08$ & 8G01001 & PNNL-AGG-ICP-AES \\
\hline $7440-23-5$ & Sodium & $2.01 \mathrm{E} 3$ & ug/g dry & $1.05 \mathrm{E} 3$ & 7/01/08 & 8G01001 & PNNL-AGG-ICP-AES \\
\hline $7440-21-3$ & Silicon & $<1.37 \mathrm{E} 3$ & ug/g dry & 1.37E3 & $7 / 01 / 08$ & 8G01001 & PNNL-AGG-ICP-AES \\
\hline 7704-34-9 & Sulfur & $<2.32 \mathrm{E} 2$ & ug/g dry & 2.32E2 & $7 / 01 / 08$ & 8G01001 & PNNL-AGG-ICP-AES \\
\hline $7440-32-6$ & Titanium & 6.63E2 & ug/g dry & 3.08E1 & $7 / 01 / 08$ & 8G01001 & PNNL-AGG-ICP-AES \\
\hline $7440-67-7$ & Zirconium & $<1.99 \mathrm{E} 1$ & ug/g dry & 1.99E1 & $7 / 01 / 08$ & 8G01001 & PNNL-AGG-ICP-AES \\
\hline $7440-15-5$ & Rhenium & $<1.72 \mathrm{E} 1$ & ug/g dry & $1.72 \mathrm{E} 1$ & $7 / 01 / 08$ & 8G01001 & PNNL-AGG-ICP-AES \\
\hline $7440-36-0$ & Antimony & $<1.09 \mathrm{E} 2$ & ug/g dry & $1.09 \mathrm{E} 2$ & $7 / 01 / 08$ & 8G01001 & PNNL-AGG-ICP-AES \\
\hline HEIS No. & B1VJ58A & \multicolumn{3}{|c|}{ Lab ID: $\quad$ 0805020-12 } & & & \\
\hline $7429-90-5$ & Aluminum & $6.21 \mathrm{E} 3$ & ug/g dry & 4.02E1 & $7 / 01 / 08$ & 8G01001 & PNNL-AGG-ICP-AES \\
\hline $7440-38-2$ & Arsenic & $<9.77 \mathrm{E} 1$ & ug/g dry & $9.77 \mathrm{E} 1$ & $7 / 01 / 08$ & 8G01001 & PNNL-AGG-ICP-AES \\
\hline $7440-42-8$ & Boron & $<8.51 \mathrm{E} 2$ & ug/g dry & $8.51 \mathrm{E} 2$ & 7/01/08 & 8G01001 & PNNL-AGG-ICP-AES \\
\hline $7440-39-3$ & Barium & $7.85 \mathrm{E} 1$ & ug/g dry & 3.83E0 & $7 / 01 / 08$ & 8G01001 & PNNL-AGG-ICP-AES \\
\hline $7440-41-7$ & Beryllium & $<1.52 \mathrm{E} 0$ & ug/g dry & $1.52 \mathrm{E} 0$ & $7 / 01 / 08$ & 8G01001 & PNNL-AGG-ICP-AES \\
\hline $7440-69-9$ & Bismuth & $<2.03 \mathrm{E} 1$ & ug/g dry & 2.03E1 & $7 / 01 / 08$ & 8G01001 & PNNL-AGG-ICP-AES \\
\hline $7440-70-2$ & Calcium & $8.81 \mathrm{E} 3$ & ug/g dry & $1.49 \mathrm{E} 2$ & $7 / 01 / 08$ & 8G01001 & PNNL-AGG-ICP-AES \\
\hline $7440-43-9$ & Cadmium & $<2.28 \mathrm{E} 0$ & ug/g dry & $2.28 \mathrm{E} 0$ & $7 / 01 / 08$ & 8G01001 & PNNL-AGG-ICP-AES \\
\hline $7440-48-4$ & Cobalt & $<8.35 \mathrm{E} 0$ & ug/g dry & 8.35E0 & $7 / 01 / 08$ & 8G01001 & PNNL-AGG-ICP-AES \\
\hline $7440-47-3$ & Chromium & $6.14 \mathrm{E} 0$ & ug/g dry & 3.34E0 & 7/01/08 & 8G01001 & PNNL-AGG-ICP-AES \\
\hline $7440-50-8$ & Copper & $<3.56 \mathrm{E} 1$ & ug/g dry & $3.56 \mathrm{E} 1$ & 7/01/08 & 8G01001 & PNNL-AGG-ICP-AES \\
\hline 7439-89-6 & Iron & $1.36 \mathrm{E} 4$ & ug/g dry & $1.02 \mathrm{E} 2$ & $7 / 01 / 08$ & 8G01001 & PNNL-AGG-ICP-AES \\
\hline 7440-09-7 & Potassium & $1.34 \mathrm{E} 3$ & ug/g dry & 9.49E1 & $7 / 01 / 08$ & 8G01001 & PNNL-AGG-ICP-AES \\
\hline $7439-93-2$ & Lithium & $<1.45 \mathrm{E} 1$ & ug/g dry & $1.45 \mathrm{E} 1$ & 7/01/08 & 8G01001 & PNNL-AGG-ICP-AES \\
\hline 7439-95-4 & Magnesium & 4.43E3 & ug/g dry & $3.16 \mathrm{E} 1$ & $7 / 01 / 08$ & 8G01001 & PNNL-AGG-ICP-AES \\
\hline $7439-96-5$ & Manganese & $2.82 \mathrm{E} 2$ & ug/g dry & $1.20 \mathrm{E} 0$ & $7 / 01 / 08$ & 8G01001 & PNNL-AGG-ICP-AES \\
\hline 7439-98-7 & Molybdenum & $<2.27 \mathrm{E} 1$ & ug/g dry & $2.27 \mathrm{E} 1$ & $7 / 01 / 08$ & 8G01001 & PNNL-AGG-ICP-AES \\
\hline $7440-02-0$ & Nickel & $9.21 \mathrm{E} 0$ & ug/g dry & 8.23E0 & $7 / 01 / 08$ & 8G01001 & PNNL-AGG-ICP-AES \\
\hline $7723-14-0$ & Phosphorus & $7.04 \mathrm{E} 2$ & ug/g dry & 7.53E1 & 7/01/08 & 8G01001 & PNNL-AGG-ICP-AES \\
\hline $7439-92-1$ & Lead & $<3.18 \mathrm{E} 1$ & ug/g dry & $3.18 \mathrm{E} 1$ & $7 / 01 / 08$ & 8G01001 & PNNL-AGG-ICP-AES \\
\hline $7440-24-6$ & Strontium & $2.80 \mathrm{E} 1$ & ug/g dry & $1.45 \mathrm{E} 0$ & $7 / 01 / 08$ & 8G01001 & PNNL-AGG-ICP-AES \\
\hline $7440-66-6$ & Zinc & $3.55 \mathrm{E} 1$ & ug/g dry & $6.27 \mathrm{E} 0$ & 7/01/08 & 8G01001 & PNNL-AGG-ICP-AES \\
\hline $7440-23-5$ & Sodium & $2.04 \mathrm{E} 3$ & ug/g dry & $1.07 \mathrm{E} 3$ & 7/01/08 & 8G01001 & PNNL-AGG-ICP-AES \\
\hline $7440-21-3$ & Silicon & $<1.40 \mathrm{E} 3$ & ug/g dry & $1.40 \mathrm{E} 3$ & $7 / 01 / 08$ & 8G01001 & PNNL-AGG-ICP-AES \\
\hline $7704-34-9$ & Sulfur & $<2.37 \mathrm{E} 2$ & ug/g dry & $2.37 \mathrm{E} 2$ & 7/01/08 & 8G01001 & PNNL-AGG-ICP-AES \\
\hline $7440-32-6$ & Titanium & $6.80 \mathrm{E} 2$ & ug/g dry & 3.16E1 & $7 / 01 / 08$ & 8G01001 & PNNL-AGG-ICP-AES \\
\hline $7440-67-7$ & Zirconium & $<2.04 \mathrm{E} 1$ & ug/g dry & $2.04 \mathrm{E} 1$ & $7 / 01 / 08$ & 8G01001 & PNNL-AGG-ICP-AES \\
\hline $7440-15-5$ & Rhenium & $<1.76 \mathrm{E} 1$ & ug/g dry & $1.76 \mathrm{E} 1$ & $7 / 01 / 08$ & 8G01001 & PNNL-AGG-ICP-AES \\
\hline $7440-36-0$ & Antimony & $<1.12 \mathrm{E} 2$ & ug/g dry & $1.12 \mathrm{E} 2$ & 7/01/08 & 8G01001 & PNNL-AGG-ICP-AES \\
\hline HEIS No. & B1VJ59C & $\mathbf{L a}$ & b ID: & $5020-14$ & & & \\
\hline
\end{tabular}


Total Metals by PNNL-AGG-ICP-AES/Acid Extract

\begin{tabular}{|c|c|c|c|c|c|c|c|}
\hline CAS \# & Analyte & Results & Units & EQL & Analyzed & Batch & Method \\
\hline HEIS No. & B1VJ59C & \multicolumn{3}{|c|}{ Lab ID: $\quad$ 0805020-14 } & & & \\
\hline $7429-90-5$ & Aluminum & $5.12 \mathrm{E} 3$ & ug/g dry & 3.75E1 & 7/01/08 & 8G01001 & PNNL-AGG-ICP-AES \\
\hline $7440-38-2$ & Arsenic & $<9.10 \mathrm{E} 1$ & ug/g dry & $9.10 \mathrm{E} 1$ & $7 / 01 / 08$ & 8G01001 & PNNL-AGG-ICP-AES \\
\hline $7440-42-8$ & Boron & $<7.93 \mathrm{E} 2$ & ug/g dry & 7.93E2 & 7/01/08 & 8G01001 & PNNL-AGG-ICP-AES \\
\hline $7440-39-3$ & Barium & 5.90E1 & ug/g dry & 3.57E0 & $7 / 01 / 08$ & 8G01001 & PNNL-AGG-ICP-AES \\
\hline $7440-41-7$ & Beryllium & $<1.41 \mathrm{E} 0$ & ug/g dry & $1.41 \mathrm{E} 0$ & $7 / 01 / 08$ & 8G01001 & PNNL-AGG-ICP-AES \\
\hline $7440-69-9$ & Bismuth & $<1.89 \mathrm{E} 1$ & ug/g dry & $1.89 \mathrm{E} 1$ & 7/01/08 & 8G01001 & PNNL-AGG-ICP-AES \\
\hline $7440-70-2$ & Calcium & 7.29E3 & ug/g dry & 1.39E2 & $7 / 01 / 08$ & 8G01001 & PNNL-AGG-ICP-AES \\
\hline $7440-43-9$ & Cadmium & $<2.13 \mathrm{E} 0$ & ug/g dry & 2.13E0 & 7/01/08 & 8G01001 & PNNL-AGG-ICP-AES \\
\hline $7440-48-4$ & Cobalt & $<7.77 \mathrm{E} 0$ & ug/g dry & 7.77E0 & $7 / 01 / 08$ & 8G01001 & PNNL-AGG-ICP-AES \\
\hline $7440-47-3$ & Chromium & $1.20 \mathrm{E} 1$ & ug/g dry & 3.11E0 & $7 / 01 / 08$ & 8G01001 & PNNL-AGG-ICP-AES \\
\hline $7440-50-8$ & Copper & $<3.32 \mathrm{E} 1$ & ug/g dry & 3.32E1 & $7 / 01 / 08$ & 8G01001 & PNNL-AGG-ICP-AES \\
\hline 7439-89-6 & Iron & $1.03 \mathrm{E} 4$ & ug/g dry & $9.52 \mathrm{E} 1$ & 7/01/08 & 8G01001 & PNNL-AGG-ICP-AES \\
\hline 7440-09-7 & Potassium & $1.04 \mathrm{E} 3$ & ug/g dry & 8.84E1 & $7 / 01 / 08$ & 8G01001 & PNNL-AGG-ICP-AES \\
\hline 7439-93-2 & Lithium & $<1.35 \mathrm{E} 1$ & ug/g dry & $1.35 \mathrm{E} 1$ & $7 / 01 / 08$ & 8G01001 & PNNL-AGG-ICP-AES \\
\hline $7439-95-4$ & Magnesium & $3.54 \mathrm{E} 3$ & ug/g dry & 2.95E1 & 7/01/08 & 8G01001 & PNNL-AGG-ICP-AES \\
\hline 7439-96-5 & Manganese & 2.19E2 & ug/g dry & $1.11 \mathrm{E} 0$ & $7 / 01 / 08$ & 8G01001 & PNNL-AGG-ICP-AES \\
\hline 7439-98-7 & Molybdenum & $<2.11 \mathrm{E} 1$ & ug/g dry & $2.11 \mathrm{E} 1$ & $7 / 01 / 08$ & 8G01001 & PNNL-AGG-ICP-AES \\
\hline $7440-02-0$ & Nickel & $1.11 \mathrm{E} 1$ & ug/g dry & 7.66E0 & $7 / 01 / 08$ & 8G01001 & PNNL-AGG-ICP-AES \\
\hline $7723-14-0$ & Phosphorus & 3.95E2 & ug/g dry & 7.02E1 & 7/01/08 & 8G01001 & PNNL-AGG-ICP-AES \\
\hline $7439-92-1$ & Lead & $<2.96 \mathrm{E} 1$ & ug/g dry & $2.96 \mathrm{E} 1$ & 7/01/08 & 8G01001 & PNNL-AGG-ICP-AES \\
\hline $7440-24-6$ & Strontium & $3.21 \mathrm{E} 1$ & ug/g dry & $1.35 \mathrm{E} 0$ & $7 / 01 / 08$ & 8G01001 & PNNL-AGG-ICP-AES \\
\hline $7440-66-6$ & Zinc & 2.82E1 & ug/g dry & $5.84 \mathrm{E} 0$ & $7 / 01 / 08$ & 8G01001 & PNNL-AGG-ICP-AES \\
\hline $7440-23-5$ & Sodium & $<9.98 \mathrm{E} 2$ & ug/g dry & $9.98 \mathrm{E} 2$ & 7/01/08 & 8G01001 & PNNL-AGG-ICP-AES \\
\hline $7440-21-3$ & Silicon & $<1.30 \mathrm{E} 3$ & ug/g dry & $1.30 \mathrm{E} 3$ & $7 / 01 / 08$ & 8G01001 & PNNL-AGG-ICP-AES \\
\hline $7704-34-9$ & Sulfur & $<2.21 \mathrm{E} 2$ & ug/g dry & $2.21 \mathrm{E} 2$ & $7 / 01 / 08$ & 8G01001 & PNNL-AGG-ICP-AES \\
\hline $7440-32-6$ & Titanium & 4.52E2 & ug/g dry & 2.94E1 & $7 / 01 / 08$ & 8G01001 & PNNL-AGG-ICP-AES \\
\hline $7440-67-7$ & Zirconium & $<1.90 \mathrm{E} 1$ & ug/g dry & $1.90 \mathrm{E} 1$ & 7/01/08 & 8G01001 & PNNL-AGG-ICP-AES \\
\hline $7440-15-5$ & Rhenium & $<1.64 \mathrm{E} 1$ & ug/g dry & $1.64 \mathrm{E} 1$ & 7/01/08 & 8G01001 & PNNL-AGG-ICP-AES \\
\hline $7440-36-0$ & Antimony & $<1.04 \mathrm{E} 2$ & ug/g dry & $1.04 \mathrm{E} 2$ & $7 / 01 / 08$ & 8G01001 & PNNL-AGG-ICP-AES \\
\hline HEIS No. & B1VJ59B & & b ID: & 5020-15 & & & \\
\hline $7429-90-5$ & Aluminum & $4.54 \mathrm{E} 3$ & ug/g dry & 3.55E1 & $7 / 01 / 08$ & 8G01001 & PNNL-AGG-ICP-AES \\
\hline $7440-38-2$ & Arsenic & $<8.63 \mathrm{E} 1$ & ug/g dry & 8.63E1 & 7/01/08 & 8G01001 & PNNL-AGG-ICP-AES \\
\hline $7440-42-8$ & Boron & $<7.51 \mathrm{E} 2$ & ug/g dry & $7.51 \mathrm{E} 2$ & $7 / 01 / 08$ & 8G01001 & PNNL-AGG-ICP-AES \\
\hline $7440-39-3$ & Barium & 4.63E1 & ug/g dry & 3.38E0 & $7 / 01 / 08$ & 8G01001 & PNNL-AGG-ICP-AES \\
\hline $7440-41-7$ & Beryllium & $<1.34 \mathrm{E} 0$ & ug/g dry & $1.34 \mathrm{E} 0$ & 7/01/08 & 8G01001 & PNNL-AGG-ICP-AES \\
\hline 7440-69-9 & Bismuth & $<1.79 \mathrm{E} 1$ & ug/g dry & $1.79 \mathrm{E} 1$ & 7/01/08 & 8G01001 & PNNL-AGG-ICP-AES \\
\hline $7440-70-2$ & Calcium & $7.42 \mathrm{E} 3$ & ug/g dry & $1.32 \mathrm{E} 2$ & $7 / 01 / 08$ & 8G01001 & PNNL-AGG-ICP-AES \\
\hline $7440-43-9$ & Cadmium & $<2.02 \mathrm{E} 0$ & ug/g dry & $2.02 \mathrm{E} 0$ & $7 / 01 / 08$ & 8G01001 & PNNL-AGG-ICP-AES \\
\hline $7440-48-4$ & Cobalt & $<7.37 \mathrm{E} 0$ & ug/g dry & 7.37E0 & 7/01/08 & 8G01001 & PNNL-AGG-ICP-AES \\
\hline $7440-47-3$ & Chromium & $1.26 \mathrm{E} 1$ & ug/g dry & $2.95 \mathrm{E} 0$ & 7/01/08 & 8G01001 & PNNL-AGG-ICP-AES \\
\hline $7440-50-8$ & Copper & $<3.14 \mathrm{E} 1$ & ug/g dry & $3.14 \mathrm{E} 1$ & 7/01/08 & 8G01001 & PNNL-AGG-ICP-AES \\
\hline 7439-89-6 & Iron & 9.03E3 & ug/g dry & $9.02 \mathrm{E} 1$ & 7/01/08 & 8G01001 & PNNL-AGG-ICP-AES \\
\hline 7440-09-7 & Potassium & $8.50 \mathrm{E} 2$ & ug/g dry & 8.37E1 & 7/01/08 & 8G01001 & PNNL-AGG-ICP-AES \\
\hline 7439-93-2 & Lithium & $<1.28 \mathrm{E} 1$ & ug/g dry & $1.28 \mathrm{E} 1$ & $7 / 01 / 08$ & 8G01001 & PNNL-AGG-ICP-AES \\
\hline 7439-95-4 & Magnesium & $3.55 \mathrm{E} 3$ & ug/g dry & 2.79E1 & $7 / 01 / 08$ & 8G01001 & PNNL-AGG-ICP-AES \\
\hline 7439-96-5 & Manganese & $1.94 \mathrm{E} 2$ & ug/g dry & $1.06 \mathrm{E} 0$ & $7 / 01 / 08$ & 8G01001 & PNNL-AGG-ICP-AES \\
\hline 7439-98-7 & Molybdenum & $<2.00 \mathrm{E} 1$ & ug/g dry & $2.00 \mathrm{E} 1$ & 7/01/08 & 8G01001 & PNNL-AGG-ICP-AES \\
\hline $7440-02-0$ & Nickel & 1.07E1 & ug/g dry & $7.26 \mathrm{E} 0$ & 7/01/08 & 8G01001 & PNNL-AGG-ICP-AES \\
\hline
\end{tabular}


Total Metals by PNNL-AGG-ICP-AES/Acid Extract

\begin{tabular}{|c|c|c|c|c|c|c|c|}
\hline CAS \# & Analyte & Results & Units & EQL & Analyzed & Batch & Method \\
\hline HEIS No. & B1VJ59B & \multicolumn{3}{|c|}{ Lab ID: $\quad$ 0805020-15 } & & & \\
\hline $7723-14-0$ & Phosphorus & 3.86E2 & ug/g dry & $6.65 \mathrm{E} 1$ & 7/01/08 & 8G01001 & PNNL-AGG-ICP-AES \\
\hline 7439-92-1 & Lead & $<2.81 \mathrm{E} 1$ & ug/g dry & $2.81 \mathrm{E} 1$ & $7 / 01 / 08$ & 8G01001 & PNNL-AGG-ICP-AES \\
\hline $7440-24-6$ & Strontium & 2.61E1 & ug/g dry & $1.28 \mathrm{E} 0$ & $7 / 01 / 08$ & 8G01001 & PNNL-AGG-ICP-AES \\
\hline $7440-66-6$ & Zinc & 2.34E1 & ug/g dry & 5.53E0 & $7 / 01 / 08$ & 8G01001 & PNNL-AGG-ICP-AES \\
\hline $7440-23-5$ & Sodium & $<9.46 \mathrm{E} 2$ & ug/g dry & $9.46 \mathrm{E} 2$ & 7/01/08 & 8G01001 & PNNL-AGG-ICP-AES \\
\hline $7440-21-3$ & Silicon & $<1.23 \mathrm{E} 3$ & ug/g dry & $1.23 \mathrm{E} 3$ & $7 / 01 / 08$ & 8G01001 & PNNL-AGG-ICP-AES \\
\hline $7704-34-9$ & Sulfur & $<2.09 \mathrm{E} 2$ & ug/g dry & 2.09E2 & $7 / 01 / 08$ & 8G01001 & PNNL-AGG-ICP-AES \\
\hline $7440-32-6$ & Titanium & $4.00 \mathrm{E} 2$ & ug/g dry & 2.79E1 & $7 / 01 / 08$ & 8G01001 & PNNL-AGG-ICP-AES \\
\hline $7440-67-7$ & Zirconium & $<1.80 \mathrm{E} 1$ & ug/g dry & $1.80 \mathrm{E} 1$ & 7/01/08 & 8G01001 & PNNL-AGG-ICP-AES \\
\hline $7440-15-5$ & Rhenium & $<1.56 \mathrm{E} 1$ & ug/g dry & $1.56 \mathrm{E} 1$ & $7 / 01 / 08$ & 8G01001 & PNNL-AGG-ICP-AES \\
\hline $7440-36-0$ & Antimony & $<9.88 \mathrm{E} 1$ & ug/g dry & $9.88 \mathrm{E} 1$ & 7/01/08 & 8G01001 & PNNL-AGG-ICP-AES \\
\hline HEIS No. & B1VJ59A & \multicolumn{3}{|c|}{ Lab ID: $\quad$ 0805020-16 } & & & \\
\hline $7429-90-5$ & Aluminum & 4.45E3 & ug/g dry & $3.51 \mathrm{E} 1$ & $7 / 01 / 08$ & 8G01001 & PNNL-AGG-ICP-AES \\
\hline $7440-38-2$ & Arsenic & $<8.53 \mathrm{E} 1$ & ug/g dry & 8.53E1 & $7 / 01 / 08$ & 8G01001 & PNNL-AGG-ICP-AES \\
\hline $7440-42-8$ & Boron & $<7.43 \mathrm{E} 2$ & ug/g dry & 7.43E2 & $7 / 01 / 08$ & 8G01001 & PNNL-AGG-ICP-AES \\
\hline $7440-39-3$ & Barium & 4.32E1 & ug/g dry & $3.34 \mathrm{E} 0$ & $7 / 01 / 08$ & 8G01001 & PNNL-AGG-ICP-AES \\
\hline $7440-41-7$ & Beryllium & $<1.33 \mathrm{E} 0$ & ug/g dry & $1.33 \mathrm{E} 0$ & $7 / 01 / 08$ & 8G01001 & PNNL-AGG-ICP-AES \\
\hline $7440-69-9$ & Bismuth & $<1.77 \mathrm{E} 1$ & ug/g dry & $1.77 \mathrm{E} 1$ & $7 / 01 / 08$ & 8G01001 & PNNL-AGG-ICP-AES \\
\hline $7440-70-2$ & Calcium & 7.77E3 & ug/g dry & $1.30 \mathrm{E} 2$ & $7 / 01 / 08$ & 8G01001 & PNNL-AGG-ICP-AES \\
\hline 7440-43-9 & Cadmium & $<1.99 \mathrm{E} 0$ & ug/g dry & 1.99E0 & $7 / 01 / 08$ & 8G01001 & PNNL-AGG-ICP-AES \\
\hline $7440-48-4$ & Cobalt & $<7.29 \mathrm{E} 0$ & ug/g dry & $7.29 \mathrm{E} 0$ & 7/01/08 & 8G01001 & PNNL-AGG-ICP-AES \\
\hline $7440-47-3$ & Chromium & $1.25 \mathrm{E} 1$ & ug/g dry & 2.92E0 & 7/01/08 & 8G01001 & PNNL-AGG-ICP-AES \\
\hline $7440-50-8$ & Copper & $<3.11 \mathrm{E} 1$ & ug/g dry & 3.11E1 & $7 / 01 / 08$ & 8G01001 & PNNL-AGG-ICP-AES \\
\hline 7439-89-6 & Iron & $9.02 \mathrm{E} 3$ & ug/g dry & 8.92E1 & $7 / 01 / 08$ & 8G01001 & PNNL-AGG-ICP-AES \\
\hline $7440-09-7$ & Potassium & 8.22E2 & ug/g dry & $8.28 \mathrm{E} 1$ & $7 / 01 / 08$ & 8G01001 & PNNL-AGG-ICP-AES \\
\hline 7439-93-2 & Lithium & $<1.27 \mathrm{E} 1$ & ug/g dry & $1.27 \mathrm{E} 1$ & $7 / 01 / 08$ & 8G01001 & PNNL-AGG-ICP-AES \\
\hline $7439-95-4$ & Magnesium & 3.62E3 & ug/g dry & $2.76 \mathrm{E} 1$ & $7 / 01 / 08$ & 8G01001 & PNNL-AGG-ICP-AES \\
\hline $7439-96-5$ & Manganese & $1.85 \mathrm{E} 2$ & ug/g dry & $1.05 \mathrm{E} 0$ & 7/01/08 & 8G01001 & PNNL-AGG-ICP-AES \\
\hline 7439-98-7 & Molybdenum & $<1.98 \mathrm{E} 1$ & ug/g dry & $1.98 \mathrm{E} 1$ & 7/01/08 & 8G01001 & PNNL-AGG-ICP-AES \\
\hline $7440-02-0$ & Nickel & 1.10E1 & ug/g dry & 7.18E0 & $7 / 01 / 08$ & 8G01001 & PNNL-AGG-ICP-AES \\
\hline 7723-14-0 & Phosphorus & 4.05E2 & ug/g dry & $6.58 \mathrm{E} 1$ & $7 / 01 / 08$ & 8G01001 & PNNL-AGG-ICP-AES \\
\hline $7439-92-1$ & Lead & $<2.78 \mathrm{E} 1$ & ug/g dry & $2.78 \mathrm{E} 1$ & 7/01/08 & 8G01001 & PNNL-AGG-ICP-AES \\
\hline $7440-24-6$ & Strontium & $2.88 \mathrm{E} 1$ & ug/g dry & $1.27 \mathrm{E} 0$ & $7 / 01 / 08$ & 8G01001 & PNNL-AGG-ICP-AES \\
\hline $7440-66-6$ & Zinc & $2.45 \mathrm{E} 1$ & ug/g dry & 5.47E0 & $7 / 01 / 08$ & 8G01001 & PNNL-AGG-ICP-AES \\
\hline $7440-23-5$ & Sodium & $<9.36 \mathrm{E} 2$ & ug/g dry & $9.36 \mathrm{E} 2$ & $7 / 01 / 08$ & 8G01001 & PNNL-AGG-ICP-AES \\
\hline $7440-21-3$ & Silicon & $<1.22 \mathrm{E} 3$ & ug/g dry & $1.22 \mathrm{E} 3$ & $7 / 01 / 08$ & 8G01001 & PNNL-AGG-ICP-AES \\
\hline $7704-34-9$ & Sulfur & $<2.07 \mathrm{E} 2$ & ug/g dry & 2.07E2 & 7/01/08 & 8G01001 & PNNL-AGG-ICP-AES \\
\hline $7440-32-6$ & Titanium & 4.27E2 & ug/g dry & $2.75 \mathrm{E} 1$ & $7 / 01 / 08$ & 8G01001 & PNNL-AGG-ICP-AES \\
\hline $7440-67-7$ & Zirconium & $<1.78 \mathrm{E} 1$ & ug/g dry & $1.78 \mathrm{E} 1$ & $7 / 01 / 08$ & 8G01001 & PNNL-AGG-ICP-AES \\
\hline $7440-15-5$ & Rhenium & $<1.54 \mathrm{E} 1$ & ug/g dry & $1.54 \mathrm{E} 1$ & 7/01/08 & 8G01001 & PNNL-AGG-ICP-AES \\
\hline $7440-36-0$ & Antimony & $<9.77 \mathrm{E} 1$ & ug/g dry & $9.77 \mathrm{E} 1$ & $7 / 01 / 08$ & 8G01001 & PNNL-AGG-ICP-AES \\
\hline HEIS No. & B1VJ60C & & b ID: & $5020-18$ & & & \\
\hline $7429-90-5$ & Aluminum & $4.76 \mathrm{E} 3$ & ug/g dry & 3.39E1 & $7 / 01 / 08$ & 8G01001 & PNNL-AGG-ICP-AES \\
\hline $7440-38-2$ & Arsenic & $<8.23 \mathrm{E} 1$ & ug/g dry & 8.23E1 & 7/01/08 & 8G01001 & PNNL-AGG-ICP-AES \\
\hline $7440-42-8$ & Boron & $<7.17 \mathrm{E} 2$ & ug/g dry & 7.17E2 & $7 / 01 / 08$ & 8G01001 & PNNL-AGG-ICP-AES \\
\hline 7440-39-3 & Barium & 5.27E1 & ug/g dry & 3.23E0 & $7 / 01 / 08$ & 8G01001 & PNNL-AGG-ICP-AES \\
\hline $7440-41-7$ & Beryllium & $<1.28 \mathrm{E} 0$ & ug/g dry & $1.28 \mathrm{E} 0$ & $7 / 01 / 08$ & 8G01001 & PNNL-AGG-ICP-AES \\
\hline 7440-69-9 & Bismuth & $<1.71 \mathrm{E} 1$ & ug/g dry & $1.71 \mathrm{E} 1$ & 7/01/08 & 8G01001 & PNNL-AGG-ICP-AES \\
\hline
\end{tabular}


Total Metals by PNNL-AGG-ICP-AES/Acid Extract

\begin{tabular}{|c|c|c|c|c|c|c|c|}
\hline CAS \# & Analyte & Results & Units & EQL & Analyzed & Batch & Method \\
\hline HEIS No. & B1VJ60C & \multicolumn{3}{|c|}{ Lab ID: $\quad$ 0805020-18 } & & & \\
\hline $7440-70-2$ & Calcium & $8.50 \mathrm{E} 3$ & ug/g dry & $1.26 \mathrm{E} 2$ & 7/01/08 & 8G01001 & PNNL-AGG-ICP-AES \\
\hline $7440-43-9$ & Cadmium & $<1.92 \mathrm{E} 0$ & ug/g dry & $1.92 \mathrm{E} 0$ & $7 / 01 / 08$ & 8G01001 & PNNL-AGG-ICP-AES \\
\hline $7440-48-4$ & Cobalt & $<7.03 \mathrm{E} 0$ & ug/g dry & 7.03E0 & 7/01/08 & 8G01001 & PNNL-AGG-ICP-AES \\
\hline $7440-47-3$ & Chromium & $2.40 \mathrm{E} 1$ & ug/g dry & 2.82E0 & $7 / 01 / 08$ & 8G01001 & PNNL-AGG-ICP-AES \\
\hline $7440-50-8$ & Copper & $<3.00 \mathrm{E} 1$ & ug/g dry & $3.00 \mathrm{E} 1$ & $7 / 01 / 08$ & 8G01001 & PNNL-AGG-ICP-AES \\
\hline 7439-89-6 & Iron & $1.10 \mathrm{E} 4$ & ug/g dry & 8.61E1 & 7/01/08 & 8G01001 & PNNL-AGG-ICP-AES \\
\hline $7440-09-7$ & Potassium & $9.70 \mathrm{E} 2$ & ug/g dry & 7.99E1 & $7 / 01 / 08$ & 8G01001 & PNNL-AGG-ICP-AES \\
\hline 7439-93-2 & Lithium & $<1.22 \mathrm{E} 1$ & ug/g dry & $1.22 \mathrm{E} 1$ & 7/01/08 & 8G01001 & PNNL-AGG-ICP-AES \\
\hline 7439-95-4 & Magnesium & 3.84E3 & ug/g dry & $2.66 \mathrm{E} 1$ & $7 / 01 / 08$ & 8G01001 & PNNL-AGG-ICP-AES \\
\hline $7439-96-5$ & Manganese & $2.35 \mathrm{E} 2$ & ug/g dry & $1.01 \mathrm{E} 0$ & $7 / 01 / 08$ & 8G01001 & PNNL-AGG-ICP-AES \\
\hline 7439-98-7 & Molybdenum & $<1.91 \mathrm{E} 1$ & ug/g dry & $1.91 \mathrm{E} 1$ & $7 / 01 / 08$ & 8G01001 & PNNL-AGG-ICP-AES \\
\hline $7440-02-0$ & Nickel & $1.59 \mathrm{E} 1$ & ug/g dry & $6.93 \mathrm{E} 0$ & 7/01/08 & 8G01001 & PNNL-AGG-ICP-AES \\
\hline $7723-14-0$ & Phosphorus & 3.91E2 & ug/g dry & 6.34E1 & $7 / 01 / 08$ & 8G01001 & PNNL-AGG-ICP-AES \\
\hline 7439-92-1 & Lead & $<2.68 \mathrm{E} 1$ & ug/g dry & $2.68 \mathrm{E} 1$ & $7 / 01 / 08$ & 8G01001 & PNNL-AGG-ICP-AES \\
\hline $7440-24-6$ & Strontium & 3.02E1 & ug/g dry & $1.22 \mathrm{E} 0$ & 7/01/08 & 8G01001 & PNNL-AGG-ICP-AES \\
\hline $7440-66-6$ & Zinc & $5.00 \mathrm{E} 1$ & ug/g dry & $5.28 \mathrm{E} 0$ & $7 / 01 / 08$ & 8G01001 & PNNL-AGG-ICP-AES \\
\hline $7440-23-5$ & Sodium & $<9.02 \mathrm{E} 2$ & ug/g dry & $9.02 \mathrm{E} 2$ & $7 / 01 / 08$ & 8G01001 & PNNL-AGG-ICP-AES \\
\hline $7440-21-3$ & Silicon & $<1.18 \mathrm{E} 3$ & ug/g dry & $1.18 \mathrm{E} 3$ & $7 / 01 / 08$ & 8G01001 & PNNL-AGG-ICP-AES \\
\hline 7704-34-9 & Sulfur & $<2.00 \mathrm{E} 2$ & ug/g dry & $2.00 \mathrm{E} 2$ & 7/01/08 & 8G01001 & PNNL-AGG-ICP-AES \\
\hline $7440-32-6$ & Titanium & $4.40 \mathrm{E} 2$ & ug/g dry & $2.66 \mathrm{E} 1$ & 7/01/08 & 8G01001 & PNNL-AGG-ICP-AES \\
\hline $7440-67-7$ & Zirconium & $<1.71 \mathrm{E} 1$ & ug/g dry & $1.71 \mathrm{E} 1$ & $7 / 01 / 08$ & 8G01001 & PNNL-AGG-ICP-AES \\
\hline $7440-15-5$ & Rhenium & $<1.48 \mathrm{E} 1$ & ug/g dry & $1.48 \mathrm{E} 1$ & $7 / 01 / 08$ & 8G01001 & PNNL-AGG-ICP-AES \\
\hline $7440-36-0$ & Antimony & $<9.43 \mathrm{E} 1$ & ug/g dry & $9.43 \mathrm{E} 1$ & 7/01/08 & 8G01001 & PNNL-AGG-ICP-AES \\
\hline HEIS No. & B1VJ60B & \multicolumn{3}{|c|}{ Lab ID: $\quad$ 0805020-19 } & & & \\
\hline $7429-90-5$ & Aluminum & $4.59 \mathrm{E} 3$ & ug/g dry & 3.58E1 & $7 / 01 / 08$ & 8G01001 & PNNL-AGG-ICP-AES \\
\hline $7440-38-2$ & Arsenic & $<8.69 \mathrm{E} 1$ & ug/g dry & 8.69E1 & $7 / 01 / 08$ & 8G01001 & PNNL-AGG-ICP-AES \\
\hline $7440-42-8$ & Boron & $<7.57 \mathrm{E} 2$ & ug/g dry & 7.57E2 & $7 / 01 / 08$ & 8G01001 & PNNL-AGG-ICP-AES \\
\hline $7440-39-3$ & Barium & 5.49E1 & ug/g dry & $3.41 \mathrm{E} 0$ & 7/01/08 & 8G01001 & PNNL-AGG-ICP-AES \\
\hline $7440-41-7$ & Beryllium & $<1.35 \mathrm{E} 0$ & ug/g dry & $1.35 \mathrm{E} 0$ & 7/01/08 & 8G01001 & PNNL-AGG-ICP-AES \\
\hline $7440-69-9$ & Bismuth & $<1.81 \mathrm{E} 1$ & ug/g dry & $1.81 \mathrm{E} 1$ & $7 / 01 / 08$ & 8G01001 & PNNL-AGG-ICP-AES \\
\hline $7440-70-2$ & Calcium & 8.60E3 & ug/g dry & 1.33E2 & $7 / 01 / 08$ & 8G01001 & PNNL-AGG-ICP-AES \\
\hline $7440-43-9$ & Cadmium & $<2.03 \mathrm{E} 0$ & ug/g dry & 2.03E0 & 7/01/08 & 8G01001 & PNNL-AGG-ICP-AES \\
\hline $7440-48-4$ & Cobalt & $<7.43 \mathrm{E} 0$ & ug/g dry & 7.43E0 & $7 / 01 / 08$ & 8G01001 & PNNL-AGG-ICP-AES \\
\hline $7440-47-3$ & Chromium & $1.02 \mathrm{E} 1$ & ug/g dry & 2.97E0 & $7 / 01 / 08$ & 8G01001 & PNNL-AGG-ICP-AES \\
\hline $7440-50-8$ & Copper & $<3.17 \mathrm{E} 1$ & ug/g dry & 3.17E1 & 7/01/08 & 8G01001 & PNNL-AGG-ICP-AES \\
\hline 7439-89-6 & Iron & 8.67E3 & ug/g dry & $9.09 \mathrm{E} 1$ & 7/01/08 & 8G01001 & PNNL-AGG-ICP-AES \\
\hline $7440-09-7$ & Potassium & $1.16 \mathrm{E} 3$ & ug/g dry & 8.44E1 & $7 / 01 / 08$ & 8G01001 & PNNL-AGG-ICP-AES \\
\hline 7439-93-2 & Lithium & $<1.29 \mathrm{E} 1$ & ug/g dry & $1.29 \mathrm{E} 1$ & $7 / 01 / 08$ & 8G01001 & PNNL-AGG-ICP-AES \\
\hline 7439-95-4 & Magnesium & 3.68E3 & ug/g dry & $2.81 \mathrm{E} 1$ & 7/01/08 & 8G01001 & PNNL-AGG-ICP-AES \\
\hline 7439-96-5 & Manganese & 2.13E2 & ug/g dry & $1.06 \mathrm{E} 0$ & 7/01/08 & 8G01001 & PNNL-AGG-ICP-AES \\
\hline 7439-98-7 & Molybdenum & $<2.02 \mathrm{E} 1$ & ug/g dry & $2.02 \mathrm{E} 1$ & 7/01/08 & 8G01001 & PNNL-AGG-ICP-AES \\
\hline $7440-02-0$ & Nickel & 9.00E0 & ug/g dry & 7.32E0 & 7/01/08 & 8G01001 & PNNL-AGG-ICP-AES \\
\hline $7723-14-0$ & Phosphorus & $3.24 \mathrm{E} 2$ & ug/g dry & $6.70 \mathrm{E} 1$ & 7/01/08 & 8G01001 & PNNL-AGG-ICP-AES \\
\hline 7439-92-1 & Lead & $<2.83 \mathrm{E} 1$ & ug/g dry & $2.83 \mathrm{E} 1$ & $7 / 01 / 08$ & 8G01001 & PNNL-AGG-ICP-AES \\
\hline $7440-24-6$ & Strontium & 2.70E1 & ug/g dry & $1.29 \mathrm{E} 0$ & $7 / 01 / 08$ & 8G01001 & PNNL-AGG-ICP-AES \\
\hline $7440-66-6$ & Zinc & $2.54 \mathrm{E} 1$ & ug/g dry & $5.58 \mathrm{E} 0$ & $7 / 01 / 08$ & 8G01001 & PNNL-AGG-ICP-AES \\
\hline $7440-23-5$ & Sodium & $<9.53 \mathrm{E} 2$ & ug/g dry & $9.53 \mathrm{E} 2$ & 7/01/08 & 8G01001 & PNNL-AGG-ICP-AES \\
\hline $7440-21-3$ & Silicon & $<1.24 \mathrm{E} 3$ & ug/g dry & $1.24 \mathrm{E} 3$ & 7/01/08 & 8G01001 & PNNL-AGG-ICP-AES \\
\hline
\end{tabular}


Total Metals by PNNL-AGG-ICP-AES/Acid Extract

\begin{tabular}{|c|c|c|c|c|c|c|c|}
\hline CAS \# & Analyte & Results & Units & EQL & Analyzed & Batch & Method \\
\hline HEIS No. & B1VJ60B & \multicolumn{3}{|c|}{ Lab ID: $\quad$ 0805020-19 } & & & \\
\hline 7704-34-9 & Sulfur & $<2.11 \mathrm{E} 2$ & ug/g dry & $2.11 \mathrm{E} 2$ & 7/01/08 & 8G01001 & PNNL-AGG-ICP-AES \\
\hline $7440-32-6$ & Titanium & 3.60E2 & ug/g dry & $2.81 \mathrm{E} 1$ & $7 / 01 / 08$ & 8G01001 & PNNL-AGG-ICP-AES \\
\hline 7440-67-7 & Zirconium & $<1.81 \mathrm{E} 1$ & ug/g dry & $1.81 \mathrm{E} 1$ & $7 / 01 / 08$ & 8G01001 & PNNL-AGG-ICP-AES \\
\hline $7440-15-5$ & Rhenium & $<1.57 \mathrm{E} 1$ & ug/g dry & $1.57 \mathrm{E} 1$ & $7 / 01 / 08$ & 8G01001 & PNNL-AGG-ICP-AES \\
\hline $7440-36-0$ & Antimony & $<9.96 \mathrm{E} 1$ & ug/g dry & 9.96E1 & $7 / 01 / 08$ & 8G01001 & PNNL-AGG-ICP-AES \\
\hline HEIS No. & B1VJ60A & \multicolumn{3}{|c|}{ Lab ID: $\quad$ 0805020-20 } & & & \\
\hline 7429-90-5 & Aluminum & 4.37E3 & ug/g dry & 3.51E1 & $7 / 01 / 08$ & 8G01001 & PNNL-AGG-ICP-AES \\
\hline $7440-38-2$ & Arsenic & $<8.53 \mathrm{E} 1$ & ug/g dry & 8.53E1 & $7 / 01 / 08$ & 8G01001 & PNNL-AGG-ICP-AES \\
\hline $7440-42-8$ & Boron & $<7.43 \mathrm{E} 2$ & ug/g dry & 7.43E2 & $7 / 01 / 08$ & 8G01001 & PNNL-AGG-ICP-AES \\
\hline $7440-39-3$ & Barium & 4.96E1 & ug/g dry & 3.34E0 & $7 / 01 / 08$ & 8G01001 & PNNL-AGG-ICP-AES \\
\hline $7440-41-7$ & Beryllium & $<1.33 \mathrm{E} 0$ & ug/g dry & 1.33E0 & $7 / 01 / 08$ & 8G01001 & PNNL-AGG-ICP-AES \\
\hline 7440-69-9 & Bismuth & $<1.77 \mathrm{E} 1$ & ug/g dry & 1.77E1 & $7 / 01 / 08$ & 8G01001 & PNNL-AGG-ICP-AES \\
\hline $7440-70-2$ & Calcium & 7.82E3 & ug/g dry & $1.30 \mathrm{E} 2$ & $7 / 01 / 08$ & 8G01001 & PNNL-AGG-ICP-AES \\
\hline $7440-43-9$ & Cadmium & $<1.99 \mathrm{E} 0$ & ug/g dry & $1.99 \mathrm{E} 0$ & 7/01/08 & 8G01001 & PNNL-AGG-ICP-AES \\
\hline $7440-48-4$ & Cobalt & $<7.29 \mathrm{E} 0$ & ug/g dry & 7.29E0 & $7 / 01 / 08$ & 8G01001 & PNNL-AGG-ICP-AES \\
\hline $7440-47-3$ & Chromium & 9.17E0 & ug/g dry & 2.92E0 & $7 / 01 / 08$ & 8G01001 & PNNL-AGG-ICP-AES \\
\hline $7440-50-8$ & Copper & $<3.11 \mathrm{E} 1$ & ug/g dry & $3.11 \mathrm{E} 1$ & 7/01/08 & 8G01001 & PNNL-AGG-ICP-AES \\
\hline 7439-89-6 & Iron & $9.13 \mathrm{E} 3$ & ug/g dry & 8.92E1 & 7/01/08 & 8G01001 & PNNL-AGG-ICP-AES \\
\hline $7440-09-7$ & Potassium & $9.14 \mathrm{E} 2$ & ug/g dry & $8.28 \mathrm{E} 1$ & $7 / 01 / 08$ & 8G01001 & PNNL-AGG-ICP-AES \\
\hline 7439-93-2 & Lithium & $<1.27 \mathrm{E} 1$ & ug/g dry & $1.27 \mathrm{E} 1$ & $7 / 01 / 08$ & 8G01001 & PNNL-AGG-ICP-AES \\
\hline $7439-95-4$ & Magnesium & 3.49E3 & ug/g dry & $2.76 \mathrm{E} 1$ & 7/01/08 & 8G01001 & PNNL-AGG-ICP-AES \\
\hline 7439-96-5 & Manganese & $1.90 \mathrm{E} 2$ & ug/g dry & $1.04 \mathrm{E} 0$ & 7/01/08 & 8G01001 & PNNL-AGG-ICP-AES \\
\hline 7439-98-7 & Molybdenum & $<1.98 \mathrm{E} 1$ & ug/g dry & $1.98 \mathrm{E} 1$ & $7 / 01 / 08$ & 8G01001 & PNNL-AGG-ICP-AES \\
\hline $7440-02-0$ & Nickel & 8.23E0 & ug/g dry & 7.18E0 & $7 / 01 / 08$ & 8G01001 & PNNL-AGG-ICP-AES \\
\hline 7723-14-0 & Phosphorus & 4.10E2 & ug/g dry & $6.57 \mathrm{E} 1$ & $7 / 01 / 08$ & 8G01001 & PNNL-AGG-ICP-AES \\
\hline 7439-92-1 & Lead & $<2.78 \mathrm{E} 1$ & ug/g dry & $2.78 \mathrm{E} 1$ & $7 / 01 / 08$ & 8G01001 & PNNL-AGG-ICP-AES \\
\hline $7440-24-6$ & Strontium & 2.59E1 & ug/g dry & $1.27 \mathrm{E} 0$ & $7 / 01 / 08$ & 8G01001 & PNNL-AGG-ICP-AES \\
\hline $7440-66-6$ & Zinc & $2.41 \mathrm{E} 1$ & ug/g dry & 5.47E0 & 7/01/08 & 8G01001 & PNNL-AGG-ICP-AES \\
\hline $7440-23-5$ & Sodium & $<9.35 \mathrm{E} 2$ & ug/g dry & 9.35E2 & $7 / 01 / 08$ & 8G01001 & PNNL-AGG-ICP-AES \\
\hline $7440-21-3$ & Silicon & $<1.22 \mathrm{E} 3$ & ug/g dry & $1.22 \mathrm{E} 3$ & $7 / 01 / 08$ & 8G01001 & PNNL-AGG-ICP-AES \\
\hline 7704-34-9 & Sulfur & $<2.07 \mathrm{E} 2$ & ug/g dry & 2.07E2 & $7 / 01 / 08$ & 8G01001 & PNNL-AGG-ICP-AES \\
\hline $7440-32-6$ & Titanium & $4.04 \mathrm{E} 2$ & ug/g dry & $2.75 \mathrm{E} 1$ & 7/01/08 & 8G01001 & PNNL-AGG-ICP-AES \\
\hline $7440-67-7$ & Zirconium & $<1.78 \mathrm{E} 1$ & ug/g dry & $1.78 \mathrm{E} 1$ & $7 / 01 / 08$ & 8G01001 & PNNL-AGG-ICP-AES \\
\hline $7440-15-5$ & Rhenium & $<1.54 \mathrm{E} 1$ & ug/g dry & $1.54 \mathrm{E} 1$ & $7 / 01 / 08$ & 8G01001 & PNNL-AGG-ICP-AES \\
\hline $7440-36-0$ & Antimony & $<9.77 \mathrm{E} 1$ & ug/g dry & $9.77 \mathrm{E} 1$ & $7 / 01 / 08$ & 8G01001 & PNNL-AGG-ICP-AES \\
\hline HEIS No. & B1VJ61C & \multicolumn{3}{|c|}{$\begin{array}{ll}\text { Lab ID: } & 0805020-22\end{array}$} & & & \\
\hline $7429-90-5$ & Aluminum & $4.15 \mathrm{E} 3$ & ug/g dry & 3.15E1 & $7 / 01 / 08$ & 8G01001 & PNNL-AGG-ICP-AES \\
\hline $7440-38-2$ & Arsenic & $<7.65 \mathrm{E} 1$ & ug/g dry & 7.65E1 & $7 / 01 / 08$ & 8G01001 & PNNL-AGG-ICP-AES \\
\hline $7440-42-8$ & Boron & $<6.66 \mathrm{E} 2$ & ug/g dry & 6.66E2 & $7 / 01 / 08$ & 8G01001 & PNNL-AGG-ICP-AES \\
\hline 7440-39-3 & Barium & 4.50E1 & ug/g dry & 3.00E0 & $7 / 01 / 08$ & 8G01001 & PNNL-AGG-ICP-AES \\
\hline $7440-41-7$ & Beryllium & $<1.19 \mathrm{E} 0$ & ug/g dry & 1.19E0 & 7/01/08 & 8G01001 & PNNL-AGG-ICP-AES \\
\hline $7440-69-9$ & Bismuth & $<1.59 \mathrm{E} 1$ & ug/g dry & $1.59 \mathrm{E} 1$ & $7 / 01 / 08$ & 8G01001 & PNNL-AGG-ICP-AES \\
\hline $7440-70-2$ & Calcium & 7.32E3 & ug/g dry & 1.17E2 & $7 / 01 / 08$ & 8G01001 & PNNL-AGG-ICP-AES \\
\hline $7440-43-9$ & Cadmium & $<1.79 \mathrm{E} 0$ & ug/g dry & $1.79 \mathrm{E} 0$ & $7 / 01 / 08$ & 8G01001 & PNNL-AGG-ICP-AES \\
\hline $7440-48-4$ & Cobalt & $<6.53 \mathrm{E} 0$ & ug/g dry & $6.53 \mathrm{E} 0$ & $7 / 01 / 08$ & 8G01001 & PNNL-AGG-ICP-AES \\
\hline $7440-47-3$ & Chromium & 9.33E0 & ug/g dry & 2.62E0 & $7 / 01 / 08$ & 8G01001 & PNNL-AGG-ICP-AES \\
\hline $7440-50-8$ & Copper & $<2.79 \mathrm{E} 1$ & ug/g dry & $2.79 \mathrm{E} 1$ & $7 / 01 / 08$ & 8G01001 & PNNL-AGG-ICP-AES \\
\hline 7439-89-6 & Iron & 8.92E3 & ug/g dry & $8.00 \mathrm{E} 1$ & 7/01/08 & 8G01001 & PNNL-AGG-ICP-AES \\
\hline
\end{tabular}


Total Metals by PNNL-AGG-ICP-AES/Acid Extract

\begin{tabular}{|c|c|c|c|c|c|c|c|}
\hline CAS \# & Analyte & Results & Units & EQL & Analyzed & Batch & Method \\
\hline HEIS No. & B1VJ61C & \multicolumn{3}{|c|}{ Lab ID: $\quad$ 0805020-22 } & & & \\
\hline $7440-09-7$ & Potassium & 8.33E2 & ug/g dry & 7.42E1 & 7/01/08 & 8G01001 & PNNL-AGG-ICP-AES \\
\hline 7439-93-2 & Lithium & $<1.13 \mathrm{E} 1$ & ug/g dry & 1.13E1 & $7 / 01 / 08$ & 8G01001 & PNNL-AGG-ICP-AES \\
\hline 7439-95-4 & Magnesium & 3.32E3 & ug/g dry & 2.48E1 & $7 / 01 / 08$ & 8G01001 & PNNL-AGG-ICP-AES \\
\hline 7439-96-5 & Manganese & 1.69E2 & ug/g dry & $9.37 \mathrm{E}-1$ & 7/01/08 & 8G01001 & PNNL-AGG-ICP-AES \\
\hline 7439-98-7 & Molybdenum & $<1.77 \mathrm{E} 1$ & ug/g dry & $1.77 \mathrm{E} 1$ & 7/01/08 & 8G01001 & PNNL-AGG-ICP-AES \\
\hline $7440-02-0$ & Nickel & 9.60E0 & ug/g dry & $6.44 \mathrm{E} 0$ & $7 / 01 / 08$ & 8G01001 & PNNL-AGG-ICP-AES \\
\hline 7723-14-0 & Phosphorus & 3.96E2 & ug/g dry & $5.89 \mathrm{E} 1$ & 7/01/08 & 8G01001 & PNNL-AGG-ICP-AES \\
\hline 7439-92-1 & Lead & $<2.49 \mathrm{E} 1$ & ug/g dry & 2.49E1 & $7 / 01 / 08$ & 8G01001 & PNNL-AGG-ICP-AES \\
\hline $7440-24-6$ & Strontium & $2.62 \mathrm{E} 1$ & ug/g dry & $1.13 \mathrm{E} 0$ & 7/01/08 & 8G01001 & PNNL-AGG-ICP-AES \\
\hline $7440-66-6$ & Zinc & $2.96 \mathrm{E} 1$ & ug/g dry & 4.91E0 & $7 / 01 / 08$ & 8G01001 & PNNL-AGG-ICP-AES \\
\hline $7440-23-5$ & Sodium & $<8.39 \mathrm{E} 2$ & ug/g dry & 8.39E2 & 7/01/08 & 8G01001 & PNNL-AGG-ICP-AES \\
\hline $7440-21-3$ & Silicon & $<1.09 \mathrm{E} 3$ & ug/g dry & $1.09 \mathrm{E} 3$ & 7/01/08 & 8G01001 & PNNL-AGG-ICP-AES \\
\hline 7704-34-9 & Sulfur & $<1.85 \mathrm{E} 2$ & ug/g dry & $1.85 \mathrm{E} 2$ & $7 / 01 / 08$ & 8G01001 & PNNL-AGG-ICP-AES \\
\hline $7440-32-6$ & Titanium & 4.37E2 & ug/g dry & $2.47 \mathrm{E} 1$ & 7/01/08 & 8G01001 & PNNL-AGG-ICP-AES \\
\hline $7440-67-7$ & Zirconium & $<1.59 \mathrm{E} 1$ & ug/g dry & $1.59 \mathrm{E} 1$ & $7 / 01 / 08$ & 8G01001 & PNNL-AGG-ICP-AES \\
\hline $7440-15-5$ & Rhenium & $<1.38 \mathrm{E} 1$ & ug/g dry & $1.38 \mathrm{E} 1$ & $7 / 01 / 08$ & 8G01001 & PNNL-AGG-ICP-AES \\
\hline $7440-36-0$ & Antimony & $<8.76 \mathrm{E} 1$ & ug/g dry & 8.76E1 & $7 / 01 / 08$ & 8G01001 & PNNL-AGG-ICP-AES \\
\hline HEIS No. & B1VJ61B & \multicolumn{3}{|c|}{$\begin{array}{ll}\text { Lab ID: } & \text { 0805020-23 }\end{array}$} & & & \\
\hline $7429-90-5$ & Aluminum & 4.17E3 & ug/g dry & 3.06E1 & $7 / 01 / 08$ & 8G01001 & PNNL-AGG-ICP-AES \\
\hline $7440-38-2$ & Arsenic & $<7.44 \mathrm{E} 1$ & ug/g dry & 7.44E1 & $7 / 01 / 08$ & 8G01001 & PNNL-AGG-ICP-AES \\
\hline $7440-42-8$ & Boron & $<6.48 \mathrm{E} 2$ & ug/g dry & $6.48 \mathrm{E} 2$ & 7/01/08 & 8G01001 & PNNL-AGG-ICP-AES \\
\hline $7440-39-3$ & Barium & 4.19E1 & ug/g dry & 2.92E0 & $7 / 01 / 08$ & 8G01001 & PNNL-AGG-ICP-AES \\
\hline $7440-41-7$ & Beryllium & $<1.16 \mathrm{E} 0$ & ug/g dry & $1.16 \mathrm{E} 0$ & $7 / 01 / 08$ & 8G01001 & PNNL-AGG-ICP-AES \\
\hline 7440-69-9 & Bismuth & $<1.55 \mathrm{E} 1$ & ug/g dry & $1.55 \mathrm{E} 1$ & $7 / 01 / 08$ & 8G01001 & PNNL-AGG-ICP-AES \\
\hline $7440-70-2$ & Calcium & 7.23E3 & ug/g dry & $1.14 \mathrm{E} 2$ & 7/01/08 & 8G01001 & PNNL-AGG-ICP-AES \\
\hline $7440-43-9$ & Cadmium & $<1.74 \mathrm{E} 0$ & ug/g dry & $1.74 \mathrm{E} 0$ & 7/01/08 & 8G01001 & PNNL-AGG-ICP-AES \\
\hline $7440-48-4$ & Cobalt & $<6.36 \mathrm{E} 0$ & ug/g dry & $6.36 \mathrm{E} 0$ & $7 / 01 / 08$ & 8G01001 & PNNL-AGG-ICP-AES \\
\hline $7440-47-3$ & Chromium & 7.00E0 & ug/g dry & $2.55 \mathrm{E} 0$ & 7/01/08 & 8G01001 & PNNL-AGG-ICP-AES \\
\hline $7440-50-8$ & Copper & $<2.71 \mathrm{E} 1$ & ug/g dry & $2.71 \mathrm{E} 1$ & 7/01/08 & 8G01001 & PNNL-AGG-ICP-AES \\
\hline 7439-89-6 & Iron & $8.80 \mathrm{E} 3$ & ug/g dry & $7.78 \mathrm{E} 1$ & $7 / 01 / 08$ & 8G01001 & PNNL-AGG-ICP-AES \\
\hline 7440-09-7 & Potassium & 8.09E2 & ug/g dry & 7.22E1 & $7 / 01 / 08$ & 8G01001 & PNNL-AGG-ICP-AES \\
\hline $7439-93-2$ & Lithium & $1.11 \mathrm{E} 1$ & ug/g dry & $1.10 \mathrm{E} 1$ & 7/01/08 & 8G01001 & PNNL-AGG-ICP-AES \\
\hline $7439-95-4$ & Magnesium & $3.48 \mathrm{E} 3$ & ug/g dry & $2.41 \mathrm{E} 1$ & 7/01/08 & 8G01001 & PNNL-AGG-ICP-AES \\
\hline 7439-96-5 & Manganese & $1.70 \mathrm{E} 2$ & ug/g dry & $9.11 \mathrm{E}-1$ & $7 / 01 / 08$ & 8G01001 & PNNL-AGG-ICP-AES \\
\hline 7439-98-7 & Molybdenum & $<1.73 \mathrm{E} 1$ & ug/g dry & $1.73 \mathrm{E} 1$ & $7 / 01 / 08$ & 8G01001 & PNNL-AGG-ICP-AES \\
\hline $7440-02-0$ & Nickel & 8.98E0 & ug/g dry & $6.26 \mathrm{E} 0$ & 7/01/08 & 8G01001 & PNNL-AGG-ICP-AES \\
\hline 7723-14-0 & Phosphorus & 3.92E2 & ug/g dry & $5.74 \mathrm{E} 1$ & 7/01/08 & 8G01001 & PNNL-AGG-ICP-AES \\
\hline $7439-92-1$ & Lead & $<2.42 \mathrm{E} 1$ & ug/g dry & $2.42 \mathrm{E} 1$ & $7 / 01 / 08$ & 8G01001 & PNNL-AGG-ICP-AES \\
\hline $7440-24-6$ & Strontium & 2.54E1 & ug/g dry & $1.10 \mathrm{E} 0$ & $7 / 01 / 08$ & 8G01001 & PNNL-AGG-ICP-AES \\
\hline $7440-66-6$ & Zinc & $2.28 \mathrm{E} 1$ & ug/g dry & 4.77E0 & 7/01/08 & 8G01001 & PNNL-AGG-ICP-AES \\
\hline $7440-23-5$ & Sodium & $<8.16 \mathrm{E} 2$ & ug/g dry & 8.16E2 & $7 / 01 / 08$ & 8G01001 & PNNL-AGG-ICP-AES \\
\hline $7440-21-3$ & Silicon & $<1.06 \mathrm{E} 3$ & ug/g dry & $1.06 \mathrm{E} 3$ & $7 / 01 / 08$ & 8G01001 & PNNL-AGG-ICP-AES \\
\hline $7704-34-9$ & Sulfur & $<1.80 \mathrm{E} 2$ & ug/g dry & $1.80 \mathrm{E} 2$ & 7/01/08 & 8G01001 & PNNL-AGG-ICP-AES \\
\hline $7440-32-6$ & Titanium & $4.08 \mathrm{E} 2$ & ug/g dry & $2.40 \mathrm{E} 1$ & 7/01/08 & 8G01001 & PNNL-AGG-ICP-AES \\
\hline $7440-67-7$ & Zirconium & $<1.55 \mathrm{E} 1$ & ug/g dry & $1.55 \mathrm{E} 1$ & 7/01/08 & 8G01001 & PNNL-AGG-ICP-AES \\
\hline $7440-15-5$ & Rhenium & $<1.34 \mathrm{E} 1$ & ug/g dry & $1.34 \mathrm{E} 1$ & $7 / 01 / 08$ & 8G01001 & PNNL-AGG-ICP-AES \\
\hline $7440-36-0$ & Antimony & $<8.53 \mathrm{E} 1$ & ug/g dry & 8.53E1 & 7/01/08 & 8G01001 & PNNL-AGG-ICP-AES \\
\hline HEIS No. & B1VJ61A & $\mathbf{L a}$ & b ID: & $5020-24$ & & & \\
\hline
\end{tabular}


Total Metals by PNNL-AGG-ICP-AES/Acid Extract

\begin{tabular}{|c|c|c|c|c|c|c|c|}
\hline CAS \# & Analyte & Results & Units & EQL & Analyzed & Batch & Method \\
\hline HEIS No. & B1VJ61A & \multicolumn{3}{|c|}{ Lab ID: $\quad$ 0805020-24 } & & & \\
\hline $7429-90-5$ & Aluminum & 4.37E3 & ug/g dry & $3.40 \mathrm{E} 1$ & 7/01/08 & 8G01001 & PNNL-AGG-ICP-AES \\
\hline $7440-38-2$ & Arsenic & $<8.27 \mathrm{E} 1$ & ug/g dry & 8.27E1 & $7 / 01 / 08$ & 8G01001 & PNNL-AGG-ICP-AES \\
\hline $7440-42-8$ & Boron & $<7.20 \mathrm{E} 2$ & ug/g dry & $7.20 \mathrm{E} 2$ & 7/01/08 & 8G01001 & PNNL-AGG-ICP-AES \\
\hline $7440-39-3$ & Barium & 4.54E1 & ug/g dry & $3.24 \mathrm{E} 0$ & $7 / 01 / 08$ & 8G01001 & PNNL-AGG-ICP-AES \\
\hline $7440-41-7$ & Beryllium & $<1.29 \mathrm{E} 0$ & ug/g dry & $1.29 \mathrm{E} 0$ & $7 / 01 / 08$ & 8G01001 & PNNL-AGG-ICP-AES \\
\hline $7440-69-9$ & Bismuth & $<1.72 \mathrm{E} 1$ & ug/g dry & $1.72 \mathrm{E} 1$ & 7/01/08 & 8G01001 & PNNL-AGG-ICP-AES \\
\hline $7440-70-2$ & Calcium & $8.24 \mathrm{E} 3$ & ug/g dry & $1.26 \mathrm{E} 2$ & $7 / 01 / 08$ & 8G01001 & PNNL-AGG-ICP-AES \\
\hline $7440-43-9$ & Cadmium & $<1.93 \mathrm{E} 0$ & ug/g dry & $1.93 \mathrm{E} 0$ & 7/01/08 & 8G01001 & PNNL-AGG-ICP-AES \\
\hline $7440-48-4$ & Cobalt & $<7.06 \mathrm{E} 0$ & ug/g dry & 7.06E0 & $7 / 01 / 08$ & 8G01001 & PNNL-AGG-ICP-AES \\
\hline $7440-47-3$ & Chromium & 7.28E0 & ug/g dry & 2.83E0 & $7 / 01 / 08$ & 8G01001 & PNNL-AGG-ICP-AES \\
\hline $7440-50-8$ & Copper & $<3.02 \mathrm{E} 1$ & ug/g dry & 3.02E1 & $7 / 01 / 08$ & 8G01001 & PNNL-AGG-ICP-AES \\
\hline 7439-89-6 & Iron & 9.17E3 & ug/g dry & 8.65E1 & 7/01/08 & 8G01001 & PNNL-AGG-ICP-AES \\
\hline 7440-09-7 & Potassium & 8.43E2 & ug/g dry & 8.03E1 & $7 / 01 / 08$ & 8G01001 & PNNL-AGG-ICP-AES \\
\hline 7439-93-2 & Lithium & $<1.23 \mathrm{E} 1$ & ug/g dry & $1.23 \mathrm{E} 1$ & $7 / 01 / 08$ & 8G01001 & PNNL-AGG-ICP-AES \\
\hline $7439-95-4$ & Magnesium & 3.47E3 & ug/g dry & $2.68 \mathrm{E} 1$ & 7/01/08 & 8G01001 & PNNL-AGG-ICP-AES \\
\hline 7439-96-5 & Manganese & $2.00 \mathrm{E} 2$ & ug/g dry & $1.01 \mathrm{E} 0$ & $7 / 01 / 08$ & 8G01001 & PNNL-AGG-ICP-AES \\
\hline 7439-98-7 & Molybdenum & $<1.92 \mathrm{E} 1$ & ug/g dry & $1.92 \mathrm{E} 1$ & $7 / 01 / 08$ & 8G01001 & PNNL-AGG-ICP-AES \\
\hline $7440-02-0$ & Nickel & 9.34E0 & ug/g dry & 6.96E0 & $7 / 01 / 08$ & 8G01001 & PNNL-AGG-ICP-AES \\
\hline $7723-14-0$ & Phosphorus & $4.08 \mathrm{E} 2$ & ug/g dry & $6.38 \mathrm{E} 1$ & 7/01/08 & 8G01001 & PNNL-AGG-ICP-AES \\
\hline $7439-92-1$ & Lead & $<2.69 \mathrm{E} 1$ & ug/g dry & 2.69E1 & 7/01/08 & 8G01001 & PNNL-AGG-ICP-AES \\
\hline $7440-24-6$ & Strontium & 2.74E1 & ug/g dry & $1.23 \mathrm{E} 0$ & $7 / 01 / 08$ & 8G01001 & PNNL-AGG-ICP-AES \\
\hline $7440-66-6$ & Zinc & $2.40 \mathrm{E} 1$ & ug/g dry & 5.31E0 & $7 / 01 / 08$ & 8G01001 & PNNL-AGG-ICP-AES \\
\hline $7440-23-5$ & Sodium & $<9.07 \mathrm{E} 2$ & ug/g dry & $9.07 \mathrm{E} 2$ & 7/01/08 & 8G01001 & PNNL-AGG-ICP-AES \\
\hline $7440-21-3$ & Silicon & $<1.18 \mathrm{E} 3$ & ug/g dry & $1.18 \mathrm{E} 3$ & $7 / 01 / 08$ & 8G01001 & PNNL-AGG-ICP-AES \\
\hline $7704-34-9$ & Sulfur & $<2.01 \mathrm{E} 2$ & ug/g dry & $2.01 \mathrm{E} 2$ & $7 / 01 / 08$ & 8G01001 & PNNL-AGG-ICP-AES \\
\hline $7440-32-6$ & Titanium & 4.73E2 & ug/g dry & 2.67E1 & $7 / 01 / 08$ & 8G01001 & PNNL-AGG-ICP-AES \\
\hline $7440-67-7$ & Zirconium & $<1.72 \mathrm{E} 1$ & ug/g dry & $1.72 \mathrm{E} 1$ & 7/01/08 & 8G01001 & PNNL-AGG-ICP-AES \\
\hline $7440-15-5$ & Rhenium & $<1.49 \mathrm{E} 1$ & ug/g dry & $1.49 \mathrm{E} 1$ & 7/01/08 & 8G01001 & PNNL-AGG-ICP-AES \\
\hline $7440-36-0$ & Antimony & $<9.48 \mathrm{E} 1$ & ug/g dry & $9.48 \mathrm{E} 1$ & $7 / 01 / 08$ & 8G01001 & PNNL-AGG-ICP-AES \\
\hline HEIS No. & B1VJ62C & & b ID: & $5020-26$ & & & \\
\hline $7429-90-5$ & Aluminum & $4.80 \mathrm{E} 3$ & ug/g dry & 3.58E1 & $7 / 01 / 08$ & 8G01001 & PNNL-AGG-ICP-AES \\
\hline $7440-38-2$ & Arsenic & $<8.71 \mathrm{E} 1$ & ug/g dry & $8.71 \mathrm{E} 1$ & 7/01/08 & 8G01001 & PNNL-AGG-ICP-AES \\
\hline $7440-42-8$ & Boron & $<7.58 \mathrm{E} 2$ & ug/g dry & $7.58 \mathrm{E} 2$ & $7 / 01 / 08$ & 8G01001 & PNNL-AGG-ICP-AES \\
\hline $7440-39-3$ & Barium & $5.20 \mathrm{E} 1$ & ug/g dry & $3.41 \mathrm{E} 0$ & $7 / 01 / 08$ & 8G01001 & PNNL-AGG-ICP-AES \\
\hline $7440-41-7$ & Beryllium & $<1.35 \mathrm{E} 0$ & ug/g dry & $1.35 \mathrm{E} 0$ & 7/01/08 & 8G01001 & PNNL-AGG-ICP-AES \\
\hline 7440-69-9 & Bismuth & $<1.81 \mathrm{E} 1$ & ug/g dry & $1.81 \mathrm{E} 1$ & 7/01/08 & 8G01001 & PNNL-AGG-ICP-AES \\
\hline $7440-70-2$ & Calcium & 8.30E3 & ug/g dry & 1.33E2 & $7 / 01 / 08$ & 8G01001 & PNNL-AGG-ICP-AES \\
\hline $7440-43-9$ & Cadmium & $<2.03 \mathrm{E} 0$ & ug/g dry & 2.03E0 & $7 / 01 / 08$ & 8G01001 & PNNL-AGG-ICP-AES \\
\hline $7440-48-4$ & Cobalt & $<7.44 \mathrm{E} 0$ & ug/g dry & 7.44E0 & 7/01/08 & 8G01001 & PNNL-AGG-ICP-AES \\
\hline $7440-47-3$ & Chromium & 7.56Е0 & ug/g dry & $2.98 \mathrm{E} 0$ & 7/01/08 & 8G01001 & PNNL-AGG-ICP-AES \\
\hline $7440-50-8$ & Copper & $<3.17 \mathrm{E} 1$ & ug/g dry & $3.17 \mathrm{E} 1$ & 7/01/08 & 8G01001 & PNNL-AGG-ICP-AES \\
\hline 7439-89-6 & Iron & 8.69E3 & ug/g dry & $9.10 \mathrm{E} 1$ & 7/01/08 & 8G01001 & PNNL-AGG-ICP-AES \\
\hline 7440-09-7 & Potassium & 1.19E3 & ug/g dry & 8.45E1 & 7/01/08 & 8G01001 & PNNL-AGG-ICP-AES \\
\hline 7439-93-2 & Lithium & $<1.29 \mathrm{E} 1$ & ug/g dry & $1.29 \mathrm{E} 1$ & $7 / 01 / 08$ & 8G01001 & PNNL-AGG-ICP-AES \\
\hline 7439-95-4 & Magnesium & $3.64 \mathrm{E} 3$ & ug/g dry & $2.82 \mathrm{E} 1$ & $7 / 01 / 08$ & 8G01001 & PNNL-AGG-ICP-AES \\
\hline 7439-96-5 & Manganese & 2.22E2 & ug/g dry & $1.07 \mathrm{E} 0$ & $7 / 01 / 08$ & 8G01001 & PNNL-AGG-ICP-AES \\
\hline 7439-98-7 & Molybdenum & $<2.02 \mathrm{E} 1$ & ug/g dry & $2.02 \mathrm{E} 1$ & 7/01/08 & 8G01001 & PNNL-AGG-ICP-AES \\
\hline $7440-02-0$ & Nickel & $<7.33 \mathrm{E} 0$ & ug/g dry & 7.33E0 & 7/01/08 & 8G01001 & PNNL-AGG-ICP-AES \\
\hline
\end{tabular}


Total Metals by PNNL-AGG-ICP-AES/Acid Extract

\begin{tabular}{|c|c|c|c|c|c|c|c|}
\hline CAS \# & Analyte & Results & Units & EQL & Analyzed & Batch & Method \\
\hline HEIS No. & B1VJ62C & \multicolumn{3}{|c|}{ Lab ID: $\quad$ 0805020-26 } & & & \\
\hline $7723-14-0$ & Phosphorus & $3.27 \mathrm{E} 2$ & ug/g dry & $6.71 \mathrm{E} 1$ & 7/01/08 & 8G01001 & PNNL-AGG-ICP-AES \\
\hline 7439-92-1 & Lead & $<2.83 \mathrm{E} 1$ & ug/g dry & 2.83E1 & $7 / 01 / 08$ & 8G01001 & PNNL-AGG-ICP-AES \\
\hline $7440-24-6$ & Strontium & 2.95E1 & ug/g dry & $1.29 \mathrm{E} 0$ & $7 / 01 / 08$ & 8G01001 & PNNL-AGG-ICP-AES \\
\hline $7440-66-6$ & Zinc & $2.50 \mathrm{E} 1$ & ug/g dry & 5.59E0 & $7 / 01 / 08$ & 8G01001 & PNNL-AGG-ICP-AES \\
\hline $7440-23-5$ & Sodium & $<9.55 \mathrm{E} 2$ & ug/g dry & $9.55 \mathrm{E} 2$ & 7/01/08 & 8G01001 & PNNL-AGG-ICP-AES \\
\hline $7440-21-3$ & Silicon & $<1.24 \mathrm{E} 3$ & ug/g dry & $1.24 \mathrm{E} 3$ & $7 / 01 / 08$ & 8G01001 & PNNL-AGG-ICP-AES \\
\hline $7704-34-9$ & Sulfur & $<2.11 \mathrm{E} 2$ & ug/g dry & 2.11E2 & $7 / 01 / 08$ & 8G01001 & PNNL-AGG-ICP-AES \\
\hline $7440-32-6$ & Titanium & 3.90E2 & ug/g dry & $2.81 \mathrm{E} 1$ & $7 / 01 / 08$ & 8G01001 & PNNL-AGG-ICP-AES \\
\hline $7440-67-7$ & Zirconium & $<1.81 \mathrm{E} 1$ & ug/g dry & $1.81 \mathrm{E} 1$ & 7/01/08 & 8G01001 & PNNL-AGG-ICP-AES \\
\hline $7440-15-5$ & Rhenium & $<1.57 \mathrm{E} 1$ & ug/g dry & $1.57 \mathrm{E} 1$ & $7 / 01 / 08$ & 8G01001 & PNNL-AGG-ICP-AES \\
\hline $7440-36-0$ & Antimony & $<9.98 \mathrm{E} 1$ & ug/g dry & $9.98 \mathrm{E} 1$ & 7/01/08 & 8G01001 & PNNL-AGG-ICP-AES \\
\hline HEIS No. & B1VJ62B & \multicolumn{3}{|c|}{ Lab ID: $\quad$ 0805020-27 } & & & \\
\hline $7429-90-5$ & Aluminum & $4.61 \mathrm{E} 3$ & ug/g dry & 3.68E1 & $7 / 01 / 08$ & 8G01001 & PNNL-AGG-ICP-AES \\
\hline $7440-38-2$ & Arsenic & $<8.94 \mathrm{E} 1$ & ug/g dry & 8.94E1 & $7 / 01 / 08$ & 8G01001 & PNNL-AGG-ICP-AES \\
\hline $7440-42-8$ & Boron & $<7.79 \mathrm{E} 2$ & ug/g dry & 7.79E2 & $7 / 01 / 08$ & 8G01001 & PNNL-AGG-ICP-AES \\
\hline $7440-39-3$ & Barium & $5.14 \mathrm{E} 1$ & ug/g dry & $3.51 \mathrm{E} 0$ & $7 / 02 / 08$ & 8G01001 & PNNL-AGG-ICP-AES \\
\hline $7440-41-7$ & Beryllium & $<1.39 \mathrm{E} 0$ & ug/g dry & $1.39 \mathrm{E} 0$ & $7 / 01 / 08$ & 8G01001 & PNNL-AGG-ICP-AES \\
\hline $7440-69-9$ & Bismuth & $<1.86 \mathrm{E} 1$ & ug/g dry & $1.86 \mathrm{E} 1$ & $7 / 01 / 08$ & 8G01001 & PNNL-AGG-ICP-AES \\
\hline $7440-70-2$ & Calcium & $7.56 \mathrm{E} 3$ & ug/g dry & $1.37 \mathrm{E} 2$ & $7 / 02 / 08$ & 8G01001 & PNNL-AGG-ICP-AES \\
\hline 7440-43-9 & Cadmium & $<2.09 \mathrm{E} 0$ & ug/g dry & 2.09E0 & $7 / 01 / 08$ & 8G01001 & PNNL-AGG-ICP-AES \\
\hline $7440-48-4$ & Cobalt & $<7.64 \mathrm{E} 0$ & ug/g dry & $7.64 \mathrm{E} 0$ & 7/01/08 & 8G01001 & PNNL-AGG-ICP-AES \\
\hline $7440-47-3$ & Chromium & $9.90 \mathrm{E} 0$ & ug/g dry & $3.06 \mathrm{E} 0$ & 7/01/08 & 8G01001 & PNNL-AGG-ICP-AES \\
\hline $7440-50-8$ & Copper & $<3.26 \mathrm{E} 1$ & ug/g dry & $3.26 \mathrm{E} 1$ & $7 / 01 / 08$ & 8G01001 & PNNL-AGG-ICP-AES \\
\hline 7439-89-6 & Iron & $9.05 \mathrm{E} 3$ & ug/g dry & 9.35E1 & $7 / 02 / 08$ & 8G01001 & PNNL-AGG-ICP-AES \\
\hline 7440-09-7 & Potassium & $1.12 \mathrm{E} 3$ & ug/g dry & 8.68E1 & $7 / 02 / 08$ & 8G01001 & PNNL-AGG-ICP-AES \\
\hline 7439-93-2 & Lithium & $<1.33 \mathrm{E} 1$ & ug/g dry & $1.33 \mathrm{E} 1$ & $7 / 01 / 08$ & 8G01001 & PNNL-AGG-ICP-AES \\
\hline $7439-95-4$ & Magnesium & $3.51 \mathrm{E} 3$ & ug/g dry & 2.89E1 & $7 / 02 / 08$ & 8G01001 & PNNL-AGG-ICP-AES \\
\hline $7439-96-5$ & Manganese & $2.14 \mathrm{E} 2$ & ug/g dry & $1.10 \mathrm{E} 0$ & 7/01/08 & 8G01001 & PNNL-AGG-ICP-AES \\
\hline 7439-98-7 & Molybdenum & $<2.07 \mathrm{E} 1$ & ug/g dry & 2.07E1 & 7/01/08 & 8G01001 & PNNL-AGG-ICP-AES \\
\hline $7440-02-0$ & Nickel & 8.89E0 & ug/g dry & 7.52E0 & $7 / 01 / 08$ & 8G01001 & PNNL-AGG-ICP-AES \\
\hline 7723-14-0 & Phosphorus & $3.44 \mathrm{E} 2$ & ug/g dry & 6.89E1 & $7 / 01 / 08$ & 8G01001 & PNNL-AGG-ICP-AES \\
\hline $7439-92-1$ & Lead & $<2.91 \mathrm{E} 1$ & ug/g dry & 2.91E1 & 7/01/08 & 8G01001 & PNNL-AGG-ICP-AES \\
\hline $7440-24-6$ & Strontium & 2.68E1 & ug/g dry & $1.33 \mathrm{E} 0$ & $7 / 02 / 08$ & 8G01001 & PNNL-AGG-ICP-AES \\
\hline $7440-66-6$ & Zinc & $2.43 \mathrm{E} 1$ & ug/g dry & $5.74 \mathrm{E} 0$ & $7 / 01 / 08$ & 8G01001 & PNNL-AGG-ICP-AES \\
\hline $7440-23-5$ & Sodium & $<9.80 \mathrm{E} 2$ & ug/g dry & $9.80 \mathrm{E} 2$ & $7 / 01 / 08$ & 8G01001 & PNNL-AGG-ICP-AES \\
\hline $7440-21-3$ & Silicon & $<1.28 \mathrm{E} 3$ & ug/g dry & $1.28 \mathrm{E} 3$ & $7 / 01 / 08$ & 8G01001 & PNNL-AGG-ICP-AES \\
\hline $7704-34-9$ & Sulfur & $<2.17 \mathrm{E} 2$ & ug/g dry & 2.17E2 & 7/01/08 & 8G01001 & PNNL-AGG-ICP-AES \\
\hline $7440-32-6$ & Titanium & 3.39E2 & ug/g dry & $2.89 \mathrm{E} 1$ & $7 / 02 / 08$ & 8G01001 & PNNL-AGG-ICP-AES \\
\hline $7440-67-7$ & Zirconium & $<1.86 \mathrm{E} 1$ & ug/g dry & $1.86 \mathrm{E} 1$ & $7 / 01 / 08$ & 8G01001 & PNNL-AGG-ICP-AES \\
\hline $7440-15-5$ & Rhenium & $<1.61 \mathrm{E} 1$ & ug/g dry & $1.61 \mathrm{E} 1$ & 7/01/08 & 8G01001 & PNNL-AGG-ICP-AES \\
\hline $7440-36-0$ & Antimony & $<1.02 \mathrm{E} 2$ & ug/g dry & $1.02 \mathrm{E} 2$ & $7 / 01 / 08$ & 8G01001 & PNNL-AGG-ICP-AES \\
\hline HEIS No. & B1VJ62A & & b ID: & $5020-28$ & & & \\
\hline $7429-90-5$ & Aluminum & $4.87 \mathrm{E} 3$ & ug/g dry & 3.73E1 & $7 / 01 / 08$ & 8G01001 & PNNL-AGG-ICP-AES \\
\hline $7440-38-2$ & Arsenic & $<9.05 \mathrm{E} 1$ & ug/g dry & $9.05 \mathrm{E} 1$ & 7/01/08 & 8G01001 & PNNL-AGG-ICP-AES \\
\hline $7440-42-8$ & Boron & $<7.88 \mathrm{E} 2$ & ug/g dry & 7.88E2 & $7 / 01 / 08$ & 8G01001 & PNNL-AGG-ICP-AES \\
\hline 7440-39-3 & Barium & 6.10E1 & ug/g dry & 3.55E0 & $7 / 01 / 08$ & 8G01001 & PNNL-AGG-ICP-AES \\
\hline $7440-41-7$ & Beryllium & $<1.41 \mathrm{E} 0$ & ug/g dry & $1.41 \mathrm{E} 0$ & $7 / 01 / 08$ & 8G01001 & PNNL-AGG-ICP-AES \\
\hline $7440-69-9$ & Bismuth & $<1.88 \mathrm{E} 1$ & ug/g dry & $1.88 \mathrm{E} 1$ & 7/01/08 & 8G01001 & PNNL-AGG-ICP-AES \\
\hline
\end{tabular}


Total Metals by PNNL-AGG-ICP-AES/Acid Extract

\begin{tabular}{|c|c|c|c|c|c|c|c|}
\hline CAS \# & Analyte & Results & Units & EQL & Analyzed & Batch & Method \\
\hline HEIS No. & B1VJ62A & \multicolumn{3}{|c|}{ Lab ID: $\quad$ 0805020-28 } & & & \\
\hline $7440-70-2$ & Calcium & $8.58 \mathrm{E} 3$ & ug/g dry & $1.38 \mathrm{E} 2$ & 7/01/08 & 8G01001 & PNNL-AGG-ICP-AES \\
\hline $7440-43-9$ & Cadmium & $<2.12 \mathrm{E} 0$ & ug/g dry & 2.12E0 & $7 / 01 / 08$ & 8G01001 & PNNL-AGG-ICP-AES \\
\hline $7440-48-4$ & Cobalt & $<7.73 \mathrm{E} 0$ & ug/g dry & 7.73E0 & 7/01/08 & 8G01001 & PNNL-AGG-ICP-AES \\
\hline $7440-47-3$ & Chromium & 7.56E0 & ug/g dry & 3.10E0 & $7 / 01 / 08$ & 8G01001 & PNNL-AGG-ICP-AES \\
\hline $7440-50-8$ & Copper & $<3.30 \mathrm{E} 1$ & ug/g dry & 3.30E1 & $7 / 01 / 08$ & 8G01001 & PNNL-AGG-ICP-AES \\
\hline 7439-89-6 & Iron & 9.13E3 & ug/g dry & $9.47 \mathrm{E} 1$ & 7/01/08 & 8G01001 & PNNL-AGG-ICP-AES \\
\hline $7440-09-7$ & Potassium & $1.04 \mathrm{E} 3$ & ug/g dry & 8.79E1 & $7 / 01 / 08$ & 8G01001 & PNNL-AGG-ICP-AES \\
\hline 7439-93-2 & Lithium & $<1.34 \mathrm{E} 1$ & ug/g dry & $1.34 \mathrm{E} 1$ & 7/01/08 & 8G01001 & PNNL-AGG-ICP-AES \\
\hline 7439-95-4 & Magnesium & 3.83E3 & ug/g dry & 2.93E1 & $7 / 01 / 08$ & 8G01001 & PNNL-AGG-ICP-AES \\
\hline $7439-96-5$ & Manganese & $2.11 \mathrm{E} 2$ & ug/g dry & $1.11 \mathrm{E} 0$ & $7 / 01 / 08$ & 8G01001 & PNNL-AGG-ICP-AES \\
\hline 7439-98-7 & Molybdenum & $<2.10 \mathrm{E} 1$ & ug/g dry & $2.10 \mathrm{E} 1$ & $7 / 01 / 08$ & 8G01001 & PNNL-AGG-ICP-AES \\
\hline $7440-02-0$ & Nickel & 7.95E0 & ug/g dry & 7.62E0 & 7/01/08 & 8G01001 & PNNL-AGG-ICP-AES \\
\hline $7723-14-0$ & Phosphorus & 3.98E2 & ug/g dry & 6.98E1 & $7 / 01 / 08$ & 8G01001 & PNNL-AGG-ICP-AES \\
\hline $7439-92-1$ & Lead & $<2.95 \mathrm{E} 1$ & ug/g dry & 2.95E1 & $7 / 01 / 08$ & 8G01001 & PNNL-AGG-ICP-AES \\
\hline $7440-24-6$ & Strontium & 2.82E1 & ug/g dry & $1.34 \mathrm{E} 0$ & 7/01/08 & 8G01001 & PNNL-AGG-ICP-AES \\
\hline $7440-66-6$ & Zinc & $2.55 \mathrm{E} 1$ & ug/g dry & $5.81 \mathrm{E} 0$ & 7/01/08 & 8G01001 & PNNL-AGG-ICP-AES \\
\hline $7440-23-5$ & Sodium & $<9.93 \mathrm{E} 2$ & ug/g dry & 9.93E2 & $7 / 01 / 08$ & 8G01001 & PNNL-AGG-ICP-AES \\
\hline $7440-21-3$ & Silicon & $<1.29 \mathrm{E} 3$ & ug/g dry & $1.29 \mathrm{E} 3$ & $7 / 01 / 08$ & 8G01001 & PNNL-AGG-ICP-AES \\
\hline 7704-34-9 & Sulfur & $<2.20 \mathrm{E} 2$ & ug/g dry & $2.20 \mathrm{E} 2$ & 7/01/08 & 8G01001 & PNNL-AGG-ICP-AES \\
\hline $7440-32-6$ & Titanium & 3.93E2 & ug/g dry & $2.92 \mathrm{E} 1$ & 7/01/08 & 8G01001 & PNNL-AGG-ICP-AES \\
\hline $7440-67-7$ & Zirconium & $<1.89 \mathrm{E} 1$ & ug/g dry & $1.89 \mathrm{E} 1$ & $7 / 01 / 08$ & 8G01001 & PNNL-AGG-ICP-AES \\
\hline $7440-15-5$ & Rhenium & $<1.63 \mathrm{E} 1$ & ug/g dry & $1.63 \mathrm{E} 1$ & $7 / 01 / 08$ & 8G01001 & PNNL-AGG-ICP-AES \\
\hline $7440-36-0$ & Antimony & $<1.04 \mathrm{E} 2$ & ug/g dry & $1.04 \mathrm{E} 2$ & 7/01/08 & 8G01001 & PNNL-AGG-ICP-AES \\
\hline HEIS No. & B1VJ64C & \multicolumn{3}{|c|}{ Lab ID: $\quad 0805020-30$} & & & \\
\hline $7429-90-5$ & Aluminum & 4.58E3 & ug/g dry & 3.76E1 & $7 / 01 / 08$ & 8G01001 & PNNL-AGG-ICP-AES \\
\hline $7440-38-2$ & Arsenic & $<9.14 \mathrm{E} 1$ & ug/g dry & $9.14 \mathrm{E} 1$ & $7 / 01 / 08$ & 8G01001 & PNNL-AGG-ICP-AES \\
\hline $7440-42-8$ & Boron & $<7.96 \mathrm{E} 2$ & ug/g dry & 7.96E2 & $7 / 01 / 08$ & 8G01001 & PNNL-AGG-ICP-AES \\
\hline $7440-39-3$ & Barium & 4.62E1 & ug/g dry & 3.59E0 & 7/01/08 & 8G01001 & PNNL-AGG-ICP-AES \\
\hline $7440-41-7$ & Beryllium & $<1.42 \mathrm{E} 0$ & ug/g dry & $1.42 \mathrm{E} 0$ & 7/01/08 & 8G01001 & PNNL-AGG-ICP-AES \\
\hline $7440-69-9$ & Bismuth & $<1.90 \mathrm{E} 1$ & ug/g dry & $1.90 \mathrm{E} 1$ & $7 / 01 / 08$ & 8G01001 & PNNL-AGG-ICP-AES \\
\hline $7440-70-2$ & Calcium & 6.96E3 & ug/g dry & $1.40 \mathrm{E} 2$ & $7 / 01 / 08$ & 8G01001 & PNNL-AGG-ICP-AES \\
\hline $7440-43-9$ & Cadmium & $<2.14 \mathrm{E} 0$ & ug/g dry & $2.14 \mathrm{E} 0$ & 7/01/08 & 8G01001 & PNNL-AGG-ICP-AES \\
\hline $7440-48-4$ & Cobalt & $<7.81 \mathrm{E} 0$ & ug/g dry & $7.81 \mathrm{E} 0$ & $7 / 01 / 08$ & 8G01001 & PNNL-AGG-ICP-AES \\
\hline $7440-47-3$ & Chromium & 1.12E1 & ug/g dry & 3.13E0 & $7 / 01 / 08$ & 8G01001 & PNNL-AGG-ICP-AES \\
\hline $7440-50-8$ & Copper & $<3.33 \mathrm{E} 1$ & ug/g dry & 3.33E1 & 7/01/08 & 8G01001 & PNNL-AGG-ICP-AES \\
\hline 7439-89-6 & Iron & $9.44 \mathrm{E} 3$ & ug/g dry & $9.56 \mathrm{E} 1$ & 7/01/08 & 8G01001 & PNNL-AGG-ICP-AES \\
\hline $7440-09-7$ & Potassium & $9.31 \mathrm{E} 2$ & ug/g dry & 8.88E1 & $7 / 01 / 08$ & 8G01001 & PNNL-AGG-ICP-AES \\
\hline 7439-93-2 & Lithium & $<1.36 \mathrm{E} 1$ & ug/g dry & $1.36 \mathrm{E} 1$ & $7 / 01 / 08$ & 8G01001 & PNNL-AGG-ICP-AES \\
\hline 7439-95-4 & Magnesium & 3.58E3 & ug/g dry & 2.96E1 & 7/01/08 & 8G01001 & PNNL-AGG-ICP-AES \\
\hline 7439-96-5 & Manganese & $1.92 \mathrm{E} 2$ & ug/g dry & $1.12 \mathrm{E} 0$ & 7/01/08 & 8G01001 & PNNL-AGG-ICP-AES \\
\hline 7439-98-7 & Molybdenum & $<2.12 \mathrm{E} 1$ & ug/g dry & $2.12 \mathrm{E} 1$ & 7/01/08 & 8G01001 & PNNL-AGG-ICP-AES \\
\hline $7440-02-0$ & Nickel & 1.03E1 & ug/g dry & 7.70E0 & 7/01/08 & 8G01001 & PNNL-AGG-ICP-AES \\
\hline $7723-14-0$ & Phosphorus & 3.84E2 & ug/g dry & 7.05E1 & 7/01/08 & 8G01001 & PNNL-AGG-ICP-AES \\
\hline 7439-92-1 & Lead & $<2.98 \mathrm{E} 1$ & ug/g dry & $2.98 \mathrm{E} 1$ & $7 / 01 / 08$ & 8G01001 & PNNL-AGG-ICP-AES \\
\hline $7440-24-6$ & Strontium & $2.26 \mathrm{E} 1$ & ug/g dry & $1.36 \mathrm{E} 0$ & $7 / 01 / 08$ & 8G01001 & PNNL-AGG-ICP-AES \\
\hline $7440-66-6$ & Zinc & $2.55 \mathrm{E} 1$ & ug/g dry & $5.87 \mathrm{E} 0$ & $7 / 01 / 08$ & 8G01001 & PNNL-AGG-ICP-AES \\
\hline $7440-23-5$ & Sodium & $<1.00 \mathrm{E} 3$ & ug/g dry & $1.00 \mathrm{E} 3$ & 7/01/08 & 8G01001 & PNNL-AGG-ICP-AES \\
\hline $7440-21-3$ & Silicon & $<1.31 \mathrm{E} 3$ & ug/g dry & $1.31 \mathrm{E} 3$ & 7/01/08 & 8G01001 & PNNL-AGG-ICP-AES \\
\hline
\end{tabular}


Total Metals by PNNL-AGG-ICP-AES/Acid Extract

\begin{tabular}{|c|c|c|c|c|c|c|c|}
\hline CAS \# & Analyte & Results & Units & EQL & Analyzed & Batch & Method \\
\hline HEIS No. & B1VJ64C & \multicolumn{3}{|c|}{ Lab ID: $\quad$ 0805020-30 } & & & \\
\hline $7704-34-9$ & Sulfur & $<2.22 \mathrm{E} 2$ & ug/g dry & $2.22 \mathrm{E} 2$ & $7 / 01 / 08$ & 8G01001 & PNNL-AGG-ICP-AES \\
\hline $7440-32-6$ & Titanium & 4.42E2 & ug/g dry & 2.95E1 & $7 / 01 / 08$ & 8G01001 & PNNL-AGG-ICP-AES \\
\hline $7440-67-7$ & Zirconium & $<1.91 \mathrm{E} 1$ & ug/g dry & $1.91 \mathrm{E} 1$ & $7 / 01 / 08$ & 8G01001 & PNNL-AGG-ICP-AES \\
\hline $7440-15-5$ & Rhenium & $<1.65 \mathrm{E} 1$ & ug/g dry & $1.65 \mathrm{E} 1$ & $7 / 01 / 08$ & 8G01001 & PNNL-AGG-ICP-AES \\
\hline $7440-36-0$ & Antimony & $<1.05 \mathrm{E} 2$ & ug/g dry & $1.05 \mathrm{E} 2$ & $7 / 01 / 08$ & 8G01001 & PNNL-AGG-ICP-AES \\
\hline HEIS No. & B1VJ64B & \multicolumn{3}{|c|}{ Lab ID: $\quad$ 0805020-31 } & & & \\
\hline $7429-90-5$ & Aluminum & $4.41 \mathrm{E} 3$ & ug/g dry & 3.34E1 & $7 / 01 / 08$ & 8G01001 & PNNL-AGG-ICP-AES \\
\hline $7440-38-2$ & Arsenic & $<8.12 \mathrm{E} 1$ & ug/g dry & 8.12E1 & $7 / 01 / 08$ & 8G01001 & PNNL-AGG-ICP-AES \\
\hline $7440-42-8$ & Boron & $<7.07 \mathrm{E} 2$ & ug/g dry & 7.07E2 & $7 / 01 / 08$ & 8G01001 & PNNL-AGG-ICP-AES \\
\hline $7440-39-3$ & Barium & 4.34E1 & ug/g dry & 3.18E0 & $7 / 01 / 08$ & 8G01001 & PNNL-AGG-ICP-AES \\
\hline $7440-41-7$ & Beryllium & $<1.26 \mathrm{E} 0$ & ug/g dry & $1.26 \mathrm{E} 0$ & $7 / 01 / 08$ & 8G01001 & PNNL-AGG-ICP-AES \\
\hline 7440-69-9 & Bismuth & $<1.69 \mathrm{E} 1$ & ug/g dry & 1.69E1 & $7 / 01 / 08$ & 8G01001 & PNNL-AGG-ICP-AES \\
\hline $7440-70-2$ & Calcium & $6.45 \mathrm{E} 3$ & ug/g dry & $1.24 \mathrm{E} 2$ & 7/01/08 & 8G01001 & PNNL-AGG-ICP-AES \\
\hline $7440-43-9$ & Cadmium & $<1.90 \mathrm{E} 0$ & ug/g dry & $1.90 \mathrm{E} 0$ & 7/01/08 & 8G01001 & PNNL-AGG-ICP-AES \\
\hline $7440-48-4$ & Cobalt & $<6.94 \mathrm{E} 0$ & ug/g dry & 6.94E0 & $7 / 01 / 08$ & 8G01001 & PNNL-AGG-ICP-AES \\
\hline $7440-47-3$ & Chromium & $1.56 \mathrm{E} 1$ & ug/g dry & $2.78 \mathrm{E} 0$ & $7 / 01 / 08$ & 8G01001 & PNNL-AGG-ICP-AES \\
\hline $7440-50-8$ & Copper & $<2.96 \mathrm{E} 1$ & ug/g dry & $2.96 \mathrm{E} 1$ & $7 / 01 / 08$ & 8G01001 & PNNL-AGG-ICP-AES \\
\hline 7439-89-6 & Iron & $9.28 \mathrm{E} 3$ & ug/g dry & 8.49E1 & 7/01/08 & 8G01001 & PNNL-AGG-ICP-AES \\
\hline $7440-09-7$ & Potassium & $9.00 \mathrm{E} 2$ & ug/g dry & $7.88 \mathrm{E} 1$ & $7 / 01 / 08$ & 8G01001 & PNNL-AGG-ICP-AES \\
\hline 7439-93-2 & Lithium & $<1.20 \mathrm{E} 1$ & ug/g dry & $1.20 \mathrm{E} 1$ & $7 / 01 / 08$ & 8G01001 & PNNL-AGG-ICP-AES \\
\hline $7439-95-4$ & Magnesium & 3.67E3 & ug/g dry & 2.63E1 & 7/01/08 & 8G01001 & PNNL-AGG-ICP-AES \\
\hline 7439-96-5 & Manganese & $1.88 \mathrm{E} 2$ & ug/g dry & $9.95 \mathrm{E}-1$ & 7/01/08 & 8G01001 & PNNL-AGG-ICP-AES \\
\hline 7439-98-7 & Molybdenum & $<1.88 \mathrm{E} 1$ & ug/g dry & $1.88 \mathrm{E} 1$ & $7 / 01 / 08$ & 8G01001 & PNNL-AGG-ICP-AES \\
\hline $7440-02-0$ & Nickel & 1.39E1 & ug/g dry & $6.83 \mathrm{E} 0$ & $7 / 01 / 08$ & 8G01001 & PNNL-AGG-ICP-AES \\
\hline 7723-14-0 & Phosphorus & 3.95E2 & ug/g dry & $6.26 \mathrm{E} 1$ & $7 / 01 / 08$ & 8G01001 & PNNL-AGG-ICP-AES \\
\hline 7439-92-1 & Lead & $<2.64 \mathrm{E} 1$ & ug/g dry & 2.64E1 & $7 / 01 / 08$ & 8G01001 & PNNL-AGG-ICP-AES \\
\hline $7440-24-6$ & Strontium & 2.36E1 & ug/g dry & $1.20 \mathrm{E} 0$ & $7 / 01 / 08$ & 8G01001 & PNNL-AGG-ICP-AES \\
\hline $7440-66-6$ & Zinc & 2.31E1 & ug/g dry & $5.21 \mathrm{E} 0$ & 7/01/08 & 8G01001 & PNNL-AGG-ICP-AES \\
\hline $7440-23-5$ & Sodium & $<8.91 \mathrm{E} 2$ & ug/g dry & 8.91E2 & 7/01/08 & 8G01001 & PNNL-AGG-ICP-AES \\
\hline $7440-21-3$ & Silicon & $<1.16 \mathrm{E} 3$ & ug/g dry & $1.16 \mathrm{E} 3$ & $7 / 01 / 08$ & 8G01001 & PNNL-AGG-ICP-AES \\
\hline 7704-34-9 & Sulfur & $<1.97 \mathrm{E} 2$ & ug/g dry & 1.97E2 & $7 / 01 / 08$ & 8G01001 & PNNL-AGG-ICP-AES \\
\hline $7440-32-6$ & Titanium & $4.26 \mathrm{E} 2$ & ug/g dry & $2.62 \mathrm{E} 1$ & 7/01/08 & 8G01001 & PNNL-AGG-ICP-AES \\
\hline $7440-67-7$ & Zirconium & $<1.69 \mathrm{E} 1$ & ug/g dry & $1.69 \mathrm{E} 1$ & $7 / 01 / 08$ & 8G01001 & PNNL-AGG-ICP-AES \\
\hline $7440-15-5$ & Rhenium & $<1.47 \mathrm{E} 1$ & ug/g dry & $1.47 \mathrm{E} 1$ & $7 / 01 / 08$ & 8G01001 & PNNL-AGG-ICP-AES \\
\hline $7440-36-0$ & Antimony & $<9.30 \mathrm{E} 1$ & ug/g dry & $9.30 \mathrm{E} 1$ & $7 / 01 / 08$ & 8G01001 & PNNL-AGG-ICP-AES \\
\hline HEIS No. & B1VJ64A & \multicolumn{3}{|c|}{$\begin{array}{ll}\text { Lab ID: } & 0805020-32\end{array}$} & & & \\
\hline $7429-90-5$ & Aluminum & 4.77E3 & ug/g dry & 3.55E1 & $7 / 01 / 08$ & 8G01001 & PNNL-AGG-ICP-AES \\
\hline $7440-38-2$ & Arsenic & $<8.62 \mathrm{E} 1$ & ug/g dry & 8.62E1 & $7 / 01 / 08$ & 8G01001 & PNNL-AGG-ICP-AES \\
\hline $7440-42-8$ & Boron & $<7.51 \mathrm{E} 2$ & ug/g dry & 7.51E2 & $7 / 01 / 08$ & 8G01001 & PNNL-AGG-ICP-AES \\
\hline $7440-39-3$ & Barium & $4.52 \mathrm{E} 1$ & ug/g dry & 3.38E0 & $7 / 01 / 08$ & 8G01001 & PNNL-AGG-ICP-AES \\
\hline $7440-41-7$ & Beryllium & $<1.34 \mathrm{E} 0$ & ug/g dry & $1.34 \mathrm{E} 0$ & $7 / 01 / 08$ & 8G01001 & PNNL-AGG-ICP-AES \\
\hline $7440-69-9$ & Bismuth & $<1.79 \mathrm{E} 1$ & ug/g dry & $1.79 \mathrm{E} 1$ & $7 / 01 / 08$ & 8G01001 & PNNL-AGG-ICP-AES \\
\hline $7440-70-2$ & Calcium & 6.59E3 & ug/g dry & 1.32E2 & $7 / 01 / 08$ & 8G01001 & PNNL-AGG-ICP-AES \\
\hline $7440-43-9$ & Cadmium & $<2.01 \mathrm{E} 0$ & ug/g dry & 2.01E0 & $7 / 01 / 08$ & 8G01001 & PNNL-AGG-ICP-AES \\
\hline $7440-48-4$ & Cobalt & $<7.36 \mathrm{E} 0$ & ug/g dry & 7.36E0 & $7 / 01 / 08$ & 8G01001 & PNNL-AGG-ICP-AES \\
\hline $7440-47-3$ & Chromium & $1.11 \mathrm{E} 1$ & ug/g dry & 2.95E0 & $7 / 01 / 08$ & 8G01001 & PNNL-AGG-ICP-AES \\
\hline $7440-50-8$ & Copper & $<3.14 \mathrm{E} 1$ & ug/g dry & 3.14E1 & $7 / 01 / 08$ & 8G01001 & PNNL-AGG-ICP-AES \\
\hline 7439-89-6 & Iron & $9.52 \mathrm{E} 3$ & ug/g dry & $9.01 \mathrm{E} 1$ & 7/01/08 & 8G01001 & PNNL-AGG-ICP-AES \\
\hline
\end{tabular}


Total Metals by PNNL-AGG-ICP-AES/Acid Extract

\begin{tabular}{|c|c|c|c|c|c|c|c|}
\hline CAS \# & Analyte & Results & Units & EQL & Analyzed & Batch & Method \\
\hline HEIS No. & B1VJ64A & \multicolumn{3}{|c|}{ Lab ID: $\quad$ 0805020-32 } & & & \\
\hline 7440-09-7 & Potassium & 8.94E2 & ug/g dry & 8.37E1 & 7/01/08 & 8G01001 & PNNL-AGG-ICP-AES \\
\hline 7439-93-2 & Lithium & $<1.28 \mathrm{E} 1$ & ug/g dry & $1.28 \mathrm{E} 1$ & 7/01/08 & 8G01001 & PNNL-AGG-ICP-AES \\
\hline 7439-95-4 & Magnesium & 3.89E3 & ug/g dry & 2.79E1 & 7/01/08 & 8G01001 & PNNL-AGG-ICP-AES \\
\hline 7439-96-5 & Manganese & $1.98 \mathrm{E} 2$ & ug/g dry & $1.06 \mathrm{E} 0$ & 7/01/08 & 8G01001 & PNNL-AGG-ICP-AES \\
\hline 7439-98-7 & Molybdenum & $<2.00 \mathrm{E} 1$ & ug/g dry & 2.00E1 & 7/01/08 & 8G01001 & PNNL-AGG-ICP-AES \\
\hline 7440-02-0 & Nickel & 1.23E1 & ug/g dry & 7.25E0 & 7/01/08 & 8G01001 & PNNL-AGG-ICP-AES \\
\hline 7723-14-0 & Phosphorus & 4.16E2 & ug/g dry & 6.64E1 & 7/01/08 & 8G01001 & PNNL-AGG-ICP-AES \\
\hline 7439-92-1 & Lead & $<2.80 \mathrm{E} 1$ & ug/g dry & 2.80E1 & 7/01/08 & 8G01001 & PNNL-AGG-ICP-AES \\
\hline $7440-24-6$ & Strontium & 2.35E1 & ug/g dry & $1.28 \mathrm{E} 0$ & 7/01/08 & 8G01001 & PNNL-AGG-ICP-AES \\
\hline 7440-66-6 & Zinc & 2.30E1 & ug/g dry & 5.53E0 & 7/01/08 & 8G01001 & PNNL-AGG-ICP-AES \\
\hline $7440-23-5$ & Sodium & $<9.45 \mathrm{E} 2$ & ug/g dry & $9.45 \mathrm{E} 2$ & 7/01/08 & 8G01001 & PNNL-AGG-ICP-AES \\
\hline $7440-21-3$ & Silicon & $<1.23 \mathrm{E} 3$ & ug/g dry & $1.23 \mathrm{E} 3$ & 7/01/08 & 8G01001 & PNNL-AGG-ICP-AES \\
\hline 7704-34-9 & Sulfur & $<2.09 \mathrm{E} 2$ & ug/g dry & 2.09E2 & 7/01/08 & 8G01001 & PNNL-AGG-ICP-AES \\
\hline $7440-32-6$ & Titanium & 4.21E2 & ug/g dry & 2.78E1 & 7/01/08 & 8G01001 & PNNL-AGG-ICP-AES \\
\hline $7440-67-7$ & Zirconium & $<1.80 \mathrm{E} 1$ & ug/g dry & $1.80 \mathrm{E} 1$ & 7/01/08 & 8G01001 & PNNL-AGG-ICP-AES \\
\hline $7440-15-5$ & Rhenium & $<1.56 \mathrm{E} 1$ & ug/g dry & $1.56 \mathrm{E} 1$ & 7/01/08 & 8G01001 & PNNL-AGG-ICP-AES \\
\hline $7440-36-0$ & Antimony & $<9.88 \mathrm{E} 1$ & ug/g dry & 9.88E1 & 7/01/08 & 8G01001 & PNNL-AGG-ICP-AES \\
\hline
\end{tabular}




\section{Radionuclides by ICP-MS/Acid Extract}

\begin{tabular}{|c|c|c|c|c|c|c|c|}
\hline CAS \# & Analyte & Results & Units & EQL & Analyzed & Batch & Method \\
\hline HEIS No. & B1VJ54B & \multicolumn{3}{|c|}{ Lab ID: $\quad$ 0805020-01 } & & & \\
\hline 13994-20-2 & Neptunium-237 & $<3.19 \mathrm{E}-3$ & ug/g dry & $3.19 E-3$ & $7 / 17 / 08$ & 8G15009 & PNNL-AGG-415 \\
\hline $15117-48-3$ & Plutonium-239 & $<7.94 \mathrm{E}-3$ & ug/g dry & 7.94E-3 & $7 / 17 / 08$ & $8 G 15009$ & PNNL-AGG-415 \\
\hline $14596-10-2$ & Americium-241 & $<6.86 \mathrm{E}-3$ & ug/g dry & $6.86 \mathrm{E}-3$ & 7/17/08 & 8G15009 & PNNL-AGG-415 \\
\hline HEIS No. & B1VJ55C & \multicolumn{3}{|c|}{ Lab ID: $\quad$ 0805020-05 } & & & \\
\hline 13994-20-2 & Neptunium-237 & $<3.73 E-3$ & ug/g dry & 3.73E-3 & $7 / 17 / 08$ & 8G15009 & PNNL-AGG-415 \\
\hline $15117-48-3$ & Plutonium-239 & $<9.27 \mathrm{E}-3$ & ug/g dry & $9.27 \mathrm{E}-3$ & 7/17/08 & 8G15009 & PNNL-AGG-415 \\
\hline $14596-10-2$ & Americium-241 & $<8.02 \mathrm{E}-3$ & ug/g dry & $8.02 \mathrm{E}-3$ & $7 / 17 / 08$ & $8 G 15009$ & PNNL-AGG-415 \\
\hline HEIS No. & B1VJ56A & \multicolumn{3}{|c|}{$\begin{array}{ll}\text { Lab ID: } & \text { 0805020-07 }\end{array}$} & & & \\
\hline 13994-20-2 & Neptunium-237 & $<3.10 \mathrm{E}-3$ & ug/g dry & 3.10E-3 & $7 / 17 / 08$ & $8 G 15010$ & PNNL-AGG-415 \\
\hline $15117-48-3$ & Plutonium-239 & $<7.72 \mathrm{E}-3$ & ug/g dry & 7.72E-3 & $7 / 17 / 08$ & 8G15010 & PNNL-AGG-415 \\
\hline $14596-10-2$ & Americium-241 & $<6.68 \mathrm{E}-3$ & ug/g dry & $6.68 \mathrm{E}-3$ & $7 / 17 / 08$ & $8 G 15010$ & PNNL-AGG-415 \\
\hline HEIS No. & B1VJ56B & \multicolumn{3}{|c|}{ Lab ID: $\quad$ 0805020-08 } & & & \\
\hline 13994-20-2 & Neptunium-237 & $<3.33 \mathrm{E}-3$ & ug/g dry & 3.33E-3 & $7 / 17 / 08$ & $8 G 15009$ & PNNL-AGG-415 \\
\hline $15117-48-3$ & Plutonium-239 & $<8.27 \mathrm{E}-3$ & ug/g dry & 8.27E-3 & $7 / 17 / 08$ & 8G15009 & PNNL-AGG-415 \\
\hline $14596-10-2$ & Americium-241 & $<7.15 \mathrm{E}-3$ & ug/g dry & $7.15 \mathrm{E}-3$ & $7 / 17 / 08$ & $8 G 15009$ & PNNL-AGG-415 \\
\hline HEIS No. & B1VJ56C & \multicolumn{3}{|c|}{ Lab ID: $\quad$ 0805020-09 } & & & \\
\hline 13994-20-2 & Neptunium-237 & $<3.22 \mathrm{E}-3$ & ug/g dry & $3.22 \mathrm{E}-3$ & $7 / 17 / 08$ & 8G15009 & PNNL-AGG-415 \\
\hline 15117-48-3 & Plutonium-239 & $<8.00 \mathrm{E}-3$ & ug/g dry & $8.00 \mathrm{E}-3$ & $7 / 17 / 08$ & 8G15009 & PNNL-AGG-415 \\
\hline $14596-10-2$ & Americium-241 & $<6.92 \mathrm{E}-3$ & ug/g dry & $6.92 \mathrm{E}-3$ & 7/17/08 & 8G15009 & PNNL-AGG-415 \\
\hline HEIS No. & B1VJ58C & \multicolumn{3}{|c|}{ Lab ID: $\quad$ 0805020-10 } & & & \\
\hline 13994-20-2 & Neptunium-237 & $<3.44 \mathrm{E}-3$ & ug/g dry & $3.44 \mathrm{E}-3$ & 7/17/08 & 8G15009 & PNNL-AGG-415 \\
\hline $15117-48-3$ & Plutonium-239 & $<8.54 \mathrm{E}-3$ & ug/g dry & $8.54 \mathrm{E}-3$ & $7 / 17 / 08$ & 8G15009 & PNNL-AGG-415 \\
\hline $14596-10-2$ & Americium-241 & $<7.39 \mathrm{E}-3$ & ug/g dry & 7.39E-3 & $7 / 17 / 08$ & 8G15009 & PNNL-AGG-415 \\
\hline HEIS No. & B1VJ58B & \multicolumn{3}{|c|}{ Lab ID: $\quad$ 0805020-11 } & & & \\
\hline $13994-20-2$ & Neptunium-237 & $<3.66 \mathrm{E}-3$ & ug/g dry & $3.66 \mathrm{E}-3$ & $7 / 17 / 08$ & 8G15009 & PNNL-AGG-415 \\
\hline $15117-48-3$ & Plutonium-239 & $<9.09 \mathrm{E}-3$ & ug/g dry & $9.09 \mathrm{E}-3$ & $7 / 17 / 08$ & 8G15009 & PNNL-AGG-415 \\
\hline $14596-10-2$ & Americium-241 & $<7.87 \mathrm{E}-3$ & ug/g dry & 7.87E-3 & 7/17/08 & 8G15009 & PNNL-AGG-415 \\
\hline HEIS No. & B1VJ58A & \multicolumn{3}{|c|}{ Lab ID: $\quad$ 0805020-12 } & & & \\
\hline 13994-20-2 & Neptunium-237 & $<3.74 \mathrm{E}-3$ & ug/g dry & $3.74 \mathrm{E}-3$ & 7/17/08 & 8G15009 & PNNL-AGG-415 \\
\hline $15117-48-3$ & Plutonium-239 & $<9.31 \mathrm{E}-3$ & ug/g dry & $9.31 \mathrm{E}-3$ & $7 / 17 / 08$ & 8G15009 & PNNL-AGG-415 \\
\hline $14596-10-2$ & Americium-241 & $<8.05 \mathrm{E}-3$ & ug/g dry & 8.05E-3 & $7 / 17 / 08$ & 8G15009 & PNNL-AGG-415 \\
\hline HEIS No. & B1VJ59C & \multicolumn{3}{|c|}{ Lab ID: $\quad$ 0805020-14 } & & & \\
\hline $13994-20-2$ & Neptunium-237 & $<3.49 \mathrm{E}-3$ & ug/g dry & $3.49 \mathrm{E}-3$ & $7 / 17 / 08$ & 8G15009 & PNNL-AGG-415 \\
\hline $15117-48-3$ & Plutonium-239 & $<8.67 \mathrm{E}-3$ & ug/g dry & 8.67E-3 & $7 / 17 / 08$ & 8G15009 & PNNL-AGG-415 \\
\hline $14596-10-2$ & Americium-241 & $<7.50 \mathrm{E}-3$ & ug/g dry & $7.50 \mathrm{E}-3$ & $7 / 17 / 08$ & $8 G 15009$ & PNNL-AGG-415 \\
\hline HEIS No. & B1VJ59B & \multicolumn{3}{|c|}{ Lab ID: $\quad$ 0805020-15 } & & & \\
\hline $13994-20-2$ & Neptunium-237 & $<3.30 \mathrm{E}-3$ & ug/g dry & 3.30E-3 & $7 / 17 / 08$ & 8G15009 & PNNL-AGG-415 \\
\hline $15117-48-3$ & Plutonium-239 & $<8.21 \mathrm{E}-3$ & ug/g dry & $8.21 \mathrm{E}-3$ & $7 / 17 / 08$ & 8G15009 & PNNL-AGG-415 \\
\hline $14596-10-2$ & Americium-241 & $<7.10 \mathrm{E}-3$ & ug/g dry & $7.10 \mathrm{E}-3$ & $7 / 17 / 08$ & 8G15009 & PNNL-AGG-415 \\
\hline HEIS No. & B1VJ59A & \multicolumn{3}{|c|}{ Lab ID: $\quad$ 0805020-16 } & & & \\
\hline $13994-20-2$ & Neptunium-237 & $<3.27 \mathrm{E}-3$ & ug/g dry & $3.27 \mathrm{E}-3$ & $7 / 17 / 08$ & 8G15009 & PNNL-AGG-415 \\
\hline $15117-48-3$ & Plutonium-239 & $<8.12 \mathrm{E}-3$ & ug/g dry & $8.12 \mathrm{E}-3$ & $7 / 17 / 08$ & $8 G 15009$ & PNNL-AGG-415 \\
\hline $14596-10-2$ & Americium-241 & $<7.03 \mathrm{E}-3$ & ug/g dry & 7.03E-3 & $7 / 17 / 08$ & 8G15009 & PNNL-AGG-415 \\
\hline HEIS No. & B1VJ60C & \multicolumn{3}{|c|}{ Lab ID: $\quad$ 0805020-18 } & & & \\
\hline $13994-20-2$ & Neptunium-237 & $<3.15 \mathrm{E}-3$ & ug/g dry & $3.15 \mathrm{E}-3$ & $7 / 17 / 08$ & 8G15009 & PNNL-AGG-415 \\
\hline $15117-48-3$ & Plutonium-239 & $<7.83 \mathrm{E}-3$ & ug/g dry & 7.83E-3 & $7 / 17 / 08$ & 8G15009 & PNNL-AGG-415 \\
\hline 14596-10-2 & Americium-241 & $<6.78 \mathrm{E}-3$ & ug/g dry & $6.78 \mathrm{E}-3$ & $7 / 17 / 08$ & 8G15009 & PNNL-AGG-415 \\
\hline
\end{tabular}




\section{Radionuclides by ICP-MS/Acid Extract}

\begin{tabular}{|c|c|c|c|c|c|c|c|}
\hline CAS \# & Analyte & Results & Units & EQL & Analyzed & Batch & Method \\
\hline HEIS No. & B1VJ60B & \multicolumn{3}{|c|}{ Lab ID: $\quad$ 0805020-19 } & & & \\
\hline 13994-20-2 & Neptunium-237 & $<3.33 \mathrm{E}-3$ & ug/g dry & 3.33E-3 & 7/17/08 & 8G15009 & PNNL-AGG-415 \\
\hline $15117-48-3$ & Plutonium-239 & $<8.28 \mathrm{E}-3$ & ug/g dry & 8.28E-3 & 7/17/08 & 8G15009 & PNNL-AGG-415 \\
\hline $14596-10-2$ & Americium-241 & $<7.16 \mathrm{E}-3$ & ug/g dry & 7.16E-3 & 7/17/08 & 8G15009 & PNNL-AGG-415 \\
\hline HEIS No. & B1VJ60A & \multicolumn{3}{|c|}{ Lab ID: $\quad$ 0805020-20 } & & & \\
\hline $13994-20-2$ & Neptunium-237 & $<3.27 \mathrm{E}-3$ & ug/g dry & $3.27 \mathrm{E}-3$ & 7/17/08 & 8G15009 & PNNL-AGG-415 \\
\hline $15117-48-3$ & Plutonium-239 & $<8.12 \mathrm{E}-3$ & ug/g dry & $8.12 \mathrm{E}-3$ & $7 / 17 / 08$ & 8G15009 & PNNL-AGG-415 \\
\hline $14596-10-2$ & Americium-241 & $<7.02 \mathrm{E}-3$ & ug/g dry & $7.02 \mathrm{E}-3$ & $7 / 17 / 08$ & 8G15009 & PNNL-AGG-415 \\
\hline HEIS No. & B1VJ61C & \multicolumn{3}{|c|}{ Lab ID: $\quad$ 0805020-22 } & & & \\
\hline 13994-20-2 & Neptunium-237 & $<2.93 \mathrm{E}-3$ & ug/g dry & 2.93E-3 & $7 / 17 / 08$ & 8G15009 & PNNL-AGG-415 \\
\hline $15117-48-3$ & Plutonium-239 & $<7.28 \mathrm{E}-3$ & ug/g dry & $7.28 \mathrm{E}-3$ & $7 / 17 / 08$ & 8G15009 & PNNL-AGG-415 \\
\hline $14596-10-2$ & Americium-241 & $<6.30 \mathrm{E}-3$ & ug/g dry & $6.30 \mathrm{E}-3$ & 7/17/08 & 8G15009 & PNNL-AGG-415 \\
\hline HEIS No. & B1VJ61B & \multicolumn{3}{|c|}{ Lab ID: $\quad$ 0805020-23 } & & & \\
\hline $13994-20-2$ & Neptunium-237 & $<2.85 \mathrm{E}-3$ & ug/g dry & $2.85 \mathrm{E}-3$ & 7/17/08 & 8G15009 & PNNL-AGG-415 \\
\hline $15117-48-3$ & Plutonium-239 & $<7.08 \mathrm{E}-3$ & ug/g dry & $7.08 \mathrm{E}-3$ & $7 / 17 / 08$ & 8G15009 & PNNL-AGG-415 \\
\hline $14596-10-2$ & Americium-241 & $<6.13 \mathrm{E}-3$ & ug/g dry & $6.13 \mathrm{E}-3$ & $7 / 17 / 08$ & 8G15009 & PNNL-AGG-415 \\
\hline HEIS No. & B1VJ61A & \multicolumn{3}{|c|}{ Lab ID: $\quad$ 0805020-24 } & & & \\
\hline 13994-20-2 & Neptunium-237 & $<3.17 \mathrm{E}-3$ & ug/g dry & 3.17E-3 & 7/17/08 & 8G15009 & PNNL-AGG-415 \\
\hline $15117-48-3$ & Plutonium-239 & $<7.87 \mathrm{E}-3$ & ug/g dry & 7.87E-3 & $7 / 17 / 08$ & 8G15009 & PNNL-AGG-415 \\
\hline $14596-10-2$ & Americium-241 & $<6.81 \mathrm{E}-3$ & ug/g dry & $6.81 \mathrm{E}-3$ & 7/17/08 & 8G15009 & PNNL-AGG-415 \\
\hline HEIS No. & B1VJ62C & \multicolumn{3}{|c|}{ Lab ID: $\quad$ 0805020-26 } & & & \\
\hline $13994-20-2$ & Neptunium-237 & $<3.33 \mathrm{E}-3$ & ug/g dry & 3.33E-3 & $7 / 17 / 08$ & 8G15009 & PNNL-AGG-415 \\
\hline $15117-48-3$ & Plutonium-239 & $<8.29 \mathrm{E}-3$ & ug/g dry & $8.29 \mathrm{E}-3$ & $7 / 17 / 08$ & 8G15009 & PNNL-AGG-415 \\
\hline $14596-10-2$ & Americium-241 & $<7.17 \mathrm{E}-3$ & ug/g dry & 7.17E-3 & $7 / 17 / 08$ & 8G15009 & PNNL-AGG-415 \\
\hline HEIS No. & B1VJ62B & \multicolumn{3}{|c|}{ Lab ID: $\quad$ 0805020-27 } & & & \\
\hline $13994-20-2$ & Neptunium-237 & $<3.42 \mathrm{E}-3$ & ug/g dry & $3.42 \mathrm{E}-3$ & 7/17/08 & 8G15010 & PNNL-AGG-415 \\
\hline $15117-48-3$ & Plutonium-239 & $<8.51 \mathrm{E}-3$ & ug/g dry & 8.51E-3 & $7 / 17 / 08$ & $8 G 15010$ & PNNL-AGG-415 \\
\hline $14596-10-2$ & Americium-241 & $<7.36 \mathrm{E}-3$ & ug/g dry & 7.36E-3 & 7/17/08 & 8G15010 & PNNL-AGG-415 \\
\hline HEIS No. & B1VJ62A & \multicolumn{3}{|c|}{ Lab ID: $\quad$ 0805020-28 } & & & \\
\hline 13994-20-2 & Neptunium-237 & $<3.47 \mathrm{E}-3$ & ug/g dry & $3.47 \mathrm{E}-3$ & 7/18/08 & 8G15010 & PNNL-AGG-415 \\
\hline $15117-48-3$ & Plutonium-239 & $<8.62 \mathrm{E}-3$ & ug/g dry & $8.62 \mathrm{E}-3$ & $7 / 18 / 08$ & $8 G 15010$ & PNNL-AGG-415 \\
\hline $14596-10-2$ & Americium-241 & $<7.46 \mathrm{E}-3$ & ug/g dry & $7.46 \mathrm{E}-3$ & 7/18/08 & $8 G 15010$ & PNNL-AGG-415 \\
\hline HEIS No. & B1VJ64C & \multicolumn{3}{|c|}{ Lab ID: $\quad$ 0805020-30 } & & & \\
\hline $13994-20-2$ & Neptunium-237 & $<3.50 \mathrm{E}-3$ & ug/g dry & $3.50 \mathrm{E}-3$ & 7/18/08 & 8G15010 & PNNL-AGG-415 \\
\hline $15117-48-3$ & Plutonium-239 & $<8.71 \mathrm{E}-3$ & ug/g dry & 8.71E-3 & 7/18/08 & 8G15010 & PNNL-AGG-415 \\
\hline $14596-10-2$ & Americium-241 & $<7.53 \mathrm{E}-3$ & ug/g dry & 7.53E-3 & $7 / 18 / 08$ & 8G15010 & PNNL-AGG-415 \\
\hline HEIS No. & B1VJ64B & \multicolumn{3}{|c|}{ Lab ID: $\quad$ 0805020-31 } & & & \\
\hline 13994-20-2 & Neptunium-237 & $<3.11 \mathrm{E}-3$ & ug/g dry & $3.11 \mathrm{E}-3$ & 7/18/08 & $8 \mathrm{G} 15010$ & PNNL-AGG-415 \\
\hline $15117-48-3$ & Plutonium-239 & $<7.73 E-3$ & ug/g dry & 7.73E-3 & $7 / 18 / 08$ & 8G15010 & PNNL-AGG-415 \\
\hline $14596-10-2$ & Americium-241 & $<6.69 \mathrm{E}-3$ & ug/g dry & $6.69 \mathrm{E}-3$ & $7 / 18 / 08$ & $8 G 15010$ & PNNL-AGG-415 \\
\hline HEIS No. & B1VJ64A & \multicolumn{3}{|c|}{ Lab ID: $\quad$ 0805020-32 } & & & \\
\hline $13994-20-2$ & Neptunium-237 & $<3.30 \mathrm{E}-3$ & ug/g dry & $3.30 \mathrm{E}-3$ & 7/18/08 & 8G15010 & PNNL-AGG-415 \\
\hline $15117-48-3$ & Plutonium-239 & $<8.21 \mathrm{E}-3$ & ug/g dry & $8.21 \mathrm{E}-3$ & $7 / 18 / 08$ & $8 G 15010$ & PNNL-AGG-415 \\
\hline $14596-10-2$ & Americium-241 & $<7.10 \mathrm{E}-3$ & ug/g dry & $7.10 \mathrm{E}-3$ & 7/18/08 & $8 G 15010$ & PNNL-AGG-415 \\
\hline
\end{tabular}




\section{Radionuclides by ICP-MS/Acid Extract}

\begin{tabular}{|c|c|c|c|c|c|c|c|}
\hline CAS \# & Analyte & Results & Units & EQL & Analyzed & Batch & Method \\
\hline \multirow{3}{*}{$\begin{array}{l}\text { HEIS No. } \\
14133-76-7\end{array}$} & B1VJ54B & \multicolumn{3}{|c|}{ Lab ID: $\quad$ 0805020-01 } & & & \\
\hline & Technetium-99 & $<4.13 \mathrm{E}-3$ & ug/g dry & 4.13E-3 & $7 / 02 / 08$ & 8G02004 & PNNL-AGG-415 \\
\hline & Uranium 238 & $1.01 \mathrm{E} 0$ & ug/g dry & $3.00 \mathrm{E}-2$ & $7 / 02 / 08$ & 8G02004 & PNNL-AGG-415 \\
\hline \multirow{3}{*}{$\begin{array}{l}\text { HEIS No. } \\
14133-76-7\end{array}$} & B1VJ55C & \multicolumn{3}{|c|}{ Lab ID: $\quad$ 0805020-05 } & & & \\
\hline & Technetium-99 & $<4.83 \mathrm{E}-3$ & ug/g dry & 4.83E-3 & $7 / 02 / 08$ & 8G02004 & PNNL-AGG-415 \\
\hline & Uranium 238 & 3.84E-1 & ug/g dry & $3.50 \mathrm{E}-2$ & 7/02/08 & 8G02004 & PNNL-AGG-415 \\
\hline \multirow{3}{*}{$\begin{array}{l}\text { HEIS No. } \\
\text { 14133-76-7 }\end{array}$} & B1VJ56A & \multicolumn{3}{|c|}{ Lab ID: $\quad$ 0805020-07 } & & & \\
\hline & Technetium-99 & $<4.02 \mathrm{E}-3$ & ug/g dry & $4.02 \mathrm{E}-3$ & $7 / 02 / 08$ & 8G02006 & PNNL-AGG-415 \\
\hline & Uranium 238 & $2.88 \mathrm{E}-1$ & ug/g dry & $2.92 \mathrm{E}-2$ & $7 / 02 / 08$ & 8G02006 & PNNL-AGG-415 \\
\hline \multirow{3}{*}{$\begin{array}{l}\text { HEIS No. } \\
14133-76-7\end{array}$} & B1VJ56B & \multicolumn{3}{|c|}{ Lab ID: $\quad$ 0805020-08 } & & & \\
\hline & Technetium-99 & $<4.31 \mathrm{E}-3$ & ug/g dry & 4.31E-3 & $7 / 02 / 08$ & 8G02004 & PNNL-AGG-415 \\
\hline & Uranium 238 & 3.18E-1 & ug/g dry & 3.13E-2 & $7 / 02 / 08$ & 8G02004 & PNNL-AGG-415 \\
\hline \multirow{3}{*}{$\begin{array}{l}\text { HEIS No. } \\
14133-76-7\end{array}$} & B1VJ56C & \multicolumn{3}{|c|}{ Lab ID: $\quad$ 0805020-09 } & & & \\
\hline & Technetium-99 & $<4.17 \mathrm{E}-3$ & ug/g dry & 4.17E-3 & $7 / 02 / 08$ & 8G02004 & PNNL-AGG-415 \\
\hline & Uranium 238 & 3.11E-1 & ug/g dry & 3.03E-2 & $7 / 02 / 08$ & 8G02004 & PNNL-AGG-415 \\
\hline \multirow{3}{*}{$\begin{array}{l}\text { HEIS No. } \\
14133-76-7\end{array}$} & B1VJ58C & \multicolumn{3}{|c|}{ Lab ID: $\quad$ 0805020-10 } & & & \\
\hline & Technetium-99 & $<4.45 \mathrm{E}-3$ & ug/g dry & $4.45 \mathrm{E}-3$ & $7 / 02 / 08$ & 8G02004 & PNNL-AGG-415 \\
\hline & Uranium 238 & $5.01 \mathrm{E}-1$ & ug/g dry & 3.23E-2 & 7/02/08 & 8G02004 & PNNL-AGG-415 \\
\hline \multirow{3}{*}{$\begin{array}{l}\text { HEIS No. } \\
14133-76-7\end{array}$} & B1VJ58B & \multicolumn{3}{|c|}{ Lab ID: $\quad$ 0805020-11 } & & & \\
\hline & Technetium-99 & $<4.74 \mathrm{E}-3$ & ug/g dry & $4.74 \mathrm{E}-3$ & $7 / 02 / 08$ & 8G02004 & PNNL-AGG-415 \\
\hline & Uranium 238 & $5.27 \mathrm{E}-1$ & ug/g dry & $3.44 \mathrm{E}-2$ & 7/02/08 & 8G02004 & PNNL-AGG-415 \\
\hline \multirow{3}{*}{$\begin{array}{l}\text { HEIS No. } \\
14133-76-7\end{array}$} & B1VJ58A & \multicolumn{3}{|c|}{ Lab ID: $\quad$ 0805020-12 } & & & \\
\hline & Technetium-99 & $<4.85 \mathrm{E}-3$ & ug/g dry & 4.85E-3 & $7 / 02 / 08$ & 8G02004 & PNNL-AGG-415 \\
\hline & Uranium 238 & 7.53E-1 & ug/g dry & $3.52 \mathrm{E}-2$ & $7 / 02 / 08$ & 8G02004 & PNNL-AGG-415 \\
\hline \multirow{3}{*}{$\begin{array}{l}\text { HEIS No. } \\
\text { 14133-76-7 }\end{array}$} & B1VJ59C & \multicolumn{3}{|c|}{ Lab ID: $\quad$ 0805020-14 } & & & \\
\hline & Technetium-99 & $<4.51 \mathrm{E}-3$ & ug/g dry & $4.51 \mathrm{E}-3$ & $7 / 02 / 08$ & 8G02004 & PNNL-AGG-415 \\
\hline & Uranium 238 & $3.16 \mathrm{E}-1$ & ug/g dry & $3.28 \mathrm{E}-2$ & $7 / 02 / 08$ & 8G02004 & PNNL-AGG-415 \\
\hline \multirow{3}{*}{$\begin{array}{l}\text { HEIS No. } \\
\text { 14133-76-7 }\end{array}$} & B1VJ59B & \multicolumn{3}{|c|}{ Lab ID: $\quad$ 0805020-15 } & & & \\
\hline & Technetium-99 & $<4.28 \mathrm{E}-3$ & ug/g dry & $4.28 \mathrm{E}-3$ & 7/02/08 & 8G02004 & PNNL-AGG-415 \\
\hline & Uranium 238 & 3.15E-1 & ug/g dry & $3.11 \mathrm{E}-2$ & 7/02/08 & 8G02004 & PNNL-AGG-415 \\
\hline \multirow{3}{*}{$\begin{array}{l}\text { HEIS No. } \\
14133-76-7\end{array}$} & B1VJ59A & & b ID: & $5020-16$ & & & \\
\hline & Technetium-99 & $<4.23 \mathrm{E}-3$ & ug/g dry & $4.23 \mathrm{E}-3$ & $7 / 02 / 08$ & 8G02004 & PNNL-AGG-415 \\
\hline & Uranium 238 & 3.65E-1 & ug/g dry & 3.07E-2 & 7/02/08 & 8G02004 & PNNL-AGG-415 \\
\hline HEIS No. & B1VJ60C & & b ID: & $5020-18$ & & & \\
\hline $14133-76-7$ & Technetium-99 & $<4.08 \mathrm{E}-3$ & ug/g dry & $4.08 \mathrm{E}-3$ & 7/02/08 & 8G02004 & PNNL-AGG-415 \\
\hline & Uranium 238 & 3.76E-1 & ug/g dry & $2.96 \mathrm{E}-2$ & 7/02/08 & 8G02004 & PNNL-AGG-415 \\
\hline HEIS No. & B1VJ60B & & b ID: & $5020-19$ & & & \\
\hline $14133-76-7$ & Technetium-99 & $<4.31 \mathrm{E}-3$ & ug/g dry & $4.31 \mathrm{E}-3$ & $7 / 02 / 08$ & 8G02004 & PNNL-AGG-415 \\
\hline & Uranium 238 & 3.34E-1 & ug/g dry & $3.13 \mathrm{E}-2$ & 7/02/08 & 8G02004 & PNNL-AGG-415 \\
\hline HEIS No. & B1VJ60A & & b ID: & $5020-20$ & & & \\
\hline 14133-76-7 & Technetium-99 & $<4.23 \mathrm{E}-3$ & ug/g dry & $4.23 \mathrm{E}-3$ & 7/02/08 & 8G02004 & PNNL-AGG-415 \\
\hline & Uranium 238 & 3.66E-1 & ug/g dry & $3.07 \mathrm{E}-2$ & 7/02/08 & 8G02004 & PNNL-AGG-415 \\
\hline HEIS No. & B1VJ61C & & b ID: & $5020-22$ & & & \\
\hline 14133-76-7 & Technetium-99 & $<3.79 \mathrm{E}-3$ & ug/g dry & 3.79E-3 & 7/02/08 & 8G02004 & PNNL-AGG-415 \\
\hline & Uranium 238 & 3.11E-1 & ug/g dry & $2.75 \mathrm{E}-2$ & 7/02/08 & 8G02004 & PNNL-AGG-415 \\
\hline HEIS No. & B1VJ61B & & b ID: & $5020-23$ & & & \\
\hline $14133-76-7$ & Technetium-99 & $<3.69 \mathrm{E}-3$ & ug/g dry & $3.69 \mathrm{E}-3$ & $7 / 02 / 08$ & 8G02004 & PNNL-AGG-415 \\
\hline
\end{tabular}




\section{Radionuclides by ICP-MS/Acid Extract}

\begin{tabular}{|c|c|c|c|c|c|c|c|}
\hline CAS \# & Analyte & Results & Units & EQL & Analyzed & Batch & Method \\
\hline \multirow[t]{2}{*}{ HEIS No. } & B1VJ61B & \multicolumn{3}{|c|}{ Lab ID: $\quad$ 0805020-23 } & & & \\
\hline & Uranium 238 & 2.93E-1 & ug/g dry & $2.68 \mathrm{E}-2$ & 7/02/08 & 8G02004 & PNNL-AGG-415 \\
\hline \multirow{3}{*}{$\begin{array}{l}\text { HEIS No. } \\
14133-76-7\end{array}$} & B1VJ61A & \multicolumn{3}{|c|}{ Lab ID: $\quad$ 0805020-24 } & & & \\
\hline & Technetium-99 & $<4.10 \mathrm{E}-3$ & ug/g dry & 4.10E-3 & 7/02/08 & 8G02004 & PNNL-AGG-415 \\
\hline & Uranium 238 & 3.39E-1 & ug/g dry & 2.98E-2 & 7/02/08 & 8G02004 & PNNL-AGG-415 \\
\hline \multirow{3}{*}{$\begin{array}{l}\text { HEIS No. } \\
14133-76-7\end{array}$} & B1VJ62C & \multicolumn{3}{|c|}{ Lab ID: $\quad$ 0805020-26 } & & & \\
\hline & Technetium-99 & $<4.32 \mathrm{E}-3$ & ug/g dry & 4.32E-3 & 7/02/08 & 8G02004 & PNNL-AGG-415 \\
\hline & Uranium 238 & $2.94 \mathrm{E}-1$ & ug/g dry & 3.13E-2 & $7 / 02 / 08$ & 8G02004 & PNNL-AGG-415 \\
\hline \multirow{3}{*}{$\begin{array}{l}\text { HEIS No. } \\
14133-76-7\end{array}$} & B1VJ62B & \multicolumn{3}{|c|}{ Lab ID: $\quad$ 0805020-27 } & & & \\
\hline & Technetium-99 & $<4.43 \mathrm{E}-3$ & ug/g dry & 4.43E-3 & $7 / 02 / 08$ & 8G02006 & PNNL-AGG-415 \\
\hline & Uranium 238 & $3.59 \mathrm{E}-1$ & ug/g dry & 3.22E-2 & 7/02/08 & 8G02006 & PNNL-AGG-415 \\
\hline \multirow{3}{*}{$\begin{array}{l}\text { HEIS No. } \\
14133-76-7\end{array}$} & B1VJ62A & \multicolumn{3}{|c|}{ Lab ID: $\quad$ 0805020-28 } & & & \\
\hline & Technetium-99 & $<4.49 \mathrm{E}-3$ & ug/g dry & 4.49E-3 & 7/02/08 & 8G02006 & PNNL-AGG-415 \\
\hline & Uranium 238 & 3.63E-1 & ug/g dry & $3.26 \mathrm{E}-2$ & $7 / 02 / 08$ & 8G02006 & PNNL-AGG-415 \\
\hline \multirow{3}{*}{$\begin{array}{l}\text { HEIS No. } \\
14133-76-7\end{array}$} & B1VJ64C & \multicolumn{3}{|c|}{ Lab ID: $\quad$ 0805020-30 } & & & \\
\hline & Technetium-99 & $<4.53 \mathrm{E}-3$ & ug/g dry & 4.53E-3 & 7/02/08 & 8G02006 & PNNL-AGG-415 \\
\hline & Uranium 238 & 3.53E-1 & ug/g dry & $3.29 \mathrm{E}-2$ & 7/02/08 & $8 G 02006$ & PNNL-AGG-415 \\
\hline \multirow{3}{*}{$\begin{array}{l}\text { HEIS No. } \\
14133-76-7\end{array}$} & B1VJ64B & \multicolumn{3}{|c|}{ Lab ID: $\quad 0805020-31$} & & & \\
\hline & Technetium-99 & $<4.03 \mathrm{E}-3$ & ug/g dry & 4.03E-3 & 7/02/08 & 8G02006 & PNNL-AGG-415 \\
\hline & Uranium 238 & 3.16E-1 & ug/g dry & 2.92E-2 & 7/02/08 & 8G02006 & PNNL-AGG-415 \\
\hline \multirow{3}{*}{$\begin{array}{l}\text { HEIS No. } \\
14133-76-7\end{array}$} & B1VJ64A & \multicolumn{3}{|c|}{ Lab ID: $\quad$ 0805020-32 } & & & \\
\hline & Technetium-99 & $<4.27 \mathrm{E}-3$ & ug/g dry & 4.27E-3 & $7 / 02 / 08$ & 8G02006 & PNNL-AGG-415 \\
\hline & Uranium 238 & 2.31E-1 & ug/g dry & $3.10 \mathrm{E}-2$ & $7 / 02 / 08$ & 8G02006 & PNNL-AGG-415 \\
\hline
\end{tabular}




\section{Radionuclides by ICP-MS/Water Extract}

\begin{tabular}{|c|c|c|c|c|c|c|c|}
\hline CAS \# & Analyte & Results & Units & EQL & Analyzed & Batch & Method \\
\hline \multirow{3}{*}{$\begin{array}{l}\text { HEIS No. } \\
14133-76-7\end{array}$} & B1VJ54B & \multicolumn{3}{|c|}{ Lab ID: $\quad$ 0805020-01 } & & & \\
\hline & Technetium-99 & $<2.34 \mathrm{E}-5$ & ug/g dry & $2.34 \mathrm{E}-5$ & 7/01/08 & 8G01002 & PNNL-AGG-415 \\
\hline & Uranium 238 & $9.84 \mathrm{E}-2$ & ug/g dry & $5.72 \mathrm{E}-4$ & 7/01/08 & 8G01002 & PNNL-AGG-415 \\
\hline \multirow{3}{*}{$\begin{array}{l}\text { HEIS No. } \\
\text { 14133-76-7 }\end{array}$} & B1VJ55C & \multicolumn{3}{|c|}{ Lab ID: $\quad$ 0805020-05 } & & & \\
\hline & Technetium-99 & $<2.30 \mathrm{E}-5$ & ug/g dry & $2.30 \mathrm{E}-5$ & 7/01/08 & 8G01002 & PNNL-AGG-415 \\
\hline & Uranium 238 & 3.93E-3 & ug/g dry & $5.63 \mathrm{E}-4$ & $7 / 01 / 08$ & 8G01002 & PNNL-AGG-415 \\
\hline \multirow{3}{*}{$\begin{array}{l}\text { HEIS No. } \\
14133-76-7\end{array}$} & B1VJ56A & \multicolumn{3}{|c|}{ Lab ID: $\quad$ 0805020-07 } & & & \\
\hline & Technetium-99 & $<2.47 \mathrm{E}-5$ & ug/g dry & $2.47 \mathrm{E}-5$ & 7/01/08 & 8G01002 & PNNL-AGG-415 \\
\hline & Uranium 238 & $9.66 \mathrm{E}-3$ & ug/g dry & $6.04 \mathrm{E}-4$ & $7 / 01 / 08$ & 8G01002 & PNNL-AGG-415 \\
\hline \multirow{3}{*}{$\begin{array}{l}\text { HEIS No. } \\
14133-76-7\end{array}$} & B1VJ56B & \multicolumn{3}{|c|}{ Lab ID: $\quad$ 0805020-08 } & & & \\
\hline & Technetium-99 & $<2.30 \mathrm{E}-5$ & ug/g dry & $2.30 \mathrm{E}-5$ & $7 / 01 / 08$ & 8G01002 & PNNL-AGG-415 \\
\hline & Uranium 238 & $2.90 \mathrm{E}-2$ & ug/g dry & $5.64 \mathrm{E}-4$ & $7 / 01 / 08$ & 8G01002 & PNNL-AGG-415 \\
\hline \multirow{3}{*}{$\begin{array}{l}\text { HEIS No. } \\
14133-76-7\end{array}$} & B1VJ56C & \multicolumn{3}{|c|}{ Lab ID: $\quad$ 0805020-09 } & & & \\
\hline & Technetium-99 & $<2.30 \mathrm{E}-5$ & ug/g dry & $2.30 \mathrm{E}-5$ & 7/01/08 & 8G01002 & PNNL-AGG-415 \\
\hline & Uranium 238 & $6.90 \mathrm{E}-3$ & ug/g dry & $5.64 \mathrm{E}-4$ & $7 / 01 / 08$ & 8G01002 & PNNL-AGG-415 \\
\hline \multirow{3}{*}{$\begin{array}{l}\text { HEIS No. } \\
\text { 14133-76-7 }\end{array}$} & B1VJ58C & \multicolumn{3}{|c|}{ Lab ID: $\quad 0805020-10$} & & & \\
\hline & Technetium-99 & $3.26 \mathrm{E}-5$ & ug/g dry & $2.30 \mathrm{E}-5$ & 7/01/08 & 8G01002 & PNNL-AGG-415 \\
\hline & Uranium 238 & $1.88 \mathrm{E}-1$ & ug/g dry & $5.64 \mathrm{E}-4$ & 7/01/08 & 8G01002 & PNNL-AGG-415 \\
\hline \multirow{3}{*}{$\begin{array}{l}\text { HEIS No. } \\
14133-76-7\end{array}$} & B1VJ58B & \multicolumn{3}{|c|}{ Lab ID: $\quad$ 0805020-11 } & & & \\
\hline & Technetium-99 & 4.93E-5 & ug/g dry & $2.31 \mathrm{E}-5$ & $7 / 22 / 08$ & 8G01002 & PNNL-AGG-415 \\
\hline & Uranium 238 & $3.18 \mathrm{E}-1$ & ug/g dry & $5.67 \mathrm{E}-4$ & $7 / 22 / 08$ & 8G01002 & PNNL-AGG-415 \\
\hline \multirow{3}{*}{$\begin{array}{l}\text { HEIS No. } \\
14133-76-7\end{array}$} & B1VJ58A & \multicolumn{3}{|c|}{ Lab ID: $\quad$ 0805020-12 } & & & \\
\hline & Technetium-99 & $6.83 \mathrm{E}-5$ & ug/g dry & $2.30 \mathrm{E}-5$ & $7 / 01 / 08$ & 8G01002 & PNNL-AGG-415 \\
\hline & Uranium 238 & $4.59 \mathrm{E}-1$ & ug/g dry & 5.63E-4 & $7 / 01 / 08$ & 8G01002 & PNNL-AGG-415 \\
\hline \multirow{3}{*}{$\begin{array}{l}\text { HEIS No. } \\
14133-76-7\end{array}$} & B1VJ59C & \multicolumn{3}{|c|}{ Lab ID: $\quad$ 0805020-14 } & & & \\
\hline & Technetium-99 & $<2.30 \mathrm{E}-5$ & ug/g dry & $2.30 \mathrm{E}-5$ & 7/01/08 & 8G01002 & PNNL-AGG-415 \\
\hline & Uranium 238 & $1.70 \mathrm{E}-3$ & & & $7 / 01 / 08$ & 8G01002 & PNNL-AGG-415 \\
\hline \multirow{3}{*}{$\begin{array}{l}\text { HEIS No. } \\
14133-76-7\end{array}$} & B1VJ59B & \multicolumn{3}{|c|}{ Lab ID: $\quad$ 0805020-15 } & & & \\
\hline & Technetium-99 & $<2.30 \mathrm{E}-5$ & ug/g dry & $2.30 \mathrm{E}-5$ & 7/01/08 & 8G01002 & PNNL-AGG-415 \\
\hline & Uranium 238 & $<5.64 \mathrm{E}-4$ & ug/g dry & $5.64 \mathrm{E}-4$ & $7 / 01 / 08$ & 8G01002 & PNNL-AGG-415 \\
\hline HEIS No. & B1VJ59A & & b ID: & $5020-16$ & & & \\
\hline 14133-76-7 & Technetium-99 & $<2.32 \mathrm{E}-5$ & ug/g dry & $2.32 \mathrm{E}-5$ & 7/01/08 & 8G01002 & PNNL-AGG-415 \\
\hline & Uranium 238 & $<5.68 \mathrm{E}-4$ & ug/g dry & $5.68 \mathrm{E}-4$ & 7/01/08 & 8G01002 & PNNL-AGG-415 \\
\hline HEIS No. & B1VJ60C & & b ID: & 5020-18 & & & \\
\hline 14133-76-7 & Technetium-99 & $<2.30 \mathrm{E}-5$ & ug/g dry & $2.30 \mathrm{E}-5$ & 7/01/08 & 8G01002 & PNNL-AGG-415 \\
\hline & Uranium 238 & 7.66E-4 & ug/g dry & 5.63E-4 & $7 / 01 / 08$ & 8G01002 & PNNL-AGG-415 \\
\hline HEIS No. & B1VJ60B & & b ID: & $5020-19$ & & & \\
\hline 14133-76-7 & Technetium-99 & $<2.30 \mathrm{E}-5$ & ug/g dry & $2.30 \mathrm{E}-5$ & 7/01/08 & 8G01002 & PNNL-AGG-415 \\
\hline & Uranium 238 & $6.47 \mathrm{E}-4$ & ug/g dry & $5.64 \mathrm{E}-4$ & 7/01/08 & 8G01002 & PNNL-AGG-415 \\
\hline HEIS No. & B1VJ60A & & b ID: & 5020-20 & & & \\
\hline 14133-76-7 & Technetium-99 & $<2.30 \mathrm{E}-5$ & ug/g dry & $2.30 \mathrm{E}-5$ & 7/01/08 & 8G01002 & PNNL-AGG-415 \\
\hline & Uranium 238 & $<5.64 \mathrm{E}-4$ & ug/g dry & $5.64 \mathrm{E}-4$ & 7/01/08 & 8G01002 & PNNL-AGG-415 \\
\hline HEIS No. & B1VJ61C & & b ID: & $5020-22$ & & & \\
\hline 14133-76-7 & Technetium-99 & $<2.30 \mathrm{E}-5$ & ug/g dry & $2.30 \mathrm{E}-5$ & 7/01/08 & 8G01002 & PNNL-AGG-415 \\
\hline & Uranium 238 & $<5.63 \mathrm{E}-4$ & ug/g dry & 5.63E-4 & 7/01/08 & 8G01002 & PNNL-AGG-415 \\
\hline HEIS No. & B1VJ61B & & b ID: & $5020-23$ & & & \\
\hline 14133-76-7 & Technetium-99 & $<2.32 \mathrm{E}-5$ & ug/g dry & $2.32 \mathrm{E}-5$ & 7/01/08 & 8G01002 & PNNL-AGG-415 \\
\hline
\end{tabular}




\section{Radionuclides by ICP-MS/Water Extract}

\begin{tabular}{|c|c|c|c|c|c|c|c|}
\hline CAS \# & Analyte & Results & Units & EQL & Analyzed & Batch & Method \\
\hline \multirow[t]{2}{*}{ HEIS No. } & B1VJ61B & \multicolumn{3}{|c|}{ Lab ID: $\quad$ 0805020-23 } & & & \\
\hline & Uranium 238 & $<5.69 \mathrm{E}-4$ & ug/g dry & $5.69 \mathrm{E}-4$ & 7/01/08 & 8G01002 & PNNL-AGG-415 \\
\hline \multirow{3}{*}{$\begin{array}{l}\text { HEIS No. } \\
14133-76-7\end{array}$} & B1VJ61A & \multicolumn{3}{|c|}{ Lab ID: $\quad$ 0805020-24 } & & & \\
\hline & Technetium-99 & $<2.39 \mathrm{E}-5$ & ug/g dry & $2.39 \mathrm{E}-5$ & 7/01/08 & 8G01002 & PNNL-AGG-415 \\
\hline & Uranium 238 & $<5.85 \mathrm{E}-4$ & ug/g dry & $5.85 \mathrm{E}-4$ & 7/01/08 & 8G01002 & PNNL-AGG-415 \\
\hline \multirow{3}{*}{$\begin{array}{l}\text { HEIS No. } \\
14133-76-7\end{array}$} & B1VJ62C & \multicolumn{3}{|c|}{ Lab ID: $\quad$ 0805020-26 } & & & \\
\hline & Technetium-99 & $<2.30 \mathrm{E}-5$ & ug/g dry & $2.30 \mathrm{E}-5$ & 7/01/08 & 8G01002 & PNNL-AGG-415 \\
\hline & Uranium 238 & 8.87E-4 & ug/g dry & 5.63E-4 & $7 / 01 / 08$ & 8G01002 & PNNL-AGG-415 \\
\hline \multirow{3}{*}{$\begin{array}{l}\text { HEIS No. } \\
14133-76-7\end{array}$} & B1VJ62B & \multicolumn{3}{|c|}{ Lab ID: $\quad$ 0805020-27 } & & & \\
\hline & Technetium-99 & $<2.30 \mathrm{E}-5$ & ug/g dry & $2.30 \mathrm{E}-5$ & 7/01/08 & 8G01003 & PNNL-AGG-415 \\
\hline & Uranium 238 & $<5.64 \mathrm{E}-4$ & ug/g dry & 5.64E-4 & 7/01/08 & 8G01003 & PNNL-AGG-415 \\
\hline \multirow{3}{*}{$\begin{array}{l}\text { HEIS No. } \\
14133-76-7\end{array}$} & B1VJ62A & \multicolumn{3}{|c|}{ Lab ID: $\quad$ 0805020-28 } & & & \\
\hline & Technetium-99 & $<2.30 \mathrm{E}-5$ & ug/g dry & $2.30 \mathrm{E}-5$ & 7/01/08 & 8G01003 & PNNL-AGG-415 \\
\hline & Uranium 238 & $<5.64 \mathrm{E}-4$ & ug/g dry & $5.64 \mathrm{E}-4$ & 7/01/08 & 8G01003 & PNNL-AGG-415 \\
\hline \multirow{3}{*}{$\begin{array}{l}\text { HEIS No. } \\
14133-76-7\end{array}$} & B1VJ64C & \multicolumn{3}{|c|}{ Lab ID: $\quad$ 0805020-30 } & & & \\
\hline & Technetium-99 & $<2.30 \mathrm{E}-5$ & ug/g dry & $2.30 \mathrm{E}-5$ & 7/01/08 & 8G01003 & PNNL-AGG-415 \\
\hline & Uranium 238 & $5.66 \mathrm{E}-4$ & ug/g dry & $5.64 \mathrm{E}-4$ & 7/01/08 & 8G01003 & PNNL-AGG-415 \\
\hline \multirow{3}{*}{$\begin{array}{l}\text { HEIS No. } \\
14133-76-7\end{array}$} & B1VJ64B & \multicolumn{3}{|c|}{ Lab ID: $\quad$ 0805020-31 } & & & \\
\hline & Technetium-99 & $<2.30 \mathrm{E}-5$ & ug/g dry & $2.30 \mathrm{E}-5$ & 7/01/08 & 8G01003 & PNNL-AGG-415 \\
\hline & Uranium 238 & $<5.63 \mathrm{E}-4$ & ug/g dry & 5.63E-4 & 7/01/08 & 8G01003 & PNNL-AGG-415 \\
\hline \multirow{3}{*}{$\begin{array}{l}\text { HEIS No. } \\
14133-76-7\end{array}$} & B1VJ64A & \multicolumn{3}{|c|}{ Lab ID: $\quad$ 0805020-32 } & & & \\
\hline & Technetium-99 & $<2.18 \mathrm{E}-5$ & ug/g dry & $2.18 \mathrm{E}-5$ & 7/01/08 & 8G01003 & PNNL-AGG-415 \\
\hline & Uranium 238 & $<5.35 \mathrm{E}-4$ & ug/g dry & 5.35E-4 & 7/01/08 & 8G01003 & PNNL-AGG-415 \\
\hline
\end{tabular}


RCRA Metals By PNNL-AGG-415/Water Extract

\begin{tabular}{|c|c|c|c|c|c|c|c|}
\hline CAS \# & Analyte & Results & Units & EQL & Analyzed & Batch & Method \\
\hline HEIS No. & B1VJ54B & \multicolumn{3}{|c|}{ Lab ID: $\quad 0805020-01$} & & & \\
\hline $14092-98-9$ & Chromium 52 & 2.23E-3 & ug/g dry & $2.09 \mathrm{E}-3$ & 7/01/08 & 8G01004 & PNNL-AGG-415 \\
\hline 13981-78-7 & Chromium 53 & $<6.50 \mathrm{E}-3$ & ug/g dry & $6.50 \mathrm{E}-3$ & 7/01/08 & 8G01004 & PNNL-AGG-415 \\
\hline 14191-84-5 & Copper 63 & 4.46E-3 & ug/g dry & $3.53 \mathrm{E}-3$ & $7 / 01 / 08$ & 8G01004 & PNNL-AGG-415 \\
\hline 14119-06-3 & Copper 65 & $<4.92 \mathrm{E}-3$ & ug/g dry & $4.92 \mathrm{E}-3$ & 7/01/08 & 8G01004 & PNNL-AGG-415 \\
\hline 14687-58-2 & Selenium 82 & $<1.12 \mathrm{E}-2$ & ug/g dry & $1.12 \mathrm{E}-2$ & 7/01/08 & 8G01004 & PNNL-AGG-415 \\
\hline $14392-17-7$ & Molybdenum 95 & 6.73E-3 & ug/g dry & $1.63 \mathrm{E}-3$ & 7/01/08 & 8G01004 & PNNL-AGG-415 \\
\hline 14392-19-9 & Molybdenum 97 & $6.69 \mathrm{E}-3$ & ug/g dry & $2.08 \mathrm{E}-3$ & $7 / 01 / 08$ & 8G01004 & PNNL-AGG-415 \\
\hline 14392-20-2 & Molybdenum 98 & $6.54 \mathrm{E}-3$ & ug/g dry & $1.63 \mathrm{E}-3$ & 7/01/08 & 8G01004 & PNNL-AGG-415 \\
\hline $14914-61-5$ & Ruthenium 101 & $<8.33 \mathrm{E}-4$ & ug/g dry & 8.33E-4 & 7/01/08 & 8G01004 & PNNL-AGG-415 \\
\hline $14914-62-6$ & Ruthenium 102 & $<5.74 \mathrm{E}-4$ & ug/g dry & $5.74 \mathrm{E}-4$ & 7/01/08 & 8G01004 & PNNL-AGG-415 \\
\hline 15766-01-5 & Ruthenium 104 & $<3.71 \mathrm{E}-4$ & ug/g dry & $3.71 \mathrm{E}-4$ & $7 / 01 / 08$ & 8G01004 & PNNL-AGG-415 \\
\hline $14378-37-1$ & Silver 107 & $<9.40 \mathrm{E}-4$ & ug/g dry & $9.40 \mathrm{E}-4$ & 7/01/08 & 8G01004 & PNNL-AGG-415 \\
\hline $14378-38-2$ & Silver 109 & $<1.09 \mathrm{E}-3$ & ug/g dry & $1.09 \mathrm{E}-3$ & 7/01/08 & 8G01004 & PNNL-AGG-415 \\
\hline 14336-64-2 & Cadmium 111 & $<3.00 \mathrm{E}-4$ & ug/g dry & $3.00 \mathrm{E}-4$ & $7 / 01 / 08$ & 8G01004 & PNNL-AGG-415 \\
\hline $14041-58-8$ & Cadmium 114 & $<6.35 \mathrm{E}-4$ & ug/g dry & $6.35 \mathrm{E}-4$ & $7 / 01 / 08$ & 8G01004 & PNNL-AGG-415 \\
\hline $14265-72-6$ & Antimony 121 & 6.63E-4 & ug/g dry & $5.48 \mathrm{E}-4$ & $7 / 01 / 08$ & 8G01004 & PNNL-AGG-415 \\
\hline $13966-27-3$ & Lead 206 & $1.39 \mathrm{E}-3$ & ug/g dry & 7.31E-4 & $7 / 01 / 08$ & 8G01004 & PNNL-AGG-415 \\
\hline 13966-28-4 & Lead 208 & $1.41 \mathrm{E}-3$ & ug/g dry & $5.69 \mathrm{E}-4$ & $7 / 01 / 08$ & 8G01004 & PNNL-AGG-415 \\
\hline HEIS No. & B1VJ55C & \multicolumn{3}{|c|}{ Lab ID: $\quad$ 0805020-05 } & & & \\
\hline $14092-98-9$ & Chromium 52 & 2.23E-3 & ug/g dry & 2.05E-3 & 7/01/08 & 8G01004 & PNNL-AGG-415 \\
\hline 13981-78-7 & Chromium 53 & $<6.40 \mathrm{E}-3$ & ug/g dry & $6.40 \mathrm{E}-3$ & 7/01/08 & 8G01004 & PNNL-AGG-415 \\
\hline $14191-84-5$ & Copper 63 & $5.62 \mathrm{E}-3$ & ug/g dry & $3.48 \mathrm{E}-3$ & $7 / 01 / 08$ & 8G01004 & PNNL-AGG-415 \\
\hline 14119-06-3 & Copper 65 & $5.29 \mathrm{E}-3$ & ug/g dry & $4.84 \mathrm{E}-3$ & $7 / 01 / 08$ & 8G01004 & PNNL-AGG-415 \\
\hline $14687-58-2$ & Selenium 82 & $<1.10 \mathrm{E}-2$ & ug/g dry & $1.10 \mathrm{E}-2$ & $7 / 01 / 08$ & 8G01004 & PNNL-AGG-415 \\
\hline $14392-17-7$ & Molybdenum 95 & 7.95E-3 & ug/g dry & $1.60 \mathrm{E}-3$ & $7 / 01 / 08$ & 8G01004 & PNNL-AGG-415 \\
\hline 14392-19-9 & Molybdenum 97 & $8.16 \mathrm{E}-3$ & ug/g dry & $2.04 \mathrm{E}-3$ & $7 / 01 / 08$ & 8G01004 & PNNL-AGG-415 \\
\hline $14392-20-2$ & Molybdenum 98 & 7.92E-3 & ug/g dry & $1.60 \mathrm{E}-3$ & $7 / 01 / 08$ & 8G01004 & PNNL-AGG-415 \\
\hline $14914-61-5$ & Ruthenium 101 & $<8.20 \mathrm{E}-4$ & ug/g dry & $8.20 \mathrm{E}-4$ & 7/01/08 & 8G01004 & PNNL-AGG-415 \\
\hline $14914-62-6$ & Ruthenium 102 & $<5.65 \mathrm{E}-4$ & ug/g dry & $5.65 \mathrm{E}-4$ & 7/01/08 & 8G01004 & PNNL-AGG-415 \\
\hline $15766-01-5$ & Ruthenium 104 & $<3.65 \mathrm{E}-4$ & ug/g dry & 3.65E-4 & $7 / 01 / 08$ & 8G01004 & PNNL-AGG-415 \\
\hline 14378-37-1 & Silver 107 & $<9.25 \mathrm{E}-4$ & ug/g dry & $9.25 \mathrm{E}-4$ & $7 / 01 / 08$ & 8G01004 & PNNL-AGG-415 \\
\hline $14378-38-2$ & Silver 109 & $<1.07 \mathrm{E}-3$ & ug/g dry & $1.07 \mathrm{E}-3$ & 7/01/08 & 8G01004 & PNNL-AGG-415 \\
\hline 14336-64-2 & Cadmium 111 & $<2.95 \mathrm{E}-4$ & ug/g dry & 2.95E-4 & $7 / 01 / 08$ & 8G01004 & PNNL-AGG-415 \\
\hline 14041-58-8 & Cadmium 114 & $<6.25 \mathrm{E}-4$ & ug/g dry & $6.25 \mathrm{E}-4$ & $7 / 01 / 08$ & 8G01004 & PNNL-AGG-415 \\
\hline $14265-72-6$ & Antimony 121 & $1.29 \mathrm{E}-3$ & ug/g dry & $5.40 \mathrm{E}-4$ & 7/01/08 & 8G01004 & PNNL-AGG-415 \\
\hline 13966-27-3 & Lead 206 & $1.32 \mathrm{E}-3$ & ug/g dry & $7.20 \mathrm{E}-4$ & $7 / 01 / 08$ & 8G01004 & PNNL-AGG-415 \\
\hline 13966-28-4 & Lead 208 & $1.32 \mathrm{E}-3$ & ug/g dry & $5.60 \mathrm{E}-4$ & 7/01/08 & 8G01004 & PNNL-AGG-415 \\
\hline HEIS No. & B1VJ56A & \multicolumn{3}{|c|}{ Lab ID: $\quad$ 0805020-07 } & & & \\
\hline $14092-98-9$ & Chromium 52 & $1.19 \mathrm{E}-2$ & ug/g dry & $2.20 \mathrm{E}-3$ & $7 / 01 / 08$ & 8G01004 & PNNL-AGG-415 \\
\hline 13981-78-7 & Chromium 53 & $1.18 \mathrm{E}-2$ & ug/g dry & $6.86 \mathrm{E}-3$ & $7 / 01 / 08$ & 8G01004 & PNNL-AGG-415 \\
\hline 14191-84-5 & Copper 63 & 2.86E-2 & ug/g dry & 3.73E-3 & $7 / 01 / 08$ & 8G01004 & PNNL-AGG-415 \\
\hline 14119-06-3 & Copper 65 & $2.70 \mathrm{E}-2$ & ug/g dry & $5.19 \mathrm{E}-3$ & $7 / 01 / 08$ & 8G01004 & PNNL-AGG-415 \\
\hline $14687-58-2$ & Selenium 82 & $<1.18 \mathrm{E}-2$ & ug/g dry & $1.18 \mathrm{E}-2$ & $7 / 01 / 08$ & 8G01004 & PNNL-AGG-415 \\
\hline $14392-17-7$ & Molybdenum 95 & $9.71 \mathrm{E}-2$ & ug/g dry & $1.72 \mathrm{E}-3$ & $7 / 01 / 08$ & 8G01004 & PNNL-AGG-415 \\
\hline 14392-19-9 & Molybdenum 97 & $9.69 \mathrm{E}-2$ & ug/g dry & $2.19 \mathrm{E}-3$ & $7 / 01 / 08$ & 8G01004 & PNNL-AGG-415 \\
\hline 14392-20-2 & Molybdenum 98 & $9.72 \mathrm{E}-2$ & ug/g dry & $1.72 \mathrm{E}-3$ & $7 / 01 / 08$ & 8G01004 & PNNL-AGG-415 \\
\hline $14914-61-5$ & Ruthenium 101 & $<8.79 \mathrm{E}-4$ & ug/g dry & 8.79E-4 & 7/01/08 & 8G01004 & PNNL-AGG-415 \\
\hline $14914-62-6$ & Ruthenium 102 & $<6.06 \mathrm{E}-4$ & ug/g dry & 6.06E-4 & 7/01/08 & 8G01004 & PNNL-AGG-415 \\
\hline
\end{tabular}


RCRA Metals By PNNL-AGG-415/Water Extract

\begin{tabular}{|c|c|c|c|c|c|c|c|}
\hline CAS \# & Analyte & Results & Units & EQL & Analyzed & Batch & Method \\
\hline HEIS No. & B1VJ56A & \multicolumn{3}{|c|}{ Lab ID: $\quad$ 0805020-07 } & & & \\
\hline $15766-01-5$ & Ruthenium 104 & $<3.91 \mathrm{E}-4$ & ug/g dry & $3.91 \mathrm{E}-4$ & 7/01/08 & 8G01004 & PNNL-AGG-415 \\
\hline 14378-37-1 & Silver 107 & $<9.91 \mathrm{E}-4$ & ug/g dry & $9.91 \mathrm{E}-4$ & $7 / 01 / 08$ & 8G01004 & PNNL-AGG-415 \\
\hline $14378-38-2$ & Silver 109 & $<1.15 \mathrm{E}-3$ & ug/g dry & $1.15 \mathrm{E}-3$ & $7 / 01 / 08$ & 8G01004 & PNNL-AGG-415 \\
\hline 14336-64-2 & Cadmium 111 & 4.07E-4 & ug/g dry & $3.16 \mathrm{E}-4$ & $7 / 01 / 08$ & 8G01004 & PNNL-AGG-415 \\
\hline $14041-58-8$ & Cadmium 114 & $<6.70 \mathrm{E}-4$ & ug/g dry & $6.70 \mathrm{E}-4$ & 7/01/08 & 8G01004 & PNNL-AGG-415 \\
\hline $14265-72-6$ & Antimony 121 & 2.29E-3 & ug/g dry & 5.79E-4 & $7 / 01 / 08$ & 8G01004 & PNNL-AGG-415 \\
\hline $13966-27-3$ & Lead 206 & 3.37E-3 & ug/g dry & 7.72E-4 & $7 / 01 / 08$ & 8G01004 & PNNL-AGG-415 \\
\hline 13966-28-4 & Lead 208 & $3.26 \mathrm{E}-3$ & ug/g dry & $6.00 \mathrm{E}-4$ & $7 / 01 / 08$ & 8G01004 & PNNL-AGG-415 \\
\hline HEIS No. & B1VJ56B & \multicolumn{3}{|c|}{ Lab ID: $\quad$ 0805020-08 } & & & \\
\hline $14092-98-9$ & Chromium 52 & $6.05 \mathrm{E}-3$ & ug/g dry & $2.06 \mathrm{E}-3$ & $7 / 01 / 08$ & 8G01004 & PNNL-AGG-415 \\
\hline 13981-78-7 & Chromium 53 & $<6.41 \mathrm{E}-3$ & ug/g dry & $6.41 \mathrm{E}-3$ & $7 / 01 / 08$ & 8G01004 & PNNL-AGG-415 \\
\hline $14191-84-5$ & Copper 63 & 2.09E-2 & ug/g dry & $3.49 \mathrm{E}-3$ & 7/01/08 & 8G01004 & PNNL-AGG-415 \\
\hline $14119-06-3$ & Copper 65 & $1.90 \mathrm{E}-2$ & ug/g dry & $4.85 \mathrm{E}-3$ & 7/01/08 & 8G01004 & PNNL-AGG-415 \\
\hline $14687-58-2$ & Selenium 82 & $<1.11 \mathrm{E}-2$ & ug/g dry & $1.11 \mathrm{E}-2$ & 7/01/08 & 8G01004 & PNNL-AGG-415 \\
\hline $14392-17-7$ & Molybdenum 95 & $1.81 \mathrm{E}-1$ & ug/g dry & $1.61 \mathrm{E}-3$ & 7/01/08 & 8G01004 & PNNL-AGG-415 \\
\hline 14392-19-9 & Molybdenum 97 & $1.80 \mathrm{E}-1$ & ug/g dry & $2.05 \mathrm{E}-3$ & $7 / 01 / 08$ & 8G01004 & PNNL-AGG-415 \\
\hline $14392-20-2$ & Molybdenum 98 & $1.82 \mathrm{E}-1$ & ug/g dry & $1.61 \mathrm{E}-3$ & 7/01/08 & 8G01004 & PNNL-AGG-415 \\
\hline $14914-61-5$ & Ruthenium 101 & $<8.21 \mathrm{E}-4$ & ug/g dry & $8.21 \mathrm{E}-4$ & $7 / 01 / 08$ & 8G01004 & PNNL-AGG-415 \\
\hline $14914-62-6$ & Ruthenium 102 & $<5.66 \mathrm{E}-4$ & ug/g dry & $5.66 \mathrm{E}-4$ & $7 / 01 / 08$ & 8G01004 & PNNL-AGG-415 \\
\hline $15766-01-5$ & Ruthenium 104 & $<3.66 \mathrm{E}-4$ & ug/g dry & $3.66 \mathrm{E}-4$ & $7 / 01 / 08$ & 8G01004 & PNNL-AGG-415 \\
\hline $14378-37-1$ & Silver 107 & $<9.26 \mathrm{E}-4$ & ug/g dry & $9.26 \mathrm{E}-4$ & 7/01/08 & 8G01004 & PNNL-AGG-415 \\
\hline $14378-38-2$ & Silver 109 & $<1.07 \mathrm{E}-3$ & ug/g dry & $1.07 \mathrm{E}-3$ & $7 / 01 / 08$ & 8G01004 & PNNL-AGG-415 \\
\hline 14336-64-2 & Cadmium 111 & $6.45 \mathrm{E}-4$ & ug/g dry & 2.95E-4 & 7/01/08 & 8G01004 & PNNL-AGG-415 \\
\hline $14041-58-8$ & Cadmium 114 & $<6.26 \mathrm{E}-4$ & ug/g dry & $6.26 \mathrm{E}-4$ & 7/01/08 & 8G01004 & PNNL-AGG-415 \\
\hline $14265-72-6$ & Antimony 121 & 2.29E-3 & ug/g dry & $5.41 \mathrm{E}-4$ & 7/01/08 & 8G01004 & PNNL-AGG-415 \\
\hline 13966-27-3 & Lead 206 & $1.89 \mathrm{E}-3$ & ug/g dry & $7.21 \mathrm{E}-4$ & $7 / 01 / 08$ & 8G01004 & PNNL-AGG-415 \\
\hline 13966-28-4 & Lead 208 & $1.90 \mathrm{E}-3$ & ug/g dry & $5.61 \mathrm{E}-4$ & $7 / 01 / 08$ & 8G01004 & PNNL-AGG-415 \\
\hline HEIS No. & B1VJ56C & \multicolumn{3}{|c|}{$\begin{array}{ll}\text { Lab ID: } & \text { 0805020-09 }\end{array}$} & & & \\
\hline 14092-98-9 & Chromium 52 & $6.06 \mathrm{E}-3$ & ug/g dry & $2.06 \mathrm{E}-3$ & $7 / 01 / 08$ & 8G01004 & PNNL-AGG-415 \\
\hline 13981-78-7 & Chromium 53 & $<6.40 \mathrm{E}-3$ & ug/g dry & $6.40 \mathrm{E}-3$ & $7 / 01 / 08$ & 8G01004 & PNNL-AGG-415 \\
\hline 14191-84-5 & Copper 63 & 3.39E-2 & ug/g dry & $3.48 \mathrm{E}-3$ & $7 / 01 / 08$ & 8G01004 & PNNL-AGG-415 \\
\hline 14119-06-3 & Copper 65 & 3.24E-2 & ug/g dry & $4.84 \mathrm{E}-3$ & $7 / 01 / 08$ & 8G01004 & PNNL-AGG-415 \\
\hline $14687-58-2$ & Selenium 82 & $<1.11 \mathrm{E}-2$ & ug/g dry & $1.11 \mathrm{E}-2$ & $7 / 01 / 08$ & 8G01004 & PNNL-AGG-415 \\
\hline 14392-17-7 & Molybdenum 95 & 1.03E-1 & ug/g dry & $1.61 \mathrm{E}-3$ & $7 / 01 / 08$ & 8G01004 & PNNL-AGG-415 \\
\hline 14392-19-9 & Molybdenum 97 & $1.03 \mathrm{E}-1$ & ug/g dry & $2.05 \mathrm{E}-3$ & 7/01/08 & 8G01004 & PNNL-AGG-415 \\
\hline $14392-20-2$ & Molybdenum 98 & $1.04 \mathrm{E}-1$ & ug/g dry & $1.61 \mathrm{E}-3$ & 7/01/08 & 8G01004 & PNNL-AGG-415 \\
\hline $14914-61-5$ & Ruthenium 101 & $<8.20 \mathrm{E}-4$ & ug/g dry & $8.20 \mathrm{E}-4$ & 7/01/08 & 8G01004 & PNNL-AGG-415 \\
\hline $14914-62-6$ & Ruthenium 102 & $<5.65 \mathrm{E}-4$ & ug/g dry & $5.65 \mathrm{E}-4$ & 7/01/08 & 8G01004 & PNNL-AGG-415 \\
\hline 15766-01-5 & Ruthenium 104 & $<3.65 E-4$ & ug/g dry & $3.65 \mathrm{E}-4$ & $7 / 01 / 08$ & 8G01004 & PNNL-AGG-415 \\
\hline $14378-37-1$ & Silver 107 & $<9.25 \mathrm{E}-4$ & ug/g dry & $9.25 \mathrm{E}-4$ & 7/01/08 & 8G01004 & PNNL-AGG-415 \\
\hline $14378-38-2$ & Silver 109 & $<1.07 \mathrm{E}-3$ & ug/g dry & $1.07 \mathrm{E}-3$ & $7 / 01 / 08$ & 8G01004 & PNNL-AGG-415 \\
\hline $14336-64-2$ & Cadmium 111 & $3.98 \mathrm{E}-4$ & ug/g dry & $2.95 \mathrm{E}-4$ & 7/01/08 & 8G01004 & PNNL-AGG-415 \\
\hline $14041-58-8$ & Cadmium 114 & $<6.25 \mathrm{E}-4$ & ug/g dry & $6.25 \mathrm{E}-4$ & 7/01/08 & 8G01004 & PNNL-AGG-415 \\
\hline $14265-72-6$ & Antimony 121 & 2.09E-3 & ug/g dry & $5.40 \mathrm{E}-4$ & 7/01/08 & 8G01004 & PNNL-AGG-415 \\
\hline $13966-27-3$ & Lead 206 & $1.76 \mathrm{E}-3$ & ug/g dry & $7.20 \mathrm{E}-4$ & $7 / 01 / 08$ & 8G01004 & PNNL-AGG-415 \\
\hline 13966-28-4 & Lead 208 & $1.76 \mathrm{E}-3$ & ug/g dry & $5.60 \mathrm{E}-4$ & 7/01/08 & 8G01004 & PNNL-AGG-415 \\
\hline HEIS No. & B1VJ58C & & b ID: & 55020-10 & & & \\
\hline $14092-98-9$ & Chromium 52 & $8.10 \mathrm{E}-3$ & ug/g dry & 2.06E-3 & 7/01/08 & 8G01004 & PNNL-AGG-415 \\
\hline
\end{tabular}


RCRA Metals By PNNL-AGG-415/Water Extract

\begin{tabular}{|c|c|c|c|c|c|c|c|}
\hline CAS \# & Analyte & Results & Units & EQL & Analyzed & Batch & Method \\
\hline HEIS No. & B1VJ58C & \multicolumn{3}{|c|}{ Lab ID: $\quad 0805020-10$} & & & \\
\hline 13981-78-7 & Chromium 53 & 7.71E-3 & ug/g dry & $6.41 \mathrm{E}-3$ & 7/01/08 & 8G01004 & PNNL-AGG-415 \\
\hline 14191-84-5 & Copper 63 & $1.39 \mathrm{E}-1$ & ug/g dry & $3.48 \mathrm{E}-3$ & 7/01/08 & 8G01004 & PNNL-AGG-415 \\
\hline 14119-06-3 & Copper 65 & $1.34 \mathrm{E}-1$ & ug/g dry & $4.84 \mathrm{E}-3$ & $7 / 01 / 08$ & 8G01004 & PNNL-AGG-415 \\
\hline $14687-58-2$ & Selenium 82 & $<1.11 \mathrm{E}-2$ & ug/g dry & $1.11 \mathrm{E}-2$ & 7/01/08 & 8G01004 & PNNL-AGG-415 \\
\hline 14392-17-7 & Molybdenum 95 & 1.94E-1 & ug/g dry & $1.61 \mathrm{E}-3$ & $7 / 01 / 08$ & 8G01004 & PNNL-AGG-415 \\
\hline 14392-19-9 & Molybdenum 97 & $1.91 \mathrm{E}-1$ & ug/g dry & $2.05 \mathrm{E}-3$ & 7/01/08 & 8G01004 & PNNL-AGG-415 \\
\hline 14392-20-2 & Molybdenum 98 & $1.91 \mathrm{E}-1$ & ug/g dry & $1.61 \mathrm{E}-3$ & $7 / 01 / 08$ & 8G01004 & PNNL-AGG-415 \\
\hline 14914-61-5 & Ruthenium 101 & $8.48 \mathrm{E}-4$ & ug/g dry & $8.21 \mathrm{E}-4$ & 7/01/08 & 8G01004 & PNNL-AGG-415 \\
\hline $14914-62-6$ & Ruthenium 102 & $<5.66 \mathrm{E}-4$ & ug/g dry & $5.66 \mathrm{E}-4$ & 7/01/08 & 8G01004 & PNNL-AGG-415 \\
\hline $15766-01-5$ & Ruthenium 104 & $<3.65 \mathrm{E}-4$ & ug/g dry & $3.65 \mathrm{E}-4$ & 7/01/08 & 8G01004 & PNNL-AGG-415 \\
\hline 14378-37-1 & Silver 107 & 2.52E-3 & ug/g dry & $9.26 \mathrm{E}-4$ & $7 / 01 / 08$ & 8G01004 & PNNL-AGG-415 \\
\hline $14378-38-2$ & Silver 109 & $<1.07 \mathrm{E}-3$ & ug/g dry & $1.07 \mathrm{E}-3$ & 7/01/08 & 8G01004 & PNNL-AGG-415 \\
\hline 14336-64-2 & Cadmium 111 & 7.61E-4 & ug/g dry & 2.95E-4 & 7/01/08 & 8G01004 & PNNL-AGG-415 \\
\hline 14041-58-8 & Cadmium 114 & $<6.26 \mathrm{E}-4$ & ug/g dry & $6.26 \mathrm{E}-4$ & $7 / 01 / 08$ & 8G01004 & PNNL-AGG-415 \\
\hline $14265-72-6$ & Antimony 121 & 2.29E-3 & ug/g dry & $5.41 \mathrm{E}-4$ & $7 / 01 / 08$ & 8G01004 & PNNL-AGG-415 \\
\hline 13966-27-3 & Lead 206 & $2.47 \mathrm{E}-3$ & ug/g dry & $7.21 \mathrm{E}-4$ & $7 / 01 / 08$ & 8G01004 & PNNL-AGG-415 \\
\hline 13966-28-4 & Lead 208 & $2.40 \mathrm{E}-3$ & ug/g dry & $5.61 \mathrm{E}-4$ & $7 / 01 / 08$ & 8G01004 & PNNL-AGG-415 \\
\hline HEIS No. & B1VJ58B & \multicolumn{3}{|c|}{ Lab ID: $\quad$ 0805020-11 } & & & \\
\hline 14092-98-9 & Chromium 52 & $1.23 \mathrm{E}-2$ & ug/g dry & $2.07 \mathrm{E}-3$ & $7 / 01 / 08$ & 8G01004 & PNNL-AGG-415 \\
\hline 13981-78-7 & Chromium 53 & $1.22 \mathrm{E}-2$ & ug/g dry & $6.44 \mathrm{E}-3$ & $7 / 01 / 08$ & 8G01004 & PNNL-AGG-415 \\
\hline $14191-84-5$ & Copper 63 & $1.25 \mathrm{E}-1$ & ug/g dry & $3.50 \mathrm{E}-3$ & 7/01/08 & 8G01004 & PNNL-AGG-415 \\
\hline 14119-06-3 & Copper 65 & 1.19E-1 & ug/g dry & $4.87 \mathrm{E}-3$ & $7 / 01 / 08$ & 8G01004 & PNNL-AGG-415 \\
\hline $14687-58-2$ & Selenium 82 & $<1.11 \mathrm{E}-2$ & ug/g dry & $1.11 \mathrm{E}-2$ & $7 / 01 / 08$ & 8G01004 & PNNL-AGG-415 \\
\hline $14392-17-7$ & Molybdenum 95 & $6.55 \mathrm{E}-2$ & ug/g dry & $1.62 \mathrm{E}-3$ & $7 / 01 / 08$ & 8G01004 & PNNL-AGG-415 \\
\hline $14392-19-9$ & Molybdenum 97 & $6.21 \mathrm{E}-2$ & ug/g dry & 2.06E-3 & $7 / 01 / 08$ & 8G01004 & PNNL-AGG-415 \\
\hline $14392-20-2$ & Molybdenum 98 & $6.14 \mathrm{E}-2$ & ug/g dry & $1.62 \mathrm{E}-3$ & $7 / 01 / 08$ & 8G01004 & PNNL-AGG-415 \\
\hline $14914-61-5$ & Ruthenium 101 & 1.03E-3 & ug/g dry & $8.25 \mathrm{E}-4$ & $7 / 01 / 08$ & 8G01004 & PNNL-AGG-415 \\
\hline 14914-62-6 & Ruthenium 102 & $<5.69 \mathrm{E}-4$ & ug/g dry & $5.69 \mathrm{E}-4$ & $7 / 01 / 08$ & 8G01004 & PNNL-AGG-415 \\
\hline 15766-01-5 & Ruthenium 104 & $<3.67 \mathrm{E}-4$ & ug/g dry & $3.67 \mathrm{E}-4$ & 7/01/08 & 8G01004 & PNNL-AGG-415 \\
\hline 14378-37-1 & Silver 107 & 1.77E-3 & ug/g dry & $9.31 \mathrm{E}-4$ & $7 / 01 / 08$ & 8G01004 & PNNL-AGG-415 \\
\hline 14378-38-2 & Silver 109 & $<1.08 \mathrm{E}-3$ & ug/g dry & $1.08 \mathrm{E}-3$ & $7 / 01 / 08$ & 8G01004 & PNNL-AGG-415 \\
\hline $14336-64-2$ & Cadmium 111 & 4.68E-4 & ug/g dry & $2.97 \mathrm{E}-4$ & 7/01/08 & 8G01004 & PNNL-AGG-415 \\
\hline 14041-58-8 & Cadmium 114 & $<6.29 \mathrm{E}-4$ & ug/g dry & $6.29 \mathrm{E}-4$ & $7 / 01 / 08$ & 8G01004 & PNNL-AGG-415 \\
\hline $14265-72-6$ & Antimony 121 & $1.76 \mathrm{E}-3$ & ug/g dry & $5.43 \mathrm{E}-4$ & $7 / 01 / 08$ & 8G01004 & PNNL-AGG-415 \\
\hline 13966-27-3 & Lead 206 & $3.85 \mathrm{E}-3$ & ug/g dry & 7.25E-4 & 7/01/08 & 8G01004 & PNNL-AGG-415 \\
\hline 13966-28-4 & Lead 208 & $3.84 \mathrm{E}-3$ & ug/g dry & $5.64 \mathrm{E}-4$ & 7/01/08 & 8G01004 & PNNL-AGG-415 \\
\hline HEIS No. & B1VJ58A & \multicolumn{3}{|c|}{ Lab ID: $\quad$ 0805020-12 } & & & \\
\hline 14092-98-9 & Chromium 52 & 1.63E-2 & ug/g dry & $2.05 \mathrm{E}-3$ & $7 / 01 / 08$ & 8G01004 & PNNL-AGG-415 \\
\hline 13981-78-7 & Chromium 53 & $1.63 \mathrm{E}-2$ & ug/g dry & $6.40 \mathrm{E}-3$ & $7 / 01 / 08$ & 8G01004 & PNNL-AGG-415 \\
\hline $14191-84-5$ & Copper 63 & $1.24 \mathrm{E}-1$ & ug/g dry & $3.48 \mathrm{E}-3$ & $7 / 01 / 08$ & 8G01004 & PNNL-AGG-415 \\
\hline 14119-06-3 & Copper 65 & $1.20 \mathrm{E}-1$ & ug/g dry & $4.84 \mathrm{E}-3$ & $7 / 01 / 08$ & 8G01004 & PNNL-AGG-415 \\
\hline $14687-58-2$ & Selenium 82 & $<1.10 \mathrm{E}-2$ & ug/g dry & $1.10 \mathrm{E}-2$ & $7 / 01 / 08$ & 8G01004 & PNNL-AGG-415 \\
\hline 14392-17-7 & Molybdenum 95 & 2.30E-2 & ug/g dry & $1.60 \mathrm{E}-3$ & $7 / 01 / 08$ & 8G01004 & PNNL-AGG-415 \\
\hline $14392-19-9$ & Molybdenum 97 & $2.02 \mathrm{E}-2$ & ug/g dry & $2.04 \mathrm{E}-3$ & $7 / 01 / 08$ & 8G01004 & PNNL-AGG-415 \\
\hline $14392-20-2$ & Molybdenum 98 & $1.90 \mathrm{E}-2$ & ug/g dry & $1.60 \mathrm{E}-3$ & $7 / 01 / 08$ & 8G01004 & PNNL-AGG-415 \\
\hline 14914-61-5 & Ruthenium 101 & $9.94 \mathrm{E}-4$ & ug/g dry & $8.20 \mathrm{E}-4$ & $7 / 01 / 08$ & 8G01004 & PNNL-AGG-415 \\
\hline $14914-62-6$ & Ruthenium 102 & $<5.65 \mathrm{E}-4$ & ug/g dry & $5.65 \mathrm{E}-4$ & 7/01/08 & 8G01004 & PNNL-AGG-415 \\
\hline $15766-01-5$ & Ruthenium 104 & $<3.65 \mathrm{E}-4$ & ug/g dry & 3.65E-4 & 7/01/08 & 8G01004 & PNNL-AGG-415 \\
\hline
\end{tabular}


RCRA Metals By PNNL-AGG-415/Water Extract

\begin{tabular}{|c|c|c|c|c|c|c|c|}
\hline CAS \# & Analyte & Results & Units & EQL & Analyzed & Batch & Method \\
\hline HEIS No. & B1VJ58A & \multicolumn{3}{|c|}{ Lab ID: $\quad 0805020-12$} & & & \\
\hline 14378-37-1 & Silver 107 & $1.58 \mathrm{E}-3$ & ug/g dry & $9.24 \mathrm{E}-4$ & 7/01/08 & 8G01004 & PNNL-AGG-415 \\
\hline 14378-38-2 & Silver 109 & $<1.07 \mathrm{E}-3$ & ug/g dry & $1.07 \mathrm{E}-3$ & $7 / 01 / 08$ & 8G01004 & PNNL-AGG-415 \\
\hline $14336-64-2$ & Cadmium 111 & $<2.95 \mathrm{E}-4$ & ug/g dry & 2.95E-4 & $7 / 01 / 08$ & 8G01004 & PNNL-AGG-415 \\
\hline $14041-58-8$ & Cadmium 114 & $<6.25 \mathrm{E}-4$ & ug/g dry & $6.25 \mathrm{E}-4$ & $7 / 01 / 08$ & 8G01004 & PNNL-AGG-415 \\
\hline $14265-72-6$ & Antimony 121 & 1.17E-3 & ug/g dry & $5.40 \mathrm{E}-4$ & 7/01/08 & 8G01004 & PNNL-AGG-415 \\
\hline 13966-27-3 & Lead 206 & 2.43E-3 & ug/g dry & $7.20 \mathrm{E}-4$ & $7 / 01 / 08$ & 8G01004 & PNNL-AGG-415 \\
\hline 13966-28-4 & Lead 208 & 2.37E-3 & ug/g dry & $5.60 \mathrm{E}-4$ & $7 / 01 / 08$ & 8G01004 & PNNL-AGG-415 \\
\hline HEIS No. & B1VJ59C & \multicolumn{3}{|c|}{ Lab ID: $\quad 0805020-14$} & & & \\
\hline 14092-98-9 & Chromium 52 & $<2.06 \mathrm{E}-3$ & ug/g dry & 2.06E-3 & $7 / 01 / 08$ & 8G01004 & PNNL-AGG-415 \\
\hline 13981-78-7 & Chromium 53 & $<6.40 \mathrm{E}-3$ & ug/g dry & $6.40 \mathrm{E}-3$ & $7 / 01 / 08$ & 8G01004 & PNNL-AGG-415 \\
\hline $14191-84-5$ & Copper 63 & 8.71E-3 & ug/g dry & $3.48 \mathrm{E}-3$ & $7 / 01 / 08$ & 8G01004 & PNNL-AGG-415 \\
\hline 14119-06-3 & Copper 65 & 8.69E-3 & ug/g dry & $4.84 \mathrm{E}-3$ & 7/01/08 & 8G01004 & PNNL-AGG-415 \\
\hline 14687-58-2 & Selenium 82 & $<1.11 \mathrm{E}-2$ & ug/g dry & $1.11 \mathrm{E}-2$ & 7/01/08 & 8G01004 & PNNL-AGG-415 \\
\hline $14392-17-7$ & Molybdenum 95 & $1.07 \mathrm{E}-1$ & ug/g dry & $1.61 \mathrm{E}-3$ & 7/01/08 & 8G01004 & PNNL-AGG-415 \\
\hline 14392-19-9 & Molybdenum 97 & $1.06 \mathrm{E}-1$ & ug/g dry & $2.05 \mathrm{E}-3$ & 7/01/08 & 8G01004 & PNNL-AGG-415 \\
\hline $14392-20-2$ & Molybdenum 98 & $1.07 \mathrm{E}-1$ & ug/g dry & $1.61 \mathrm{E}-3$ & $7 / 01 / 08$ & 8G01004 & PNNL-AGG-415 \\
\hline $14914-61-5$ & Ruthenium 101 & $<8.20 \mathrm{E}-4$ & ug/g dry & $8.20 \mathrm{E}-4$ & 7/01/08 & 8G01004 & PNNL-AGG-415 \\
\hline $14914-62-6$ & Ruthenium 102 & $<5.65 \mathrm{E}-4$ & ug/g dry & $5.65 \mathrm{E}-4$ & $7 / 01 / 08$ & 8G01004 & PNNL-AGG-415 \\
\hline 15766-01-5 & Ruthenium 104 & $<3.65 \mathrm{E}-4$ & ug/g dry & 3.65E-4 & $7 / 01 / 08$ & 8G01004 & PNNL-AGG-415 \\
\hline 14378-37-1 & Silver 107 & $<9.25 \mathrm{E}-4$ & ug/g dry & $9.25 \mathrm{E}-4$ & $7 / 01 / 08$ & 8G01004 & PNNL-AGG-415 \\
\hline 14378-38-2 & Silver 109 & $<1.07 \mathrm{E}-3$ & ug/g dry & $1.07 \mathrm{E}-3$ & 7/01/08 & 8G01004 & PNNL-AGG-415 \\
\hline $14336-64-2$ & Cadmium 111 & $3.78 \mathrm{E}-4$ & ug/g dry & 2.95E-4 & $7 / 01 / 08$ & 8G01004 & PNNL-AGG-415 \\
\hline 14041-58-8 & Cadmium 114 & $<6.25 \mathrm{E}-4$ & ug/g dry & $6.25 \mathrm{E}-4$ & 7/01/08 & 8G01004 & PNNL-AGG-415 \\
\hline $14265-72-6$ & Antimony 121 & $1.08 \mathrm{E}-3$ & ug/g dry & $5.40 \mathrm{E}-4$ & 7/01/08 & 8G01004 & PNNL-AGG-415 \\
\hline $13966-27-3$ & Lead 206 & $<7.20 \mathrm{E}-4$ & ug/g dry & $7.20 \mathrm{E}-4$ & $7 / 01 / 08$ & 8G01004 & PNNL-AGG-415 \\
\hline 13966-28-4 & Lead 208 & $<5.60 \mathrm{E}-4$ & ug/g dry & $5.60 \mathrm{E}-4$ & $7 / 01 / 08$ & 8G01004 & PNNL-AGG-415 \\
\hline HEIS No. & B1VJ59B & \multicolumn{3}{|c|}{$\begin{array}{ll}\text { Lab ID: } & \text { 0805020-15 }\end{array}$} & & & \\
\hline 14092-98-9 & Chromium 52 & $<2.06 \mathrm{E}-3$ & ug/g dry & $2.06 \mathrm{E}-3$ & 7/01/08 & 8G01004 & PNNL-AGG-415 \\
\hline 13981-78-7 & Chromium 53 & $<6.40 \mathrm{E}-3$ & ug/g dry & $6.40 \mathrm{E}-3$ & 7/01/08 & 8G01004 & PNNL-AGG-415 \\
\hline 14191-84-5 & Copper 63 & $<3.48 \mathrm{E}-3$ & ug/g dry & $3.48 \mathrm{E}-3$ & $7 / 01 / 08$ & 8G01004 & PNNL-AGG-415 \\
\hline 14119-06-3 & Copper 65 & $<4.84 \mathrm{E}-3$ & ug/g dry & $4.84 \mathrm{E}-3$ & $7 / 01 / 08$ & 8G01004 & PNNL-AGG-415 \\
\hline $14687-58-2$ & Selenium 82 & $<1.11 \mathrm{E}-2$ & ug/g dry & $1.11 \mathrm{E}-2$ & $7 / 01 / 08$ & 8G01004 & PNNL-AGG-415 \\
\hline 14392-17-7 & Molybdenum 95 & 4.33E-2 & ug/g dry & $1.61 \mathrm{E}-3$ & $7 / 01 / 08$ & 8G01004 & PNNL-AGG-415 \\
\hline 14392-19-9 & Molybdenum 97 & $4.31 \mathrm{E}-2$ & ug/g dry & 2.05E-3 & $7 / 01 / 08$ & 8G01004 & PNNL-AGG-415 \\
\hline 14392-20-2 & Molybdenum 98 & 4.27E-2 & ug/g dry & $1.61 \mathrm{E}-3$ & 7/01/08 & 8G01004 & PNNL-AGG-415 \\
\hline $14914-61-5$ & Ruthenium 101 & $<8.21 \mathrm{E}-4$ & ug/g dry & $8.21 \mathrm{E}-4$ & 7/01/08 & 8G01004 & PNNL-AGG-415 \\
\hline $14914-62-6$ & Ruthenium 102 & $<5.65 \mathrm{E}-4$ & ug/g dry & 5.65E-4 & 7/01/08 & 8G01004 & PNNL-AGG-415 \\
\hline $15766-01-5$ & Ruthenium 104 & $<3.65 \mathrm{E}-4$ & ug/g dry & $3.65 \mathrm{E}-4$ & 7/01/08 & 8G01004 & PNNL-AGG-415 \\
\hline 14378-37-1 & Silver 107 & $<9.26 \mathrm{E}-4$ & ug/g dry & $9.26 \mathrm{E}-4$ & 7/01/08 & 8G01004 & PNNL-AGG-415 \\
\hline 14378-38-2 & Silver 109 & $<1.07 \mathrm{E}-3$ & ug/g dry & $1.07 \mathrm{E}-3$ & 7/01/08 & 8G01004 & PNNL-AGG-415 \\
\hline 14336-64-2 & Cadmium 111 & $<2.95 \mathrm{E}-4$ & ug/g dry & 2.95E-4 & $7 / 01 / 08$ & 8G01004 & PNNL-AGG-415 \\
\hline $14041-58-8$ & Cadmium 114 & $<6.25 \mathrm{E}-4$ & ug/g dry & $6.25 \mathrm{E}-4$ & 7/01/08 & 8G01004 & PNNL-AGG-415 \\
\hline $14265-72-6$ & Antimony 121 & $<5.40 \mathrm{E}-4$ & ug/g dry & $5.40 \mathrm{E}-4$ & 7/01/08 & 8G01004 & PNNL-AGG-415 \\
\hline $13966-27-3$ & Lead 206 & $<7.20 \mathrm{E}-4$ & ug/g dry & $7.20 \mathrm{E}-4$ & 7/01/08 & 8G01004 & PNNL-AGG-415 \\
\hline 13966-28-4 & Lead 208 & $<5.60 \mathrm{E}-4$ & ug/g dry & $5.60 \mathrm{E}-4$ & $7 / 01 / 08$ & 8G01004 & PNNL-AGG-415 \\
\hline HEIS No. & B1VJ59A & \multicolumn{3}{|c|}{ Lab ID: $\quad$ 0805020-16 } & & & \\
\hline $14092-98-9$ & Chromium 52 & $<2.07 \mathrm{E}-3$ & ug/g dry & 2.07E-3 & $7 / 01 / 08$ & 8G01004 & PNNL-AGG-415 \\
\hline 13981-78-7 & Chromium 53 & $<6.45 \mathrm{E}-3$ & ug/g dry & $6.45 \mathrm{E}-3$ & 7/01/08 & 8G01004 & PNNL-AGG-415 \\
\hline
\end{tabular}


RCRA Metals By PNNL-AGG-415/Water Extract

\begin{tabular}{|c|c|c|c|c|c|c|c|}
\hline CAS \# & Analyte & Results & Units & EQL & Analyzed & Batch & Method \\
\hline HEIS No. & B1VJ59A & \multicolumn{3}{|c|}{ Lab ID: $\quad$ 0805020-16 } & & & \\
\hline 14191-84-5 & Copper 63 & $<3.51 \mathrm{E}-3$ & ug/g dry & $3.51 \mathrm{E}-3$ & 7/01/08 & 8G01004 & PNNL-AGG-415 \\
\hline 14119-06-3 & Copper 65 & $<4.88 \mathrm{E}-3$ & ug/g dry & $4.88 \mathrm{E}-3$ & $7 / 01 / 08$ & 8G01004 & PNNL-AGG-415 \\
\hline $14687-58-2$ & Selenium 82 & $<1.11 \mathrm{E}-2$ & ug/g dry & $1.11 \mathrm{E}-2$ & $7 / 01 / 08$ & 8G01004 & PNNL-AGG-415 \\
\hline $14392-17-7$ & Molybdenum 95 & 3.54E-2 & ug/g dry & $1.62 \mathrm{E}-3$ & $7 / 01 / 08$ & 8G01004 & PNNL-AGG-415 \\
\hline 14392-19-9 & Molybdenum 97 & $3.52 \mathrm{E}-2$ & ug/g dry & $2.06 \mathrm{E}-3$ & 7/01/08 & 8G01004 & PNNL-AGG-415 \\
\hline $14392-20-2$ & Molybdenum 98 & 3.53E-2 & ug/g dry & $1.62 \mathrm{E}-3$ & $7 / 01 / 08$ & 8G01004 & PNNL-AGG-415 \\
\hline $14914-61-5$ & Ruthenium 101 & $<8.26 \mathrm{E}-4$ & ug/g dry & 8.26E-4 & $7 / 01 / 08$ & 8G01004 & PNNL-AGG-415 \\
\hline $14914-62-6$ & Ruthenium 102 & $<5.69 \mathrm{E}-4$ & ug/g dry & $5.69 \mathrm{E}-4$ & $7 / 01 / 08$ & 8G01004 & PNNL-AGG-415 \\
\hline 15766-01-5 & Ruthenium 104 & $<3.68 \mathrm{E}-4$ & ug/g dry & $3.68 \mathrm{E}-4$ & 7/01/08 & 8G01004 & PNNL-AGG-415 \\
\hline $14378-37-1$ & Silver 107 & $<9.32 \mathrm{E}-4$ & ug/g dry & $9.32 \mathrm{E}-4$ & $7 / 01 / 08$ & 8G01004 & PNNL-AGG-415 \\
\hline $14378-38-2$ & Silver 109 & $<1.08 \mathrm{E}-3$ & ug/g dry & $1.08 \mathrm{E}-3$ & $7 / 01 / 08$ & 8G01004 & PNNL-AGG-415 \\
\hline $14336-64-2$ & Cadmium 111 & $<2.97 \mathrm{E}-4$ & ug/g dry & $2.97 \mathrm{E}-4$ & 7/01/08 & 8G01004 & PNNL-AGG-415 \\
\hline 14041-58-8 & Cadmium 114 & $<6.30 \mathrm{E}-4$ & ug/g dry & $6.30 \mathrm{E}-4$ & 7/01/08 & 8G01004 & PNNL-AGG-415 \\
\hline $14265-72-6$ & Antimony 121 & $<5.44 \mathrm{E}-4$ & ug/g dry & $5.44 \mathrm{E}-4$ & $7 / 01 / 08$ & 8G01004 & PNNL-AGG-415 \\
\hline 13966-27-3 & Lead 206 & $<7.25 \mathrm{E}-4$ & ug/g dry & 7.25E-4 & $7 / 01 / 08$ & 8G01004 & PNNL-AGG-415 \\
\hline 13966-28-4 & Lead 208 & $<5.64 \mathrm{E}-4$ & ug/g dry & $5.64 \mathrm{E}-4$ & 7/01/08 & 8G01004 & PNNL-AGG-415 \\
\hline HEIS No. & B1VJ60C & \multicolumn{3}{|c|}{$\begin{array}{ll}\text { Lab ID: } & 0805020-18\end{array}$} & & & \\
\hline 14092-98-9 & Chromium 52 & $<2.05 \mathrm{E}-3$ & ug/g dry & 2.05E-3 & $7 / 01 / 08$ & 8G01004 & PNNL-AGG-415 \\
\hline 13981-78-7 & Chromium 53 & $<6.40 \mathrm{E}-3$ & ug/g dry & $6.40 \mathrm{E}-3$ & $7 / 01 / 08$ & 8G01004 & PNNL-AGG-415 \\
\hline 14191-84-5 & Copper 63 & $1.08 \mathrm{E}-2$ & ug/g dry & $3.48 \mathrm{E}-3$ & 7/01/08 & 8G01004 & PNNL-AGG-415 \\
\hline 14119-06-3 & Copper 65 & $1.09 \mathrm{E}-2$ & ug/g dry & $4.84 \mathrm{E}-3$ & 7/01/08 & 8G01004 & PNNL-AGG-415 \\
\hline 14687-58-2 & Selenium 82 & $<1.10 \mathrm{E}-2$ & ug/g dry & $1.10 \mathrm{E}-2$ & 7/01/08 & 8G01004 & PNNL-AGG-415 \\
\hline 14392-17-7 & Molybdenum 95 & 2.22E-1 & ug/g dry & $1.60 \mathrm{E}-3$ & 7/01/08 & 8G01004 & PNNL-AGG-415 \\
\hline 14392-19-9 & Molybdenum 97 & $2.22 \mathrm{E}-1$ & ug/g dry & $2.04 \mathrm{E}-3$ & 7/01/08 & 8G01004 & PNNL-AGG-415 \\
\hline $14392-20-2$ & Molybdenum 98 & 2.23E-1 & ug/g dry & $1.60 \mathrm{E}-3$ & 7/01/08 & 8G01004 & PNNL-AGG-415 \\
\hline $14914-61-5$ & Ruthenium 101 & $<8.20 \mathrm{E}-4$ & ug/g dry & $8.20 \mathrm{E}-4$ & $7 / 01 / 08$ & 8G01004 & PNNL-AGG-415 \\
\hline 14914-62-6 & Ruthenium 102 & $<5.65 \mathrm{E}-4$ & ug/g dry & $5.65 \mathrm{E}-4$ & $7 / 01 / 08$ & 8G01004 & PNNL-AGG-415 \\
\hline 15766-01-5 & Ruthenium 104 & $<3.65 \mathrm{E}-4$ & ug/g dry & 3.65E-4 & 7/01/08 & 8G01004 & PNNL-AGG-415 \\
\hline 14378-37-1 & Silver 107 & $<9.25 \mathrm{E}-4$ & ug/g dry & $9.25 \mathrm{E}-4$ & 7/01/08 & 8G01004 & PNNL-AGG-415 \\
\hline 14378-38-2 & Silver 109 & $<1.07 \mathrm{E}-3$ & ug/g dry & $1.07 \mathrm{E}-3$ & $7 / 01 / 08$ & 8G01004 & PNNL-AGG-415 \\
\hline $14336-64-2$ & Cadmium 111 & 8.02E-4 & ug/g dry & 2.95E-4 & 7/01/08 & 8G01004 & PNNL-AGG-415 \\
\hline 14041-58-8 & Cadmium 114 & $<6.25 \mathrm{E}-4$ & ug/g dry & $6.25 \mathrm{E}-4$ & 7/01/08 & 8G01004 & PNNL-AGG-415 \\
\hline $14265-72-6$ & Antimony 121 & $1.25 \mathrm{E}-3$ & ug/g dry & $5.40 \mathrm{E}-4$ & 7/01/08 & 8G01004 & PNNL-AGG-415 \\
\hline 13966-27-3 & Lead 206 & $<7.20 \mathrm{E}-4$ & ug/g dry & $7.20 \mathrm{E}-4$ & 7/01/08 & 8G01004 & PNNL-AGG-415 \\
\hline 13966-28-4 & Lead 208 & $6.77 \mathrm{E}-4$ & ug/g dry & $5.60 \mathrm{E}-4$ & $7 / 01 / 08$ & 8G01004 & PNNL-AGG-415 \\
\hline HEIS No. & B1VJ60B & \multicolumn{3}{|c|}{$\begin{array}{ll}\text { Lab ID: } & 0805020-19\end{array}$} & & & \\
\hline 14092-98-9 & Chromium 52 & $<2.06 \mathrm{E}-3$ & ug/g dry & 2.06E-3 & $7 / 01 / 08$ & 8G01004 & PNNL-AGG-415 \\
\hline 13981-78-7 & Chromium 53 & $<6.40 \mathrm{E}-3$ & ug/g dry & $6.40 \mathrm{E}-3$ & $7 / 01 / 08$ & 8G01004 & PNNL-AGG-415 \\
\hline 14191-84-5 & Copper 63 & $<3.48 \mathrm{E}-3$ & ug/g dry & $3.48 \mathrm{E}-3$ & $7 / 01 / 08$ & 8G01004 & PNNL-AGG-415 \\
\hline 14119-06-3 & Copper 65 & $<4.84 \mathrm{E}-3$ & ug/g dry & $4.84 \mathrm{E}-3$ & 7/01/08 & 8G01004 & PNNL-AGG-415 \\
\hline 14687-58-2 & Selenium 82 & $<1.11 \mathrm{E}-2$ & ug/g dry & $1.11 \mathrm{E}-2$ & $7 / 01 / 08$ & 8G01004 & PNNL-AGG-415 \\
\hline $14392-17-7$ & Molybdenum 95 & 5.37E-2 & ug/g dry & $1.61 \mathrm{E}-3$ & $7 / 01 / 08$ & 8G01004 & PNNL-AGG-415 \\
\hline 14392-19-9 & Molybdenum 97 & $5.31 \mathrm{E}-2$ & ug/g dry & 2.05E-3 & 7/01/08 & 8G01004 & PNNL-AGG-415 \\
\hline $14392-20-2$ & Molybdenum 98 & $5.31 \mathrm{E}-2$ & ug/g dry & $1.61 \mathrm{E}-3$ & 7/01/08 & 8G01004 & PNNL-AGG-415 \\
\hline $14914-61-5$ & Ruthenium 101 & $<8.20 \mathrm{E}-4$ & ug/g dry & $8.20 \mathrm{E}-4$ & $7 / 01 / 08$ & 8G01004 & PNNL-AGG-415 \\
\hline $14914-62-6$ & Ruthenium 102 & $<5.65 \mathrm{E}-4$ & ug/g dry & $5.65 \mathrm{E}-4$ & 7/01/08 & 8G01004 & PNNL-AGG-415 \\
\hline $15766-01-5$ & Ruthenium 104 & $<3.65 \mathrm{E}-4$ & ug/g dry & 3.65E-4 & 7/01/08 & 8G01004 & PNNL-AGG-415 \\
\hline 14378-37-1 & Silver 107 & $<9.26 \mathrm{E}-4$ & ug/g dry & $9.26 \mathrm{E}-4$ & 7/01/08 & 8G01004 & PNNL-AGG-415 \\
\hline
\end{tabular}


RCRA Metals By PNNL-AGG-415/Water Extract

\begin{tabular}{|c|c|c|c|c|c|c|c|}
\hline CAS \# & Analyte & Results & Units & EQL & Analyzed & Batch & Method \\
\hline HEIS No. & B1VJ60B & \multicolumn{3}{|c|}{ Lab ID: $\quad 0805020-19$} & & & \\
\hline $14378-38-2$ & Silver 109 & $<1.07 \mathrm{E}-3$ & ug/g dry & $1.07 \mathrm{E}-3$ & 7/01/08 & 8G01004 & PNNL-AGG-415 \\
\hline 14336-64-2 & Cadmium 111 & $<2.95 \mathrm{E}-4$ & ug/g dry & 2.95E-4 & $7 / 01 / 08$ & 8G01004 & PNNL-AGG-415 \\
\hline $14041-58-8$ & Cadmium 114 & $<6.25 \mathrm{E}-4$ & ug/g dry & $6.25 \mathrm{E}-4$ & $7 / 01 / 08$ & 8G01004 & PNNL-AGG-415 \\
\hline $14265-72-6$ & Antimony 121 & $<5.40 \mathrm{E}-4$ & ug/g dry & $5.40 \mathrm{E}-4$ & $7 / 01 / 08$ & 8G01004 & PNNL-AGG-415 \\
\hline $13966-27-3$ & Lead 206 & $<7.20 \mathrm{E}-4$ & ug/g dry & $7.20 \mathrm{E}-4$ & 7/01/08 & 8G01004 & PNNL-AGG-415 \\
\hline 13966-28-4 & Lead 208 & $<5.60 \mathrm{E}-4$ & ug/g dry & $5.60 \mathrm{E}-4$ & $7 / 01 / 08$ & 8G01004 & PNNL-AGG-415 \\
\hline HEIS No. & B1VJ60A & \multicolumn{3}{|c|}{ Lab ID: $\quad$ 0805020-20 } & & & \\
\hline $14092-98-9$ & Chromium 52 & $<2.06 \mathrm{E}-3$ & ug/g dry & 2.06E-3 & $7 / 01 / 08$ & 8G01004 & PNNL-AGG-415 \\
\hline 13981-78-7 & Chromium 53 & $<6.40 \mathrm{E}-3$ & ug/g dry & $6.40 \mathrm{E}-3$ & 7/01/08 & 8G01004 & PNNL-AGG-415 \\
\hline 14191-84-5 & Copper 63 & $<3.48 \mathrm{E}-3$ & ug/g dry & $3.48 \mathrm{E}-3$ & $7 / 01 / 08$ & 8G01004 & PNNL-AGG-415 \\
\hline 14119-06-3 & Copper 65 & $<4.84 \mathrm{E}-3$ & ug/g dry & $4.84 \mathrm{E}-3$ & $7 / 01 / 08$ & 8G01004 & PNNL-AGG-415 \\
\hline $14687-58-2$ & Selenium 82 & $<1.11 \mathrm{E}-2$ & ug/g dry & $1.11 \mathrm{E}-2$ & 7/01/08 & 8G01004 & PNNL-AGG-415 \\
\hline $14392-17-7$ & Molybdenum 95 & 3.47E-2 & ug/g dry & $1.61 \mathrm{E}-3$ & 7/01/08 & 8G01004 & PNNL-AGG-415 \\
\hline 14392-19-9 & Molybdenum 97 & $3.44 \mathrm{E}-2$ & ug/g dry & 2.05E-3 & 7/01/08 & 8G01004 & PNNL-AGG-415 \\
\hline 14392-20-2 & Molybdenum 98 & 3.43E-2 & ug/g dry & $1.61 \mathrm{E}-3$ & 7/01/08 & 8G01004 & PNNL-AGG-415 \\
\hline $14914-61-5$ & Ruthenium 101 & $<8.20 \mathrm{E}-4$ & ug/g dry & $8.20 \mathrm{E}-4$ & $7 / 01 / 08$ & 8G01004 & PNNL-AGG-415 \\
\hline 14914-62-6 & Ruthenium 102 & $<5.65 \mathrm{E}-4$ & ug/g dry & $5.65 \mathrm{E}-4$ & 7/01/08 & 8G01004 & PNNL-AGG-415 \\
\hline 15766-01-5 & Ruthenium 104 & $<3.65 \mathrm{E}-4$ & ug/g dry & 3.65E-4 & $7 / 01 / 08$ & 8G01004 & PNNL-AGG-415 \\
\hline $14378-37-1$ & Silver 107 & $<9.25 \mathrm{E}-4$ & ug/g dry & $9.25 \mathrm{E}-4$ & $7 / 01 / 08$ & 8G01004 & PNNL-AGG-415 \\
\hline $14378-38-2$ & Silver 109 & $<1.07 \mathrm{E}-3$ & ug/g dry & $1.07 \mathrm{E}-3$ & $7 / 01 / 08$ & 8G01004 & PNNL-AGG-415 \\
\hline 14336-64-2 & Cadmium 111 & $<2.95 \mathrm{E}-4$ & ug/g dry & 2.95E-4 & 7/01/08 & 8G01004 & PNNL-AGG-415 \\
\hline $14041-58-8$ & Cadmium 114 & $<6.25 \mathrm{E}-4$ & ug/g dry & $6.25 \mathrm{E}-4$ & $7 / 01 / 08$ & 8G01004 & PNNL-AGG-415 \\
\hline $14265-72-6$ & Antimony 121 & $<5.40 \mathrm{E}-4$ & ug/g dry & $5.40 \mathrm{E}-4$ & 7/01/08 & 8G01004 & PNNL-AGG-415 \\
\hline $13966-27-3$ & Lead 206 & $<7.20 \mathrm{E}-4$ & ug/g dry & $7.20 \mathrm{E}-4$ & 7/01/08 & 8G01004 & PNNL-AGG-415 \\
\hline $13966-28-4$ & Lead 208 & $<5.60 \mathrm{E}-4$ & ug/g dry & $5.60 \mathrm{E}-4$ & $7 / 01 / 08$ & 8G01004 & PNNL-AGG-415 \\
\hline HEIS No. & B1VJ61C & \multicolumn{3}{|c|}{$\begin{array}{ll}\text { Lab ID: } & 0805020-22\end{array}$} & & & \\
\hline 14092-98-9 & Chromium 52 & $<2.05 \mathrm{E}-3$ & ug/g dry & 2.05E-3 & $7 / 01 / 08$ & 8G01004 & PNNL-AGG-415 \\
\hline 13981-78-7 & Chromium 53 & $<6.40 \mathrm{E}-3$ & ug/g dry & $6.40 \mathrm{E}-3$ & $7 / 01 / 08$ & 8G01004 & PNNL-AGG-415 \\
\hline $14191-84-5$ & Copper 63 & $<3.48 \mathrm{E}-3$ & ug/g dry & $3.48 \mathrm{E}-3$ & 7/01/08 & 8G01004 & PNNL-AGG-415 \\
\hline 14119-06-3 & Copper 65 & $<4.84 \mathrm{E}-3$ & ug/g dry & $4.84 \mathrm{E}-3$ & $7 / 01 / 08$ & 8G01004 & PNNL-AGG-415 \\
\hline $14687-58-2$ & Selenium 82 & $<1.10 \mathrm{E}-2$ & ug/g dry & $1.10 \mathrm{E}-2$ & $7 / 01 / 08$ & 8G01004 & PNNL-AGG-415 \\
\hline 14392-17-7 & Molybdenum 95 & $5.52 \mathrm{E}-2$ & ug/g dry & $1.60 \mathrm{E}-3$ & $7 / 01 / 08$ & 8G01004 & PNNL-AGG-415 \\
\hline 14392-19-9 & Molybdenum 97 & $5.49 \mathrm{E}-2$ & ug/g dry & $2.04 \mathrm{E}-3$ & $7 / 01 / 08$ & 8G01004 & PNNL-AGG-415 \\
\hline $14392-20-2$ & Molybdenum 98 & $5.52 \mathrm{E}-2$ & ug/g dry & $1.60 \mathrm{E}-3$ & $7 / 01 / 08$ & 8G01004 & PNNL-AGG-415 \\
\hline 14914-61-5 & Ruthenium 101 & $<8.20 \mathrm{E}-4$ & ug/g dry & $8.20 \mathrm{E}-4$ & 7/01/08 & 8G01004 & PNNL-AGG-415 \\
\hline $14914-62-6$ & Ruthenium 102 & $<5.65 \mathrm{E}-4$ & ug/g dry & $5.65 \mathrm{E}-4$ & 7/01/08 & 8G01004 & PNNL-AGG-415 \\
\hline 15766-01-5 & Ruthenium 104 & $<3.65 E-4$ & ug/g dry & 3.65E-4 & 7/01/08 & 8G01004 & PNNL-AGG-415 \\
\hline $14378-37-1$ & Silver 107 & $<9.25 \mathrm{E}-4$ & ug/g dry & $9.25 \mathrm{E}-4$ & 7/01/08 & 8G01004 & PNNL-AGG-415 \\
\hline $14378-38-2$ & Silver 109 & $<1.07 E-3$ & ug/g dry & $1.07 \mathrm{E}-3$ & $7 / 01 / 08$ & 8G01004 & PNNL-AGG-415 \\
\hline $14336-64-2$ & Cadmium 111 & $<2.95 \mathrm{E}-4$ & ug/g dry & 2.95E-4 & 7/01/08 & 8G01004 & PNNL-AGG-415 \\
\hline $14041-58-8$ & Cadmium 114 & $<6.25 \mathrm{E}-4$ & ug/g dry & $6.25 \mathrm{E}-4$ & $7 / 01 / 08$ & 8G01004 & PNNL-AGG-415 \\
\hline $14265-72-6$ & Antimony 121 & $5.84 \mathrm{E}-4$ & ug/g dry & $5.40 \mathrm{E}-4$ & 7/01/08 & 8G01004 & PNNL-AGG-415 \\
\hline $13966-27-3$ & Lead 206 & $<7.20 \mathrm{E}-4$ & ug/g dry & $7.20 \mathrm{E}-4$ & 7/01/08 & 8G01004 & PNNL-AGG-415 \\
\hline 13966-28-4 & Lead 208 & $<5.60 \mathrm{E}-4$ & ug/g dry & $5.60 \mathrm{E}-4$ & 7/01/08 & 8G01004 & PNNL-AGG-415 \\
\hline HEIS No. & B1VJ61B & \multicolumn{3}{|c|}{$\begin{array}{ll}\text { Lab ID: } & 0805020-23\end{array}$} & & & \\
\hline 14092-98-9 & Chromium 52 & $<2.08 \mathrm{E}-3$ & ug/g dry & $2.08 \mathrm{E}-3$ & $7 / 01 / 08$ & 8G01004 & PNNL-AGG-415 \\
\hline 13981-78-7 & Chromium 53 & $<6.46 \mathrm{E}-3$ & ug/g dry & $6.46 \mathrm{E}-3$ & 7/01/08 & 8G01004 & PNNL-AGG-415 \\
\hline $14191-84-5$ & Copper 63 & $<3.51 \mathrm{E}-3$ & ug/g dry & $3.51 \mathrm{E}-3$ & 7/01/08 & 8G01004 & PNNL-AGG-415 \\
\hline
\end{tabular}


RCRA Metals By PNNL-AGG-415/Water Extract

\begin{tabular}{|c|c|c|c|c|c|c|c|}
\hline CAS \# & Analyte & Results & Units & EQL & Analyzed & Batch & Method \\
\hline HEIS No. & B1VJ61B & \multicolumn{3}{|c|}{ Lab ID: $\quad 0805020-23$} & & & \\
\hline 14119-06-3 & Copper 65 & $<4.89 \mathrm{E}-3$ & ug/g dry & $4.89 \mathrm{E}-3$ & 7/01/08 & 8G01004 & PNNL-AGG-415 \\
\hline $14687-58-2$ & Selenium 82 & $<1.12 \mathrm{E}-2$ & ug/g dry & $1.12 \mathrm{E}-2$ & $7 / 01 / 08$ & 8G01004 & PNNL-AGG-415 \\
\hline $14392-17-7$ & Molybdenum 95 & 3.17E-2 & ug/g dry & $1.62 \mathrm{E}-3$ & $7 / 01 / 08$ & 8G01004 & PNNL-AGG-415 \\
\hline 14392-19-9 & Molybdenum 97 & $3.13 \mathrm{E}-2$ & ug/g dry & 2.07E-3 & $7 / 01 / 08$ & 8G01004 & PNNL-AGG-415 \\
\hline $14392-20-2$ & Molybdenum 98 & $3.14 \mathrm{E}-2$ & ug/g dry & $1.62 \mathrm{E}-3$ & 7/01/08 & 8G01004 & PNNL-AGG-415 \\
\hline $14914-61-5$ & Ruthenium 101 & $<8.28 \mathrm{E}-4$ & ug/g dry & $8.28 \mathrm{E}-4$ & $7 / 01 / 08$ & 8G01004 & PNNL-AGG-415 \\
\hline $14914-62-6$ & Ruthenium 102 & $<5.71 \mathrm{E}-4$ & ug/g dry & $5.71 \mathrm{E}-4$ & $7 / 01 / 08$ & 8G01004 & PNNL-AGG-415 \\
\hline $15766-01-5$ & Ruthenium 104 & $<3.69 \mathrm{E}-4$ & ug/g dry & $3.69 \mathrm{E}-4$ & $7 / 01 / 08$ & 8G01004 & PNNL-AGG-415 \\
\hline 14378-37-1 & Silver 107 & $<9.34 \mathrm{E}-4$ & ug/g dry & $9.34 \mathrm{E}-4$ & 7/01/08 & 8G01004 & PNNL-AGG-415 \\
\hline $14378-38-2$ & Silver 109 & $<1.08 \mathrm{E}-3$ & ug/g dry & $1.08 \mathrm{E}-3$ & $7 / 01 / 08$ & 8G01004 & PNNL-AGG-415 \\
\hline 14336-64-2 & Cadmium 111 & $<2.98 \mathrm{E}-4$ & ug/g dry & $2.98 \mathrm{E}-4$ & $7 / 01 / 08$ & 8G01004 & PNNL-AGG-415 \\
\hline 14041-58-8 & Cadmium 114 & $<6.31 \mathrm{E}-4$ & ug/g dry & $6.31 \mathrm{E}-4$ & 7/01/08 & 8G01004 & PNNL-AGG-415 \\
\hline $14265-72-6$ & Antimony 121 & $<5.45 \mathrm{E}-4$ & ug/g dry & $5.45 \mathrm{E}-4$ & 7/01/08 & 8G01004 & PNNL-AGG-415 \\
\hline 13966-27-3 & Lead 206 & $<7.27 \mathrm{E}-4$ & ug/g dry & 7.27E-4 & $7 / 01 / 08$ & 8G01004 & PNNL-AGG-415 \\
\hline 13966-28-4 & Lead 208 & $<5.66 \mathrm{E}-4$ & ug/g dry & $5.66 \mathrm{E}-4$ & $7 / 01 / 08$ & 8G01004 & PNNL-AGG-415 \\
\hline HEIS No. & $\overline{\text { B1VJ61A }}$ & \multicolumn{3}{|c|}{$\begin{array}{ll}\text { Lab ID: } & 0805020-24\end{array}$} & & & \\
\hline 14092-98-9 & Chromium 52 & $<2.13 \mathrm{E}-3$ & ug/g dry & 2.13E-3 & $7 / 01 / 08$ & 8G01004 & PNNL-AGG-415 \\
\hline 13981-78-7 & Chromium 53 & $<6.64 \mathrm{E}-3$ & ug/g dry & $6.64 \mathrm{E}-3$ & $7 / 01 / 08$ & 8G01004 & PNNL-AGG-415 \\
\hline 14191-84-5 & Copper 63 & $<3.61 \mathrm{E}-3$ & ug/g dry & $3.61 \mathrm{E}-3$ & $7 / 01 / 08$ & 8G01004 & PNNL-AGG-415 \\
\hline 14119-06-3 & Copper 65 & $<5.02 \mathrm{E}-3$ & ug/g dry & $5.02 \mathrm{E}-3$ & 7/01/08 & 8G01004 & PNNL-AGG-415 \\
\hline 14687-58-2 & Selenium 82 & $<1.15 \mathrm{E}-2$ & ug/g dry & $1.15 \mathrm{E}-2$ & 7/01/08 & 8G01004 & PNNL-AGG-415 \\
\hline $14392-17-7$ & Molybdenum 95 & $1.95 \mathrm{E}-2$ & ug/g dry & $1.67 \mathrm{E}-3$ & 7/01/08 & 8G01004 & PNNL-AGG-415 \\
\hline 14392-19-9 & Molybdenum 97 & $1.95 \mathrm{E}-2$ & ug/g dry & $2.12 \mathrm{E}-3$ & 7/01/08 & 8G01004 & PNNL-AGG-415 \\
\hline $14392-20-2$ & Molybdenum 98 & $1.94 \mathrm{E}-2$ & ug/g dry & $1.67 \mathrm{E}-3$ & 7/01/08 & 8G01004 & PNNL-AGG-415 \\
\hline 14914-61-5 & Ruthenium 101 & $<8.51 \mathrm{E}-4$ & ug/g dry & $8.51 \mathrm{E}-4$ & 7/01/08 & 8G01004 & PNNL-AGG-415 \\
\hline $14914-62-6$ & Ruthenium 102 & $<5.86 \mathrm{E}-4$ & ug/g dry & $5.86 \mathrm{E}-4$ & $7 / 01 / 08$ & 8G01004 & PNNL-AGG-415 \\
\hline 15766-01-5 & Ruthenium 104 & $<3.79 \mathrm{E}-4$ & ug/g dry & $3.79 \mathrm{E}-4$ & 7/01/08 & 8G01004 & PNNL-AGG-415 \\
\hline $14378-37-1$ & Silver 107 & $<9.60 \mathrm{E}-4$ & ug/g dry & $9.60 \mathrm{E}-4$ & 7/01/08 & 8G01004 & PNNL-AGG-415 \\
\hline 14378-38-2 & Silver 109 & $<1.11 \mathrm{E}-3$ & ug/g dry & $1.11 \mathrm{E}-3$ & 7/01/08 & 8G01004 & PNNL-AGG-415 \\
\hline $14336-64-2$ & Cadmium 111 & $<3.06 \mathrm{E}-4$ & ug/g dry & 3.06E-4 & $7 / 01 / 08$ & 8G01004 & PNNL-AGG-415 \\
\hline 14041-58-8 & Cadmium 114 & $<6.49 \mathrm{E}-4$ & ug/g dry & $6.49 \mathrm{E}-4$ & 7/01/08 & 8G01004 & PNNL-AGG-415 \\
\hline $14265-72-6$ & Antimony 121 & $<5.61 \mathrm{E}-4$ & ug/g dry & $5.61 \mathrm{E}-4$ & 7/01/08 & 8G01004 & PNNL-AGG-415 \\
\hline $13966-27-3$ & Lead 206 & $<7.47 \mathrm{E}-4$ & ug/g dry & 7.47E-4 & 7/01/08 & 8G01004 & PNNL-AGG-415 \\
\hline 13966-28-4 & Lead 208 & $<5.81 \mathrm{E}-4$ & ug/g dry & $5.81 \mathrm{E}-4$ & 7/01/08 & 8G01004 & PNNL-AGG-415 \\
\hline HEIS No. & B1VJ62C & \multicolumn{3}{|c|}{$\begin{array}{ll}\text { Lab ID: } & 0805020-26\end{array}$} & & & \\
\hline 14092-98-9 & Chromium 52 & $<2.05 \mathrm{E}-3$ & ug/g dry & 2.05E-3 & $7 / 01 / 08$ & 8G01004 & PNNL-AGG-415 \\
\hline 13981-78-7 & Chromium 53 & $<6.40 \mathrm{E}-3$ & ug/g dry & $6.40 \mathrm{E}-3$ & $7 / 01 / 08$ & 8G01004 & PNNL-AGG-415 \\
\hline 14191-84-5 & Copper 63 & $5.71 \mathrm{E}-3$ & ug/g dry & $3.48 \mathrm{E}-3$ & $7 / 01 / 08$ & 8G01004 & PNNL-AGG-415 \\
\hline 14119-06-3 & Copper 65 & 6.72E-3 & ug/g dry & $4.84 \mathrm{E}-3$ & $7 / 01 / 08$ & 8G01004 & PNNL-AGG-415 \\
\hline 14687-58-2 & Selenium 82 & $<1.10 \mathrm{E}-2$ & ug/g dry & $1.10 \mathrm{E}-2$ & 7/01/08 & 8G01004 & PNNL-AGG-415 \\
\hline 14392-17-7 & Molybdenum 95 & 1.57E-1 & ug/g dry & $1.60 \mathrm{E}-3$ & $7 / 01 / 08$ & 8G01004 & PNNL-AGG-415 \\
\hline 14392-19-9 & Molybdenum 97 & $1.55 \mathrm{E}-1$ & ug/g dry & 2.04E-3 & $7 / 01 / 08$ & 8G01004 & PNNL-AGG-415 \\
\hline $14392-20-2$ & Molybdenum 98 & $1.55 \mathrm{E}-1$ & ug/g dry & $1.60 \mathrm{E}-3$ & $7 / 01 / 08$ & 8G01004 & PNNL-AGG-415 \\
\hline 14914-61-5 & Ruthenium 101 & $<8.20 \mathrm{E}-4$ & ug/g dry & $8.20 \mathrm{E}-4$ & 7/01/08 & 8G01004 & PNNL-AGG-415 \\
\hline $14914-62-6$ & Ruthenium 102 & $<5.65 \mathrm{E}-4$ & ug/g dry & 5.65E-4 & $7 / 01 / 08$ & 8G01004 & PNNL-AGG-415 \\
\hline 15766-01-5 & Ruthenium 104 & 4.35E-4 & ug/g dry & 3.65E-4 & 7/01/08 & 8G01004 & PNNL-AGG-415 \\
\hline 14378-37-1 & Silver 107 & $<9.25 \mathrm{E}-4$ & ug/g dry & $9.25 \mathrm{E}-4$ & 7/01/08 & 8G01004 & PNNL-AGG-415 \\
\hline 14378-38-2 & Silver 109 & $<1.07 \mathrm{E}-3$ & ug/g dry & $1.07 \mathrm{E}-3$ & 7/01/08 & 8G01004 & PNNL-AGG-415 \\
\hline
\end{tabular}


RCRA Metals By PNNL-AGG-415/Water Extract

\begin{tabular}{|c|c|c|c|c|c|c|c|}
\hline CAS \# & Analyte & Results & Units & EQL & Analyzed & Batch & Method \\
\hline HEIS No. & B1VJ62C & \multicolumn{3}{|c|}{ Lab ID: $\quad 0805020-26$} & & & \\
\hline $14336-64-2$ & Cadmium 111 & $5.71 \mathrm{E}-4$ & ug/g dry & $2.95 \mathrm{E}-4$ & 7/01/08 & 8G01004 & PNNL-AGG-415 \\
\hline $14041-58-8$ & Cadmium 114 & $<6.25 \mathrm{E}-4$ & ug/g dry & $6.25 \mathrm{E}-4$ & $7 / 01 / 08$ & 8G01004 & PNNL-AGG-415 \\
\hline $14265-72-6$ & Antimony 121 & 9.17E-4 & ug/g dry & $5.40 \mathrm{E}-4$ & $7 / 01 / 08$ & 8G01004 & PNNL-AGG-415 \\
\hline $13966-27-3$ & Lead 206 & $<7.20 \mathrm{E}-4$ & ug/g dry & $7.20 \mathrm{E}-4$ & $7 / 01 / 08$ & 8G01004 & PNNL-AGG-415 \\
\hline 13966-28-4 & Lead 208 & $<5.60 \mathrm{E}-4$ & ug/g dry & $5.60 \mathrm{E}-4$ & 7/01/08 & 8G01004 & PNNL-AGG-415 \\
\hline HEIS No. & B1VJ62B & \multicolumn{3}{|c|}{$\begin{array}{ll}\text { Lab ID: } & \text { 0805020-27 }\end{array}$} & & & \\
\hline 14092-98-9 & Chromium 52 & $<2.06 \mathrm{E}-3$ & ug/g dry & $2.06 \mathrm{E}-3$ & $7 / 01 / 08$ & 8G01005 & PNNL-AGG-415 \\
\hline 13981-78-7 & Chromium 53 & $<6.41 \mathrm{E}-3$ & ug/g dry & $6.41 \mathrm{E}-3$ & $7 / 01 / 08$ & 8G01005 & PNNL-AGG-415 \\
\hline $14191-84-5$ & Copper 63 & $<3.48 \mathrm{E}-3$ & ug/g dry & $3.48 \mathrm{E}-3$ & 7/01/08 & 8G01005 & PNNL-AGG-415 \\
\hline 14119-06-3 & Copper 65 & $<4.84 \mathrm{E}-3$ & ug/g dry & $4.84 \mathrm{E}-3$ & $7 / 01 / 08$ & 8G01005 & PNNL-AGG-415 \\
\hline $14687-58-2$ & Selenium 82 & $<1.11 \mathrm{E}-2$ & ug/g dry & $1.11 \mathrm{E}-2$ & $7 / 01 / 08$ & 8G01005 & PNNL-AGG-415 \\
\hline $14392-17-7$ & Molybdenum 95 & 5.95E-2 & ug/g dry & $1.61 \mathrm{E}-3$ & 7/01/08 & 8G01005 & PNNL-AGG-415 \\
\hline $14392-19-9$ & Molybdenum 97 & $5.93 \mathrm{E}-2$ & ug/g dry & 2.05E-3 & 7/01/08 & 8G01005 & PNNL-AGG-415 \\
\hline $14392-20-2$ & Molybdenum 98 & $5.94 \mathrm{E}-2$ & ug/g dry & $1.61 \mathrm{E}-3$ & 7/01/08 & $8 G 01005$ & PNNL-AGG-415 \\
\hline $14914-61-5$ & Ruthenium 101 & $<8.21 \mathrm{E}-4$ & ug/g dry & $8.21 \mathrm{E}-4$ & 7/01/08 & 8G01005 & PNNL-AGG-415 \\
\hline $14914-62-6$ & Ruthenium 102 & $<5.65 \mathrm{E}-4$ & ug/g dry & $5.65 \mathrm{E}-4$ & $7 / 01 / 08$ & 8G01005 & PNNL-AGG-415 \\
\hline $15766-01-5$ & Ruthenium 104 & $<3.65 \mathrm{E}-4$ & ug/g dry & 3.65E-4 & 7/01/08 & 8G01005 & PNNL-AGG-415 \\
\hline 14378-37-1 & Silver 107 & $<9.26 \mathrm{E}-4$ & ug/g dry & $9.26 \mathrm{E}-4$ & $7 / 01 / 08$ & 8G01005 & PNNL-AGG-415 \\
\hline $14378-38-2$ & Silver 109 & $<1.07 \mathrm{E}-3$ & ug/g dry & $1.07 \mathrm{E}-3$ & $7 / 01 / 08$ & 8G01005 & PNNL-AGG-415 \\
\hline 14336-64-2 & Cadmium 111 & $<2.95 \mathrm{E}-4$ & ug/g dry & $2.95 \mathrm{E}-4$ & $7 / 01 / 08$ & 8G01005 & PNNL-AGG-415 \\
\hline 14041-58-8 & Cadmium 114 & $<6.26 \mathrm{E}-4$ & ug/g dry & $6.26 \mathrm{E}-4$ & 7/01/08 & 8G01005 & PNNL-AGG-415 \\
\hline $14265-72-6$ & Antimony 121 & $<5.40 \mathrm{E}-4$ & ug/g dry & $5.40 \mathrm{E}-4$ & $7 / 01 / 08$ & 8G01005 & PNNL-AGG-415 \\
\hline 13966-27-3 & Lead 206 & $<7.21 \mathrm{E}-4$ & ug/g dry & 7.21E-4 & 7/01/08 & 8G01005 & PNNL-AGG-415 \\
\hline 13966-28-4 & Lead 208 & $<5.60 \mathrm{E}-4$ & ug/g dry & $5.60 \mathrm{E}-4$ & 7/01/08 & 8G01005 & PNNL-AGG-415 \\
\hline HEIS No. & B1VJ62A & \multicolumn{3}{|c|}{$\begin{array}{ll}\text { Lab ID: } & \text { 0805020-28 }\end{array}$} & & & \\
\hline 14092-98-9 & Chromium 52 & $<2.06 \mathrm{E}-3$ & ug/g dry & $2.06 \mathrm{E}-3$ & $7 / 01 / 08$ & $8 G 01005$ & PNNL-AGG-415 \\
\hline 13981-78-7 & Chromium 53 & $<6.41 \mathrm{E}-3$ & ug/g dry & $6.41 \mathrm{E}-3$ & $7 / 01 / 08$ & 8G01005 & PNNL-AGG-415 \\
\hline $14191-84-5$ & Copper 63 & $<3.49 \mathrm{E}-3$ & ug/g dry & $3.49 \mathrm{E}-3$ & $7 / 01 / 08$ & 8G01005 & PNNL-AGG-415 \\
\hline 14119-06-3 & Copper 65 & $<4.85 \mathrm{E}-3$ & ug/g dry & $4.85 \mathrm{E}-3$ & 7/01/08 & 8G01005 & PNNL-AGG-415 \\
\hline $14687-58-2$ & Selenium 82 & $<1.11 \mathrm{E}-2$ & ug/g dry & $1.11 \mathrm{E}-2$ & $7 / 01 / 08$ & 8G01005 & PNNL-AGG-415 \\
\hline $14392-17-7$ & Molybdenum 95 & $5.46 \mathrm{E}-2$ & ug/g dry & $1.61 \mathrm{E}-3$ & $7 / 01 / 08$ & 8G01005 & PNNL-AGG-415 \\
\hline 14392-19-9 & Molybdenum 97 & $5.45 \mathrm{E}-2$ & ug/g dry & $2.05 \mathrm{E}-3$ & $7 / 01 / 08$ & 8G01005 & PNNL-AGG-415 \\
\hline $14392-20-2$ & Molybdenum 98 & $5.46 \mathrm{E}-2$ & ug/g dry & $1.61 \mathrm{E}-3$ & $7 / 01 / 08$ & 8G01005 & PNNL-AGG-415 \\
\hline 14914-61-5 & Ruthenium 101 & $<8.21 \mathrm{E}-4$ & ug/g dry & $8.21 \mathrm{E}-4$ & 7/01/08 & 8G01005 & PNNL-AGG-415 \\
\hline $14914-62-6$ & Ruthenium 102 & $<5.66 \mathrm{E}-4$ & ug/g dry & $5.66 \mathrm{E}-4$ & 7/01/08 & 8G01005 & PNNL-AGG-415 \\
\hline 15766-01-5 & Ruthenium 104 & $<3.66 \mathrm{E}-4$ & ug/g dry & 3.66E-4 & 7/01/08 & 8G01005 & PNNL-AGG-415 \\
\hline $14378-37-1$ & Silver 107 & $<9.26 \mathrm{E}-4$ & ug/g dry & $9.26 \mathrm{E}-4$ & 7/01/08 & $8 G 01005$ & PNNL-AGG-415 \\
\hline $14378-38-2$ & Silver 109 & $<1.07 \mathrm{E}-3$ & ug/g dry & $1.07 \mathrm{E}-3$ & 7/01/08 & 8G01005 & PNNL-AGG-415 \\
\hline 14336-64-2 & Cadmium 111 & $<2.95 \mathrm{E}-4$ & ug/g dry & $2.95 \mathrm{E}-4$ & 7/01/08 & $8 G 01005$ & PNNL-AGG-415 \\
\hline $14041-58-8$ & Cadmium 114 & $<6.26 \mathrm{E}-4$ & ug/g dry & $6.26 \mathrm{E}-4$ & 7/01/08 & 8G01005 & PNNL-AGG-415 \\
\hline $14265-72-6$ & Antimony 121 & $<5.41 \mathrm{E}-4$ & ug/g dry & $5.41 \mathrm{E}-4$ & $7 / 01 / 08$ & 8G01005 & PNNL-AGG-415 \\
\hline 13966-27-3 & Lead 206 & $<7.21 \mathrm{E}-4$ & ug/g dry & $7.21 \mathrm{E}-4$ & 7/01/08 & 8G01005 & PNNL-AGG-415 \\
\hline $13966-28-4$ & Lead 208 & $<5.61 \mathrm{E}-4$ & ug/g dry & $5.61 \mathrm{E}-4$ & 7/01/08 & 8G01005 & PNNL-AGG-415 \\
\hline HEIS No. & B1VJ64C & \multicolumn{3}{|c|}{$\begin{array}{ll}\text { Lab ID: } & 0805020-30\end{array}$} & & & \\
\hline 14092-98-9 & Chromium 52 & $<2.06 \mathrm{E}-3$ & ug/g dry & $2.06 \mathrm{E}-3$ & $7 / 01 / 08$ & 8G01005 & PNNL-AGG-415 \\
\hline 13981-78-7 & Chromium 53 & $<6.40 \mathrm{E}-3$ & ug/g dry & $6.40 \mathrm{E}-3$ & $7 / 01 / 08$ & 8G01005 & PNNL-AGG-415 \\
\hline 14191-84-5 & Copper 63 & $<3.48 \mathrm{E}-3$ & ug/g dry & $3.48 \mathrm{E}-3$ & 7/01/08 & 8G01005 & PNNL-AGG-415 \\
\hline $14119-06-3$ & Copper 65 & $<4.84 \mathrm{E}-3$ & ug/g dry & $4.84 \mathrm{E}-3$ & 7/01/08 & 8G01005 & PNNL-AGG-415 \\
\hline
\end{tabular}


RCRA Metals By PNNL-AGG-415/Water Extract

\begin{tabular}{|c|c|c|c|c|c|c|c|}
\hline CAS \# & Analyte & Results & Units & EQL & Analyzed & Batch & Method \\
\hline HEIS No. & B1VJ64C & \multicolumn{3}{|c|}{ Lab ID: $\quad$ 0805020-30 } & & & \\
\hline 14687-58-2 & Selenium 82 & $<1.11 \mathrm{E}-2$ & ug/g dry & $1.11 \mathrm{E}-2$ & 7/01/08 & 8G01005 & PNNL-AGG-415 \\
\hline 14392-17-7 & Molybdenum 95 & 9.73E-2 & ug/g dry & $1.61 \mathrm{E}-3$ & $7 / 01 / 08$ & 8G01005 & PNNL-AGG-415 \\
\hline 14392-19-9 & Molybdenum 97 & $9.67 \mathrm{E}-2$ & ug/g dry & 2.05E-3 & $7 / 01 / 08$ & 8G01005 & PNNL-AGG-415 \\
\hline $14392-20-2$ & Molybdenum 98 & $9.75 \mathrm{E}-2$ & ug/g dry & $1.61 \mathrm{E}-3$ & $7 / 01 / 08$ & 8G01005 & PNNL-AGG-415 \\
\hline 14914-61-5 & Ruthenium 101 & $<8.20 \mathrm{E}-4$ & ug/g dry & $8.20 \mathrm{E}-4$ & 7/01/08 & 8G01005 & PNNL-AGG-415 \\
\hline $14914-62-6$ & Ruthenium 102 & $<5.65 \mathrm{E}-4$ & ug/g dry & 5.65E-4 & $7 / 01 / 08$ & 8G01005 & PNNL-AGG-415 \\
\hline $15766-01-5$ & Ruthenium 104 & $<3.65 \mathrm{E}-4$ & ug/g dry & 3.65E-4 & $7 / 01 / 08$ & 8G01005 & PNNL-AGG-415 \\
\hline 14378-37-1 & Silver 107 & $<9.25 \mathrm{E}-4$ & ug/g dry & $9.25 \mathrm{E}-4$ & $7 / 01 / 08$ & 8G01005 & PNNL-AGG-415 \\
\hline 14378-38-2 & Silver 109 & $<1.07 \mathrm{E}-3$ & ug/g dry & $1.07 \mathrm{E}-3$ & 7/01/08 & 8G01005 & PNNL-AGG-415 \\
\hline $14336-64-2$ & Cadmium 111 & $3.78 \mathrm{E}-4$ & ug/g dry & 2.95E-4 & $7 / 01 / 08$ & 8G01005 & PNNL-AGG-415 \\
\hline 14041-58-8 & Cadmium 114 & $<6.25 \mathrm{E}-4$ & ug/g dry & $6.25 \mathrm{E}-4$ & $7 / 01 / 08$ & 8G01005 & PNNL-AGG-415 \\
\hline $14265-72-6$ & Antimony 121 & 7.36E-4 & ug/g dry & $5.40 \mathrm{E}-4$ & 7/01/08 & 8G01005 & PNNL-AGG-415 \\
\hline $13966-27-3$ & Lead 206 & $<7.20 \mathrm{E}-4$ & ug/g dry & $7.20 \mathrm{E}-4$ & 7/01/08 & 8G01005 & PNNL-AGG-415 \\
\hline 13966-28-4 & Lead 208 & $<5.60 \mathrm{E}-4$ & ug/g dry & $5.60 \mathrm{E}-4$ & $7 / 01 / 08$ & 8G01005 & PNNL-AGG-415 \\
\hline HEIS No. & B1VJ64B & \multicolumn{3}{|c|}{ Lab ID: $\quad$ 0805020-31 } & & & \\
\hline 14092-98-9 & Chromium 52 & $<2.05 \mathrm{E}-3$ & ug/g dry & 2.05E-3 & $7 / 01 / 08$ & 8G01005 & PNNL-AGG-415 \\
\hline 13981-78-7 & Chromium 53 & $<6.40 \mathrm{E}-3$ & ug/g dry & $6.40 \mathrm{E}-3$ & 7/01/08 & 8G01005 & PNNL-AGG-415 \\
\hline 14191-84-5 & Copper 63 & $<3.48 \mathrm{E}-3$ & ug/g dry & $3.48 \mathrm{E}-3$ & $7 / 01 / 08$ & 8G01005 & PNNL-AGG-415 \\
\hline 14119-06-3 & Copper 65 & $<4.84 \mathrm{E}-3$ & ug/g dry & $4.84 \mathrm{E}-3$ & $7 / 01 / 08$ & 8G01005 & PNNL-AGG-415 \\
\hline 14687-58-2 & Selenium 82 & $<1.10 \mathrm{E}-2$ & ug/g dry & $1.10 \mathrm{E}-2$ & 7/01/08 & 8G01005 & PNNL-AGG-415 \\
\hline $14392-17-7$ & Molybdenum 95 & 5.75E-2 & ug/g dry & $1.60 \mathrm{E}-3$ & 7/01/08 & 8G01005 & PNNL-AGG-415 \\
\hline 14392-19-9 & Molybdenum 97 & $5.64 \mathrm{E}-2$ & ug/g dry & $2.04 \mathrm{E}-3$ & 7/01/08 & $8 G 01005$ & PNNL-AGG-415 \\
\hline $14392-20-2$ & Molybdenum 98 & 5.72E-2 & ug/g dry & $1.60 \mathrm{E}-3$ & $7 / 01 / 08$ & 8G01005 & PNNL-AGG-415 \\
\hline $14914-61-5$ & Ruthenium 101 & $<8.20 \mathrm{E}-4$ & ug/g dry & $8.20 \mathrm{E}-4$ & 7/01/08 & $8 G 01005$ & PNNL-AGG-415 \\
\hline $14914-62-6$ & Ruthenium 102 & $<5.65 \mathrm{E}-4$ & ug/g dry & $5.65 \mathrm{E}-4$ & 7/01/08 & $8 G 01005$ & PNNL-AGG-415 \\
\hline 15766-01-5 & Ruthenium 104 & $<3.65 \mathrm{E}-4$ & ug/g dry & 3.65E-4 & $7 / 01 / 08$ & 8G01005 & PNNL-AGG-415 \\
\hline $14378-37-1$ & Silver 107 & $<9.25 \mathrm{E}-4$ & ug/g dry & $9.25 \mathrm{E}-4$ & 7/01/08 & 8G01005 & PNNL-AGG-415 \\
\hline 14378-38-2 & Silver 109 & $<1.07 \mathrm{E}-3$ & ug/g dry & $1.07 \mathrm{E}-3$ & 7/01/08 & 8G01005 & PNNL-AGG-415 \\
\hline $14336-64-2$ & Cadmium 111 & $<2.95 \mathrm{E}-4$ & ug/g dry & 2.95E-4 & 7/01/08 & 8G01005 & PNNL-AGG-415 \\
\hline 14041-58-8 & Cadmium 114 & $<6.25 \mathrm{E}-4$ & ug/g dry & $6.25 \mathrm{E}-4$ & $7 / 01 / 08$ & 8G01005 & PNNL-AGG-415 \\
\hline $14265-72-6$ & Antimony 121 & $<5.40 \mathrm{E}-4$ & ug/g dry & $5.40 \mathrm{E}-4$ & 7/01/08 & $8 G 01005$ & PNNL-AGG-415 \\
\hline $13966-27-3$ & Lead 206 & $<7.20 \mathrm{E}-4$ & ug/g dry & $7.20 \mathrm{E}-4$ & 7/01/08 & 8G01005 & PNNL-AGG-415 \\
\hline $13966-28-4$ & Lead 208 & $<5.60 \mathrm{E}-4$ & ug/g dry & $5.60 \mathrm{E}-4$ & $7 / 01 / 08$ & $8 G 01005$ & PNNL-AGG-415 \\
\hline HEIS No. & B1VJ64A & \multicolumn{3}{|c|}{ Lab ID: $\quad$ 0805020-32 } & & & \\
\hline 14092-98-9 & Chromium 52 & $<1.95 \mathrm{E}-3$ & ug/g dry & $1.95 \mathrm{E}-3$ & $7 / 01 / 08$ & 8G01005 & PNNL-AGG-415 \\
\hline 13981-78-7 & Chromium 53 & $<6.07 \mathrm{E}-3$ & ug/g dry & $6.07 \mathrm{E}-3$ & $7 / 01 / 08$ & 8G01005 & PNNL-AGG-415 \\
\hline 14191-84-5 & Copper 63 & $<3.30 \mathrm{E}-3$ & ug/g dry & $3.30 \mathrm{E}-3$ & 7/01/08 & 8G01005 & PNNL-AGG-415 \\
\hline 14119-06-3 & Copper 65 & $<4.59 \mathrm{E}-3$ & ug/g dry & $4.59 \mathrm{E}-3$ & $7 / 01 / 08$ & 8G01005 & PNNL-AGG-415 \\
\hline $14687-58-2$ & Selenium 82 & $<1.05 \mathrm{E}-2$ & ug/g dry & $1.05 \mathrm{E}-2$ & $7 / 01 / 08$ & 8G01005 & PNNL-AGG-415 \\
\hline $14392-17-7$ & Molybdenum 95 & 2.99E-2 & ug/g dry & $1.52 \mathrm{E}-3$ & 7/01/08 & 8G01005 & PNNL-AGG-415 \\
\hline 14392-19-9 & Molybdenum 97 & $2.97 \mathrm{E}-2$ & ug/g dry & $1.94 \mathrm{E}-3$ & $7 / 01 / 08$ & 8G01005 & PNNL-AGG-415 \\
\hline 14392-20-2 & Molybdenum 98 & 2.97E-2 & ug/g dry & $1.52 \mathrm{E}-3$ & $7 / 01 / 08$ & 8G01005 & PNNL-AGG-415 \\
\hline $14914-61-5$ & Ruthenium 101 & $<7.78 \mathrm{E}-4$ & ug/g dry & $7.78 \mathrm{E}-4$ & 7/01/08 & $8 G 01005$ & PNNL-AGG-415 \\
\hline $14914-62-6$ & Ruthenium 102 & $<5.36 \mathrm{E}-4$ & ug/g dry & 5.36E-4 & 7/01/08 & 8G01005 & PNNL-AGG-415 \\
\hline 15766-01-5 & Ruthenium 104 & $<3.46 \mathrm{E}-4$ & ug/g dry & $3.46 \mathrm{E}-4$ & $7 / 01 / 08$ & 8G01005 & PNNL-AGG-415 \\
\hline $14378-37-1$ & Silver 107 & $<8.78 \mathrm{E}-4$ & ug/g dry & $8.78 \mathrm{E}-4$ & 7/01/08 & 8G01005 & PNNL-AGG-415 \\
\hline 14378-38-2 & Silver 109 & $<1.02 \mathrm{E}-3$ & ug/g dry & $1.02 \mathrm{E}-3$ & 7/01/08 & $8 G 01005$ & PNNL-AGG-415 \\
\hline $14336-64-2$ & Cadmium 111 & $<2.80 \mathrm{E}-4$ & ug/g dry & $2.80 \mathrm{E}-4$ & 7/01/08 & $8 G 01005$ & PNNL-AGG-415 \\
\hline
\end{tabular}


RCRA Metals By PNNL-AGG-415/Water Extract

\begin{tabular}{|c|c|c|c|c|c|c|c|}
\hline CAS \# & Analyte & Results & Units & EQL & Analyzed & Batch & Method \\
\hline HEIS No. & B1VJ64A & & b ID: & $5020-32$ & & & \\
\hline $14041-58-8$ & Cadmium 114 & $<5.93 \mathrm{E}-4$ & ug/g dry & $5.93 E-4$ & 7/01/08 & 8G01005 & PNNL-AGG-415 \\
\hline $14265-72-6$ & Antimony 121 & $<5.12 \mathrm{E}-4$ & ug/g dry & $5.12 \mathrm{E}-4$ & 7/01/08 & 8G01005 & PNNL-AGG-415 \\
\hline 13966-27-3 & Lead 206 & $<6.83 \mathrm{E}-4$ & ug/g dry & $6.83 \mathrm{E}-4$ & 7/01/08 & 8G01005 & PNNL-AGG-415 \\
\hline 13966-28-4 & Lead 208 & $<5.31 \mathrm{E}-4$ & ug/g dry & $5.31 \mathrm{E}-4$ & 7/01/08 & 8G01005 & PNNL-AGG-415 \\
\hline
\end{tabular}


RCRA Metals By PNNL-AGG-415/Acid Extract

\begin{tabular}{|c|c|c|c|c|c|c|c|}
\hline CAS \# & Analyte & Results & Units & EQL & Analyzed & Batch & Method \\
\hline HEIS No. & B1VJ54B & \multicolumn{3}{|c|}{ Lab ID: $\quad$ 0805020-01 } & & & \\
\hline $14687-58-2$ & Selenium 82 & $<1.14 \mathrm{E} 0$ & ug/g dry & 1.14E0 & 7/02/08 & 8G02005 & PNNL-AGG-415 \\
\hline 14392-17-7 & Molybdenum 95 & $<2.33 \mathrm{E}-1$ & ug/g dry & 2.33E-1 & $7 / 02 / 08$ & 8G02005 & PNNL-AGG-415 \\
\hline 14392-19-9 & Molybdenum 97 & $<2.14 \mathrm{E}-1$ & ug/g dry & $2.14 \mathrm{E}-1$ & $7 / 02 / 08$ & 8G02005 & PNNL-AGG-415 \\
\hline $14392-20-2$ & Molybdenum 98 & 1.93E-1 & ug/g dry & 8.86E-2 & $7 / 02 / 08$ & 8G02005 & PNNL-AGG-415 \\
\hline 14914-61-5 & Ruthenium 101 & $<4.48 \mathrm{E}-2$ & ug/g dry & 4.48E-2 & $7 / 02 / 08$ & 8G02005 & PNNL-AGG-415 \\
\hline $14914-62-6$ & Ruthenium 102 & $<2.71 \mathrm{E}-2$ & ug/g dry & $2.71 \mathrm{E}-2$ & $7 / 02 / 08$ & 8G02005 & PNNL-AGG-415 \\
\hline $15766-01-5$ & Ruthenium 104 & $<7.57 \mathrm{E}-2$ & ug/g dry & 7.57E-2 & $7 / 02 / 08$ & 8G02005 & PNNL-AGG-415 \\
\hline $14336-64-2$ & Cadmium 111 & 9.63E-2 & ug/g dry & $4.90 \mathrm{E}-2$ & $7 / 02 / 08$ & 8G02005 & PNNL-AGG-415 \\
\hline 14041-58-8 & Cadmium 114 & $7.24 \mathrm{E}-2$ & ug/g dry & $1.11 \mathrm{E}-2$ & $7 / 02 / 08$ & 8G02005 & PNNL-AGG-415 \\
\hline $14265-72-6$ & Antimony 121 & $<7.92 \mathrm{E}-2$ & ug/g dry & 7.92E-2 & $7 / 02 / 08$ & 8G02005 & PNNL-AGG-415 \\
\hline HEIS No. & B1VJ55C & \multicolumn{3}{|c|}{ Lab ID: $\quad$ 0805020-05 } & & & \\
\hline $14687-58-2$ & Selenium 82 & $<1.33 \mathrm{E} 0$ & ug/g dry & 1.33E0 & $7 / 02 / 08$ & 8G02005 & PNNL-AGG-415 \\
\hline $14392-17-7$ & Molybdenum 95 & $<2.73 \mathrm{E}-1$ & ug/g dry & $2.73 \mathrm{E}-1$ & 7/02/08 & 8G02005 & PNNL-AGG-415 \\
\hline 14392-19-9 & Molybdenum 97 & $<2.50 \mathrm{E}-1$ & ug/g dry & $2.50 \mathrm{E}-1$ & $7 / 02 / 08$ & $8 G 02005$ & PNNL-AGG-415 \\
\hline $14392-20-2$ & Molybdenum 98 & 2.10E-1 & ug/g dry & 1.03E-1 & $7 / 02 / 08$ & 8G02005 & PNNL-AGG-415 \\
\hline $14914-61-5$ & Ruthenium 101 & $<5.23 \mathrm{E}-2$ & ug/g dry & $5.23 \mathrm{E}-2$ & $7 / 02 / 08$ & 8G02005 & PNNL-AGG-415 \\
\hline $14914-62-6$ & Ruthenium 102 & $<3.16 \mathrm{E}-2$ & ug/g dry & $3.16 \mathrm{E}-2$ & $7 / 02 / 08$ & 8G02005 & PNNL-AGG-415 \\
\hline 15766-01-5 & Ruthenium 104 & $<8.84 \mathrm{E}-2$ & ug/g dry & 8.84E-2 & $7 / 02 / 08$ & 8G02005 & PNNL-AGG-415 \\
\hline $14336-64-2$ & Cadmium 111 & $9.14 \mathrm{E}-2$ & ug/g dry & $5.72 \mathrm{E}-2$ & $7 / 02 / 08$ & 8G02005 & PNNL-AGG-415 \\
\hline $14041-58-8$ & Cadmium 114 & 6.63E-2 & ug/g dry & $1.30 \mathrm{E}-2$ & $7 / 02 / 08$ & 8G02005 & PNNL-AGG-415 \\
\hline $14265-72-6$ & Antimony 121 & $<9.25 \mathrm{E}-2$ & ug/g dry & $9.25 \mathrm{E}-2$ & $7 / 02 / 08$ & 8G02005 & PNNL-AGG-415 \\
\hline HEIS No. & B1VJ56A & \multicolumn{3}{|c|}{$\begin{array}{ll}\text { Lab ID: } & \text { 0805020-07 }\end{array}$} & & & \\
\hline $14687-58-2$ & Selenium 82 & $<1.11 \mathrm{E} 0$ & ug/g dry & $1.11 \mathrm{E} 0$ & $7 / 02 / 08$ & 8G02007 & PNNL-AGG-415 \\
\hline 14392-17-7 & Molybdenum 95 & 2.93E-1 & ug/g dry & 2.27E-1 & $7 / 02 / 08$ & 8G02007 & PNNL-AGG-415 \\
\hline $14392-19-9$ & Molybdenum 97 & $2.76 \mathrm{E}-1$ & ug/g dry & $2.08 \mathrm{E}-1$ & 7/02/08 & 8G02007 & PNNL-AGG-415 \\
\hline $14392-20-2$ & Molybdenum 98 & $2.70 \mathrm{E}-1$ & ug/g dry & $8.61 \mathrm{E}-2$ & $7 / 02 / 08$ & 8G02007 & PNNL-AGG-415 \\
\hline 14914-61-5 & Ruthenium 101 & $<4.36 \mathrm{E}-2$ & ug/g dry & 4.36E-2 & $7 / 02 / 08$ & 8G02007 & PNNL-AGG-415 \\
\hline $14914-62-6$ & Ruthenium 102 & $<2.63 \mathrm{E}-2$ & ug/g dry & 2.63E-2 & $7 / 02 / 08$ & 8G02007 & PNNL-AGG-415 \\
\hline 15766-01-5 & Ruthenium 104 & $<7.36 \mathrm{E}-2$ & ug/g dry & 7.36E-2 & $7 / 02 / 08$ & 8G02007 & PNNL-AGG-415 \\
\hline $14336-64-2$ & Cadmium 111 & $6.41 \mathrm{E}-2$ & ug/g dry & 4.76E-2 & $7 / 02 / 08$ & $8 G 02007$ & PNNL-AGG-415 \\
\hline 14041-58-8 & Cadmium 114 & $4.00 \mathrm{E}-2$ & ug/g dry & $1.08 \mathrm{E}-2$ & $7 / 02 / 08$ & 8G02007 & PNNL-AGG-415 \\
\hline $14265-72-6$ & Antimony 121 & $<7.70 \mathrm{E}-2$ & ug/g dry & $7.70 \mathrm{E}-2$ & $7 / 02 / 08$ & 8G02007 & PNNL-AGG-415 \\
\hline HEIS No. & B1VJ56B & \multicolumn{3}{|c|}{ Lab ID: $\quad$ 0805020-08 } & & & \\
\hline 14687-58-2 & Selenium 82 & $<1.19 \mathrm{E} 0$ & ug/g dry & 1.19E0 & $7 / 02 / 08$ & 8G02005 & PNNL-AGG-415 \\
\hline $14392-17-7$ & Molybdenum 95 & $3.00 \mathrm{E}-1$ & ug/g dry & 2.43E-1 & $7 / 02 / 08$ & 8G02005 & PNNL-AGG-415 \\
\hline 14392-19-9 & Molybdenum 97 & 2.89E-1 & ug/g dry & 2.23E-1 & $7 / 02 / 08$ & 8G02005 & PNNL-AGG-415 \\
\hline $14392-20-2$ & Molybdenum 98 & 2.83E-1 & ug/g dry & $9.23 \mathrm{E}-2$ & 7/02/08 & 8G02005 & PNNL-AGG-415 \\
\hline 14914-61-5 & Ruthenium 101 & $<4.67 \mathrm{E}-2$ & ug/g dry & 4.67E-2 & $7 / 02 / 08$ & 8G02005 & PNNL-AGG-415 \\
\hline 14914-62-6 & Ruthenium 102 & $<2.82 \mathrm{E}-2$ & ug/g dry & 2.82E-2 & $7 / 02 / 08$ & 8G02005 & PNNL-AGG-415 \\
\hline 15766-01-5 & Ruthenium 104 & $<7.89 \mathrm{E}-2$ & ug/g dry & 7.89E-2 & $7 / 02 / 08$ & 8G02005 & PNNL-AGG-415 \\
\hline $14336-64-2$ & Cadmium 111 & $6.15 \mathrm{E}-2$ & ug/g dry & $5.10 \mathrm{E}-2$ & $7 / 02 / 08$ & 8G02005 & PNNL-AGG-415 \\
\hline 14041-58-8 & Cadmium 114 & 3.93E-2 & ug/g dry & $1.16 \mathrm{E}-2$ & $7 / 02 / 08$ & 8G02005 & PNNL-AGG-415 \\
\hline $14265-72-6$ & Antimony 121 & $<8.25 \mathrm{E}-2$ & ug/g dry & 8.25E-2 & $7 / 02 / 08$ & 8G02005 & PNNL-AGG-415 \\
\hline HEIS No. & B1VJ56C & \multicolumn{3}{|c|}{ Lab ID: $\quad$ 0805020-09 } & & & \\
\hline $14687-58-2$ & Selenium 82 & $<1.15 \mathrm{E} 0$ & ug/g dry & $1.15 \mathrm{E} 0$ & $7 / 02 / 08$ & 8G02005 & PNNL-AGG-415 \\
\hline $14392-17-7$ & Molybdenum 95 & $1.04 \mathrm{E} 0$ & ug/g dry & 2.35E-1 & $7 / 02 / 08$ & 8G02005 & PNNL-AGG-415 \\
\hline 14392-19-9 & Molybdenum 97 & $1.02 \mathrm{E} 0$ & ug/g dry & $2.16 \mathrm{E}-1$ & $7 / 02 / 08$ & 8G02005 & PNNL-AGG-415 \\
\hline 14392-20-2 & Molybdenum 98 & $1.02 \mathrm{E} 0$ & ug/g dry & 8.93E-2 & $7 / 02 / 08$ & 8G02005 & PNNL-AGG-415 \\
\hline
\end{tabular}


RCRA Metals By PNNL-AGG-415/Acid Extract

\begin{tabular}{|c|c|c|c|c|c|c|c|}
\hline CAS \# & Analyte & Results & Units & EQL & Analyzed & Batch & Method \\
\hline HEIS No. & B1VJ56C & \multicolumn{3}{|c|}{ Lab ID: $\quad$ 0805020-09 } & & & \\
\hline $14914-61-5$ & Ruthenium 101 & $<4.52 \mathrm{E}-2$ & ug/g dry & $4.52 \mathrm{E}-2$ & 7/02/08 & 8G02005 & PNNL-AGG-415 \\
\hline $14914-62-6$ & Ruthenium 102 & $<2.73 \mathrm{E}-2$ & ug/g dry & 2.73E-2 & $7 / 02 / 08$ & 8G02005 & PNNL-AGG-415 \\
\hline $15766-01-5$ & Ruthenium 104 & $<7.63 \mathrm{E}-2$ & ug/g dry & 7.63E-2 & $7 / 02 / 08$ & 8G02005 & PNNL-AGG-415 \\
\hline 14336-64-2 & Cadmium 111 & 5.87E-2 & ug/g dry & $4.94 \mathrm{E}-2$ & $7 / 02 / 08$ & 8G02005 & PNNL-AGG-415 \\
\hline $14041-58-8$ & Cadmium 114 & 4.17E-2 & ug/g dry & $1.12 \mathrm{E}-2$ & 7/02/08 & 8G02005 & PNNL-AGG-415 \\
\hline $14265-72-6$ & Antimony 121 & $<7.98 \mathrm{E}-2$ & ug/g dry & $7.98 \mathrm{E}-2$ & $7 / 02 / 08$ & 8G02005 & PNNL-AGG-415 \\
\hline HEIS No. & B1VJ58C & \multicolumn{3}{|c|}{ Lab ID: $\quad$ 0805020-10 } & & & \\
\hline $14687-58-2$ & Selenium 82 & $<1.23 \mathrm{E} 0$ & ug/g dry & $1.23 \mathrm{E} 0$ & $7 / 02 / 08$ & 8G02005 & PNNL-AGG-415 \\
\hline $14392-17-7$ & Molybdenum 95 & $7.80 \mathrm{E}-1$ & ug/g dry & $2.51 \mathrm{E}-1$ & $7 / 02 / 08$ & 8G02005 & PNNL-AGG-415 \\
\hline 14392-19-9 & Molybdenum 97 & $7.64 \mathrm{E}-1$ & ug/g dry & $2.30 \mathrm{E}-1$ & $7 / 02 / 08$ & 8G02005 & PNNL-AGG-415 \\
\hline $14392-20-2$ & Molybdenum 98 & 7.63E-1 & ug/g dry & $9.53 \mathrm{E}-2$ & $7 / 02 / 08$ & 8G02005 & PNNL-AGG-415 \\
\hline 14914-61-5 & Ruthenium 101 & $<4.82 \mathrm{E}-2$ & ug/g dry & $4.82 \mathrm{E}-2$ & $7 / 02 / 08$ & 8G02005 & PNNL-AGG-415 \\
\hline $14914-62-6$ & Ruthenium 102 & $<2.91 \mathrm{E}-2$ & ug/g dry & $2.91 \mathrm{E}-2$ & $7 / 02 / 08$ & 8G02005 & PNNL-AGG-415 \\
\hline 15766-01-5 & Ruthenium 104 & $<8.15 \mathrm{E}-2$ & ug/g dry & $8.15 \mathrm{E}-2$ & $7 / 02 / 08$ & $8 G 02005$ & PNNL-AGG-415 \\
\hline 14336-64-2 & Cadmium 111 & $9.42 \mathrm{E}-2$ & ug/g dry & $5.27 \mathrm{E}-2$ & $7 / 02 / 08$ & $8 G 02005$ & PNNL-AGG-415 \\
\hline $14041-58-8$ & Cadmium 114 & 7.21E-2 & ug/g dry & $1.20 \mathrm{E}-2$ & $7 / 02 / 08$ & 8G02005 & PNNL-AGG-415 \\
\hline $14265-72-6$ & Antimony 121 & $<8.52 \mathrm{E}-2$ & ug/g dry & $8.52 \mathrm{E}-2$ & $7 / 02 / 08$ & 8G02005 & PNNL-AGG-415 \\
\hline HEIS No. & B1VJ58B & \multicolumn{3}{|c|}{$\begin{array}{ll}\text { Lab ID: } & \text { 0805020-11 }\end{array}$} & & & \\
\hline $14687-58-2$ & Selenium 82 & $<1.31 \mathrm{E} 0$ & ug/g dry & $1.31 \mathrm{E} 0$ & 7/02/08 & 8G02005 & PNNL-AGG-415 \\
\hline $14392-17-7$ & Molybdenum 95 & $3.03 \mathrm{E}-1$ & ug/g dry & 2.67E-1 & $7 / 02 / 08$ & 8G02005 & PNNL-AGG-415 \\
\hline 14392-19-9 & Molybdenum 97 & $2.91 \mathrm{E}-1$ & ug/g dry & $2.45 \mathrm{E}-1$ & $7 / 02 / 08$ & 8G02005 & PNNL-AGG-415 \\
\hline $14392-20-2$ & Molybdenum 98 & $2.78 \mathrm{E}-1$ & ug/g dry & $1.01 \mathrm{E}-1$ & $7 / 02 / 08$ & $8 G 02005$ & PNNL-AGG-415 \\
\hline $14914-61-5$ & Ruthenium 101 & $<5.13 \mathrm{E}-2$ & ug/g dry & $5.13 \mathrm{E}-2$ & $7 / 02 / 08$ & 8G02005 & PNNL-AGG-415 \\
\hline $14914-62-6$ & Ruthenium 102 & $<3.10 \mathrm{E}-2$ & ug/g dry & $3.10 \mathrm{E}-2$ & $7 / 02 / 08$ & 8G02005 & PNNL-AGG-415 \\
\hline $15766-01-5$ & Ruthenium 104 & $<8.68 \mathrm{E}-2$ & ug/g dry & $8.68 \mathrm{E}-2$ & 7/02/08 & 8G02005 & PNNL-AGG-415 \\
\hline 14336-64-2 & Cadmium 111 & 8.95E-2 & ug/g dry & $5.61 \mathrm{E}-2$ & 7/02/08 & 8G02005 & PNNL-AGG-415 \\
\hline 14041-58-8 & Cadmium 114 & $6.85 \mathrm{E}-2$ & ug/g dry & $1.27 \mathrm{E}-2$ & $7 / 02 / 08$ & 8G02005 & PNNL-AGG-415 \\
\hline $14265-72-6$ & Antimony 121 & $<9.07 \mathrm{E}-2$ & ug/g dry & $9.07 \mathrm{E}-2$ & 7/02/08 & 8G02005 & PNNL-AGG-415 \\
\hline HEIS No. & B1VJ58A & \multicolumn{3}{|c|}{$\begin{array}{ll}\text { Lab ID: } & 0805020-12\end{array}$} & & & \\
\hline $14687-58-2$ & Selenium 82 & $<1.34 \mathrm{E} 0$ & ug/g dry & $1.34 \mathrm{E} 0$ & $7 / 02 / 08$ & 8G02005 & PNNL-AGG-415 \\
\hline 14392-17-7 & Molybdenum 95 & $<2.74 \mathrm{E}-1$ & ug/g dry & $2.74 \mathrm{E}-1$ & $7 / 02 / 08$ & 8G02005 & PNNL-AGG-415 \\
\hline 14392-19-9 & Molybdenum 97 & $<2.51 \mathrm{E}-1$ & ug/g dry & $2.51 \mathrm{E}-1$ & $7 / 02 / 08$ & 8G02005 & PNNL-AGG-415 \\
\hline $14392-20-2$ & Molybdenum 98 & $1.68 \mathrm{E}-1$ & ug/g dry & $1.04 \mathrm{E}-1$ & $7 / 02 / 08$ & 8G02005 & PNNL-AGG-415 \\
\hline 14914-61-5 & Ruthenium 101 & $<5.25 \mathrm{E}-2$ & ug/g dry & $5.25 \mathrm{E}-2$ & $7 / 02 / 08$ & 8G02005 & PNNL-AGG-415 \\
\hline $14914-62-6$ & Ruthenium 102 & $<3.18 \mathrm{E}-2$ & ug/g dry & $3.18 \mathrm{E}-2$ & $7 / 02 / 08$ & 8G02005 & PNNL-AGG-415 \\
\hline $15766-01-5$ & Ruthenium 104 & $<8.88 \mathrm{E}-2$ & ug/g dry & 8.88E-2 & $7 / 02 / 08$ & 8G02005 & PNNL-AGG-415 \\
\hline $14336-64-2$ & Cadmium 111 & $9.41 \mathrm{E}-2$ & ug/g dry & $5.74 \mathrm{E}-2$ & 7/02/08 & 8G02005 & PNNL-AGG-415 \\
\hline $14041-58-8$ & Cadmium 114 & 7.11E-2 & ug/g dry & $1.30 \mathrm{E}-2$ & $7 / 02 / 08$ & 8G02005 & PNNL-AGG-415 \\
\hline $14265-72-6$ & Antimony 121 & $<9.29 \mathrm{E}-2$ & ug/g dry & $9.29 \mathrm{E}-2$ & 7/02/08 & 8G02005 & PNNL-AGG-415 \\
\hline HEIS No. & B1VJ59C & \multicolumn{3}{|c|}{ Lab ID: $\quad$ 0805020-14 } & & & \\
\hline $14687-58-2$ & Selenium 82 & $<1.24 \mathrm{E} 0$ & ug/g dry & $1.24 \mathrm{E} 0$ & $7 / 02 / 08$ & 8G02005 & PNNL-AGG-415 \\
\hline $14392-17-7$ & Molybdenum 95 & $1.27 \mathrm{E} 0$ & ug/g dry & $2.55 \mathrm{E}-1$ & $7 / 02 / 08$ & $8 G 02005$ & PNNL-AGG-415 \\
\hline 14392-19-9 & Molybdenum 97 & $1.25 \mathrm{E} 0$ & ug/g dry & $2.34 \mathrm{E}-1$ & $7 / 02 / 08$ & 8G02005 & PNNL-AGG-415 \\
\hline $14392-20-2$ & Molybdenum 98 & $1.28 \mathrm{E} 0$ & ug/g dry & $9.67 \mathrm{E}-2$ & $7 / 02 / 08$ & $8 G 02005$ & PNNL-AGG-415 \\
\hline $14914-61-5$ & Ruthenium 101 & $<4.89 \mathrm{E}-2$ & ug/g dry & 4.89E-2 & $7 / 02 / 08$ & 8G02005 & PNNL-AGG-415 \\
\hline $14914-62-6$ & Ruthenium 102 & $<2.96 \mathrm{E}-2$ & ug/g dry & $2.96 \mathrm{E}-2$ & $7 / 02 / 08$ & 8G02005 & PNNL-AGG-415 \\
\hline $15766-01-5$ & Ruthenium 104 & $<8.27 \mathrm{E}-2$ & ug/g dry & 8.27E-2 & $7 / 02 / 08$ & 8G02005 & PNNL-AGG-415 \\
\hline 14336-64-2 & Cadmium 111 & $6.25 \mathrm{E}-2$ & ug/g dry & $5.35 \mathrm{E}-2$ & 7/02/08 & 8G02005 & PNNL-AGG-415 \\
\hline
\end{tabular}


RCRA Metals By PNNL-AGG-415/Acid Extract

\begin{tabular}{|c|c|c|c|c|c|c|c|}
\hline CAS \# & Analyte & Results & Units & EQL & Analyzed & Batch & Method \\
\hline HEIS No. & B1VJ59C & \multicolumn{3}{|c|}{ Lab ID: $\quad$ 0805020-14 } & & & \\
\hline $14041-58-8$ & Cadmium 114 & 4.36E-2 & ug/g dry & $1.21 \mathrm{E}-2$ & 7/02/08 & 8G02005 & PNNL-AGG-415 \\
\hline $14265-72-6$ & Antimony 121 & $<8.65 \mathrm{E}-2$ & ug/g dry & 8.65E-2 & 7/02/08 & 8G02005 & PNNL-AGG-415 \\
\hline HEIS No. & B1VJ59B & \multicolumn{3}{|c|}{ Lab ID: $\quad$ 0805020-15 } & & & \\
\hline $14687-58-2$ & Selenium 82 & $<1.18 \mathrm{E} 0$ & ug/g dry & $1.18 \mathrm{E} 0$ & $7 / 02 / 08$ & 8G02005 & PNNL-AGG-415 \\
\hline 14392-17-7 & Molybdenum 95 & $3.04 \mathrm{E}-1$ & ug/g dry & $2.42 \mathrm{E}-1$ & $7 / 02 / 08$ & 8G02005 & PNNL-AGG-415 \\
\hline 14392-19-9 & Molybdenum 97 & $2.94 \mathrm{E}-1$ & ug/g dry & $2.21 \mathrm{E}-1$ & $7 / 02 / 08$ & 8G02005 & PNNL-AGG-415 \\
\hline $14392-20-2$ & Molybdenum 98 & $2.91 \mathrm{E}-1$ & ug/g dry & $9.16 \mathrm{E}-2$ & 7/02/08 & 8G02005 & PNNL-AGG-415 \\
\hline 14914-61-5 & Ruthenium 101 & $<4.64 \mathrm{E}-2$ & ug/g dry & 4.64E-2 & $7 / 02 / 08$ & 8G02005 & PNNL-AGG-415 \\
\hline 14914-62-6 & Ruthenium 102 & $<2.80 \mathrm{E}-2$ & ug/g dry & $2.80 \mathrm{E}-2$ & $7 / 02 / 08$ & 8G02005 & PNNL-AGG-415 \\
\hline $15766-01-5$ & Ruthenium 104 & $<7.83 \mathrm{E}-2$ & ug/g dry & 7.83E-2 & 7/02/08 & 8G02005 & PNNL-AGG-415 \\
\hline 14336-64-2 & Cadmium 111 & $<5.07 \mathrm{E}-2$ & ug/g dry & 5.07E-2 & $7 / 02 / 08$ & 8G02005 & PNNL-AGG-415 \\
\hline $14041-58-8$ & Cadmium 114 & $3.51 \mathrm{E}-2$ & ug/g dry & $1.15 \mathrm{E}-2$ & $7 / 02 / 08$ & 8G02005 & PNNL-AGG-415 \\
\hline $14265-72-6$ & Antimony 121 & $<8.19 \mathrm{E}-2$ & ug/g dry & $8.19 \mathrm{E}-2$ & 7/02/08 & 8G02005 & PNNL-AGG-415 \\
\hline HEIS No. & B1VJ59A & \multicolumn{3}{|c|}{ Lab ID: $\quad$ 0805020-16 } & & & \\
\hline $14687-58-2$ & Selenium 82 & $<1.17 \mathrm{E} 0$ & ug/g dry & $1.17 \mathrm{E} 0$ & $7 / 02 / 08$ & 8G02005 & PNNL-AGG-415 \\
\hline 14392-17-7 & Molybdenum 95 & 3.36E-1 & ug/g dry & 2.39E-1 & $7 / 02 / 08$ & 8G02005 & PNNL-AGG-415 \\
\hline 14392-19-9 & Molybdenum 97 & $3.29 \mathrm{E}-1$ & ug/g dry & $2.19 \mathrm{E}-1$ & 7/02/08 & 8G02005 & PNNL-AGG-415 \\
\hline $14392-20-2$ & Molybdenum 98 & 3.26E-1 & ug/g dry & $9.06 \mathrm{E}-2$ & 7/02/08 & 8G02005 & PNNL-AGG-415 \\
\hline 14914-61-5 & Ruthenium 101 & $<4.59 \mathrm{E}-2$ & ug/g dry & $4.59 \mathrm{E}-2$ & 7/02/08 & 8G02005 & PNNL-AGG-415 \\
\hline $14914-62-6$ & Ruthenium 102 & $<2.77 \mathrm{E}-2$ & ug/g dry & 2.77E-2 & $7 / 02 / 08$ & 8G02005 & PNNL-AGG-415 \\
\hline 15766-01-5 & Ruthenium 104 & $<7.75 \mathrm{E}-2$ & ug/g dry & 7.75E-2 & 7/02/08 & 8G02005 & PNNL-AGG-415 \\
\hline 14336-64-2 & Cadmium 111 & $<5.01 \mathrm{E}-2$ & ug/g dry & $5.01 \mathrm{E}-2$ & 7/02/08 & 8G02005 & PNNL-AGG-415 \\
\hline 14041-58-8 & Cadmium 114 & 3.47E-2 & ug/g dry & $1.14 \mathrm{E}-2$ & $7 / 02 / 08$ & 8G02005 & PNNL-AGG-415 \\
\hline $14265-72-6$ & Antimony 121 & $<8.10 \mathrm{E}-2$ & ug/g dry & $8.10 \mathrm{E}-2$ & 7/02/08 & 8G02005 & PNNL-AGG-415 \\
\hline HEIS No. & B1VJ60C & \multicolumn{3}{|c|}{ Lab ID: $\quad$ 0805020-18 } & & & \\
\hline $14687-58-2$ & Selenium 82 & $<1.12 \mathrm{E} 0$ & ug/g dry & $1.12 \mathrm{E} 0$ & 7/02/08 & 8G02005 & PNNL-AGG-415 \\
\hline $14392-17-7$ & Molybdenum 95 & 2.55E0 & ug/g dry & $2.30 \mathrm{E}-1$ & $7 / 02 / 08$ & 8G02005 & PNNL-AGG-415 \\
\hline 14392-19-9 & Molybdenum 97 & $2.56 \mathrm{E} 0$ & ug/g dry & $2.11 \mathrm{E}-1$ & 7/02/08 & 8G02005 & PNNL-AGG-415 \\
\hline $14392-20-2$ & Molybdenum 98 & $2.58 \mathrm{E} 0$ & ug/g dry & 8.74E-2 & $7 / 02 / 08$ & 8G02005 & PNNL-AGG-415 \\
\hline 14914-61-5 & Ruthenium 101 & $<4.42 \mathrm{E}-2$ & ug/g dry & $4.42 \mathrm{E}-2$ & 7/02/08 & 8G02005 & PNNL-AGG-415 \\
\hline $14914-62-6$ & Ruthenium 102 & $<2.67 \mathrm{E}-2$ & ug/g dry & $2.67 \mathrm{E}-2$ & $7 / 02 / 08$ & 8G02005 & PNNL-AGG-415 \\
\hline $15766-01-5$ & Ruthenium 104 & $<7.47 \mathrm{E}-2$ & ug/g dry & 7.47E-2 & $7 / 02 / 08$ & $8 G 02005$ & PNNL-AGG-415 \\
\hline 14336-64-2 & Cadmium 111 & 6.13E-2 & ug/g dry & 4.83E-2 & 7/02/08 & 8G02005 & PNNL-AGG-415 \\
\hline 14041-58-8 & Cadmium 114 & 4.93E-2 & ug/g dry & $1.10 \mathrm{E}-2$ & $7 / 02 / 08$ & 8G02005 & PNNL-AGG-415 \\
\hline $14265-72-6$ & Antimony 121 & $<7.82 \mathrm{E}-2$ & ug/g dry & $7.82 \mathrm{E}-2$ & $7 / 02 / 08$ & 8G02005 & PNNL-AGG-415 \\
\hline HEIS No. & B1VJ60B & \multicolumn{3}{|c|}{ Lab ID: $\quad$ 0805020-19 } & & & \\
\hline $14687-58-2$ & Selenium 82 & $<1.19 \mathrm{E} 0$ & ug/g dry & 1.19E0 & $7 / 02 / 08$ & 8G02005 & PNNL-AGG-415 \\
\hline 14392-17-7 & Molybdenum 95 & 2.69E-1 & ug/g dry & 2.43E-1 & $7 / 02 / 08$ & 8G02005 & PNNL-AGG-415 \\
\hline 14392-19-9 & Molybdenum 97 & $2.53 \mathrm{E}-1$ & ug/g dry & 2.23E-1 & 7/02/08 & 8G02005 & PNNL-AGG-415 \\
\hline $14392-20-2$ & Molybdenum 98 & 2.47E-1 & ug/g dry & $9.24 \mathrm{E}-2$ & 7/02/08 & 8G02005 & PNNL-AGG-415 \\
\hline 14914-61-5 & Ruthenium 101 & $<4.67 \mathrm{E}-2$ & ug/g dry & 4.67E-2 & 7/02/08 & 8G02005 & PNNL-AGG-415 \\
\hline 14914-62-6 & Ruthenium 102 & $<2.83 \mathrm{E}-2$ & ug/g dry & $2.83 \mathrm{E}-2$ & 7/02/08 & 8G02005 & PNNL-AGG-415 \\
\hline 15766-01-5 & Ruthenium 104 & $<7.90 \mathrm{E}-2$ & ug/g dry & $7.90 \mathrm{E}-2$ & 7/02/08 & 8G02005 & PNNL-AGG-415 \\
\hline $14336-64-2$ & Cadmium 111 & $5.42 \mathrm{E}-2$ & ug/g dry & $5.11 \mathrm{E}-2$ & 7/02/08 & 8G02005 & PNNL-AGG-415 \\
\hline 14041-58-8 & Cadmium 114 & 3.92E-2 & ug/g dry & $1.16 \mathrm{E}-2$ & 7/02/08 & 8G02005 & PNNL-AGG-415 \\
\hline $14265-72-6$ & Antimony 121 & $<8.26 \mathrm{E}-2$ & ug/g dry & 8.26E-2 & $7 / 02 / 08$ & $8 G 02005$ & PNNL-AGG-415 \\
\hline HEIS No. & B1VJ60A & & ID: & $5020-20$ & & & \\
\hline 14687-58-2 & Selenium 82 & $<1.17 \mathrm{E} 0$ & ug/g dry & $1.17 \mathrm{E} 0$ & 7/02/08 & 8G02005 & PNNL-AGG-415 \\
\hline
\end{tabular}


RCRA Metals By PNNL-AGG-415/Acid Extract

\begin{tabular}{|c|c|c|c|c|c|c|c|}
\hline CAS \# & Analyte & Results & Units & EQL & Analyzed & Batch & Method \\
\hline HEIS No. & B1VJ60A & \multicolumn{3}{|c|}{ Lab ID: $\quad$ 0805020-20 } & & & \\
\hline $14392-17-7$ & Molybdenum 95 & $2.90 \mathrm{E}-1$ & ug/g dry & $2.39 \mathrm{E}-1$ & $7 / 02 / 08$ & 8G02005 & PNNL-AGG-415 \\
\hline 14392-19-9 & Molybdenum 97 & 2.77E-1 & ug/g dry & 2.19E-1 & 7/02/08 & 8G02005 & PNNL-AGG-415 \\
\hline $14392-20-2$ & Molybdenum 98 & $2.78 \mathrm{E}-1$ & ug/g dry & $9.06 \mathrm{E}-2$ & $7 / 02 / 08$ & 8G02005 & PNNL-AGG-415 \\
\hline $14914-61-5$ & Ruthenium 101 & $<4.58 \mathrm{E}-2$ & ug/g dry & $4.58 \mathrm{E}-2$ & $7 / 02 / 08$ & 8G02005 & PNNL-AGG-415 \\
\hline $14914-62-6$ & Ruthenium 102 & $<2.77 \mathrm{E}-2$ & ug/g dry & $2.77 \mathrm{E}-2$ & 7/02/08 & 8G02005 & PNNL-AGG-415 \\
\hline 15766-01-5 & Ruthenium 104 & $<7.75 \mathrm{E}-2$ & ug/g dry & 7.75E-2 & $7 / 02 / 08$ & 8G02005 & PNNL-AGG-415 \\
\hline $14336-64-2$ & Cadmium 111 & $5.55 \mathrm{E}-2$ & ug/g dry & $5.01 \mathrm{E}-2$ & $7 / 02 / 08$ & 8G02005 & PNNL-AGG-415 \\
\hline 14041-58-8 & Cadmium 114 & 3.77E-2 & ug/g dry & $1.14 \mathrm{E}-2$ & $7 / 02 / 08$ & 8G02005 & PNNL-AGG-415 \\
\hline $14265-72-6$ & Antimony 121 & $<8.10 \mathrm{E}-2$ & ug/g dry & $8.10 \mathrm{E}-2$ & $7 / 02 / 08$ & 8G02005 & PNNL-AGG-415 \\
\hline HEIS No. & B1VJ61C & \multicolumn{3}{|c|}{$\begin{array}{ll}\text { Lab ID: } & \text { 0805020-22 }\end{array}$} & & & \\
\hline $14687-58-2$ & Selenium 82 & $<1.05 \mathrm{E} 0$ & ug/g dry & $1.05 \mathrm{E} 0$ & $7 / 02 / 08$ & 8G02005 & PNNL-AGG-415 \\
\hline $14392-17-7$ & Molybdenum 95 & 8.64E-1 & ug/g dry & $2.14 \mathrm{E}-1$ & $7 / 02 / 08$ & 8G02005 & PNNL-AGG-415 \\
\hline $14392-19-9$ & Molybdenum 97 & $8.31 \mathrm{E}-1$ & ug/g dry & $1.96 \mathrm{E}-1$ & 7/02/08 & 8G02005 & PNNL-AGG-415 \\
\hline $14392-20-2$ & Molybdenum 98 & $8.56 \mathrm{E}-1$ & ug/g dry & 8.13E-2 & $7 / 02 / 08$ & $8 G 02005$ & PNNL-AGG-415 \\
\hline 14914-61-5 & Ruthenium 101 & $<4.11 \mathrm{E}-2$ & ug/g dry & $4.11 \mathrm{E}-2$ & $7 / 02 / 08$ & $8 G 02005$ & PNNL-AGG-415 \\
\hline $14914-62-6$ & Ruthenium 102 & $<2.49 \mathrm{E}-2$ & ug/g dry & 2.49E-2 & $7 / 02 / 08$ & 8G02005 & PNNL-AGG-415 \\
\hline $15766-01-5$ & Ruthenium 104 & $<6.95 \mathrm{E}-2$ & ug/g dry & $6.95 \mathrm{E}-2$ & $7 / 02 / 08$ & $8 G 02005$ & PNNL-AGG-415 \\
\hline 14336-64-2 & Cadmium 111 & 5.47E-2 & ug/g dry & 4.49E-2 & $7 / 02 / 08$ & 8G02005 & PNNL-AGG-415 \\
\hline 14041-58-8 & Cadmium 114 & 3.63E-2 & ug/g dry & $1.02 \mathrm{E}-2$ & $7 / 02 / 08$ & 8G02005 & PNNL-AGG-415 \\
\hline $14265-72-6$ & Antimony 121 & $<7.26 \mathrm{E}-2$ & ug/g dry & 7.26E-2 & $7 / 02 / 08$ & $8 G 02005$ & PNNL-AGG-415 \\
\hline HEIS No. & B1VJ61B & \multicolumn{3}{|c|}{$\begin{array}{ll}\text { Lab ID: } & 0805020-23\end{array}$} & & & \\
\hline $14687-58-2$ & Selenium 82 & $<1.02 \mathrm{E} 0$ & ug/g dry & $1.02 \mathrm{E} 0$ & $7 / 02 / 08$ & 8G02005 & PNNL-AGG-415 \\
\hline $14392-17-7$ & Molybdenum 95 & $2.57 \mathrm{E}-1$ & ug/g dry & $2.08 \mathrm{E}-1$ & $7 / 02 / 08$ & $8 G 02005$ & PNNL-AGG-415 \\
\hline 14392-19-9 & Molybdenum 97 & $2.58 \mathrm{E}-1$ & ug/g dry & $1.91 \mathrm{E}-1$ & $7 / 02 / 08$ & 8G02005 & PNNL-AGG-415 \\
\hline $14392-20-2$ & Molybdenum 98 & $2.50 \mathrm{E}-1$ & ug/g dry & $7.91 \mathrm{E}-2$ & 7/02/08 & 8G02005 & PNNL-AGG-415 \\
\hline $14914-61-5$ & Ruthenium 101 & $<4.00 \mathrm{E}-2$ & ug/g dry & 4.00E-2 & $7 / 02 / 08$ & 8G02005 & PNNL-AGG-415 \\
\hline 14914-62-6 & Ruthenium 102 & $<2.42 \mathrm{E}-2$ & ug/g dry & 2.42E-2 & $7 / 02 / 08$ & 8G02005 & PNNL-AGG-415 \\
\hline $15766-01-5$ & Ruthenium 104 & $<6.76 \mathrm{E}-2$ & ug/g dry & $6.76 \mathrm{E}-2$ & $7 / 02 / 08$ & 8G02005 & PNNL-AGG-415 \\
\hline $14336-64-2$ & Cadmium 111 & 4.57E-2 & ug/g dry & 4.37E-2 & $7 / 02 / 08$ & 8G02005 & PNNL-AGG-415 \\
\hline 14041-58-8 & Cadmium 114 & $3.26 \mathrm{E}-2$ & ug/g dry & $9.92 \mathrm{E}-3$ & $7 / 02 / 08$ & 8G02005 & PNNL-AGG-415 \\
\hline $14265-72-6$ & Antimony 121 & $<7.07 \mathrm{E}-2$ & ug/g dry & 7.07E-2 & 7/02/08 & 8G02005 & PNNL-AGG-415 \\
\hline HEIS No. & B1VJ61A & \multicolumn{3}{|c|}{ Lab ID: $\quad$ 0805020-24 } & & & \\
\hline $14687-58-2$ & Selenium 82 & $<1.13 \mathrm{E} 0$ & ug/g dry & 1.13E0 & 7/02/08 & 8G02005 & PNNL-AGG-415 \\
\hline $14392-17-7$ & Molybdenum 95 & 2.39E-1 & ug/g dry & 2.32E-1 & 7/02/08 & 8G02005 & PNNL-AGG-415 \\
\hline $14392-19-9$ & Molybdenum 97 & $2.27 \mathrm{E}-1$ & ug/g dry & $2.12 \mathrm{E}-1$ & $7 / 02 / 08$ & 8G02005 & PNNL-AGG-415 \\
\hline 14392-20-2 & Molybdenum 98 & $2.26 \mathrm{E}-1$ & ug/g dry & 8.79E-2 & $7 / 02 / 08$ & 8G02005 & PNNL-AGG-415 \\
\hline $14914-61-5$ & Ruthenium 101 & $<4.45 \mathrm{E}-2$ & ug/g dry & 4.45E-2 & 7/02/08 & 8G02005 & PNNL-AGG-415 \\
\hline $14914-62-6$ & Ruthenium 102 & $<2.69 \mathrm{E}-2$ & ug/g dry & $2.69 \mathrm{E}-2$ & $7 / 02 / 08$ & 8G02005 & PNNL-AGG-415 \\
\hline 15766-01-5 & Ruthenium 104 & $<7.51 \mathrm{E}-2$ & ug/g dry & $7.51 \mathrm{E}-2$ & $7 / 02 / 08$ & 8G02005 & PNNL-AGG-415 \\
\hline $14336-64-2$ & Cadmium 111 & 5.49E-2 & ug/g dry & $4.86 \mathrm{E}-2$ & 7/02/08 & 8G02005 & PNNL-AGG-415 \\
\hline 14041-58-8 & Cadmium 114 & $4.21 \mathrm{E}-2$ & ug/g dry & $1.10 \mathrm{E}-2$ & 7/02/08 & 8G02005 & PNNL-AGG-415 \\
\hline $14265-72-6$ & Antimony 121 & $<7.86 \mathrm{E}-2$ & ug/g dry & $7.86 \mathrm{E}-2$ & 7/02/08 & 8G02005 & PNNL-AGG-415 \\
\hline HEIS No. & B1VJ62C & \multicolumn{3}{|c|}{$\begin{array}{ll}\text { Lab ID: } & 0805020-26\end{array}$} & & & \\
\hline $14687-58-2$ & Selenium 82 & $<1.19 \mathrm{E} 0$ & ug/g dry & 1.19E0 & $7 / 02 / 08$ & 8G02005 & PNNL-AGG-415 \\
\hline $14392-17-7$ & Molybdenum 95 & $2.49 \mathrm{E}-1$ & ug/g dry & $2.44 \mathrm{E}-1$ & $7 / 02 / 08$ & 8G02005 & PNNL-AGG-415 \\
\hline 14392-19-9 & Molybdenum 97 & 2.49E-1 & ug/g dry & 2.23E-1 & $7 / 02 / 08$ & 8G02005 & PNNL-AGG-415 \\
\hline $14392-20-2$ & Molybdenum 98 & $2.46 \mathrm{E}-1$ & ug/g dry & $9.25 \mathrm{E}-2$ & $7 / 02 / 08$ & 8G02005 & PNNL-AGG-415 \\
\hline $14914-61-5$ & Ruthenium 101 & $<4.68 \mathrm{E}-2$ & ug/g dry & 4.68E-2 & $7 / 02 / 08$ & 8G02005 & PNNL-AGG-415 \\
\hline
\end{tabular}


RCRA Metals By PNNL-AGG-415/Acid Extract

\begin{tabular}{|c|c|c|c|c|c|c|c|}
\hline CAS \# & Analyte & Results & Units & EQL & Analyzed & Batch & Method \\
\hline HEIS No. & B1VJ62C & \multicolumn{3}{|c|}{ Lab ID: $\quad$ 0805020-26 } & & & \\
\hline $14914-62-6$ & Ruthenium 102 & $<2.83 \mathrm{E}-2$ & ug/g dry & $2.83 \mathrm{E}-2$ & 7/02/08 & 8G02005 & PNNL-AGG-415 \\
\hline $15766-01-5$ & Ruthenium 104 & $<7.91 \mathrm{E}-2$ & ug/g dry & $7.91 \mathrm{E}-2$ & 7/02/08 & 8G02005 & PNNL-AGG-415 \\
\hline 14336-64-2 & Cadmium 111 & 5.83E-2 & ug/g dry & $5.11 \mathrm{E}-2$ & $7 / 02 / 08$ & 8G02005 & PNNL-AGG-415 \\
\hline $14041-58-8$ & Cadmium 114 & 4.05E-2 & ug/g dry & $1.16 \mathrm{E}-2$ & $7 / 02 / 08$ & 8G02005 & PNNL-AGG-415 \\
\hline $14265-72-6$ & Antimony 121 & $<8.27 \mathrm{E}-2$ & ug/g dry & $8.27 \mathrm{E}-2$ & 7/02/08 & 8G02005 & PNNL-AGG-415 \\
\hline HEIS No. & B1VJ62B & \multicolumn{3}{|c|}{$\begin{array}{ll}\text { Lab ID: } & 0805020-27\end{array}$} & & & \\
\hline $14687-58-2$ & Selenium 82 & $<1.22 \mathrm{E} 0$ & ug/g dry & $1.22 \mathrm{E} 0$ & $7 / 02 / 08$ & 8G02007 & PNNL-AGG-415 \\
\hline $14392-17-7$ & Molybdenum 95 & $2.66 \mathrm{E}-1$ & ug/g dry & $2.50 \mathrm{E}-1$ & $7 / 02 / 08$ & 8G02007 & PNNL-AGG-415 \\
\hline 14392-19-9 & Molybdenum 97 & $2.64 \mathrm{E}-1$ & ug/g dry & $2.29 \mathrm{E}-1$ & $7 / 02 / 08$ & 8G02007 & PNNL-AGG-415 \\
\hline $14392-20-2$ & Molybdenum 98 & $2.56 \mathrm{E}-1$ & ug/g dry & $9.50 \mathrm{E}-2$ & $7 / 02 / 08$ & 8G02007 & PNNL-AGG-415 \\
\hline 14914-61-5 & Ruthenium 101 & $<4.81 \mathrm{E}-2$ & ug/g dry & $4.81 \mathrm{E}-2$ & $7 / 02 / 08$ & 8G02007 & PNNL-AGG-415 \\
\hline $14914-62-6$ & Ruthenium 102 & $<2.91 \mathrm{E}-2$ & ug/g dry & $2.91 \mathrm{E}-2$ & $7 / 02 / 08$ & 8G02007 & PNNL-AGG-415 \\
\hline $15766-01-5$ & Ruthenium 104 & $<8.12 \mathrm{E}-2$ & ug/g dry & $8.12 \mathrm{E}-2$ & $7 / 02 / 08$ & 8G02007 & PNNL-AGG-415 \\
\hline 14336-64-2 & Cadmium 111 & $<5.25 \mathrm{E}-2$ & ug/g dry & $5.25 \mathrm{E}-2$ & $7 / 02 / 08$ & $8 G 02007$ & PNNL-AGG-415 \\
\hline 14041-58-8 & Cadmium 114 & 3.67E-2 & ug/g dry & 1.19E-2 & $7 / 02 / 08$ & 8G02007 & PNNL-AGG-415 \\
\hline $14265-72-6$ & Antimony 121 & $<8.49 \mathrm{E}-2$ & ug/g dry & $8.49 \mathrm{E}-2$ & $7 / 02 / 08$ & 8G02007 & PNNL-AGG-415 \\
\hline HEIS No. & B1VJ62A & \multicolumn{3}{|c|}{$\begin{array}{ll}\text { Lab ID: } & 0805020-28\end{array}$} & & & \\
\hline $14687-58-2$ & Selenium 82 & $<1.24 \mathrm{E} 0$ & ug/g dry & $1.24 \mathrm{E} 0$ & $7 / 02 / 08$ & 8G02007 & PNNL-AGG-415 \\
\hline $14392-17-7$ & Molybdenum 95 & $<2.53 \mathrm{E}-1$ & ug/g dry & 2.53E-1 & $7 / 02 / 08$ & 8G02007 & PNNL-AGG-415 \\
\hline 14392-19-9 & Molybdenum 97 & $<2.32 \mathrm{E}-1$ & ug/g dry & 2.32E-1 & $7 / 02 / 08$ & 8G02007 & PNNL-AGG-415 \\
\hline $14392-20-2$ & Molybdenum 98 & $1.94 \mathrm{E}-1$ & ug/g dry & $9.62 \mathrm{E}-2$ & $7 / 02 / 08$ & 8G02007 & PNNL-AGG-415 \\
\hline $14914-61-5$ & Ruthenium 101 & $<4.87 \mathrm{E}-2$ & ug/g dry & $4.87 \mathrm{E}-2$ & $7 / 02 / 08$ & 8G02007 & PNNL-AGG-415 \\
\hline $14914-62-6$ & Ruthenium 102 & $<2.94 \mathrm{E}-2$ & ug/g dry & $2.94 \mathrm{E}-2$ & $7 / 02 / 08$ & 8G02007 & PNNL-AGG-415 \\
\hline $15766-01-5$ & Ruthenium 104 & $<8.22 \mathrm{E}-2$ & ug/g dry & $8.22 \mathrm{E}-2$ & $7 / 02 / 08$ & 8G02007 & PNNL-AGG-415 \\
\hline $14336-64-2$ & Cadmium 111 & $5.62 \mathrm{E}-2$ & ug/g dry & $5.32 \mathrm{E}-2$ & 7/02/08 & 8G02007 & PNNL-AGG-415 \\
\hline $14041-58-8$ & Cadmium 114 & 4.17E-2 & ug/g dry & $1.21 \mathrm{E}-2$ & $7 / 02 / 08$ & $8 G 02007$ & PNNL-AGG-415 \\
\hline $14265-72-6$ & Antimony 121 & $<8.60 \mathrm{E}-2$ & ug/g dry & $8.60 \mathrm{E}-2$ & 7/02/08 & 8G02007 & PNNL-AGG-415 \\
\hline HEIS No. & B1VJ64C & \multicolumn{3}{|c|}{$\begin{array}{ll}\text { Lab ID: } & 0805020-30\end{array}$} & & & \\
\hline $14687-58-2$ & Selenium 82 & $<1.25 \mathrm{E} 0$ & ug/g dry & $1.25 \mathrm{E} 0$ & $7 / 02 / 08$ & 8G02007 & PNNL-AGG-415 \\
\hline $14392-17-7$ & Molybdenum 95 & $5.25 \mathrm{E}-1$ & ug/g dry & $2.56 \mathrm{E}-1$ & $7 / 02 / 08$ & 8G02007 & PNNL-AGG-415 \\
\hline 14392-19-9 & Molybdenum 97 & $5.04 \mathrm{E}-1$ & ug/g dry & $2.35 \mathrm{E}-1$ & $7 / 02 / 08$ & 8G02007 & PNNL-AGG-415 \\
\hline $14392-20-2$ & Molybdenum 98 & 5.10E-1 & ug/g dry & $9.72 \mathrm{E}-2$ & $7 / 02 / 08$ & 8G02007 & PNNL-AGG-415 \\
\hline 14914-61-5 & Ruthenium 101 & $<4.92 \mathrm{E}-2$ & ug/g dry & $4.92 \mathrm{E}-2$ & $7 / 02 / 08$ & 8G02007 & PNNL-AGG-415 \\
\hline $14914-62-6$ & Ruthenium 102 & $<2.97 \mathrm{E}-2$ & ug/g dry & 2.97E-2 & $7 / 02 / 08$ & 8G02007 & PNNL-AGG-415 \\
\hline $15766-01-5$ & Ruthenium 104 & $<8.31 \mathrm{E}-2$ & ug/g dry & $8.31 \mathrm{E}-2$ & $7 / 02 / 08$ & 8G02007 & PNNL-AGG-415 \\
\hline 14336-64-2 & Cadmium 111 & 6.33E-2 & ug/g dry & 5.37E-2 & $7 / 02 / 08$ & 8G02007 & PNNL-AGG-415 \\
\hline $14041-58-8$ & Cadmium 114 & 4.36E-2 & ug/g dry & $1.22 \mathrm{E}-2$ & 7/02/08 & 8G02007 & PNNL-AGG-415 \\
\hline $14265-72-6$ & Antimony 121 & $<8.69 \mathrm{E}-2$ & ug/g dry & $8.69 \mathrm{E}-2$ & $7 / 02 / 08$ & 8G02007 & PNNL-AGG-415 \\
\hline HEIS No. & B1VJ64B & \multicolumn{3}{|c|}{ Lab ID: $\quad$ 0805020-31 } & & & \\
\hline $14687-58-2$ & Selenium 82 & $<1.11 \mathrm{E} 0$ & ug/g dry & $1.11 \mathrm{E} 0$ & $7 / 02 / 08$ & 8G02007 & PNNL-AGG-415 \\
\hline $14392-17-7$ & Molybdenum 95 & $4.61 \mathrm{E}-1$ & ug/g dry & $2.27 \mathrm{E}-1$ & $7 / 02 / 08$ & 8G02007 & PNNL-AGG-415 \\
\hline 14392-19-9 & Molybdenum 97 & $4.52 \mathrm{E}-1$ & ug/g dry & $2.08 \mathrm{E}-1$ & $7 / 02 / 08$ & 8G02007 & PNNL-AGG-415 \\
\hline $14392-20-2$ & Molybdenum 98 & $4.48 \mathrm{E}-1$ & ug/g dry & 8.63E-2 & $7 / 02 / 08$ & 8G02007 & PNNL-AGG-415 \\
\hline $14914-61-5$ & Ruthenium 101 & $<4.36 \mathrm{E}-2$ & ug/g dry & 4.36E-2 & $7 / 02 / 08$ & 8G02007 & PNNL-AGG-415 \\
\hline $14914-62-6$ & Ruthenium 102 & $<2.64 \mathrm{E}-2$ & ug/g dry & $2.64 \mathrm{E}-2$ & $7 / 02 / 08$ & 8G02007 & PNNL-AGG-415 \\
\hline $15766-01-5$ & Ruthenium 104 & $<7.38 \mathrm{E}-2$ & ug/g dry & 7.38E-2 & $7 / 02 / 08$ & 8G02007 & PNNL-AGG-415 \\
\hline $14336-64-2$ & Cadmium 111 & $5.11 \mathrm{E}-2$ & ug/g dry & 4.77E-2 & $7 / 02 / 08$ & 8G02007 & PNNL-AGG-415 \\
\hline 14041-58-8 & Cadmium 114 & 3.92E-2 & ug/g dry & $1.08 \mathrm{E}-2$ & 7/02/08 & 8G02007 & PNNL-AGG-415 \\
\hline
\end{tabular}


RCRA Metals By PNNL-AGG-415/Acid Extract

\begin{tabular}{|c|c|c|c|c|c|c|c|}
\hline CAS \# & Analyte & Results & Units & EQL & Analyzed & Batch & Method \\
\hline HEIS No. & B1VJ64B & \multicolumn{3}{|c|}{ Lab ID: $\quad$ 0805020-31 } & & & \\
\hline $14265-72-6$ & Antimony 121 & $<7.71 \mathrm{E}-2$ & ug/g dry & 7.71E-2 & $7 / 02 / 08$ & 8G02007 & PNNL-AGG-415 \\
\hline HEIS No. & B1VJ64A & \multicolumn{3}{|c|}{ Lab ID: $\quad$ 0805020-32 } & & & \\
\hline $14687-58-2$ & Selenium 82 & $<1.18 \mathrm{E} 0$ & ug/g dry & $1.18 \mathrm{E} 0$ & $7 / 02 / 08$ & $8 G 02007$ & PNNL-AGG-415 \\
\hline 14392-17-7 & Molybdenum 95 & $<2.41 \mathrm{E}-1$ & ug/g dry & $2.41 \mathrm{E}-1$ & $7 / 02 / 08$ & 8G02007 & PNNL-AGG-415 \\
\hline 14392-19-9 & Molybdenum 97 & $2.21 \mathrm{E}-1$ & ug/g dry & 2.21E-1 & $7 / 02 / 08$ & 8G02007 & PNNL-AGG-415 \\
\hline $14392-20-2$ & Molybdenum 98 & $2.15 \mathrm{E}-1$ & ug/g dry & $9.16 \mathrm{E}-2$ & $7 / 02 / 08$ & 8G02007 & PNNL-AGG-415 \\
\hline $14914-61-5$ & Ruthenium 101 & $<4.63 \mathrm{E}-2$ & ug/g dry & 4.63E-2 & $7 / 02 / 08$ & 8G02007 & PNNL-AGG-415 \\
\hline $14914-62-6$ & Ruthenium 102 & $<2.80 \mathrm{E}-2$ & ug/g dry & $2.80 \mathrm{E}-2$ & $7 / 02 / 08$ & 8G02007 & PNNL-AGG-415 \\
\hline $15766-01-5$ & Ruthenium 104 & $<7.83 \mathrm{E}-2$ & ug/g dry & 7.83E-2 & 7/02/08 & 8G02007 & PNNL-AGG-415 \\
\hline $14336-64-2$ & Cadmium 111 & $5.48 \mathrm{E}-2$ & ug/g dry & $5.06 \mathrm{E}-2$ & $7 / 02 / 08$ & 8G02007 & PNNL-AGG-415 \\
\hline $14041-58-8$ & Cadmium 114 & $3.74 \mathrm{E}-2$ & ug/g dry & $1.15 \mathrm{E}-2$ & $7 / 02 / 08$ & 8G02007 & PNNL-AGG-415 \\
\hline $14265-72-6$ & Antimony 121 & $<8.19 \mathrm{E}-2$ & ug/g dry & 8.19E-2 & $7 / 02 / 08$ & 8G02007 & PNNL-AGG-415 \\
\hline
\end{tabular}




\section{Carbon Analysis/Soil}

\begin{tabular}{|c|c|c|c|c|c|}
\hline $\begin{array}{l}\text { Total Org } \\
\text { Lab ID }\end{array}$ & $\begin{array}{c}\text { c Carbon } \\
\text { HEIS No. }\end{array}$ & $\begin{array}{l}\text { D01 } \\
\text { Results }\end{array}$ & EQL & Analyzed & Batch \\
\hline 0805020-01 & B1VJ54B & 7.97E2 & $2.00 \mathrm{E} 2$ & $7 / 01 / 08$ & [CALC] \\
\hline 0805020-05 & B1VJ55C & 6.36E2 & $2.00 \mathrm{E} 2$ & 7/01/08 & [CALC] \\
\hline 0805020-07 & B1VJ56A & $2.94 \mathrm{E} 2$ & $2.00 \mathrm{E} 2$ & 7/01/08 & [CALC] \\
\hline 0805020-08 & B1VJ56B & 3.53E2 & $2.00 \mathrm{E} 2$ & 7/01/08 & [CALC] \\
\hline 0805020-09 & B1VJ56C & $6.70 \mathrm{E} 2$ & $2.00 \mathrm{E} 2$ & 7/01/08 & [CALC] \\
\hline 0805020-10 & B1VJ58C & 8.88E2 & $2.00 \mathrm{E} 2$ & 7/01/08 & [CALC] \\
\hline 0805020-11 & B1VJ58B & 6.65E2 & $2.00 \mathrm{E} 2$ & 7/01/08 & [CALC] \\
\hline 0805020-12 & B1VJ58A & 8.46E2 & $2.00 \mathrm{E} 2$ & 7/01/08 & [CALC] \\
\hline 0805020-14 & B1VJ59C & 5.55E2 & $2.00 \mathrm{E} 2$ & 7/01/08 & [CALC] \\
\hline 0805020-15 & B1VJ59B & $3.10 \mathrm{E} 2$ & $2.00 \mathrm{E} 2$ & 7/01/08 & [CALC] \\
\hline 0805020-16 & B1VJ59A & 2.62E2 & $2.00 \mathrm{E} 2$ & 7/02/08 & [CALC] \\
\hline 0805020-18 & B1VJ60C & $9.71 \mathrm{E} 2$ & $2.00 \mathrm{E} 2$ & $7 / 02 / 08$ & [CALC] \\
\hline 0805020-19 & B1VJ60B & $<2.00 \mathrm{E} 2$ & $2.00 \mathrm{E} 2$ & $7 / 02 / 08$ & [CALC] \\
\hline 0805020-20 & B1VJ60A & 2.36E2 & $2.00 \mathrm{E} 2$ & $7 / 02 / 08$ & [CALC] \\
\hline 0805020-22 & B1VJ61C & 6.93E2 & $2.00 \mathrm{E} 2$ & $7 / 02 / 08$ & [CALC] \\
\hline 0805020-23 & B1VJ61B & 3.07E2 & $2.00 \mathrm{E} 2$ & $7 / 02 / 08$ & [CALC] \\
\hline 0805020-24 & B1VJ61A & $2.40 \mathrm{E} 2$ & $2.00 \mathrm{E} 2$ & $7 / 02 / 08$ & [CALC] \\
\hline 0805020-26 & B1VJ62C & 3.66E2 & $2.00 \mathrm{E} 2$ & $7 / 02 / 08$ & [CALC] \\
\hline 0805020-27 & B1VJ62B & $<2.00 \mathrm{E} 2$ & $2.00 \mathrm{E} 2$ & $7 / 02 / 08$ & [CALC] \\
\hline 0805020-28 & B1VJ62A & $4.01 \mathrm{E} 2$ & $2.00 \mathrm{E} 2$ & $7 / 02 / 08$ & [CALC] \\
\hline 0805020-30 & B1VJ64C & $3.70 \mathrm{E} 2$ & $2.00 \mathrm{E} 2$ & $7 / 02 / 08$ & [CALC] \\
\hline 0805020-31 & B1VJ64B & 4.13E2 & $2.00 \mathrm{E} 2$ & $7 / 02 / 08$ & [CALC] \\
\hline 0805020-32 & B1VJ64A & $3.54 \mathrm{E} 2$ & $2.00 \mathrm{E} 2$ & $7 / 02 / 08$ & [CALC] \\
\hline
\end{tabular}




\section{Carbon Analysis/Soil}

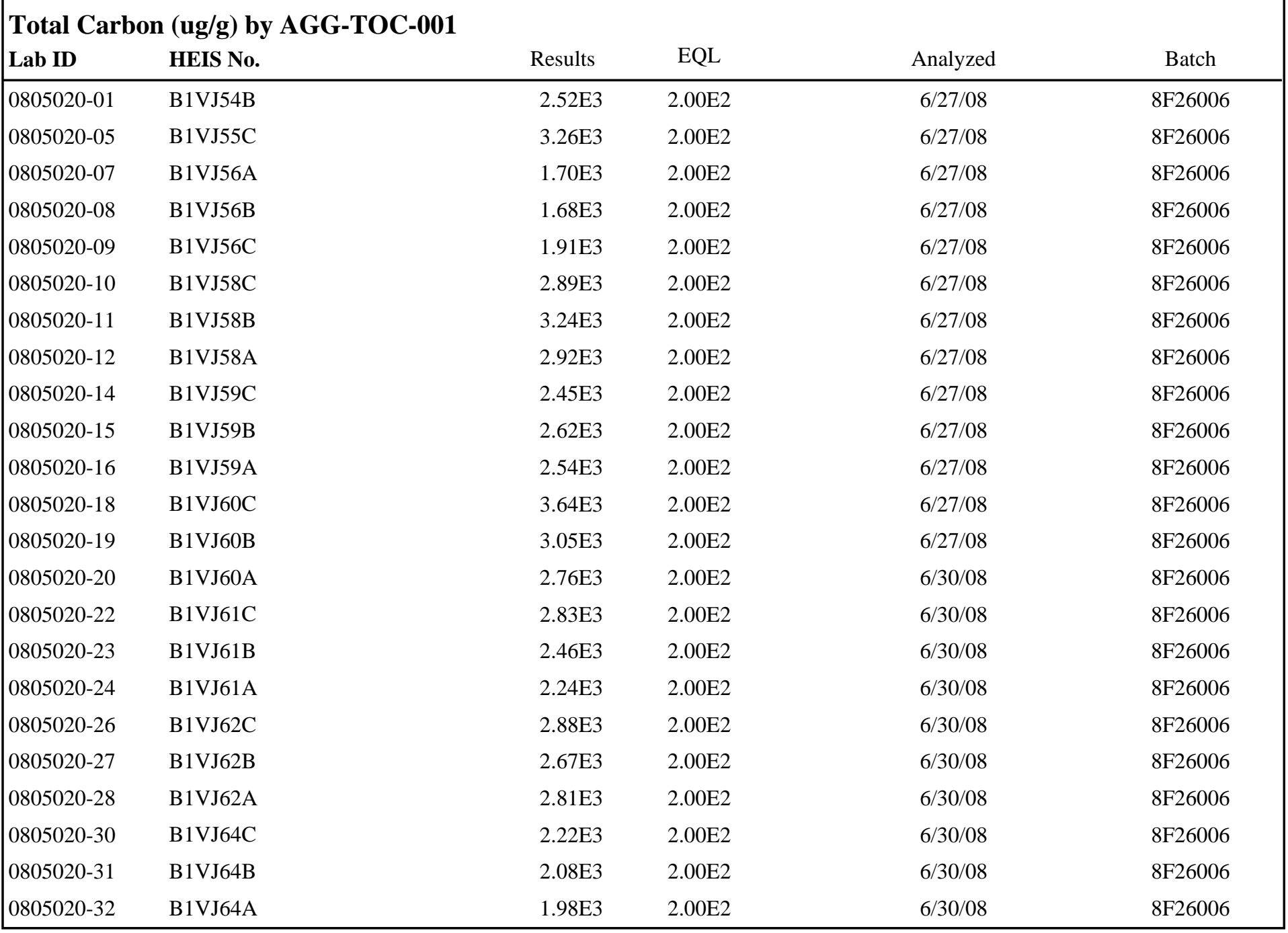




\begin{tabular}{|c|c|c|c|c|c|}
\hline \multicolumn{6}{|c|}{ Carbon Analysis/Soil } \\
\hline \multicolumn{6}{|c|}{ Total Inorganic Carbon (ug/g) by AGG-TOC-001 } \\
\hline Lab ID & HEIS No. & Results & EQL & Analyzed & Batch \\
\hline 0805020-01 & B1VJ54B & $1.73 \mathrm{E} 3$ & $2.00 \mathrm{E} 2$ & 7/01/08 & $8 \mathrm{~F} 30010$ \\
\hline 0805020-05 & B1VJ55C & $2.63 \mathrm{E} 3$ & $2.00 \mathrm{E} 2$ & 7/01/08 & $8 \mathrm{~F} 30010$ \\
\hline 0805020-07 & B1VJ56A & $1.41 \mathrm{E} 3$ & $2.00 \mathrm{E} 2$ & 7/01/08 & $8 \mathrm{~F} 30010$ \\
\hline 0805020-08 & B1VJ56B & $1.33 \mathrm{E} 3$ & $2.00 \mathrm{E} 2$ & 7/01/08 & $8 \mathrm{~F} 30010$ \\
\hline 0805020-09 & B1VJ56C & $1.24 \mathrm{E} 3$ & $2.00 \mathrm{E} 2$ & $7 / 01 / 08$ & $8 \mathrm{~F} 30010$ \\
\hline 0805020-10 & B1VJ58C & $2.00 \mathrm{E} 3$ & $2.00 \mathrm{E} 2$ & 7/01/08 & $8 \mathrm{~F} 30010$ \\
\hline 0805020-11 & B1VJ58B & $2.57 \mathrm{E} 3$ & $2.00 \mathrm{E} 2$ & 7/01/08 & $8 \mathrm{~F} 30010$ \\
\hline 0805020-12 & B1VJ58A & $2.07 \mathrm{E} 3$ & $2.00 \mathrm{E} 2$ & 7/01/08 & $8 \mathrm{~F} 30010$ \\
\hline 0805020-14 & B1VJ59C & $1.90 \mathrm{E} 3$ & $2.00 \mathrm{E} 2$ & 7/01/08 & $8 \mathrm{~F} 30010$ \\
\hline 0805020-15 & B1VJ59B & $2.31 \mathrm{E} 3$ & $2.00 \mathrm{E} 2$ & 7/01/08 & $8 \mathrm{~F} 30010$ \\
\hline 0805020-16 & B1VJ59A & $2.28 \mathrm{E} 3$ & $2.00 \mathrm{E} 2$ & $7 / 02 / 08$ & $8 F 30010$ \\
\hline 0805020-18 & B1VJ60C & $2.67 \mathrm{E} 3$ & $2.00 \mathrm{E} 2$ & $7 / 02 / 08$ & $8 \mathrm{~F} 30010$ \\
\hline 0805020-19 & B1VJ60B & $2.90 \mathrm{E} 3$ & $2.00 \mathrm{E} 2$ & 7/02/08 & $8 \mathrm{~F} 30010$ \\
\hline 0805020-20 & B1VJ60A & $2.52 \mathrm{E} 3$ & $2.00 \mathrm{E} 2$ & 7/02/08 & $8 \mathrm{~F} 30010$ \\
\hline 0805020-22 & B1VJ61C & $2.14 \mathrm{E} 3$ & $2.00 \mathrm{E} 2$ & 7/02/08 & $8 \mathrm{~F} 30010$ \\
\hline 0805020-23 & B1VJ61B & $2.15 \mathrm{E} 3$ & $2.00 \mathrm{E} 2$ & 7/02/08 & $8 \mathrm{~F} 30010$ \\
\hline 0805020-24 & B1VJ61A & $2.00 \mathrm{E} 3$ & $2.00 \mathrm{E} 2$ & $7 / 02 / 08$ & $8 \mathrm{~F} 30010$ \\
\hline 0805020-26 & B1VJ62C & $2.51 \mathrm{E} 3$ & $2.00 \mathrm{E} 2$ & 7/02/08 & $8 \mathrm{~F} 30010$ \\
\hline 0805020-27 & B1VJ62B & $2.52 \mathrm{E} 3$ & $2.00 \mathrm{E} 2$ & 7/02/08 & $8 \mathrm{~F} 30010$ \\
\hline 0805020-28 & B1VJ62A & $2.41 \mathrm{E} 3$ & $2.00 \mathrm{E} 2$ & 7/02/08 & $8 \mathrm{~F} 30010$ \\
\hline 0805020-30 & B1VJ64C & $1.85 \mathrm{E} 3$ & $2.00 \mathrm{E} 2$ & 7/02/08 & $8 \mathrm{~F} 30010$ \\
\hline 0805020-31 & B1VJ64B & $1.66 \mathrm{E} 3$ & $2.00 \mathrm{E} 2$ & 7/02/08 & $8 \mathrm{~F} 30010$ \\
\hline 0805020-32 & B1VJ64A & $1.62 \mathrm{E} 3$ & $2.00 \mathrm{E} 2$ & $7 / 02 / 08$ & $8 \mathrm{~F} 30010$ \\
\hline
\end{tabular}


GEA/Soil

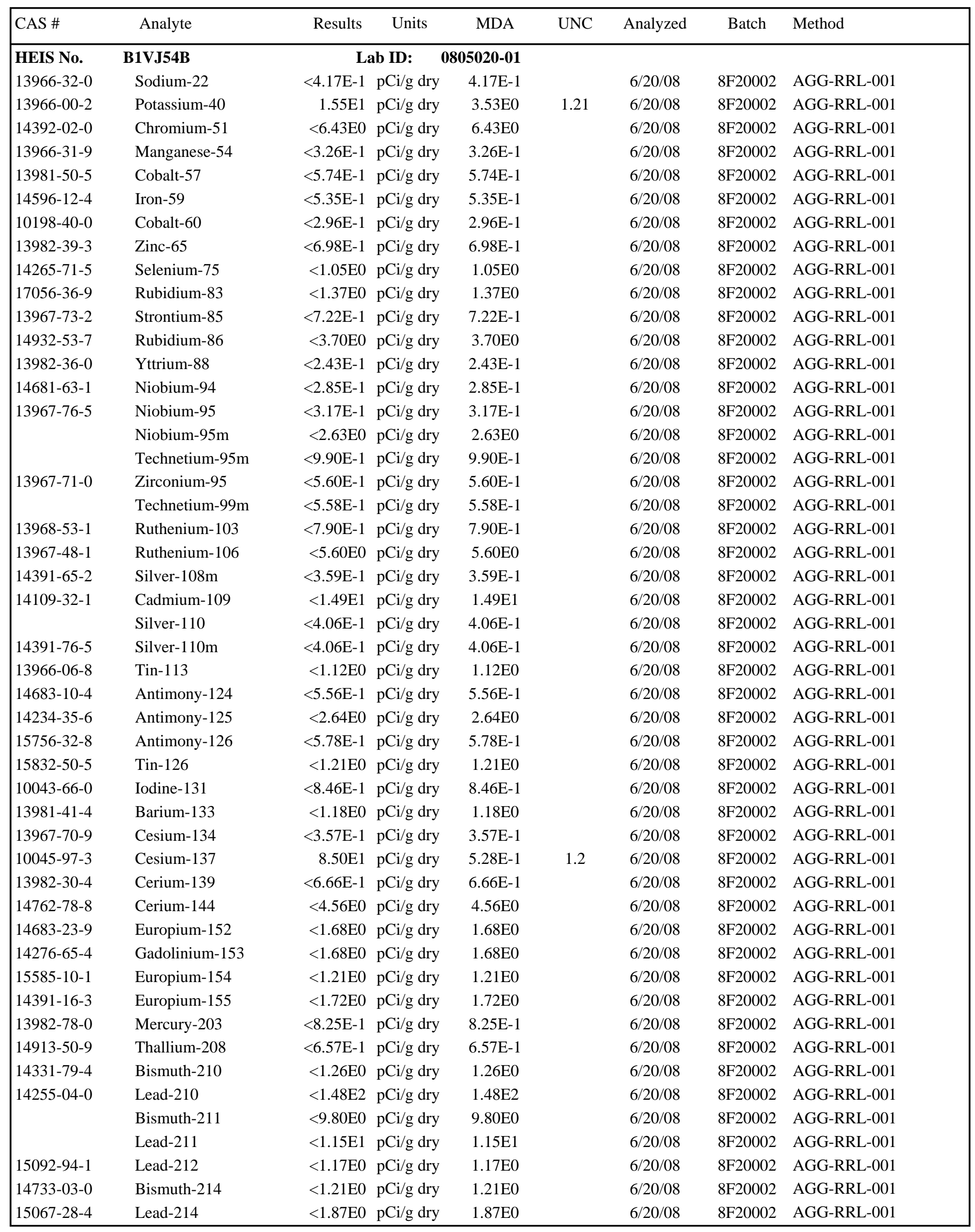


GEA/Soil

\begin{tabular}{|c|c|c|c|c|c|c|c|c|}
\hline CAS \# & Analyte & Results & Units & MDA & UNC & Analyzed & Batch & Method \\
\hline HEIS No. & B1VJ54B & \multicolumn{3}{|c|}{ Lab ID: $\quad$ 0805020-01 } & & & & \\
\hline $14835-02-0$ & Radon-219 & $<6.40 \mathrm{E} 0$ & pCi/g dry & $6.40 \mathrm{E} 0$ & & 6/20/08 & 8F20002 & AGG-RRL-001 \\
\hline 22481-48-7 & Radon-220 & $<5.68 \mathrm{E} 2$ & pCi/g dry & $5.68 \mathrm{E} 2$ & & 6/20/08 & 8F20002 & AGG-RRL-001 \\
\hline 28522-20-5 & Radon-221 & $<2.79 \mathrm{E} 0$ & pCi/g dry & 2.79E0 & & 6/20/08 & $8 F 20002$ & AGG-RRL-001 \\
\hline 15756-98-6 & Francium-223 & $<1.93 \mathrm{E} 1$ & pCi/g dry & $1.93 \mathrm{E} 1$ & & 6/20/08 & 8F20002 & AGG-RRL-001 \\
\hline 15623-45-7 & Radium-223 & $<4.70 \mathrm{E} 0$ & pCi/g dry & 4.70E0 & & 6/20/08 & 8F20002 & AGG-RRL-001 \\
\hline 13233-32-4 & Radium-224 & $<1.67 \mathrm{E} 1$ & pCi/g dry & $1.67 \mathrm{E} 1$ & & 6/20/08 & 8F20002 & AGG-RRL-001 \\
\hline 13982-63-3 & Radium-226 & $<1.72 \mathrm{E} 1$ & pCi/g dry & $1.72 \mathrm{E} 1$ & & 6/20/08 & 8F20002 & AGG-RRL-001 \\
\hline 15623-47-9 & Thorium-227 & $<5.88 \mathrm{E} 0$ & pCi/g dry & $5.88 \mathrm{E} 0$ & & $6 / 20 / 08$ & 8F20002 & AGG-RRL-001 \\
\hline 14331-83-0 & Actinium-228 & $<1.44 \mathrm{E} 0$ & pCi/g dry & $1.44 \mathrm{E} 0$ & & 6/20/08 & 8F20002 & AGG-RRL-001 \\
\hline 14274-82-9 & Thorium-228 & $<2.31 \mathrm{E} 2$ & pCi/g dry & 2.31E2 & & $6 / 20 / 08$ & 8F20002 & AGG-RRL-001 \\
\hline 14269-63-7 & Thorium-230 & $<2.22 \mathrm{E} 2$ & pCi/g dry & 2.22E2 & & 6/20/08 & 8F20002 & AGG-RRL-001 \\
\hline 14331-85-2 & Protactinium-231 & $<2.76 \mathrm{E} 1$ & pCi/g dry & $2.76 \mathrm{E} 1$ & & 6/20/08 & $8 F 20002$ & AGG-RRL-001 \\
\hline $14932-40-2$ & Thorium-231 & $<1.19 \mathrm{E} 2$ & pCi/g dry & $1.19 \mathrm{E} 2$ & & 6/20/08 & 8F20002 & AGG-RRL-001 \\
\hline $7440-29-1$ & Thorium-232 & $<6.91 \mathrm{E} 2$ & pCi/g dry & 6.91E2 & & 6/20/08 & $8 F 20002$ & AGG-RRL-001 \\
\hline 13981-14-1 & Protactinium-233 & $<1.73 \mathrm{E} 0$ & pCi/g dry & $1.73 \mathrm{E} 0$ & & 6/20/08 & 8F20002 & AGG-RRL-001 \\
\hline \multirow[t]{2}{*}{ 15100-28-4 } & Protactinium-234 & $<1.93 \mathrm{E} 0$ & pCi/g dry & 1.93E0 & & 6/20/08 & 8F20002 & AGG-RRL-001 \\
\hline & Protactinium-234m & $<3.90 \mathrm{E} 1$ & pCi/g dry & $3.90 \mathrm{E} 1$ & & 6/20/08 & 8F20002 & AGG-RRL-001 \\
\hline 15065-10-8 & Thorium-234 & $<1.90 \mathrm{E} 1$ & pCi/g dry & $1.90 \mathrm{E} 1$ & & 6/20/08 & 8F20002 & AGG-RRL-001 \\
\hline 15117-96-1 & Uranium 235 & $<4.80 \mathrm{E} 0$ & pCi/g dry & $4.80 \mathrm{E} 0$ & & 6/20/08 & 8F20002 & AGG-RRL-001 \\
\hline 13994-20-2 & Neptunium-237 & $<4.47 \mathrm{E} 0$ & pCi/g dry & 4.47E0 & & 6/20/08 & 8F20002 & AGG-RRL-001 \\
\hline \multirow[t]{2}{*}{ 13981-16-3 } & Plutonium-238 & $<6.84 \mathrm{E} 3$ & pCi/g dry & $6.84 \mathrm{E} 3$ & & 6/20/08 & 8F20002 & AGG-RRL-001 \\
\hline & Uranium 238 & $<9.84 \mathrm{E} 0$ & pCi/g dry & $9.84 \mathrm{E} 0$ & & 6/20/08 & 8F20002 & AGG-RRL-001 \\
\hline $15117-48-3$ & Plutonium-239 & $<7.96 \mathrm{E} 3$ & pCi/g dry & 7.96E3 & & 6/20/08 & 8F20002 & AGG-RRL-001 \\
\hline 14119-33-6 & Plutonium-240 & $<6.98 \mathrm{E} 3$ & pCi/g dry & $6.98 \mathrm{E} 3$ & & $6 / 20 / 08$ & 8F20002 & AGG-RRL-001 \\
\hline $14596-10-2$ & Americium-241 & $<3.48 \mathrm{E} 0$ & pCi/g dry & $3.48 \mathrm{E} 0$ & & 6/20/08 & 8F20002 & AGG-RRL-001 \\
\hline 14993-75-0 & Americium-243 & $<1.16 \mathrm{E} 0$ & pCi/g dry & $1.16 \mathrm{E} 0$ & & 6/20/08 & 8F20002 & AGG-RRL-001 \\
\hline $15757-87-6$ & Curium-243 & $<2.13 \mathrm{E} 0$ & pCi/g dry & 2.13E0 & & $6 / 20 / 08$ & 8 F20002 & AGG-RRL-001 \\
\hline 15621-76-8 & Curium-245 & $<1.75 \mathrm{E} 0$ & pCi/g dry & $1.75 \mathrm{E} 0$ & & 6/20/08 & 8F20002 & AGG-RRL-001 \\
\hline HEIS No. & B1VJ55C & \multicolumn{3}{|c|}{ Lab ID: $\quad$ 0805020-05 } & & & & \\
\hline 13966-32-0 & Sodium-22 & $<4.74 \mathrm{E}-1$ & pCi/g dry & $4.74 \mathrm{E}-1$ & & $6 / 20 / 08$ & 8F20002 & AGG-RRL-001 \\
\hline 13966-00-2 & Potassium-40 & $<1.02 \mathrm{E} 1$ & pCi/g dry & $1.02 \mathrm{E} 1$ & & 6/20/08 & 8F20002 & AGG-RRL-001 \\
\hline 14392-02-0 & Chromium-51 & $<4.48 \mathrm{E} 0$ & pCi/g dry & 4.48E0 & & $6 / 20 / 08$ & 8F20002 & AGG-RRL-001 \\
\hline 13966-31-9 & Manganese-54 & $<5.12 \mathrm{E}-1$ & pCi/g dry & $5.12 \mathrm{E}-1$ & & 6/20/08 & 8F20002 & AGG-RRL-001 \\
\hline 13981-50-5 & Cobalt-57 & $<4.64 \mathrm{E}-1$ & pCi/g dry & 4.64E-1 & & 6/20/08 & 8F20002 & AGG-RRL-001 \\
\hline $14596-12-4$ & Iron-59 & $<8.10 \mathrm{E}-1$ & pCi/g dry & $8.10 \mathrm{E}-1$ & & $6 / 20 / 08$ & 8F20002 & AGG-RRL-001 \\
\hline 10198-40-0 & Cobalt-60 & $<4.78 \mathrm{E}-1$ & pCi/g dry & $4.78 \mathrm{E}-1$ & & 6/20/08 & 8F20002 & AGG-RRL-001 \\
\hline 13982-39-3 & Zinc-65 & $<1.01 \mathrm{E} 0$ & pCi/g dry & $1.01 \mathrm{E} 0$ & & 6/20/08 & 8F20002 & AGG-RRL-001 \\
\hline $14265-71-5$ & Selenium-75 & $<7.58 \mathrm{E}-1$ & pCi/g dry & $7.58 \mathrm{E}-1$ & & 6/20/08 & 8F20002 & AGG-RRL-001 \\
\hline 17056-36-9 & Rubidium-83 & $<1.18 \mathrm{E} 0$ & pCi/g dry & $1.18 \mathrm{E} 0$ & & $6 / 20 / 08$ & 8F20002 & AGG-RRL-001 \\
\hline 13967-73-2 & Strontium-85 & $<5.49 \mathrm{E}-1$ & pCi/g dry & $5.49 \mathrm{E}-1$ & & 6/20/08 & 8F20002 & AGG-RRL-001 \\
\hline $14932-53-7$ & Rubidium-86 & $<5.75 \mathrm{E} 0$ & pCi/g dry & $5.75 \mathrm{E} 0$ & & 6/20/08 & 8F20002 & AGG-RRL-001 \\
\hline 13982-36-0 & Yttrium-88 & $<4.00 \mathrm{E}-1$ & pCi/g dry & 4.00E-1 & & 6/20/08 & 8F20002 & AGG-RRL-001 \\
\hline 14681-63-1 & Niobium-94 & $<4.56 \mathrm{E}-1$ & pCi/g dry & $4.56 \mathrm{E}-1$ & & 6/20/08 & 8F20002 & AGG-RRL-001 \\
\hline \multirow[t]{3}{*}{$13967-76-5$} & Niobium-95 & $<4.85 \mathrm{E}-1$ & pCi/g dry & $4.85 \mathrm{E}-1$ & & 6/20/08 & 8F20002 & AGG-RRL-001 \\
\hline & Niobium-95m & $<2.08 \mathrm{E} 0$ & pCi/g dry & $2.08 \mathrm{E} 0$ & & 6/20/08 & 8F20002 & AGG-RRL-001 \\
\hline & Technetium-95m & $<7.19 \mathrm{E}-1$ & pCi/g dry & 7.19E-1 & & 6/20/08 & 8F20002 & AGG-RRL-001 \\
\hline \multirow[t]{2}{*}{ 13967-71-0 } & Zirconium-95 & $<8.90 \mathrm{E}-1$ & pCi/g dry & 8.90E-1 & & 6/20/08 & 8F20002 & AGG-RRL-001 \\
\hline & Technetium-99m & $<4.66 \mathrm{E}-1$ & pCi/g dry & $4.66 \mathrm{E}-1$ & & 6/20/08 & 8F20002 & AGG-RRL-001 \\
\hline
\end{tabular}


GEA/Soil

\begin{tabular}{|c|c|c|c|c|c|c|c|c|}
\hline CAS \# & Analyte & Results & Units & MDA & UNC & Analyzed & Batch & Method \\
\hline HEIS No. & B1VJ55C & & ab ID: & 5020-05 & & & & \\
\hline 13968-53-1 & Ruthenium-103 & $<5.58 \mathrm{E}-1$ & pCi/g dry & $5.58 \mathrm{E}-1$ & & 6/20/08 & 8F20002 & AGG-RRL-001 \\
\hline 13967-48-1 & Ruthenium-106 & $<5.28 \mathrm{E} 0$ & pCi/g dry & $5.28 \mathrm{E} 0$ & & 6/20/08 & $8 \mathrm{~F} 20002$ & AGG-RRL-001 \\
\hline 14391-65-2 & Silver-108m & $<4.74 \mathrm{E}-1$ & pCi/g dry & $4.74 \mathrm{E}-1$ & & $6 / 20 / 08$ & $8 \mathrm{~F} 20002$ & AGG-RRL-001 \\
\hline \multirow[t]{2}{*}{ 14109-32-1 } & Cadmium-109 & $<1.29 \mathrm{E} 1$ & pCi/g dry & $1.29 \mathrm{E} 1$ & & 6/20/08 & $8 \mathrm{~F} 20002$ & AGG-RRL-001 \\
\hline & Silver-110 & $<4.80 \mathrm{E}-1$ & pCi/g dry & $4.80 \mathrm{E}-1$ & & 6/20/08 & $8 \mathrm{~F} 20002$ & AGG-RRL-001 \\
\hline 14391-76-5 & Silver-110m & $<4.81 \mathrm{E}-1$ & pCi/g dry & $4.81 \mathrm{E}-1$ & & 6/20/08 & 8 F20002 & AGG-RRL-001 \\
\hline 13966-06-8 & Tin-113 & $<6.56 \mathrm{E}-1$ & pCi/g dry & $6.56 \mathrm{E}-1$ & & 6/20/08 & $8 \mathrm{~F} 20002$ & AGG-RRL-001 \\
\hline 14683-10-4 & Antimony-124 & $<5.46 \mathrm{E}-1$ & pCi/g dry & $5.46 \mathrm{E}-1$ & & 6/20/08 & $8 \mathrm{~F} 20002$ & AGG-RRL-001 \\
\hline $14234-35-6$ & Antimony-125 & $<1.49 \mathrm{E} 0$ & pCi/g dry & $1.49 \mathrm{E} 0$ & & 6/20/08 & $8 \mathrm{~F} 20002$ & AGG-RRL-001 \\
\hline 15756-32-8 & Antimony-126 & $<4.51 \mathrm{E}-1$ & pCi/g dry & $4.51 \mathrm{E}-1$ & & 6/20/08 & $8 \mathrm{~F} 20002$ & AGG-RRL-001 \\
\hline $15832-50-5$ & Tin-126 & $<1.06 \mathrm{E} 0$ & pCi/g dry & $1.06 \mathrm{E} 0$ & & 6/20/08 & $8 \mathrm{~F} 20002$ & AGG-RRL-001 \\
\hline 10043-66-0 & Iodine-131 & $<5.78 \mathrm{E}-1$ & pCi/g dry & $5.78 \mathrm{E}-1$ & & 6/20/08 & $8 \mathrm{~F} 20002$ & AGG-RRL-001 \\
\hline 13981-41-4 & Barium-133 & $<8.12 \mathrm{E}-1$ & pCi/g dry & $8.12 \mathrm{E}-1$ & & 6/20/08 & $8 \mathrm{~F} 20002$ & AGG-RRL-001 \\
\hline 13967-70-9 & Cesium-134 & $<5.55 \mathrm{E}-1$ & pCi/g dry & $5.55 \mathrm{E}-1$ & & 6/20/08 & $8 \mathrm{~F} 20002$ & AGG-RRL-001 \\
\hline $10045-97-3$ & Cesium-137 & $<5.14 \mathrm{E}-1$ & pCi/g dry & $5.14 \mathrm{E}-1$ & & 6/20/08 & $8 \mathrm{~F} 20002$ & AGG-RRL-001 \\
\hline 13982-30-4 & Cerium-139 & $<5.49 \mathrm{E}-1$ & pCi/g dry & $5.49 \mathrm{E}-1$ & & 6/20/08 & $8 \mathrm{~F} 20002$ & AGG-RRL-001 \\
\hline 14762-78-8 & Cerium-144 & $<3.77 \mathrm{E} 0$ & pCi/g dry & 3.77E0 & & 6/20/08 & $8 \mathrm{~F} 20002$ & AGG-RRL-001 \\
\hline 14683-23-9 & Europium-152 & $<1.90 \mathrm{E} 0$ & pCi/g dry & $1.90 \mathrm{E} 0$ & & 6/20/08 & $8 \mathrm{~F} 20002$ & AGG-RRL-001 \\
\hline 14276-65-4 & Gadolinium-153 & $<1.42 \mathrm{E} 0$ & pCi/g dry & $1.42 \mathrm{E} 0$ & & 6/20/08 & $8 \mathrm{~F} 20002$ & AGG-RRL-001 \\
\hline 15585-10-1 & Europium-154 & $<9.85 \mathrm{E}-1$ & pCi/g dry & $9.85 \mathrm{E}-1$ & & 6/20/08 & $8 \mathrm{~F} 20002$ & AGG-RRL-001 \\
\hline 14391-16-3 & Europium-155 & $<1.50 \mathrm{E} 0$ & pCi/g dry & $1.50 \mathrm{E} 0$ & & 6/20/08 & 8 F20002 & AGG-RRL-001 \\
\hline 13982-78-0 & Mercury-203 & $<5.96 \mathrm{E}-1$ & pCi/g dry & $5.96 \mathrm{E}-1$ & & 6/20/08 & $8 \mathrm{~F} 20002$ & AGG-RRL-001 \\
\hline 14913-50-9 & Thallium-208 & $<5.63 \mathrm{E}-1$ & pCi/g dry & $5.63 \mathrm{E}-1$ & & 6/20/08 & $8 \mathrm{~F} 20002$ & AGG-RRL-001 \\
\hline 14331-79-4 & Bismuth-210 & $<9.04 \mathrm{E}-1$ & pCi/g dry & $9.04 \mathrm{E}-1$ & & 6/20/08 & $8 \mathrm{~F} 20002$ & AGG-RRL-001 \\
\hline \multirow[t]{3}{*}{$14255-04-0$} & Lead-210 & $<6.89 \mathrm{E} 1$ & pCi/g dry & $6.89 \mathrm{E} 1$ & & 6/20/08 & $8 \mathrm{~F} 20002$ & AGG-RRL-001 \\
\hline & Bismuth-211 & $<1.05 \mathrm{E} 1$ & pCi/g dry & $1.05 \mathrm{E} 1$ & & 6/20/08 & $8 \mathrm{~F} 20002$ & AGG-RRL-001 \\
\hline & Lead-211 & $<1.44 \mathrm{E} 1$ & pCi/g dry & $1.44 \mathrm{E} 1$ & & 6/20/08 & $8 \mathrm{~F} 20002$ & AGG-RRL-001 \\
\hline 15092-94-1 & Lead-212 & $<1.22 \mathrm{E} 0$ & pCi/g dry & $1.22 \mathrm{E} 0$ & & 6/20/08 & $8 \mathrm{~F} 20002$ & AGG-RRL-001 \\
\hline $14733-03-0$ & Bismuth-214 & $<1.19 \mathrm{E} 0$ & pCi/g dry & $1.19 \mathrm{E} 0$ & & 6/20/08 & $8 \mathrm{~F} 20002$ & AGG-RRL-001 \\
\hline $15067-28-4$ & Lead-214 & $<1.32 \mathrm{E} 0$ & pCi/g dry & 1.32E0 & & 6/20/08 & $8 \mathrm{~F} 20002$ & AGG-RRL-001 \\
\hline $14835-02-0$ & Radon-219 & $<4.56 \mathrm{E} 0$ & pCi/g dry & 4.56E0 & & 6/20/08 & $8 \mathrm{~F} 20002$ & AGG-RRL-001 \\
\hline 22481-48-7 & Radon-220 & $<4.52 \mathrm{E} 2$ & pCi/g dry & $4.52 \mathrm{E} 2$ & & 6/20/08 & 8 F20002 & AGG-RRL-001 \\
\hline 28522-20-5 & Radon-221 & $<2.15 \mathrm{E} 0$ & pCi/g dry & 2.15E0 & & 6/20/08 & $8 \mathrm{~F} 20002$ & AGG-RRL-001 \\
\hline 15756-98-6 & Francium-223 & $<1.53 \mathrm{E} 1$ & pCi/g dry & $1.53 \mathrm{E} 1$ & & 6/20/08 & $8 \mathrm{~F} 20002$ & AGG-RRL-001 \\
\hline $15623-45-7$ & Radium-223 & $<3.29 \mathrm{E} 0$ & pCi/g dry & $3.29 \mathrm{E} 0$ & & 6/20/08 & $8 \mathrm{~F} 20002$ & AGG-RRL-001 \\
\hline 13233-32-4 & Radium-224 & $<1.33 \mathrm{E} 1$ & pCi/g dry & $1.33 \mathrm{E} 1$ & & 6/20/08 & $8 \mathrm{~F} 20002$ & AGG-RRL-001 \\
\hline 13982-63-3 & Radium-226 & $<1.32 \mathrm{E} 1$ & pCi/g dry & $1.32 \mathrm{E} 1$ & & 6/20/08 & $8 \mathrm{~F} 20002$ & AGG-RRL-001 \\
\hline $15623-47-9$ & Thorium-227 & $<4.65 \mathrm{E} 0$ & pCi/g dry & 4.65E0 & & 6/20/08 & $8 \mathrm{~F} 20002$ & AGG-RRL-001 \\
\hline 14331-83-0 & Actinium-228 & $<1.96 \mathrm{E} 0$ & pCi/g dry & $1.96 \mathrm{E} 0$ & & 6/20/08 & $8 \mathrm{~F} 20002$ & AGG-RRL-001 \\
\hline $14274-82-9$ & Thorium-228 & $<1.66 \mathrm{E} 2$ & pCi/g dry & $1.66 \mathrm{E} 2$ & & 6/20/08 & $8 \mathrm{~F} 20002$ & AGG-RRL-001 \\
\hline $14269-63-7$ & Thorium-230 & $<1.76 \mathrm{E} 2$ & pCi/g dry & $1.76 \mathrm{E} 2$ & & 6/20/08 & $8 \mathrm{~F} 20002$ & AGG-RRL-001 \\
\hline $14331-85-2$ & Protactinium-231 & $<2.03 \mathrm{E} 1$ & pCi/g dry & 2.03E1 & & 6/20/08 & $8 \mathrm{~F} 20002$ & AGG-RRL-001 \\
\hline $14932-40-2$ & Thorium-231 & $<1.02 \mathrm{E} 2$ & pCi/g dry & $1.02 \mathrm{E} 2$ & & 6/20/08 & $8 \mathrm{~F} 20002$ & AGG-RRL-001 \\
\hline 7440-29-1 & Thorium-232 & $<4.44 \mathrm{E} 2$ & pCi/g dry & $4.44 \mathrm{E} 2$ & & $6 / 20 / 08$ & $8 \mathrm{~F} 20002$ & AGG-RRL-001 \\
\hline 13981-14-1 & Protactinium-233 & $<1.23 \mathrm{E} 0$ & pCi/g dry & $1.23 \mathrm{E} 0$ & & 6/20/08 & $8 \mathrm{~F} 20002$ & AGG-RRL-001 \\
\hline \multirow[t]{2}{*}{$15100-28-4$} & Protactinium-234 & $<1.86 \mathrm{E} 0$ & pCi/g dry & $1.86 \mathrm{E} 0$ & & 6/20/08 & $8 \mathrm{~F} 20002$ & AGG-RRL-001 \\
\hline & Protactinium-234m & $<5.42 \mathrm{E} 1$ & pCi/g dry & $5.42 \mathrm{E} 1$ & & 6/20/08 & 8F20002 & AGG-RRL-001 \\
\hline 15065-10-8 & Thorium-234 & $<1.54 \mathrm{E} 1$ & pCi/g dry & $1.54 \mathrm{E} 1$ & & 6/20/08 & $8 \mathrm{~F} 20002$ & AGG-RRL-001 \\
\hline 15117-96-1 & Uranium 235 & $<4.05 \mathrm{E} 0$ & pCi/g dry & $4.05 \mathrm{E} 0$ & & 6/20/08 & $8 F 20002$ & AGG-RRL-001 \\
\hline
\end{tabular}


GEA/Soil

\begin{tabular}{|c|c|c|c|c|c|c|c|c|}
\hline CAS \# & Analyte & Results & Units & MDA & UNC & Analyzed & Batch & Method \\
\hline HEIS No. & B1VJ55C & & ab ID: & 5020-05 & & & & \\
\hline 13994-20-2 & Neptunium-237 & $<3.91 \mathrm{E} 0$ & pCi/g dry & 3.91E0 & & 6/20/08 & $8 \mathrm{~F} 20002$ & AGG-RRL-001 \\
\hline \multirow[t]{2}{*}{ 13981-16-3 } & Plutonium-238 & $<5.88 \mathrm{E} 3$ & pCi/g dry & $5.88 \mathrm{E} 3$ & & 6/20/08 & 8 F20002 & AGG-RRL-001 \\
\hline & Uranium 238 & $<8.26 \mathrm{E} 0$ & pCi/g dry & 8.26Е0 & & 6/20/08 & 8 F20002 & AGG-RRL-001 \\
\hline 15117-48-3 & Plutonium-239 & $<6.14 \mathrm{E} 3$ & pCi/g dry & 6.14E3 & & $6 / 20 / 08$ & $8 \mathrm{~F} 20002$ & AGG-RRL-001 \\
\hline 14119-33-6 & Plutonium-240 & $<5.89 \mathrm{E} 3$ & pCi/g dry & 5.89E3 & & 6/20/08 & $8 \mathrm{~F} 20002$ & AGG-RRL-001 \\
\hline $14596-10-2$ & Americium-241 & $<2.27 \mathrm{E} 0$ & pCi/g dry & $2.27 \mathrm{E} 0$ & & 6/20/08 & 8 F20002 & AGG-RRL-001 \\
\hline 14993-75-0 & Americium-243 & $<9.59 \mathrm{E}-1$ & pCi/g dry & $9.59 \mathrm{E}-1$ & & 6/20/08 & $8 \mathrm{~F} 20002$ & AGG-RRL-001 \\
\hline 15757-87-6 & Curium-243 & $<1.80 \mathrm{E} 0$ & pCi/g dry & $1.80 \mathrm{E} 0$ & & 6/20/08 & $8 \mathrm{~F} 20002$ & AGG-RRL-001 \\
\hline 15621-76-8 & Curium-245 & $<1.48 \mathrm{E} 0$ & pCi/g dry & $1.48 \mathrm{E} 0$ & & 6/20/08 & $8 \mathrm{~F} 20002$ & AGG-RRL-001 \\
\hline HEIS No. & B1VJ56A & & ab ID: & $5020-07$ & & & & \\
\hline 13966-32-0 & Sodium-22 & $<3.46 \mathrm{E}-1$ & pCi/g dry & $3.46 \mathrm{E}-1$ & & 6/20/08 & 8F20002 & AGG-RRL-001 \\
\hline 13966-00-2 & Potassium-40 & 1.76E1 & pCi/g dry & 2.79E0 & 1.17 & 6/20/08 & $8 \mathrm{~F} 20002$ & AGG-RRL-001 \\
\hline 14392-02-0 & Chromium-51 & $<2.86 \mathrm{E} 0$ & pCi/g dry & 2.86E0 & & 6/20/08 & $8 \mathrm{~F} 20002$ & AGG-RRL-001 \\
\hline 13966-31-9 & Manganese-54 & $<3.01 \mathrm{E}-1$ & pCi/g dry & $3.01 \mathrm{E}-1$ & & 6/20/08 & 8 F20002 & AGG-RRL-001 \\
\hline 13981-50-5 & Cobalt-57 & $<2.91 \mathrm{E}-1$ & pCi/g dry & 2.91E-1 & & 6/20/08 & 8 F20002 & AGG-RRL-001 \\
\hline $14596-12-4$ & Iron-59 & $<5.79 \mathrm{E}-1$ & pCi/g dry & 5.79E-1 & & 6/20/08 & 8 F20002 & AGG-RRL-001 \\
\hline 10198-40-0 & Cobalt-60 & $<4.10 \mathrm{E}-1$ & pCi/g dry & 4.10E-1 & & 6/20/08 & $8 \mathrm{~F} 20002$ & AGG-RRL-001 \\
\hline 13982-39-3 & Zinc-65 & $<6.44 \mathrm{E}-1$ & pCi/g dry & $6.44 \mathrm{E}-1$ & & 6/20/08 & 8 F20002 & AGG-RRL-001 \\
\hline 14265-71-5 & Selenium-75 & $<4.97 \mathrm{E}-1$ & pCi/g dry & 4.97E-1 & & 6/20/08 & $8 \mathrm{~F} 20002$ & AGG-RRL-001 \\
\hline 17056-36-9 & Rubidium-83 & $<6.06 \mathrm{E}-1$ & pCi/g dry & $6.06 \mathrm{E}-1$ & & 6/20/08 & $8 \mathrm{~F} 20002$ & AGG-RRL-001 \\
\hline 13967-73-2 & Strontium-85 & $<3.90 \mathrm{E}-1$ & pCi/g dry & 3.90E-1 & & 6/20/08 & $8 \mathrm{~F} 20002$ & AGG-RRL-001 \\
\hline $14932-53-7$ & Rubidium-86 & $<3.57 \mathrm{E} 0$ & pCi/g dry & 3.57E0 & & 6/20/08 & 8 F20002 & AGG-RRL-001 \\
\hline 13982-36-0 & Yttrium-88 & $<2.47 \mathrm{E}-1$ & pCi/g dry & 2.47E-1 & & 6/20/08 & $8 \mathrm{~F} 20002$ & AGG-RRL-001 \\
\hline 14681-63-1 & Niobium-94 & $<3.08 \mathrm{E}-1$ & pCi/g dry & $3.08 \mathrm{E}-1$ & & 6/20/08 & $8 \mathrm{~F} 20002$ & AGG-RRL-001 \\
\hline \multirow[t]{3}{*}{ 13967-76-5 } & Niobium-95 & $<3.23 \mathrm{E}-1$ & pCi/g dry & 3.23E-1 & & 6/20/08 & $8 \mathrm{~F} 20002$ & AGG-RRL-001 \\
\hline & Niobium-95m & $<1.33 \mathrm{E} 0$ & pCi/g dry & $1.33 \mathrm{E} 0$ & & 6/20/08 & 8 F20002 & AGG-RRL-001 \\
\hline & Technetium-95m & $<4.64 \mathrm{E}-1$ & pCi/g dry & 4.64E-1 & & 6/20/08 & $8 \mathrm{~F} 20002$ & AGG-RRL-001 \\
\hline \multirow[t]{2}{*}{ 13967-71-0 } & Zirconium-95 & $<5.29 \mathrm{E}-1$ & pCi/g dry & $5.29 \mathrm{E}-1$ & & 6/20/08 & $8 \mathrm{~F} 20002$ & AGG-RRL-001 \\
\hline & Technetium-99m & $<2.95 \mathrm{E}-1$ & pCi/g dry & 2.95E-1 & & 6/20/08 & 8 F20002 & AGG-RRL-001 \\
\hline 13968-53-1 & Ruthenium-103 & $<2.97 \mathrm{E}-1$ & pCi/g dry & 2.97E-1 & & 6/20/08 & $8 \mathrm{~F} 20002$ & AGG-RRL-001 \\
\hline 13967-48-1 & Ruthenium-106 & $<3.24 \mathrm{E} 0$ & pCi/g dry & $3.24 \mathrm{E} 0$ & & 6/20/08 & 8 F20002 & AGG-RRL-001 \\
\hline 14391-65-2 & Silver-108m & $<3.20 \mathrm{E}-1$ & pCi/g dry & $3.20 \mathrm{E}-1$ & & 6/20/08 & $8 \mathrm{~F} 20002$ & AGG-RRL-001 \\
\hline \multirow[t]{2}{*}{ 14109-32-1 } & Cadmium-109 & $<7.95 \mathrm{E} 0$ & pCi/g dry & 7.95E0 & & 6/20/08 & 8 F20002 & AGG-RRL-001 \\
\hline & Silver-110 & $<3.18 \mathrm{E}-1$ & pCi/g dry & $3.18 \mathrm{E}-1$ & & 6/20/08 & $8 \mathrm{~F} 20002$ & AGG-RRL-001 \\
\hline 14391-76-5 & Silver-110m & $<3.19 \mathrm{E}-1$ & pCi/g dry & $3.19 \mathrm{E}-1$ & & 6/20/08 & $8 \mathrm{~F} 20002$ & AGG-RRL-001 \\
\hline 13966-06-8 & Tin-113 & $<4.55 \mathrm{E}-1$ & pCi/g dry & 4.55E-1 & & 6/20/08 & $8 \mathrm{~F} 20002$ & AGG-RRL-001 \\
\hline $14683-10-4$ & Antimony-124 & $<3.04 \mathrm{E}-1$ & pCi/g dry & $3.04 \mathrm{E}-1$ & & 6/20/08 & $8 \mathrm{~F} 20002$ & AGG-RRL-001 \\
\hline $14234-35-6$ & Antimony-125 & $<9.82 \mathrm{E}-1$ & pCi/g dry & $9.82 \mathrm{E}-1$ & & 6/20/08 & $8 \mathrm{~F} 20002$ & AGG-RRL-001 \\
\hline 15756-32-8 & Antimony-126 & $<3.12 \mathrm{E}-1$ & pCi/g dry & $3.12 \mathrm{E}-1$ & & 6/20/08 & $8 \mathrm{~F} 20002$ & AGG-RRL-001 \\
\hline 15832-50-5 & Tin-126 & $<6.38 \mathrm{E}-1$ & pCi/g dry & $6.38 \mathrm{E}-1$ & & 6/20/08 & $8 \mathrm{~F} 20002$ & AGG-RRL-001 \\
\hline 10043-66-0 & Iodine-131 & $<3.59 \mathrm{E}-1$ & pCi/g dry & $3.59 \mathrm{E}-1$ & & 6/20/08 & $8 \mathrm{~F} 20002$ & AGG-RRL-001 \\
\hline 13981-41-4 & Barium-133 & $<5.31 \mathrm{E}-1$ & pCi/g dry & $5.31 \mathrm{E}-1$ & & 6/20/08 & $8 \mathrm{~F} 20002$ & AGG-RRL-001 \\
\hline 13967-70-9 & Cesium-134 & $<3.51 \mathrm{E}-1$ & pCi/g dry & $3.51 \mathrm{E}-1$ & & 6/20/08 & $8 \mathrm{~F} 20002$ & AGG-RRL-001 \\
\hline 10045-97-3 & Cesium-137 & $<3.50 \mathrm{E}-1$ & pCi/g dry & $3.50 \mathrm{E}-1$ & & 6/20/08 & 8 F20002 & AGG-RRL-001 \\
\hline 13982-30-4 & Cerium-139 & $<3.42 \mathrm{E}-1$ & pCi/g dry & 3.42E-1 & & 6/20/08 & $8 \mathrm{~F} 20002$ & AGG-RRL-001 \\
\hline 14762-78-8 & Cerium-144 & $<2.41 \mathrm{E} 0$ & pCi/g dry & $2.41 \mathrm{E} 0$ & & 6/20/08 & $8 \mathrm{~F} 20002$ & AGG-RRL-001 \\
\hline 14683-23-9 & Europium-152 & $<1.12 \mathrm{E} 0$ & pCi/g dry & $1.12 \mathrm{E} 0$ & & 6/20/08 & $8 \mathrm{~F} 20002$ & AGG-RRL-001 \\
\hline $14276-65-4$ & Gadolinium-153 & $<8.58 \mathrm{E}-1$ & pCi/g dry & $8.58 \mathrm{E}-1$ & & 6/20/08 & 8F20002 & AGG-RRL-001 \\
\hline
\end{tabular}


GEA/Soil

\begin{tabular}{|c|c|c|c|c|c|c|c|c|}
\hline CAS \# & Analyte & Results & Units & MDA & UNC & Analyzed & Batch & Method \\
\hline HEIS No. & B1VJ56A & & b ID: & 05020-07 & & & & \\
\hline $15585-10-1$ & Europium-154 & $<6.24 \mathrm{E}-1$ & pCi/g dry & $6.24 \mathrm{E}-1$ & & 6/20/08 & $8 F 20002$ & AGG-RRL-001 \\
\hline 14391-16-3 & Europium-155 & $<8.97 \mathrm{E}-1$ & pCi/g dry & 8.97E-1 & & 6/20/08 & $8 \mathrm{~F} 20002$ & AGG-RRL-001 \\
\hline 13982-78-0 & Mercury-203 & $<3.80 \mathrm{E}-1$ & pCi/g dry & $3.80 \mathrm{E}-1$ & & $6 / 20 / 08$ & $8 \mathrm{~F} 20002$ & AGG-RRL-001 \\
\hline 14913-50-9 & Thallium-208 & $<2.69 \mathrm{E}-1$ & pCi/g dry & 2.69E-1 & & 6/20/08 & $8 \mathrm{~F} 20002$ & AGG-RRL-001 \\
\hline 14331-79-4 & Bismuth-210 & $<6.00 \mathrm{E}-1$ & pCi/g dry & $6.00 \mathrm{E}-1$ & & 6/20/08 & $8 \mathrm{~F} 20002$ & AGG-RRL-001 \\
\hline \multirow[t]{3}{*}{$14255-04-0$} & Lead-210 & $<1.17 \mathrm{E} 2$ & pCi/g dry & $1.17 \mathrm{E} 2$ & & 6/20/08 & $8 \mathrm{~F} 20002$ & AGG-RRL-001 \\
\hline & Bismuth-211 & $<7.04 \mathrm{E} 0$ & pCi/g dry & 7.04E0 & & 6/20/08 & $8 \mathrm{~F} 20002$ & AGG-RRL-001 \\
\hline & Lead-211 & $<9.62 \mathrm{E} 0$ & pCi/g dry & 9.62E0 & & 6/20/08 & $8 \mathrm{~F} 20002$ & AGG-RRL-001 \\
\hline 15092-94-1 & Lead-212 & $<7.92 \mathrm{E}-1$ & pCi/g dry & $7.92 \mathrm{E}-1$ & & 6/20/08 & $8 \mathrm{~F} 20002$ & AGG-RRL-001 \\
\hline 14733-03-0 & Bismuth-214 & $<7.53 \mathrm{E}-1$ & pCi/g dry & 7.53E-1 & & 6/20/08 & $8 \mathrm{~F} 20002$ & AGG-RRL-001 \\
\hline $15067-28-4$ & Lead-214 & $<8.50 \mathrm{E}-1$ & pCi/g dry & $8.50 \mathrm{E}-1$ & & 6/20/08 & $8 \mathrm{~F} 20002$ & AGG-RRL-001 \\
\hline $14835-02-0$ & Radon-219 & $<3.03 \mathrm{E} 0$ & pCi/g dry & 3.03E0 & & 6/20/08 & $8 \mathrm{~F} 20002$ & AGG-RRL-001 \\
\hline 22481-48-7 & Radon-220 & $<2.52 \mathrm{E} 2$ & pCi/g dry & $2.52 \mathrm{E} 2$ & & 6/20/08 & $8 \mathrm{~F} 20002$ & AGG-RRL-001 \\
\hline $28522-20-5$ & Radon-221 & $<1.37 \mathrm{E} 0$ & pCi/g dry & $1.37 \mathrm{E} 0$ & & 6/20/08 & $8 \mathrm{~F} 20002$ & AGG-RRL-001 \\
\hline 15756-98-6 & Francium-223 & $<9.80 \mathrm{E} 0$ & pCi/g dry & $9.80 \mathrm{E} 0$ & & 6/20/08 & $8 \mathrm{~F} 20002$ & AGG-RRL-001 \\
\hline $15623-45-7$ & Radium-223 & $<2.20 \mathrm{E} 0$ & pCi/g dry & $2.20 \mathrm{E} 0$ & & 6/20/08 & $8 \mathrm{~F} 20002$ & AGG-RRL-001 \\
\hline 13233-32-4 & Radium-224 & $<8.49 \mathrm{E} 0$ & pCi/g dry & 8.49E0 & & $6 / 20 / 08$ & $8 \mathrm{~F} 20002$ & AGG-RRL-001 \\
\hline 13982-63-3 & Radium-226 & $<8.51 \mathrm{E} 0$ & pCi/g dry & 8.51E0 & & 6/20/08 & $8 \mathrm{~F} 20002$ & AGG-RRL-001 \\
\hline $15623-47-9$ & Thorium-227 & $<2.98 \mathrm{E} 0$ & pCi/g dry & 2.98E0 & & 6/20/08 & $8 \mathrm{~F} 20002$ & AGG-RRL-001 \\
\hline 14331-83-0 & Actinium-228 & $<1.31 \mathrm{E} 0$ & pCi/g dry & $1.31 \mathrm{E} 0$ & & 6/20/08 & $8 \mathrm{~F} 20002$ & AGG-RRL-001 \\
\hline $14274-82-9$ & Thorium-228 & $<1.10 \mathrm{E} 2$ & pCi/g dry & $1.10 \mathrm{E} 2$ & & 6/20/08 & $8 \mathrm{~F} 20002$ & AGG-RRL-001 \\
\hline 14269-63-7 & Thorium-230 & $<1.09 \mathrm{E} 2$ & pCi/g dry & $1.09 \mathrm{E} 2$ & & $6 / 20 / 08$ & $8 \mathrm{~F} 20002$ & AGG-RRL-001 \\
\hline $14331-85-2$ & Protactinium-231 & $<1.28 \mathrm{E} 1$ & pCi/g dry & $1.28 \mathrm{E} 1$ & & 6/20/08 & $8 \mathrm{~F} 20002$ & AGG-RRL-001 \\
\hline $14932-40-2$ & Thorium-231 & $<6.05 \mathrm{E} 1$ & pCi/g dry & $6.05 \mathrm{E} 1$ & & 6/20/08 & $8 \mathrm{~F} 20002$ & AGG-RRL-001 \\
\hline 7440-29-1 & Thorium-232 & $<3.59 \mathrm{E} 2$ & pCi/g dry & 3.59E2 & & 6/20/08 & $8 \mathrm{~F} 20002$ & AGG-RRL-001 \\
\hline 13981-14-1 & Protactinium-233 & $<7.73 \mathrm{E}-1$ & pCi/g dry & 7.73E-1 & & 6/20/08 & $8 \mathrm{~F} 20002$ & AGG-RRL-001 \\
\hline \multirow[t]{2}{*}{$15100-28-4$} & Protactinium-234 & $<1.09 \mathrm{E} 0$ & pCi/g dry & $1.09 \mathrm{E} 0$ & & $6 / 20 / 08$ & $8 \mathrm{~F} 20002$ & AGG-RRL-001 \\
\hline & Protactinium-234m & $<4.06 \mathrm{E} 1$ & pCi/g dry & $4.06 \mathrm{E} 1$ & & 6/20/08 & $8 \mathrm{~F} 20002$ & AGG-RRL-001 \\
\hline $15065-10-8$ & Thorium-234 & $<1.00 \mathrm{E} 1$ & pCi/g dry & $1.00 \mathrm{E} 1$ & & 6/20/08 & $8 \mathrm{~F} 20002$ & AGG-RRL-001 \\
\hline 15117-96-1 & Uranium 235 & $<2.55 \mathrm{E} 0$ & pCi/g dry & $2.55 \mathrm{E} 0$ & & 6/20/08 & $8 \mathrm{~F} 20002$ & AGG-RRL-001 \\
\hline 13994-20-2 & Neptunium-237 & $<2.33 \mathrm{E} 0$ & pCi/g dry & 2.33E0 & & 6/20/08 & $8 \mathrm{~F} 20002$ & AGG-RRL-001 \\
\hline \multirow[t]{2}{*}{ 13981-16-3 } & Plutonium-238 & $<3.39 \mathrm{E} 3$ & pCi/g dry & 3.39E3 & & 6/20/08 & $8 \mathrm{~F} 20002$ & AGG-RRL-001 \\
\hline & Uranium 238 & $<5.19 \mathrm{E} 0$ & pCi/g dry & 5.19E0 & & 6/20/08 & $8 \mathrm{~F} 20002$ & AGG-RRL-001 \\
\hline 15117-48-3 & Plutonium-239 & $<4.21 \mathrm{E} 3$ & pCi/g dry & $4.21 \mathrm{E} 3$ & & 6/20/08 & $8 \mathrm{~F} 20002$ & AGG-RRL-001 \\
\hline 14119-33-6 & Plutonium-240 & $<3.54 \mathrm{E} 3$ & pCi/g dry & $3.54 \mathrm{E} 3$ & & 6/20/08 & $8 \mathrm{~F} 20002$ & AGG-RRL-001 \\
\hline $14596-10-2$ & Americium-241 & $<1.80 \mathrm{E} 0$ & pCi/g dry & $1.80 \mathrm{E} 0$ & & 6/20/08 & $8 \mathrm{~F} 20002$ & AGG-RRL-001 \\
\hline $14993-75-0$ & Americium-243 & $<6.02 \mathrm{E}-1$ & pCi/g dry & $6.02 \mathrm{E}-1$ & & 6/20/08 & $8 \mathrm{~F} 20002$ & AGG-RRL-001 \\
\hline 15757-87-6 & Curium-243 & $<1.08 \mathrm{E} 0$ & pCi/g dry & $1.08 \mathrm{E} 0$ & & 6/20/08 & $8 \mathrm{~F} 20002$ & AGG-RRL-001 \\
\hline 15621-76-8 & Curium-245 & $<8.86 \mathrm{E}-1$ & pCi/g dry & 8.86E-1 & & $6 / 20 / 08$ & 8F20002 & AGG-RRL-001 \\
\hline HEIS No. & B1VJ56B & & b ID: & 05020-08 & & & & \\
\hline 13966-32-0 & Sodium-22 & $<3.33 \mathrm{E}-1$ & pCi/g dry & 3.33E-1 & & 6/20/08 & 8F20002 & AGG-RRL-001 \\
\hline 13966-00-2 & Potassium-40 & $<6.74 \mathrm{E} 0$ & pCi/g dry & $6.74 \mathrm{E} 0$ & & 6/20/08 & $8 \mathrm{~F} 20002$ & AGG-RRL-001 \\
\hline $14392-02-0$ & Chromium-51 & $<2.95 \mathrm{E} 0$ & pCi/g dry & 2.95E0 & & 6/20/08 & $8 \mathrm{~F} 20002$ & AGG-RRL-001 \\
\hline 13966-31-9 & Manganese-54 & $<3.47 \mathrm{E}-1$ & pCi/g dry & 3.47E-1 & & 6/20/08 & $8 \mathrm{~F} 20002$ & AGG-RRL-001 \\
\hline 13981-50-5 & Cobalt-57 & $<3.19 \mathrm{E}-1$ & pCi/g dry & $3.19 \mathrm{E}-1$ & & 6/20/08 & $8 \mathrm{~F} 20002$ & AGG-RRL-001 \\
\hline $14596-12-4$ & Iron-59 & $<6.15 \mathrm{E}-1$ & pCi/g dry & $6.15 \mathrm{E}-1$ & & 6/20/08 & $8 \mathrm{~F} 20002$ & AGG-RRL-001 \\
\hline 10198-40-0 & Cobalt-60 & $<4.01 \mathrm{E}-1$ & pCi/g dry & $4.01 \mathrm{E}-1$ & & 6/20/08 & $8 \mathrm{~F} 20002$ & AGG-RRL-001 \\
\hline 13982-39-3 & Zinc-65 & $<7.19 \mathrm{E}-1$ & pCi/g dry & 7.19E-1 & & 6/20/08 & 8F20002 & AGG-RRL-001 \\
\hline
\end{tabular}


GEA/Soil

\begin{tabular}{|c|c|c|c|c|c|c|c|c|}
\hline CAS \# & Analyte & Results & Units & MDA & UNC & Analyzed & Batch & Method \\
\hline HEIS No. & B1VJ56B & & ab ID: $\quad 0$ & $5020-08$ & & & & \\
\hline 14265-71-5 & Selenium-75 & $<4.99 \mathrm{E}-1$ & pCi/g dry & 4.99E-1 & & 6/20/08 & 8F20002 & AGG-RRL-001 \\
\hline 17056-36-9 & Rubidium-83 & $<8.07 \mathrm{E}-1$ & pCi/g dry & 8.07E-1 & & 6/20/08 & $8 \mathrm{~F} 20002$ & AGG-RRL-001 \\
\hline 13967-73-2 & Strontium-85 & $<3.75 \mathrm{E}-1$ & pCi/g dry & 3.75E-1 & & $6 / 20 / 08$ & $8 \mathrm{~F} 20002$ & AGG-RRL-001 \\
\hline 14932-53-7 & Rubidium-86 & $<4.46 \mathrm{E} 0$ & pCi/g dry & 4.46E0 & & 6/20/08 & $8 \mathrm{~F} 20002$ & AGG-RRL-001 \\
\hline 13982-36-0 & Yttrium-88 & $<2.99 \mathrm{E}-1$ & pCi/g dry & 2.99E-1 & & 6/20/08 & $8 \mathrm{~F} 20002$ & AGG-RRL-001 \\
\hline 14681-63-1 & Niobium-94 & $<3.23 \mathrm{E}-1$ & pCi/g dry & 3.23E-1 & & 6/20/08 & 8 F20002 & AGG-RRL-001 \\
\hline \multirow[t]{3}{*}{ 13967-76-5 } & Niobium-95 & $<3.29 \mathrm{E}-1$ & pCi/g dry & $3.29 \mathrm{E}-1$ & & 6/20/08 & $8 \mathrm{~F} 20002$ & AGG-RRL-001 \\
\hline & Niobium-95m & $<1.42 \mathrm{E} 0$ & pCi/g dry & $1.42 \mathrm{E} 0$ & & 6/20/08 & $8 \mathrm{~F} 20002$ & AGG-RRL-001 \\
\hline & Technetium-95m & $<4.92 \mathrm{E}-1$ & pCi/g dry & 4.92E-1 & & 6/20/08 & $8 \mathrm{~F} 20002$ & AGG-RRL-001 \\
\hline \multirow[t]{2}{*}{ 13967-71-0 } & Zirconium-95 & $<5.81 \mathrm{E}-1$ & pCi/g dry & $5.81 \mathrm{E}-1$ & & 6/20/08 & $8 \mathrm{~F} 20002$ & AGG-RRL-001 \\
\hline & Technetium-99m & $<3.12 \mathrm{E}-1$ & pCi/g dry & $3.12 \mathrm{E}-1$ & & 6/20/08 & $8 \mathrm{~F} 20002$ & AGG-RRL-001 \\
\hline 13968-53-1 & Ruthenium-103 & $<3.75 \mathrm{E}-1$ & pCi/g dry & 3.75E-1 & & 6/20/08 & $8 \mathrm{~F} 20002$ & AGG-RRL-001 \\
\hline 13967-48-1 & Ruthenium-106 & $<3.66 \mathrm{E} 0$ & pCi/g dry & $3.66 \mathrm{E} 0$ & & 6/20/08 & $8 \mathrm{~F} 20002$ & AGG-RRL-001 \\
\hline 14391-65-2 & Silver-108m & $<3.07 \mathrm{E}-1$ & pCi/g dry & 3.07E-1 & & 6/20/08 & $8 \mathrm{~F} 20002$ & AGG-RRL-001 \\
\hline \multirow[t]{2}{*}{ 14109-32-1 } & Cadmium-109 & $<8.87 \mathrm{E} 0$ & pCi/g dry & 8.87E0 & & 6/20/08 & $8 \mathrm{~F} 20002$ & AGG-RRL-001 \\
\hline & Silver-110 & $<3.20 \mathrm{E}-1$ & pCi/g dry & $3.20 \mathrm{E}-1$ & & 6/20/08 & $8 \mathrm{~F} 20002$ & AGG-RRL-001 \\
\hline 14391-76-5 & Silver-110m & $<3.21 \mathrm{E}-1$ & pCi/g dry & $3.21 \mathrm{E}-1$ & & 6/20/08 & $8 \mathrm{~F} 20002$ & AGG-RRL-001 \\
\hline 13966-06-8 & Tin-113 & $<4.69 \mathrm{E}-1$ & pCi/g dry & 4.69E-1 & & 6/20/08 & $8 \mathrm{~F} 20002$ & AGG-RRL-001 \\
\hline 14683-10-4 & Antimony-124 & $<3.92 \mathrm{E}-1$ & pCi/g dry & $3.92 \mathrm{E}-1$ & & 6/20/08 & $8 \mathrm{~F} 20002$ & AGG-RRL-001 \\
\hline 14234-35-6 & Antimony-125 & $<9.63 \mathrm{E}-1$ & pCi/g dry & 9.63E-1 & & 6/20/08 & $8 \mathrm{~F} 20002$ & AGG-RRL-001 \\
\hline 15756-32-8 & Antimony-126 & $<3.00 \mathrm{E}-1$ & pCi/g dry & $3.00 \mathrm{E}-1$ & & 6/20/08 & $8 \mathrm{~F} 20002$ & AGG-RRL-001 \\
\hline 15832-50-5 & Tin-126 & $<7.30 \mathrm{E}-1$ & pCi/g dry & 7.30E-1 & & 6/20/08 & $8 \mathrm{~F} 20002$ & AGG-RRL-001 \\
\hline 10043-66-0 & Iodine-131 & $<3.85 \mathrm{E}-1$ & pCi/g dry & 3.85E-1 & & 6/20/08 & $8 \mathrm{~F} 20002$ & AGG-RRL-001 \\
\hline 13981-41-4 & Barium-133 & $<5.25 \mathrm{E}-1$ & pCi/g dry & $5.25 \mathrm{E}-1$ & & 6/20/08 & $8 \mathrm{~F} 20002$ & AGG-RRL-001 \\
\hline 13967-70-9 & Cesium-134 & $<3.67 \mathrm{E}-1$ & pCi/g dry & 3.67E-1 & & 6/20/08 & $8 \mathrm{~F} 20002$ & AGG-RRL-001 \\
\hline $10045-97-3$ & Cesium-137 & $<3.56 \mathrm{E}-1$ & pCi/g dry & $3.56 \mathrm{E}-1$ & & 6/20/08 & $8 \mathrm{~F} 20002$ & AGG-RRL-001 \\
\hline 13982-30-4 & Cerium-139 & $<3.64 \mathrm{E}-1$ & pCi/g dry & $3.64 \mathrm{E}-1$ & & 6/20/08 & $8 \mathrm{~F} 20002$ & AGG-RRL-001 \\
\hline 14762-78-8 & Cerium-144 & $<2.54 \mathrm{E} 0$ & pCi/g dry & $2.54 \mathrm{E} 0$ & & 6/20/08 & $8 \mathrm{~F} 20002$ & AGG-RRL-001 \\
\hline 14683-23-9 & Europium-152 & $<1.27 \mathrm{E} 0$ & pCi/g dry & $1.27 \mathrm{E} 0$ & & 6/20/08 & $8 \mathrm{~F} 20002$ & AGG-RRL-001 \\
\hline 14276-65-4 & Gadolinium-153 & $<9.78 \mathrm{E}-1$ & pCi/g dry & $9.78 \mathrm{E}-1$ & & 6/20/08 & $8 \mathrm{~F} 20002$ & AGG-RRL-001 \\
\hline 15585-10-1 & Europium-154 & $<6.70 \mathrm{E}-1$ & pCi/g dry & $6.70 \mathrm{E}-1$ & & 6/20/08 & $8 \mathrm{~F} 20002$ & AGG-RRL-001 \\
\hline 14391-16-3 & Europium-155 & $<1.04 \mathrm{E} 0$ & pCi/g dry & $1.04 \mathrm{E} 0$ & & 6/20/08 & 8 F20002 & AGG-RRL-001 \\
\hline 13982-78-0 & Mercury-203 & $<3.85 \mathrm{E}-1$ & pCi/g dry & 3.85E-1 & & $6 / 20 / 08$ & $8 \mathrm{~F} 20002$ & AGG-RRL-001 \\
\hline 14913-50-9 & Thallium-208 & $<4.07 \mathrm{E}-1$ & pCi/g dry & 4.07E-1 & & 6/20/08 & $8 \mathrm{~F} 20002$ & AGG-RRL-001 \\
\hline 14331-79-4 & Bismuth-210 & $<5.97 \mathrm{E}-1$ & pCi/g dry & 5.97E-1 & & 6/20/08 & $8 \mathrm{~F} 20002$ & AGG-RRL-001 \\
\hline \multirow[t]{3}{*}{$14255-04-0$} & Lead-210 & $<4.59 \mathrm{E} 1$ & pCi/g dry & 4.59E1 & & 6/20/08 & 8 F20002 & AGG-RRL-001 \\
\hline & Bismuth-211 & $<7.40 \mathrm{E} 0$ & pCi/g dry & 7.40E0 & & 6/20/08 & $8 \mathrm{~F} 20002$ & AGG-RRL-001 \\
\hline & Lead-211 & $<1.01 \mathrm{E} 1$ & pCi/g dry & $1.01 \mathrm{E} 1$ & & 6/20/08 & $8 \mathrm{~F} 20002$ & AGG-RRL-001 \\
\hline 15092-94-1 & Lead-212 & $<8.24 \mathrm{E}-1$ & pCi/g dry & $8.24 \mathrm{E}-1$ & & 6/20/08 & $8 \mathrm{~F} 20002$ & AGG-RRL-001 \\
\hline 14733-03-0 & Bismuth-214 & $<8.26 \mathrm{E}-1$ & pCi/g dry & $8.26 \mathrm{E}-1$ & & 6/20/08 & $8 \mathrm{~F} 20002$ & AGG-RRL-001 \\
\hline $15067-28-4$ & Lead-214 & $<8.66 \mathrm{E}-1$ & pCi/g dry & 8.66E-1 & & 6/20/08 & $8 \mathrm{~F} 20002$ & AGG-RRL-001 \\
\hline $14835-02-0$ & Radon-219 & $<3.00 \mathrm{E} 0$ & pCi/g dry & $3.00 \mathrm{E} 0$ & & 6/20/08 & $8 \mathrm{~F} 20002$ & AGG-RRL-001 \\
\hline 22481-48-7 & Radon-220 & $<3.12 \mathrm{E} 2$ & pCi/g dry & 3.12E2 & & 6/20/08 & $8 \mathrm{~F} 20002$ & AGG-RRL-001 \\
\hline 28522-20-5 & Radon-221 & $<1.47 \mathrm{E} 0$ & pCi/g dry & $1.47 \mathrm{E} 0$ & & 6/20/08 & $8 \mathrm{~F} 20002$ & AGG-RRL-001 \\
\hline 15756-98-6 & Francium-223 & $<1.04 \mathrm{E} 1$ & pCi/g dry & $1.04 \mathrm{E} 1$ & & 6/20/08 & $8 \mathrm{~F} 20002$ & AGG-RRL-001 \\
\hline $15623-45-7$ & Radium-223 & $<2.20 \mathrm{E} 0$ & pCi/g dry & $2.20 \mathrm{E} 0$ & & 6/20/08 & $8 \mathrm{~F} 20002$ & AGG-RRL-001 \\
\hline 13233-32-4 & Radium-224 & $<8.98 \mathrm{E} 0$ & pCi/g dry & 8.98E0 & & 6/20/08 & 8F20002 & AGG-RRL-001 \\
\hline 13982-63-3 & Radium-226 & $<9.05 \mathrm{E} 0$ & pCi/g dry & $9.05 \mathrm{E} 0$ & & 6/20/08 & $8 \mathrm{~F} 20002$ & AGG-RRL-001 \\
\hline $15623-47-9$ & Thorium-227 & $<3.17 \mathrm{E} 0$ & pCi/g dry & 3.17E0 & & 6/20/08 & $8 F 20002$ & AGG-RRL-001 \\
\hline
\end{tabular}


GEA/Soil

\begin{tabular}{|c|c|c|c|c|c|c|c|c|}
\hline CAS \# & Analyte & Results & Units & MDA & UNC & Analyzed & Batch & Method \\
\hline HEIS No. & B1VJ56B & & ab ID: & $5020-08$ & & & & \\
\hline 14331-83-0 & Actinium-228 & $<1.39 \mathrm{E} 0$ & pCi/g dry & 1.39E0 & & 6/20/08 & $8 \mathrm{~F} 20002$ & AGG-RRL-001 \\
\hline $14274-82-9$ & Thorium-228 & $<1.15 \mathrm{E} 2$ & pCi/g dry & $1.15 \mathrm{E} 2$ & & 6/20/08 & $8 \mathrm{~F} 20002$ & AGG-RRL-001 \\
\hline $14269-63-7$ & Thorium-230 & $<1.21 \mathrm{E} 2$ & pCi/g dry & $1.21 \mathrm{E} 2$ & & $6 / 20 / 08$ & $8 \mathrm{~F} 20002$ & AGG-RRL-001 \\
\hline $14331-85-2$ & Protactinium-231 & $<1.35 \mathrm{E} 1$ & pCi/g dry & $1.35 \mathrm{E} 1$ & & 6/20/08 & $8 \mathrm{~F} 20002$ & AGG-RRL-001 \\
\hline $14932-40-2$ & Thorium-231 & $<6.93 \mathrm{E} 1$ & pCi/g dry & 6.93E1 & & 6/20/08 & $8 \mathrm{~F} 20002$ & AGG-RRL-001 \\
\hline $7440-29-1$ & Thorium-232 & $<3.01 \mathrm{E} 2$ & pCi/g dry & 3.01E2 & & 6/20/08 & $8 \mathrm{~F} 20002$ & AGG-RRL-001 \\
\hline 13981-14-1 & Protactinium-233 & $<8.23 \mathrm{E}-1$ & pCi/g dry & 8.23E-1 & & 6/20/08 & $8 \mathrm{~F} 20002$ & AGG-RRL-001 \\
\hline \multirow{2}{*}{$15100-28-4$} & Protactinium-234 & $<1.27 \mathrm{E} 0$ & pCi/g dry & $1.27 \mathrm{E} 0$ & & $6 / 20 / 08$ & $8 \mathrm{~F} 20002$ & AGG-RRL-001 \\
\hline & Protactinium-234m & $<3.66 \mathrm{E} 1$ & pCi/g dry & $3.66 \mathrm{E} 1$ & & $6 / 20 / 08$ & $8 \mathrm{~F} 20002$ & AGG-RRL-001 \\
\hline $15065-10-8$ & Thorium-234 & $<1.05 \mathrm{E} 1$ & pCi/g dry & $1.05 \mathrm{E} 1$ & & $6 / 20 / 08$ & $8 \mathrm{~F} 20002$ & AGG-RRL-001 \\
\hline 15117-96-1 & Uranium 235 & $<2.63 \mathrm{E} 0$ & pCi/g dry & 2.63E0 & & 6/20/08 & $8 \mathrm{~F} 20002$ & AGG-RRL-001 \\
\hline 13994-20-2 & Neptunium-237 & $<2.70 \mathrm{E} 0$ & pCi/g dry & $2.70 \mathrm{E} 0$ & & $6 / 20 / 08$ & $8 \mathrm{~F} 20002$ & AGG-RRL-001 \\
\hline \multirow[t]{2}{*}{ 13981-16-3 } & Plutonium-238 & $<3.98 \mathrm{E} 3$ & pCi/g dry & 3.98E3 & & 6/20/08 & $8 \mathrm{~F} 20002$ & AGG-RRL-001 \\
\hline & Uranium 238 & $<5.65 \mathrm{E} 0$ & pCi/g dry & $5.65 \mathrm{E} 0$ & & $6 / 20 / 08$ & $8 \mathrm{~F} 20002$ & AGG-RRL-001 \\
\hline $15117-48-3$ & Plutonium-239 & $<4.15 \mathrm{E} 3$ & pCi/g dry & 4.15E3 & & 6/20/08 & 8 F20002 & AGG-RRL-001 \\
\hline 14119-33-6 & Plutonium-240 & $<3.99 \mathrm{E} 3$ & pCi/g dry & 3.99E3 & & 6/20/08 & 8 F20002 & AGG-RRL-001 \\
\hline $14596-10-2$ & Americium-241 & $<1.54 \mathrm{E} 0$ & pCi/g dry & $1.54 \mathrm{E} 0$ & & $6 / 20 / 08$ & $8 \mathrm{~F} 20002$ & AGG-RRL-001 \\
\hline $14993-75-0$ & Americium-243 & $<6.64 \mathrm{E}-1$ & pCi/g dry & $6.64 \mathrm{E}-1$ & & 6/20/08 & $8 \mathrm{~F} 20002$ & AGG-RRL-001 \\
\hline $15757-87-6$ & Curium-243 & $<1.22 \mathrm{E} 0$ & pCi/g dry & $1.22 \mathrm{E} 0$ & & 6/20/08 & $8 \mathrm{~F} 20002$ & AGG-RRL-001 \\
\hline 15621-76-8 & Curium-245 & $<1.00 \mathrm{E} 0$ & pCi/g dry & $1.00 \mathrm{E} 0$ & & 6/20/08 & $8 \mathrm{~F} 20002$ & AGG-RRL-001 \\
\hline HEIS No. & B1VJ56C & & ab ID: & $55020-09$ & & & & \\
\hline 13966-32-0 & Sodium-22 & $<2.87 \mathrm{E}-1$ & pCi/g dry & 2.87E-1 & & 6/20/08 & $8 \mathrm{~F} 20002$ & AGG-RRL-001 \\
\hline $13966-00-2$ & Potassium-40 & 1.65E1 & pCi/g dry & 2.83E0 & 1.1 & 6/20/08 & $8 \mathrm{~F} 20002$ & AGG-RRL-001 \\
\hline $14392-02-0$ & Chromium-51 & $<2.45 \mathrm{E} 0$ & pCi/g dry & $2.45 \mathrm{E} 0$ & & 6/20/08 & 8 F20002 & AGG-RRL-001 \\
\hline 13966-31-9 & Manganese-54 & $<2.45 \mathrm{E}-1$ & pCi/g dry & $2.45 \mathrm{E}-1$ & & 6/20/08 & 8F20002 & AGG-RRL-001 \\
\hline 13981-50-5 & Cobalt-57 & $<2.61 \mathrm{E}-1$ & pCi/g dry & $2.61 \mathrm{E}-1$ & & 6/20/08 & 8 F20002 & AGG-RRL-001 \\
\hline $14596-12-4$ & Iron-59 & $<5.03 \mathrm{E}-1$ & pCi/g dry & 5.03E-1 & & 6/20/08 & 8 F20002 & AGG-RRL-001 \\
\hline $10198-40-0$ & Cobalt-60 & $<2.80 \mathrm{E}-1$ & pCi/g dry & $2.80 \mathrm{E}-1$ & & 6/20/08 & 8 F20002 & AGG-RRL-001 \\
\hline 13982-39-3 & Zinc-65 & $<5.94 \mathrm{E}-1$ & pCi/g dry & $5.94 \mathrm{E}-1$ & & 6/20/08 & $8 \mathrm{~F} 20002$ & AGG-RRL-001 \\
\hline $14265-71-5$ & Selenium-75 & $<4.18 \mathrm{E}-1$ & pCi/g dry & 4.18E-1 & & 6/20/08 & 8 F20002 & AGG-RRL-001 \\
\hline $17056-36-9$ & Rubidium-83 & $<4.80 \mathrm{E}-1$ & pCi/g dry & $4.80 \mathrm{E}-1$ & & 6/20/08 & $8 \mathrm{~F} 20002$ & AGG-RRL-001 \\
\hline 13967-73-2 & Strontium-85 & $<3.35 \mathrm{E}-1$ & pCi/g dry & 3.35E-1 & & 6/20/08 & 8 F20002 & AGG-RRL-001 \\
\hline 14932-53-7 & Rubidium-86 & $<2.99 \mathrm{E} 0$ & pCi/g dry & $2.99 \mathrm{E} 0$ & & 6/20/08 & $8 F 20002$ & AGG-RRL-001 \\
\hline 13982-36-0 & Yttrium-88 & $<1.87 \mathrm{E}-1$ & pCi/g dry & $1.87 \mathrm{E}-1$ & & 6/20/08 & $8 \mathrm{~F} 20002$ & AGG-RRL-001 \\
\hline 14681-63-1 & Niobium-94 & $<2.53 \mathrm{E}-1$ & pCi/g dry & 2.53E-1 & & 6/20/08 & 8 F20002 & AGG-RRL-001 \\
\hline \multirow[t]{3}{*}{ 13967-76-5 } & Niobium-95 & $<2.76 \mathrm{E}-1$ & pCi/g dry & 2.76E-1 & & 6/20/08 & $8 \mathrm{~F} 20002$ & AGG-RRL-001 \\
\hline & Niobium-95m & $<1.20 \mathrm{E} 0$ & pCi/g dry & $1.20 \mathrm{E} 0$ & & 6/20/08 & 8 F20002 & AGG-RRL-001 \\
\hline & Technetium-95m & $<4.13 \mathrm{E}-1$ & pCi/g dry & 4.13E-1 & & 6/20/08 & $8 \mathrm{~F} 20002$ & AGG-RRL-001 \\
\hline \multirow[t]{2}{*}{ 13967-71-0 } & Zirconium-95 & $<4.65 \mathrm{E}-1$ & pCi/g dry & 4.65E-1 & & 6/20/08 & 8 F20002 & AGG-RRL-001 \\
\hline & Technetium-99m & $<2.66 \mathrm{E}-1$ & pCi/g dry & 2.66E-1 & & $6 / 20 / 08$ & $8 \mathrm{~F} 20002$ & AGG-RRL-001 \\
\hline 13968-53-1 & Ruthenium-103 & $<2.73 \mathrm{E}-1$ & pCi/g dry & 2.73E-1 & & 6/20/08 & 8 F20002 & AGG-RRL-001 \\
\hline $13967-48-1$ & Ruthenium-106 & $<2.45 \mathrm{E} 0$ & pCi/g dry & $2.45 \mathrm{E} 0$ & & 6/20/08 & 8 F20002 & AGG-RRL-001 \\
\hline $14391-65-2$ & Silver-108m & $<2.53 \mathrm{E}-1$ & pCi/g dry & 2.53E-1 & & 6/20/08 & $8 \mathrm{~F} 20002$ & AGG-RRL-001 \\
\hline \multirow[t]{2}{*}{ 14109-32-1 } & Cadmium-109 & $<7.04 \mathrm{E} 0$ & pCi/g dry & 7.04E0 & & $6 / 20 / 08$ & $8 \mathrm{~F} 20002$ & AGG-RRL-001 \\
\hline & Silver-110 & $<2.69 \mathrm{E}-1$ & pCi/g dry & 2.69E-1 & & $6 / 20 / 08$ & 8 F20002 & AGG-RRL-001 \\
\hline $14391-76-5$ & Silver-110m & $<2.70 \mathrm{E}-1$ & pCi/g dry & 2.70E-1 & & 6/20/08 & 8 F20002 & AGG-RRL-001 \\
\hline 13966-06-8 & Tin-113 & $<3.65 \mathrm{E}-1$ & pCi/g dry & 3.65E-1 & & 6/20/08 & 8 F20002 & AGG-RRL-001 \\
\hline $14683-10-4$ & Antimony-124 & $<2.71 \mathrm{E}-1$ & pCi/g dry & $2.71 \mathrm{E}-1$ & & $6 / 20 / 08$ & 8F20002 & AGG-RRL-001 \\
\hline
\end{tabular}


GEA/Soil

\begin{tabular}{|c|c|c|c|c|c|c|c|c|}
\hline CAS \# & Analyte & Results & Units & MDA & UNC & Analyzed & Batch & Method \\
\hline HEIS No. & B1VJ56C & \multicolumn{3}{|c|}{ Lab ID: $\quad$ 0805020-09 } & & & & \\
\hline 14234-35-6 & Antimony-125 & $<7.72 \mathrm{E}-1$ & pCi/g dry & $7.72 \mathrm{E}-1$ & & 6/20/08 & $8 \mathrm{~F} 20002$ & AGG-RRL-001 \\
\hline 15756-32-8 & Antimony-126 & $<2.66 \mathrm{E}-1$ & pCi/g dry & $2.66 \mathrm{E}-1$ & & 6/20/08 & $8 \mathrm{~F} 20002$ & AGG-RRL-001 \\
\hline $15832-50-5$ & Tin-126 & $<5.55 \mathrm{E}-1$ & pCi/g dry & $5.55 \mathrm{E}-1$ & & $6 / 20 / 08$ & $8 \mathrm{~F} 20002$ & AGG-RRL-001 \\
\hline 10043-66-0 & Iodine-131 & $<2.86 \mathrm{E}-1$ & pCi/g dry & $2.86 \mathrm{E}-1$ & & 6/20/08 & $8 \mathrm{~F} 20002$ & AGG-RRL-001 \\
\hline 13981-41-4 & Barium-133 & $<4.76 \mathrm{E}-1$ & pCi/g dry & 4.76E-1 & & 6/20/08 & $8 \mathrm{~F} 20002$ & AGG-RRL-001 \\
\hline 13967-70-9 & Cesium-134 & $<3.08 \mathrm{E}-1$ & pCi/g dry & $3.08 \mathrm{E}-1$ & & 6/20/08 & 8 F20002 & AGG-RRL-001 \\
\hline 10045-97-3 & Cesium-137 & $<3.06 \mathrm{E}-1$ & pCi/g dry & 3.06E-1 & & 6/20/08 & $8 \mathrm{~F} 20002$ & AGG-RRL-001 \\
\hline 13982-30-4 & Cerium-139 & $<2.91 \mathrm{E}-1$ & pCi/g dry & 2.91E-1 & & 6/20/08 & $8 \mathrm{~F} 20002$ & AGG-RRL-001 \\
\hline 14762-78-8 & Cerium-144 & $<2.14 \mathrm{E} 0$ & pCi/g dry & $2.14 \mathrm{E} 0$ & & 6/20/08 & $8 \mathrm{~F} 20002$ & AGG-RRL-001 \\
\hline 14683-23-9 & Europium-152 & $<1.00 \mathrm{E} 0$ & pCi/g dry & $1.00 \mathrm{E} 0$ & & $6 / 20 / 08$ & $8 \mathrm{~F} 20002$ & AGG-RRL-001 \\
\hline $14276-65-4$ & Gadolinium-153 & $<8.07 \mathrm{E}-1$ & pCi/g dry & 8.07E-1 & & 6/20/08 & $8 \mathrm{~F} 20002$ & AGG-RRL-001 \\
\hline 15585-10-1 & Europium-154 & $<5.49 \mathrm{E}-1$ & pCi/g dry & 5.49E-1 & & $6 / 20 / 08$ & $8 \mathrm{~F} 20002$ & AGG-RRL-001 \\
\hline 14391-16-3 & Europium-155 & $<7.86 \mathrm{E}-1$ & pCi/g dry & 7.86E-1 & & 6/20/08 & $8 \mathrm{~F} 20002$ & AGG-RRL-001 \\
\hline 13982-78-0 & Mercury-203 & $<3.19 \mathrm{E}-1$ & pCi/g dry & 3.19E-1 & & 6/20/08 & $8 \mathrm{~F} 20002$ & AGG-RRL-001 \\
\hline 14913-50-9 & Thallium-208 & 4.38E-1 & pCi/g dry & $2.92 \mathrm{E}-1$ & 0.331 & 6/20/08 & $8 \mathrm{~F} 20002$ & AGG-RRL-001 \\
\hline 14331-79-4 & Bismuth-210 & $<5.03 \mathrm{E}-1$ & pCi/g dry & 5.03E-1 & & 6/20/08 & $8 \mathrm{~F} 20002$ & AGG-RRL-001 \\
\hline \multirow[t]{3}{*}{$14255-04-0$} & Lead-210 & $<9.94 \mathrm{E} 1$ & pCi/g dry & 9.94E1 & & 6/20/08 & $8 \mathrm{~F} 20002$ & AGG-RRL-001 \\
\hline & Bismuth-211 & $<6.04 \mathrm{E} 0$ & pCi/g dry & $6.04 \mathrm{E} 0$ & & 6/20/08 & $8 \mathrm{~F} 20002$ & AGG-RRL-001 \\
\hline & Lead-211 & $<8.26$ E0 & pCi/g dry & $8.26 \mathrm{E} 0$ & & 6/20/08 & $8 \mathrm{~F} 20002$ & AGG-RRL-001 \\
\hline 15092-94-1 & Lead-212 & $<7.14 \mathrm{E}-1$ & pCi/g dry & 7.14E-1 & & 6/20/08 & $8 \mathrm{~F} 20002$ & AGG-RRL-001 \\
\hline 14733-03-0 & Bismuth-214 & $<6.74 \mathrm{E}-1$ & pCi/g dry & $6.74 \mathrm{E}-1$ & & 6/20/08 & $8 \mathrm{~F} 20002$ & AGG-RRL-001 \\
\hline 15067-28-4 & Lead-214 & 1.66E0 & pCi/g dry & $6.06 \mathrm{E}-1$ & 1.81 & 6/20/08 & $8 \mathrm{~F} 20002$ & AGG-RRL-001 \\
\hline 14835-02-0 & Radon-219 & $<2.56 \mathrm{E} 0$ & pCi/g dry & $2.56 \mathrm{E} 0$ & & 6/20/08 & $8 \mathrm{~F} 20002$ & AGG-RRL-001 \\
\hline 22481-48-7 & Radon-220 & $<2.22 \mathrm{E} 2$ & pCi/g dry & 2.22E2 & & 6/20/08 & $8 \mathrm{~F} 20002$ & AGG-RRL-001 \\
\hline 28522-20-5 & Radon-221 & $<1.24 \mathrm{E} 0$ & pCi/g dry & $1.24 \mathrm{E} 0$ & & 6/20/08 & $8 \mathrm{~F} 20002$ & AGG-RRL-001 \\
\hline 15756-98-6 & Francium-223 & $<8.81 \mathrm{E} 0$ & pCi/g dry & 8.81E0 & & 6/20/08 & $8 \mathrm{~F} 20002$ & AGG-RRL-001 \\
\hline 15623-45-7 & Radium-223 & $<1.86 \mathrm{E} 0$ & pCi/g dry & $1.86 \mathrm{E} 0$ & & 6/20/08 & $8 \mathrm{~F} 20002$ & AGG-RRL-001 \\
\hline 13233-32-4 & Radium-224 & $<7.51 \mathrm{E} 0$ & pCi/g dry & 7.51E0 & & 6/20/08 & $8 \mathrm{~F} 20002$ & AGG-RRL-001 \\
\hline 13982-63-3 & Radium-226 & $<7.67 \mathrm{E} 0$ & pCi/g dry & 7.67E0 & & 6/20/08 & $8 \mathrm{~F} 20002$ & AGG-RRL-001 \\
\hline 15623-47-9 & Thorium-227 & $<2.67 \mathrm{E} 0$ & pCi/g dry & 2.67E0 & & 6/20/08 & $8 \mathrm{~F} 20002$ & AGG-RRL-001 \\
\hline 14331-83-0 & Actinium-228 & $<1.16 \mathrm{E} 0$ & pCi/g dry & $1.16 \mathrm{E} 0$ & & 6/20/08 & $8 \mathrm{~F} 20002$ & AGG-RRL-001 \\
\hline 14274-82-9 & Thorium-228 & $<9.57 \mathrm{E} 1$ & pCi/g dry & $9.57 \mathrm{E} 1$ & & 6/20/08 & 8 F20002 & AGG-RRL-001 \\
\hline 14269-63-7 & Thorium-230 & $<9.72 \mathrm{E} 1$ & pCi/g dry & $9.72 \mathrm{E} 1$ & & 6/20/08 & $8 \mathrm{~F} 20002$ & AGG-RRL-001 \\
\hline 14331-85-2 & Protactinium-231 & $<1.11 \mathrm{E} 1$ & pCi/g dry & $1.11 \mathrm{E} 1$ & & 6/20/08 & $8 \mathrm{~F} 20002$ & AGG-RRL-001 \\
\hline 14932-40-2 & Thorium-231 & $<5.47 \mathrm{E} 1$ & pCi/g dry & $5.47 \mathrm{E} 1$ & & 6/20/08 & $8 \mathrm{~F} 20002$ & AGG-RRL-001 \\
\hline 7440-29-1 & Thorium-232 & $<3.09 \mathrm{E} 2$ & pCi/g dry & 3.09E2 & & 6/20/08 & $8 \mathrm{~F} 20002$ & AGG-RRL-001 \\
\hline 13981-14-1 & Protactinium-233 & $<7.01 \mathrm{E}-1$ & pCi/g dry & 7.01E-1 & & 6/20/08 & $8 \mathrm{~F} 20002$ & AGG-RRL-001 \\
\hline \multirow[t]{2}{*}{$15100-28-4$} & Protactinium-234 & $<1.03 \mathrm{E} 0$ & pCi/g dry & 1.03E0 & & 6/20/08 & $8 \mathrm{~F} 20002$ & AGG-RRL-001 \\
\hline & Protactinium-234m & $<3.32 \mathrm{E} 1$ & pCi/g dry & 3.32E1 & & 6/20/08 & $8 \mathrm{~F} 20002$ & AGG-RRL-001 \\
\hline $15065-10-8$ & Thorium-234 & $<9.22 \mathrm{E} 0$ & pCi/g dry & $9.22 \mathrm{E} 0$ & & 6/20/08 & $8 \mathrm{~F} 20002$ & AGG-RRL-001 \\
\hline 15117-96-1 & Uranium 235 & $<2.25 \mathrm{E} 0$ & pCi/g dry & $2.25 \mathrm{E} 0$ & & 6/20/08 & $8 \mathrm{~F} 20002$ & AGG-RRL-001 \\
\hline 13994-20-2 & Neptunium-237 & $<2.05 \mathrm{E} 0$ & pCi/g dry & 2.05E0 & & 6/20/08 & $8 \mathrm{~F} 20002$ & AGG-RRL-001 \\
\hline \multirow[t]{2}{*}{ 13981-16-3 } & Plutonium-238 & $<3.20 \mathrm{E} 3$ & pCi/g dry & $3.20 \mathrm{E} 3$ & & 6/20/08 & $8 \mathrm{~F} 20002$ & AGG-RRL-001 \\
\hline & Uranium 238 & $<4.77 \mathrm{E} 0$ & pCi/g dry & 4.77E0 & & $6 / 20 / 08$ & $8 \mathrm{~F} 20002$ & AGG-RRL-001 \\
\hline 15117-48-3 & Plutonium-239 & $<3.66 \mathrm{E} 3$ & pCi/g dry & 3.66E3 & & 6/20/08 & $8 \mathrm{~F} 20002$ & AGG-RRL-001 \\
\hline 14119-33-6 & Plutonium-240 & $<3.20 \mathrm{E} 3$ & pCi/g dry & $3.20 \mathrm{E} 3$ & & 6/20/08 & $8 \mathrm{~F} 20002$ & AGG-RRL-001 \\
\hline $14596-10-2$ & Americium-241 & $<1.54 \mathrm{E} 0$ & pCi/g dry & $1.54 \mathrm{E} 0$ & & 6/20/08 & 8F20002 & AGG-RRL-001 \\
\hline 14993-75-0 & Americium-243 & $<3.44 \mathrm{E}-1$ & pCi/g dry & 3.44E-1 & & 6/20/08 & $8 \mathrm{~F} 20002$ & AGG-RRL-001 \\
\hline 15757-87-6 & Curium-243 & $<9.74 \mathrm{E}-1$ & pCi/g dry & $9.74 \mathrm{E}-1$ & & 6/20/08 & $8 \mathrm{~F} 20002$ & AGG-RRL-001 \\
\hline
\end{tabular}




\section{GEA/Soil}

\begin{tabular}{|c|c|c|c|c|c|c|c|c|}
\hline CAS \# & Analyte & Results & Units & MDA & UNC & Analyzed & Batch & Method \\
\hline HEIS No. & B1VJ56C & \multicolumn{4}{|c|}{ Lab ID: $\quad$ 0805020-09 } & & & \\
\hline 15621-76-8 & Curium-245 & $<8.00 \mathrm{E}-1$ & pCi/g dry & 8.00E-1 & & 6/20/08 & 8F20002 & AGG-RRL-001 \\
\hline HEIS No. & B1VJ58C & \multicolumn{4}{|c|}{ Lab ID: $\quad$ 0805020-10 } & & & \\
\hline 13966-32-0 & Sodium-22 & $<3.62 \mathrm{E}-1$ & pCi/g dry & $3.62 \mathrm{E}-1$ & & 6/20/08 & 8F20002 & AGG-RRL-001 \\
\hline $13966-00-2$ & Potassium-40 & 2.06E1 & pCi/g dry & 2.94E0 & 1.18 & 6/20/08 & $8 \mathrm{~F} 20002$ & AGG-RRL-001 \\
\hline $14392-02-0$ & Chromium-51 & $<3.24 \mathrm{E} 0$ & pCi/g dry & $3.24 \mathrm{E} 0$ & & 6/20/08 & 8F20002 & AGG-RRL-001 \\
\hline 13966-31-9 & Manganese-54 & $<3.75 \mathrm{E}-1$ & pCi/g dry & 3.75E-1 & & $6 / 20 / 08$ & 8 F20002 & AGG-RRL-001 \\
\hline $13981-50-5$ & Cobalt-57 & $<3.35 \mathrm{E}-1$ & pCi/g dry & 3.35E-1 & & $6 / 20 / 08$ & $8 \mathrm{~F} 20002$ & AGG-RRL-001 \\
\hline $14596-12-4$ & Iron-59 & $<7.00 \mathrm{E}-1$ & pCi/g dry & $7.00 \mathrm{E}-1$ & & 6/20/08 & $8 \mathrm{~F} 20002$ & AGG-RRL-001 \\
\hline $10198-40-0$ & Cobalt-60 & $<6.37 \mathrm{E}-1$ & pCi/g dry & 6.37E-1 & & 6/20/08 & 8F20002 & AGG-RRL-001 \\
\hline 13982-39-3 & Zinc-65 & $<8.73 \mathrm{E}-1$ & pCi/g dry & 8.73E-1 & & 6/20/08 & 8F20002 & AGG-RRL-001 \\
\hline $14265-71-5$ & Selenium-75 & $<5.54 \mathrm{E}-1$ & pCi/g dry & $5.54 \mathrm{E}-1$ & & $6 / 20 / 08$ & $8 \mathrm{~F} 20002$ & AGG-RRL-001 \\
\hline 17056-36-9 & Rubidium-83 & $<8.43 \mathrm{E}-1$ & pCi/g dry & 8.43E-1 & & 6/20/08 & 8 F20002 & AGG-RRL-001 \\
\hline 13967-73-2 & Strontium-85 & $<3.97 \mathrm{E}-1$ & pCi/g dry & $3.97 \mathrm{E}-1$ & & 6/20/08 & 8F20002 & AGG-RRL-001 \\
\hline 14932-53-7 & Rubidium-86 & $<4.69 \mathrm{E} 0$ & pCi/g dry & 4.69E0 & & $6 / 20 / 08$ & $8 \mathrm{~F} 20002$ & AGG-RRL-001 \\
\hline $13982-36-0$ & Yttrium-88 & $<2.99 \mathrm{E}-1$ & pCi/g dry & $2.99 \mathrm{E}-1$ & & $6 / 20 / 08$ & $8 \mathrm{~F} 20002$ & AGG-RRL-001 \\
\hline 14681-63-1 & Niobium-94 & $<3.51 \mathrm{E}-1$ & pCi/g dry & $3.51 \mathrm{E}-1$ & & 6/20/08 & $8 \mathrm{~F} 20002$ & AGG-RRL-001 \\
\hline \multirow[t]{3}{*}{ 13967-76-5 } & Niobium-95 & $<3.39 \mathrm{E}-1$ & pCi/g dry & 3.39E-1 & & 6/20/08 & 8F20002 & AGG-RRL-001 \\
\hline & Niobium-95m & $<1.52 \mathrm{E} 0$ & pCi/g dry & $1.52 \mathrm{E} 0$ & & 6/20/08 & 8F20002 & AGG-RRL-001 \\
\hline & Technetium-95m & $<5.15 \mathrm{E}-1$ & pCi/g dry & $5.15 \mathrm{E}-1$ & & 6/20/08 & 8 F20002 & AGG-RRL-001 \\
\hline \multirow[t]{2}{*}{ 13967-71-0 } & Zirconium-95 & $<6.29 \mathrm{E}-1$ & pCi/g dry & $6.29 \mathrm{E}-1$ & & 6/20/08 & 8F20002 & AGG-RRL-001 \\
\hline & Technetium-99m & $<3.39 \mathrm{E}-1$ & pCi/g dry & 3.39E-1 & & 6/20/08 & 8 F20002 & AGG-RRL-001 \\
\hline 13968-53-1 & Ruthenium-103 & $<3.89 \mathrm{E}-1$ & pCi/g dry & 3.89E-1 & & 6/20/08 & 8F20002 & AGG-RRL-001 \\
\hline 13967-48-1 & Ruthenium-106 & $<4.06 \mathrm{E} 0$ & pCi/g dry & 4.06E0 & & 6/20/08 & $8 \mathrm{~F} 20002$ & AGG-RRL-001 \\
\hline 14391-65-2 & Silver-108m & $<3.53 \mathrm{E}-1$ & pCi/g dry & 3.53E-1 & & 6/20/08 & 8F20002 & AGG-RRL-001 \\
\hline \multirow[t]{2}{*}{$14109-32-1$} & Cadmium-109 & $<9.28 \mathrm{E} 0$ & pCi/g dry & $9.28 \mathrm{E} 0$ & & 6/20/08 & 8F20002 & AGG-RRL-001 \\
\hline & Silver-110 & $<3.61 \mathrm{E}-1$ & pCi/g dry & $3.61 \mathrm{E}-1$ & & 6/20/08 & 8F20002 & AGG-RRL-001 \\
\hline $14391-76-5$ & Silver-110m & $<3.62 \mathrm{E}-1$ & pCi/g dry & $3.62 \mathrm{E}-1$ & & 6/20/08 & 8F20002 & AGG-RRL-001 \\
\hline 13966-06-8 & Tin-113 & $<4.91 \mathrm{E}-1$ & pCi/g dry & $4.91 \mathrm{E}-1$ & & 6/20/08 & 8F20002 & AGG-RRL-001 \\
\hline $14683-10-4$ & Antimony-124 & $<4.22 \mathrm{E}-1$ & pCi/g dry & $4.22 \mathrm{E}-1$ & & 6/20/08 & 8 F20002 & AGG-RRL-001 \\
\hline 14234-35-6 & Antimony-125 & $<1.09 \mathrm{E} 0$ & pCi/g dry & $1.09 \mathrm{E} 0$ & & 6/20/08 & 8F20002 & AGG-RRL-001 \\
\hline 15756-32-8 & Antimony-126 & $<3.39 \mathrm{E}-1$ & pCi/g dry & 3.39E-1 & & 6/20/08 & 8F20002 & AGG-RRL-001 \\
\hline $15832-50-5$ & Tin-126 & $<7.58 \mathrm{E}-1$ & pCi/g dry & $7.58 \mathrm{E}-1$ & & 6/20/08 & 8F20002 & AGG-RRL-001 \\
\hline $10043-66-0$ & Iodine-131 & $<4.25 \mathrm{E}-1$ & pCi/g dry & 4.25E-1 & & 6/20/08 & $8 \mathrm{~F} 20002$ & AGG-RRL-001 \\
\hline 13981-41-4 & Barium-133 & $<5.90 \mathrm{E}-1$ & pCi/g dry & $5.90 \mathrm{E}-1$ & & 6/20/08 & $8 \mathrm{~F} 20002$ & AGG-RRL-001 \\
\hline 13967-70-9 & Cesium-134 & $<4.28 \mathrm{E}-1$ & pCi/g dry & $4.28 \mathrm{E}-1$ & & 6/20/08 & 8F20002 & AGG-RRL-001 \\
\hline 10045-97-3 & Cesium-137 & $<4.02 \mathrm{E}-1$ & pCi/g dry & $4.02 \mathrm{E}-1$ & & 6/20/08 & 8F20002 & AGG-RRL-001 \\
\hline 13982-30-4 & Cerium-139 & $<3.86 \mathrm{E}-1$ & pCi/g dry & 3.86E-1 & & 6/20/08 & 8F20002 & AGG-RRL-001 \\
\hline 14762-78-8 & Cerium-144 & $<2.77 \mathrm{E} 0$ & pCi/g dry & 2.77E0 & & 6/20/08 & 8F20002 & AGG-RRL-001 \\
\hline 14683-23-9 & Europium-152 & $<1.37 \mathrm{E} 0$ & pCi/g dry & $1.37 \mathrm{E} 0$ & & 6/20/08 & 8F20002 & AGG-RRL-001 \\
\hline $14276-65-4$ & Gadolinium-153 & $<1.03 \mathrm{E} 0$ & pCi/g dry & $1.03 \mathrm{E} 0$ & & 6/20/08 & 8 F20002 & AGG-RRL-001 \\
\hline 15585-10-1 & Europium-154 & $<7.10 \mathrm{E}-1$ & pCi/g dry & $7.10 \mathrm{E}-1$ & & 6/20/08 & 8F20002 & AGG-RRL-001 \\
\hline 14391-16-3 & Europium-155 & $<1.08 \mathrm{E} 0$ & pCi/g dry & $1.08 \mathrm{E} 0$ & & 6/20/08 & $8 \mathrm{~F} 20002$ & AGG-RRL-001 \\
\hline 13982-78-0 & Mercury-203 & $<4.22 \mathrm{E}-1$ & pCi/g dry & $4.22 \mathrm{E}-1$ & & 6/20/08 & 8F20002 & AGG-RRL-001 \\
\hline $14913-50-9$ & Thallium-208 & $<4.32 \mathrm{E}-1$ & pCi/g dry & 4.32E-1 & & 6/20/08 & 8F20002 & AGG-RRL-001 \\
\hline 14331-79-4 & Bismuth-210 & $<6.58 \mathrm{E}-1$ & pCi/g dry & $6.58 \mathrm{E}-1$ & & 6/20/08 & 8F20002 & AGG-RRL-001 \\
\hline \multirow[t]{3}{*}{ 14255-04-0 } & Lead-210 & $<4.53 \mathrm{E} 1$ & pCi/g dry & 4.53E1 & & 6/20/08 & 8F20002 & AGG-RRL-001 \\
\hline & Bismuth-211 & $<7.48 \mathrm{E} 0$ & pCi/g dry & $7.48 \mathrm{E} 0$ & & 6/20/08 & 8F20002 & AGG-RRL-001 \\
\hline & Lead-211 & $<1.02 \mathrm{E} 1$ & pCi/g dry & $1.02 \mathrm{E} 1$ & & 6/20/08 & $8 F 20002$ & AGG-RRL-001 \\
\hline
\end{tabular}


GEA/Soil

\begin{tabular}{|c|c|c|c|c|c|c|c|c|}
\hline CAS \# & Analyte & Results & Units & MDA & UNC & Analyzed & Batch & Method \\
\hline HEIS No. & B1VJ58C & \multicolumn{4}{|c|}{ Lab ID: $\quad$ 0805020-10 } & & & \\
\hline 15092-94-1 & Lead-212 & $<8.83 \mathrm{E}-1$ & pCi/g dry & 8.83E-1 & & 6/20/08 & 8F20002 & AGG-RRL-001 \\
\hline 14733-03-0 & Bismuth-214 & $<9.08 \mathrm{E}-1$ & pCi/g dry & $9.08 \mathrm{E}-1$ & & 6/20/08 & 8F20002 & AGG-RRL-001 \\
\hline $15067-28-4$ & Lead-214 & $<9.50 \mathrm{E}-1$ & pCi/g dry & $9.50 \mathrm{E}-1$ & & 6/20/08 & 8F20002 & AGG-RRL-001 \\
\hline $14835-02-0$ & Radon-219 & $<3.28 \mathrm{E} 0$ & pCi/g dry & $3.28 \mathrm{E} 0$ & & 6/20/08 & 8F20002 & AGG-RRL-001 \\
\hline 22481-48-7 & Radon-220 & $<3.27 \mathrm{E} 2$ & pCi/g dry & 3.27E2 & & 6/20/08 & 8F20002 & AGG-RRL-001 \\
\hline 28522-20-5 & Radon-221 & $<1.52 \mathrm{E} 0$ & pCi/g dry & $1.52 \mathrm{E} 0$ & & 6/20/08 & 8F20002 & AGG-RRL-001 \\
\hline 15756-98-6 & Francium-223 & $<1.12 \mathrm{E} 1$ & pCi/g dry & $1.12 \mathrm{E} 1$ & & 6/20/08 & 8F20002 & AGG-RRL-001 \\
\hline 15623-45-7 & Radium-223 & $<2.42 \mathrm{E} 0$ & pCi/g dry & 2.42E0 & & $6 / 20 / 08$ & 8F20002 & AGG-RRL-001 \\
\hline 13233-32-4 & Radium-224 & $<9.54 \mathrm{E} 0$ & pCi/g dry & $9.54 \mathrm{E} 0$ & & 6/20/08 & 8F20002 & AGG-RRL-001 \\
\hline 13982-63-3 & Radium-226 & $<9.36 \mathrm{E} 0$ & pCi/g dry & $9.36 \mathrm{E} 0$ & & $6 / 20 / 08$ & 8F20002 & AGG-RRL-001 \\
\hline 15623-47-9 & Thorium-227 & $<3.39 \mathrm{E} 0$ & pCi/g dry & 3.39E0 & & 6/20/08 & 8F20002 & AGG-RRL-001 \\
\hline $14331-83-0$ & Actinium-228 & $<1.60 \mathrm{E} 0$ & pCi/g dry & $1.60 \mathrm{E} 0$ & & $6 / 20 / 08$ & 8 F20002 & AGG-RRL-001 \\
\hline $14274-82-9$ & Thorium-228 & $<1.23 \mathrm{E} 2$ & pCi/g dry & 1.23E2 & & 6/20/08 & 8F20002 & AGG-RRL-001 \\
\hline $14269-63-7$ & Thorium-230 & $<1.25 \mathrm{E} 2$ & pCi/g dry & $1.25 \mathrm{E} 2$ & & 6/20/08 & $8 F 20002$ & AGG-RRL-001 \\
\hline 14331-85-2 & Protactinium-231 & $<1.48 \mathrm{E} 1$ & pCi/g dry & $1.48 \mathrm{E} 1$ & & 6/20/08 & 8F20002 & AGG-RRL-001 \\
\hline $14932-40-2$ & Thorium-231 & $<7.15 \mathrm{E} 1$ & pCi/g dry & 7.15E1 & & 6/20/08 & 8F20002 & AGG-RRL-001 \\
\hline 7440-29-1 & Thorium-232 & $<3.16 \mathrm{E} 2$ & pCi/g dry & 3.16E2 & & 6/20/08 & 8F20002 & AGG-RRL-001 \\
\hline 13981-14-1 & Protactinium-233 & $<9.00 \mathrm{E}-1$ & pCi/g dry & $9.00 \mathrm{E}-1$ & & 6/20/08 & 8F20002 & AGG-RRL-001 \\
\hline \multirow[t]{2}{*}{ 15100-28-4 } & Protactinium-234 & $<1.33 \mathrm{E} 0$ & pCi/g dry & $1.33 \mathrm{E} 0$ & & 6/20/08 & 8F20002 & AGG-RRL-001 \\
\hline & Protactinium-234m & $<4.89 \mathrm{E} 1$ & pCi/g dry & 4.89E1 & & 6/20/08 & 8F20002 & AGG-RRL-001 \\
\hline $15065-10-8$ & Thorium-234 & $<1.11 \mathrm{E} 1$ & pCi/g dry & $1.11 \mathrm{E} 1$ & & $6 / 20 / 08$ & 8F20002 & AGG-RRL-001 \\
\hline 15117-96-1 & Uranium 235 & $<2.89 \mathrm{E} 0$ & pCi/g dry & 2.89E0 & & $6 / 20 / 08$ & 8F20002 & AGG-RRL-001 \\
\hline 13994-20-2 & Neptunium-237 & $<2.81 \mathrm{E} 0$ & pCi/g dry & $2.81 \mathrm{E} 0$ & & 6/20/08 & $8 F 20002$ & AGG-RRL-001 \\
\hline \multirow[t]{2}{*}{ 13981-16-3 } & Plutonium-238 & $<4.15 \mathrm{E} 3$ & pCi/g dry & 4.15E3 & & $6 / 20 / 08$ & 8F20002 & AGG-RRL-001 \\
\hline & Uranium 238 & $<5.94 \mathrm{E} 0$ & pCi/g dry & 5.94E0 & & $6 / 20 / 08$ & $8 F 20002$ & AGG-RRL-001 \\
\hline $15117-48-3$ & Plutonium-239 & $<4.38 \mathrm{E} 3$ & pCi/g dry & 4.38E3 & & $6 / 20 / 08$ & 8F20002 & AGG-RRL-001 \\
\hline 14119-33-6 & Plutonium-240 & $<4.12 \mathrm{E} 3$ & pCi/g dry & $4.12 \mathrm{E} 3$ & & $6 / 20 / 08$ & 8 F20002 & AGG-RRL-001 \\
\hline 14596-10-2 & Americium-241 & $<1.61 \mathrm{E} 0$ & pCi/g dry & $1.61 \mathrm{E} 0$ & & $6 / 20 / 08$ & 8F20002 & AGG-RRL-001 \\
\hline 14993-75-0 & Americium-243 & $<6.84 \mathrm{E}-1$ & pCi/g dry & $6.84 \mathrm{E}-1$ & & $6 / 20 / 08$ & $8 F 20002$ & AGG-RRL-001 \\
\hline 15757-87-6 & Curium-243 & $<1.26 \mathrm{E} 0$ & pCi/g dry & $1.26 \mathrm{E} 0$ & & 6/20/08 & 8F20002 & AGG-RRL-001 \\
\hline 15621-76-8 & Curium-245 & $<1.04 \mathrm{E} 0$ & pCi/g dry & $1.04 \mathrm{E} 0$ & & $6 / 20 / 08$ & 8F20002 & AGG-RRL-001 \\
\hline HEIS No. & B1VJ58B & \multicolumn{3}{|c|}{ Lab ID: $\quad$ 0805020-11 } & \multirow{7}{*}{1.2} & & & \\
\hline 13966-32-0 & Sodium-22 & $<3.16 \mathrm{E}-1$ & pCi/g dry & $3.16 \mathrm{E}-1$ & & $6 / 23 / 08$ & 8F20002 & AGG-RRL-001 \\
\hline 13966-00-2 & Potassium-40 & 2.09E1 & pCi/g dry & 2.93E0 & & 6/23/08 & 8F20002 & AGG-RRL-001 \\
\hline $14392-02-0$ & Chromium-51 & $<2.71 \mathrm{E} 0$ & pCi/g dry & $2.71 \mathrm{E} 0$ & & 6/23/08 & 8F20002 & AGG-RRL-001 \\
\hline 13966-31-9 & Manganese-54 & $<2.90 \mathrm{E}-1$ & pCi/g dry & $2.90 \mathrm{E}-1$ & & 6/23/08 & 8F20002 & AGG-RRL-001 \\
\hline 13981-50-5 & Cobalt-57 & $<2.71 \mathrm{E}-1$ & pCi/g dry & $2.71 \mathrm{E}-1$ & & 6/23/08 & 8F20002 & AGG-RRL-001 \\
\hline $14596-12-4$ & Iron-59 & $<6.06 \mathrm{E}-1$ & pCi/g dry & $6.06 \mathrm{E}-1$ & & 6/23/08 & 8F20002 & AGG-RRL-001 \\
\hline $10198-40-0$ & Cobalt-60 & $1.72 \mathrm{E} 0$ & pCi/g dry & $2.74 \mathrm{E}-1$ & \multirow[t]{10}{*}{0.154} & 6/23/08 & 8F20002 & AGG-RRL-001 \\
\hline 13982-39-3 & Zinc-65 & $<8.02 \mathrm{E}-1$ & pCi/g dry & 8.02E-1 & & 6/23/08 & 8F20002 & AGG-RRL-001 \\
\hline 14265-71-5 & Selenium-75 & $<4.60 \mathrm{E}-1$ & pCi/g dry & $4.60 \mathrm{E}-1$ & & 6/23/08 & $8 F 20002$ & AGG-RRL-001 \\
\hline 17056-36-9 & Rubidium-83 & $<5.60 \mathrm{E}-1$ & pCi/g dry & $5.60 \mathrm{E}-1$ & & 6/23/08 & 8F20002 & AGG-RRL-001 \\
\hline 13967-73-2 & Strontium-85 & $<1.60 \mathrm{E}-1$ & pCi/g dry & $1.60 \mathrm{E}-1$ & & 6/23/08 & 8F20002 & AGG-RRL-001 \\
\hline 14932-53-7 & Rubidium-86 & $<3.58 \mathrm{E} 0$ & pCi/g dry & 3.58E0 & & 6/23/08 & 8F20002 & AGG-RRL-001 \\
\hline 13982-36-0 & Yttrium-88 & $<1.93 \mathrm{E}-1$ & pCi/g dry & $1.93 \mathrm{E}-1$ & & 6/23/08 & 8F20002 & AGG-RRL-001 \\
\hline 14681-63-1 & Niobium-94 & $<2.93 \mathrm{E}-1$ & pCi/g dry & 2.93E-1 & & 6/23/08 & 8F20002 & AGG-RRL-001 \\
\hline \multirow[t]{2}{*}{$13967-76-5$} & Niobium-95 & $<2.98 \mathrm{E}-1$ & pCi/g dry & $2.98 \mathrm{E}-1$ & & 6/23/08 & 8F20002 & AGG-RRL-001 \\
\hline & Niobium-95m & $<1.33 \mathrm{E} 0$ & pCi/g dry & 1.33E0 & & 6/23/08 & 8F20002 & AGG-RRL-001 \\
\hline
\end{tabular}


GEA/Soil

\begin{tabular}{|c|c|c|c|c|c|c|c|c|}
\hline CAS \# & Analyte & Results & Units & MDA & UNC & Analyzed & Batch & Method \\
\hline \multirow[t]{2}{*}{ HEIS No. } & B1VJ58B & \multicolumn{4}{|c|}{ Lab ID: $\quad$ 0805020-11 } & & & \\
\hline & Technetium-95m & $<4.42 \mathrm{E}-1$ & pCi/g dry & 4.42E-1 & & 6/23/08 & 8F20002 & AGG-RRL-001 \\
\hline \multirow[t]{2}{*}{ 13967-71-0 } & Zirconium-95 & $<5.00 \mathrm{E}-1$ & pCi/g dry & $5.00 \mathrm{E}-1$ & & 6/23/08 & 8 F20002 & AGG-RRL-001 \\
\hline & Technetium-99m & $<2.80 \mathrm{E}-1$ & pCi/g dry & $2.80 \mathrm{E}-1$ & & 6/23/08 & $8 \mathrm{~F} 20002$ & AGG-RRL-001 \\
\hline 13968-53-1 & Ruthenium-103 & $<2.91 \mathrm{E}-1$ & pCi/g dry & $2.91 \mathrm{E}-1$ & & 6/23/08 & $8 \mathrm{~F} 20002$ & AGG-RRL-001 \\
\hline 13967-48-1 & Ruthenium-106 & $<2.99 \mathrm{E} 0$ & pCi/g dry & 2.99E0 & & 6/23/08 & $8 \mathrm{~F} 20002$ & AGG-RRL-001 \\
\hline 14391-65-2 & Silver-108m & $<2.92 \mathrm{E}-1$ & pCi/g dry & $2.92 \mathrm{E}-1$ & & 6/23/08 & 8 F20002 & AGG-RRL-001 \\
\hline \multirow[t]{2}{*}{ 14109-32-1 } & Cadmium-109 & $<7.48 \mathrm{E} 0$ & pCi/g dry & 7.48E0 & & 6/23/08 & $8 \mathrm{~F} 20002$ & AGG-RRL-001 \\
\hline & Silver-110 & $<3.07 \mathrm{E}-1$ & pCi/g dry & 3.07E-1 & & 6/23/08 & $8 \mathrm{~F} 20002$ & AGG-RRL-001 \\
\hline 14391-76-5 & Silver-110m & $<3.08 \mathrm{E}-1$ & pCi/g dry & $3.08 \mathrm{E}-1$ & & 6/23/08 & $8 \mathrm{~F} 20002$ & AGG-RRL-001 \\
\hline 13966-06-8 & Tin-113 & $<3.95 \mathrm{E}-1$ & pCi/g dry & 3.95E-1 & & 6/23/08 & $8 \mathrm{~F} 20002$ & AGG-RRL-001 \\
\hline 14683-10-4 & Antimony-124 & $<2.94 \mathrm{E}-1$ & pCi/g dry & 2.94E-1 & & $6 / 23 / 08$ & $8 \mathrm{~F} 20002$ & AGG-RRL-001 \\
\hline 14234-35-6 & Antimony-125 & $<8.95 \mathrm{E}-1$ & pCi/g dry & 8.95E-1 & & 6/23/08 & $8 \mathrm{~F} 20002$ & AGG-RRL-001 \\
\hline 15756-32-8 & Antimony-126 & $<3.03 \mathrm{E}-1$ & pCi/g dry & 3.03E-1 & & 6/23/08 & $8 \mathrm{~F} 20002$ & AGG-RRL-001 \\
\hline 15832-50-5 & Tin-126 & $<5.92 \mathrm{E}-1$ & pCi/g dry & $5.92 \mathrm{E}-1$ & & 6/23/08 & $8 \mathrm{~F} 20002$ & AGG-RRL-001 \\
\hline 10043-66-0 & Iodine-131 & $<3.13 \mathrm{E}-1$ & pCi/g dry & 3.13E-1 & & 6/23/08 & $8 \mathrm{~F} 20002$ & AGG-RRL-001 \\
\hline 13981-41-4 & Barium-133 & $<5.14 \mathrm{E}-1$ & pCi/g dry & $5.14 \mathrm{E}-1$ & & 6/23/08 & $8 \mathrm{~F} 20002$ & AGG-RRL-001 \\
\hline 13967-70-9 & Cesium-134 & $<3.46 \mathrm{E}-1$ & pCi/g dry & $3.46 \mathrm{E}-1$ & & 6/23/08 & $8 \mathrm{~F} 20002$ & AGG-RRL-001 \\
\hline 10045-97-3 & Cesium-137 & $<3.49 \mathrm{E}-1$ & pCi/g dry & 3.49E-1 & & 6/23/08 & $8 \mathrm{~F} 20002$ & AGG-RRL-001 \\
\hline 13982-30-4 & Cerium-139 & $<3.21 \mathrm{E}-1$ & pCi/g dry & $3.21 \mathrm{E}-1$ & & 6/23/08 & $8 \mathrm{~F} 20002$ & AGG-RRL-001 \\
\hline 14762-78-8 & Cerium-144 & $<2.16 \mathrm{E} 0$ & pCi/g dry & $2.16 \mathrm{E} 0$ & & 6/23/08 & $8 \mathrm{~F} 20002$ & AGG-RRL-001 \\
\hline 14683-23-9 & Europium-152 & $<1.07 \mathrm{E} 0$ & pCi/g dry & 1.07E0 & & 6/23/08 & 8 F20002 & AGG-RRL-001 \\
\hline 14276-65-4 & Gadolinium-153 & $<8.18 \mathrm{E}-1$ & pCi/g dry & $8.18 \mathrm{E}-1$ & & 6/23/08 & $8 \mathrm{~F} 20002$ & AGG-RRL-001 \\
\hline 15585-10-1 & Europium-154 & $<5.80 \mathrm{E}-1$ & pCi/g dry & $5.80 \mathrm{E}-1$ & & 6/23/08 & $8 \mathrm{~F} 20002$ & AGG-RRL-001 \\
\hline 14391-16-3 & Europium-155 & $<8.28 \mathrm{E}-1$ & pCi/g dry & $8.28 \mathrm{E}-1$ & & 6/23/08 & $8 \mathrm{~F} 20002$ & AGG-RRL-001 \\
\hline 13982-78-0 & Mercury-203 & $<3.64 \mathrm{E}-1$ & pCi/g dry & 3.64E-1 & & 6/23/08 & $8 \mathrm{~F} 20002$ & AGG-RRL-001 \\
\hline 14913-50-9 & Thallium-208 & $<3.20 \mathrm{E}-1$ & pCi/g dry & $3.20 \mathrm{E}-1$ & & 6/23/08 & $8 \mathrm{~F} 20002$ & AGG-RRL-001 \\
\hline 14331-79-4 & Bismuth-210 & $<5.49 \mathrm{E}-1$ & pCi/g dry & $5.49 \mathrm{E}-1$ & & 6/23/08 & $8 \mathrm{~F} 20002$ & AGG-RRL-001 \\
\hline \multirow[t]{3}{*}{$14255-04-0$} & Lead-210 & $<1.02 \mathrm{E} 2$ & pCi/g dry & $1.02 \mathrm{E} 2$ & & 6/23/08 & $8 \mathrm{~F} 20002$ & AGG-RRL-001 \\
\hline & Bismuth-211 & $<6.55 \mathrm{E} 0$ & pCi/g dry & $6.55 \mathrm{E} 0$ & & 6/23/08 & $8 \mathrm{~F} 20002$ & AGG-RRL-001 \\
\hline & Lead-211 & $<8.96 \mathrm{E} 0$ & pCi/g dry & 8.96Е0 & & $6 / 23 / 08$ & $8 \mathrm{~F} 20002$ & AGG-RRL-001 \\
\hline 15092-94-1 & Lead-212 & $<6.43 \mathrm{E}-1$ & pCi/g dry & 6.43E-1 & & 6/23/08 & $8 \mathrm{~F} 20002$ & AGG-RRL-001 \\
\hline 14733-03-0 & Bismuth-214 & $<7.50 \mathrm{E}-1$ & pCi/g dry & $7.50 \mathrm{E}-1$ & & 6/23/08 & $8 \mathrm{~F} 20002$ & AGG-RRL-001 \\
\hline $15067-28-4$ & Lead-214 & 2.00E0 & pCi/g dry & 7.02E-1 & 0.925 & 6/23/08 & $8 \mathrm{~F} 20002$ & AGG-RRL-001 \\
\hline $14835-02-0$ & Radon-219 & $<2.90 \mathrm{E} 0$ & pCi/g dry & $2.90 \mathrm{E} 0$ & & 6/23/08 & $8 \mathrm{~F} 20002$ & AGG-RRL-001 \\
\hline 22481-48-7 & Radon-220 & $<2.53 \mathrm{E} 2$ & pCi/g dry & 2.53E2 & & 6/23/08 & $8 \mathrm{~F} 20002$ & AGG-RRL-001 \\
\hline 28522-20-5 & Radon-221 & $<1.32 \mathrm{E} 0$ & pCi/g dry & $1.32 \mathrm{E} 0$ & & 6/23/08 & 8 F20002 & AGG-RRL-001 \\
\hline 15756-98-6 & Francium-223 & $<9.70 \mathrm{E} 0$ & pCi/g dry & $9.70 \mathrm{E} 0$ & & 6/23/08 & $8 \mathrm{~F} 20002$ & AGG-RRL-001 \\
\hline 15623-45-7 & Radium-223 & $<2.06 \mathrm{E} 0$ & pCi/g dry & $2.06 \mathrm{E} 0$ & & 6/23/08 & $8 \mathrm{~F} 20002$ & AGG-RRL-001 \\
\hline 13233-32-4 & Radium-224 & $<8.43 \mathrm{E} 0$ & pCi/g dry & 8.43E0 & & 6/23/08 & $8 \mathrm{~F} 20002$ & AGG-RRL-001 \\
\hline 13982-63-3 & Radium-226 & $<8.13 \mathrm{E} 0$ & pCi/g dry & 8.13E0 & & 6/23/08 & $8 \mathrm{~F} 20002$ & AGG-RRL-001 \\
\hline 15623-47-9 & Thorium-227 & $<2.97 \mathrm{E} 0$ & pCi/g dry & 2.97E0 & & 6/23/08 & $8 \mathrm{~F} 20002$ & AGG-RRL-001 \\
\hline 14331-83-0 & Actinium-228 & $<1.46 \mathrm{E} 0$ & pCi/g dry & $1.46 \mathrm{E} 0$ & & 6/23/08 & $8 \mathrm{~F} 20002$ & AGG-RRL-001 \\
\hline $14274-82-9$ & Thorium-228 & $<1.01 \mathrm{E} 2$ & pCi/g dry & $1.01 \mathrm{E} 2$ & & 6/23/08 & $8 \mathrm{~F} 20002$ & AGG-RRL-001 \\
\hline 14269-63-7 & Thorium-230 & $<1.07 \mathrm{E} 2$ & pCi/g dry & $1.07 \mathrm{E} 2$ & & 6/23/08 & $8 \mathrm{~F} 20002$ & AGG-RRL-001 \\
\hline 14331-85-2 & Protactinium-231 & $<1.20 \mathrm{E} 1$ & pCi/g dry & $1.20 \mathrm{E} 1$ & & 6/23/08 & $8 \mathrm{~F} 20002$ & AGG-RRL-001 \\
\hline $14932-40-2$ & Thorium-231 & $<5.57 \mathrm{E} 1$ & pCi/g dry & 5.57E1 & & 6/23/08 & $8 \mathrm{~F} 20002$ & AGG-RRL-001 \\
\hline 7440-29-1 & Thorium-232 & $<3.52 \mathrm{E} 2$ & pCi/g dry & 3.52E2 & & 6/23/08 & 8F20002 & AGG-RRL-001 \\
\hline 13981-14-1 & Protactinium-233 & $<7.53 \mathrm{E}-1$ & pCi/g dry & 7.53E-1 & & 6/23/08 & $8 \mathrm{~F} 20002$ & AGG-RRL-001 \\
\hline $15100-28-4$ & Protactinium-234 & $<1.04 \mathrm{E} 0$ & pCi/g dry & $1.04 \mathrm{E} 0$ & & 6/23/08 & $8 F 20002$ & AGG-RRL-001 \\
\hline
\end{tabular}




\section{GEA/Soil}

\begin{tabular}{|c|c|c|c|c|c|c|c|c|}
\hline CAS \# & Analyte & Results & Units & MDA & UNC & Analyzed & Batch & Method \\
\hline \multirow[t]{2}{*}{ HEIS No. } & B1VJ58B & \multicolumn{3}{|c|}{ Lab ID: $\quad$ 0805020-11 } & & & & \\
\hline & Protactinium-234m & $<3.96 \mathrm{E} 1$ & pCi/g dry & 3.96E1 & & 6/23/08 & 8F20002 & AGG-RRL-001 \\
\hline 15065-10-8 & Thorium-234 & $<9.64 \mathrm{E} 0$ & pCi/g dry & 9.64E0 & & 6/23/08 & 8F20002 & AGG-RRL-001 \\
\hline 15117-96-1 & Uranium 235 & $<2.34 \mathrm{E} 0$ & pCi/g dry & $2.34 \mathrm{E} 0$ & & $6 / 23 / 08$ & $8 F 20002$ & AGG-RRL-001 \\
\hline 13994-20-2 & Neptunium-237 & $<2.16 \mathrm{E} 0$ & pCi/g dry & $2.16 \mathrm{E} 0$ & & $6 / 23 / 08$ & $8 F 20002$ & AGG-RRL-001 \\
\hline \multirow[t]{2}{*}{ 13981-16-3 } & Plutonium-238 & $<3.23 \mathrm{E} 3$ & pCi/g dry & 3.23E3 & & 6/23/08 & $8 \mathrm{~F} 20002$ & AGG-RRL-001 \\
\hline & Uranium 238 & $<4.99 \mathrm{E} 0$ & pCi/g dry & 4.99E0 & & 6/23/08 & $8 F 20002$ & AGG-RRL-001 \\
\hline $15117-48-3$ & Plutonium-239 & $<3.78 \mathrm{E} 3$ & pCi/g dry & 3.78E3 & & 6/23/08 & 8F20002 & AGG-RRL-001 \\
\hline 14119-33-6 & Plutonium-240 & $<3.25 \mathrm{E} 3$ & pCi/g dry & $3.25 \mathrm{E} 3$ & & $6 / 23 / 08$ & 8F20002 & AGG-RRL-001 \\
\hline $14596-10-2$ & Americium-241 & $<1.71 \mathrm{E} 0$ & pCi/g dry & $1.71 \mathrm{E} 0$ & & 6/23/08 & 8F20002 & AGG-RRL-001 \\
\hline 14993-75-0 & Americium-243 & $<6.05 \mathrm{E}-1$ & pCi/g dry & $6.05 \mathrm{E}-1$ & & 6/23/08 & $8 F 20002$ & AGG-RRL-001 \\
\hline 15757-87-6 & Curium-243 & $<9.90 \mathrm{E}-1$ & pCi/g dry & $9.90 \mathrm{E}-1$ & & $6 / 23 / 08$ & $8 F 20002$ & AGG-RRL-001 \\
\hline 15621-76-8 & Curium-245 & $<8.13 \mathrm{E}-1$ & pCi/g dry & 8.13E-1 & & 6/23/08 & 8F20002 & AGG-RRL-001 \\
\hline HEIS No. & B1VJ58A & \multicolumn{3}{|c|}{ Lab ID: $\quad$ 0805020-12 } & & & & \\
\hline 13966-32-0 & Sodium-22 & $<3.43 \mathrm{E}-1$ & pCi/g dry & 3.43E-1 & & 6/23/08 & 8F20002 & AGG-RRL-001 \\
\hline 13966-00-2 & Potassium-40 & $<6.41 \mathrm{E} 0$ & pCi/g dry & $6.41 \mathrm{E} 0$ & & 6/23/08 & 8F20002 & AGG-RRL-001 \\
\hline 14392-02-0 & Chromium-51 & $<3.06 \mathrm{E} 0$ & pCi/g dry & 3.06E0 & & 6/23/08 & 8F20002 & AGG-RRL-001 \\
\hline 13966-31-9 & Manganese-54 & $<3.75 \mathrm{E}-1$ & pCi/g dry & 3.75E-1 & & 6/23/08 & 8F20002 & AGG-RRL-001 \\
\hline 13981-50-5 & Cobalt-57 & $<3.19 \mathrm{E}-1$ & pCi/g dry & 3.19E-1 & & 6/23/08 & 8F20002 & AGG-RRL-001 \\
\hline $14596-12-4$ & Iron-59 & $<7.07 \mathrm{E}-1$ & pCi/g dry & 7.07E-1 & & 6/23/08 & 8F20002 & AGG-RRL-001 \\
\hline 10198-40-0 & Cobalt-60 & $<6.26 \mathrm{E}-1$ & pCi/g dry & $6.26 \mathrm{E}-1$ & & $6 / 23 / 08$ & 8 F20002 & AGG-RRL-001 \\
\hline 13982-39-3 & Zinc-65 & $<7.89 \mathrm{E}-1$ & pCi/g dry & 7.89E-1 & & 6/23/08 & 8F20002 & AGG-RRL-001 \\
\hline 14265-71-5 & Selenium-75 & $<5.25 \mathrm{E}-1$ & pCi/g dry & $5.25 \mathrm{E}-1$ & & 6/23/08 & 8F20002 & AGG-RRL-001 \\
\hline 17056-36-9 & Rubidium-83 & $<7.37 \mathrm{E}-1$ & pCi/g dry & 7.37E-1 & & 6/23/08 & 8F20002 & AGG-RRL-001 \\
\hline 13967-73-2 & Strontium-85 & $<3.39 \mathrm{E}-1$ & pCi/g dry & 3.39E-1 & & 6/23/08 & 8F20002 & AGG-RRL-001 \\
\hline $14932-53-7$ & Rubidium-86 & $<4.42 \mathrm{E} 0$ & pCi/g dry & 4.42E0 & & 6/23/08 & 8F20002 & AGG-RRL-001 \\
\hline 13982-36-0 & Yttrium-88 & $<2.74 \mathrm{E}-1$ & pCi/g dry & $2.74 \mathrm{E}-1$ & & 6/23/08 & 8F20002 & AGG-RRL-001 \\
\hline 14681-63-1 & Niobium-94 & $<3.36 \mathrm{E}-1$ & pCi/g dry & 3.36E-1 & & 6/23/08 & 8F20002 & AGG-RRL-001 \\
\hline \multirow{3}{*}{ 13967-76-5 } & Niobium-95 & $<3.37 \mathrm{E}-1$ & pCi/g dry & 3.37E-1 & & 6/23/08 & $8 \mathrm{~F} 20002$ & AGG-RRL-001 \\
\hline & Niobium-95m & $<1.41 \mathrm{E} 0$ & pCi/g dry & $1.41 \mathrm{E} 0$ & & 6/23/08 & 8F20002 & AGG-RRL-001 \\
\hline & Technetium-95m & $<4.78 \mathrm{E}-1$ & pCi/g dry & $4.78 \mathrm{E}-1$ & & 6/23/08 & $8 \mathrm{~F} 20002$ & AGG-RRL-001 \\
\hline \multirow[t]{2}{*}{ 13967-71-0 } & Zirconium-95 & $<5.74 \mathrm{E}-1$ & pCi/g dry & $5.74 \mathrm{E}-1$ & & 6/23/08 & 8F20002 & AGG-RRL-001 \\
\hline & Technetium-99m & $<3.24 \mathrm{E}-1$ & pCi/g dry & $3.24 \mathrm{E}-1$ & & 6/23/08 & 8F20002 & AGG-RRL-001 \\
\hline 13968-53-1 & Ruthenium-103 & $<3.50 \mathrm{E}-1$ & pCi/g dry & $3.50 \mathrm{E}-1$ & & 6/23/08 & 8F20002 & AGG-RRL-001 \\
\hline 13967-48-1 & Ruthenium-106 & $<3.77 \mathrm{E} 0$ & pCi/g dry & 3.77E0 & & 6/23/08 & 8F20002 & AGG-RRL-001 \\
\hline 14391-65-2 & Silver-108m & $<3.26 \mathrm{E}-1$ & pCi/g dry & $3.26 \mathrm{E}-1$ & & 6/23/08 & 8F20002 & AGG-RRL-001 \\
\hline \multirow[t]{2}{*}{ 14109-32-1 } & Cadmium-109 & $<9.00 \mathrm{E} 0$ & pCi/g dry & $9.00 \mathrm{E} 0$ & & 6/23/08 & 8F20002 & AGG-RRL-001 \\
\hline & Silver-110 & $<3.12 \mathrm{E}-1$ & pCi/g dry & $3.12 \mathrm{E}-1$ & & 6/23/08 & 8F20002 & AGG-RRL-001 \\
\hline 14391-76-5 & Silver-110m & $<3.13 \mathrm{E}-1$ & pCi/g dry & 3.13E-1 & & 6/23/08 & 8F20002 & AGG-RRL-001 \\
\hline 13966-06-8 & Tin-113 & $<4.46 \mathrm{E}-1$ & pCi/g dry & 4.46E-1 & & 6/23/08 & 8F20002 & AGG-RRL-001 \\
\hline 14683-10-4 & Antimony-124 & $<3.80 \mathrm{E}-1$ & pCi/g dry & 3.80E-1 & & 6/23/08 & 8F20002 & AGG-RRL-001 \\
\hline $14234-35-6$ & Antimony-125 & $<9.93 \mathrm{E}-1$ & pCi/g dry & $9.93 \mathrm{E}-1$ & & 6/23/08 & $8 F 20002$ & AGG-RRL-001 \\
\hline 15756-32-8 & Antimony-126 & $<3.11 \mathrm{E}-1$ & pCi/g dry & $3.11 \mathrm{E}-1$ & & 6/23/08 & 8F20002 & AGG-RRL-001 \\
\hline 15832-50-5 & Tin-126 & $<7.35 \mathrm{E}-1$ & pCi/g dry & 7.35E-1 & & 6/23/08 & 8F20002 & AGG-RRL-001 \\
\hline 10043-66-0 & Iodine-131 & $<4.06 \mathrm{E}-1$ & pCi/g dry & 4.06E-1 & & 6/23/08 & 8F20002 & AGG-RRL-001 \\
\hline 13981-41-4 & Barium-133 & $<5.44 \mathrm{E}-1$ & pCi/g dry & $5.44 \mathrm{E}-1$ & & 6/23/08 & 8F20002 & AGG-RRL-001 \\
\hline 13967-70-9 & Cesium-134 & $<3.82 \mathrm{E}-1$ & pCi/g dry & 3.82E-1 & & 6/23/08 & 8F20002 & AGG-RRL-001 \\
\hline 10045-97-3 & Cesium-137 & $<3.53 \mathrm{E}-1$ & pCi/g dry & 3.53E-1 & & 6/23/08 & 8F20002 & AGG-RRL-001 \\
\hline 13982-30-4 & Cerium-139 & $<3.66 \mathrm{E}-1$ & pCi/g dry & 3.66E-1 & & 6/23/08 & $8 F 20002$ & AGG-RRL-001 \\
\hline
\end{tabular}


GEA/Soil

\begin{tabular}{|c|c|c|c|c|c|c|c|c|}
\hline CAS \# & Analyte & Results & Units & MDA & UNC & Analyzed & Batch & Method \\
\hline HEIS No. & B1VJ58A & & b ID: & 5020-12 & & & & \\
\hline 14762-78-8 & Cerium-144 & $<2.59 \mathrm{E} 0$ & pCi/g dry & $2.59 \mathrm{E} 0$ & & 6/23/08 & $8 F 20002$ & AGG-RRL-001 \\
\hline 14683-23-9 & Europium-152 & $<1.27 \mathrm{E} 0$ & pCi/g dry & $1.27 \mathrm{E} 0$ & & 6/23/08 & $8 \mathrm{~F} 20002$ & AGG-RRL-001 \\
\hline 14276-65-4 & Gadolinium-153 & $<9.93 \mathrm{E}-1$ & pCi/g dry & $9.93 \mathrm{E}-1$ & & 6/23/08 & $8 \mathrm{~F} 20002$ & AGG-RRL-001 \\
\hline 15585-10-1 & Europium-154 & $<6.76 \mathrm{E}-1$ & pCi/g dry & $6.76 \mathrm{E}-1$ & & 6/23/08 & $8 \mathrm{~F} 20002$ & AGG-RRL-001 \\
\hline 14391-16-3 & Europium-155 & $<1.04 \mathrm{E} 0$ & pCi/g dry & $1.04 \mathrm{E} 0$ & & 6/23/08 & $8 \mathrm{~F} 20002$ & AGG-RRL-001 \\
\hline 13982-78-0 & Mercury-203 & $<4.00 \mathrm{E}-1$ & pCi/g dry & $4.00 \mathrm{E}-1$ & & 6/23/08 & $8 \mathrm{~F} 20002$ & AGG-RRL-001 \\
\hline 14913-50-9 & Thallium-208 & $<4.04 \mathrm{E}-1$ & pCi/g dry & 4.04E-1 & & 6/23/08 & $8 \mathrm{~F} 20002$ & AGG-RRL-001 \\
\hline 14331-79-4 & Bismuth-210 & $<6.29 \mathrm{E}-1$ & pCi/g dry & $6.29 \mathrm{E}-1$ & & 6/23/08 & $8 \mathrm{~F} 20002$ & AGG-RRL-001 \\
\hline \multirow[t]{3}{*}{$14255-04-0$} & Lead-210 & $<4.30 \mathrm{E} 1$ & pCi/g dry & 4.30E1 & & 6/23/08 & $8 \mathrm{~F} 20002$ & AGG-RRL-001 \\
\hline & Bismuth-211 & $<7.21 \mathrm{E} 0$ & pCi/g dry & 7.21E0 & & 6/23/08 & $8 \mathrm{~F} 20002$ & AGG-RRL-001 \\
\hline & Lead-211 & $<9.86 \mathrm{E} 0$ & pCi/g dry & $9.86 \mathrm{E} 0$ & & 6/23/08 & $8 \mathrm{~F} 20002$ & AGG-RRL-001 \\
\hline 15092-94-1 & Lead-212 & $<8.20 \mathrm{E}-1$ & pCi/g dry & $8.20 \mathrm{E}-1$ & & 6/23/08 & $8 \mathrm{~F} 20002$ & AGG-RRL-001 \\
\hline 14733-03-0 & Bismuth-214 & $<8.35 \mathrm{E}-1$ & pCi/g dry & 8.35E-1 & & 6/23/08 & $8 \mathrm{~F} 20002$ & AGG-RRL-001 \\
\hline $15067-28-4$ & Lead-214 & $<8.84 \mathrm{E}-1$ & pCi/g dry & 8.84E-1 & & 6/23/08 & $8 \mathrm{~F} 20002$ & AGG-RRL-001 \\
\hline $14835-02-0$ & Radon-219 & $<3.16 \mathrm{E} 0$ & pCi/g dry & $3.16 \mathrm{E} 0$ & & 6/23/08 & $8 \mathrm{~F} 20002$ & AGG-RRL-001 \\
\hline 22481-48-7 & Radon-220 & $<3.20 \mathrm{E} 2$ & pCi/g dry & $3.20 \mathrm{E} 2$ & & 6/23/08 & $8 \mathrm{~F} 20002$ & AGG-RRL-001 \\
\hline 28522-20-5 & Radon-221 & $<1.44 \mathrm{E} 0$ & pCi/g dry & $1.44 \mathrm{E} 0$ & & 6/23/08 & $8 \mathrm{~F} 20002$ & AGG-RRL-001 \\
\hline 15756-98-6 & Francium-223 & $<1.04 \mathrm{E} 1$ & pCi/g dry & $1.04 \mathrm{E} 1$ & & 6/23/08 & $8 \mathrm{~F} 20002$ & AGG-RRL-001 \\
\hline $15623-45-7$ & Radium-223 & $<2.31 \mathrm{E} 0$ & pCi/g dry & 2.31E0 & & 6/23/08 & $8 \mathrm{~F} 20002$ & AGG-RRL-001 \\
\hline 13233-32-4 & Radium-224 & $<8.88 \mathrm{E} 0$ & pCi/g dry & 8.88E0 & & 6/23/08 & $8 \mathrm{~F} 20002$ & AGG-RRL-001 \\
\hline 13982-63-3 & Radium-226 & $<8.90 \mathrm{E} 0$ & pCi/g dry & 8.90E0 & & 6/23/08 & $8 \mathrm{~F} 20002$ & AGG-RRL-001 \\
\hline $15623-47-9$ & Thorium-227 & $<3.15 \mathrm{E} 0$ & pCi/g dry & $3.15 \mathrm{E} 0$ & & 6/23/08 & $8 \mathrm{~F} 20002$ & AGG-RRL-001 \\
\hline 14331-83-0 & Actinium-228 & $<1.53 \mathrm{E} 0$ & pCi/g dry & $1.53 \mathrm{E} 0$ & & 6/23/08 & $8 \mathrm{~F} 20002$ & AGG-RRL-001 \\
\hline 14274-82-9 & Thorium-228 & $<1.13 \mathrm{E} 2$ & pCi/g dry & 1.13E2 & & 6/23/08 & $8 \mathrm{~F} 20002$ & AGG-RRL-001 \\
\hline 14269-63-7 & Thorium-230 & $<1.17 \mathrm{E} 2$ & pCi/g dry & $1.17 \mathrm{E} 2$ & & 6/23/08 & $8 \mathrm{~F} 20002$ & AGG-RRL-001 \\
\hline 14331-85-2 & Protactinium-231 & $<1.41 \mathrm{E} 1$ & pCi/g dry & $1.41 \mathrm{E} 1$ & & 6/23/08 & $8 \mathrm{~F} 20002$ & AGG-RRL-001 \\
\hline 14932-40-2 & Thorium-231 & $<7.07 \mathrm{E} 1$ & pCi/g dry & 7.07E1 & & 6/23/08 & 8F20002 & AGG-RRL-001 \\
\hline 7440-29-1 & Thorium-232 & $<2.93 \mathrm{E} 2$ & pCi/g dry & 2.93E2 & & 6/23/08 & $8 \mathrm{~F} 20002$ & AGG-RRL-001 \\
\hline 13981-14-1 & Protactinium-233 & $<8.54 \mathrm{E}-1$ & pCi/g dry & 8.54E-1 & & 6/23/08 & $8 \mathrm{~F} 20002$ & AGG-RRL-001 \\
\hline \multirow[t]{2}{*}{$15100-28-4$} & Protactinium-234 & $<1.30 \mathrm{E} 0$ & pCi/g dry & $1.30 \mathrm{E} 0$ & & 6/23/08 & $8 \mathrm{~F} 20002$ & AGG-RRL-001 \\
\hline & Protactinium-234m & $<4.43 \mathrm{E} 1$ & pCi/g dry & 4.43E1 & & 6/23/08 & $8 \mathrm{~F} 20002$ & AGG-RRL-001 \\
\hline 15065-10-8 & Thorium-234 & $<1.03 \mathrm{E} 1$ & pCi/g dry & $1.03 \mathrm{E} 1$ & & 6/23/08 & $8 \mathrm{~F} 20002$ & AGG-RRL-001 \\
\hline 15117-96-1 & Uranium 235 & $<2.78 \mathrm{E} 0$ & pCi/g dry & $2.78 \mathrm{E} 0$ & & 6/23/08 & $8 \mathrm{~F} 20002$ & AGG-RRL-001 \\
\hline 13994-20-2 & Neptunium-237 & $<2.72 \mathrm{E} 0$ & pCi/g dry & 2.72E0 & & 6/23/08 & $8 \mathrm{~F} 20002$ & AGG-RRL-001 \\
\hline \multirow[t]{2}{*}{ 13981-16-3 } & Plutonium-238 & $<4.09 \mathrm{E} 3$ & pCi/g dry & 4.09E3 & & 6/23/08 & $8 \mathrm{~F} 20002$ & AGG-RRL-001 \\
\hline & Uranium 238 & $<5.81 \mathrm{E} 0$ & pCi/g dry & $5.81 \mathrm{E} 0$ & & 6/23/08 & $8 \mathrm{~F} 20002$ & AGG-RRL-001 \\
\hline $15117-48-3$ & Plutonium-239 & $<4.08 \mathrm{E} 3$ & pCi/g dry & $4.08 \mathrm{E} 3$ & & 6/23/08 & $8 \mathrm{~F} 20002$ & AGG-RRL-001 \\
\hline 14119-33-6 & Plutonium-240 & $<4.07 \mathrm{E} 3$ & pCi/g dry & 4.07E3 & & 6/23/08 & $8 \mathrm{~F} 20002$ & AGG-RRL-001 \\
\hline $14596-10-2$ & Americium-241 & $<1.49 \mathrm{E} 0$ & pCi/g dry & $1.49 \mathrm{E} 0$ & & 6/23/08 & $8 \mathrm{~F} 20002$ & AGG-RRL-001 \\
\hline 14993-75-0 & Americium-243 & $<6.53 \mathrm{E}-1$ & pCi/g dry & $6.53 \mathrm{E}-1$ & & 6/23/08 & $8 \mathrm{~F} 20002$ & AGG-RRL-001 \\
\hline $15757-87-6$ & Curium-243 & $<1.24 \mathrm{E} 0$ & pCi/g dry & $1.24 \mathrm{E} 0$ & & 6/23/08 & $8 \mathrm{~F} 20002$ & AGG-RRL-001 \\
\hline 15621-76-8 & Curium-245 & $<1.02 \mathrm{E} 0$ & pCi/g dry & $1.02 \mathrm{E} 0$ & & 6/23/08 & $8 F 20002$ & AGG-RRL-001 \\
\hline HEIS No. & B1VJ59C & & b ID: & $55020-14$ & & & & \\
\hline 13966-32-0 & Sodium-22 & $<3.62 \mathrm{E}-1$ & pCi/g dry & $3.62 \mathrm{E}-1$ & & 6/23/08 & $8 \mathrm{~F} 20002$ & AGG-RRL-001 \\
\hline 13966-00-2 & Potassium-40 & 2.43E1 & pCi/g dry & 2.99E0 & 1.35 & 6/23/08 & $8 \mathrm{~F} 20002$ & AGG-RRL-001 \\
\hline 14392-02-0 & Chromium-51 & $<2.94 \mathrm{E} 0$ & pCi/g dry & 2.94E0 & & 6/23/08 & $8 \mathrm{~F} 20002$ & AGG-RRL-001 \\
\hline 13966-31-9 & Manganese-54 & $<2.90 \mathrm{E}-1$ & pCi/g dry & 2.90E-1 & & 6/23/08 & $8 \mathrm{~F} 20002$ & AGG-RRL-001 \\
\hline 13981-50-5 & Cobalt-57 & $<2.85 \mathrm{E}-1$ & pCi/g dry & 2.85E-1 & & 6/23/08 & 8F20002 & AGG-RRL-001 \\
\hline
\end{tabular}


GEA/Soil

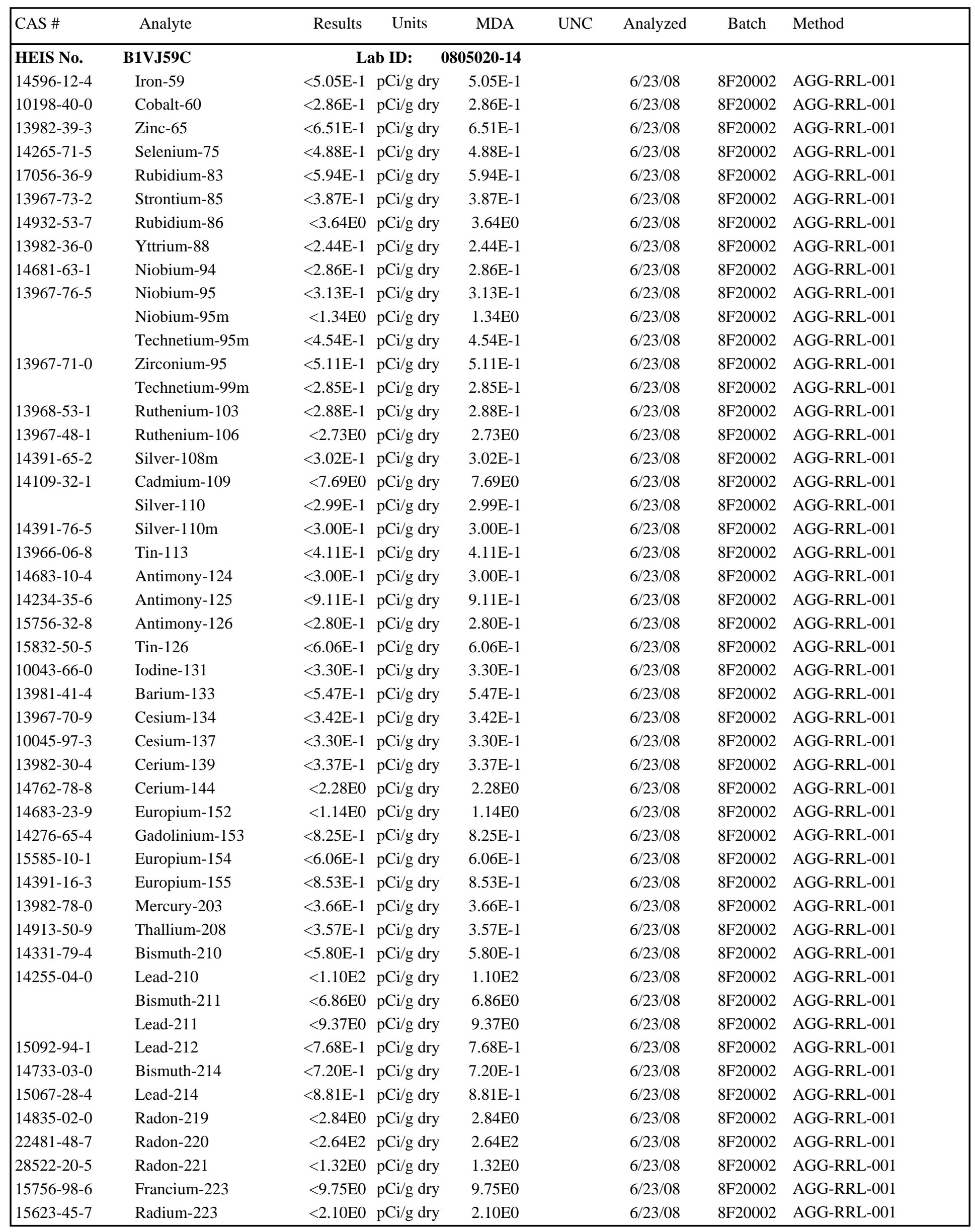


GEA/Soil

\begin{tabular}{|c|c|c|c|c|c|c|c|c|}
\hline CAS \# & Analyte & Results & Units & MDA & UNC & Analyzed & Batch & Method \\
\hline HEIS No. & B1VJ59C & \multicolumn{3}{|c|}{ Lab ID: $\quad$ 0805020-14 } & & & & \\
\hline 13233-32-4 & Radium-224 & $<8.52 \mathrm{E} 0$ & pCi/g dry & $8.52 \mathrm{E} 0$ & & $6 / 23 / 08$ & $8 F 20002$ & AGG-RRL-001 \\
\hline 13982-63-3 & Radium-226 & $<8.16 \mathrm{E} 0$ & pCi/g dry & $8.16 \mathrm{E} 0$ & & $6 / 23 / 08$ & 8F20002 & AGG-RRL-001 \\
\hline $15623-47-9$ & Thorium-227 & $<3.00 \mathrm{E} 0$ & pCi/g dry & $3.00 \mathrm{E} 0$ & & $6 / 23 / 08$ & 8 F20002 & AGG-RRL-001 \\
\hline 14331-83-0 & Actinium-228 & $<1.32 \mathrm{E} 0$ & pCi/g dry & $1.32 \mathrm{E} 0$ & & $6 / 23 / 08$ & 8F20002 & AGG-RRL-001 \\
\hline 14274-82-9 & Thorium-228 & $<1.04 \mathrm{E} 2$ & pCi/g dry & $1.04 \mathrm{E} 2$ & & 6/23/08 & 8F20002 & AGG-RRL-001 \\
\hline 14269-63-7 & Thorium-230 & $<1.15 \mathrm{E} 2$ & pCi/g dry & $1.15 \mathrm{E} 2$ & & 6/23/08 & $8 F 20002$ & AGG-RRL-001 \\
\hline $14331-85-2$ & Protactinium-231 & $<1.20 \mathrm{E} 1$ & pCi/g dry & $1.20 \mathrm{E} 1$ & & 6/23/08 & $8 F 20002$ & AGG-RRL-001 \\
\hline $14932-40-2$ & Thorium-231 & $<5.94 \mathrm{E} 1$ & pCi/g dry & $5.94 \mathrm{E} 1$ & & $6 / 23 / 08$ & 8F20002 & AGG-RRL-001 \\
\hline $7440-29-1$ & Thorium-232 & $<3.61 \mathrm{E} 2$ & pCi/g dry & 3.61E2 & & $6 / 23 / 08$ & 8F20002 & AGG-RRL-001 \\
\hline 13981-14-1 & Protactinium-233 & $<7.93 \mathrm{E}-1$ & pCi/g dry & 7.93E-1 & & 6/23/08 & 8 F20002 & AGG-RRL-001 \\
\hline \multirow[t]{2}{*}{$15100-28-4$} & Protactinium-234 & $<1.05 \mathrm{E} 0$ & pCi/g dry & $1.05 \mathrm{E} 0$ & & 6/23/08 & 8F20002 & AGG-RRL-001 \\
\hline & Protactinium-234m & $<3.75 \mathrm{E} 1$ & pCi/g dry & 3.75E1 & & $6 / 23 / 08$ & $8 F 20002$ & AGG-RRL-001 \\
\hline $15065-10-8$ & Thorium-234 & $<9.62 \mathrm{E} 0$ & pCi/g dry & $9.62 \mathrm{E} 0$ & & $6 / 23 / 08$ & 8F20002 & AGG-RRL-001 \\
\hline 15117-96-1 & Uranium 235 & $<2.42 \mathrm{E} 0$ & pCi/g dry & $2.42 \mathrm{E} 0$ & & 6/23/08 & 8F20002 & AGG-RRL-001 \\
\hline 13994-20-2 & Neptunium-237 & $<2.22 \mathrm{E} 0$ & pCi/g dry & $2.22 \mathrm{E} 0$ & & 6/23/08 & $8 F 20002$ & AGG-RRL-001 \\
\hline \multirow[t]{2}{*}{ 13981-16-3 } & Plutonium-238 & $<3.36 \mathrm{E} 3$ & pCi/g dry & $3.36 \mathrm{E} 3$ & & 6/23/08 & $8 F 20002$ & AGG-RRL-001 \\
\hline & Uranium 238 & $<4.98 \mathrm{E} 0$ & pCi/g dry & 4.98E0 & & $6 / 23 / 08$ & 8F20002 & AGG-RRL-001 \\
\hline $15117-48-3$ & Plutonium-239 & $<3.99 \mathrm{E} 3$ & pCi/g dry & 3.99E3 & & $6 / 23 / 08$ & 8F20002 & AGG-RRL-001 \\
\hline 14119-33-6 & Plutonium-240 & $<3.47 \mathrm{E} 3$ & pCi/g dry & $3.47 \mathrm{E} 3$ & & $6 / 23 / 08$ & 8F20002 & AGG-RRL-001 \\
\hline $14596-10-2$ & Americium-241 & $<1.79 \mathrm{E} 0$ & pCi/g dry & $1.79 \mathrm{E} 0$ & & $6 / 23 / 08$ & $8 F 20002$ & AGG-RRL-001 \\
\hline $14993-75-0$ & Americium-243 & $<6.05 \mathrm{E}-1$ & pCi/g dry & $6.05 \mathrm{E}-1$ & & 6/23/08 & $8 F 20002$ & AGG-RRL-001 \\
\hline 15757-87-6 & Curium-243 & $<1.06 \mathrm{E} 0$ & pCi/g dry & $1.06 \mathrm{E} 0$ & & 6/23/08 & 8F20002 & AGG-RRL-001 \\
\hline 15621-76-8 & Curium-245 & $<8.69 \mathrm{E}-1$ & pCi/g dry & $8.69 \mathrm{E}-1$ & & $6 / 23 / 08$ & $8 F 20002$ & AGG-RRL-001 \\
\hline HEIS No. & B1VJ59B & \multicolumn{3}{|c|}{ Lab ID: $\quad$ 0805020-15 } & & & & \\
\hline 13966-32-0 & Sodium-22 & $<3.81 \mathrm{E}-1$ & pCi/g dry & 3.81E-1 & & 6/23/08 & $8 F 20002$ & AGG-RRL-001 \\
\hline $13966-00-2$ & Potassium-40 & 2.34E1 & pCi/g dry & 3.78E0 & 1.45 & 6/23/08 & $8 F 20002$ & AGG-RRL-001 \\
\hline $14392-02-0$ & Chromium-51 & $<3.44 \mathrm{E} 0$ & pCi/g dry & $3.44 \mathrm{E} 0$ & & $6 / 23 / 08$ & $8 F 20002$ & AGG-RRL-001 \\
\hline 13966-31-9 & Manganese-54 & $<3.94 \mathrm{E}-1$ & pCi/g dry & $3.94 \mathrm{E}-1$ & & $6 / 23 / 08$ & $8 F 20002$ & AGG-RRL-001 \\
\hline $13981-50-5$ & Cobalt-57 & $<3.67 \mathrm{E}-1$ & pCi/g dry & $3.67 \mathrm{E}-1$ & & 6/23/08 & 8F20002 & AGG-RRL-001 \\
\hline $14596-12-4$ & Iron-59 & $<6.87 \mathrm{E}-1$ & pCi/g dry & $6.87 \mathrm{E}-1$ & & 6/23/08 & $8 F 20002$ & AGG-RRL-001 \\
\hline 10198-40-0 & Cobalt-60 & $<3.83 \mathrm{E}-1$ & pCi/g dry & 3.83E-1 & & 6/23/08 & 8F20002 & AGG-RRL-001 \\
\hline 13982-39-3 & Zinc-65 & $<8.56 \mathrm{E}-1$ & pCi/g dry & 8.56E-1 & & $6 / 23 / 08$ & 8F20002 & AGG-RRL-001 \\
\hline $14265-71-5$ & Selenium-75 & $<5.80 \mathrm{E}-1$ & pCi/g dry & $5.80 \mathrm{E}-1$ & & 6/23/08 & $8 F 20002$ & AGG-RRL-001 \\
\hline 17056-36-9 & Rubidium-83 & $<8.79 \mathrm{E}-1$ & pCi/g dry & $8.79 \mathrm{E}-1$ & & 6/23/08 & 8F20002 & AGG-RRL-001 \\
\hline 13967-73-2 & Strontium-85 & $<4.22 \mathrm{E}-1$ & pCi/g dry & $4.22 \mathrm{E}-1$ & & 6/23/08 & $8 F 20002$ & AGG-RRL-001 \\
\hline $14932-53-7$ & Rubidium-86 & $<4.72 \mathrm{E} 0$ & pCi/g dry & 4.72E0 & & 6/23/08 & 8F20002 & AGG-RRL-001 \\
\hline 13982-36-0 & Yttrium-88 & $<3.00 \mathrm{E}-1$ & pCi/g dry & $3.00 \mathrm{E}-1$ & & 6/23/08 & 8F20002 & AGG-RRL-001 \\
\hline 14681-63-1 & Niobium-94 & $<3.48 \mathrm{E}-1$ & pCi/g dry & $3.48 \mathrm{E}-1$ & & 6/23/08 & $8 F 20002$ & AGG-RRL-001 \\
\hline \multirow[t]{3}{*}{ 13967-76-5 } & Niobium-95 & $<3.59 \mathrm{E}-1$ & pCi/g dry & $3.59 \mathrm{E}-1$ & & 6/23/08 & 8F20002 & AGG-RRL-001 \\
\hline & Niobium-95m & $<1.56 \mathrm{E} 0$ & pCi/g dry & $1.56 \mathrm{E} 0$ & & $6 / 23 / 08$ & 8F20002 & AGG-RRL-001 \\
\hline & Technetium-95m & $<5.38 \mathrm{E}-1$ & pCi/g dry & $5.38 \mathrm{E}-1$ & & 6/23/08 & $8 F 20002$ & AGG-RRL-001 \\
\hline \multirow[t]{2}{*}{ 13967-71-0 } & Zirconium-95 & $<6.40 \mathrm{E}-1$ & pCi/g dry & $6.40 \mathrm{E}-1$ & & 6/23/08 & $8 F 20002$ & AGG-RRL-001 \\
\hline & Technetium-99m & $<3.62 \mathrm{E}-1$ & pCi/g dry & $3.62 \mathrm{E}-1$ & & 6/23/08 & 8F20002 & AGG-RRL-001 \\
\hline 13968-53-1 & Ruthenium-103 & $<4.15 \mathrm{E}-1$ & pCi/g dry & 4.15E-1 & & 6/23/08 & 8F20002 & AGG-RRL-001 \\
\hline 13967-48-1 & Ruthenium-106 & $<4.20 \mathrm{E} 0$ & pCi/g dry & $4.20 \mathrm{E} 0$ & & 6/23/08 & $8 F 20002$ & AGG-RRL-001 \\
\hline $14391-65-2$ & Silver-108m & $<3.53 \mathrm{E}-1$ & pCi/g dry & 3.53E-1 & & 6/23/08 & 8F20002 & AGG-RRL-001 \\
\hline \multirow[t]{2}{*}{ 14109-32-1 } & Cadmium-109 & $<1.02 \mathrm{E} 1$ & pCi/g dry & $1.02 \mathrm{E} 1$ & & 6/23/08 & 8F20002 & AGG-RRL-001 \\
\hline & Silver-110 & $<3.48 \mathrm{E}-1$ & pCi/g dry & $3.48 \mathrm{E}-1$ & & 6/23/08 & 8 F20002 & AGG-RRL-001 \\
\hline
\end{tabular}


GEA/Soil

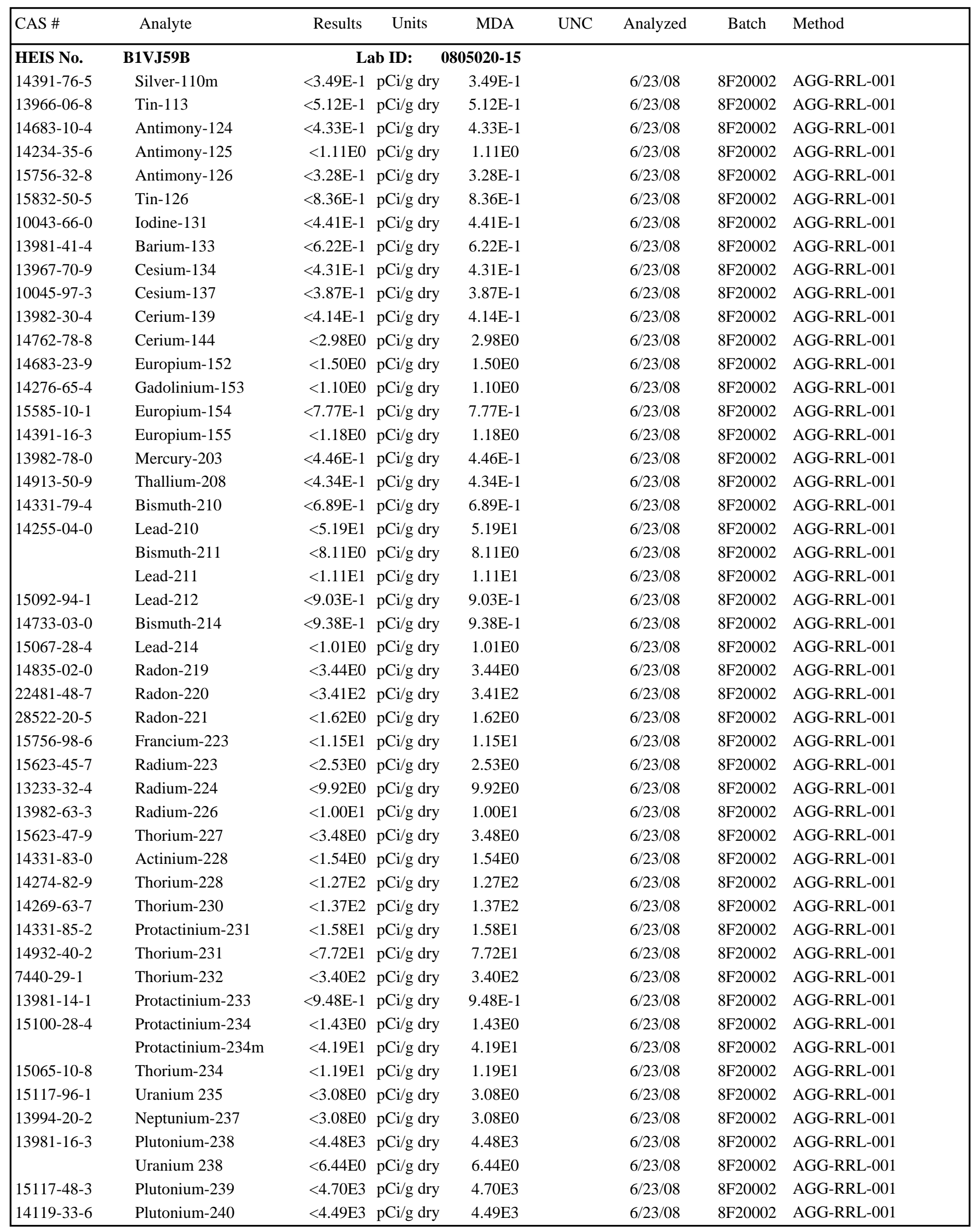




\section{GEA/Soil}

\begin{tabular}{|c|c|c|c|c|c|c|c|c|}
\hline CAS \# & Analyte & Results & Units & MDA & UNC & Analyzed & Batch & Method \\
\hline HEIS No. & B1VJ59B & \multicolumn{4}{|c|}{ Lab ID: $\quad$ 0805020-15 } & & & \\
\hline $14596-10-2$ & Americium-241 & $<1.74 \mathrm{E} 0$ & pCi/g dry & $1.74 \mathrm{E} 0$ & & 6/23/08 & $8 F 20002$ & AGG-RRL-001 \\
\hline $14993-75-0$ & Americium-243 & $<7.51 \mathrm{E}-1$ & pCi/g dry & $7.51 \mathrm{E}-1$ & & 6/23/08 & 8F20002 & AGG-RRL-001 \\
\hline $15757-87-6$ & Curium-243 & $<1.36 \mathrm{E} 0$ & pCi/g dry & $1.36 \mathrm{E} 0$ & & 6/23/08 & 8F20002 & AGG-RRL-001 \\
\hline 15621-76-8 & Curium-245 & $<1.12 \mathrm{E} 0$ & pCi/g dry & $1.12 \mathrm{E} 0$ & & 6/23/08 & 8F20002 & AGG-RRL-001 \\
\hline HEIS No. & B1VJ59A & \multicolumn{3}{|c|}{ Lab ID: $\quad$ 0805020-16 } & & & & \\
\hline $13966-32-0$ & Sodium-22 & $<3.54 \mathrm{E}-1$ & pCi/g dry & $3.54 \mathrm{E}-1$ & & 6/23/08 & $8 F 20002$ & AGG-RRL-001 \\
\hline $13966-00-2$ & Potassium-40 & 2.07E1 & pCi/g dry & 3.64E0 & 1.36 & $6 / 23 / 08$ & $8 F 20002$ & AGG-RRL-001 \\
\hline $14392-02-0$ & Chromium-51 & $<2.95 \mathrm{E} 0$ & pCi/g dry & 2.95E0 & & 6/23/08 & 8F20002 & AGG-RRL-001 \\
\hline 13966-31-9 & Manganese-54 & $<2.84 \mathrm{E}-1$ & pCi/g dry & $2.84 \mathrm{E}-1$ & & $6 / 23 / 08$ & 8F20002 & AGG-RRL-001 \\
\hline $13981-50-5$ & Cobalt-57 & $<2.96 \mathrm{E}-1$ & pCi/g dry & $2.96 \mathrm{E}-1$ & & 6/23/08 & 8F20002 & AGG-RRL-001 \\
\hline $14596-12-4$ & Iron-59 & $<5.14 \mathrm{E}-1$ & pCi/g dry & $5.14 \mathrm{E}-1$ & & 6/23/08 & 8F20002 & AGG-RRL-001 \\
\hline $10198-40-0$ & Cobalt-60 & $<3.38 \mathrm{E}-1$ & pCi/g dry & $3.38 \mathrm{E}-1$ & & 6/23/08 & 8F20002 & AGG-RRL-001 \\
\hline 13982-39-3 & Zinc-65 & $<7.24 \mathrm{E}-1$ & pCi/g dry & $7.24 \mathrm{E}-1$ & & 6/23/08 & $8 F 20002$ & AGG-RRL-001 \\
\hline $14265-71-5$ & Selenium-75 & $<4.76 \mathrm{E}-1$ & pCi/g dry & 4.76E-1 & & $6 / 23 / 08$ & 8F20002 & AGG-RRL-001 \\
\hline 17056-36-9 & Rubidium-83 & $<5.93 \mathrm{E}-1$ & pCi/g dry & 5.93E-1 & & $6 / 23 / 08$ & $8 F 20002$ & AGG-RRL-001 \\
\hline 13967-73-2 & Strontium-85 & $<3.86 \mathrm{E}-1$ & pCi/g dry & $3.86 \mathrm{E}-1$ & & 6/23/08 & 8F20002 & AGG-RRL-001 \\
\hline 14932-53-7 & Rubidium-86 & $<3.26 \mathrm{E} 0$ & pCi/g dry & $3.26 \mathrm{E} 0$ & & 6/23/08 & 8F20002 & AGG-RRL-001 \\
\hline 13982-36-0 & Yttrium-88 & $<2.48 \mathrm{E}-1$ & pCi/g dry & $2.48 \mathrm{E}-1$ & & 6/23/08 & 8F20002 & AGG-RRL-001 \\
\hline $14681-63-1$ & Niobium-94 & $<2.78 \mathrm{E}-1$ & pCi/g dry & $2.78 \mathrm{E}-1$ & & 6/23/08 & 8F20002 & AGG-RRL-001 \\
\hline \multirow[t]{3}{*}{$13967-76-5$} & Niobium-95 & $<3.17 \mathrm{E}-1$ & pCi/g dry & 3.17E-1 & & 6/23/08 & 8F20002 & AGG-RRL-001 \\
\hline & Niobium-95m & $<1.37 \mathrm{E} 0$ & pCi/g dry & $1.37 \mathrm{E} 0$ & & 6/23/08 & $8 F 20002$ & AGG-RRL-001 \\
\hline & Technetium-95m & $<4.74 \mathrm{E}-1$ & pCi/g dry & $4.74 \mathrm{E}-1$ & & 6/23/08 & $8 F 20002$ & AGG-RRL-001 \\
\hline \multirow[t]{2}{*}{ 13967-71-0 } & Zirconium-95 & $<5.04 \mathrm{E}-1$ & pCi/g dry & $5.04 \mathrm{E}-1$ & & 6/23/08 & 8F20002 & AGG-RRL-001 \\
\hline & Technetium-99m & $<2.99 \mathrm{E}-1$ & pCi/g dry & $2.99 \mathrm{E}-1$ & & 6/23/08 & 8F20002 & AGG-RRL-001 \\
\hline 13968-53-1 & Ruthenium-103 & $<3.26 \mathrm{E}-1$ & pCi/g dry & 3.26E-1 & & 6/23/08 & 8F20002 & AGG-RRL-001 \\
\hline 13967-48-1 & Ruthenium-106 & $<3.03 \mathrm{E} 0$ & pCi/g dry & 3.03E0 & & 6/23/08 & $8 F 20002$ & AGG-RRL-001 \\
\hline $14391-65-2$ & Silver-108m & $<2.98 \mathrm{E}-1$ & pCi/g dry & $2.98 \mathrm{E}-1$ & & 6/23/08 & 8F20002 & AGG-RRL-001 \\
\hline \multirow[t]{2}{*}{$14109-32-1$} & Cadmium-109 & $<7.86 \mathrm{E} 0$ & pCi/g dry & 7.86E0 & & 6/23/08 & $8 F 20002$ & AGG-RRL-001 \\
\hline & Silver-110 & $<2.94 \mathrm{E}-1$ & pCi/g dry & $2.94 \mathrm{E}-1$ & & 6/23/08 & 8F20002 & AGG-RRL-001 \\
\hline 14391-76-5 & Silver-110m & $<2.94 \mathrm{E}-1$ & pCi/g dry & $2.94 \mathrm{E}-1$ & & 6/23/08 & 8F20002 & AGG-RRL-001 \\
\hline 13966-06-8 & Tin-113 & $<4.46 \mathrm{E}-1$ & pCi/g dry & 4.46E-1 & & 6/23/08 & 8F20002 & AGG-RRL-001 \\
\hline $14683-10-4$ & Antimony-124 & $<3.02 \mathrm{E}-1$ & pCi/g dry & $3.02 \mathrm{E}-1$ & & 6/23/08 & $8 F 20002$ & AGG-RRL-001 \\
\hline $14234-35-6$ & Antimony-125 & $<9.43 \mathrm{E}-1$ & pCi/g dry & $9.43 \mathrm{E}-1$ & & 6/23/08 & 8F20002 & AGG-RRL-001 \\
\hline 15756-32-8 & Antimony-126 & $<3.12 \mathrm{E}-1$ & pCi/g dry & 3.12E-1 & & 6/23/08 & 8F20002 & AGG-RRL-001 \\
\hline $15832-50-5$ & Tin-126 & $<6.19 \mathrm{E}-1$ & pCi/g dry & $6.19 \mathrm{E}-1$ & & 6/23/08 & 8F20002 & AGG-RRL-001 \\
\hline 10043-66-0 & Iodine-131 & $<3.54 \mathrm{E}-1$ & pCi/g dry & $3.54 \mathrm{E}-1$ & & 6/23/08 & 8F20002 & AGG-RRL-001 \\
\hline 13981-41-4 & Barium-133 & $<5.47 \mathrm{E}-1$ & pCi/g dry & $5.47 \mathrm{E}-1$ & & 6/23/08 & $8 F 20002$ & AGG-RRL-001 \\
\hline 13967-70-9 & Cesium-134 & $<3.37 \mathrm{E}-1$ & pCi/g dry & 3.37E-1 & & 6/23/08 & 8F20002 & AGG-RRL-001 \\
\hline $10045-97-3$ & Cesium-137 & $<3.28 \mathrm{E}-1$ & pCi/g dry & $3.28 \mathrm{E}-1$ & & 6/23/08 & 8F20002 & AGG-RRL-001 \\
\hline 13982-30-4 & Cerium-139 & $<3.52 \mathrm{E}-1$ & pCi/g dry & $3.52 \mathrm{E}-1$ & & 6/23/08 & $8 F 20002$ & AGG-RRL-001 \\
\hline $14762-78-8$ & Cerium-144 & $<2.38 \mathrm{E} 0$ & pCi/g dry & $2.38 \mathrm{E} 0$ & & 6/23/08 & $8 F 20002$ & AGG-RRL-001 \\
\hline 14683-23-9 & Europium-152 & $<1.13 \mathrm{E} 0$ & pCi/g dry & $1.13 \mathrm{E} 0$ & & 6/23/08 & 8F20002 & AGG-RRL-001 \\
\hline $14276-65-4$ & Gadolinium-153 & $<9.06 \mathrm{E}-1$ & pCi/g dry & $9.06 \mathrm{E}-1$ & & 6/23/08 & $8 F 20002$ & AGG-RRL-001 \\
\hline 15585-10-1 & Europium-154 & $<6.19 \mathrm{E}-1$ & pCi/g dry & $6.19 \mathrm{E}-1$ & & 6/23/08 & 8F20002 & AGG-RRL-001 \\
\hline 14391-16-3 & Europium-155 & $<8.75 \mathrm{E}-1$ & pCi/g dry & 8.75E-1 & & 6/23/08 & 8F20002 & AGG-RRL-001 \\
\hline $13982-78-0$ & Mercury-203 & $<3.75 \mathrm{E}-1$ & pCi/g dry & 3.75E-1 & & 6/23/08 & 8F20002 & AGG-RRL-001 \\
\hline $14913-50-9$ & Thallium-208 & $<2.87 \mathrm{E}-1$ & pCi/g dry & $2.87 \mathrm{E}-1$ & & 6/23/08 & $8 F 20002$ & AGG-RRL-001 \\
\hline $14331-79-4$ & Bismuth-210 & $<5.82 \mathrm{E}-1$ & pCi/g dry & $5.82 \mathrm{E}-1$ & & 6/23/08 & $8 F 20002$ & AGG-RRL-001 \\
\hline
\end{tabular}


GEA/Soil

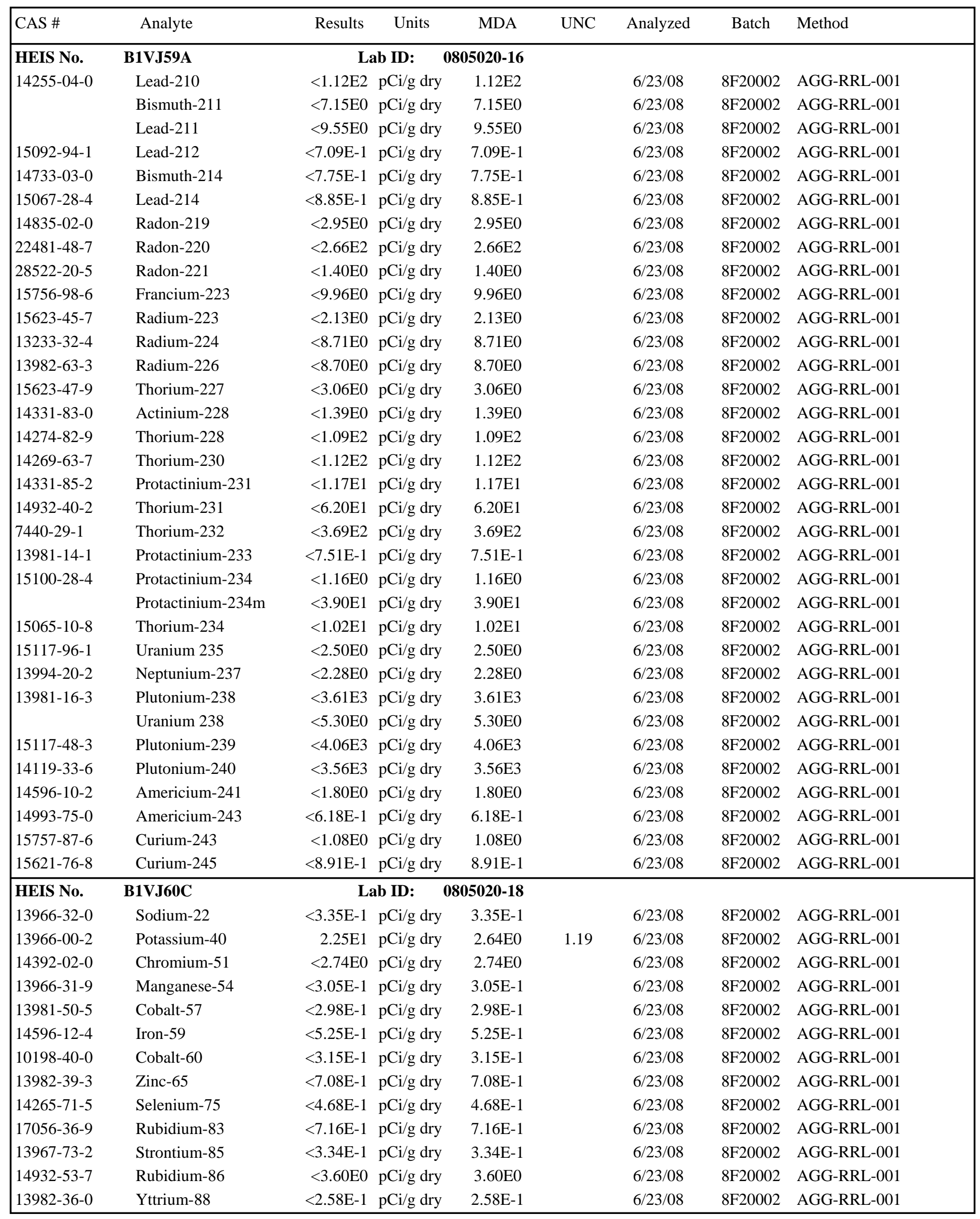


GEA/Soil

\begin{tabular}{|c|c|c|c|c|c|c|c|c|}
\hline CAS \# & Analyte & Results & Units & MDA & UNC & Analyzed & Batch & Method \\
\hline HEIS No. & B1VJ60C & & ab ID: & $5020-18$ & & & & \\
\hline 14681-63-1 & Niobium-94 & $<2.89 \mathrm{E}-1$ & pCi/g dry & $2.89 \mathrm{E}-1$ & & 6/23/08 & 8F20002 & AGG-RRL-001 \\
\hline \multirow[t]{3}{*}{ 13967-76-5 } & Niobium-95 & $<2.80 \mathrm{E}-1$ & pCi/g dry & $2.80 \mathrm{E}-1$ & & 6/23/08 & $8 \mathrm{~F} 20002$ & AGG-RRL-001 \\
\hline & Niobium-95m & $<1.32 \mathrm{E} 0$ & pCi/g dry & $1.32 \mathrm{E} 0$ & & 6/23/08 & $8 \mathrm{~F} 20002$ & AGG-RRL-001 \\
\hline & Technetium-95m & $<4.33 \mathrm{E}-1$ & pCi/g dry & 4.33E-1 & & 6/23/08 & $8 \mathrm{~F} 20002$ & AGG-RRL-001 \\
\hline \multirow[t]{2}{*}{ 13967-71-0 } & Zirconium-95 & $<5.09 \mathrm{E}-1$ & pCi/g dry & $5.09 \mathrm{E}-1$ & & 6/23/08 & $8 \mathrm{~F} 20002$ & AGG-RRL-001 \\
\hline & Technetium-99m & $<3.01 \mathrm{E}-1$ & pCi/g dry & 3.01E-1 & & 6/23/08 & 8 F20002 & AGG-RRL-001 \\
\hline 13968-53-1 & Ruthenium-103 & $<3.42 \mathrm{E}-1$ & pCi/g dry & $3.42 \mathrm{E}-1$ & & 6/23/08 & $8 \mathrm{~F} 20002$ & AGG-RRL-001 \\
\hline 13967-48-1 & Ruthenium-106 & $<3.28 \mathrm{E} 0$ & pCi/g dry & $3.28 \mathrm{E} 0$ & & 6/23/08 & $8 \mathrm{~F} 20002$ & AGG-RRL-001 \\
\hline $14391-65-2$ & Silver-108m & $<2.80 \mathrm{E}-1$ & pCi/g dry & $2.80 \mathrm{E}-1$ & & 6/23/08 & $8 \mathrm{~F} 20002$ & AGG-RRL-001 \\
\hline \multirow[t]{2}{*}{ 14109-32-1 } & Cadmium-109 & $<8.52 \mathrm{E} 0$ & pCi/g dry & $8.52 \mathrm{E} 0$ & & 6/23/08 & $8 \mathrm{~F} 20002$ & AGG-RRL-001 \\
\hline & Silver-110 & $<3.03 \mathrm{E}-1$ & pCi/g dry & 3.03E-1 & & 6/23/08 & $8 \mathrm{~F} 20002$ & AGG-RRL-001 \\
\hline 14391-76-5 & Silver-110m & $<3.04 \mathrm{E}-1$ & pCi/g dry & $3.04 \mathrm{E}-1$ & & 6/23/08 & $8 \mathrm{~F} 20002$ & AGG-RRL-001 \\
\hline 13966-06-8 & Tin-113 & $<3.90 \mathrm{E}-1$ & pCi/g dry & $3.90 \mathrm{E}-1$ & & 6/23/08 & $8 \mathrm{~F} 20002$ & AGG-RRL-001 \\
\hline 14683-10-4 & Antimony-124 & $<3.49 \mathrm{E}-1$ & pCi/g dry & 3.49E-1 & & 6/23/08 & $8 \mathrm{~F} 20002$ & AGG-RRL-001 \\
\hline $14234-35-6$ & Antimony-125 & $<8.85 \mathrm{E}-1$ & pCi/g dry & 8.85E-1 & & 6/23/08 & $8 \mathrm{~F} 20002$ & AGG-RRL-001 \\
\hline 15756-32-8 & Antimony-126 & $<2.92 \mathrm{E}-1$ & pCi/g dry & 2.92E-1 & & 6/23/08 & $8 \mathrm{~F} 20002$ & AGG-RRL-001 \\
\hline $15832-50-5$ & Tin-126 & $<6.95 \mathrm{E}-1$ & pCi/g dry & $6.95 \mathrm{E}-1$ & & 6/23/08 & $8 \mathrm{~F} 20002$ & AGG-RRL-001 \\
\hline 10043-66-0 & Iodine-131 & $<3.51 \mathrm{E}-1$ & pCi/g dry & $3.51 \mathrm{E}-1$ & & 6/23/08 & $8 \mathrm{~F} 20002$ & AGG-RRL-001 \\
\hline 13981-41-4 & Barium-133 & $<4.92 \mathrm{E}-1$ & pCi/g dry & $4.92 \mathrm{E}-1$ & & 6/23/08 & $8 \mathrm{~F} 20002$ & AGG-RRL-001 \\
\hline 13967-70-9 & Cesium-134 & $<3.32 \mathrm{E}-1$ & pCi/g dry & 3.32E-1 & & 6/23/08 & $8 \mathrm{~F} 20002$ & AGG-RRL-001 \\
\hline 10045-97-3 & Cesium-137 & $<3.44 \mathrm{E}-1$ & pCi/g dry & $3.44 \mathrm{E}-1$ & & 6/23/08 & $8 \mathrm{~F} 20002$ & AGG-RRL-001 \\
\hline 13982-30-4 & Cerium-139 & $<3.33 \mathrm{E}-1$ & pCi/g dry & 3.33E-1 & & 6/23/08 & $8 \mathrm{~F} 20002$ & AGG-RRL-001 \\
\hline 14762-78-8 & Cerium-144 & $<2.46 \mathrm{E} 0$ & pCi/g dry & $2.46 \mathrm{E} 0$ & & 6/23/08 & $8 \mathrm{~F} 20002$ & AGG-RRL-001 \\
\hline 14683-23-9 & Europium-152 & $<1.17 \mathrm{E} 0$ & pCi/g dry & $1.17 \mathrm{E} 0$ & & 6/23/08 & $8 \mathrm{~F} 20002$ & AGG-RRL-001 \\
\hline $14276-65-4$ & Gadolinium-153 & $<9.09 \mathrm{E}-1$ & pCi/g dry & $9.09 \mathrm{E}-1$ & & 6/23/08 & $8 \mathrm{~F} 20002$ & AGG-RRL-001 \\
\hline 15585-10-1 & Europium-154 & $<6.30 \mathrm{E}-1$ & pCi/g dry & $6.30 \mathrm{E}-1$ & & 6/23/08 & $8 \mathrm{~F} 20002$ & AGG-RRL-001 \\
\hline 14391-16-3 & Europium-155 & $<9.89 \mathrm{E}-1$ & pCi/g dry & $9.89 \mathrm{E}-1$ & & 6/23/08 & $8 \mathrm{~F} 20002$ & AGG-RRL-001 \\
\hline 13982-78-0 & Mercury-203 & $<3.58 \mathrm{E}-1$ & pCi/g dry & $3.58 \mathrm{E}-1$ & & 6/23/08 & $8 \mathrm{~F} 20002$ & AGG-RRL-001 \\
\hline $14913-50-9$ & Thallium-208 & $<3.59 \mathrm{E}-1$ & pCi/g dry & $3.59 \mathrm{E}-1$ & & 6/23/08 & $8 \mathrm{~F} 20002$ & AGG-RRL-001 \\
\hline 14331-79-4 & Bismuth-210 & $<5.56 \mathrm{E}-1$ & pCi/g dry & $5.56 \mathrm{E}-1$ & & 6/23/08 & $8 \mathrm{~F} 20002$ & AGG-RRL-001 \\
\hline \multirow[t]{3}{*}{$14255-04-0$} & Lead-210 & $<4.02 \mathrm{E} 1$ & pCi/g dry & 4.02E1 & & 6/23/08 & $8 \mathrm{~F} 20002$ & AGG-RRL-001 \\
\hline & Bismuth-211 & $<6.29 \mathrm{E} 0$ & pCi/g dry & $6.29 \mathrm{E} 0$ & & 6/23/08 & $8 \mathrm{~F} 20002$ & AGG-RRL-001 \\
\hline & Lead-211 & $<8.59 \mathrm{E} 0$ & pCi/g dry & 8.59E0 & & 6/23/08 & $8 \mathrm{~F} 20002$ & AGG-RRL-001 \\
\hline 15092-94-1 & Lead-212 & $<7.65 \mathrm{E}-1$ & pCi/g dry & 7.65E-1 & & 6/23/08 & $8 \mathrm{~F} 20002$ & AGG-RRL-001 \\
\hline 14733-03-0 & Bismuth-214 & $<7.38 \mathrm{E}-1$ & pCi/g dry & 7.38E-1 & & 6/23/08 & $8 \mathrm{~F} 20002$ & AGG-RRL-001 \\
\hline $15067-28-4$ & Lead-214 & $<8.07 \mathrm{E}-1$ & pCi/g dry & 8.07E-1 & & 6/23/08 & $8 \mathrm{~F} 20002$ & AGG-RRL-001 \\
\hline 14835-02-0 & Radon-219 & $<2.81 \mathrm{E} 0$ & pCi/g dry & 2.81E0 & & 6/23/08 & $8 \mathrm{~F} 20002$ & AGG-RRL-001 \\
\hline 22481-48-7 & Radon-220 & $<2.79 \mathrm{E} 2$ & pCi/g dry & 2.79E2 & & 6/23/08 & $8 \mathrm{~F} 20002$ & AGG-RRL-001 \\
\hline 28522-20-5 & Radon-221 & $<1.29 \mathrm{E} 0$ & pCi/g dry & $1.29 \mathrm{E} 0$ & & 6/23/08 & $8 \mathrm{~F} 20002$ & AGG-RRL-001 \\
\hline 15756-98-6 & Francium-223 & $<9.69 \mathrm{E} 0$ & pCi/g dry & 9.69E0 & & 6/23/08 & $8 \mathrm{~F} 20002$ & AGG-RRL-001 \\
\hline $15623-45-7$ & Radium-223 & $<2.05 \mathrm{E} 0$ & pCi/g dry & 2.05E0 & & 6/23/08 & $8 \mathrm{~F} 20002$ & AGG-RRL-001 \\
\hline 13233-32-4 & Radium-224 & $<8.31 \mathrm{E} 0$ & pCi/g dry & 8.31E0 & & 6/23/08 & $8 \mathrm{~F} 20002$ & AGG-RRL-001 \\
\hline 13982-63-3 & Radium-226 & $<7.98 \mathrm{E} 0$ & pCi/g dry & 7.98E0 & & 6/23/08 & $8 \mathrm{~F} 20002$ & AGG-RRL-001 \\
\hline $15623-47-9$ & Thorium-227 & $<2.94 \mathrm{E} 0$ & pCi/g dry & $2.94 \mathrm{E} 0$ & & 6/23/08 & $8 \mathrm{~F} 20002$ & AGG-RRL-001 \\
\hline 14331-83-0 & Actinium-228 & $<1.36 \mathrm{E} 0$ & pCi/g dry & $1.36 \mathrm{E} 0$ & & 6/23/08 & $8 \mathrm{~F} 20002$ & AGG-RRL-001 \\
\hline 14274-82-9 & Thorium-228 & $<1.04 \mathrm{E} 2$ & pCi/g dry & $1.04 \mathrm{E} 2$ & & 6/23/08 & $8 \mathrm{~F} 20002$ & AGG-RRL-001 \\
\hline 14269-63-7 & Thorium-230 & $<1.12 \mathrm{E} 2$ & pCi/g dry & $1.12 \mathrm{E} 2$ & & 6/23/08 & 8F20002 & AGG-RRL-001 \\
\hline 14331-85-2 & Protactinium-231 & $<1.26 \mathrm{E} 1$ & pCi/g dry & $1.26 \mathrm{E} 1$ & & 6/23/08 & 8F20002 & AGG-RRL-001 \\
\hline $14932-40-2$ & Thorium-231 & $<6.45 \mathrm{E} 1$ & pCi/g dry & $6.45 \mathrm{E} 1$ & & 6/23/08 & $8 F 20002$ & AGG-RRL-001 \\
\hline
\end{tabular}


GEA/Soil

\begin{tabular}{|c|c|c|c|c|c|c|c|c|}
\hline CAS \# & Analyte & Results & Units & MDA & UNC & Analyzed & Batch & Method \\
\hline HEIS No. & B1VJ60C & & ab ID: & $5020-18$ & & & & \\
\hline 7440-29-1 & Thorium-232 & $<2.75 \mathrm{E} 2$ & pCi/g dry & 2.75E2 & & 6/23/08 & $8 \mathrm{~F} 20002$ & AGG-RRL-001 \\
\hline 13981-14-1 & Protactinium-233 & $<7.45 \mathrm{E}-1$ & pCi/g dry & 7.45E-1 & & 6/23/08 & $8 \mathrm{~F} 20002$ & AGG-RRL-001 \\
\hline \multirow[t]{2}{*}{$15100-28-4$} & Protactinium-234 & $<1.19 \mathrm{E} 0$ & pCi/g dry & 1.19E0 & & 6/23/08 & 8 F20002 & AGG-RRL-001 \\
\hline & Protactinium-234m & $<3.66 \mathrm{E} 1$ & pCi/g dry & 3.66E1 & & 6/23/08 & $8 \mathrm{~F} 20002$ & AGG-RRL-001 \\
\hline 15065-10-8 & Thorium-234 & $<9.72 \mathrm{E} 0$ & pCi/g dry & 9.72E0 & & 6/23/08 & $8 \mathrm{~F} 20002$ & AGG-RRL-001 \\
\hline 15117-96-1 & Uranium 235 & $<2.53 \mathrm{E} 0$ & pCi/g dry & 2.53E0 & & 6/23/08 & $8 \mathrm{~F} 20002$ & AGG-RRL-001 \\
\hline 13994-20-2 & Neptunium-237 & $<2.57 \mathrm{E} 0$ & pCi/g dry & 2.57E0 & & 6/23/08 & $8 \mathrm{~F} 20002$ & AGG-RRL-001 \\
\hline \multirow{2}{*}{ 13981-16-3 } & Plutonium-238 & $<3.74 \mathrm{E} 3$ & pCi/g dry & $3.74 \mathrm{E} 3$ & & 6/23/08 & $8 \mathrm{~F} 20002$ & AGG-RRL-001 \\
\hline & Uranium 238 & $<5.38 \mathrm{E} 0$ & pCi/g dry & 5.38E0 & & 6/23/08 & $8 \mathrm{~F} 20002$ & AGG-RRL-001 \\
\hline $15117-48-3$ & Plutonium-239 & $<3.71 \mathrm{E} 3$ & pCi/g dry & $3.71 \mathrm{E} 3$ & & 6/23/08 & $8 \mathrm{~F} 20002$ & AGG-RRL-001 \\
\hline 14119-33-6 & Plutonium-240 & $<3.70 \mathrm{E} 3$ & pCi/g dry & 3.70E3 & & 6/23/08 & $8 \mathrm{~F} 20002$ & AGG-RRL-001 \\
\hline 14596-10-2 & Americium-241 & $<1.41 \mathrm{E} 0$ & pCi/g dry & $1.41 \mathrm{E} 0$ & & 6/23/08 & $8 \mathrm{~F} 20002$ & AGG-RRL-001 \\
\hline 14993-75-0 & Americium-243 & $<6.22 \mathrm{E}-1$ & pCi/g dry & $6.22 \mathrm{E}-1$ & & 6/23/08 & $8 \mathrm{~F} 20002$ & AGG-RRL-001 \\
\hline 15757-87-6 & Curium-243 & $<1.13 \mathrm{E} 0$ & pCi/g dry & 1.13E0 & & 6/23/08 & 8F20002 & AGG-RRL-001 \\
\hline 15621-76-8 & Curium-245 & $<9.28 \mathrm{E}-1$ & pCi/g dry & $9.28 \mathrm{E}-1$ & & 6/23/08 & $8 \mathrm{~F} 20002$ & AGG-RRL-001 \\
\hline HEIS No. & B1VJ60B & & ab ID: & 5020-19 & & & & \\
\hline 13966-32-0 & Sodium-22 & $<3.24 \mathrm{E}-1$ & pCi/g dry & $3.24 \mathrm{E}-1$ & & 6/23/08 & $8 \mathrm{~F} 20002$ & AGG-RRL-001 \\
\hline 13966-00-2 & Potassium-40 & 2.17E1 & pCi/g dry & 2.91E0 & 1.29 & 6/23/08 & 8 F20002 & AGG-RRL-001 \\
\hline 14392-02-0 & Chromium-51 & $<2.72 \mathrm{E} 0$ & pCi/g dry & 2.72E0 & & 6/23/08 & $8 \mathrm{~F} 20002$ & AGG-RRL-001 \\
\hline 13966-31-9 & Manganese-54 & $<2.72 \mathrm{E}-1$ & pCi/g dry & $2.72 \mathrm{E}-1$ & & 6/23/08 & $8 \mathrm{~F} 20002$ & AGG-RRL-001 \\
\hline 13981-50-5 & Cobalt-57 & $<3.04 \mathrm{E}-1$ & pCi/g dry & 3.04E-1 & & 6/23/08 & $8 \mathrm{~F} 20002$ & AGG-RRL-001 \\
\hline $14596-12-4$ & Iron-59 & $<5.17 \mathrm{E}-1$ & pCi/g dry & $5.17 \mathrm{E}-1$ & & 6/23/08 & 8 F20002 & AGG-RRL-001 \\
\hline 10198-40-0 & Cobalt-60 & $<2.97 \mathrm{E}-1$ & pCi/g dry & 2.97E-1 & & 6/23/08 & $8 \mathrm{~F} 20002$ & AGG-RRL-001 \\
\hline 13982-39-3 & Zinc-65 & $<7.23 \mathrm{E}-1$ & pCi/g dry & 7.23E-1 & & 6/23/08 & $8 \mathrm{~F} 20002$ & AGG-RRL-001 \\
\hline 14265-71-5 & Selenium-75 & $<4.71 \mathrm{E}-1$ & pCi/g dry & $4.71 \mathrm{E}-1$ & & 6/23/08 & $8 \mathrm{~F} 20002$ & AGG-RRL-001 \\
\hline 17056-36-9 & Rubidium-83 & $<5.87 \mathrm{E}-1$ & pCi/g dry & $5.87 \mathrm{E}-1$ & & 6/23/08 & 8 F20002 & AGG-RRL-001 \\
\hline 13967-73-2 & Strontium-85 & $<3.88 \mathrm{E}-1$ & pCi/g dry & $3.88 \mathrm{E}-1$ & & 6/23/08 & $8 \mathrm{~F} 20002$ & AGG-RRL-001 \\
\hline 14932-53-7 & Rubidium-86 & $<3.64 \mathrm{E} 0$ & pCi/g dry & 3.64E0 & & 6/23/08 & $8 \mathrm{~F} 20002$ & AGG-RRL-001 \\
\hline 13982-36-0 & Yttrium-88 & $<2.26 \mathrm{E}-1$ & pCi/g dry & $2.26 \mathrm{E}-1$ & & 6/23/08 & 8 F20002 & AGG-RRL-001 \\
\hline 14681-63-1 & Niobium-94 & $<2.86 \mathrm{E}-1$ & pCi/g dry & 2.86E-1 & & 6/23/08 & $8 \mathrm{~F} 20002$ & AGG-RRL-001 \\
\hline \multirow[t]{3}{*}{ 13967-76-5 } & Niobium-95 & $<2.97 \mathrm{E}-1$ & pCi/g dry & 2.97E-1 & & 6/23/08 & 8 F20002 & AGG-RRL-001 \\
\hline & Niobium-95m & $<1.36 \mathrm{E} 0$ & pCi/g dry & $1.36 \mathrm{E} 0$ & & 6/23/08 & $8 \mathrm{~F} 20002$ & AGG-RRL-001 \\
\hline & Technetium-95m & $<4.45 \mathrm{E}-1$ & pCi/g dry & 4.45E-1 & & 6/23/08 & 8 F20002 & AGG-RRL-001 \\
\hline \multirow[t]{2}{*}{ 13967-71-0 } & Zirconium-95 & $<5.26 \mathrm{E}-1$ & pCi/g dry & $5.26 \mathrm{E}-1$ & & 6/23/08 & $8 \mathrm{~F} 20002$ & AGG-RRL-001 \\
\hline & Technetium-99m & $<2.89 \mathrm{E}-1$ & pCi/g dry & 2.89E-1 & & 6/23/08 & 8 F20002 & AGG-RRL-001 \\
\hline 13968-53-1 & Ruthenium-103 & $<2.78 \mathrm{E}-1$ & pCi/g dry & $2.78 \mathrm{E}-1$ & & 6/23/08 & $8 \mathrm{~F} 20002$ & AGG-RRL-001 \\
\hline 13967-48-1 & Ruthenium-106 & $<2.59 \mathrm{E} 0$ & pCi/g dry & 2.59E0 & & 6/23/08 & $8 \mathrm{~F} 20002$ & AGG-RRL-001 \\
\hline 14391-65-2 & Silver-108m & $<2.97 \mathrm{E}-1$ & pCi/g dry & 2.97E-1 & & 6/23/08 & $8 \mathrm{~F} 20002$ & AGG-RRL-001 \\
\hline \multirow[t]{2}{*}{ 14109-32-1 } & Cadmium-109 & $<8.10 \mathrm{E} 0$ & pCi/g dry & 8.10E0 & & 6/23/08 & $8 \mathrm{~F} 20002$ & AGG-RRL-001 \\
\hline & Silver-110 & $<3.05 \mathrm{E}-1$ & pCi/g dry & 3.05E-1 & & 6/23/08 & $8 \mathrm{~F} 20002$ & AGG-RRL-001 \\
\hline 14391-76-5 & Silver-110m & $<3.06 \mathrm{E}-1$ & pCi/g dry & $3.06 \mathrm{E}-1$ & & 6/23/08 & $8 \mathrm{~F} 20002$ & AGG-RRL-001 \\
\hline 13966-06-8 & Tin-113 & $<4.24 \mathrm{E}-1$ & pCi/g dry & $4.24 \mathrm{E}-1$ & & 6/23/08 & $8 \mathrm{~F} 20002$ & AGG-RRL-001 \\
\hline $14683-10-4$ & Antimony-124 & $<2.91 \mathrm{E}-1$ & pCi/g dry & $2.91 \mathrm{E}-1$ & & 6/23/08 & $8 \mathrm{~F} 20002$ & AGG-RRL-001 \\
\hline $14234-35-6$ & Antimony-125 & $<9.48 \mathrm{E}-1$ & pCi/g dry & $9.48 \mathrm{E}-1$ & & 6/23/08 & 8 F20002 & AGG-RRL-001 \\
\hline 15756-32-8 & Antimony-126 & $<2.82 \mathrm{E}-1$ & pCi/g dry & $2.82 \mathrm{E}-1$ & & 6/23/08 & $8 \mathrm{~F} 20002$ & AGG-RRL-001 \\
\hline $15832-50-5$ & Tin-126 & $<6.33 \mathrm{E}-1$ & pCi/g dry & 6.33E-1 & & 6/23/08 & $8 \mathrm{~F} 20002$ & AGG-RRL-001 \\
\hline 10043-66-0 & Iodine-131 & $<3.45 \mathrm{E}-1$ & pCi/g dry & 3.45E-1 & & 6/23/08 & $8 \mathrm{~F} 20002$ & AGG-RRL-001 \\
\hline 13981-41-4 & Barium-133 & $<5.54 \mathrm{E}-1$ & pCi/g dry & $5.54 \mathrm{E}-1$ & & 6/23/08 & 8F20002 & AGG-RRL-001 \\
\hline
\end{tabular}


GEA/Soil

\begin{tabular}{|c|c|c|c|c|c|c|c|c|}
\hline CAS \# & Analyte & Results & Units & MDA & UNC & Analyzed & Batch & Method \\
\hline HEIS No. & B1VJ60B & \multicolumn{3}{|c|}{ Lab ID: $\quad$ 0805020-19 } & & & & \\
\hline 13967-70-9 & Cesium-134 & $<3.51 \mathrm{E}-1$ & pCi/g dry & $3.51 \mathrm{E}-1$ & & $6 / 23 / 08$ & $8 F 20002$ & AGG-RRL-001 \\
\hline 10045-97-3 & Cesium-137 & $<3.23 E-1$ & pCi/g dry & $3.23 E-1$ & & $6 / 23 / 08$ & $8 \mathrm{~F} 20002$ & AGG-RRL-001 \\
\hline 13982-30-4 & Cerium-139 & $<3.40 \mathrm{E}-1$ & pCi/g dry & $3.40 \mathrm{E}-1$ & & 6/23/08 & $8 \mathrm{~F} 20002$ & AGG-RRL-001 \\
\hline 14762-78-8 & Cerium-144 & $<2.35 \mathrm{E} 0$ & pCi/g dry & $2.35 \mathrm{E} 0$ & & 6/23/08 & $8 \mathrm{~F} 20002$ & AGG-RRL-001 \\
\hline 14683-23-9 & Europium-152 & $<1.12 \mathrm{E} 0$ & pCi/g dry & $1.12 \mathrm{E} 0$ & & 6/23/08 & $8 \mathrm{~F} 20002$ & AGG-RRL-001 \\
\hline 14276-65-4 & Gadolinium-153 & $<8.91 \mathrm{E}-1$ & pCi/g dry & 8.91E-1 & & 6/23/08 & $8 \mathrm{~F} 20002$ & AGG-RRL-001 \\
\hline 15585-10-1 & Europium-154 & $<6.33 \mathrm{E}-1$ & pCi/g dry & 6.33E-1 & & 6/23/08 & $8 \mathrm{~F} 20002$ & AGG-RRL-001 \\
\hline 14391-16-3 & Europium-155 & $<8.88 \mathrm{E}-1$ & pCi/g dry & $8.88 \mathrm{E}-1$ & & $6 / 23 / 08$ & $8 \mathrm{~F} 20002$ & AGG-RRL-001 \\
\hline 13982-78-0 & Mercury-203 & $<3.48 \mathrm{E}-1$ & pCi/g dry & $3.48 \mathrm{E}-1$ & & 6/23/08 & $8 \mathrm{~F} 20002$ & AGG-RRL-001 \\
\hline 14913-50-9 & Thallium-208 & $<3.08 \mathrm{E}-1$ & pCi/g dry & $3.08 \mathrm{E}-1$ & & $6 / 23 / 08$ & $8 \mathrm{~F} 20002$ & AGG-RRL-001 \\
\hline 14331-79-4 & Bismuth-210 & $<5.56 \mathrm{E}-1$ & pCi/g dry & $5.56 \mathrm{E}-1$ & & 6/23/08 & $8 \mathrm{~F} 20002$ & AGG-RRL-001 \\
\hline \multirow[t]{3}{*}{$14255-04-0$} & Lead-210 & $<1.10 \mathrm{E} 2$ & pCi/g dry & $1.10 \mathrm{E} 2$ & & 6/23/08 & $8 \mathrm{~F} 20002$ & AGG-RRL-001 \\
\hline & Bismuth-211 & $<6.80 \mathrm{E} 0$ & pCi/g dry & $6.80 \mathrm{E} 0$ & & 6/23/08 & $8 \mathrm{~F} 20002$ & AGG-RRL-001 \\
\hline & Lead-211 & $<9.30 \mathrm{E} 0$ & pCi/g dry & $9.30 \mathrm{E} 0$ & & 6/23/08 & $8 \mathrm{~F} 20002$ & AGG-RRL-001 \\
\hline 15092-94-1 & Lead-212 & 5.65E-1 & pCi/g dry & $5.32 \mathrm{E}-1$ & 0.123 & 6/23/08 & $8 \mathrm{~F} 20002$ & AGG-RRL-001 \\
\hline 14733-03-0 & Bismuth-214 & $<7.45 \mathrm{E}-1$ & pCi/g dry & 7.45E-1 & & 6/23/08 & $8 \mathrm{~F} 20002$ & AGG-RRL-001 \\
\hline $15067-28-4$ & Lead-214 & $<8.83 \mathrm{E}-1$ & pCi/g dry & 8.83E-1 & & 6/23/08 & $8 \mathrm{~F} 20002$ & AGG-RRL-001 \\
\hline $14835-02-0$ & Radon-219 & $<2.78 \mathrm{E} 0$ & pCi/g dry & $2.78 \mathrm{E} 0$ & & 6/23/08 & $8 \mathrm{~F} 20002$ & AGG-RRL-001 \\
\hline 22481-48-7 & Radon-220 & $<2.53 \mathrm{E} 2$ & pCi/g dry & 2.53E2 & & 6/23/08 & $8 \mathrm{~F} 20002$ & AGG-RRL-001 \\
\hline 28522-20-5 & Radon-221 & $<1.34 \mathrm{E} 0$ & pCi/g dry & $1.34 \mathrm{E} 0$ & & 6/23/08 & $8 \mathrm{~F} 20002$ & AGG-RRL-001 \\
\hline 15756-98-6 & Francium-223 & $<9.80 \mathrm{E} 0$ & pCi/g dry & $9.80 \mathrm{E} 0$ & & 6/23/08 & $8 \mathrm{~F} 20002$ & AGG-RRL-001 \\
\hline $15623-45-7$ & Radium-223 & $<2.03 \mathrm{E} 0$ & pCi/g dry & 2.03E0 & & 6/23/08 & $8 \mathrm{~F} 20002$ & AGG-RRL-001 \\
\hline 13233-32-4 & Radium-224 & $<8.65 \mathrm{E} 0$ & pCi/g dry & 8.65E0 & & 6/23/08 & $8 \mathrm{~F} 20002$ & AGG-RRL-001 \\
\hline 13982-63-3 & Radium-226 & $<8.35 \mathrm{E} 0$ & pCi/g dry & 8.35E0 & & 6/23/08 & $8 \mathrm{~F} 20002$ & AGG-RRL-001 \\
\hline $15623-47-9$ & Thorium-227 & $<3.04 \mathrm{E} 0$ & pCi/g dry & $3.04 \mathrm{E} 0$ & & 6/23/08 & $8 \mathrm{~F} 20002$ & AGG-RRL-001 \\
\hline 14331-83-0 & Actinium-228 & $<1.36 \mathrm{E} 0$ & pCi/g dry & $1.36 \mathrm{E} 0$ & & 6/23/08 & $8 \mathrm{~F} 20002$ & AGG-RRL-001 \\
\hline 14274-82-9 & Thorium-228 & $<1.08 \mathrm{E} 2$ & pCi/g dry & $1.08 \mathrm{E} 2$ & & 6/23/08 & $8 \mathrm{~F} 20002$ & AGG-RRL-001 \\
\hline 14269-63-7 & Thorium-230 & $<1.16 \mathrm{E} 2$ & pCi/g dry & $1.16 \mathrm{E} 2$ & & 6/23/08 & $8 \mathrm{~F} 20002$ & AGG-RRL-001 \\
\hline 14331-85-2 & Protactinium-231 & $<1.22 \mathrm{E} 1$ & pCi/g dry & $1.22 \mathrm{E} 1$ & & 6/23/08 & $8 \mathrm{~F} 20002$ & AGG-RRL-001 \\
\hline $14932-40-2$ & Thorium-231 & $<6.28 \mathrm{E} 1$ & pCi/g dry & $6.28 \mathrm{E} 1$ & & 6/23/08 & $8 \mathrm{~F} 20002$ & AGG-RRL-001 \\
\hline 7440-29-1 & Thorium-232 & $<3.64 \mathrm{E} 2$ & pCi/g dry & 3.64E2 & & 6/23/08 & $8 \mathrm{~F} 20002$ & AGG-RRL-001 \\
\hline 13981-14-1 & Protactinium-233 & $<7.74 \mathrm{E}-1$ & pCi/g dry & 7.74E-1 & & 6/23/08 & $8 \mathrm{~F} 20002$ & AGG-RRL-001 \\
\hline \multirow[t]{2}{*}{$15100-28-4$} & Protactinium-234 & $<1.14 \mathrm{E} 0$ & pCi/g dry & $1.14 \mathrm{E} 0$ & & 6/23/08 & $8 \mathrm{~F} 20002$ & AGG-RRL-001 \\
\hline & Protactinium-234m & $<3.66 \mathrm{E} 1$ & pCi/g dry & 3.66E1 & & 6/23/08 & $8 \mathrm{~F} 20002$ & AGG-RRL-001 \\
\hline 15065-10-8 & Thorium-234 & $<1.01 \mathrm{E} 1$ & pCi/g dry & $1.01 \mathrm{E} 1$ & & 6/23/08 & $8 \mathrm{~F} 20002$ & AGG-RRL-001 \\
\hline 15117-96-1 & Uranium 235 & $<2.48 \mathrm{E} 0$ & pCi/g dry & $2.48 \mathrm{E} 0$ & & 6/23/08 & $8 \mathrm{~F} 20002$ & AGG-RRL-001 \\
\hline 13994-20-2 & Neptunium-237 & $<2.31 \mathrm{E} 0$ & pCi/g dry & $2.31 \mathrm{E} 0$ & & 6/23/08 & $8 \mathrm{~F} 20002$ & AGG-RRL-001 \\
\hline \multirow[t]{2}{*}{ 13981-16-3 } & Plutonium-238 & $<3.63 \mathrm{E} 3$ & pCi/g dry & 3.63E3 & & 6/23/08 & $8 \mathrm{~F} 20002$ & AGG-RRL-001 \\
\hline & Uranium 238 & $<5.22 \mathrm{E} 0$ & pCi/g dry & 5.22E0 & & 6/23/08 & $8 \mathrm{~F} 20002$ & AGG-RRL-001 \\
\hline 15117-48-3 & Plutonium-239 & $<4.15 \mathrm{E} 3$ & pCi/g dry & 4.15E3 & & 6/23/08 & $8 \mathrm{~F} 20002$ & AGG-RRL-001 \\
\hline 14119-33-6 & Plutonium-240 & $<3.58 \mathrm{E} 3$ & pCi/g dry & 3.58E3 & & 6/23/08 & $8 \mathrm{~F} 20002$ & AGG-RRL-001 \\
\hline $14596-10-2$ & Americium-241 & $<1.78 \mathrm{E} 0$ & pCi/g dry & $1.78 \mathrm{E} 0$ & & 6/23/08 & $8 \mathrm{~F} 20002$ & AGG-RRL-001 \\
\hline 14993-75-0 & Americium-243 & $<6.24 \mathrm{E}-1$ & pCi/g dry & $6.24 \mathrm{E}-1$ & & 6/23/08 & $8 \mathrm{~F} 20002$ & AGG-RRL-001 \\
\hline $15757-87-6$ & Curium-243 & $<1.09 \mathrm{E} 0$ & pCi/g dry & 1.09E0 & & $6 / 23 / 08$ & $8 \mathrm{~F} 20002$ & AGG-RRL-001 \\
\hline 15621-76-8 & Curium-245 & $<8.96 \mathrm{E}-1$ & pCi/g dry & 8.96E-1 & & 6/23/08 & $8 F 20002$ & AGG-RRL-001 \\
\hline HEIS No. & B1VJ60A & \multicolumn{3}{|c|}{ Lab ID: $\quad 0805020-20$} & & & & \\
\hline 13966-32-0 & Sodium-22 & $<4.13 \mathrm{E}-1$ & pCi/g dry & 4.13E-1 & & 6/23/08 & $8 \mathrm{~F} 20002$ & AGG-RRL-001 \\
\hline 13966-00-2 & Potassium-40 & 2.22E1 & pCi/g dry & 3.91E0 & 1.43 & $6 / 23 / 08$ & 8F20002 & AGG-RRL-001 \\
\hline
\end{tabular}


GEA/Soil

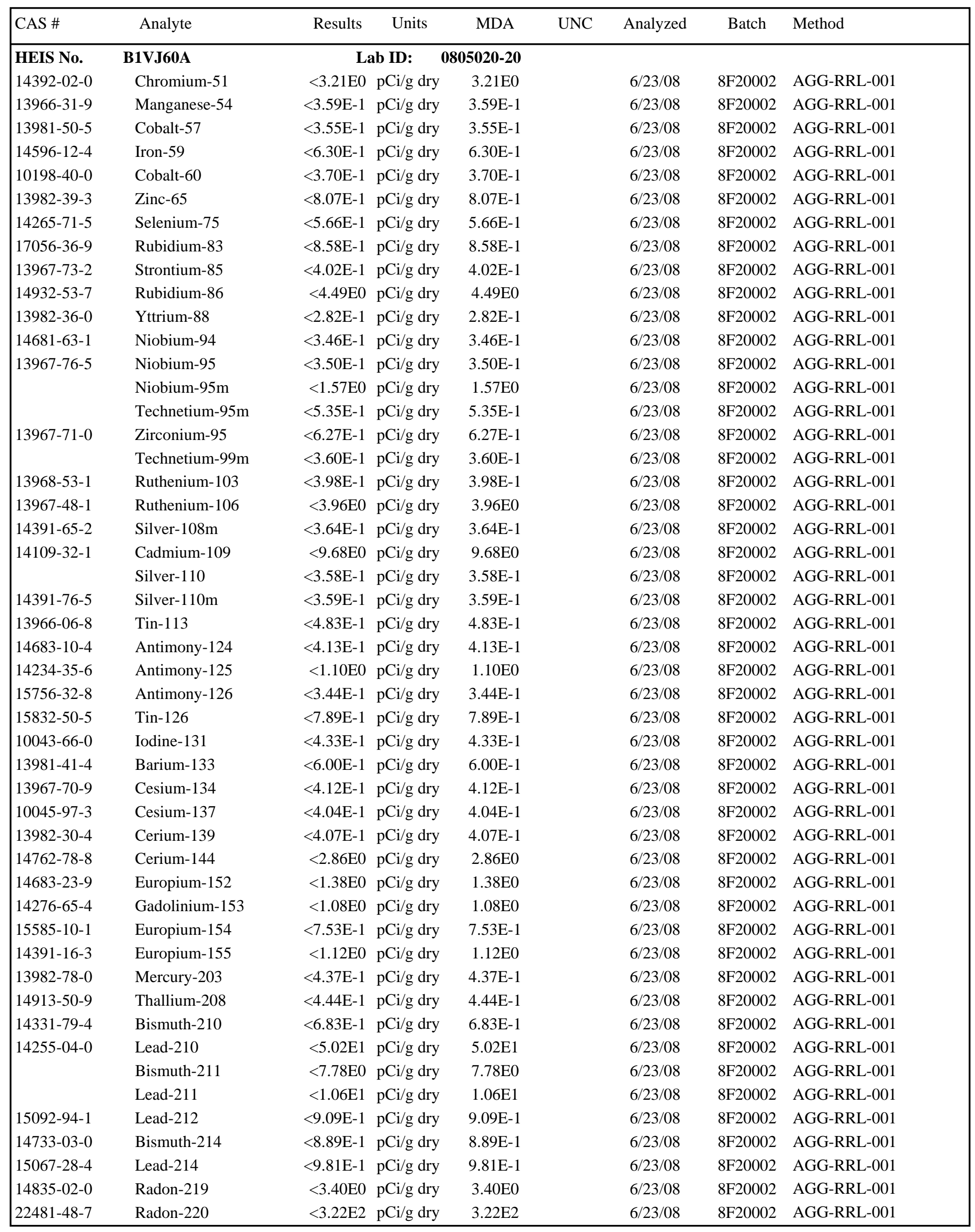


GEA/Soil

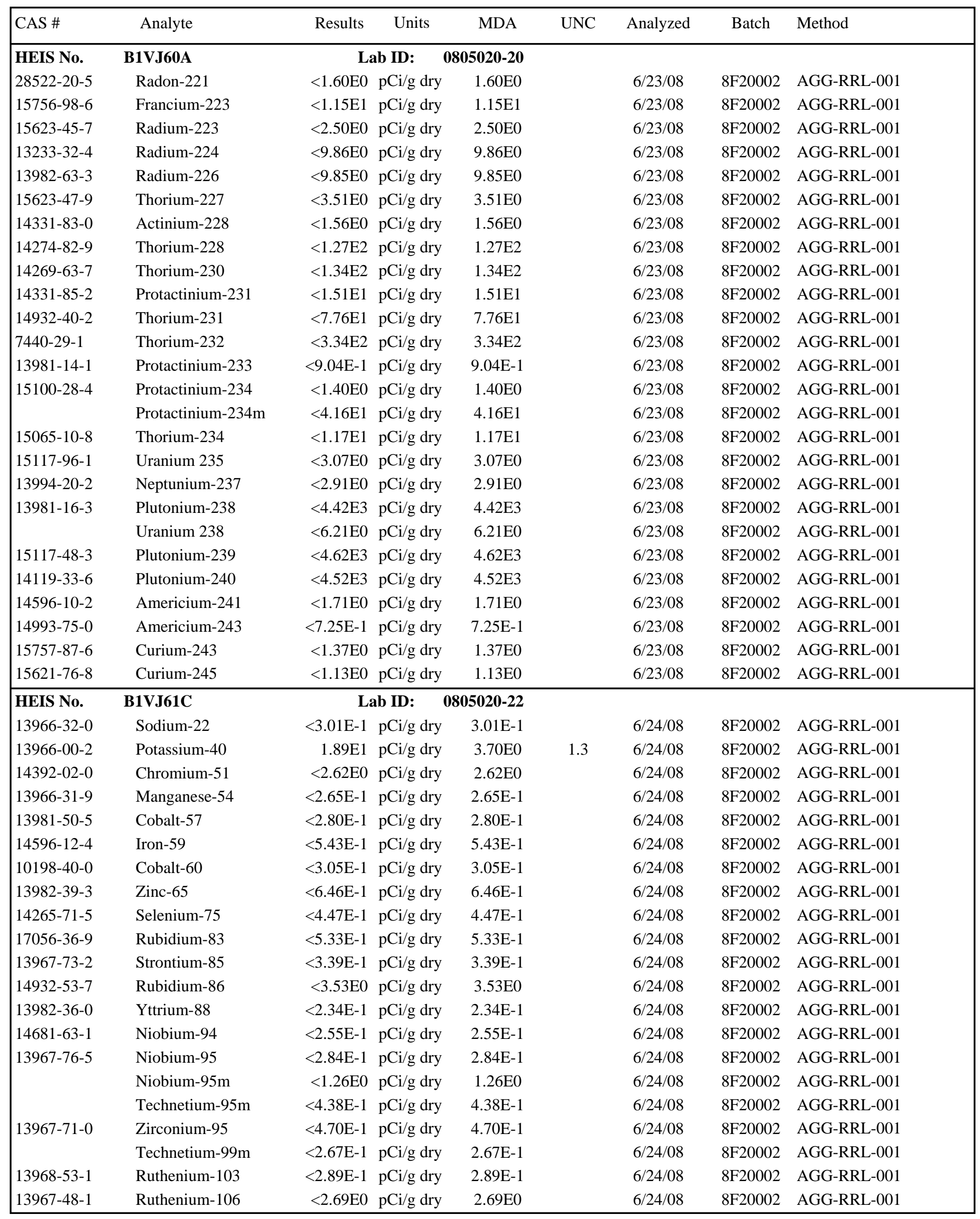


GEA/Soil

\begin{tabular}{|c|c|c|c|c|c|c|c|c|}
\hline CAS \# & Analyte & Results & Units & MDA & UNC & Analyzed & Batch & Method \\
\hline HEIS No. & B1VJ61C & & ab ID: $\quad 0$ & $5020-22$ & & & & \\
\hline $14391-65-2$ & Silver-108m & $<2.81 \mathrm{E}-1$ & pCi/g dry & $2.81 \mathrm{E}-1$ & & $6 / 24 / 08$ & 8F20002 & AGG-RRL-001 \\
\hline \multirow[t]{2}{*}{ 14109-32-1 } & Cadmium-109 & $<7.30 \mathrm{E} 0$ & pCi/g dry & 7.30E0 & & $6 / 24 / 08$ & $8 \mathrm{~F} 20002$ & AGG-RRL-001 \\
\hline & Silver-110 & $<2.80 \mathrm{E}-1$ & pCi/g dry & $2.80 \mathrm{E}-1$ & & $6 / 24 / 08$ & $8 \mathrm{~F} 20002$ & AGG-RRL-001 \\
\hline 14391-76-5 & Silver-110m & $<2.81 \mathrm{E}-1$ & pCi/g dry & $2.81 \mathrm{E}-1$ & & 6/24/08 & $8 \mathrm{~F} 20002$ & AGG-RRL-001 \\
\hline 13966-06-8 & Tin-113 & $<3.92 \mathrm{E}-1$ & pCi/g dry & 3.92E-1 & & $6 / 24 / 08$ & $8 \mathrm{~F} 20002$ & AGG-RRL-001 \\
\hline 14683-10-4 & Antimony-124 & $<2.75 \mathrm{E}-1$ & pCi/g dry & 2.75E-1 & & 6/24/08 & 8 F20002 & AGG-RRL-001 \\
\hline $14234-35-6$ & Antimony-125 & $<9.07 \mathrm{E}-1$ & pCi/g dry & 9.07E-1 & & 6/24/08 & $8 \mathrm{~F} 20002$ & AGG-RRL-001 \\
\hline 15756-32-8 & Antimony-126 & $<2.56 \mathrm{E}-1$ & pCi/g dry & $2.56 \mathrm{E}-1$ & & 6/24/08 & $8 \mathrm{~F} 20002$ & AGG-RRL-001 \\
\hline 15832-50-5 & Tin-126 & $<5.77 \mathrm{E}-1$ & pCi/g dry & 5.77E-1 & & 6/24/08 & $8 \mathrm{~F} 20002$ & AGG-RRL-001 \\
\hline 10043-66-0 & Iodine-131 & $<3.14 \mathrm{E}-1$ & pCi/g dry & $3.14 \mathrm{E}-1$ & & 6/24/08 & $8 \mathrm{~F} 20002$ & AGG-RRL-001 \\
\hline 13981-41-4 & Barium-133 & $<5.25 \mathrm{E}-1$ & pCi/g dry & $5.25 \mathrm{E}-1$ & & 6/24/08 & $8 \mathrm{~F} 20002$ & AGG-RRL-001 \\
\hline 13967-70-9 & Cesium-134 & $<3.24 \mathrm{E}-1$ & pCi/g dry & $3.24 \mathrm{E}-1$ & & $6 / 24 / 08$ & $8 \mathrm{~F} 20002$ & AGG-RRL-001 \\
\hline 10045-97-3 & Cesium-137 & $<3.02 \mathrm{E}-1$ & pCi/g dry & $3.02 \mathrm{E}-1$ & & 6/24/08 & $8 \mathrm{~F} 20002$ & AGG-RRL-001 \\
\hline 13982-30-4 & Cerium-139 & $<3.29 \mathrm{E}-1$ & pCi/g dry & $3.29 \mathrm{E}-1$ & & $6 / 24 / 08$ & $8 \mathrm{~F} 20002$ & AGG-RRL-001 \\
\hline 14762-78-8 & Cerium-144 & $<2.20 \mathrm{E} 0$ & pCi/g dry & $2.20 \mathrm{E} 0$ & & 6/24/08 & $8 \mathrm{~F} 20002$ & AGG-RRL-001 \\
\hline 14683-23-9 & Europium-152 & $<1.03 \mathrm{E} 0$ & pCi/g dry & $1.03 \mathrm{E} 0$ & & $6 / 24 / 08$ & $8 \mathrm{~F} 20002$ & AGG-RRL-001 \\
\hline $14276-65-4$ & Gadolinium-153 & $<8.12 \mathrm{E}-1$ & pCi/g dry & $8.12 \mathrm{E}-1$ & & 6/24/08 & $8 \mathrm{~F} 20002$ & AGG-RRL-001 \\
\hline 15585-10-1 & Europium-154 & $<5.92 \mathrm{E}-1$ & pCi/g dry & 5.92E-1 & & $6 / 24 / 08$ & $8 \mathrm{~F} 20002$ & AGG-RRL-001 \\
\hline 14391-16-3 & Europium-155 & $<8.12 \mathrm{E}-1$ & pCi/g dry & 8.12E-1 & & 6/24/08 & $8 \mathrm{~F} 20002$ & AGG-RRL-001 \\
\hline 13982-78-0 & Mercury-203 & $<3.35 \mathrm{E}-1$ & pCi/g dry & 3.35E-1 & & $6 / 24 / 08$ & $8 \mathrm{~F} 20002$ & AGG-RRL-001 \\
\hline 14913-50-9 & Thallium-208 & $<3.48 \mathrm{E}-1$ & pCi/g dry & $3.48 \mathrm{E}-1$ & & 6/24/08 & 8 F20002 & AGG-RRL-001 \\
\hline 14331-79-4 & Bismuth-210 & $<5.40 \mathrm{E}-1$ & pCi/g dry & $5.40 \mathrm{E}-1$ & & 6/24/08 & $8 \mathrm{~F} 20002$ & AGG-RRL-001 \\
\hline \multirow[t]{3}{*}{$14255-04-0$} & Lead-210 & $<1.04 \mathrm{E} 2$ & pCi/g dry & $1.04 \mathrm{E} 2$ & & $6 / 24 / 08$ & $8 \mathrm{~F} 20002$ & AGG-RRL-001 \\
\hline & Bismuth-211 & $<6.22 \mathrm{E} 0$ & pCi/g dry & $6.22 \mathrm{E} 0$ & & $6 / 24 / 08$ & $8 \mathrm{~F} 20002$ & AGG-RRL-001 \\
\hline & Lead-211 & $<8.50 \mathrm{E} 0$ & pCi/g dry & 8.50E0 & & $6 / 24 / 08$ & $8 \mathrm{~F} 20002$ & AGG-RRL-001 \\
\hline 15092-94-1 & Lead-212 & $<6.22 \mathrm{E}-1$ & pCi/g dry & $6.22 \mathrm{E}-1$ & & 6/24/08 & $8 \mathrm{~F} 20002$ & AGG-RRL-001 \\
\hline 14733-03-0 & Bismuth-214 & $<7.00 \mathrm{E}-1$ & pCi/g dry & 7.00E-1 & & $6 / 24 / 08$ & $8 \mathrm{~F} 20002$ & AGG-RRL-001 \\
\hline $15067-28-4$ & Lead-214 & $<8.47 \mathrm{E}-1$ & pCi/g dry & $8.47 \mathrm{E}-1$ & & 6/24/08 & $8 \mathrm{~F} 20002$ & AGG-RRL-001 \\
\hline $14835-02-0$ & Radon-219 & $<2.74 \mathrm{E} 0$ & pCi/g dry & $2.74 \mathrm{E} 0$ & & 6/24/08 & $8 \mathrm{~F} 20002$ & AGG-RRL-001 \\
\hline 22481-48-7 & Radon-220 & $<2.41 \mathrm{E} 2$ & pCi/g dry & $2.41 \mathrm{E} 2$ & & 6/24/08 & $8 \mathrm{~F} 20002$ & AGG-RRL-001 \\
\hline 28522-20-5 & Radon-221 & $<1.25 \mathrm{E} 0$ & pCi/g dry & $1.25 \mathrm{E} 0$ & & $6 / 24 / 08$ & $8 \mathrm{~F} 20002$ & AGG-RRL-001 \\
\hline 15756-98-6 & Francium-223 & $<9.12 \mathrm{E} 0$ & pCi/g dry & $9.12 \mathrm{E} 0$ & & 6/24/08 & 8 F20002 & AGG-RRL-001 \\
\hline $15623-45-7$ & Radium-223 & $<1.99 \mathrm{E} 0$ & pCi/g dry & $1.99 \mathrm{E} 0$ & & $6 / 24 / 08$ & $8 \mathrm{~F} 20002$ & AGG-RRL-001 \\
\hline 13233-32-4 & Radium-224 & $<8.15 \mathrm{E} 0$ & pCi/g dry & 8.15E0 & & 6/24/08 & $8 \mathrm{~F} 20002$ & AGG-RRL-001 \\
\hline 13982-63-3 & Radium-226 & $<7.77 \mathrm{E} 0$ & pCi/g dry & 7.77E0 & & $6 / 24 / 08$ & $8 \mathrm{~F} 20002$ & AGG-RRL-001 \\
\hline $15623-47-9$ & Thorium-227 & $<2.81 \mathrm{E} 0$ & pCi/g dry & $2.81 \mathrm{E} 0$ & & 6/24/08 & $8 \mathrm{~F} 20002$ & AGG-RRL-001 \\
\hline 14331-83-0 & Actinium-228 & $<1.21 \mathrm{E} 0$ & pCi/g dry & $1.21 \mathrm{E} 0$ & & $6 / 24 / 08$ & $8 \mathrm{~F} 20002$ & AGG-RRL-001 \\
\hline 14274-82-9 & Thorium-228 & $<9.72 \mathrm{E} 1$ & pCi/g dry & $9.72 \mathrm{E} 1$ & & $6 / 24 / 08$ & $8 \mathrm{~F} 20002$ & AGG-RRL-001 \\
\hline $14269-63-7$ & Thorium-230 & $<1.04 \mathrm{E} 2$ & pCi/g dry & $1.04 \mathrm{E} 2$ & & 6/24/08 & $8 \mathrm{~F} 20002$ & AGG-RRL-001 \\
\hline $14331-85-2$ & Protactinium-231 & $<1.12 \mathrm{E} 1$ & pCi/g dry & $1.12 \mathrm{E} 1$ & & $6 / 24 / 08$ & $8 \mathrm{~F} 20002$ & AGG-RRL-001 \\
\hline $14932-40-2$ & Thorium-231 & $<5.71 \mathrm{E} 1$ & pCi/g dry & $5.71 \mathrm{E} 1$ & & 6/24/08 & $8 \mathrm{~F} 20002$ & AGG-RRL-001 \\
\hline 7440-29-1 & Thorium-232 & $<3.48 \mathrm{E} 2$ & pCi/g dry & $3.48 \mathrm{E} 2$ & & $6 / 24 / 08$ & $8 \mathrm{~F} 20002$ & AGG-RRL-001 \\
\hline 13981-14-1 & Protactinium-233 & $<7.02 \mathrm{E}-1$ & pCi/g dry & $7.02 \mathrm{E}-1$ & & 6/24/08 & $8 \mathrm{~F} 20002$ & AGG-RRL-001 \\
\hline \multirow[t]{2}{*}{$15100-28-4$} & Protactinium-234 & $<1.01 \mathrm{E} 0$ & pCi/g dry & $1.01 \mathrm{E} 0$ & & $6 / 24 / 08$ & $8 \mathrm{~F} 20002$ & AGG-RRL-001 \\
\hline & Protactinium-234m & $<3.65 \mathrm{E} 1$ & pCi/g dry & 3.65E1 & & $6 / 24 / 08$ & $8 \mathrm{~F} 20002$ & AGG-RRL-001 \\
\hline 15065-10-8 & Thorium-234 & $<9.51 \mathrm{E} 0$ & pCi/g dry & $9.51 \mathrm{E} 0$ & & $6 / 24 / 08$ & $8 \mathrm{~F} 20002$ & AGG-RRL-001 \\
\hline 15117-96-1 & Uranium 235 & $<2.31 \mathrm{E} 0$ & pCi/g dry & 2.31E0 & & $6 / 24 / 08$ & 8F20002 & AGG-RRL-001 \\
\hline 13994-20-2 & Neptunium-237 & $<2.11 \mathrm{E} 0$ & pCi/g dry & 2.11E0 & & $6 / 24 / 08$ & $8 \mathrm{~F} 20002$ & AGG-RRL-001 \\
\hline 13981-16-3 & Plutonium-238 & $<3.21 \mathrm{E} 3$ & pCi/g dry & $3.21 \mathrm{E} 3$ & & $6 / 24 / 08$ & $8 F 20002$ & AGG-RRL-001 \\
\hline
\end{tabular}




\section{GEA/Soil}

\begin{tabular}{|c|c|c|c|c|c|c|c|c|}
\hline CAS \# & Analyte & Results & Units & MDA & UNC & Analyzed & Batch & Method \\
\hline \multirow[t]{2}{*}{ HEIS No. } & B1VJ61C & \multicolumn{4}{|c|}{ Lab ID: $\quad 0805020-22$} & & & \\
\hline & Uranium 238 & $<4.93 \mathrm{E} 0$ & pCi/g dry & 4.93E0 & & 6/24/08 & 8F20002 & AGG-RRL-001 \\
\hline 15117-48-3 & Plutonium-239 & $<3.88 \mathrm{E} 3$ & pCi/g dry & 3.88E3 & & 6/24/08 & 8F20002 & AGG-RRL-001 \\
\hline 14119-33-6 & Plutonium-240 & $<3.31 \mathrm{E} 3$ & pCi/g dry & 3.31E3 & & 6/24/08 & $8 \mathrm{~F} 20002$ & AGG-RRL-001 \\
\hline $14596-10-2$ & Americium-241 & $<1.70 \mathrm{E} 0$ & pCi/g dry & $1.70 \mathrm{E} 0$ & & $6 / 24 / 08$ & $8 F 20002$ & AGG-RRL-001 \\
\hline 14993-75-0 & Americium-243 & $<5.68 \mathrm{E}-1$ & pCi/g dry & $5.68 \mathrm{E}-1$ & & $6 / 24 / 08$ & $8 \mathrm{~F} 20002$ & AGG-RRL-001 \\
\hline 15757-87-6 & Curium-243 & $<1.01 \mathrm{E} 0$ & pCi/g dry & $1.01 \mathrm{E} 0$ & & 6/24/08 & 8 F20002 & AGG-RRL-001 \\
\hline 15621-76-8 & Curium-245 & $<8.28 \mathrm{E}-1$ & pCi/g dry & $8.28 \mathrm{E}-1$ & & $6 / 24 / 08$ & 8F20002 & AGG-RRL-001 \\
\hline HEIS No. & B1VJ61B & \multicolumn{4}{|c|}{ Lab ID: $\quad$ 0805020-23 } & & & \\
\hline 13966-32-0 & Sodium-22 & $<3.29 \mathrm{E}-1$ & pCi/g dry & $3.29 \mathrm{E}-1$ & & $6 / 24 / 08$ & 8F20002 & AGG-RRL-001 \\
\hline $13966-00-2$ & Potassium-40 & 2.16E1 & pCi/g dry & $2.22 \mathrm{E} 0$ & 1.18 & $6 / 24 / 08$ & 8F20002 & AGG-RRL-001 \\
\hline $14392-02-0$ & Chromium-51 & $<2.95 \mathrm{E} 0$ & pCi/g dry & 2.95E0 & & $6 / 24 / 08$ & $8 \mathrm{~F} 20002$ & AGG-RRL-001 \\
\hline 13966-31-9 & Manganese-54 & $<3.42 \mathrm{E}-1$ & pCi/g dry & $3.42 \mathrm{E}-1$ & & $6 / 24 / 08$ & $8 \mathrm{~F} 20002$ & AGG-RRL-001 \\
\hline $13981-50-5$ & Cobalt-57 & $<3.07 \mathrm{E}-1$ & pCi/g dry & 3.07E-1 & & $6 / 24 / 08$ & $8 \mathrm{~F} 20002$ & AGG-RRL-001 \\
\hline $14596-12-4$ & Iron-59 & $<5.89 \mathrm{E}-1$ & pCi/g dry & $5.89 \mathrm{E}-1$ & & $6 / 24 / 08$ & 8F20002 & AGG-RRL-001 \\
\hline $10198-40-0$ & Cobalt-60 & $<2.99 \mathrm{E}-1$ & pCi/g dry & $2.99 \mathrm{E}-1$ & & 6/24/08 & 8F20002 & AGG-RRL-001 \\
\hline 13982-39-3 & Zinc-65 & $<7.55 \mathrm{E}-1$ & pCi/g dry & 7.55E-1 & & $6 / 24 / 08$ & 8F20002 & AGG-RRL-001 \\
\hline 14265-71-5 & Selenium-75 & $<5.00 \mathrm{E}-1$ & pCi/g dry & $5.00 \mathrm{E}-1$ & & 6/24/08 & $8 \mathrm{~F} 20002$ & AGG-RRL-001 \\
\hline 17056-36-9 & Rubidium-83 & $<7.39 \mathrm{E}-1$ & pCi/g dry & 7.39E-1 & & 6/24/08 & 8F20002 & AGG-RRL-001 \\
\hline 13967-73-2 & Strontium-85 & $<3.48 \mathrm{E}-1$ & pCi/g dry & 3.48E-1 & & $6 / 24 / 08$ & 8F20002 & AGG-RRL-001 \\
\hline 14932-53-7 & Rubidium-86 & $<3.78 \mathrm{E} 0$ & pCi/g dry & 3.78E0 & & $6 / 24 / 08$ & 8 F20002 & AGG-RRL-001 \\
\hline $13982-36-0$ & Yttrium-88 & $<2.48 \mathrm{E}-1$ & pCi/g dry & $2.48 \mathrm{E}-1$ & & $6 / 24 / 08$ & 8F20002 & AGG-RRL-001 \\
\hline 14681-63-1 & Niobium-94 & $<3.01 \mathrm{E}-1$ & pCi/g dry & $3.01 \mathrm{E}-1$ & & $6 / 24 / 08$ & 8F20002 & AGG-RRL-001 \\
\hline \multirow[t]{3}{*}{ 13967-76-5 } & Niobium-95 & $<2.95 \mathrm{E}-1$ & pCi/g dry & 2.95E-1 & & 6/24/08 & 8F20002 & AGG-RRL-001 \\
\hline & Niobium-95m & $<1.32 \mathrm{E} 0$ & pCi/g dry & $1.32 \mathrm{E} 0$ & & 6/24/08 & 8F20002 & AGG-RRL-001 \\
\hline & Technetium-95m & $<4.75 \mathrm{E}-1$ & pCi/g dry & 4.75E-1 & & $6 / 24 / 08$ & 8F20002 & AGG-RRL-001 \\
\hline \multirow[t]{2}{*}{ 13967-71-0 } & Zirconium-95 & $<5.22 \mathrm{E}-1$ & pCi/g dry & $5.22 \mathrm{E}-1$ & & 6/24/08 & 8F20002 & AGG-RRL-001 \\
\hline & Technetium-99m & $<3.12 \mathrm{E}-1$ & pCi/g dry & $3.12 \mathrm{E}-1$ & & 6/24/08 & 8F20002 & AGG-RRL-001 \\
\hline 13968-53-1 & Ruthenium-103 & $<3.34 \mathrm{E}-1$ & pCi/g dry & 3.34E-1 & & 6/24/08 & $8 F 20002$ & AGG-RRL-001 \\
\hline 13967-48-1 & Ruthenium-106 & $<3.53 \mathrm{E} 0$ & pCi/g dry & 3.53E0 & & 6/24/08 & 8F20002 & AGG-RRL-001 \\
\hline 14391-65-2 & Silver-108m & $<3.07 \mathrm{E}-1$ & pCi/g dry & 3.07E-1 & & 6/24/08 & $8 \mathrm{~F} 20002$ & AGG-RRL-001 \\
\hline \multirow[t]{2}{*}{ 14109-32-1 } & Cadmium-109 & $<8.78 \mathrm{E} 0$ & pCi/g dry & 8.78E0 & & 6/24/08 & 8F20002 & AGG-RRL-001 \\
\hline & Silver-110 & $<3.21 \mathrm{E}-1$ & pCi/g dry & $3.21 \mathrm{E}-1$ & & 6/24/08 & $8 \mathrm{~F} 20002$ & AGG-RRL-001 \\
\hline 14391-76-5 & Silver-110m & $<3.22 \mathrm{E}-1$ & pCi/g dry & $3.22 \mathrm{E}-1$ & & 6/24/08 & 8F20002 & AGG-RRL-001 \\
\hline 13966-06-8 & Tin-113 & $<4.39 \mathrm{E}-1$ & pCi/g dry & 4.39E-1 & & $6 / 24 / 08$ & 8F20002 & AGG-RRL-001 \\
\hline $14683-10-4$ & Antimony-124 & $<3.87 \mathrm{E}-1$ & pCi/g dry & $3.87 \mathrm{E}-1$ & & 6/24/08 & 8F20002 & AGG-RRL-001 \\
\hline $14234-35-6$ & Antimony-125 & $<9.48 \mathrm{E}-1$ & pCi/g dry & $9.48 \mathrm{E}-1$ & & 6/24/08 & 8F20002 & AGG-RRL-001 \\
\hline 15756-32-8 & Antimony-126 & $<3.08 \mathrm{E}-1$ & pCi/g dry & $3.08 \mathrm{E}-1$ & & 6/24/08 & 8F20002 & AGG-RRL-001 \\
\hline $15832-50-5$ & Tin-126 & $<7.17 \mathrm{E}-1$ & pCi/g dry & 7.17E-1 & & 6/24/08 & 8F20002 & AGG-RRL-001 \\
\hline 10043-66-0 & Iodine-131 & $<3.73 \mathrm{E}-1$ & pCi/g dry & 3.73E-1 & & 6/24/08 & 8F20002 & AGG-RRL-001 \\
\hline 13981-41-4 & Barium-133 & $<5.36 \mathrm{E}-1$ & pCi/g dry & 5.36E-1 & & $6 / 24 / 08$ & 8F20002 & AGG-RRL-001 \\
\hline 13967-70-9 & Cesium-134 & $<3.49 \mathrm{E}-1$ & pCi/g dry & $3.49 \mathrm{E}-1$ & & 6/24/08 & $8 F 20002$ & AGG-RRL-001 \\
\hline $10045-97-3$ & Cesium-137 & $<3.58 \mathrm{E}-1$ & pCi/g dry & $3.58 \mathrm{E}-1$ & & 6/24/08 & 8F20002 & AGG-RRL-001 \\
\hline 13982-30-4 & Cerium-139 & $<3.45 \mathrm{E}-1$ & pCi/g dry & $3.45 \mathrm{E}-1$ & & 6/24/08 & 8F20002 & AGG-RRL-001 \\
\hline 14762-78-8 & Cerium-144 & $<2.53 \mathrm{E} 0$ & pCi/g dry & 2.53E0 & & 6/24/08 & 8F20002 & AGG-RRL-001 \\
\hline 14683-23-9 & Europium-152 & $<1.28 \mathrm{E} 0$ & pCi/g dry & $1.28 \mathrm{E} 0$ & & 6/24/08 & 8F20002 & AGG-RRL-001 \\
\hline $14276-65-4$ & Gadolinium-153 & $<9.67 \mathrm{E}-1$ & pCi/g dry & $9.67 \mathrm{E}-1$ & & 6/24/08 & 8F20002 & AGG-RRL-001 \\
\hline 15585-10-1 & Europium-154 & $<6.53 \mathrm{E}-1$ & pCi/g dry & $6.53 \mathrm{E}-1$ & & 6/24/08 & $8 \mathrm{~F} 20002$ & AGG-RRL-001 \\
\hline 14391-16-3 & Europium-155 & $<1.01 \mathrm{E} 0$ & pCi/g dry & $1.01 \mathrm{E} 0$ & & 6/24/08 & 8F20002 & AGG-RRL-001 \\
\hline
\end{tabular}


GEA/Soil

\begin{tabular}{|c|c|c|c|c|c|c|c|c|}
\hline CAS \# & Analyte & Results & Units & MDA & UNC & Analyzed & Batch & Method \\
\hline HEIS No. & B1VJ61B & & b ID: & $5020-23$ & & & & \\
\hline 13982-78-0 & Mercury-203 & $<3.82 \mathrm{E}-1$ & pCi/g dry & 3.82E-1 & & $6 / 24 / 08$ & 8F20002 & AGG-RRL-001 \\
\hline 14913-50-9 & Thallium-208 & $<3.87 E-1$ & pCi/g dry & $3.87 \mathrm{E}-1$ & & $6 / 24 / 08$ & $8 \mathrm{~F} 20002$ & AGG-RRL-001 \\
\hline 14331-79-4 & Bismuth-210 & $<5.96 \mathrm{E}-1$ & pCi/g dry & 5.96E-1 & & $6 / 24 / 08$ & $8 \mathrm{~F} 20002$ & AGG-RRL-001 \\
\hline \multirow[t]{3}{*}{ 14255-04-0 } & Lead-210 & $<4.28 \mathrm{E} 1$ & pCi/g dry & $4.28 \mathrm{E} 1$ & & $6 / 24 / 08$ & $8 \mathrm{~F} 20002$ & AGG-RRL-001 \\
\hline & Bismuth-211 & $<7.03 \mathrm{E} 0$ & pCi/g dry & 7.03E0 & & $6 / 24 / 08$ & $8 \mathrm{~F} 20002$ & AGG-RRL-001 \\
\hline & Lead-211 & $<9.61 \mathrm{E} 0$ & pCi/g dry & $9.61 \mathrm{E} 0$ & & $6 / 24 / 08$ & $8 \mathrm{~F} 20002$ & AGG-RRL-001 \\
\hline 15092-94-1 & Lead-212 & $<7.67 \mathrm{E}-1$ & pCi/g dry & 7.67E-1 & & $6 / 24 / 08$ & $8 \mathrm{~F} 20002$ & AGG-RRL-001 \\
\hline 14733-03-0 & Bismuth-214 & $<8.19 \mathrm{E}-1$ & pCi/g dry & 8.19E-1 & & $6 / 24 / 08$ & $8 \mathrm{~F} 20002$ & AGG-RRL-001 \\
\hline $15067-28-4$ & Lead-214 & $<8.82 \mathrm{E}-1$ & pCi/g dry & 8.82E-1 & & $6 / 24 / 08$ & $8 \mathrm{~F} 20002$ & AGG-RRL-001 \\
\hline $14835-02-0$ & Radon-219 & $<2.99 \mathrm{E} 0$ & pCi/g dry & $2.99 \mathrm{E} 0$ & & 6/24/08 & $8 \mathrm{~F} 20002$ & AGG-RRL-001 \\
\hline 22481-48-7 & Radon-220 & $<2.89 \mathrm{E} 2$ & pCi/g dry & $2.89 \mathrm{E} 2$ & & $6 / 24 / 08$ & $8 \mathrm{~F} 20002$ & AGG-RRL-001 \\
\hline 28522-20-5 & Radon-221 & $<1.42 \mathrm{E} 0$ & pCi/g dry & $1.42 \mathrm{E} 0$ & & $6 / 24 / 08$ & $8 \mathrm{~F} 20002$ & AGG-RRL-001 \\
\hline 15756-98-6 & Francium-223 & $<9.75 \mathrm{E} 0$ & pCi/g dry & $9.75 \mathrm{E} 0$ & & 6/24/08 & $8 \mathrm{~F} 20002$ & AGG-RRL-001 \\
\hline $15623-45-7$ & Radium-223 & $<2.19 \mathrm{E} 0$ & pCi/g dry & 2.19E0 & & $6 / 24 / 08$ & $8 \mathrm{~F} 20002$ & AGG-RRL-001 \\
\hline 13233-32-4 & Radium-224 & $<8.27 \mathrm{E} 0$ & pCi/g dry & 8.27E0 & & 6/24/08 & $8 \mathrm{~F} 20002$ & AGG-RRL-001 \\
\hline 13982-63-3 & Radium-226 & $<8.77 \mathrm{E} 0$ & pCi/g dry & 8.77E0 & & $6 / 24 / 08$ & $8 \mathrm{~F} 20002$ & AGG-RRL-001 \\
\hline $15623-47-9$ & Thorium-227 & $<2.96 \mathrm{E} 0$ & pCi/g dry & 2.96E0 & & $6 / 24 / 08$ & $8 \mathrm{~F} 20002$ & AGG-RRL-001 \\
\hline 14331-83-0 & Actinium-228 & $<1.39 \mathrm{E} 0$ & pCi/g dry & $1.39 \mathrm{E} 0$ & & 6/24/08 & $8 \mathrm{~F} 20002$ & AGG-RRL-001 \\
\hline $14274-82-9$ & Thorium-228 & $<1.12 \mathrm{E} 2$ & pCi/g dry & $1.12 \mathrm{E} 2$ & & $6 / 24 / 08$ & $8 \mathrm{~F} 20002$ & AGG-RRL-001 \\
\hline $14269-63-7$ & Thorium-230 & $<1.16 \mathrm{E} 2$ & pCi/g dry & 1.16E2 & & $6 / 24 / 08$ & $8 \mathrm{~F} 20002$ & AGG-RRL-001 \\
\hline $14331-85-2$ & Protactinium-231 & $<1.31 \mathrm{E} 1$ & pCi/g dry & $1.31 \mathrm{E} 1$ & & $6 / 24 / 08$ & $8 \mathrm{~F} 20002$ & AGG-RRL-001 \\
\hline $14932-40-2$ & Thorium-231 & $<6.85 \mathrm{E} 1$ & pCi/g dry & $6.85 \mathrm{E} 1$ & & $6 / 24 / 08$ & $8 \mathrm{~F} 20002$ & AGG-RRL-001 \\
\hline $7440-29-1$ & Thorium-232 & $<2.92 \mathrm{E} 2$ & pCi/g dry & $2.92 \mathrm{E} 2$ & & $6 / 24 / 08$ & $8 \mathrm{~F} 20002$ & AGG-RRL-001 \\
\hline 13981-14-1 & Protactinium-233 & $<8.04 \mathrm{E}-1$ & pCi/g dry & 8.04E-1 & & $6 / 24 / 08$ & $8 \mathrm{~F} 20002$ & AGG-RRL-001 \\
\hline \multirow[t]{2}{*}{$15100-28-4$} & Protactinium-234 & $<1.26 \mathrm{E} 0$ & pCi/g dry & $1.26 \mathrm{E} 0$ & & 6/24/08 & $8 \mathrm{~F} 20002$ & AGG-RRL-001 \\
\hline & Protactinium-234m & $<3.82 \mathrm{E} 1$ & pCi/g dry & 3.82E1 & & $6 / 24 / 08$ & $8 \mathrm{~F} 20002$ & AGG-RRL-001 \\
\hline $15065-10-8$ & Thorium-234 & $<1.02 \mathrm{E} 1$ & pCi/g dry & $1.02 \mathrm{E} 1$ & & $6 / 24 / 08$ & $8 \mathrm{~F} 20002$ & AGG-RRL-001 \\
\hline 15117-96-1 & Uranium 235 & $<2.62 \mathrm{E} 0$ & pCi/g dry & 2.62E0 & & 6/24/08 & $8 \mathrm{~F} 20002$ & AGG-RRL-001 \\
\hline $13994-20-2$ & Neptunium-237 & $<2.64 \mathrm{E} 0$ & pCi/g dry & 2.64E0 & & 6/24/08 & $8 \mathrm{~F} 20002$ & AGG-RRL-001 \\
\hline \multirow[t]{2}{*}{ 13981-16-3 } & Plutonium-238 & $<3.96 \mathrm{E} 3$ & pCi/g dry & 3.96E3 & & $6 / 24 / 08$ & $8 \mathrm{~F} 20002$ & AGG-RRL-001 \\
\hline & Uranium 238 & $<5.64 \mathrm{E} 0$ & pCi/g dry & $5.64 \mathrm{E} 0$ & & $6 / 24 / 08$ & $8 \mathrm{~F} 20002$ & AGG-RRL-001 \\
\hline $15117-48-3$ & Plutonium-239 & $<4.04 \mathrm{E} 3$ & pCi/g dry & $4.04 \mathrm{E} 3$ & & 6/24/08 & $8 \mathrm{~F} 20002$ & AGG-RRL-001 \\
\hline $14119-33-6$ & Plutonium-240 & $<3.94 \mathrm{E} 3$ & pCi/g dry & 3.94E3 & & 6/24/08 & $8 \mathrm{~F} 20002$ & AGG-RRL-001 \\
\hline $14596-10-2$ & Americium-241 & $<1.50 \mathrm{E} 0$ & pCi/g dry & $1.50 \mathrm{E} 0$ & & 6/24/08 & $8 \mathrm{~F} 20002$ & AGG-RRL-001 \\
\hline 14993-75-0 & Americium-243 & $<6.43 \mathrm{E}-1$ & pCi/g dry & 6.43E-1 & & $6 / 24 / 08$ & $8 \mathrm{~F} 20002$ & AGG-RRL-001 \\
\hline $15757-87-6$ & Curium-243 & $<1.21 \mathrm{E} 0$ & pCi/g dry & $1.21 \mathrm{E} 0$ & & 6/24/08 & $8 \mathrm{~F} 20002$ & AGG-RRL-001 \\
\hline 15621-76-8 & Curium-245 & $<9.94 \mathrm{E}-1$ & pCi/g dry & $9.94 \mathrm{E}-1$ & & 6/24/08 & $8 F 20002$ & AGG-RRL-001 \\
\hline HEIS No. & B1VJ61A & \multicolumn{3}{|c|}{ Lab ID: $\quad 0805020-24$} & & & & \\
\hline 13966-32-0 & Sodium-22 & $<3.39 \mathrm{E}-1$ & pCi/g dry & 3.39E-1 & & $6 / 24 / 08$ & $8 \mathrm{~F} 20002$ & AGG-RRL-001 \\
\hline $13966-00-2$ & Potassium-40 & 1.93E1 & pCi/g dry & 3.37E0 & 1.24 & 6/24/08 & $8 \mathrm{~F} 20002$ & AGG-RRL-001 \\
\hline $14392-02-0$ & Chromium-51 & $<2.93 \mathrm{E} 0$ & pCi/g dry & 2.93E0 & & $6 / 24 / 08$ & $8 \mathrm{~F} 20002$ & AGG-RRL-001 \\
\hline 13966-31-9 & Manganese-54 & $<3.01 \mathrm{E}-1$ & pCi/g dry & $3.01 \mathrm{E}-1$ & & $6 / 24 / 08$ & $8 \mathrm{~F} 20002$ & AGG-RRL-001 \\
\hline 13981-50-5 & Cobalt-57 & $<3.11 \mathrm{E}-1$ & pCi/g dry & $3.11 \mathrm{E}-1$ & & $6 / 24 / 08$ & $8 \mathrm{~F} 20002$ & AGG-RRL-001 \\
\hline $14596-12-4$ & Iron-59 & $<5.66 \mathrm{E}-1$ & pCi/g dry & $5.66 \mathrm{E}-1$ & & $6 / 24 / 08$ & $8 \mathrm{~F} 20002$ & AGG-RRL-001 \\
\hline $10198-40-0$ & Cobalt-60 & $<3.27 \mathrm{E}-1$ & pCi/g dry & $3.27 \mathrm{E}-1$ & & 6/24/08 & $8 \mathrm{~F} 20002$ & AGG-RRL-001 \\
\hline 13982-39-3 & Zinc-65 & $<7.12 \mathrm{E}-1$ & pCi/g dry & $7.12 \mathrm{E}-1$ & & $6 / 24 / 08$ & $8 \mathrm{~F} 20002$ & AGG-RRL-001 \\
\hline 14265-71-5 & Selenium-75 & $<4.99 \mathrm{E}-1$ & pCi/g dry & 4.99E-1 & & $6 / 24 / 08$ & $8 \mathrm{~F} 20002$ & AGG-RRL-001 \\
\hline 17056-36-9 & Rubidium-83 & $<7.65 \mathrm{E}-1$ & pCi/g dry & 7.65E-1 & & $6 / 24 / 08$ & 8F20002 & AGG-RRL-001 \\
\hline
\end{tabular}


GEA/Soil

\begin{tabular}{|c|c|c|c|c|c|c|c|c|}
\hline CAS \# & Analyte & Results & Units & MDA & UNC & Analyzed & Batch & Method \\
\hline HEIS No. & B1VJ61A & & ab ID: & $5020-24$ & & & & \\
\hline 13967-73-2 & Strontium-85 & $<3.48 \mathrm{E}-1$ & pCi/g dry & $3.48 \mathrm{E}-1$ & & 6/24/08 & 8F20002 & AGG-RRL-001 \\
\hline 14932-53-7 & Rubidium-86 & $<3.75 \mathrm{E} 0$ & pCi/g dry & 3.75E0 & & 6/24/08 & $8 \mathrm{~F} 20002$ & AGG-RRL-001 \\
\hline 13982-36-0 & Yttrium-88 & $<2.30 \mathrm{E}-1$ & pCi/g dry & $2.30 \mathrm{E}-1$ & & $6 / 24 / 08$ & $8 \mathrm{~F} 20002$ & AGG-RRL-001 \\
\hline 14681-63-1 & Niobium-94 & $<3.11 \mathrm{E}-1$ & pCi/g dry & $3.11 \mathrm{E}-1$ & & 6/24/08 & $8 \mathrm{~F} 20002$ & AGG-RRL-001 \\
\hline \multirow[t]{3}{*}{ 13967-76-5 } & Niobium-95 & $<3.06 \mathrm{E}-1$ & pCi/g dry & 3.06E-1 & & $6 / 24 / 08$ & $8 \mathrm{~F} 20002$ & AGG-RRL-001 \\
\hline & Niobium-95m & $<1.37 \mathrm{E} 0$ & pCi/g dry & 1.37E0 & & 6/24/08 & 8 F20002 & AGG-RRL-001 \\
\hline & Technetium-95m & $<4.66 \mathrm{E}-1$ & pCi/g dry & 4.66E-1 & & 6/24/08 & $8 \mathrm{~F} 20002$ & AGG-RRL-001 \\
\hline \multirow[t]{2}{*}{ 13967-71-0 } & Zirconium-95 & $<5.32 \mathrm{E}-1$ & pCi/g dry & $5.32 \mathrm{E}-1$ & & 6/24/08 & $8 \mathrm{~F} 20002$ & AGG-RRL-001 \\
\hline & Technetium-99m & $<3.12 \mathrm{E}-1$ & pCi/g dry & $3.12 \mathrm{E}-1$ & & 6/24/08 & $8 \mathrm{~F} 20002$ & AGG-RRL-001 \\
\hline 13968-53-1 & Ruthenium-103 & $<3.35 \mathrm{E}-1$ & pCi/g dry & 3.35E-1 & & $6 / 24 / 08$ & $8 \mathrm{~F} 20002$ & AGG-RRL-001 \\
\hline 13967-48-1 & Ruthenium-106 & $<3.55 \mathrm{E} 0$ & pCi/g dry & $3.55 \mathrm{E} 0$ & & 6/24/08 & $8 \mathrm{~F} 20002$ & AGG-RRL-001 \\
\hline 14391-65-2 & Silver-108m & $<3.03 \mathrm{E}-1$ & pCi/g dry & 3.03E-1 & & $6 / 24 / 08$ & $8 \mathrm{~F} 20002$ & AGG-RRL-001 \\
\hline \multirow[t]{2}{*}{ 14109-32-1 } & Cadmium-109 & $<8.67 \mathrm{E} 0$ & pCi/g dry & 8.67Е0 & & 6/24/08 & $8 \mathrm{~F} 20002$ & AGG-RRL-001 \\
\hline & Silver-110 & $<3.25 \mathrm{E}-1$ & pCi/g dry & $3.25 \mathrm{E}-1$ & & $6 / 24 / 08$ & $8 \mathrm{~F} 20002$ & AGG-RRL-001 \\
\hline 14391-76-5 & Silver-110m & $<3.26 \mathrm{E}-1$ & pCi/g dry & $3.26 \mathrm{E}-1$ & & $6 / 24 / 08$ & $8 \mathrm{~F} 20002$ & AGG-RRL-001 \\
\hline 13966-06-8 & Tin-113 & $<4.31 \mathrm{E}-1$ & pCi/g dry & $4.31 \mathrm{E}-1$ & & $6 / 24 / 08$ & $8 \mathrm{~F} 20002$ & AGG-RRL-001 \\
\hline $14683-10-4$ & Antimony-124 & $<3.77 \mathrm{E}-1$ & pCi/g dry & 3.77E-1 & & 6/24/08 & $8 \mathrm{~F} 20002$ & AGG-RRL-001 \\
\hline 14234-35-6 & Antimony-125 & $<9.40 \mathrm{E}-1$ & pCi/g dry & $9.40 \mathrm{E}-1$ & & $6 / 24 / 08$ & $8 \mathrm{~F} 20002$ & AGG-RRL-001 \\
\hline 15756-32-8 & Antimony-126 & $<3.12 \mathrm{E}-1$ & pCi/g dry & $3.12 \mathrm{E}-1$ & & 6/24/08 & $8 \mathrm{~F} 20002$ & AGG-RRL-001 \\
\hline 15832-50-5 & Tin-126 & $<7.11 \mathrm{E}-1$ & pCi/g dry & 7.11E-1 & & $6 / 24 / 08$ & $8 \mathrm{~F} 20002$ & AGG-RRL-001 \\
\hline 10043-66-0 & Iodine-131 & $<3.77 \mathrm{E}-1$ & pCi/g dry & 3.77E-1 & & $6 / 24 / 08$ & $8 \mathrm{~F} 20002$ & AGG-RRL-001 \\
\hline 13981-41-4 & Barium-133 & $<5.28 \mathrm{E}-1$ & pCi/g dry & $5.28 \mathrm{E}-1$ & & $6 / 24 / 08$ & $8 \mathrm{~F} 20002$ & AGG-RRL-001 \\
\hline 13967-70-9 & Cesium-134 & $<3.65 \mathrm{E}-1$ & pCi/g dry & 3.65E-1 & & $6 / 24 / 08$ & $8 \mathrm{~F} 20002$ & AGG-RRL-001 \\
\hline $10045-97-3$ & Cesium-137 & $<3.69 \mathrm{E}-1$ & pCi/g dry & 3.69E-1 & & 6/24/08 & $8 \mathrm{~F} 20002$ & AGG-RRL-001 \\
\hline 13982-30-4 & Cerium-139 & $<3.55 \mathrm{E}-1$ & pCi/g dry & 3.55E-1 & & $6 / 24 / 08$ & $8 \mathrm{~F} 20002$ & AGG-RRL-001 \\
\hline 14762-78-8 & Cerium-144 & $<2.53 \mathrm{E} 0$ & pCi/g dry & 2.53E0 & & 6/24/08 & $8 \mathrm{~F} 20002$ & AGG-RRL-001 \\
\hline 14683-23-9 & Europium-152 & $<1.24 \mathrm{E} 0$ & pCi/g dry & $1.24 \mathrm{E} 0$ & & $6 / 24 / 08$ & $8 \mathrm{~F} 20002$ & AGG-RRL-001 \\
\hline $14276-65-4$ & Gadolinium-153 & $<9.59 \mathrm{E}-1$ & pCi/g dry & $9.59 \mathrm{E}-1$ & & 6/24/08 & $8 \mathrm{~F} 20002$ & AGG-RRL-001 \\
\hline 15585-10-1 & Europium-154 & $<6.57 \mathrm{E}-1$ & pCi/g dry & $6.57 \mathrm{E}-1$ & & 6/24/08 & $8 \mathrm{~F} 20002$ & AGG-RRL-001 \\
\hline 14391-16-3 & Europium-155 & $<1.01 \mathrm{E} 0$ & pCi/g dry & $1.01 \mathrm{E} 0$ & & 6/24/08 & $8 \mathrm{~F} 20002$ & AGG-RRL-001 \\
\hline 13982-78-0 & Mercury-203 & $<3.80 \mathrm{E}-1$ & pCi/g dry & 3.80E-1 & & $6 / 24 / 08$ & $8 \mathrm{~F} 20002$ & AGG-RRL-001 \\
\hline 14913-50-9 & Thallium-208 & $<3.91 \mathrm{E}-1$ & pCi/g dry & $3.91 \mathrm{E}-1$ & & $6 / 24 / 08$ & $8 \mathrm{~F} 20002$ & AGG-RRL-001 \\
\hline 14331-79-4 & Bismuth-210 & $<5.97 \mathrm{E}-1$ & pCi/g dry & 5.97E-1 & & $6 / 24 / 08$ & $8 \mathrm{~F} 20002$ & AGG-RRL-001 \\
\hline \multirow[t]{3}{*}{$14255-04-0$} & Lead-210 & $<4.21 \mathrm{E} 1$ & pCi/g dry & $4.21 \mathrm{E} 1$ & & 6/24/08 & $8 \mathrm{~F} 20002$ & AGG-RRL-001 \\
\hline & Bismuth-211 & $<6.93 \mathrm{E} 0$ & pCi/g dry & 6.93E0 & & $6 / 24 / 08$ & $8 \mathrm{~F} 20002$ & AGG-RRL-001 \\
\hline & Lead-211 & $<9.48 \mathrm{E} 0$ & pCi/g dry & $9.48 \mathrm{E} 0$ & & 6/24/08 & $8 \mathrm{~F} 20002$ & AGG-RRL-001 \\
\hline 15092-94-1 & Lead-212 & $<7.77 \mathrm{E}-1$ & pCi/g dry & 7.77E-1 & & $6 / 24 / 08$ & $8 \mathrm{~F} 20002$ & AGG-RRL-001 \\
\hline 14733-03-0 & Bismuth-214 & $<8.04 \mathrm{E}-1$ & pCi/g dry & 8.04E-1 & & $6 / 24 / 08$ & $8 \mathrm{~F} 20002$ & AGG-RRL-001 \\
\hline $15067-28-4$ & Lead-214 & $<8.55 \mathrm{E}-1$ & pCi/g dry & 8.55E-1 & & 6/24/08 & 8F20002 & AGG-RRL-001 \\
\hline $14835-02-0$ & Radon-219 & $<2.99 \mathrm{E} 0$ & pCi/g dry & $2.99 \mathrm{E} 0$ & & $6 / 24 / 08$ & $8 \mathrm{~F} 20002$ & AGG-RRL-001 \\
\hline 22481-48-7 & Radon-220 & $<2.82 \mathrm{E} 2$ & pCi/g dry & 2.82E2 & & 6/24/08 & $8 \mathrm{~F} 20002$ & AGG-RRL-001 \\
\hline 28522-20-5 & Radon-221 & $<1.41 \mathrm{E} 0$ & pCi/g dry & $1.41 \mathrm{E} 0$ & & $6 / 24 / 08$ & $8 \mathrm{~F} 20002$ & AGG-RRL-001 \\
\hline 15756-98-6 & Francium-223 & $<1.00 \mathrm{E} 1$ & pCi/g dry & $1.00 \mathrm{E} 1$ & & 6/24/08 & $8 \mathrm{~F} 20002$ & AGG-RRL-001 \\
\hline $15623-45-7$ & Radium-223 & $<2.18 \mathrm{E} 0$ & pCi/g dry & 2.18E0 & & $6 / 24 / 08$ & $8 \mathrm{~F} 20002$ & AGG-RRL-001 \\
\hline 13233-32-4 & Radium-224 & $<8.47 \mathrm{E} 0$ & pCi/g dry & 8.47E0 & & 6/24/08 & $8 \mathrm{~F} 20002$ & AGG-RRL-001 \\
\hline 13982-63-3 & Radium-226 & $<8.67 \mathrm{E} 0$ & pCi/g dry & 8.67E0 & & 6/24/08 & $8 \mathrm{~F} 20002$ & AGG-RRL-001 \\
\hline $15623-47-9$ & Thorium-227 & $<3.05 \mathrm{E} 0$ & pCi/g dry & 3.05E0 & & 6/24/08 & 8F20002 & AGG-RRL-001 \\
\hline 14331-83-0 & Actinium-228 & $<1.32 \mathrm{E} 0$ & pCi/g dry & $1.32 \mathrm{E} 0$ & & 6/24/08 & $8 F 20002$ & AGG-RRL-001 \\
\hline 14274-82-9 & Thorium-228 & $<1.12 \mathrm{E} 2$ & pCi/g dry & $1.12 \mathrm{E} 2$ & & 6/24/08 & 8F20002 & AGG-RRL-001 \\
\hline
\end{tabular}


GEA/Soil

\begin{tabular}{|c|c|c|c|c|c|c|c|c|}
\hline CAS \# & Analyte & Results & Units & MDA & UNC & Analyzed & Batch & Method \\
\hline HEIS No. & B1VJ61A & & ab ID: & $5020-24$ & & & & \\
\hline 14269-63-7 & Thorium-230 & $<1.13 \mathrm{E} 2$ & pCi/g dry & 1.13E2 & & $6 / 24 / 08$ & 8F20002 & AGG-RRL-001 \\
\hline 14331-85-2 & Protactinium-231 & $<1.32 \mathrm{E} 1$ & pCi/g dry & $1.32 \mathrm{E} 1$ & & $6 / 24 / 08$ & 8F20002 & AGG-RRL-001 \\
\hline $14932-40-2$ & Thorium-231 & $<6.87 \mathrm{E} 1$ & pCi/g dry & 6.87E1 & & $6 / 24 / 08$ & 8F20002 & AGG-RRL-001 \\
\hline 7440-29-1 & Thorium-232 & $<2.85 \mathrm{E} 2$ & pCi/g dry & 2.85E2 & & $6 / 24 / 08$ & 8F20002 & AGG-RRL-001 \\
\hline 13981-14-1 & Protactinium-233 & $<8.10 \mathrm{E}-1$ & pCi/g dry & $8.10 \mathrm{E}-1$ & & $6 / 24 / 08$ & 8F20002 & AGG-RRL-001 \\
\hline \multirow[t]{2}{*}{$15100-28-4$} & Protactinium-234 & $<1.25 \mathrm{E} 0$ & pCi/g dry & $1.25 \mathrm{E} 0$ & & $6 / 24 / 08$ & 8F20002 & AGG-RRL-001 \\
\hline & Protactinium-234m & $<3.46 \mathrm{E} 1$ & pCi/g dry & $3.46 \mathrm{E} 1$ & & $6 / 24 / 08$ & 8F20002 & AGG-RRL-001 \\
\hline 15065-10-8 & Thorium-234 & $<9.84 \mathrm{E} 0$ & pCi/g dry & $9.84 \mathrm{E} 0$ & & $6 / 24 / 08$ & $8 \mathrm{~F} 20002$ & AGG-RRL-001 \\
\hline 15117-96-1 & Uranium 235 & $<2.62 \mathrm{E} 0$ & pCi/g dry & 2.62E0 & & $6 / 24 / 08$ & 8F20002 & AGG-RRL-001 \\
\hline 13994-20-2 & Neptunium-237 & $<2.63 \mathrm{E} 0$ & pCi/g dry & 2.63E0 & & $6 / 24 / 08$ & $8 F 20002$ & AGG-RRL-001 \\
\hline \multirow[t]{2}{*}{ 13981-16-3 } & Plutonium-238 & $<3.93 \mathrm{E} 3$ & pCi/g dry & 3.93E3 & & $6 / 24 / 08$ & 8F20002 & AGG-RRL-001 \\
\hline & Uranium 238 & $<5.54 \mathrm{E} 0$ & pCi/g dry & $5.54 \mathrm{E} 0$ & & $6 / 24 / 08$ & $8 \mathrm{~F} 20002$ & AGG-RRL-001 \\
\hline 15117-48-3 & Plutonium-239 & $<3.99 \mathrm{E} 3$ & pCi/g dry & 3.99E3 & & $6 / 24 / 08$ & 8F20002 & AGG-RRL-001 \\
\hline 14119-33-6 & Plutonium-240 & $<3.98 \mathrm{E} 3$ & pCi/g dry & $3.98 \mathrm{E} 3$ & & 6/24/08 & 8F20002 & AGG-RRL-001 \\
\hline $14596-10-2$ & Americium-241 & $<1.45 \mathrm{E} 0$ & pCi/g dry & $1.45 \mathrm{E} 0$ & & $6 / 24 / 08$ & 8F20002 & AGG-RRL-001 \\
\hline 14993-75-0 & Americium-243 & $<6.27 \mathrm{E}-1$ & pCi/g dry & $6.27 \mathrm{E}-1$ & & $6 / 24 / 08$ & 8F20002 & AGG-RRL-001 \\
\hline 15757-87-6 & Curium-243 & $<1.22 \mathrm{E} 0$ & pCi/g dry & $1.22 \mathrm{E} 0$ & & $6 / 24 / 08$ & 8F20002 & AGG-RRL-001 \\
\hline 15621-76-8 & Curium-245 & $<9.99 \mathrm{E}-1$ & pCi/g dry & $9.99 \mathrm{E}-1$ & & $6 / 24 / 08$ & $8 F 20002$ & AGG-RRL-001 \\
\hline HEIS No. & B1VJ62C & \multicolumn{3}{|c|}{ Lab ID: $\quad$ 0805020-26 } & & & & \\
\hline 13966-32-0 & Sodium-22 & $<3.33 \mathrm{E}-1$ & pCi/g dry & 3.33E-1 & & $6 / 24 / 08$ & $8 \mathrm{~F} 20002$ & AGG-RRL-001 \\
\hline 13966-00-2 & Potassium-40 & 1.91E1 & pCi/g dry & 2.99E0 & 1.21 & $6 / 24 / 08$ & 8F20002 & AGG-RRL-001 \\
\hline $14392-02-0$ & Chromium-51 & $<2.69 \mathrm{E} 0$ & pCi/g dry & 2.69E0 & & $6 / 24 / 08$ & 8F20002 & AGG-RRL-001 \\
\hline 13966-31-9 & Manganese-54 & $<2.92 \mathrm{E}-1$ & pCi/g dry & $2.92 \mathrm{E}-1$ & & $6 / 24 / 08$ & 8F20002 & AGG-RRL-001 \\
\hline $13981-50-5$ & Cobalt-57 & $<2.97 \mathrm{E}-1$ & pCi/g dry & 2.97E-1 & & $6 / 24 / 08$ & 8F20002 & AGG-RRL-001 \\
\hline $14596-12-4$ & Iron-59 & $<5.10 \mathrm{E}-1$ & pCi/g dry & $5.10 \mathrm{E}-1$ & & $6 / 24 / 08$ & 8F20002 & AGG-RRL-001 \\
\hline $10198-40-0$ & Cobalt-60 & $<2.74 \mathrm{E}-1$ & pCi/g dry & $2.74 \mathrm{E}-1$ & & $6 / 24 / 08$ & 8F20002 & AGG-RRL-001 \\
\hline 13982-39-3 & Zinc-65 & $<6.22 \mathrm{E}-1$ & pCi/g dry & $6.22 \mathrm{E}-1$ & & $6 / 24 / 08$ & 8F20002 & AGG-RRL-001 \\
\hline 14265-71-5 & Selenium-75 & $<4.77 \mathrm{E}-1$ & pCi/g dry & 4.77E-1 & & $6 / 24 / 08$ & 8F20002 & AGG-RRL-001 \\
\hline 17056-36-9 & Rubidium-83 & $<5.86 \mathrm{E}-1$ & pCi/g dry & $5.86 \mathrm{E}-1$ & & $6 / 24 / 08$ & 8F20002 & AGG-RRL-001 \\
\hline 13967-73-2 & Strontium-85 & $<3.70 \mathrm{E}-1$ & pCi/g dry & $3.70 \mathrm{E}-1$ & & $6 / 24 / 08$ & 8F20002 & AGG-RRL-001 \\
\hline $14932-53-7$ & Rubidium-86 & $<3.61 \mathrm{E} 0$ & pCi/g dry & 3.61E0 & & $6 / 24 / 08$ & 8F20002 & AGG-RRL-001 \\
\hline 13982-36-0 & Yttrium-88 & $<2.12 \mathrm{E}-1$ & pCi/g dry & $2.12 \mathrm{E}-1$ & & $6 / 24 / 08$ & 8F20002 & AGG-RRL-001 \\
\hline 14681-63-1 & Niobium-94 & $<2.84 \mathrm{E}-1$ & pCi/g dry & $2.84 \mathrm{E}-1$ & & $6 / 24 / 08$ & 8F20002 & AGG-RRL-001 \\
\hline \multirow[t]{3}{*}{ 13967-76-5 } & Niobium-95 & $<2.78 \mathrm{E}-1$ & pCi/g dry & $2.78 \mathrm{E}-1$ & & 6/24/08 & $8 F 20002$ & AGG-RRL-001 \\
\hline & Niobium-95m & $<1.33 \mathrm{E} 0$ & pCi/g dry & $1.33 \mathrm{E} 0$ & & $6 / 24 / 08$ & 8F20002 & AGG-RRL-001 \\
\hline & Technetium-95m & $<4.52 \mathrm{E}-1$ & pCi/g dry & $4.52 \mathrm{E}-1$ & & $6 / 24 / 08$ & 8F20002 & AGG-RRL-001 \\
\hline \multirow[t]{2}{*}{ 13967-71-0 } & Zirconium-95 & $<4.97 \mathrm{E}-1$ & pCi/g dry & 4.97E-1 & & $6 / 24 / 08$ & 8F20002 & AGG-RRL-001 \\
\hline & Technetium-99m & $<2.86 \mathrm{E}-1$ & pCi/g dry & $2.86 \mathrm{E}-1$ & & $6 / 24 / 08$ & 8F20002 & AGG-RRL-001 \\
\hline 13968-53-1 & Ruthenium-103 & $<3.09 \mathrm{E}-1$ & pCi/g dry & $3.09 \mathrm{E}-1$ & & $6 / 24 / 08$ & 8F20002 & AGG-RRL-001 \\
\hline 13967-48-1 & Ruthenium-106 & $<2.70 \mathrm{E} 0$ & pCi/g dry & $2.70 \mathrm{E} 0$ & & $6 / 24 / 08$ & 8F20002 & AGG-RRL-001 \\
\hline 14391-65-2 & Silver-108m & $<2.90 \mathrm{E}-1$ & pCi/g dry & $2.90 \mathrm{E}-1$ & & $6 / 24 / 08$ & 8F20002 & AGG-RRL-001 \\
\hline \multirow[t]{2}{*}{ 14109-32-1 } & Cadmium-109 & $<7.83 \mathrm{E} 0$ & pCi/g dry & 7.83E0 & & $6 / 24 / 08$ & 8F20002 & AGG-RRL-001 \\
\hline & Silver-110 & $<2.94 \mathrm{E}-1$ & pCi/g dry & $2.94 \mathrm{E}-1$ & & $6 / 24 / 08$ & 8F20002 & AGG-RRL-001 \\
\hline 14391-76-5 & Silver-110m & $<2.95 \mathrm{E}-1$ & pCi/g dry & 2.95E-1 & & $6 / 24 / 08$ & 8F20002 & AGG-RRL-001 \\
\hline 13966-06-8 & Tin-113 & $<4.19 \mathrm{E}-1$ & pCi/g dry & 4.19E-1 & & $6 / 24 / 08$ & $8 F 20002$ & AGG-RRL-001 \\
\hline $14683-10-4$ & Antimony-124 & $<2.89 \mathrm{E}-1$ & pCi/g dry & $2.89 \mathrm{E}-1$ & & $6 / 24 / 08$ & 8F20002 & AGG-RRL-001 \\
\hline 14234-35-6 & Antimony-125 & $<8.95 \mathrm{E}-1$ & pCi/g dry & 8.95E-1 & & $6 / 24 / 08$ & 8F20002 & AGG-RRL-001 \\
\hline 15756-32-8 & Antimony-126 & $<2.94 \mathrm{E}-1$ & pCi/g dry & $2.94 \mathrm{E}-1$ & & 6/24/08 & 8F20002 & AGG-RRL-001 \\
\hline
\end{tabular}


GEA/Soil

\begin{tabular}{|c|c|c|c|c|c|c|c|c|}
\hline CAS \# & Analyte & Results & Units & MDA & UNC & Analyzed & Batch & Method \\
\hline HEIS No. & B1VJ62C & \multicolumn{3}{|c|}{ Lab ID: $\quad$ 0805020-26 } & & & & \\
\hline $15832-50-5$ & Tin-126 & $<6.16 \mathrm{E}-1$ & pCi/g dry & 6.16E-1 & & $6 / 24 / 08$ & 8F20002 & AGG-RRL-001 \\
\hline $10043-66-0$ & Iodine-131 & $<3.40 \mathrm{E}-1$ & pCi/g dry & $3.40 \mathrm{E}-1$ & & 6/24/08 & 8F20002 & AGG-RRL-001 \\
\hline 13981-41-4 & Barium-133 & $<5.27 \mathrm{E}-1$ & pCi/g dry & $5.27 \mathrm{E}-1$ & & $6 / 24 / 08$ & 8 F20002 & AGG-RRL-001 \\
\hline 13967-70-9 & Cesium-134 & $<3.38 \mathrm{E}-1$ & pCi/g dry & 3.38E-1 & & 6/24/08 & $8 F 20002$ & AGG-RRL-001 \\
\hline 10045-97-3 & Cesium-137 & $<3.36 \mathrm{E}-1$ & pCi/g dry & 3.36E-1 & & $6 / 24 / 08$ & 8F20002 & AGG-RRL-001 \\
\hline 13982-30-4 & Cerium-139 & $<3.30 \mathrm{E}-1$ & pCi/g dry & 3.30E-1 & & 6/24/08 & 8F20002 & AGG-RRL-001 \\
\hline 14762-78-8 & Cerium-144 & $<2.27 \mathrm{E} 0$ & pCi/g dry & 2.27E0 & & $6 / 24 / 08$ & 8F20002 & AGG-RRL-001 \\
\hline 14683-23-9 & Europium-152 & $<1.03 \mathrm{E} 0$ & pCi/g dry & 1.03E0 & & $6 / 24 / 08$ & $8 \mathrm{~F} 20002$ & AGG-RRL-001 \\
\hline $14276-65-4$ & Gadolinium-153 & $<8.19 \mathrm{E}-1$ & pCi/g dry & 8.19E-1 & & $6 / 24 / 08$ & 8F20002 & AGG-RRL-001 \\
\hline 15585-10-1 & Europium-154 & $<6.25 \mathrm{E}-1$ & pCi/g dry & $6.25 \mathrm{E}-1$ & & $6 / 24 / 08$ & $8 F 20002$ & AGG-RRL-001 \\
\hline 14391-16-3 & Europium-155 & $<8.57 \mathrm{E}-1$ & pCi/g dry & 8.57E-1 & & 6/24/08 & $8 F 20002$ & AGG-RRL-001 \\
\hline 13982-78-0 & Mercury-203 & $<3.51 \mathrm{E}-1$ & pCi/g dry & $3.51 \mathrm{E}-1$ & & $6 / 24 / 08$ & 8F20002 & AGG-RRL-001 \\
\hline $14913-50-9$ & Thallium-208 & $<2.92 \mathrm{E}-1$ & pCi/g dry & $2.92 \mathrm{E}-1$ & & $6 / 24 / 08$ & 8F20002 & AGG-RRL-001 \\
\hline 14331-79-4 & Bismuth-210 & $<5.77 \mathrm{E}-1$ & pCi/g dry & 5.77E-1 & & $6 / 24 / 08$ & 8F20002 & AGG-RRL-001 \\
\hline \multirow[t]{3}{*}{$14255-04-0$} & Lead-210 & $<1.08 \mathrm{E} 2$ & pCi/g dry & $1.08 \mathrm{E} 2$ & & $6 / 24 / 08$ & 8F20002 & AGG-RRL-001 \\
\hline & Bismuth-211 & $<6.56 \mathrm{E} 0$ & pCi/g dry & 6.56E0 & & $6 / 24 / 08$ & 8F20002 & AGG-RRL-001 \\
\hline & Lead-211 & $<8.97 \mathrm{E} 0$ & pCi/g dry & 8.97E0 & & $6 / 24 / 08$ & 8F20002 & AGG-RRL-001 \\
\hline 15092-94-1 & Lead-212 & $<6.53 \mathrm{E}-1$ & pCi/g dry & 6.53E-1 & & $6 / 24 / 08$ & 8F20002 & AGG-RRL-001 \\
\hline 14733-03-0 & Bismuth-214 & $<6.70 \mathrm{E}-1$ & pCi/g dry & $6.70 \mathrm{E}-1$ & & 6/24/08 & $8 F 20002$ & AGG-RRL-001 \\
\hline $15067-28-4$ & Lead-214 & $<8.34 \mathrm{E}-1$ & pCi/g dry & 8.34E-1 & & $6 / 24 / 08$ & 8F20002 & AGG-RRL-001 \\
\hline $14835-02-0$ & Radon-219 & $<2.89 \mathrm{E} 0$ & pCi/g dry & 2.89E0 & & $6 / 24 / 08$ & 8F20002 & AGG-RRL-001 \\
\hline 22481-48-7 & Radon-220 & $<2.35 \mathrm{E} 2$ & pCi/g dry & 2.35E2 & & $6 / 24 / 08$ & 8F20002 & AGG-RRL-001 \\
\hline 28522-20-5 & Radon-221 & $<1.32 \mathrm{E} 0$ & pCi/g dry & $1.32 \mathrm{E} 0$ & & $6 / 24 / 08$ & 8F20002 & AGG-RRL-001 \\
\hline 15756-98-6 & Francium-223 & $<9.57 \mathrm{E} 0$ & pCi/g dry & 9.57E0 & & $6 / 24 / 08$ & 8F20002 & AGG-RRL-001 \\
\hline $15623-45-7$ & Radium-223 & $<2.14 \mathrm{E} 0$ & pCi/g dry & $2.14 \mathrm{E} 0$ & & $6 / 24 / 08$ & 8F20002 & AGG-RRL-001 \\
\hline $13233-32-4$ & Radium-224 & $<8.33 \mathrm{E} 0$ & pCi/g dry & 8.33Е0 & & $6 / 24 / 08$ & 8F20002 & AGG-RRL-001 \\
\hline 13982-63-3 & Radium-226 & $<8.14 \mathrm{E} 0$ & pCi/g dry & $8.14 \mathrm{E} 0$ & & $6 / 24 / 08$ & $8 \mathrm{~F} 20002$ & AGG-RRL-001 \\
\hline $15623-47-9$ & Thorium-227 & $<2.97 \mathrm{E} 0$ & pCi/g dry & 2.97E0 & & $6 / 24 / 08$ & 8F20002 & AGG-RRL-001 \\
\hline 14331-83-0 & Actinium-228 & $<1.32 \mathrm{E} 0$ & pCi/g dry & $1.32 \mathrm{E} 0$ & & 6/24/08 & $8 F 20002$ & AGG-RRL-001 \\
\hline $14274-82-9$ & Thorium-228 & $<1.06 \mathrm{E} 2$ & pCi/g dry & $1.06 \mathrm{E} 2$ & & $6 / 24 / 08$ & $8 F 20002$ & AGG-RRL-001 \\
\hline 14269-63-7 & Thorium-230 & $<1.08 \mathrm{E} 2$ & pCi/g dry & $1.08 \mathrm{E} 2$ & & $6 / 24 / 08$ & 8F20002 & AGG-RRL-001 \\
\hline 14331-85-2 & Protactinium-231 & $<1.18 \mathrm{E} 1$ & pCi/g dry & $1.18 \mathrm{E} 1$ & & $6 / 24 / 08$ & 8F20002 & AGG-RRL-001 \\
\hline $14932-40-2$ & Thorium-231 & $<5.80 \mathrm{E} 1$ & pCi/g dry & $5.80 \mathrm{E} 1$ & & $6 / 24 / 08$ & 8F20002 & AGG-RRL-001 \\
\hline 7440-29-1 & Thorium-232 & $<3.56 \mathrm{E} 2$ & pCi/g dry & 3.56E2 & & $6 / 24 / 08$ & 8F20002 & AGG-RRL-001 \\
\hline 13981-14-1 & Protactinium-233 & $<7.09 \mathrm{E}-1$ & pCi/g dry & 7.09E-1 & & $6 / 24 / 08$ & 8F20002 & AGG-RRL-001 \\
\hline \multirow[t]{2}{*}{$15100-28-4$} & Protactinium-234 & $<1.04 \mathrm{E} 0$ & pCi/g dry & $1.04 \mathrm{E} 0$ & & $6 / 24 / 08$ & 8F20002 & AGG-RRL-001 \\
\hline & Protactinium-234m & $<3.50 \mathrm{E} 1$ & pCi/g dry & $3.50 \mathrm{E} 1$ & & $6 / 24 / 08$ & 8F20002 & AGG-RRL-001 \\
\hline 15065-10-8 & Thorium-234 & $<9.67 \mathrm{E} 0$ & pCi/g dry & 9.67E0 & & $6 / 24 / 08$ & $8 \mathrm{~F} 20002$ & AGG-RRL-001 \\
\hline 15117-96-1 & Uranium 235 & $<2.42 \mathrm{E} 0$ & pCi/g dry & $2.42 \mathrm{E} 0$ & & $6 / 24 / 08$ & 8F20002 & AGG-RRL-001 \\
\hline 13994-20-2 & Neptunium-237 & $<2.23 \mathrm{E} 0$ & pCi/g dry & 2.23E0 & & 6/24/08 & $8 F 20002$ & AGG-RRL-001 \\
\hline \multirow[t]{2}{*}{ 13981-16-3 } & Plutonium-238 & $<3.31 \mathrm{E} 3$ & pCi/g dry & 3.31E3 & & $6 / 24 / 08$ & 8F20002 & AGG-RRL-001 \\
\hline & Uranium 238 & $<5.01 \mathrm{E} 0$ & pCi/g dry & $5.01 \mathrm{E} 0$ & & $6 / 24 / 08$ & 8F20002 & AGG-RRL-001 \\
\hline 15117-48-3 & Plutonium-239 & $<4.03 \mathrm{E} 3$ & pCi/g dry & 4.03E3 & & $6 / 24 / 08$ & 8F20002 & AGG-RRL-001 \\
\hline 14119-33-6 & Plutonium-240 & $<3.41 \mathrm{E} 3$ & pCi/g dry & $3.41 \mathrm{E} 3$ & & $6 / 24 / 08$ & 8F20002 & AGG-RRL-001 \\
\hline 14596-10-2 & Americium-241 & $<1.73 \mathrm{E} 0$ & pCi/g dry & $1.73 \mathrm{E} 0$ & & $6 / 24 / 08$ & 8F20002 & AGG-RRL-001 \\
\hline 14993-75-0 & Americium-243 & $<3.56 \mathrm{E}-1$ & pCi/g dry & $3.56 \mathrm{E}-1$ & & $6 / 24 / 08$ & 8F20002 & AGG-RRL-001 \\
\hline 15757-87-6 & Curium-243 & $<1.04 \mathrm{E} 0$ & pCi/g dry & $1.04 \mathrm{E} 0$ & & $6 / 24 / 08$ & 8F20002 & AGG-RRL-001 \\
\hline 15621-76-8 & Curium-245 & $<8.54 \mathrm{E}-1$ & pCi/g dry & $8.54 \mathrm{E}-1$ & & 6/24/08 & $8 F 20002$ & AGG-RRL-001 \\
\hline
\end{tabular}


GEA/Soil

\begin{tabular}{|c|c|c|c|c|c|c|c|c|}
\hline CAS \# & Analyte & Results & Units & MDA & UNC & Analyzed & Batch & Method \\
\hline HEIS No. & B1VJ62B & \multicolumn{3}{|c|}{ Lab ID: $\quad$ 0805020-27 } & & & & \\
\hline 13966-32-0 & Sodium-22 & $<3.33 \mathrm{E}-1$ & pCi/g dry & 3.33E-1 & & 6/24/08 & 8F20002 & AGG-RRL-001 \\
\hline 13966-00-2 & Potassium-40 & $2.04 \mathrm{E} 1$ & pCi/g dry & 3.04E0 & 1.27 & 6/24/08 & $8 \mathrm{~F} 20002$ & AGG-RRL-001 \\
\hline $14392-02-0$ & Chromium-51 & $<2.86 \mathrm{E} 0$ & pCi/g dry & $2.86 \mathrm{E} 0$ & & $6 / 24 / 08$ & $8 \mathrm{~F} 20002$ & AGG-RRL-001 \\
\hline 13966-31-9 & Manganese-54 & $<3.06 \mathrm{E}-1$ & pCi/g dry & $3.06 \mathrm{E}-1$ & & $6 / 24 / 08$ & $8 \mathrm{~F} 20002$ & AGG-RRL-001 \\
\hline 13981-50-5 & Cobalt-57 & $<2.88 \mathrm{E}-1$ & pCi/g dry & $2.88 \mathrm{E}-1$ & & $6 / 24 / 08$ & $8 \mathrm{~F} 20002$ & AGG-RRL-001 \\
\hline 14596-12-4 & Iron-59 & $<5.81 \mathrm{E}-1$ & pCi/g dry & $5.81 \mathrm{E}-1$ & & 6/24/08 & 8 F20002 & AGG-RRL-001 \\
\hline 10198-40-0 & Cobalt-60 & $<3.18 \mathrm{E}-1$ & pCi/g dry & $3.18 \mathrm{E}-1$ & & 6/24/08 & $8 \mathrm{~F} 20002$ & AGG-RRL-001 \\
\hline 13982-39-3 & Zinc-65 & $<7.32 \mathrm{E}-1$ & pCi/g dry & 7.32E-1 & & $6 / 24 / 08$ & $8 \mathrm{~F} 20002$ & AGG-RRL-001 \\
\hline 14265-71-5 & Selenium-75 & $<4.83 \mathrm{E}-1$ & pCi/g dry & 4.83E-1 & & 6/24/08 & $8 \mathrm{~F} 20002$ & AGG-RRL-001 \\
\hline 17056-36-9 & Rubidium-83 & $<5.74 \mathrm{E}-1$ & pCi/g dry & $5.74 \mathrm{E}-1$ & & $6 / 24 / 08$ & $8 \mathrm{~F} 20002$ & AGG-RRL-001 \\
\hline 13967-73-2 & Strontium-85 & $<3.92 \mathrm{E}-1$ & pCi/g dry & 3.92E-1 & & $6 / 24 / 08$ & $8 \mathrm{~F} 20002$ & AGG-RRL-001 \\
\hline 14932-53-7 & Rubidium-86 & $<3.72 \mathrm{E} 0$ & pCi/g dry & 3.72E0 & & $6 / 24 / 08$ & $8 \mathrm{~F} 20002$ & AGG-RRL-001 \\
\hline 13982-36-0 & Yttrium-88 & $<2.32 \mathrm{E}-1$ & pCi/g dry & 2.32E-1 & & 6/24/08 & $8 \mathrm{~F} 20002$ & AGG-RRL-001 \\
\hline 14681-63-1 & Niobium-94 & $<2.90 \mathrm{E}-1$ & pCi/g dry & $2.90 \mathrm{E}-1$ & & 6/24/08 & 8F20002 & AGG-RRL-001 \\
\hline \multirow[t]{3}{*}{ 13967-76-5 } & Niobium-95 & $<3.02 \mathrm{E}-1$ & pCi/g dry & 3.02E-1 & & 6/24/08 & $8 \mathrm{~F} 20002$ & AGG-RRL-001 \\
\hline & Niobium-95m & $<1.40 \mathrm{E} 0$ & pCi/g dry & $1.40 \mathrm{E} 0$ & & 6/24/08 & $8 \mathrm{~F} 20002$ & AGG-RRL-001 \\
\hline & Technetium-95m & $<4.66 \mathrm{E}-1$ & pCi/g dry & $4.66 \mathrm{E}-1$ & & 6/24/08 & $8 \mathrm{~F} 20002$ & AGG-RRL-001 \\
\hline \multirow[t]{2}{*}{ 13967-71-0 } & Zirconium-95 & $<5.36 \mathrm{E}-1$ & pCi/g dry & 5.36E-1 & & $6 / 24 / 08$ & $8 \mathrm{~F} 20002$ & AGG-RRL-001 \\
\hline & Technetium-99m & $<3.05 \mathrm{E}-1$ & pCi/g dry & 3.05E-1 & & $6 / 24 / 08$ & $8 \mathrm{~F} 20002$ & AGG-RRL-001 \\
\hline 13968-53-1 & Ruthenium-103 & $<2.95 \mathrm{E}-1$ & pCi/g dry & 2.95E-1 & & $6 / 24 / 08$ & $8 \mathrm{~F} 20002$ & AGG-RRL-001 \\
\hline 13967-48-1 & Ruthenium-106 & $<2.93 \mathrm{E} 0$ & pCi/g dry & 2.93E0 & & $6 / 24 / 08$ & $8 \mathrm{~F} 20002$ & AGG-RRL-001 \\
\hline 14391-65-2 & Silver-108m & $<3.06 \mathrm{E}-1$ & pCi/g dry & 3.06E-1 & & 6/24/08 & $8 \mathrm{~F} 20002$ & AGG-RRL-001 \\
\hline \multirow[t]{2}{*}{ 14109-32-1 } & Cadmium-109 & $<8.01 \mathrm{E} 0$ & pCi/g dry & 8.01E0 & & $6 / 24 / 08$ & $8 \mathrm{~F} 20002$ & AGG-RRL-001 \\
\hline & Silver-110 & $<3.07 \mathrm{E}-1$ & pCi/g dry & 3.07E-1 & & $6 / 24 / 08$ & $8 \mathrm{~F} 20002$ & AGG-RRL-001 \\
\hline 14391-76-5 & Silver-110m & $<3.08 \mathrm{E}-1$ & pCi/g dry & 3.08E-1 & & $6 / 24 / 08$ & $8 \mathrm{~F} 20002$ & AGG-RRL-001 \\
\hline 13966-06-8 & Tin-113 & $<4.14 \mathrm{E}-1$ & pCi/g dry & $4.14 \mathrm{E}-1$ & & 6/24/08 & $8 \mathrm{~F} 20002$ & AGG-RRL-001 \\
\hline 14683-10-4 & Antimony-124 & $<2.88 \mathrm{E}-1$ & pCi/g dry & $2.88 \mathrm{E}-1$ & & $6 / 24 / 08$ & $8 \mathrm{~F} 20002$ & AGG-RRL-001 \\
\hline $14234-35-6$ & Antimony-125 & $<9.16 \mathrm{E}-1$ & pCi/g dry & $9.16 \mathrm{E}-1$ & & 6/24/08 & $8 \mathrm{~F} 20002$ & AGG-RRL-001 \\
\hline 15756-32-8 & Antimony-126 & $<3.11 \mathrm{E}-1$ & pCi/g dry & $3.11 \mathrm{E}-1$ & & 6/24/08 & $8 \mathrm{~F} 20002$ & AGG-RRL-001 \\
\hline 15832-50-5 & Tin-126 & $<6.36 \mathrm{E}-1$ & pCi/g dry & $6.36 \mathrm{E}-1$ & & $6 / 24 / 08$ & $8 \mathrm{~F} 20002$ & AGG-RRL-001 \\
\hline 10043-66-0 & Iodine-131 & $<3.53 \mathrm{E}-1$ & pCi/g dry & 3.53E-1 & & 6/24/08 & $8 \mathrm{~F} 20002$ & AGG-RRL-001 \\
\hline 13981-41-4 & Barium-133 & $<5.84 \mathrm{E}-1$ & pCi/g dry & $5.84 \mathrm{E}-1$ & & 6/24/08 & 8 F20002 & AGG-RRL-001 \\
\hline 13967-70-9 & Cesium-134 & $<3.30 \mathrm{E}-1$ & pCi/g dry & 3.30E-1 & & $6 / 24 / 08$ & $8 \mathrm{~F} 20002$ & AGG-RRL-001 \\
\hline 10045-97-3 & Cesium-137 & $<3.60 \mathrm{E}-1$ & pCi/g dry & 3.60E-1 & & $6 / 24 / 08$ & $8 \mathrm{~F} 20002$ & AGG-RRL-001 \\
\hline 13982-30-4 & Cerium-139 & $<3.52 \mathrm{E}-1$ & pCi/g dry & 3.52E-1 & & $6 / 24 / 08$ & $8 \mathrm{~F} 20002$ & AGG-RRL-001 \\
\hline 14762-78-8 & Cerium-144 & $<2.37 \mathrm{E} 0$ & pCi/g dry & 2.37E0 & & 6/24/08 & 8 F20002 & AGG-RRL-001 \\
\hline 14683-23-9 & Europium-152 & $<1.09 \mathrm{E} 0$ & pCi/g dry & 1.09E0 & & $6 / 24 / 08$ & $8 \mathrm{~F} 20002$ & AGG-RRL-001 \\
\hline 14276-65-4 & Gadolinium-153 & $<8.66 \mathrm{E}-1$ & pCi/g dry & 8.66E-1 & & $6 / 24 / 08$ & $8 \mathrm{~F} 20002$ & AGG-RRL-001 \\
\hline 15585-10-1 & Europium-154 & $<6.07 \mathrm{E}-1$ & pCi/g dry & 6.07E-1 & & 6/24/08 & 8F20002 & AGG-RRL-001 \\
\hline 14391-16-3 & Europium-155 & $<8.90 \mathrm{E}-1$ & pCi/g dry & $8.90 \mathrm{E}-1$ & & 6/24/08 & $8 F 20002$ & AGG-RRL-001 \\
\hline 13982-78-0 & Mercury-203 & $<3.74 \mathrm{E}-1$ & pCi/g dry & 3.74E-1 & & 6/24/08 & $8 \mathrm{~F} 20002$ & AGG-RRL-001 \\
\hline 14913-50-9 & Thallium-208 & $<3.16 \mathrm{E}-1$ & pCi/g dry & $3.16 \mathrm{E}-1$ & & $6 / 24 / 08$ & $8 \mathrm{~F} 20002$ & AGG-RRL-001 \\
\hline 14331-79-4 & Bismuth-210 & $<5.70 \mathrm{E}-1$ & pCi/g dry & $5.70 \mathrm{E}-1$ & & 6/24/08 & $8 \mathrm{~F} 20002$ & AGG-RRL-001 \\
\hline \multirow[t]{3}{*}{$14255-04-0$} & Lead-210 & $<1.11 \mathrm{E} 2$ & pCi/g dry & $1.11 \mathrm{E} 2$ & & 6/24/08 & $8 \mathrm{~F} 20002$ & AGG-RRL-001 \\
\hline & Bismuth-211 & $<7.17 \mathrm{E} 0$ & pCi/g dry & 7.17E0 & & 6/24/08 & $8 \mathrm{~F} 20002$ & AGG-RRL-001 \\
\hline & Lead-211 & $<9.80 \mathrm{E} 0$ & pCi/g dry & $9.80 \mathrm{E} 0$ & & 6/24/08 & $8 \mathrm{~F} 20002$ & AGG-RRL-001 \\
\hline 15092-94-1 & Lead-212 & $<6.39 \mathrm{E}-1$ & pCi/g dry & 6.39E-1 & & $6 / 24 / 08$ & 8F20002 & AGG-RRL-001 \\
\hline 14733-03-0 & Bismuth-214 & $<7.76 \mathrm{E}-1$ & pCi/g dry & 7.76E-1 & & $6 / 24 / 08$ & $8 \mathrm{~F} 20002$ & AGG-RRL-001 \\
\hline $15067-28-4$ & Lead-214 & $<9.11 \mathrm{E}-1$ & pCi/g dry & $9.11 \mathrm{E}-1$ & & $6 / 24 / 08$ & $8 F 20002$ & AGG-RRL-001 \\
\hline
\end{tabular}


GEA/Soil

\begin{tabular}{|c|c|c|c|c|c|c|c|c|}
\hline CAS \# & Analyte & Results & Units & MDA & UNC & Analyzed & Batch & Method \\
\hline HEIS No. & B1VJ62B & \multicolumn{3}{|c|}{ Lab ID: $\quad$ 0805020-27 } & & & & \\
\hline $14835-02-0$ & Radon-219 & $<2.87 \mathrm{E} 0$ & pCi/g dry & 2.87E0 & & 6/24/08 & 8F20002 & AGG-RRL-001 \\
\hline 22481-48-7 & Radon-220 & $<2.71 \mathrm{E} 2$ & pCi/g dry & $2.71 \mathrm{E} 2$ & & $6 / 24 / 08$ & 8F20002 & AGG-RRL-001 \\
\hline 28522-20-5 & Radon-221 & $<1.34 \mathrm{E} 0$ & pCi/g dry & $1.34 \mathrm{E} 0$ & & $6 / 24 / 08$ & $8 F 20002$ & AGG-RRL-001 \\
\hline 15756-98-6 & Francium-223 & $<1.02 \mathrm{E} 1$ & pCi/g dry & $1.02 \mathrm{E} 1$ & & $6 / 24 / 08$ & 8F20002 & AGG-RRL-001 \\
\hline 15623-45-7 & Radium-223 & $<2.11 \mathrm{E} 0$ & pCi/g dry & $2.11 \mathrm{E} 0$ & & $6 / 24 / 08$ & 8F20002 & AGG-RRL-001 \\
\hline 13233-32-4 & Radium-224 & $<8.83 \mathrm{E} 0$ & pCi/g dry & 8.83E0 & & $6 / 24 / 08$ & 8F20002 & AGG-RRL-001 \\
\hline 13982-63-3 & Radium-226 & $<8.34 \mathrm{E} 0$ & pCi/g dry & 8.34E0 & & $6 / 24 / 08$ & 8F20002 & AGG-RRL-001 \\
\hline 15623-47-9 & Thorium-227 & $<3.13 \mathrm{E} 0$ & pCi/g dry & 3.13E0 & & $6 / 24 / 08$ & 8F20002 & AGG-RRL-001 \\
\hline 14331-83-0 & Actinium-228 & $<1.41 \mathrm{E} 0$ & pCi/g dry & $1.41 \mathrm{E} 0$ & & $6 / 24 / 08$ & 8F20002 & AGG-RRL-001 \\
\hline 14274-82-9 & Thorium-228 & $<1.08 \mathrm{E} 2$ & pCi/g dry & $1.08 \mathrm{E} 2$ & & $6 / 24 / 08$ & 8F20002 & AGG-RRL-001 \\
\hline 14269-63-7 & Thorium-230 & $<1.13 \mathrm{E} 2$ & pCi/g dry & 1.13E2 & & $6 / 24 / 08$ & 8F20002 & AGG-RRL-001 \\
\hline 14331-85-2 & Protactinium-231 & $<1.25 \mathrm{E} 1$ & pCi/g dry & $1.25 \mathrm{E} 1$ & & $6 / 24 / 08$ & $8 F 20002$ & AGG-RRL-001 \\
\hline $14932-40-2$ & Thorium-231 & $<6.28 \mathrm{E} 1$ & pCi/g dry & $6.28 \mathrm{E} 1$ & & $6 / 24 / 08$ & 8F20002 & AGG-RRL-001 \\
\hline $7440-29-1$ & Thorium-232 & $<3.74 \mathrm{E} 2$ & pCi/g dry & $3.74 \mathrm{E} 2$ & & 6/24/08 & $8 F 20002$ & AGG-RRL-001 \\
\hline 13981-14-1 & Protactinium-233 & $<7.85 \mathrm{E}-1$ & pCi/g dry & $7.85 \mathrm{E}-1$ & & $6 / 24 / 08$ & 8F20002 & AGG-RRL-001 \\
\hline \multirow{2}{*}{$15100-28-4$} & Protactinium-234 & $<1.11 \mathrm{E} 0$ & pCi/g dry & $1.11 \mathrm{E} 0$ & & $6 / 24 / 08$ & 8F20002 & AGG-RRL-001 \\
\hline & Protactinium-234m & $<3.76 \mathrm{E} 1$ & pCi/g dry & $3.76 \mathrm{E} 1$ & & $6 / 24 / 08$ & 8F20002 & AGG-RRL-001 \\
\hline 15065-10-8 & Thorium-234 & $<1.00 \mathrm{E} 1$ & pCi/g dry & $1.00 \mathrm{E} 1$ & & $6 / 24 / 08$ & 8F20002 & AGG-RRL-001 \\
\hline 15117-96-1 & Uranium 235 & $<2.53 \mathrm{E} 0$ & pCi/g dry & 2.53E0 & & $6 / 24 / 08$ & 8F20002 & AGG-RRL-001 \\
\hline 13994-20-2 & Neptunium-237 & $<2.32 \mathrm{E} 0$ & pCi/g dry & 2.32E0 & & $6 / 24 / 08$ & 8F20002 & AGG-RRL-001 \\
\hline \multirow[t]{2}{*}{ 13981-16-3 } & Plutonium-238 & $<3.53 \mathrm{E} 3$ & pCi/g dry & 3.53E3 & & $6 / 24 / 08$ & 8F20002 & AGG-RRL-001 \\
\hline & Uranium 238 & $<5.20 \mathrm{E} 0$ & pCi/g dry & $5.20 \mathrm{E} 0$ & & $6 / 24 / 08$ & 8F20002 & AGG-RRL-001 \\
\hline $15117-48-3$ & Plutonium-239 & $<4.06 \mathrm{E} 3$ & pCi/g dry & 4.06E3 & & $6 / 24 / 08$ & 8F20002 & AGG-RRL-001 \\
\hline 14119-33-6 & Plutonium-240 & $<3.63 \mathrm{E} 3$ & pCi/g dry & 3.63E3 & & $6 / 24 / 08$ & 8F20002 & AGG-RRL-001 \\
\hline $14596-10-2$ & Americium-241 & $<1.85 \mathrm{E} 0$ & pCi/g dry & $1.85 \mathrm{E} 0$ & & $6 / 24 / 08$ & 8F20002 & AGG-RRL-001 \\
\hline 14993-75-0 & Americium-243 & $<6.22 \mathrm{E}-1$ & pCi/g dry & $6.22 \mathrm{E}-1$ & & $6 / 24 / 08$ & 8F20002 & AGG-RRL-001 \\
\hline 15757-87-6 & Curium-243 & $<1.11 \mathrm{E} 0$ & pCi/g dry & $1.11 \mathrm{E} 0$ & & $6 / 24 / 08$ & 8 F20002 & AGG-RRL-001 \\
\hline 15621-76-8 & Curium-245 & $<9.08 \mathrm{E}-1$ & pCi/g dry & $9.08 \mathrm{E}-1$ & & $6 / 24 / 08$ & 8F20002 & AGG-RRL-001 \\
\hline HEIS No. & B1VJ62A & \multicolumn{3}{|c|}{ Lab ID: $\quad 0805020-28$} & & & & \\
\hline 13966-32-0 & Sodium-22 & $<3.71 \mathrm{E}-1$ & pCi/g dry & $3.71 \mathrm{E}-1$ & & $6 / 24 / 08$ & 8F20002 & AGG-RRL-001 \\
\hline 13966-00-2 & Potassium-40 & 1.95E1 & pCi/g dry & 3.79E0 & 1.28 & $6 / 24 / 08$ & 8F20002 & AGG-RRL-001 \\
\hline 14392-02-0 & Chromium-51 & $<3.10 \mathrm{E} 0$ & pCi/g dry & 3.10E0 & & $6 / 24 / 08$ & 8F20002 & AGG-RRL-001 \\
\hline 13966-31-9 & Manganese-54 & $<3.28 \mathrm{E}-1$ & pCi/g dry & $3.28 \mathrm{E}-1$ & & $6 / 24 / 08$ & 8F20002 & AGG-RRL-001 \\
\hline 13981-50-5 & Cobalt-57 & $<3.14 \mathrm{E}-1$ & pCi/g dry & $3.14 \mathrm{E}-1$ & & $6 / 24 / 08$ & 8F20002 & AGG-RRL-001 \\
\hline $14596-12-4$ & Iron-59 & $<5.82 \mathrm{E}-1$ & pCi/g dry & $5.82 \mathrm{E}-1$ & & $6 / 24 / 08$ & 8F20002 & AGG-RRL-001 \\
\hline 10198-40-0 & Cobalt-60 & $<3.30 \mathrm{E}-1$ & pCi/g dry & 3.30E-1 & & 6/24/08 & 8F20002 & AGG-RRL-001 \\
\hline 13982-39-3 & Zinc-65 & $<7.13 \mathrm{E}-1$ & pCi/g dry & 7.13E-1 & & $6 / 24 / 08$ & $8 F 20002$ & AGG-RRL-001 \\
\hline $14265-71-5$ & Selenium-75 & $<5.04 \mathrm{E}-1$ & pCi/g dry & $5.04 \mathrm{E}-1$ & & $6 / 24 / 08$ & 8F20002 & AGG-RRL-001 \\
\hline 17056-36-9 & Rubidium-83 & $<8.08 \mathrm{E}-1$ & pCi/g dry & 8.08E-1 & & 6/24/08 & 8F20002 & AGG-RRL-001 \\
\hline 13967-73-2 & Strontium-85 & $<3.78 \mathrm{E}-1$ & pCi/g dry & $3.78 \mathrm{E}-1$ & & $6 / 24 / 08$ & 8F20002 & AGG-RRL-001 \\
\hline $14932-53-7$ & Rubidium-86 & $<4.36 \mathrm{E} 0$ & pCi/g dry & 4.36Е 0 & & 6/24/08 & 8F20002 & AGG-RRL-001 \\
\hline 13982-36-0 & Yttrium-88 & $<2.43 \mathrm{E}-1$ & pCi/g dry & 2.43E-1 & & 6/24/08 & 8F20002 & AGG-RRL-001 \\
\hline 14681-63-1 & Niobium-94 & $<3.24 \mathrm{E}-1$ & pCi/g dry & $3.24 \mathrm{E}-1$ & & 6/24/08 & 8F20002 & AGG-RRL-001 \\
\hline \multirow[t]{3}{*}{ 13967-76-5 } & Niobium-95 & $<3.00 \mathrm{E}-1$ & pCi/g dry & $3.00 \mathrm{E}-1$ & & 6/24/08 & 8F20002 & AGG-RRL-001 \\
\hline & Niobium-95m & $<1.43 \mathrm{E} 0$ & pCi/g dry & $1.43 \mathrm{E} 0$ & & 6/24/08 & 8F20002 & AGG-RRL-001 \\
\hline & Technetium-95m & $<4.93 \mathrm{E}-1$ & pCi/g dry & 4.93E-1 & & 6/24/08 & 8F20002 & AGG-RRL-001 \\
\hline \multirow[t]{2}{*}{ 13967-71-0 } & Zirconium-95 & $<5.23 \mathrm{E}-1$ & pCi/g dry & 5.23E-1 & & 6/24/08 & 8F20002 & AGG-RRL-001 \\
\hline & Technetium-99m & $<3.25 \mathrm{E}-1$ & pCi/g dry & 3.25E-1 & & 6/24/08 & 8F20002 & AGG-RRL-001 \\
\hline
\end{tabular}


GEA/Soil

\begin{tabular}{|c|c|c|c|c|c|c|c|c|}
\hline CAS \# & Analyte & Results & Units & MDA & UNC & Analyzed & Batch & Method \\
\hline HEIS No. & B1VJ62A & & ab ID: & $5020-28$ & & & & \\
\hline 13968-53-1 & Ruthenium-103 & $<3.69 \mathrm{E}-1$ & pCi/g dry & 3.69E-1 & & 6/24/08 & 8F20002 & AGG-RRL-001 \\
\hline 13967-48-1 & Ruthenium-106 & $<3.55 \mathrm{E} 0$ & pCi/g dry & 3.55E0 & & 6/24/08 & $8 \mathrm{~F} 20002$ & AGG-RRL-001 \\
\hline 14391-65-2 & Silver-108m & $<3.12 \mathrm{E}-1$ & pCi/g dry & $3.12 \mathrm{E}-1$ & & $6 / 24 / 08$ & $8 \mathrm{~F} 20002$ & AGG-RRL-001 \\
\hline \multirow[t]{2}{*}{ 14109-32-1 } & Cadmium-109 & $<9.36 \mathrm{E} 0$ & pCi/g dry & 9.36Е0 & & 6/24/08 & $8 \mathrm{~F} 20002$ & AGG-RRL-001 \\
\hline & Silver-110 & $<3.23 \mathrm{E}-1$ & pCi/g dry & 3.23E-1 & & $6 / 24 / 08$ & $8 \mathrm{~F} 20002$ & AGG-RRL-001 \\
\hline 14391-76-5 & Silver-110m & $<3.23 \mathrm{E}-1$ & pCi/g dry & 3.23E-1 & & 6/24/08 & 8 F20002 & AGG-RRL-001 \\
\hline 13966-06-8 & Tin-113 & $<4.68 \mathrm{E}-1$ & pCi/g dry & $4.68 \mathrm{E}-1$ & & 6/24/08 & $8 \mathrm{~F} 20002$ & AGG-RRL-001 \\
\hline 14683-10-4 & Antimony-124 & $<3.74 \mathrm{E}-1$ & pCi/g dry & $3.74 \mathrm{E}-1$ & & 6/24/08 & $8 \mathrm{~F} 20002$ & AGG-RRL-001 \\
\hline $14234-35-6$ & Antimony-125 & $<9.64 \mathrm{E}-1$ & pCi/g dry & $9.64 \mathrm{E}-1$ & & 6/24/08 & $8 \mathrm{~F} 20002$ & AGG-RRL-001 \\
\hline 15756-32-8 & Antimony-126 & $<3.14 \mathrm{E}-1$ & pCi/g dry & $3.14 \mathrm{E}-1$ & & $6 / 24 / 08$ & $8 \mathrm{~F} 20002$ & AGG-RRL-001 \\
\hline $15832-50-5$ & Tin-126 & $<7.66 \mathrm{E}-1$ & pCi/g dry & $7.66 \mathrm{E}-1$ & & 6/24/08 & $8 \mathrm{~F} 20002$ & AGG-RRL-001 \\
\hline 10043-66-0 & Iodine-131 & $<4.01 \mathrm{E}-1$ & pCi/g dry & $4.01 \mathrm{E}-1$ & & $6 / 24 / 08$ & $8 \mathrm{~F} 20002$ & AGG-RRL-001 \\
\hline 13981-41-4 & Barium-133 & $<5.54 \mathrm{E}-1$ & pCi/g dry & $5.54 \mathrm{E}-1$ & & 6/24/08 & $8 \mathrm{~F} 20002$ & AGG-RRL-001 \\
\hline 13967-70-9 & Cesium-134 & $<3.64 \mathrm{E}-1$ & pCi/g dry & 3.64E-1 & & $6 / 24 / 08$ & $8 \mathrm{~F} 20002$ & AGG-RRL-001 \\
\hline 10045-97-3 & Cesium-137 & $<3.63 \mathrm{E}-1$ & pCi/g dry & 3.63E-1 & & 6/24/08 & $8 \mathrm{~F} 20002$ & AGG-RRL-001 \\
\hline 13982-30-4 & Cerium-139 & $<3.63 \mathrm{E}-1$ & pCi/g dry & 3.63E-1 & & $6 / 24 / 08$ & $8 \mathrm{~F} 20002$ & AGG-RRL-001 \\
\hline 14762-78-8 & Cerium-144 & $<2.61 \mathrm{E} 0$ & pCi/g dry & 2.61E0 & & 6/24/08 & $8 \mathrm{~F} 20002$ & AGG-RRL-001 \\
\hline 14683-23-9 & Europium-152 & $<1.31 \mathrm{E} 0$ & pCi/g dry & $1.31 \mathrm{E} 0$ & & $6 / 24 / 08$ & $8 \mathrm{~F} 20002$ & AGG-RRL-001 \\
\hline 14276-65-4 & Gadolinium-153 & $<1.01 \mathrm{E} 0$ & pCi/g dry & $1.01 \mathrm{E} 0$ & & 6/24/08 & $8 \mathrm{~F} 20002$ & AGG-RRL-001 \\
\hline 15585-10-1 & Europium-154 & $<6.67 \mathrm{E}-1$ & pCi/g dry & 6.67E-1 & & $6 / 24 / 08$ & $8 \mathrm{~F} 20002$ & AGG-RRL-001 \\
\hline 14391-16-3 & Europium-155 & $<1.09 \mathrm{E} 0$ & pCi/g dry & 1.09E0 & & 6/24/08 & 8 F20002 & AGG-RRL-001 \\
\hline 13982-78-0 & Mercury-203 & $<3.90 \mathrm{E}-1$ & pCi/g dry & $3.90 \mathrm{E}-1$ & & 6/24/08 & $8 \mathrm{~F} 20002$ & AGG-RRL-001 \\
\hline 14913-50-9 & Thallium-208 & $<4.12 \mathrm{E}-1$ & pCi/g dry & 4.12E-1 & & $6 / 24 / 08$ & $8 \mathrm{~F} 20002$ & AGG-RRL-001 \\
\hline 14331-79-4 & Bismuth-210 & $<6.01 \mathrm{E}-1$ & pCi/g dry & $6.01 \mathrm{E}-1$ & & 6/24/08 & $8 \mathrm{~F} 20002$ & AGG-RRL-001 \\
\hline \multirow[t]{3}{*}{$14255-04-0$} & Lead-210 & $<4.38 \mathrm{E} 1$ & pCi/g dry & 4.38E1 & & 6/24/08 & $8 \mathrm{~F} 20002$ & AGG-RRL-001 \\
\hline & Bismuth-211 & $<7.10 \mathrm{E} 0$ & pCi/g dry & 7.10E0 & & 6/24/08 & $8 \mathrm{~F} 20002$ & AGG-RRL-001 \\
\hline & Lead-211 & $<9.70 \mathrm{E} 0$ & pCi/g dry & $9.70 \mathrm{E} 0$ & & 6/24/08 & $8 \mathrm{~F} 20002$ & AGG-RRL-001 \\
\hline 15092-94-1 & Lead-212 & $<8.33 \mathrm{E}-1$ & pCi/g dry & 8.33E-1 & & 6/24/08 & $8 \mathrm{~F} 20002$ & AGG-RRL-001 \\
\hline 14733-03-0 & Bismuth-214 & $<7.87 \mathrm{E}-1$ & pCi/g dry & $7.87 \mathrm{E}-1$ & & 6/24/08 & $8 \mathrm{~F} 20002$ & AGG-RRL-001 \\
\hline $15067-28-4$ & Lead-214 & $<8.95 \mathrm{E}-1$ & pCi/g dry & 8.95E-1 & & 6/24/08 & $8 \mathrm{~F} 20002$ & AGG-RRL-001 \\
\hline $14835-02-0$ & Radon-219 & $<3.07 \mathrm{E} 0$ & pCi/g dry & 3.07E0 & & $6 / 24 / 08$ & $8 \mathrm{~F} 20002$ & AGG-RRL-001 \\
\hline 22481-48-7 & Radon-220 & $<3.05 \mathrm{E} 2$ & pCi/g dry & 3.05E2 & & 6/24/08 & $8 \mathrm{~F} 20002$ & AGG-RRL-001 \\
\hline 28522-20-5 & Radon-221 & $<1.46 \mathrm{E} 0$ & pCi/g dry & $1.46 \mathrm{E} 0$ & & $6 / 24 / 08$ & $8 \mathrm{~F} 20002$ & AGG-RRL-001 \\
\hline 15756-98-6 & Francium-223 & $<1.06 \mathrm{E} 1$ & pCi/g dry & $1.06 \mathrm{E} 1$ & & $6 / 24 / 08$ & $8 \mathrm{~F} 20002$ & AGG-RRL-001 \\
\hline $15623-45-7$ & Radium-223 & $<2.24 \mathrm{E} 0$ & pCi/g dry & $2.24 \mathrm{E} 0$ & & $6 / 24 / 08$ & $8 \mathrm{~F} 20002$ & AGG-RRL-001 \\
\hline 13233-32-4 & Radium-224 & $<9.11 \mathrm{E} 0$ & pCi/g dry & $9.11 \mathrm{E} 0$ & & 6/24/08 & 8 F20002 & AGG-RRL-001 \\
\hline 13982-63-3 & Radium-226 & $<8.98 \mathrm{E} 0$ & pCi/g dry & 8.98E0 & & $6 / 24 / 08$ & $8 \mathrm{~F} 20002$ & AGG-RRL-001 \\
\hline $15623-47-9$ & Thorium-227 & $<3.20 \mathrm{E} 0$ & pCi/g dry & $3.20 \mathrm{E} 0$ & & 6/24/08 & $8 \mathrm{~F} 20002$ & AGG-RRL-001 \\
\hline 14331-83-0 & Actinium-228 & $<1.40 \mathrm{E} 0$ & pCi/g dry & $1.40 \mathrm{E} 0$ & & 6/24/08 & $8 \mathrm{~F} 20002$ & AGG-RRL-001 \\
\hline $14274-82-9$ & Thorium-228 & $<1.15 \mathrm{E} 2$ & pCi/g dry & $1.15 \mathrm{E} 2$ & & 6/24/08 & $8 \mathrm{~F} 20002$ & AGG-RRL-001 \\
\hline 14269-63-7 & Thorium-230 & $<1.24 \mathrm{E} 2$ & pCi/g dry & $1.24 \mathrm{E} 2$ & & 6/24/08 & $8 \mathrm{~F} 20002$ & AGG-RRL-001 \\
\hline $14331-85-2$ & Protactinium-231 & $<1.37 \mathrm{E} 1$ & pCi/g dry & 1.37E1 & & $6 / 24 / 08$ & $8 \mathrm{~F} 20002$ & AGG-RRL-001 \\
\hline $14932-40-2$ & Thorium-231 & $<7.16 \mathrm{E} 1$ & pCi/g dry & 7.16E1 & & 6/24/08 & $8 \mathrm{~F} 20002$ & AGG-RRL-001 \\
\hline 7440-29-1 & Thorium-232 & $<3.04 \mathrm{E} 2$ & pCi/g dry & $3.04 \mathrm{E} 2$ & & $6 / 24 / 08$ & $8 \mathrm{~F} 20002$ & AGG-RRL-001 \\
\hline 13981-14-1 & Protactinium-233 & $<8.21 \mathrm{E}-1$ & pCi/g dry & $8.21 \mathrm{E}-1$ & & 6/24/08 & $8 \mathrm{~F} 20002$ & AGG-RRL-001 \\
\hline \multirow[t]{2}{*}{$15100-28-4$} & Protactinium-234 & $<1.31 \mathrm{E} 0$ & pCi/g dry & $1.31 \mathrm{E} 0$ & & $6 / 24 / 08$ & $8 \mathrm{~F} 20002$ & AGG-RRL-001 \\
\hline & Protactinium-234m & $<4.18 \mathrm{E} 1$ & pCi/g dry & 4.18E1 & & $6 / 24 / 08$ & 8F20002 & AGG-RRL-001 \\
\hline 15065-10-8 & Thorium-234 & $<1.07 \mathrm{E} 1$ & pCi/g dry & $1.07 \mathrm{E} 1$ & & $6 / 24 / 08$ & $8 \mathrm{~F} 20002$ & AGG-RRL-001 \\
\hline 15117-96-1 & Uranium 235 & $<2.76 \mathrm{E} 0$ & pCi/g dry & 2.76E0 & & $6 / 24 / 08$ & $8 F 20002$ & AGG-RRL-001 \\
\hline
\end{tabular}


GEA/Soil

\begin{tabular}{|c|c|c|c|c|c|c|c|c|}
\hline CAS \# & Analyte & Results & Units & MDA & UNC & Analyzed & Batch & Method \\
\hline HEIS No. & B1VJ62A & & ab ID: & $05020-28$ & & & & \\
\hline 13994-20-2 & Neptunium-237 & $<2.83 \mathrm{E} 0$ & pCi/g dry & 2.83E0 & & $6 / 24 / 08$ & $8 F 20002$ & AGG-RRL-001 \\
\hline \multirow[t]{2}{*}{ 13981-16-3 } & Plutonium-238 & $<4.09 \mathrm{E} 3$ & pCi/g dry & 4.09E3 & & $6 / 24 / 08$ & $8 \mathrm{~F} 20002$ & AGG-RRL-001 \\
\hline & Uranium 238 & $<5.91 \mathrm{E} 0$ & pCi/g dry & 5.91E0 & & $6 / 24 / 08$ & $8 \mathrm{~F} 20002$ & AGG-RRL-001 \\
\hline $15117-48-3$ & Plutonium-239 & $<4.15 \mathrm{E} 3$ & pCi/g dry & 4.15E3 & & $6 / 24 / 08$ & $8 \mathrm{~F} 20002$ & AGG-RRL-001 \\
\hline 14119-33-6 & Plutonium-240 & $<4.10 \mathrm{E} 3$ & pCi/g dry & $4.10 \mathrm{E} 3$ & & $6 / 24 / 08$ & $8 \mathrm{~F} 20002$ & AGG-RRL-001 \\
\hline 14596-10-2 & Americium-241 & $<1.56 \mathrm{E} 0$ & pCi/g dry & $1.56 \mathrm{E} 0$ & & $6 / 24 / 08$ & $8 \mathrm{~F} 20002$ & AGG-RRL-001 \\
\hline 14993-75-0 & Americium-243 & $<6.86 \mathrm{E}-1$ & pCi/g dry & $6.86 \mathrm{E}-1$ & & 6/24/08 & $8 \mathrm{~F} 20002$ & AGG-RRL-001 \\
\hline 15757-87-6 & Curium-243 & $<1.26 \mathrm{E} 0$ & pCi/g dry & $1.26 \mathrm{E} 0$ & & $6 / 24 / 08$ & $8 \mathrm{~F} 20002$ & AGG-RRL-001 \\
\hline 15621-76-8 & Curium-245 & $<1.03 \mathrm{E} 0$ & pCi/g dry & $1.03 \mathrm{E} 0$ & & 6/24/08 & $8 \mathrm{~F} 20002$ & AGG-RRL-001 \\
\hline HEIS No. & B1VJ64C & & ab ID: & $05020-30$ & & & & \\
\hline 13966-32-0 & Sodium-22 & $<3.22 \mathrm{E}-1$ & pCi/g dry & $3.22 \mathrm{E}-1$ & & 6/25/08 & 8F20002 & AGG-RRL-001 \\
\hline 13966-00-2 & Potassium-40 & 2.01E1 & pCi/g dry & 2.69E0 & 1.18 & $6 / 25 / 08$ & $8 \mathrm{~F} 20002$ & AGG-RRL-001 \\
\hline 14392-02-0 & Chromium-51 & $<2.60 \mathrm{E} 0$ & pCi/g dry & $2.60 \mathrm{E} 0$ & & $6 / 25 / 08$ & $8 \mathrm{~F} 20002$ & AGG-RRL-001 \\
\hline 13966-31-9 & Manganese-54 & $<2.67 \mathrm{E}-1$ & pCi/g dry & $2.67 \mathrm{E}-1$ & & 6/25/08 & 8 F20002 & AGG-RRL-001 \\
\hline 13981-50-5 & Cobalt-57 & $<2.68 \mathrm{E}-1$ & pCi/g dry & $2.68 \mathrm{E}-1$ & & 6/25/08 & $8 \mathrm{~F} 20002$ & AGG-RRL-001 \\
\hline $14596-12-4$ & Iron-59 & $<5.62 \mathrm{E}-1$ & pCi/g dry & 5.62E-1 & & $6 / 25 / 08$ & $8 \mathrm{~F} 20002$ & AGG-RRL-001 \\
\hline 10198-40-0 & Cobalt-60 & $<2.83 \mathrm{E}-1$ & pCi/g dry & 2.83E-1 & & $6 / 25 / 08$ & $8 \mathrm{~F} 20002$ & AGG-RRL-001 \\
\hline 13982-39-3 & Zinc-65 & $<6.71 \mathrm{E}-1$ & pCi/g dry & $6.71 \mathrm{E}-1$ & & $6 / 25 / 08$ & $8 \mathrm{~F} 20002$ & AGG-RRL-001 \\
\hline $14265-71-5$ & Selenium-75 & $<4.35 \mathrm{E}-1$ & pCi/g dry & 4.35E-1 & & 6/25/08 & $8 \mathrm{~F} 20002$ & AGG-RRL-001 \\
\hline 17056-36-9 & Rubidium-83 & $<5.15 \mathrm{E}-1$ & pCi/g dry & $5.15 \mathrm{E}-1$ & & $6 / 25 / 08$ & $8 \mathrm{~F} 20002$ & AGG-RRL-001 \\
\hline 13967-73-2 & Strontium-85 & $<3.40 \mathrm{E}-1$ & pCi/g dry & $3.40 \mathrm{E}-1$ & & 6/25/08 & $8 \mathrm{~F} 20002$ & AGG-RRL-001 \\
\hline 14932-53-7 & Rubidium-86 & $<2.91 \mathrm{E} 0$ & pCi/g dry & 2.91E0 & & 6/25/08 & $8 \mathrm{~F} 20002$ & AGG-RRL-001 \\
\hline 13982-36-0 & Yttrium-88 & $<1.49 \mathrm{E}-1$ & pCi/g dry & $1.49 \mathrm{E}-1$ & & $6 / 25 / 08$ & $8 \mathrm{~F} 20002$ & AGG-RRL-001 \\
\hline 14681-63-1 & Niobium-94 & $<2.59 \mathrm{E}-1$ & pCi/g dry & $2.59 \mathrm{E}-1$ & & $6 / 25 / 08$ & $8 \mathrm{~F} 20002$ & AGG-RRL-001 \\
\hline \multirow[t]{3}{*}{ 13967-76-5 } & Niobium-95 & $<2.68 \mathrm{E}-1$ & pCi/g dry & $2.68 \mathrm{E}-1$ & & $6 / 25 / 08$ & $8 \mathrm{~F} 20002$ & AGG-RRL-001 \\
\hline & Niobium-95m & $<1.27 \mathrm{E} 0$ & pCi/g dry & $1.27 \mathrm{E} 0$ & & 6/25/08 & $8 \mathrm{~F} 20002$ & AGG-RRL-001 \\
\hline & Technetium-95m & $<4.25 \mathrm{E}-1$ & pCi/g dry & 4.25E-1 & & 6/25/08 & $8 \mathrm{~F} 20002$ & AGG-RRL-001 \\
\hline \multirow[t]{2}{*}{ 13967-71-0 } & Zirconium-95 & $<4.52 \mathrm{E}-1$ & pCi/g dry & $4.52 \mathrm{E}-1$ & & 6/25/08 & $8 \mathrm{~F} 20002$ & AGG-RRL-001 \\
\hline & Technetium-99m & $<2.64 \mathrm{E}-1$ & pCi/g dry & $2.64 \mathrm{E}-1$ & & 6/25/08 & $8 \mathrm{~F} 20002$ & AGG-RRL-001 \\
\hline 13968-53-1 & Ruthenium-103 & $<2.90 \mathrm{E}-1$ & pCi/g dry & $2.90 \mathrm{E}-1$ & & 6/25/08 & $8 \mathrm{~F} 20002$ & AGG-RRL-001 \\
\hline 13967-48-1 & Ruthenium-106 & $<2.38 \mathrm{E} 0$ & pCi/g dry & $2.38 \mathrm{E} 0$ & & $6 / 25 / 08$ & $8 \mathrm{~F} 20002$ & AGG-RRL-001 \\
\hline 14391-65-2 & Silver-108m & $<2.67 \mathrm{E}-1$ & pCi/g dry & 2.67E-1 & & $6 / 25 / 08$ & $8 \mathrm{~F} 20002$ & AGG-RRL-001 \\
\hline \multirow[t]{2}{*}{ 14109-32-1 } & Cadmium-109 & $<7.39 \mathrm{E} 0$ & pCi/g dry & 7.39E0 & & $6 / 25 / 08$ & $8 \mathrm{~F} 20002$ & AGG-RRL-001 \\
\hline & Silver-110 & $<2.86 \mathrm{E}-1$ & pCi/g dry & $2.86 \mathrm{E}-1$ & & 6/25/08 & $8 \mathrm{~F} 20002$ & AGG-RRL-001 \\
\hline 14391-76-5 & Silver-110m & $<2.87 \mathrm{E}-1$ & pCi/g dry & $2.87 \mathrm{E}-1$ & & $6 / 25 / 08$ & $8 \mathrm{~F} 20002$ & AGG-RRL-001 \\
\hline 13966-06-8 & Tin-113 & $<3.98 \mathrm{E}-1$ & pCi/g dry & $3.98 \mathrm{E}-1$ & & 6/25/08 & $8 \mathrm{~F} 20002$ & AGG-RRL-001 \\
\hline $14683-10-4$ & Antimony-124 & $<2.91 \mathrm{E}-1$ & pCi/g dry & $2.91 \mathrm{E}-1$ & & 6/25/08 & $8 \mathrm{~F} 20002$ & AGG-RRL-001 \\
\hline $14234-35-6$ & Antimony-125 & $<8.66 \mathrm{E}-1$ & pCi/g dry & 8.66E-1 & & 6/25/08 & $8 \mathrm{~F} 20002$ & AGG-RRL-001 \\
\hline 15756-32-8 & Antimony-126 & $<2.67 \mathrm{E}-1$ & pCi/g dry & 2.67E-1 & & $6 / 25 / 08$ & $8 \mathrm{~F} 20002$ & AGG-RRL-001 \\
\hline 15832-50-5 & Tin-126 & $<5.87 \mathrm{E}-1$ & pCi/g dry & $5.87 \mathrm{E}-1$ & & 6/25/08 & $8 \mathrm{~F} 20002$ & AGG-RRL-001 \\
\hline 10043-66-0 & Iodine-131 & $<2.97 \mathrm{E}-1$ & pCi/g dry & 2.97E-1 & & $6 / 25 / 08$ & $8 \mathrm{~F} 20002$ & AGG-RRL-001 \\
\hline 13981-41-4 & Barium-133 & $<5.23 \mathrm{E}-1$ & pCi/g dry & $5.23 \mathrm{E}-1$ & & $6 / 25 / 08$ & $8 \mathrm{~F} 20002$ & AGG-RRL-001 \\
\hline 13967-70-9 & Cesium-134 & $<3.24 \mathrm{E}-1$ & pCi/g dry & $3.24 \mathrm{E}-1$ & & 6/25/08 & $8 \mathrm{~F} 20002$ & AGG-RRL-001 \\
\hline 10045-97-3 & Cesium-137 & $<3.20 \mathrm{E}-1$ & pCi/g dry & $3.20 \mathrm{E}-1$ & & $6 / 25 / 08$ & $8 \mathrm{~F} 20002$ & AGG-RRL-001 \\
\hline 13982-30-4 & Cerium-139 & $<3.18 \mathrm{E}-1$ & pCi/g dry & $3.18 \mathrm{E}-1$ & & 6/25/08 & $8 F 20002$ & AGG-RRL-001 \\
\hline 14762-78-8 & Cerium-144 & $<2.14 \mathrm{E} 0$ & pCi/g dry & $2.14 \mathrm{E} 0$ & & 6/25/08 & $8 F 20002$ & AGG-RRL-001 \\
\hline 14683-23-9 & Europium-152 & $<1.04 \mathrm{E} 0$ & pCi/g dry & $1.04 \mathrm{E} 0$ & & 6/25/08 & $8 \mathrm{~F} 20002$ & AGG-RRL-001 \\
\hline $14276-65-4$ & Gadolinium-153 & $<8.08 \mathrm{E}-1$ & pCi/g dry & $8.08 \mathrm{E}-1$ & & $6 / 25 / 08$ & 8F20002 & AGG-RRL-001 \\
\hline
\end{tabular}


GEA/Soil

\begin{tabular}{|c|c|c|c|c|c|c|c|c|}
\hline CAS \# & Analyte & Results & Units & MDA & UNC & Analyzed & Batch & Method \\
\hline HEIS No. & B1VJ64C & & b ID: & 05020-30 & & & & \\
\hline $15585-10-1$ & Europium-154 & $<5.68 \mathrm{E}-1$ & pCi/g dry & $5.68 \mathrm{E}-1$ & & 6/25/08 & $8 F 20002$ & AGG-RRL-001 \\
\hline 14391-16-3 & Europium-155 & $<8.30 \mathrm{E}-1$ & pCi/g dry & $8.30 \mathrm{E}-1$ & & $6 / 25 / 08$ & $8 \mathrm{~F} 20002$ & AGG-RRL-001 \\
\hline 13982-78-0 & Mercury-203 & $<3.28 \mathrm{E}-1$ & pCi/g dry & $3.28 \mathrm{E}-1$ & & $6 / 25 / 08$ & $8 \mathrm{~F} 20002$ & AGG-RRL-001 \\
\hline 14913-50-9 & Thallium-208 & $<2.55 \mathrm{E}-1$ & pCi/g dry & $2.55 \mathrm{E}-1$ & & $6 / 25 / 08$ & $8 \mathrm{~F} 20002$ & AGG-RRL-001 \\
\hline 14331-79-4 & Bismuth-210 & $<5.19 \mathrm{E}-1$ & pCi/g dry & $5.19 \mathrm{E}-1$ & & 6/25/08 & $8 \mathrm{~F} 20002$ & AGG-RRL-001 \\
\hline \multirow[t]{3}{*}{$14255-04-0$} & Lead-210 & $<1.01 \mathrm{E} 2$ & pCi/g dry & $1.01 \mathrm{E} 2$ & & $6 / 25 / 08$ & $8 \mathrm{~F} 20002$ & AGG-RRL-001 \\
\hline & Bismuth-211 & $<6.33 \mathrm{E} 0$ & pCi/g dry & 6.33Е0 & & 6/25/08 & $8 \mathrm{~F} 20002$ & AGG-RRL-001 \\
\hline & Lead-211 & $<8.65 \mathrm{E} 0$ & pCi/g dry & 8.65E0 & & $6 / 25 / 08$ & $8 \mathrm{~F} 20002$ & AGG-RRL-001 \\
\hline 15092-94-1 & Lead-212 & 5.99E-1 & pCi/g dry & $5.01 \mathrm{E}-1$ & 0.22 & 6/25/08 & $8 \mathrm{~F} 20002$ & AGG-RRL-001 \\
\hline 14733-03-0 & Bismuth-214 & $<7.26 \mathrm{E}-1$ & pCi/g dry & 7.26E-1 & & $6 / 25 / 08$ & $8 \mathrm{~F} 20002$ & AGG-RRL-001 \\
\hline $15067-28-4$ & Lead-214 & $<8.48 \mathrm{E}-1$ & pCi/g dry & $8.48 \mathrm{E}-1$ & & 6/25/08 & $8 \mathrm{~F} 20002$ & AGG-RRL-001 \\
\hline $14835-02-0$ & Radon-219 & $<2.59 \mathrm{E} 0$ & pCi/g dry & $2.59 \mathrm{E} 0$ & & $6 / 25 / 08$ & $8 \mathrm{~F} 20002$ & AGG-RRL-001 \\
\hline 22481-48-7 & Radon-220 & $<2.32 \mathrm{E} 2$ & pCi/g dry & 2.32E2 & & 6/25/08 & $8 \mathrm{~F} 20002$ & AGG-RRL-001 \\
\hline $28522-20-5$ & Radon-221 & $<1.25 \mathrm{E} 0$ & pCi/g dry & $1.25 \mathrm{E} 0$ & & 6/25/08 & $8 \mathrm{~F} 20002$ & AGG-RRL-001 \\
\hline 15756-98-6 & Francium-223 & $<9.23 \mathrm{E} 0$ & pCi/g dry & $9.23 \mathrm{E} 0$ & & $6 / 25 / 08$ & $8 \mathrm{~F} 20002$ & AGG-RRL-001 \\
\hline $15623-45-7$ & Radium-223 & $<1.90 \mathrm{E} 0$ & pCi/g dry & $1.90 \mathrm{E} 0$ & & $6 / 25 / 08$ & $8 \mathrm{~F} 20002$ & AGG-RRL-001 \\
\hline 13233-32-4 & Radium-224 & $<4.71 \mathrm{E} 0$ & pCi/g dry & 4.71E0 & & $6 / 25 / 08$ & $8 \mathrm{~F} 20002$ & AGG-RRL-001 \\
\hline 13982-63-3 & Radium-226 & $<7.77 \mathrm{E} 0$ & pCi/g dry & 7.77E0 & & 6/25/08 & $8 \mathrm{~F} 20002$ & AGG-RRL-001 \\
\hline $15623-47-9$ & Thorium-227 & $<2.84 \mathrm{E} 0$ & pCi/g dry & $2.84 \mathrm{E} 0$ & & $6 / 25 / 08$ & $8 \mathrm{~F} 20002$ & AGG-RRL-001 \\
\hline 14331-83-0 & Actinium-228 & $<1.24 \mathrm{E} 0$ & pCi/g dry & $1.24 \mathrm{E} 0$ & & $6 / 25 / 08$ & $8 \mathrm{~F} 20002$ & AGG-RRL-001 \\
\hline $14274-82-9$ & Thorium-228 & $<9.99 \mathrm{E} 1$ & pCi/g dry & 9.99E1 & & $6 / 25 / 08$ & $8 \mathrm{~F} 20002$ & AGG-RRL-001 \\
\hline 14269-63-7 & Thorium-230 & $<1.05 \mathrm{E} 2$ & pCi/g dry & $1.05 \mathrm{E} 2$ & & $6 / 25 / 08$ & $8 \mathrm{~F} 20002$ & AGG-RRL-001 \\
\hline $14331-85-2$ & Protactinium-231 & $<1.13 \mathrm{E} 1$ & pCi/g dry & $1.13 \mathrm{E} 1$ & & $6 / 25 / 08$ & $8 \mathrm{~F} 20002$ & AGG-RRL-001 \\
\hline $14932-40-2$ & Thorium-231 & $<5.64 \mathrm{E} 1$ & pCi/g dry & $5.64 \mathrm{E} 1$ & & $6 / 25 / 08$ & $8 \mathrm{~F} 20002$ & AGG-RRL-001 \\
\hline 7440-29-1 & Thorium-232 & $<3.38 \mathrm{E} 2$ & pCi/g dry & $3.38 \mathrm{E} 2$ & & $6 / 25 / 08$ & $8 \mathrm{~F} 20002$ & AGG-RRL-001 \\
\hline 13981-14-1 & Protactinium-233 & $<7.05 \mathrm{E}-1$ & pCi/g dry & 7.05E-1 & & 6/25/08 & $8 \mathrm{~F} 20002$ & AGG-RRL-001 \\
\hline \multirow[t]{2}{*}{$15100-28-4$} & Protactinium-234 & $<1.03 \mathrm{E} 0$ & pCi/g dry & 1.03E0 & & $6 / 25 / 08$ & $8 \mathrm{~F} 20002$ & AGG-RRL-001 \\
\hline & Protactinium-234m & $<3.50 \mathrm{E} 1$ & pCi/g dry & $3.50 \mathrm{E} 1$ & & 6/25/08 & $8 \mathrm{~F} 20002$ & AGG-RRL-001 \\
\hline $15065-10-8$ & Thorium-234 & $<9.54 \mathrm{E} 0$ & pCi/g dry & $9.54 \mathrm{E} 0$ & & 6/25/08 & $8 \mathrm{~F} 20002$ & AGG-RRL-001 \\
\hline 15117-96-1 & Uranium 235 & $<2.23 \mathrm{E} 0$ & pCi/g dry & 2.23E0 & & $6 / 25 / 08$ & $8 \mathrm{~F} 20002$ & AGG-RRL-001 \\
\hline 13994-20-2 & Neptunium-237 & $<2.16 \mathrm{E} 0$ & pCi/g dry & 2.16E0 & & $6 / 25 / 08$ & $8 \mathrm{~F} 20002$ & AGG-RRL-001 \\
\hline \multirow[t]{2}{*}{ 13981-16-3 } & Plutonium-238 & $<3.20 \mathrm{E} 3$ & pCi/g dry & $3.20 \mathrm{E} 3$ & & 6/25/08 & $8 \mathrm{~F} 20002$ & AGG-RRL-001 \\
\hline & Uranium 238 & $<4.94 \mathrm{E} 0$ & pCi/g dry & $4.94 \mathrm{E} 0$ & & 6/25/08 & $8 \mathrm{~F} 20002$ & AGG-RRL-001 \\
\hline 15117-48-3 & Plutonium-239 & $<3.76 \mathrm{E} 3$ & pCi/g dry & 3.76E3 & & 6/25/08 & $8 \mathrm{~F} 20002$ & AGG-RRL-001 \\
\hline 14119-33-6 & Plutonium-240 & $<3.30 \mathrm{E} 3$ & pCi/g dry & 3.30E3 & & 6/25/08 & $8 \mathrm{~F} 20002$ & AGG-RRL-001 \\
\hline $14596-10-2$ & Americium-241 & $<1.65 \mathrm{E} 0$ & pCi/g dry & $1.65 \mathrm{E} 0$ & & 6/25/08 & $8 \mathrm{~F} 20002$ & AGG-RRL-001 \\
\hline $14993-75-0$ & Americium-243 & $<5.90 \mathrm{E}-1$ & pCi/g dry & $5.90 \mathrm{E}-1$ & & 6/25/08 & $8 \mathrm{~F} 20002$ & AGG-RRL-001 \\
\hline 15757-87-6 & Curium-243 & $<1.01 \mathrm{E} 0$ & pCi/g dry & $1.01 \mathrm{E} 0$ & & $6 / 25 / 08$ & $8 \mathrm{~F} 20002$ & AGG-RRL-001 \\
\hline 15621-76-8 & Curium-245 & $<8.26 \mathrm{E}-1$ & pCi/g dry & 8.26E-1 & & $6 / 25 / 08$ & 8F20002 & AGG-RRL-001 \\
\hline HEIS No. & B1VJ64B & & b ID: & 05020-31 & & & & \\
\hline 13966-32-0 & Sodium-22 & $<3.40 \mathrm{E}-1$ & pCi/g dry & $3.40 \mathrm{E}-1$ & & $6 / 25 / 08$ & 8F20002 & AGG-RRL-001 \\
\hline 13966-00-2 & Potassium-40 & 1.77E1 & pCi/g dry & $3.10 \mathrm{E} 0$ & 1.17 & $6 / 25 / 08$ & $8 \mathrm{~F} 20002$ & AGG-RRL-001 \\
\hline $14392-02-0$ & Chromium-51 & $<3.02 \mathrm{E} 0$ & pCi/g dry & 3.02E0 & & 6/25/08 & $8 \mathrm{~F} 20002$ & AGG-RRL-001 \\
\hline 13966-31-9 & Manganese-54 & $<3.12 \mathrm{E}-1$ & pCi/g dry & $3.12 \mathrm{E}-1$ & & 6/25/08 & $8 \mathrm{~F} 20002$ & AGG-RRL-001 \\
\hline 13981-50-5 & Cobalt-57 & $<3.26 \mathrm{E}-1$ & pCi/g dry & $3.26 \mathrm{E}-1$ & & 6/25/08 & $8 \mathrm{~F} 20002$ & AGG-RRL-001 \\
\hline $14596-12-4$ & Iron-59 & $<6.15 \mathrm{E}-1$ & pCi/g dry & $6.15 \mathrm{E}-1$ & & 6/25/08 & $8 \mathrm{~F} 20002$ & AGG-RRL-001 \\
\hline 10198-40-0 & Cobalt-60 & $<3.32 \mathrm{E}-1$ & pCi/g dry & 3.32E-1 & & 6/25/08 & $8 \mathrm{~F} 20002$ & AGG-RRL-001 \\
\hline 13982-39-3 & Zinc-65 & $<7.45 \mathrm{E}-1$ & pCi/g dry & 7.45E-1 & & $6 / 25 / 08$ & 8F20002 & AGG-RRL-001 \\
\hline
\end{tabular}


GEA/Soil

\begin{tabular}{|c|c|c|c|c|c|c|c|c|}
\hline CAS \# & Analyte & Results & Units & MDA & UNC & Analyzed & Batch & Method \\
\hline HEIS No. & B1VJ64B & & ab ID: $\quad 0$ & $5020-31$ & & & & \\
\hline 14265-71-5 & Selenium-75 & $<5.14 \mathrm{E}-1$ & pCi/g dry & $5.14 \mathrm{E}-1$ & & 6/25/08 & 8F20002 & AGG-RRL-001 \\
\hline 17056-36-9 & Rubidium-83 & $<7.63 \mathrm{E}-1$ & pCi/g dry & 7.63E-1 & & 6/25/08 & $8 \mathrm{~F} 20002$ & AGG-RRL-001 \\
\hline 13967-73-2 & Strontium-85 & $<3.59 \mathrm{E}-1$ & pCi/g dry & $3.59 \mathrm{E}-1$ & & $6 / 25 / 08$ & $8 \mathrm{~F} 20002$ & AGG-RRL-001 \\
\hline 14932-53-7 & Rubidium-86 & $<3.87 \mathrm{E} 0$ & pCi/g dry & 3.87E0 & & 6/25/08 & $8 \mathrm{~F} 20002$ & AGG-RRL-001 \\
\hline 13982-36-0 & Yttrium-88 & $<3.19 \mathrm{E}-1$ & pCi/g dry & 3.19E-1 & & 6/25/08 & $8 \mathrm{~F} 20002$ & AGG-RRL-001 \\
\hline 14681-63-1 & Niobium-94 & $<3.00 \mathrm{E}-1$ & pCi/g dry & $3.00 \mathrm{E}-1$ & & 6/25/08 & 8 F20002 & AGG-RRL-001 \\
\hline \multirow[t]{3}{*}{ 13967-76-5 } & Niobium-95 & $<3.15 \mathrm{E}-1$ & pCi/g dry & $3.15 \mathrm{E}-1$ & & 6/25/08 & $8 \mathrm{~F} 20002$ & AGG-RRL-001 \\
\hline & Niobium-95m & $<1.44 \mathrm{E} 0$ & pCi/g dry & $1.44 \mathrm{E} 0$ & & 6/25/08 & $8 \mathrm{~F} 20002$ & AGG-RRL-001 \\
\hline & Technetium-95m & $<4.86 \mathrm{E}-1$ & pCi/g dry & 4.86E-1 & & 6/25/08 & $8 \mathrm{~F} 20002$ & AGG-RRL-001 \\
\hline \multirow[t]{2}{*}{ 13967-71-0 } & Zirconium-95 & $<5.36 \mathrm{E}-1$ & pCi/g dry & $5.36 \mathrm{E}-1$ & & $6 / 25 / 08$ & $8 \mathrm{~F} 20002$ & AGG-RRL-001 \\
\hline & Technetium-99m & $<3.24 \mathrm{E}-1$ & pCi/g dry & $3.24 \mathrm{E}-1$ & & $6 / 25 / 08$ & $8 \mathrm{~F} 20002$ & AGG-RRL-001 \\
\hline 13968-53-1 & Ruthenium-103 & $<3.46 \mathrm{E}-1$ & pCi/g dry & $3.46 \mathrm{E}-1$ & & $6 / 25 / 08$ & $8 \mathrm{~F} 20002$ & AGG-RRL-001 \\
\hline 13967-48-1 & Ruthenium-106 & $<3.58 \mathrm{E} 0$ & pCi/g dry & 3.58E0 & & 6/25/08 & $8 \mathrm{~F} 20002$ & AGG-RRL-001 \\
\hline 14391-65-2 & Silver-108m & $<2.99 \mathrm{E}-1$ & pCi/g dry & 2.99E-1 & & 6/25/08 & $8 \mathrm{~F} 20002$ & AGG-RRL-001 \\
\hline \multirow[t]{2}{*}{ 14109-32-1 } & Cadmium-109 & $<9.15 \mathrm{E} 0$ & pCi/g dry & $9.15 \mathrm{E} 0$ & & 6/25/08 & $8 \mathrm{~F} 20002$ & AGG-RRL-001 \\
\hline & Silver-110 & $<3.13 \mathrm{E}-1$ & pCi/g dry & 3.13E-1 & & $6 / 25 / 08$ & $8 \mathrm{~F} 20002$ & AGG-RRL-001 \\
\hline 14391-76-5 & Silver-110m & $<3.14 \mathrm{E}-1$ & pCi/g dry & $3.14 \mathrm{E}-1$ & & 6/25/08 & $8 \mathrm{~F} 20002$ & AGG-RRL-001 \\
\hline 13966-06-8 & Tin-113 & $<4.44 \mathrm{E}-1$ & pCi/g dry & 4.44E-1 & & 6/25/08 & $8 \mathrm{~F} 20002$ & AGG-RRL-001 \\
\hline 14683-10-4 & Antimony-124 & $<3.75 \mathrm{E}-1$ & pCi/g dry & $3.75 \mathrm{E}-1$ & & 6/25/08 & $8 \mathrm{~F} 20002$ & AGG-RRL-001 \\
\hline 14234-35-6 & Antimony-125 & $<9.29 \mathrm{E}-1$ & pCi/g dry & $9.29 \mathrm{E}-1$ & & 6/25/08 & $8 \mathrm{~F} 20002$ & AGG-RRL-001 \\
\hline 15756-32-8 & Antimony-126 & $<2.93 \mathrm{E}-1$ & pCi/g dry & 2.93E-1 & & $6 / 25 / 08$ & $8 \mathrm{~F} 20002$ & AGG-RRL-001 \\
\hline 15832-50-5 & Tin-126 & $<7.46 \mathrm{E}-1$ & pCi/g dry & 7.46E-1 & & 6/25/08 & $8 \mathrm{~F} 20002$ & AGG-RRL-001 \\
\hline 10043-66-0 & Iodine-131 & $<3.91 \mathrm{E}-1$ & pCi/g dry & $3.91 \mathrm{E}-1$ & & $6 / 25 / 08$ & $8 \mathrm{~F} 20002$ & AGG-RRL-001 \\
\hline 13981-41-4 & Barium-133 & $<5.49 \mathrm{E}-1$ & pCi/g dry & $5.49 \mathrm{E}-1$ & & 6/25/08 & $8 \mathrm{~F} 20002$ & AGG-RRL-001 \\
\hline 13967-70-9 & Cesium-134 & $<3.76 \mathrm{E}-1$ & pCi/g dry & $3.76 \mathrm{E}-1$ & & $6 / 25 / 08$ & $8 \mathrm{~F} 20002$ & AGG-RRL-001 \\
\hline $10045-97-3$ & Cesium-137 & $<3.48 \mathrm{E}-1$ & pCi/g dry & $3.48 \mathrm{E}-1$ & & 6/25/08 & $8 \mathrm{~F} 20002$ & AGG-RRL-001 \\
\hline 13982-30-4 & Cerium-139 & $<3.60 \mathrm{E}-1$ & pCi/g dry & $3.60 \mathrm{E}-1$ & & 6/25/08 & $8 \mathrm{~F} 20002$ & AGG-RRL-001 \\
\hline 14762-78-8 & Cerium-144 & $<2.62 \mathrm{E} 0$ & pCi/g dry & 2.62E0 & & 6/25/08 & $8 \mathrm{~F} 20002$ & AGG-RRL-001 \\
\hline 14683-23-9 & Europium-152 & $<1.30 \mathrm{E} 0$ & pCi/g dry & $1.30 \mathrm{E} 0$ & & 6/25/08 & $8 \mathrm{~F} 20002$ & AGG-RRL-001 \\
\hline 14276-65-4 & Gadolinium-153 & $<9.89 \mathrm{E}-1$ & pCi/g dry & $9.89 \mathrm{E}-1$ & & $6 / 25 / 08$ & $8 \mathrm{~F} 20002$ & AGG-RRL-001 \\
\hline 15585-10-1 & Europium-154 & $<6.89 \mathrm{E}-1$ & pCi/g dry & $6.89 \mathrm{E}-1$ & & $6 / 25 / 08$ & $8 \mathrm{~F} 20002$ & AGG-RRL-001 \\
\hline 14391-16-3 & Europium-155 & $<1.06 \mathrm{E} 0$ & pCi/g dry & $1.06 \mathrm{E} 0$ & & 6/25/08 & 8 F20002 & AGG-RRL-001 \\
\hline 13982-78-0 & Mercury-203 & $<3.94 \mathrm{E}-1$ & pCi/g dry & 3.94E-1 & & 6/25/08 & $8 \mathrm{~F} 20002$ & AGG-RRL-001 \\
\hline 14913-50-9 & Thallium-208 & $<4.26 \mathrm{E}-1$ & pCi/g dry & 4.26E-1 & & $6 / 25 / 08$ & $8 \mathrm{~F} 20002$ & AGG-RRL-001 \\
\hline 14331-79-4 & Bismuth-210 & $<6.13 \mathrm{E}-1$ & pCi/g dry & 6.13E-1 & & 6/25/08 & $8 \mathrm{~F} 20002$ & AGG-RRL-001 \\
\hline \multirow[t]{3}{*}{$14255-04-0$} & Lead-210 & $<4.36 \mathrm{E} 1$ & pCi/g dry & 4.36E1 & & 6/25/08 & 8 F20002 & AGG-RRL-001 \\
\hline & Bismuth-211 & $<6.90 \mathrm{E} 0$ & pCi/g dry & 6.90E0 & & 6/25/08 & $8 \mathrm{~F} 20002$ & AGG-RRL-001 \\
\hline & Lead-211 & $<9.43 \mathrm{E} 0$ & pCi/g dry & $9.43 \mathrm{E} 0$ & & $6 / 25 / 08$ & $8 \mathrm{~F} 20002$ & AGG-RRL-001 \\
\hline 15092-94-1 & Lead-212 & $<8.37 \mathrm{E}-1$ & pCi/g dry & 8.37E-1 & & 6/25/08 & $8 \mathrm{~F} 20002$ & AGG-RRL-001 \\
\hline 14733-03-0 & Bismuth-214 & $<8.13 \mathrm{E}-1$ & pCi/g dry & 8.13E-1 & & 6/25/08 & $8 \mathrm{~F} 20002$ & AGG-RRL-001 \\
\hline $15067-28-4$ & Lead-214 & $<8.94 \mathrm{E}-1$ & pCi/g dry & 8.94E-1 & & 6/25/08 & $8 \mathrm{~F} 20002$ & AGG-RRL-001 \\
\hline $14835-02-0$ & Radon-219 & $<3.10 \mathrm{E} 0$ & pCi/g dry & $3.10 \mathrm{E} 0$ & & $6 / 25 / 08$ & $8 \mathrm{~F} 20002$ & AGG-RRL-001 \\
\hline 22481-48-7 & Radon-220 & $<2.80 \mathrm{E} 2$ & pCi/g dry & $2.80 \mathrm{E} 2$ & & 6/25/08 & $8 \mathrm{~F} 20002$ & AGG-RRL-001 \\
\hline 28522-20-5 & Radon-221 & $<1.44 \mathrm{E} 0$ & pCi/g dry & $1.44 \mathrm{E} 0$ & & $6 / 25 / 08$ & $8 \mathrm{~F} 20002$ & AGG-RRL-001 \\
\hline 15756-98-6 & Francium-223 & $<1.06 \mathrm{E} 1$ & pCi/g dry & $1.06 \mathrm{E} 1$ & & 6/25/08 & $8 \mathrm{~F} 20002$ & AGG-RRL-001 \\
\hline $15623-45-7$ & Radium-223 & $<2.24 \mathrm{E} 0$ & pCi/g dry & $2.24 \mathrm{E} 0$ & & 6/25/08 & $8 \mathrm{~F} 20002$ & AGG-RRL-001 \\
\hline 13233-32-4 & Radium-224 & $<9.08 \mathrm{E} 0$ & pCi/g dry & $9.08 \mathrm{E} 0$ & & 6/25/08 & 8F20002 & AGG-RRL-001 \\
\hline 13982-63-3 & Radium-226 & $<8.87 \mathrm{E} 0$ & pCi/g dry & 8.87E0 & & $6 / 25 / 08$ & $8 \mathrm{~F} 20002$ & AGG-RRL-001 \\
\hline $15623-47-9$ & Thorium-227 & $<3.22 \mathrm{E} 0$ & pCi/g dry & 3.22E0 & & 6/25/08 & $8 F 20002$ & AGG-RRL-001 \\
\hline
\end{tabular}


GEA/Soil

\begin{tabular}{|c|c|c|c|c|c|c|c|c|}
\hline CAS \# & Analyte & Results & Units & MDA & UNC & Analyzed & Batch & Method \\
\hline HEIS No. & B1VJ64B & & ab ID: & 05020-31 & & & & \\
\hline $14331-83-0$ & Actinium-228 & $<1.39 \mathrm{E} 0$ & pCi/g dry & $1.39 \mathrm{E} 0$ & & 6/25/08 & $8 F 20002$ & AGG-RRL-001 \\
\hline 14274-82-9 & Thorium-228 & $<1.16 \mathrm{E} 2$ & pCi/g dry & $1.16 \mathrm{E} 2$ & & $6 / 25 / 08$ & $8 \mathrm{~F} 20002$ & AGG-RRL-001 \\
\hline 14269-63-7 & Thorium-230 & $<1.19 \mathrm{E} 2$ & pCi/g dry & $1.19 \mathrm{E} 2$ & & $6 / 25 / 08$ & $8 \mathrm{~F} 20002$ & AGG-RRL-001 \\
\hline $14331-85-2$ & Protactinium-231 & $<1.34 \mathrm{E} 1$ & pCi/g dry & $1.34 \mathrm{E} 1$ & & $6 / 25 / 08$ & $8 \mathrm{~F} 20002$ & AGG-RRL-001 \\
\hline $14932-40-2$ & Thorium-231 & $<7.17 \mathrm{E} 1$ & pCi/g dry & 7.17E1 & & 6/25/08 & $8 \mathrm{~F} 20002$ & AGG-RRL-001 \\
\hline 7440-29-1 & Thorium-232 & $<2.97 \mathrm{E} 2$ & pCi/g dry & 2.97E2 & & $6 / 25 / 08$ & $8 \mathrm{~F} 20002$ & AGG-RRL-001 \\
\hline 13981-14-1 & Protactinium-233 & $<8.24 \mathrm{E}-1$ & pCi/g dry & $8.24 \mathrm{E}-1$ & & 6/25/08 & $8 F 20002$ & AGG-RRL-001 \\
\hline \multirow[t]{2}{*}{$15100-28-4$} & Protactinium-234 & $<1.29 \mathrm{E} 0$ & pCi/g dry & $1.29 \mathrm{E} 0$ & & $6 / 25 / 08$ & $8 \mathrm{~F} 20002$ & AGG-RRL-001 \\
\hline & Protactinium-234m & $<4.19 \mathrm{E} 1$ & pCi/g dry & 4.19E1 & & 6/25/08 & $8 \mathrm{~F} 20002$ & AGG-RRL-001 \\
\hline $15065-10-8$ & Thorium-234 & $<1.03 \mathrm{E} 1$ & pCi/g dry & 1.03E1 & & 6/25/08 & $8 \mathrm{~F} 20002$ & AGG-RRL-001 \\
\hline 15117-96-1 & Uranium 235 & $<2.76 \mathrm{E} 0$ & pCi/g dry & $2.76 \mathrm{E} 0$ & & $6 / 25 / 08$ & $8 \mathrm{~F} 20002$ & AGG-RRL-001 \\
\hline 13994-20-2 & Neptunium-237 & $<2.76 \mathrm{E} 0$ & pCi/g dry & 2.76E0 & & $6 / 25 / 08$ & $8 \mathrm{~F} 20002$ & AGG-RRL-001 \\
\hline \multirow[t]{2}{*}{ 13981-16-3 } & Plutonium-238 & $<4.09 \mathrm{E} 3$ & pCi/g dry & 4.09E3 & & $6 / 25 / 08$ & $8 \mathrm{~F} 20002$ & AGG-RRL-001 \\
\hline & Uranium 238 & $<5.79 \mathrm{E} 0$ & pCi/g dry & 5.79E0 & & 6/25/08 & $8 \mathrm{~F} 20002$ & AGG-RRL-001 \\
\hline 15117-48-3 & Plutonium-239 & $<4.20 \mathrm{E} 3$ & pCi/g dry & $4.20 \mathrm{E} 3$ & & 6/25/08 & $8 \mathrm{~F} 20002$ & AGG-RRL-001 \\
\hline 14119-33-6 & Plutonium-240 & $<4.11 \mathrm{E} 3$ & pCi/g dry & 4.11E3 & & $6 / 25 / 08$ & $8 \mathrm{~F} 20002$ & AGG-RRL-001 \\
\hline $14596-10-2$ & Americium-241 & $<1.51 \mathrm{E} 0$ & pCi/g dry & $1.51 \mathrm{E} 0$ & & $6 / 25 / 08$ & $8 \mathrm{~F} 20002$ & AGG-RRL-001 \\
\hline $14993-75-0$ & Americium-243 & $<6.50 \mathrm{E}-1$ & pCi/g dry & $6.50 \mathrm{E}-1$ & & 6/25/08 & $8 \mathrm{~F} 20002$ & AGG-RRL-001 \\
\hline $15757-87-6$ & Curium-243 & $<1.26 \mathrm{E} 0$ & pCi/g dry & $1.26 \mathrm{E} 0$ & & $6 / 25 / 08$ & $8 \mathrm{~F} 20002$ & AGG-RRL-001 \\
\hline 15621-76-8 & Curium-245 & $<1.03 \mathrm{E} 0$ & pCi/g dry & 1.03E0 & & $6 / 25 / 08$ & $8 \mathrm{~F} 20002$ & AGG-RRL-001 \\
\hline HEIS No. & B1VJ64A & & ab ID: & 05020-32 & & & & \\
\hline $13966-32-0$ & Sodium-22 & $<3.28 \mathrm{E}-1$ & pCi/g dry & $3.28 \mathrm{E}-1$ & & $6 / 25 / 08$ & 8F20002 & AGG-RRL-001 \\
\hline 13966-00-2 & Potassium-40 & 2.38E1 & pCi/g dry & $2.78 \mathrm{E} 0$ & 1.3 & $6 / 25 / 08$ & 8F20002 & AGG-RRL-001 \\
\hline $14392-02-0$ & Chromium-51 & $<2.52 \mathrm{E} 0$ & pCi/g dry & $2.52 \mathrm{E} 0$ & & $6 / 25 / 08$ & $8 \mathrm{~F} 20002$ & AGG-RRL-001 \\
\hline 13966-31-9 & Manganese-54 & $<2.58 \mathrm{E}-1$ & pCi/g dry & $2.58 \mathrm{E}-1$ & & $6 / 25 / 08$ & 8F20002 & AGG-RRL-001 \\
\hline 13981-50-5 & Cobalt-57 & $<2.71 \mathrm{E}-1$ & pCi/g dry & $2.71 \mathrm{E}-1$ & & 6/25/08 & $8 F 20002$ & AGG-RRL-001 \\
\hline $14596-12-4$ & Iron-59 & $<5.58 \mathrm{E}-1$ & pCi/g dry & $5.58 \mathrm{E}-1$ & & 6/25/08 & $8 \mathrm{~F} 20002$ & AGG-RRL-001 \\
\hline 10198-40-0 & Cobalt-60 & $<2.78 \mathrm{E}-1$ & pCi/g dry & $2.78 \mathrm{E}-1$ & & 6/25/08 & 8F20002 & AGG-RRL-001 \\
\hline 13982-39-3 & Zinc-65 & $<6.66 \mathrm{E}-1$ & pCi/g dry & $6.66 \mathrm{E}-1$ & & 6/25/08 & 8F20002 & AGG-RRL-001 \\
\hline $14265-71-5$ & Selenium-75 & $<4.33 \mathrm{E}-1$ & pCi/g dry & 4.33E-1 & & 6/25/08 & $8 F 20002$ & AGG-RRL-001 \\
\hline 17056-36-9 & Rubidium-83 & $<5.14 \mathrm{E}-1$ & pCi/g dry & $5.14 \mathrm{E}-1$ & & $6 / 25 / 08$ & $8 \mathrm{~F} 20002$ & AGG-RRL-001 \\
\hline 13967-73-2 & Strontium-85 & $<3.51 \mathrm{E}-1$ & pCi/g dry & $3.51 \mathrm{E}-1$ & & $6 / 25 / 08$ & 8F20002 & AGG-RRL-001 \\
\hline $14932-53-7$ & Rubidium-86 & $<3.09 \mathrm{E} 0$ & pCi/g dry & 3.09E0 & & 6/25/08 & $8 F 20002$ & AGG-RRL-001 \\
\hline 13982-36-0 & Yttrium-88 & $<2.18 \mathrm{E}-1$ & pCi/g dry & $2.18 \mathrm{E}-1$ & & $6 / 25 / 08$ & $8 \mathrm{~F} 20002$ & AGG-RRL-001 \\
\hline 14681-63-1 & Niobium-94 & $<2.61 \mathrm{E}-1$ & pCi/g dry & 2.61E-1 & & $6 / 25 / 08$ & 8F20002 & AGG-RRL-001 \\
\hline \multirow[t]{3}{*}{ 13967-76-5 } & Niobium-95 & $<2.76 \mathrm{E}-1$ & pCi/g dry & $2.76 \mathrm{E}-1$ & & 6/25/08 & $8 \mathrm{~F} 20002$ & AGG-RRL-001 \\
\hline & Niobium-95m & $<1.28 \mathrm{E} 0$ & pCi/g dry & $1.28 \mathrm{E} 0$ & & 6/25/08 & $8 \mathrm{~F} 20002$ & AGG-RRL-001 \\
\hline & Technetium-95m & $<4.23 \mathrm{E}-1$ & pCi/g dry & 4.23E-1 & & 6/25/08 & $8 \mathrm{~F} 20002$ & AGG-RRL-001 \\
\hline \multirow[t]{2}{*}{ 13967-71-0 } & Zirconium-95 & $<4.92 \mathrm{E}-1$ & pCi/g dry & $4.92 \mathrm{E}-1$ & & $6 / 25 / 08$ & $8 \mathrm{~F} 20002$ & AGG-RRL-001 \\
\hline & Technetium-99m & $<2.68 \mathrm{E}-1$ & pCi/g dry & $2.68 \mathrm{E}-1$ & & 6/25/08 & 8F20002 & AGG-RRL-001 \\
\hline 13968-53-1 & Ruthenium-103 & $<2.76 \mathrm{E}-1$ & pCi/g dry & $2.76 \mathrm{E}-1$ & & $6 / 25 / 08$ & 8F20002 & AGG-RRL-001 \\
\hline 13967-48-1 & Ruthenium-106 & $<2.52 \mathrm{E} 0$ & pCi/g dry & $2.52 \mathrm{E} 0$ & & $6 / 25 / 08$ & $8 \mathrm{~F} 20002$ & AGG-RRL-001 \\
\hline $14391-65-2$ & Silver-108m & $<2.74 \mathrm{E}-1$ & pCi/g dry & $2.74 \mathrm{E}-1$ & & 6/25/08 & $8 \mathrm{~F} 20002$ & AGG-RRL-001 \\
\hline \multirow[t]{2}{*}{ 14109-32-1 } & Cadmium-109 & $<7.22 \mathrm{E} 0$ & pCi/g dry & 7.22E0 & & 6/25/08 & $8 \mathrm{~F} 20002$ & AGG-RRL-001 \\
\hline & Silver-110 & $<2.84 \mathrm{E}-1$ & pCi/g dry & $2.84 \mathrm{E}-1$ & & 6/25/08 & $8 \mathrm{~F} 20002$ & AGG-RRL-001 \\
\hline 14391-76-5 & Silver-110m & $<2.84 \mathrm{E}-1$ & pCi/g dry & $2.84 \mathrm{E}-1$ & & 6/25/08 & $8 \mathrm{~F} 20002$ & AGG-RRL-001 \\
\hline 13966-06-8 & Tin-113 & $<4.07 \mathrm{E}-1$ & pCi/g dry & 4.07E-1 & & 6/25/08 & $8 \mathrm{~F} 20002$ & AGG-RRL-001 \\
\hline $14683-10-4$ & Antimony-124 & $<2.82 \mathrm{E}-1$ & pCi/g dry & 2.82E-1 & & $6 / 25 / 08$ & 8F20002 & AGG-RRL-001 \\
\hline
\end{tabular}


GEA/Soil

\begin{tabular}{|c|c|c|c|c|c|c|c|c|}
\hline CAS \# & Analyte & Results & Units & MDA & UNC & Analyzed & Batch & Method \\
\hline HEIS No. & B1VJ64A & \multicolumn{3}{|c|}{ Lab ID: $\quad 0805020-32$} & & & & \\
\hline 14234-35-6 & Antimony-125 & $<8.69 \mathrm{E}-1$ & pCi/g dry & 8.69E-1 & & 6/25/08 & $8 \mathrm{~F} 20002$ & AGG-RRL-001 \\
\hline 15756-32-8 & Antimony-126 & $<2.61 \mathrm{E}-1$ & pCi/g dry & $2.61 \mathrm{E}-1$ & & $6 / 25 / 08$ & $8 \mathrm{~F} 20002$ & AGG-RRL-001 \\
\hline $15832-50-5$ & Tin-126 & $<5.67 \mathrm{E}-1$ & pCi/g dry & 5.67E-1 & & $6 / 25 / 08$ & $8 \mathrm{~F} 20002$ & AGG-RRL-001 \\
\hline $10043-66-0$ & Iodine-131 & $<3.17 \mathrm{E}-1$ & pCi/g dry & 3.17E-1 & & $6 / 25 / 08$ & $8 \mathrm{~F} 20002$ & AGG-RRL-001 \\
\hline 13981-41-4 & Barium-133 & $<5.12 \mathrm{E}-1$ & pCi/g dry & $5.12 \mathrm{E}-1$ & & 6/25/08 & $8 \mathrm{~F} 20002$ & AGG-RRL-001 \\
\hline 13967-70-9 & Cesium-134 & $<3.06 \mathrm{E}-1$ & pCi/g dry & 3.06E-1 & & 6/25/08 & 8 F20002 & AGG-RRL-001 \\
\hline 10045-97-3 & Cesium-137 & $<3.19 \mathrm{E}-1$ & pCi/g dry & $3.19 \mathrm{E}-1$ & & 6/25/08 & $8 \mathrm{~F} 20002$ & AGG-RRL-001 \\
\hline 13982-30-4 & Cerium-139 & $<3.16 \mathrm{E}-1$ & pCi/g dry & $3.16 \mathrm{E}-1$ & & $6 / 25 / 08$ & $8 \mathrm{~F} 20002$ & AGG-RRL-001 \\
\hline 14762-78-8 & Cerium-144 & $<2.20 \mathrm{E} 0$ & pCi/g dry & $2.20 \mathrm{E} 0$ & & 6/25/08 & $8 \mathrm{~F} 20002$ & AGG-RRL-001 \\
\hline 14683-23-9 & Europium-152 & $<1.10 \mathrm{E} 0$ & pCi/g dry & $1.10 \mathrm{E} 0$ & & $6 / 25 / 08$ & $8 \mathrm{~F} 20002$ & AGG-RRL-001 \\
\hline 14276-65-4 & Gadolinium-153 & $<8.04 \mathrm{E}-1$ & pCi/g dry & 8.04E-1 & & $6 / 25 / 08$ & $8 \mathrm{~F} 20002$ & AGG-RRL-001 \\
\hline 15585-10-1 & Europium-154 & $<5.83 \mathrm{E}-1$ & pCi/g dry & $5.83 \mathrm{E}-1$ & & $6 / 25 / 08$ & $8 \mathrm{~F} 20002$ & AGG-RRL-001 \\
\hline 14391-16-3 & Europium-155 & $<7.97 \mathrm{E}-1$ & pCi/g dry & 7.97E-1 & & 6/25/08 & $8 \mathrm{~F} 20002$ & AGG-RRL-001 \\
\hline 13982-78-0 & Mercury-203 & $<3.41 \mathrm{E}-1$ & pCi/g dry & $3.41 \mathrm{E}-1$ & & 6/25/08 & $8 \mathrm{~F} 20002$ & AGG-RRL-001 \\
\hline 14913-50-9 & Thallium-208 & $<3.31 \mathrm{E}-1$ & pCi/g dry & 3.31E-1 & & 6/25/08 & $8 \mathrm{~F} 20002$ & AGG-RRL-001 \\
\hline 14331-79-4 & Bismuth-210 & $<5.19 \mathrm{E}-1$ & pCi/g dry & $5.19 \mathrm{E}-1$ & & 6/25/08 & $8 \mathrm{~F} 20002$ & AGG-RRL-001 \\
\hline \multirow[t]{3}{*}{$14255-04-0$} & Lead-210 & $<1.01 \mathrm{E} 2$ & pCi/g dry & $1.01 \mathrm{E} 2$ & & 6/25/08 & $8 \mathrm{~F} 20002$ & AGG-RRL-001 \\
\hline & Bismuth-211 & $<6.58 \mathrm{E} 0$ & pCi/g dry & $6.58 \mathrm{E} 0$ & & 6/25/08 & $8 \mathrm{~F} 20002$ & AGG-RRL-001 \\
\hline & Lead-211 & $<8.99 \mathrm{E} 0$ & pCi/g dry & 8.99E0 & & $6 / 25 / 08$ & $8 \mathrm{~F} 20002$ & AGG-RRL-001 \\
\hline 15092-94-1 & Lead-212 & 8.06E-1 & pCi/g dry & $6.84 \mathrm{E}-1$ & 0.157 & 6/25/08 & $8 \mathrm{~F} 20002$ & AGG-RRL-001 \\
\hline 14733-03-0 & Bismuth-214 & $<7.10 \mathrm{E}-1$ & pCi/g dry & 7.10E-1 & & 6/25/08 & $8 \mathrm{~F} 20002$ & AGG-RRL-001 \\
\hline 15067-28-4 & Lead-214 & $<8.22 \mathrm{E}-1$ & pCi/g dry & $8.22 \mathrm{E}-1$ & & 6/25/08 & $8 \mathrm{~F} 20002$ & AGG-RRL-001 \\
\hline 14835-02-0 & Radon-219 & $<2.73 \mathrm{E} 0$ & pCi/g dry & 2.73E0 & & $6 / 25 / 08$ & $8 \mathrm{~F} 20002$ & AGG-RRL-001 \\
\hline 22481-48-7 & Radon-220 & $<2.23 \mathrm{E} 2$ & pCi/g dry & 2.23E2 & & 6/25/08 & $8 \mathrm{~F} 20002$ & AGG-RRL-001 \\
\hline 28522-20-5 & Radon-221 & $<1.24 \mathrm{E} 0$ & pCi/g dry & $1.24 \mathrm{E} 0$ & & $6 / 25 / 08$ & $8 \mathrm{~F} 20002$ & AGG-RRL-001 \\
\hline 15756-98-6 & Francium-223 & $<9.36 \mathrm{E} 0$ & pCi/g dry & 9.36E0 & & 6/25/08 & $8 \mathrm{~F} 20002$ & AGG-RRL-001 \\
\hline 15623-45-7 & Radium-223 & $<1.94 \mathrm{E} 0$ & pCi/g dry & $1.94 \mathrm{E} 0$ & & $6 / 25 / 08$ & $8 \mathrm{~F} 20002$ & AGG-RRL-001 \\
\hline 13233-32-4 & Radium-224 & $<8.13 \mathrm{E} 0$ & pCi/g dry & 8.13E0 & & 6/25/08 & $8 \mathrm{~F} 20002$ & AGG-RRL-001 \\
\hline 13982-63-3 & Radium-226 & $<7.69 \mathrm{E} 0$ & pCi/g dry & 7.69E0 & & 6/25/08 & $8 \mathrm{~F} 20002$ & AGG-RRL-001 \\
\hline 15623-47-9 & Thorium-227 & $<2.87 \mathrm{E} 0$ & pCi/g dry & 2.87E0 & & $6 / 25 / 08$ & $8 \mathrm{~F} 20002$ & AGG-RRL-001 \\
\hline 14331-83-0 & Actinium-228 & $<1.27 \mathrm{E} 0$ & pCi/g dry & $1.27 \mathrm{E} 0$ & & $6 / 25 / 08$ & $8 \mathrm{~F} 20002$ & AGG-RRL-001 \\
\hline 14274-82-9 & Thorium-228 & $<9.73 \mathrm{E} 1$ & pCi/g dry & 9.73E1 & & 6/25/08 & 8 F20002 & AGG-RRL-001 \\
\hline 14269-63-7 & Thorium-230 & $<1.06 \mathrm{E} 2$ & pCi/g dry & $1.06 \mathrm{E} 2$ & & 6/25/08 & $8 \mathrm{~F} 20002$ & AGG-RRL-001 \\
\hline 14331-85-2 & Protactinium-231 & $<1.11 \mathrm{E} 1$ & pCi/g dry & $1.11 \mathrm{E} 1$ & & $6 / 25 / 08$ & $8 \mathrm{~F} 20002$ & AGG-RRL-001 \\
\hline 14932-40-2 & Thorium-231 & $<5.57 \mathrm{E} 1$ & pCi/g dry & $5.57 \mathrm{E} 1$ & & 6/25/08 & $8 \mathrm{~F} 20002$ & AGG-RRL-001 \\
\hline 7440-29-1 & Thorium-232 & $<3.34 \mathrm{E} 2$ & pCi/g dry & 3.34E2 & & 6/25/08 & 8 F20002 & AGG-RRL-001 \\
\hline 13981-14-1 & Protactinium-233 & $<7.03 \mathrm{E}-1$ & pCi/g dry & 7.03E-1 & & 6/25/08 & $8 \mathrm{~F} 20002$ & AGG-RRL-001 \\
\hline \multirow[t]{2}{*}{$15100-28-4$} & Protactinium-234 & $<1.02 \mathrm{E} 0$ & pCi/g dry & $1.02 \mathrm{E} 0$ & & $6 / 25 / 08$ & $8 \mathrm{~F} 20002$ & AGG-RRL-001 \\
\hline & Protactinium-234m & $<3.74 \mathrm{E} 1$ & pCi/g dry & 3.74E1 & & 6/25/08 & $8 \mathrm{~F} 20002$ & AGG-RRL-001 \\
\hline $15065-10-8$ & Thorium-234 & $<9.36 \mathrm{E} 0$ & pCi/g dry & 9.36Е0 & & 6/25/08 & $8 \mathrm{~F} 20002$ & AGG-RRL-001 \\
\hline 15117-96-1 & Uranium 235 & $<2.28 \mathrm{E} 0$ & pCi/g dry & $2.28 \mathrm{E} 0$ & & 6/25/08 & $8 \mathrm{~F} 20002$ & AGG-RRL-001 \\
\hline 13994-20-2 & Neptunium-237 & $<2.08 \mathrm{E} 0$ & pCi/g dry & $2.08 \mathrm{E} 0$ & & $6 / 25 / 08$ & $8 \mathrm{~F} 20002$ & AGG-RRL-001 \\
\hline \multirow[t]{2}{*}{ 13981-16-3 } & Plutonium-238 & $<3.19 \mathrm{E} 3$ & pCi/g dry & 3.19E3 & & 6/25/08 & $8 \mathrm{~F} 20002$ & AGG-RRL-001 \\
\hline & Uranium 238 & $<4.85 \mathrm{E} 0$ & pCi/g dry & 4.85E0 & & $6 / 25 / 08$ & $8 \mathrm{~F} 20002$ & AGG-RRL-001 \\
\hline 15117-48-3 & Plutonium-239 & $<3.87 \mathrm{E} 3$ & pCi/g dry & 3.87E3 & & 6/25/08 & $8 \mathrm{~F} 20002$ & AGG-RRL-001 \\
\hline 14119-33-6 & Plutonium-240 & $<3.24 \mathrm{E} 3$ & pCi/g dry & $3.24 \mathrm{E} 3$ & & 6/25/08 & $8 \mathrm{~F} 20002$ & AGG-RRL-001 \\
\hline $14596-10-2$ & Americium-241 & $<1.63 \mathrm{E} 0$ & pCi/g dry & $1.63 \mathrm{E} 0$ & & 6/25/08 & 8F20002 & AGG-RRL-001 \\
\hline 14993-75-0 & Americium-243 & $<5.93 \mathrm{E}-1$ & pCi/g dry & 5.93E-1 & & $6 / 25 / 08$ & $8 \mathrm{~F} 20002$ & AGG-RRL-001 \\
\hline 15757-87-6 & Curium-243 & $<9.88 \mathrm{E}-1$ & pCi/g dry & $9.88 \mathrm{E}-1$ & & $6 / 25 / 08$ & $8 F 20002$ & AGG-RRL-001 \\
\hline
\end{tabular}




\section{GEA/Soil}

\begin{tabular}{|c|c|c|c|c|c|c|c|c|}
\hline CAS \# & Analyte & Results & Units & MDA & UNC & Analyzed & Batch & Method \\
\hline HEIS No. & B1VJ64A & & b ID: & 05020-32 & & & & \\
\hline $15621-76-8$ & Curium-245 & $<8.12 \mathrm{E}-1$ & pCi/g dry & 8.12E-1 & & $6 / 25 / 08$ & 8F20002 & AGG-RRL-001 \\
\hline
\end{tabular}


Total Alpha Total Beta/Acid Extract

\begin{tabular}{|c|c|c|c|c|c|c|c|c|}
\hline CAS \# & Analyte & Results & Units & MDA & UNC & Analyzed & Batch & Method \\
\hline HEIS No. & B1VJ54B & \multicolumn{3}{|c|}{ Lab ID: $\quad$ 0805020-01 } & & & & \\
\hline $12587-47-2$ & Gross Beta & $2.34 \mathrm{E} 2$ & pCi/g dry & $1.30 \mathrm{E} 1$ & 7.05 & $7 / 02 / 08$ & 8G02015 & AGG-RRL-002 \\
\hline $12587-46-1$ & Gross Alpha & $<5.25 \mathrm{E} 0$ & pCi/g dry & $5.25 \mathrm{E} 0$ & & $7 / 02 / 08$ & 8G02015 & AGG-RRL-002 \\
\hline HEIS No. & B1VJ55C & \multicolumn{3}{|c|}{ Lab ID: $\quad$ 0805020-05 } & & & & \\
\hline $12587-47-2$ & Gross Beta & $<1.30 \mathrm{E} 1$ & pCi/g dry & $1.30 \mathrm{E} 1$ & & $7 / 02 / 08$ & 8G02015 & AGG-RRL-002 \\
\hline $12587-46-1$ & Gross Alpha & $<5.25 \mathrm{E} 0$ & pCi/g dry & 5.25E0 & & $7 / 02 / 08$ & 8G02015 & AGG-RRL-002 \\
\hline HEIS No. & B1VJ56A & \multicolumn{3}{|c|}{$\begin{array}{ll}\text { Lab ID: } & \text { 0805020-07 }\end{array}$} & & & & \\
\hline $12587-47-2$ & Gross Beta & $<1.30 \mathrm{E} 1$ & pCi/g dry & $1.30 \mathrm{E} 1$ & & $7 / 02 / 08$ & 8G02015 & AGG-RRL-002 \\
\hline $12587-46-1$ & Gross Alpha & $<5.25 \mathrm{E} 0$ & pCi/g dry & $5.25 \mathrm{E} 0$ & & $7 / 02 / 08$ & 8G02015 & AGG-RRL-002 \\
\hline HEIS No. & B1VJ56B & \multicolumn{3}{|c|}{ Lab ID: $\quad$ 0805020-08 } & & & & \\
\hline $12587-47-2$ & Gross Beta & $<1.30 \mathrm{E} 1$ & pCi/g dry & $1.30 \mathrm{E} 1$ & & $7 / 02 / 08$ & 8G02015 & AGG-RRL-002 \\
\hline $12587-46-1$ & Gross Alpha & $<5.25 \mathrm{E} 0$ & pCi/g dry & $5.25 \mathrm{E} 0$ & & $7 / 02 / 08$ & 8G02015 & AGG-RRL-002 \\
\hline HEIS No. & $\begin{array}{l}\text { B1VJ56C } \\
\end{array}$ & \multicolumn{3}{|c|}{$\begin{array}{ll}\text { Lab ID: } & \text { 0805020-09 }\end{array}$} & & & & \\
\hline $12587-47-2$ & Gross Beta & $<1.30 \mathrm{E} 1$ & pCi/g dry & $1.30 \mathrm{E} 1$ & & $7 / 02 / 08$ & 8G02015 & AGG-RRL-002 \\
\hline $12587-46-1$ & Gross Alpha & $<5.25 \mathrm{E} 0$ & pCi/g dry & $5.25 \mathrm{E} 0$ & & $7 / 02 / 08$ & 8G02015 & AGG-RRL-002 \\
\hline HEIS No. & B1VJ58C & \multicolumn{3}{|c|}{ Lab ID: $\quad$ 0805020-10 } & & & & \\
\hline $12587-47-2$ & Gross Beta & $<1.30 \mathrm{E} 1$ & pCi/g dry & $1.30 \mathrm{E} 1$ & & $7 / 02 / 08$ & 8G02015 & AGG-RRL-002 \\
\hline $12587-46-1$ & Gross Alpha & $<5.25 \mathrm{E} 0$ & pCi/g dry & $5.25 \mathrm{E} 0$ & & $7 / 02 / 08$ & 8G02015 & AGG-RRL-002 \\
\hline HEIS No. & B1VJ58B & \multicolumn{3}{|c|}{ Lab ID: $\quad$ 0805020-11 } & & & & \\
\hline $12587-47-2$ & Gross Beta & $<1.30 \mathrm{E} 1$ & pCi/g dry & $1.30 \mathrm{E} 1$ & & $7 / 02 / 08$ & 8G02015 & AGG-RRL-002 \\
\hline $12587-46-1$ & Gross Alpha & $<5.25 \mathrm{E} 0$ & pCi/g dry & $5.25 \mathrm{E} 0$ & & $7 / 02 / 08$ & 8G02015 & AGG-RRL-002 \\
\hline HEIS No. & B1VJ58A & \multicolumn{3}{|c|}{ Lab ID: $\quad$ 0805020-12 } & & & & \\
\hline $12587-47-2$ & Gross Beta & $<1.30 \mathrm{E} 1$ & pCi/g dry & $1.30 \mathrm{E} 1$ & & $7 / 02 / 08$ & 8G02015 & AGG-RRL-002 \\
\hline $12587-46-1$ & Gross Alpha & $<5.25 \mathrm{E} 0$ & pCi/g dry & $5.25 \mathrm{E} 0$ & & $7 / 02 / 08$ & 8G02015 & AGG-RRL-002 \\
\hline HEIS No. & B1VJ59C & \multicolumn{3}{|c|}{ Lab ID: $\quad$ 0805020-14 } & & & & \\
\hline $12587-47-2$ & Gross Beta & $<1.30 \mathrm{E} 1$ & pCi/g dry & $1.30 \mathrm{E} 1$ & & $7 / 02 / 08$ & 8G02015 & AGG-RRL-002 \\
\hline $12587-46-1$ & Gross Alpha & $<5.25 \mathrm{E} 0$ & pCi/g dry & $5.25 \mathrm{E} 0$ & & $7 / 02 / 08$ & 8G02015 & AGG-RRL-002 \\
\hline HEIS No. & B1VJ59B & \multicolumn{3}{|c|}{ Lab ID: $\quad$ 0805020-15 } & & & & \\
\hline $12587-47-2$ & Gross Beta & $<1.30 \mathrm{E} 1$ & pCi/g dry & $1.30 \mathrm{E} 1$ & & $7 / 02 / 08$ & 8G02015 & AGG-RRL-002 \\
\hline $12587-46-1$ & Gross Alpha & $<5.25 \mathrm{E} 0$ & pCi/g dry & $5.25 \mathrm{E} 0$ & & 7/02/08 & 8G02015 & AGG-RRL-002 \\
\hline HEIS No. & B1VJ59A & \multicolumn{3}{|c|}{ Lab ID: $\quad$ 0805020-16 } & & & & \\
\hline $12587-47-2$ & Gross Beta & $<1.30 \mathrm{E} 1$ & pCi/g dry & $1.30 \mathrm{E} 1$ & & $7 / 02 / 08$ & 8G02015 & AGG-RRL-002 \\
\hline $12587-46-1$ & Gross Alpha & $<5.25 \mathrm{E} 0$ & pCi/g dry & $5.25 \mathrm{E} 0$ & & $7 / 02 / 08$ & 8G02015 & AGG-RRL-002 \\
\hline HEIS No. & B1VJ60C & \multicolumn{3}{|c|}{$\begin{array}{ll}\text { Lab ID: } & \text { 0805020-18 }\end{array}$} & & & & \\
\hline $12587-47-2$ & Gross Beta & $<1.30 \mathrm{E} 1$ & pCi/g dry & $1.30 \mathrm{E} 1$ & & 7/02/08 & 8G02015 & AGG-RRL-002 \\
\hline $12587-46-1$ & Gross Alpha & $<5.25 \mathrm{E} 0$ & pCi/g dry & 5.25E0 & & $7 / 02 / 08$ & 8G02015 & AGG-RRL-002 \\
\hline HEIS No. & B1VJ60B & \multicolumn{3}{|c|}{ Lab ID: $\quad$ 0805020-19 } & & & & \\
\hline $12587-47-2$ & Gross Beta & $<1.30 \mathrm{E} 1$ & pCi/g dry & $1.30 \mathrm{E} 1$ & & $7 / 02 / 08$ & 8G02015 & AGG-RRL-002 \\
\hline $12587-46-1$ & Gross Alpha & $<5.25 \mathrm{E} 0$ & pCi/g dry & $5.25 \mathrm{E} 0$ & & $7 / 02 / 08$ & 8G02015 & AGG-RRL-002 \\
\hline HEIS No. & B1VJ60A & \multicolumn{3}{|c|}{ Lab ID: $\quad$ 0805020-20 } & & & & \\
\hline $12587-47-2$ & Gross Beta & $<1.30 \mathrm{E} 1$ & pCi/g dry & $1.30 \mathrm{E} 1$ & & $7 / 02 / 08$ & 8G02015 & AGG-RRL-002 \\
\hline $12587-46-1$ & Gross Alpha & $<5.25 \mathrm{E} 0$ & pCi/g dry & $5.25 \mathrm{E} 0$ & & 7/02/08 & 8G02015 & AGG-RRL-002 \\
\hline HEIS No. & B1VJ61C & \multicolumn{3}{|c|}{ Lab ID: $\quad$ 0805020-22 } & & & & \\
\hline $12587-47-2$ & Gross Beta & $<1.30 \mathrm{E} 1$ & pCi/g dry & $1.30 \mathrm{E} 1$ & & $7 / 02 / 08$ & 8G02015 & AGG-RRL-002 \\
\hline $12587-46-1$ & Gross Alpha & $<5.25 \mathrm{E} 0$ & pCi/g dry & $5.25 \mathrm{E} 0$ & & $7 / 02 / 08$ & 8G02015 & AGG-RRL-002 \\
\hline HEIS No. & B1VJ61B & \multicolumn{3}{|c|}{ Lab ID: $\quad$ 0805020-23 } & & & & \\
\hline $12587-47-2$ & Gross Beta & $<1.30 \mathrm{E} 1$ & pCi/g dry & $1.30 \mathrm{E} 1$ & & 7/02/08 & 8G02015 & AGG-RRL-002 \\
\hline
\end{tabular}


Total Alpha Total Beta/Acid Extract

\begin{tabular}{|c|c|c|c|c|c|c|c|c|}
\hline CAS \# & Analyte & Results & Units & MDA & UNC & Analyzed & Batch & Method \\
\hline HEIS No. & B1VJ61B & \multicolumn{4}{|c|}{ Lab ID: $\quad$ 0805020-23 } & & & \\
\hline $12587-46-1$ & Gross Alpha & $<5.25 \mathrm{E} 0$ & pCi/g dry & $5.25 \mathrm{E} 0$ & & 7/02/08 & 8G02015 & AGG-RRL-002 \\
\hline HEIS No. & B1VJ61A & \multicolumn{4}{|c|}{ Lab ID: $\quad$ 0805020-24 } & & & \\
\hline $12587-47-2$ & Gross Beta & $<1.30 \mathrm{E} 1$ & pCi/g dry & 1.30E1 & & 7/02/08 & 8G02015 & AGG-RRL-002 \\
\hline $12587-46-1$ & Gross Alpha & $<5.25 \mathrm{E} 0$ & pCi/g dry & $5.25 \mathrm{E} 0$ & & $7 / 02 / 08$ & 8G02015 & AGG-RRL-002 \\
\hline HEIS No. & B1VJ62C & \multicolumn{4}{|c|}{ Lab ID: $\quad$ 0805020-26 } & & & \\
\hline $12587-47-2$ & Gross Beta & $<1.30 \mathrm{E} 1$ & pCi/g dry & $1.30 \mathrm{E} 1$ & & 7/02/08 & 8G02015 & AGG-RRL-002 \\
\hline $12587-46-1$ & Gross Alpha & $<5.25 \mathrm{E} 0$ & pCi/g dry & $5.25 \mathrm{E} 0$ & & $7 / 02 / 08$ & $8 G 02015$ & AGG-RRL-002 \\
\hline HEIS No. & B1VJ62B & \multicolumn{4}{|c|}{ Lab ID: $\quad$ 0805020-27 } & & & \\
\hline $12587-47-2$ & Gross Beta & $<1.30 \mathrm{E} 1$ & pCi/g dry & 1.30E1 & & $7 / 02 / 08$ & 8G02015 & AGG-RRL-002 \\
\hline $12587-46-1$ & Gross Alpha & $<5.25 \mathrm{E} 0$ & pCi/g dry & 5.25E0 & & 7/02/08 & 8G02015 & AGG-RRL-002 \\
\hline HEIS No. & B1VJ62A & \multicolumn{4}{|c|}{ Lab ID: $\quad$ 0805020-28 } & & & \\
\hline $12587-47-2$ & Gross Beta & $<1.30 \mathrm{E} 1$ & pCi/g dry & $1.30 \mathrm{E} 1$ & & 7/02/08 & $8 G 02015$ & AGG-RRL-002 \\
\hline $12587-46-1$ & Gross Alpha & $<5.25 \mathrm{E} 0$ & pCi/g dry & 5.25E0 & & $7 / 02 / 08$ & 8G02015 & AGG-RRL-002 \\
\hline HEIS No. & B1VJ64C & \multicolumn{4}{|c|}{ Lab ID: $\quad$ 0805020-30 } & & & \\
\hline $12587-47-2$ & Gross Beta & $<1.30 \mathrm{E} 1$ & pCi/g dry & 1.30E1 & & 7/02/08 & 8G02015 & AGG-RRL-002 \\
\hline $12587-46-1$ & Gross Alpha & $<5.25 \mathrm{E} 0$ & pCi/g dry & $5.25 \mathrm{E} 0$ & & $7 / 02 / 08$ & $8 G 02015$ & AGG-RRL-002 \\
\hline HEIS No. & B1VJ64B & \multicolumn{4}{|c|}{ Lab ID: $\quad$ 0805020-31 } & & & \\
\hline $12587-47-2$ & Gross Beta & $<1.30 \mathrm{E} 1$ & pCi/g dry & $1.30 \mathrm{E} 1$ & & $7 / 02 / 08$ & 8G02015 & AGG-RRL-002 \\
\hline $12587-46-1$ & Gross Alpha & $<5.25 \mathrm{E} 0$ & pCi/g dry & 5.25E0 & & 7/02/08 & 8G02015 & AGG-RRL-002 \\
\hline HEIS No. & B1VJ64A & \multicolumn{4}{|c|}{ Lab ID: $\quad$ 0805020-32 } & & & \\
\hline $12587-47-2$ & Gross Beta & $<1.30 \mathrm{E} 1$ & pCi/g dry & 1.30E1 & & 7/02/08 & 8G02015 & AGG-RRL-002 \\
\hline $12587-46-1$ & Gross Alpha & $<5.25 \mathrm{E} 0$ & pCi/g dry & $5.25 \mathrm{E} 0$ & & 7/02/08 & 8G02015 & AGG-RRL-002 \\
\hline
\end{tabular}


Total Alpha Total Beta/Water Extract

\begin{tabular}{|c|c|c|c|c|c|c|c|c|}
\hline CAS \# & Analyte & Results & Units & MDA & UNC & Analyzed & Batch & Method \\
\hline HEIS No. & B1VJ54B & \multicolumn{4}{|c|}{ Lab ID: $\quad$ 0805020-01 } & & & \\
\hline $12587-47-2$ & Gross Beta & $<1.21 \mathrm{E} 1$ & pCi/g dry & $1.21 \mathrm{E} 1$ & & 7/01/08 & 8G02003 & AGG-RRL-002 \\
\hline $12587-46-1$ & Gross Alpha & $<5.56 \mathrm{E} 0$ & pCi/g dry & $5.56 \mathrm{E} 0$ & & $7 / 01 / 08$ & 8G02003 & AGG-RRL-002 \\
\hline HEIS No. & B1VJ55C & \multicolumn{4}{|c|}{ Lab ID: $\quad$ 0805020-05 } & & & \\
\hline $12587-47-2$ & Gross Beta & $<1.21 \mathrm{E} 1$ & pCi/g dry & $1.21 \mathrm{E} 1$ & & $7 / 01 / 08$ & 8G02003 & AGG-RRL-002 \\
\hline $12587-46-1$ & Gross Alpha & $<5.56 \mathrm{E} 0$ & pCi/g dry & $5.56 \mathrm{E} 0$ & & 7/01/08 & 8G02003 & AGG-RRL-002 \\
\hline HEIS No. & B1VJ56A & \multicolumn{4}{|c|}{ Lab ID: $\quad$ 0805020-07 } & & & \\
\hline $12587-47-2$ & Gross Beta & $<1.21 \mathrm{E} 1$ & pCi/g dry & $1.21 \mathrm{E} 1$ & & $7 / 01 / 08$ & 8G02003 & AGG-RRL-002 \\
\hline $12587-46-1$ & Gross Alpha & $<5.56 \mathrm{E} 0$ & pCi/g dry & $5.56 \mathrm{E} 0$ & & $7 / 01 / 08$ & 8G02003 & AGG-RRL-002 \\
\hline HEIS No. & B1VJ56B & \multicolumn{4}{|c|}{$\begin{array}{ll}\text { Lab ID: } & \text { 0805020-08 }\end{array}$} & & & \\
\hline $12587-47-2$ & Gross Beta & $<1.21 \mathrm{E} 1$ & pCi/g dry & $1.21 \mathrm{E} 1$ & & $7 / 01 / 08$ & 8G02003 & AGG-RRL-002 \\
\hline $12587-46-1$ & Gross Alpha & $<5.56 \mathrm{E} 0$ & pCi/g dry & 5.56E0 & & $7 / 01 / 08$ & 8G02003 & AGG-RRL-002 \\
\hline HEIS No. & B1VJ56C & \multicolumn{4}{|c|}{ Lab ID: $\quad$ 0805020-09 } & & & \\
\hline $12587-47-2$ & Gross Beta & $<1.21 \mathrm{E} 1$ & pCi/g dry & $1.21 \mathrm{E} 1$ & & 7/01/08 & 8G02003 & AGG-RRL-002 \\
\hline $12587-46-1$ & Gross Alpha & $<5.56 \mathrm{E} 0$ & pCi/g dry & 5.56E0 & & $7 / 01 / 08$ & 8G02003 & AGG-RRL-002 \\
\hline HEIS No. & B1VJ58C & \multicolumn{4}{|c|}{ Lab ID: $\quad$ 0805020-10 } & & & \\
\hline $12587-47-2$ & Gross Beta & $<1.21 \mathrm{E} 1$ & pCi/g dry & $1.21 \mathrm{E} 1$ & & $7 / 01 / 08$ & 8G02003 & AGG-RRL-002 \\
\hline $12587-46-1$ & Gross Alpha & $<5.56 \mathrm{E} 0$ & pCi/g dry & 5.56E0 & & $7 / 01 / 08$ & 8G02003 & AGG-RRL-002 \\
\hline HEIS No. & B1VJ58B & \multicolumn{4}{|c|}{ Lab ID: $\quad$ 0805020-11 } & & & \\
\hline $12587-47-2$ & Gross Beta & $<1.21 \mathrm{E} 1$ & pCi/g dry & $1.21 \mathrm{E} 1$ & & $7 / 01 / 08$ & 8G02003 & AGG-RRL-002 \\
\hline $12587-46-1$ & Gross Alpha & $<5.56 \mathrm{E} 0$ & pCi/g dry & 5.56E0 & & $7 / 01 / 08$ & 8G02003 & AGG-RRL-002 \\
\hline HEIS No. & B1VJ58A & \multicolumn{4}{|c|}{ Lab ID: $\quad$ 0805020-12 } & & & \\
\hline $12587-47-2$ & Gross Beta & $<1.21 \mathrm{E} 1$ & pCi/g dry & $1.21 \mathrm{E} 1$ & & $7 / 01 / 08$ & 8G02003 & AGG-RRL-002 \\
\hline $12587-46-1$ & Gross Alpha & $<5.56 \mathrm{E} 0$ & pCi/g dry & $5.56 \mathrm{E} 0$ & & $7 / 01 / 08$ & 8G02003 & AGG-RRL-002 \\
\hline HEIS No. & B1VJ59C & \multicolumn{4}{|c|}{ Lab ID: $\quad$ 0805020-14 } & & & \\
\hline $12587-47-2$ & Gross Beta & $<1.21 \mathrm{E} 1$ & pCi/g dry & $1.21 \mathrm{E} 1$ & & $7 / 01 / 08$ & 8G02003 & AGG-RRL-002 \\
\hline $12587-46-1$ & Gross Alpha & $<5.56 \mathrm{E} 0$ & pCi/g dry & 5.56E0 & & $7 / 01 / 08$ & 8G02003 & AGG-RRL-002 \\
\hline HEIS No. & B1VJ59B & \multicolumn{4}{|c|}{ Lab ID: $\quad$ 0805020-15 } & & & \\
\hline $12587-47-2$ & Gross Beta & $<1.21 \mathrm{E} 1$ & pCi/g dry & $1.21 \mathrm{E} 1$ & & $7 / 01 / 08$ & 8G02003 & AGG-RRL-002 \\
\hline $12587-46-1$ & Gross Alpha & $<5.56 \mathrm{E} 0$ & pCi/g dry & 5.56E0 & & 7/01/08 & 8G02003 & AGG-RRL-002 \\
\hline HEIS No. & B1VJ59A & \multicolumn{4}{|c|}{ Lab ID: $\quad$ 0805020-16 } & & & \\
\hline $12587-47-2$ & Gross Beta & $<1.21 \mathrm{E} 1$ & pCi/g dry & $1.21 \mathrm{E} 1$ & & $7 / 01 / 08$ & 8G02003 & AGG-RRL-002 \\
\hline $12587-46-1$ & Gross Alpha & $<5.56 \mathrm{E} 0$ & pCi/g dry & 5.56E0 & & $7 / 01 / 08$ & 8G02003 & AGG-RRL-002 \\
\hline HEIS No. & B1VJ60C & \multicolumn{4}{|c|}{$\begin{array}{ll}\text { Lab ID: } & \text { 0805020-18 }\end{array}$} & & & \\
\hline $12587-47-2$ & Gross Beta & $<1.21 \mathrm{E} 1$ & pCi/g dry & $1.21 \mathrm{E} 1$ & & 7/01/08 & 8G02003 & AGG-RRL-002 \\
\hline $12587-46-1$ & Gross Alpha & $<5.56 \mathrm{E} 0$ & pCi/g dry & 5.56E0 & & $7 / 01 / 08$ & 8G02003 & AGG-RRL-002 \\
\hline HEIS No. & B1VJ60B & \multicolumn{4}{|c|}{ Lab ID: $\quad 0805020-19$} & & & \\
\hline $12587-47-2$ & Gross Beta & $<1.21 \mathrm{E} 1$ & pCi/g dry & $1.21 \mathrm{E} 1$ & & $7 / 01 / 08$ & 8G02003 & AGG-RRL-002 \\
\hline $12587-46-1$ & Gross Alpha & $<5.56 \mathrm{E} 0$ & pCi/g dry & 5.56E0 & & $7 / 01 / 08$ & 8G02003 & AGG-RRL-002 \\
\hline HEIS No. & B1VJ60A & \multicolumn{4}{|c|}{$\begin{array}{ll}\text { Lab ID: } & \text { 0805020-20 }\end{array}$} & & & \\
\hline $12587-47-2$ & Gross Beta & $<1.21 \mathrm{E} 1$ & pCi/g dry & $1.21 \mathrm{E} 1$ & & $7 / 01 / 08$ & 8G02003 & AGG-RRL-002 \\
\hline $12587-46-1$ & Gross Alpha & $<5.56 \mathrm{E} 0$ & pCi/g dry & $5.56 \mathrm{E} 0$ & & 7/01/08 & 8G02003 & AGG-RRL-002 \\
\hline HEIS No. & B1VJ61C & \multicolumn{4}{|c|}{ Lab ID: $\quad$ 0805020-22 } & & & \\
\hline $12587-47-2$ & Gross Beta & $<1.21 \mathrm{E} 1$ & pCi/g dry & $1.21 \mathrm{E} 1$ & & $7 / 01 / 08$ & 8G02003 & AGG-RRL-002 \\
\hline $12587-46-1$ & Gross Alpha & $<5.56 \mathrm{E} 0$ & pCi/g dry & 5.56E0 & & 7/01/08 & 8G02003 & AGG-RRL-002 \\
\hline HEIS No. & B1VJ61B & \multicolumn{4}{|c|}{ Lab ID: $\quad$ 0805020-23 } & & & \\
\hline $12587-47-2$ & Gross Beta & $<1.21 \mathrm{E} 1$ & pCi/g dry & $1.21 \mathrm{E} 1$ & & 7/01/08 & 8G02003 & AGG-RRL-002 \\
\hline
\end{tabular}


Total Alpha Total Beta/Water Extract

\begin{tabular}{|c|c|c|c|c|c|c|c|c|}
\hline CAS \# & Analyte & Results & Units & MDA & UNC & Analyzed & Batch & Method \\
\hline HEIS No. & B1VJ61B & \multirow{2}{*}{\multicolumn{4}{|c|}{ Lab ID: 0805020-23 }} & & & \\
\hline $12587-46-1$ & Gross Alpha & & & & & 7/01/08 & 8G02003 & AGG-RRL-002 \\
\hline HEIS No. & B1VJ61A & \multicolumn{4}{|c|}{ Lab ID: $\quad$ 0805020-24 } & & & \\
\hline $12587-47-2$ & Gross Beta & $<1.21 \mathrm{E} 1$ & pCi/g dry & $1.21 \mathrm{E} 1$ & & $7 / 01 / 08$ & 8G02003 & AGG-RRL-002 \\
\hline $12587-46-1$ & Gross Alpha & $<5.56 \mathrm{E} 0$ & pCi/g dry & $5.56 \mathrm{E} 0$ & & $7 / 01 / 08$ & 8G02003 & AGG-RRL-002 \\
\hline HEIS No. & B1VJ62C & \multicolumn{4}{|c|}{ Lab ID: $\quad 0805020-26$} & & & \\
\hline $12587-47-2$ & Gross Beta & $<1.21 \mathrm{E} 1$ & pCi/g dry & $1.21 \mathrm{E} 1$ & & $7 / 01 / 08$ & 8G02003 & AGG-RRL-002 \\
\hline $12587-46-1$ & Gross Alpha & $<5.56 \mathrm{E} 0$ & pCi/g dry & $5.56 \mathrm{E} 0$ & & $7 / 01 / 08$ & 8G02003 & AGG-RRL-002 \\
\hline HEIS No. & B1VJ62B & \multicolumn{4}{|c|}{ Lab ID: $\quad$ 0805020-27 } & & & \\
\hline $12587-47-2$ & Gross Beta & $<1.21 \mathrm{E} 1$ & pCi/g dry & $1.21 \mathrm{E} 1$ & & $7 / 01 / 08$ & 8G02003 & AGG-RRL-002 \\
\hline $12587-46-1$ & Gross Alpha & $<5.56 \mathrm{E} 0$ & pCi/g dry & 5.56E0 & & $7 / 01 / 08$ & 8G02003 & AGG-RRL-002 \\
\hline HEIS No. & B1VJ62A & \multicolumn{4}{|c|}{ Lab ID: $\quad 0805020-28$} & & & \\
\hline $12587-47-2$ & Gross Beta & $<1.21 \mathrm{E} 1$ & pCi/g dry & $1.21 \mathrm{E} 1$ & & $7 / 01 / 08$ & 8G02003 & AGG-RRL-002 \\
\hline $12587-46-1$ & Gross Alpha & $<5.56 \mathrm{E} 0$ & pCi/g dry & $5.56 \mathrm{E} 0$ & & $7 / 01 / 08$ & 8G02003 & AGG-RRL-002 \\
\hline HEIS No. & B1VJ64C & \multicolumn{4}{|c|}{ Lab ID: $\quad$ 0805020-30 } & & & \\
\hline $12587-47-2$ & Gross Beta & $<1.21 \mathrm{E} 1$ & pCi/g dry & $1.21 \mathrm{E} 1$ & & $7 / 01 / 08$ & 8G02003 & AGG-RRL-002 \\
\hline $12587-46-1$ & Gross Alpha & $<5.56 \mathrm{E} 0$ & pCi/g dry & $5.56 \mathrm{E} 0$ & & $7 / 01 / 08$ & 8G02003 & AGG-RRL-002 \\
\hline HEIS No. & B1VJ64B & \multicolumn{4}{|c|}{ Lab ID: $\quad$ 0805020-31 } & & & \\
\hline $12587-47-2$ & Gross Beta & $<1.21 \mathrm{E} 1$ & pCi/g dry & $1.21 \mathrm{E} 1$ & & 7/01/08 & 8G02003 & AGG-RRL-002 \\
\hline $12587-46-1$ & Gross Alpha & $<5.56 \mathrm{E} 0$ & pCi/g dry & $5.56 \mathrm{E} 0$ & & $7 / 01 / 08$ & 8G02003 & AGG-RRL-002 \\
\hline HEIS No. & B1VJ64A & \multicolumn{4}{|c|}{ Lab ID: $\quad 0805020-32$} & & & \\
\hline $12587-47-2$ & Gross Beta & $<1.21 \mathrm{E} 1$ & pCi/g dry & $1.21 \mathrm{E} 1$ & & 7/01/08 & 8G02003 & AGG-RRL-002 \\
\hline $12587-46-1$ & Gross Alpha & $<5.56 \mathrm{E} 0$ & pCi/g dry & 5.56E0 & & $7 / 01 / 08$ & 8G02003 & AGG-RRL-002 \\
\hline
\end{tabular}


Wet Chemistry - Quality Control

Environmental Science Laboratory

\begin{tabular}{|lrrrrrrrrrr}
\hline & & Reporting & & Spike & Source & & \%REC & & RPD & \\
Analyte & Result & Limit & Units & Level & Result & \%REC & Limits & RPD & Limit & Notes \\
\hline
\end{tabular}

Batch 8F26007 - 1:1 Water Extract (pH_EC_Alk)

Blank (8F26007-BLK1)

Specific Conductance (EC)

Duplicate (8F26007-DUP1)

Specific Conductance (EC)

Batch 8F26008 - 1:1 Water Extract (pH_EC_Alk)

Blank (8F26008-BLK1)

Specific Conductance (EC)

Duplicate (8F26008-DUP1)

Specific Conductance (EC)

Batch 8F26009 - 1:1 Water Extract (pH_EC_Alk)

Duplicate (8F26009-DUP1)

$\mathrm{pH}$

Duplicate (8F26009-DUP2)

$\mathrm{pH}$

Batch 8F26010 - 1:1 Water Extract (pH_EC_Alk)

Duplicate (8F26010-DUP1)

$\mathrm{pH}$
Soun

$7.80 \mathrm{E} 0$
Source: 0805020-11 Prepared \& Analyzed: 06/26/08

9.68E0 N/A pH Units 9.64E0

Prepared \& Analyzed: 06/26/08

N/A pH Units 7.98E0

Prepared \& Analyzed: 06/26/08

Prepared \& Analyzed: 06/26/08

2.E0

2.58

35

Prepared \& Analyzed: 06/26/08

Prepared \& Analyzed: 06/26/08

4.52E-1

$14.8 \quad 35$
$0.414 \quad 35$

$0.881 \quad 35$
Prepared \& Analyzed: 06/26/08

7.79E0
0.128

35 
Wet Chemistry - Quality Control

Environmental Science Laboratory

\begin{tabular}{|lrrrrrrrrrrr}
\hline & & Reporting & & Spike & Source & & \%REC & & RPD & & \\
Analyte & Result & Limit & Units & Level & Result & \%REC & Limits & RPD & Limit & Notes \\
\hline
\end{tabular}

Batch 8F30007 - 1:1 Water Extract (pH_EC_Alk)

Blank (8F30007-BLK1)

Alkalinity as $\mathrm{CaCO} 3$

$<2.35 \mathrm{E} 1 \quad 2.35 \mathrm{E} 1 \mathrm{ug} / \mathrm{g}$ wet

Prepared \& Analyzed: 06/30/08

Duplicate (8F30007-DUP1)

Source: 0805020-11

Prepared \& Analyzed: 06/30/08

Alkalinity as $\mathrm{CaCO} 3$

9.39E2

2.47E1 ug/g dry

$9.25 \mathrm{E} 2$

35

Batch 8F30008 - 1:1 Water Extract (pH_EC_Alk)

Blank (8F30008-BLK1)

Alkalinity as $\mathrm{CaCO} 3$

$<2.35 \mathrm{E} 1$

Prepared \& Analyzed: 06/30/08

Duplicate (8F30008-DUP1)

Source: 0805020-27

Prepared \& Analyzed: 06/30/08

Alkalinity as $\mathrm{CaCO} 3$

3.69E1 2.33E1 ug/g dry

3.96E1

6.89

35 
Anions by Ion Chromatography - Quality Control

Environmental Science Laboratory

\begin{tabular}{|c|c|c|c|c|c|c|c|c|c|c|}
\hline Analyte & Result & $\begin{array}{c}\text { orting } \\
\text { Limit }\end{array}$ & Units & $\begin{array}{l}\text { Spike } \\
\text { Level }\end{array}$ & $\begin{array}{l}\text { Source } \\
\text { Result }\end{array}$ & \%REC & $\begin{array}{l}\text { \%REC } \\
\text { Limits }\end{array}$ & RPD & $\begin{array}{l}\text { RPD } \\
\text { Limit }\end{array}$ & Notes \\
\hline
\end{tabular}

Batch 8E29005 - 1:1 Water Extract (IC)

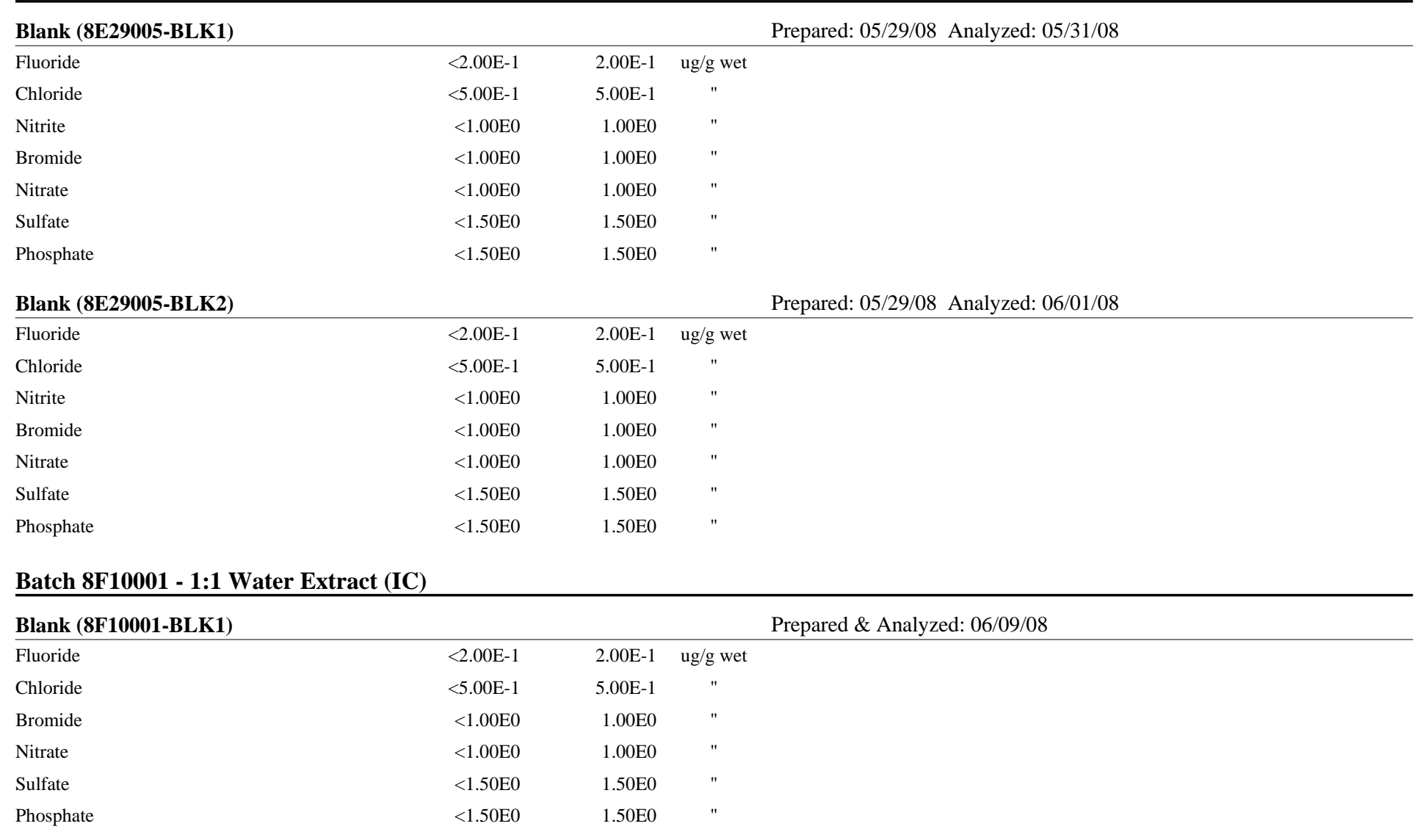


Anions by Ion Chromatography - Quality Control

Environmental Science Laboratory

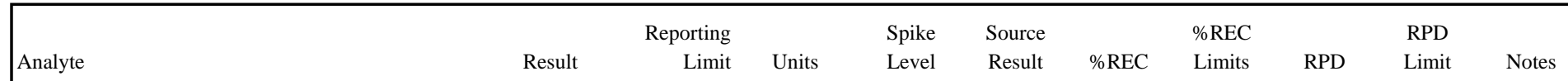

Batch 8F26011 - 1:1 Water Extract (IC)

\begin{tabular}{|c|c|c|c|c|c|c|c|c|c|}
\hline \multirow{2}{*}{$\begin{array}{l}\text { Blank (8F26011-BLK1) } \\
\text { Fluoride }\end{array}$} & \multicolumn{9}{|c|}{ Prepared: 06/26/08 Analyzed: 06/27/08 } \\
\hline & $<2.00 \mathrm{E}-1$ & $2.00 \mathrm{E}-1$ & ug/g wet & & & & & & \\
\hline Chloride & $<5.00 \mathrm{E}-1$ & $5.00 \mathrm{E}-1$ & $"$ & & & & & & \\
\hline Nitrite & $<1.00 \mathrm{E} 0$ & $1.00 \mathrm{E} 0$ & " & & & & & & \\
\hline Bromide & $<1.00 \mathrm{E} 0$ & $1.00 \mathrm{E} 0$ & $"$ & & & & & & \\
\hline Nitrate & $<1.00 \mathrm{E} 0$ & $1.00 \mathrm{E} 0$ & $"$ & & & & & & \\
\hline Sulfate & $<1.50 \mathrm{E} 0$ & $1.50 \mathrm{E} 0$ & " & & & & & & \\
\hline Phosphate & $<1.50 \mathrm{E} 0$ & $1.50 \mathrm{E} 0$ & " & & & & & & \\
\hline LCS (8F26011-BS1) & \multicolumn{9}{|c|}{ Prepared: 06/26/08 Analyzed: 06/27/08 } \\
\hline Fluoride & 2.07E0 & $2.00 \mathrm{E}-1$ & ug/g wet & 2.001 & & 103 & $80-120$ & & \\
\hline Chloride & 5.12E0 & $5.00 \mathrm{E}-1$ & $"$ & 5.002 & & 102 & $80-120$ & & \\
\hline Nitrite & 9.73E0 & $1.00 \mathrm{E} 0$ & $"$ & 10.00 & & 97.3 & $80-120$ & & \\
\hline Bromide & $1.01 \mathrm{E} 1$ & $1.00 \mathrm{E} 0$ & $"$ & 10.00 & & 101 & $80-120$ & & \\
\hline Nitrate & $1.06 \mathrm{E} 1$ & $1.00 \mathrm{E} 0$ & $"$ & 10.00 & & 106 & $80-120$ & & \\
\hline Sulfate & $1.55 \mathrm{E} 1$ & $1.50 \mathrm{E} 0$ & $"$ & 15.00 & & 103 & 80-120 & & \\
\hline Phosphate & $1.51 \mathrm{E} 1$ & $1.50 \mathrm{E} 0$ & $"$ & 15.00 & & 101 & $80-120$ & & \\
\hline Duplicate (8F26011-DUP1) & \multicolumn{3}{|c|}{ Source: 0805020-11 } & \multicolumn{6}{|c|}{ Prepared: 06/26/08 Analyzed: 07/01/08 } \\
\hline Fluoride & 2.03E1 & 2.10E0 & ug/g dry & & $2.0 \mathrm{E} 1$ & & & 1.65 & 35 \\
\hline Chloride & $<5.25 \mathrm{E} 0$ & 5.25E0 & $"$ & & ND & & & & 35 \\
\hline Nitrite & $<1.05 \mathrm{E} 1$ & $1.05 \mathrm{E} 1$ & " & & ND & & & & 35 \\
\hline Bromide & $<1.05 \mathrm{E} 1$ & $1.05 \mathrm{E} 1$ & $"$ & & ND & & & & 35 \\
\hline Nitrate & $1.93 \mathrm{E} 2$ & $1.05 \mathrm{E} 1$ & " & & $1.99 \mathrm{E} 2$ & & & 3.20 & 35 \\
\hline Sulfate & $1.83 \mathrm{E} 1$ & $1.57 \mathrm{E} 1$ & $"$ & & $1.83 \mathrm{E} 1$ & & & 0.00842 & 35 \\
\hline Phosphate & $<1.57 \mathrm{E} 1$ & $1.57 \mathrm{E} 1$ & $"$ & & ND & & & & 35 \\
\hline Post Spike (8F26011-PS1) & \multicolumn{3}{|c|}{ Source: 0805020-01 } & \multicolumn{4}{|c|}{ Prepared: 06/26/08 Analyzed: 06/27/08 } & & \\
\hline Fluoride & $1.97 \mathrm{E} 0$ & N/A & $\mathrm{ug} / \mathrm{mL}$ & 0.8 & $11.6 \mathrm{E}-1$ & 102 & $75-125$ & & \\
\hline Chloride & 2.34E0 & N/A & $"$ & 2 & $42.9 \mathrm{E}-2$ & 95.8 & $75-125$ & & \\
\hline Nitrite & 3.79E0 & N/A & $"$ & 4 & ND & 94.6 & $75-125$ & & \\
\hline Bromide & 3.8E0 & N/A & $"$ & 4 & $15.7 \mathrm{E}-2$ & 91.1 & $75-125$ & & \\
\hline Nitrate & 1.17E1 & N/A & $"$ & 4 & 80.7E-1 & 90.9 & $75-125$ & & \\
\hline Sulfate & 9.59E0 & N/A & $"$ & 6 & 46.7E-1 & 82.1 & $75-125$ & & \\
\hline Phosphate & $6.22 \mathrm{E} 0$ & N/A & " & 6 & $90.9 \mathrm{E}-2$ & 88.5 & $75-125$ & & \\
\hline
\end{tabular}


Anions by Ion Chromatography - Quality Control

Environmental Science Laboratory

\begin{tabular}{|c|c|c|c|c|c|c|c|c|c|c|}
\hline Analyte & Result & $\begin{array}{r}\text { porting } \\
\text { Limit }\end{array}$ & Units & $\begin{array}{l}\text { Spike } \\
\text { Level }\end{array}$ & $\begin{array}{l}\text { Source } \\
\text { Result }\end{array}$ & $\%$ REC & $\begin{array}{c}\text { \%REC } \\
\text { Limits }\end{array}$ & RPD & $\begin{array}{l}\text { RPD } \\
\text { Limit }\end{array}$ & Notes \\
\hline
\end{tabular}

Batch 8F26016 - 1:1 Water Extract (IC)

\begin{tabular}{|c|c|c|c|c|c|c|c|c|c|}
\hline \multirow{2}{*}{$\begin{array}{l}\text { Blank (8F26016-BLK1) } \\
\text { Fluoride }\end{array}$} & \multicolumn{9}{|c|}{ Prepared: 06/26/08 Analyzed: 06/27/08 } \\
\hline & $<2.00 \mathrm{E}-1$ & $2.00 \mathrm{E}-1$ & ug/g wet & & & & & & \\
\hline Chloride & $<5.00 \mathrm{E}-1$ & $5.00 \mathrm{E}-1$ & $"$ & & & & & & \\
\hline Nitrite & $<1.00 \mathrm{E} 0$ & $1.00 \mathrm{E} 0$ & " & & & & & & \\
\hline Bromide & $<1.00 \mathrm{E} 0$ & $1.00 \mathrm{E} 0$ & $"$ & & & & & & \\
\hline Nitrate & $<1.00 \mathrm{E} 0$ & $1.00 \mathrm{E} 0$ & $"$ & & & & & & \\
\hline Sulfate & $<1.50 \mathrm{E} 0$ & $1.50 \mathrm{E} 0$ & " & & & & & & \\
\hline Phosphate & $<1.50 \mathrm{E} 0$ & $1.50 \mathrm{E} 0$ & $"$ & & & & & & \\
\hline LCS (8F26016-BS1) & \multicolumn{9}{|c|}{ Prepared: 06/26/08 Analyzed: 06/27/08 } \\
\hline Fluoride & 2.12E0 & $2.00 \mathrm{E}-1$ & ug/g wet & 1.999 & & 106 & $80-120$ & & \\
\hline Chloride & 5.18E0 & $5.00 \mathrm{E}-1$ & $"$ & 4.997 & & 104 & $80-120$ & & \\
\hline Nitrite & $9.87 \mathrm{E} 0$ & $1.00 \mathrm{E} 0$ & $"$ & 9.993 & & 98.8 & $80-120$ & & \\
\hline Bromide & $1.01 \mathrm{E} 1$ & $1.00 \mathrm{E} 0$ & $"$ & 9.993 & & 101 & $80-120$ & & \\
\hline Nitrate & $1.08 \mathrm{E} 1$ & $1.00 \mathrm{E} 0$ & $"$ & 9.993 & & 109 & $80-120$ & & \\
\hline Sulfate & $1.55 \mathrm{E} 1$ & $1.50 \mathrm{E} 0$ & $"$ & 14.99 & & 104 & 80-120 & & \\
\hline Phosphate & $1.52 \mathrm{E} 1$ & $1.50 \mathrm{E} 0$ & $"$ & 14.99 & & 102 & $80-120$ & & \\
\hline Duplicate (8F26016-DUP1) & \multicolumn{3}{|c|}{ Source: 0805020-27 } & \multicolumn{4}{|c|}{ Prepared: 06/26/08 Analyzed: 06/27/08 } & & \\
\hline Fluoride & $<1.98 \mathrm{E} 0$ & $1.98 \mathrm{E} 0$ & ug/g dry & & ND & & & & 35 \\
\hline Chloride & $4.52 \mathrm{E} 1$ & 4.96E0 & $"$ & & $4.1 \mathrm{E} 1$ & & & 9.84 & 35 \\
\hline Nitrite & $<9.91 \mathrm{E} 0$ & $9.91 \mathrm{E} 0$ & " & & ND & & & & 35 \\
\hline Bromide & $<9.91 \mathrm{E} 0$ & $9.91 \mathrm{E} 0$ & $"$ & & ND & & & & 35 \\
\hline Nitrate & $1.28 \mathrm{E} 1$ & $9.91 \mathrm{E} 0$ & " & & $1.02 \mathrm{E} 1$ & & & 22.4 & 35 \\
\hline Sulfate & $1.44 \mathrm{E} 2$ & $1.49 \mathrm{E} 1$ & " & & $1.15 \mathrm{E} 2$ & & & 22.0 & 35 \\
\hline Phosphate & $<1.49 \mathrm{E} 1$ & $1.49 \mathrm{E} 1$ & $"$ & & ND & & & & 35 \\
\hline Post Spike (8F26016-PS1) & \multicolumn{3}{|c|}{ Source: 0805020-32 } & \multicolumn{4}{|c|}{ Prepared: 06/26/08 Analyzed: 06/27/08 } & & \\
\hline Fluoride & $1.37 \mathrm{E} 0$ & N/A & $\mathrm{ug} / \mathrm{mL}$ & 0.8 & $59.1 \mathrm{E}-2$ & 97.5 & $75-125$ & & \\
\hline Chloride & 4.22E0 & N/A & $"$ & 2 & 24.E-1 & 91.1 & $75-125$ & & \\
\hline Nitrite & 3.85E0 & N/A & $"$ & 4 & 27.E-2 & 89.4 & $75-125$ & & \\
\hline Bromide & 3.72E0 & N/A & $"$ & 4 & $11.8 \mathrm{E}-2$ & 89.9 & $75-125$ & & \\
\hline Nitrate & 1.49E1 & N/A & $"$ & 4 & 11.3E0 & 88.8 & $75-125$ & & \\
\hline Sulfate & $7.52 \mathrm{E} 1$ & N/A & $"$ & 6 & 72.6E0 & 42.5 & $75-125$ & & \\
\hline Phosphate & 5.69E0 & N/A & $"$ & 6 & $38.2 \mathrm{E}-2$ & 88.5 & $75-125$ & & \\
\hline
\end{tabular}


Total Metals by PNNL-AGG-ICP-AES/Water Extract - Quality Control

Environmental Science Laboratory

\begin{tabular}{|lrrrrrrrrrr}
\hline & & Reporting & & Spike & Source & & \%REC & & RPD & \\
Analyte & Result & Limit & Units & Level & Result & \%REC & Limits & RPD & Limit & Notes \\
\hline
\end{tabular}

Batch 8F30003 - 1:1 Water Extract (ICP/ICPMS)

\begin{tabular}{|c|c|c|c|}
\hline \multicolumn{4}{|c|}{ Blank (8F30003-BLK1) } \\
\hline Aluminum & $<8.58 \mathrm{E}-2$ & $8.58 \mathrm{E}-2$ & ug/g wet \\
\hline Arsenic & $<3.60 \mathrm{E}-1$ & 3.60E-1 & $"$ \\
\hline Boron & $<1.94 \mathrm{E} 0$ & $1.94 \mathrm{E} 0$ & $"$ \\
\hline Barium & $<8.79 \mathrm{E}-3$ & 8.79E-3 & $"$ \\
\hline Beryllium & $<2.84 \mathrm{E}-2$ & $2.84 \mathrm{E}-2$ & $"$ \\
\hline Bismuth & $<1.88 \mathrm{E}-1$ & $1.88 \mathrm{E}-1$ & $"$ \\
\hline Calcium & $<3.87 \mathrm{E}-1$ & 3.87E-1 & $"$ \\
\hline Cadmium & $<2.69 \mathrm{E}-2$ & $2.69 \mathrm{E}-2$ & $"$ \\
\hline Cobalt & $<9.60 \mathrm{E}-2$ & $9.60 \mathrm{E}-2$ & $"$ \\
\hline Chromium & $<3.33 E-2$ & 3.33E-2 & $"$ \\
\hline Copper & $<8.04 \mathrm{E}-2$ & $8.04 \mathrm{E}-2$ & $"$ \\
\hline Iron & $<1.42 \mathrm{E}-1$ & $1.42 \mathrm{E}-1$ & $"$ \\
\hline Potassium & $<2.33 \mathrm{E} 0$ & 2.33E0 & $"$ \\
\hline Lithium & $<5.40 \mathrm{E}-1$ & $5.40 \mathrm{E}-1$ & $"$ \\
\hline Magnesium & $<8.34 \mathrm{E}-2$ & 8.34E-2 & $"$ \\
\hline Manganese & $<1.71 \mathrm{E}-2$ & $1.71 \mathrm{E}-2$ & $"$ \\
\hline Molybdenum & $<1.39 \mathrm{E}-1$ & $1.39 \mathrm{E}-1$ & $"$ \\
\hline Nickel & $<9.33 \mathrm{E}-2$ & $9.33 \mathrm{E}-2$ & $"$ \\
\hline Phosphorus & $<1.03 \mathrm{E} 0$ & 1.03E0 & $"$ \\
\hline Lead & $<4.32 \mathrm{E}-1$ & 4.32E-1 & $"$ \\
\hline Selenium & $<1.95 \mathrm{E} 0$ & 1.95E0 & $"$ \\
\hline Strontium & $<5.22 \mathrm{E}-2$ & $5.22 \mathrm{E}-2$ & $"$ \\
\hline Thallium & $<1.03 \mathrm{E} 0$ & 1.03E0 & $"$ \\
\hline Vanadium & $<4.53 \mathrm{E}-2$ & $4.53 \mathrm{E}-2$ & $"$ \\
\hline Zinc & $<9.27 \mathrm{E}-2$ & $9.27 \mathrm{E}-2$ & $"$ \\
\hline Sodium & $<6.69 \mathrm{E}-1$ & $6.69 \mathrm{E}-1$ & $"$ \\
\hline Silicon & $<1.50 \mathrm{E} 0$ & $1.50 \mathrm{E} 0$ & $"$ \\
\hline Sulfur & $<3.08 \mathrm{E} 0$ & 3.08E0 & $"$ \\
\hline Titanium & $<8.76 \mathrm{E}-3$ & $8.76 \mathrm{E}-3$ & $"$ \\
\hline Zirconium & $<1.50 \mathrm{E}-1$ & $1.50 \mathrm{E}-1$ & $"$ \\
\hline Silver & $<7.71 \mathrm{E}-2$ & 7.71E-2 & $"$ \\
\hline Rhenium & $<1.52 \mathrm{E}-1$ & $1.52 \mathrm{E}-1$ & $"$ \\
\hline Antimony & $<6.33 \mathrm{E}-1$ & 6.33E-1 & $"$ \\
\hline
\end{tabular}


Total Metals by PNNL-AGG-ICP-AES/Water Extract - Quality Control

Environmental Science Laboratory

\begin{tabular}{|lrrrrrrrrrr}
\hline & & Reporting & & Spike & Source & & \%REC & & RPD & \\
Analyte & Result & Limit & Units & Level & Result & \%REC & Limits & RPD & Limit & Notes \\
\hline
\end{tabular}

Batch 8F30003 - 1:1 Water Extract (ICP/ICPMS)

\begin{tabular}{|c|c|c|c|}
\hline \multicolumn{4}{|c|}{ Blank (8F30003-BLK2) } \\
\hline Aluminum & $<8.58 \mathrm{E}-2$ & $8.58 \mathrm{E}-2$ & ug/g wet \\
\hline Arsenic & $<3.60 \mathrm{E}-1$ & $3.60 \mathrm{E}-1$ & $"$ \\
\hline Boron & $<1.94 \mathrm{E} 0$ & $1.94 \mathrm{E} 0$ & $"$ \\
\hline Barium & $<8.79 \mathrm{E}-3$ & 8.79E-3 & $"$ \\
\hline Beryllium & $<2.84 \mathrm{E}-2$ & $2.84 \mathrm{E}-2$ & $"$ \\
\hline Bismuth & $<1.88 \mathrm{E}-1$ & $1.88 \mathrm{E}-1$ & $"$ \\
\hline Calcium & $<3.87 \mathrm{E}-1$ & 3.87E-1 & $"$ \\
\hline Cadmium & $<2.69 \mathrm{E}-2$ & $2.69 \mathrm{E}-2$ & $"$ \\
\hline Cobalt & $<9.60 \mathrm{E}-2$ & $9.60 \mathrm{E}-2$ & $"$ \\
\hline Chromium & $<3.33 \mathrm{E}-2$ & 3.33E-2 & $"$ \\
\hline Copper & $<8.04 \mathrm{E}-2$ & 8.04E-2 & $"$ \\
\hline Iron & $<1.42 \mathrm{E}-1$ & $1.42 \mathrm{E}-1$ & $"$ \\
\hline Potassium & $<2.33 \mathrm{E} 0$ & 2.33E0 & $"$ \\
\hline Lithium & $<5.40 \mathrm{E}-1$ & $5.40 \mathrm{E}-1$ & $"$ \\
\hline Magnesium & $<8.34 \mathrm{E}-2$ & $8.34 \mathrm{E}-2$ & $"$ \\
\hline Manganese & $<1.71 \mathrm{E}-2$ & $1.71 \mathrm{E}-2$ & $"$ \\
\hline Molybdenum & $<1.39 \mathrm{E}-1$ & $1.39 \mathrm{E}-1$ & $"$ \\
\hline Nickel & $<9.33 \mathrm{E}-2$ & $9.33 \mathrm{E}-2$ & $"$ \\
\hline Phosphorus & $<1.03 \mathrm{E} 0$ & 1.03E0 & $"$ \\
\hline Lead & $<4.32 \mathrm{E}-1$ & 4.32E-1 & " \\
\hline Selenium & $<1.95 \mathrm{E} 0$ & 1.95E0 & $"$ \\
\hline Strontium & $<5.22 \mathrm{E}-2$ & $5.22 \mathrm{E}-2$ & $"$ \\
\hline Thallium & $<1.03 \mathrm{E} 0$ & 1.03E0 & $"$ \\
\hline Vanadium & $<4.53 \mathrm{E}-2$ & $4.53 \mathrm{E}-2$ & $"$ \\
\hline Zinc & $<9.27 \mathrm{E}-2$ & $9.27 \mathrm{E}-2$ & $"$ \\
\hline Sodium & $<6.69 \mathrm{E}-1$ & $6.69 \mathrm{E}-1$ & $"$ \\
\hline Silicon & $<1.50 \mathrm{E} 0$ & $1.50 \mathrm{E} 0$ & $"$ \\
\hline Sulfur & $<3.08 \mathrm{E} 0$ & 3.08E0 & $"$ \\
\hline Titanium & $<8.76 \mathrm{E}-3$ & $8.76 \mathrm{E}-3$ & " \\
\hline Zirconium & $<1.50 \mathrm{E}-1$ & $1.50 \mathrm{E}-1$ & $"$ \\
\hline Silver & $<7.71 \mathrm{E}-2$ & $7.71 \mathrm{E}-2$ & $"$ \\
\hline Rhenium & $<1.52 \mathrm{E}-1$ & $1.52 \mathrm{E}-1$ & $"$ \\
\hline Antimony & $<6.33 \mathrm{E}-1$ & 6.33E-1 & $"$ \\
\hline
\end{tabular}


Total Metals by PNNL-AGG-ICP-AES/Water Extract - Quality Control

Environmental Science Laboratory

\begin{tabular}{|c|c|c|c|c|c|c|c|c|c|c|}
\hline & & porting & & Spike & Source & & \%REC & & RPD & \\
\hline Analyte & Result & Limit & Units & Level & Result & $\%$ REC & Limits & RPD & Limit & Notes \\
\hline
\end{tabular}

Batch 8F30003 - 1:1 Water Extract (ICP/ICPMS)

\begin{tabular}{|c|c|c|c|c|c|c|}
\hline \multirow{2}{*}{$\begin{array}{l}\text { LCS (8F30003-BS1) } \\
\text { Aluminum }\end{array}$} & \multicolumn{6}{|c|}{ Prepared: 06/25/08 Analyzed: 06/30/08 } \\
\hline & $4.78 \mathrm{E} 0$ & $8.58 \mathrm{E}-2$ & ug/g wet & 5.002 & 95.6 & $80-120$ \\
\hline Arsenic & 4.70E0 & 3.60E-1 & $"$ & 5.002 & 94.1 & $80-120$ \\
\hline Boron & $4.82 \mathrm{E} 0$ & $1.94 \mathrm{E} 0$ & $"$ & 5.002 & 96.4 & $80-120$ \\
\hline Barium & 4.75E0 & $8.79 \mathrm{E}-3$ & $"$ & 5.002 & 95.1 & $80-120$ \\
\hline Beryllium & 4.65E0 & $2.84 \mathrm{E}-2$ & $"$ & 5.002 & 93.0 & $80-120$ \\
\hline Bismuth & $<1.88 \mathrm{E}-1$ & $1.88 \mathrm{E}-1$ & $"$ & & & $80-120$ \\
\hline Calcium & 4.67E0 & 3.87E-1 & $"$ & 5.002 & 93.4 & $80-120$ \\
\hline Cadmium & 4.64E0 & 2.69E-2 & $"$ & 5.002 & 92.7 & $80-120$ \\
\hline Cobalt & 4.47E0 & $9.60 \mathrm{E}-2$ & $"$ & 5.002 & 89.3 & $80-120$ \\
\hline Chromium & 4.74E0 & 3.33E-2 & $"$ & 5.002 & 94.8 & $80-120$ \\
\hline Copper & 4.83E0 & $8.04 \mathrm{E}-2$ & $"$ & 5.002 & 96.5 & $80-120$ \\
\hline Iron & $4.68 \mathrm{E} 0$ & $1.42 \mathrm{E}-1$ & $"$ & 5.002 & 93.5 & $80-120$ \\
\hline Potassium & $4.78 \mathrm{E} 1$ & 2.33E0 & $"$ & 50.02 & 95.6 & $80-120$ \\
\hline Lithium & $<5.40 \mathrm{E}-1$ & $5.40 \mathrm{E}-1$ & $"$ & & & $80-120$ \\
\hline Magnesium & 4.74E0 & 8.34E-2 & $"$ & 5.002 & 94.8 & $80-120$ \\
\hline Manganese & 4.64E0 & $1.71 \mathrm{E}-2$ & $"$ & 5.002 & 92.8 & $80-120$ \\
\hline Molybdenum & 4.73E0 & $1.39 \mathrm{E}-1$ & $"$ & 5.002 & 94.6 & $80-120$ \\
\hline Nickel & $4.60 \mathrm{E} 0$ & 9.33E-2 & $"$ & 5.002 & 91.9 & $80-120$ \\
\hline Phosphorus & $<1.03 \mathrm{E} 0$ & $1.03 \mathrm{E} 0$ & $"$ & & & $80-120$ \\
\hline Lead & $4.60 \mathrm{E} 0$ & 4.32E-1 & $"$ & 5.002 & 92.0 & $80-120$ \\
\hline Selenium & $4.81 \mathrm{E} 0$ & $1.95 \mathrm{E} 0$ & $"$ & 5.002 & 96.2 & $80-120$ \\
\hline Strontium & $<5.22 \mathrm{E}-2$ & $5.22 \mathrm{E}-2$ & $"$ & & & $80-120$ \\
\hline Thallium & $4.60 \mathrm{E} 0$ & 1.03E0 & $"$ & 5.002 & 91.9 & $80-120$ \\
\hline Vanadium & 4.77E0 & 4.53E-2 & $"$ & 5.002 & 95.4 & $80-120$ \\
\hline Zinc & $4.48 \mathrm{E} 0$ & $9.27 \mathrm{E}-2$ & $"$ & 5.002 & 89.6 & $80-120$ \\
\hline Sodium & $5.14 \mathrm{E} 0$ & 6.69E-1 & $"$ & 5.002 & 103 & $80-120$ \\
\hline Silicon & 2.77E0 & $1.50 \mathrm{E} 0$ & $"$ & 2.501 & 111 & $80-120$ \\
\hline Sulfur & $<3.08 \mathrm{E} 0$ & $3.08 \mathrm{E} 0$ & $"$ & & & $80-120$ \\
\hline Titanium & $4.66 \mathrm{E} 0$ & $8.76 \mathrm{E}-3$ & $"$ & 5.002 & 93.2 & $80-120$ \\
\hline Zirconium & $<1.50 \mathrm{E}-1$ & $1.50 \mathrm{E}-1$ & $"$ & & & $80-120$ \\
\hline Silver & 4.78E0 & $7.71 \mathrm{E}-2$ & $"$ & 5.002 & 95.5 & $80-120$ \\
\hline Rhenium & $<1.52 \mathrm{E}-1$ & $1.52 \mathrm{E}-1$ & $"$ & & & $80-120$ \\
\hline Antimony & $4.75 \mathrm{E} 0$ & 6.33E-1 & $"$ & 5.002 & 95.0 & $80-120$ \\
\hline
\end{tabular}


Total Metals by PNNL-AGG-ICP-AES/Water Extract - Quality Control

Environmental Science Laboratory

\begin{tabular}{|c|c|c|c|c|c|c|c|c|c|c|}
\hline & & porting & & Spike & Source & & \%REC & & RPD & \\
\hline Analyte & Result & Limit & Units & Level & Result & $\%$ REC & Limits & RPD & Limit & Notes \\
\hline
\end{tabular}

Batch 8F30003 - 1:1 Water Extract (ICP/ICPMS)

\begin{tabular}{|c|c|c|c|c|c|c|}
\hline \multirow{2}{*}{$\begin{array}{l}\text { LCS (8F30003-BS2) } \\
\text { Aluminum }\end{array}$} & \multicolumn{6}{|c|}{ Prepared: 06/25/08 Analyzed: 06/30/08 } \\
\hline & 4.97E0 & $8.58 \mathrm{E}-2$ & ug/g wet & 4.997 & 99.4 & $80-120$ \\
\hline Arsenic & $4.90 \mathrm{E} 0$ & 3.60E-1 & $"$ & 4.997 & 98.0 & $80-120$ \\
\hline Boron & 4.97E0 & $1.94 \mathrm{E} 0$ & $"$ & 4.997 & 99.5 & $80-120$ \\
\hline Barium & 4.96E0 & $8.79 \mathrm{E}-3$ & $"$ & 4.997 & 99.4 & $80-120$ \\
\hline Beryllium & $4.80 \mathrm{E} 0$ & $2.84 \mathrm{E}-2$ & $"$ & 4.997 & 96.1 & $80-120$ \\
\hline Bismuth & $<1.88 \mathrm{E}-1$ & $1.88 \mathrm{E}-1$ & $"$ & & & $80-120$ \\
\hline Calcium & 4.86Е0 & 3.87E-1 & $"$ & 4.997 & 97.3 & $80-120$ \\
\hline Cadmium & $4.80 \mathrm{E} 0$ & 2.69E-2 & $"$ & 4.997 & 96.0 & $80-120$ \\
\hline Cobalt & 4.61E0 & $9.60 \mathrm{E}-2$ & $"$ & 4.997 & 92.3 & $80-120$ \\
\hline Chromium & 4.89E0 & 3.33E-2 & $"$ & 4.997 & 98.0 & $80-120$ \\
\hline Copper & 5.03E0 & $8.04 \mathrm{E}-2$ & $"$ & 4.997 & 101 & $80-120$ \\
\hline Iron & $4.80 \mathrm{E} 0$ & $1.42 \mathrm{E}-1$ & $"$ & 4.997 & 96.0 & $80-120$ \\
\hline Potassium & $5.00 \mathrm{E} 1$ & 2.33E0 & $"$ & 49.97 & 100 & $80-120$ \\
\hline Lithium & $<5.40 \mathrm{E}-1$ & $5.40 \mathrm{E}-1$ & $"$ & & & $80-120$ \\
\hline Magnesium & $4.90 \mathrm{E} 0$ & 8.34E-2 & $"$ & 4.997 & 98.1 & $80-120$ \\
\hline Manganese & 4.85E0 & $1.71 \mathrm{E}-2$ & $"$ & 4.997 & 97.0 & $80-120$ \\
\hline Molybdenum & 4.87E0 & 1.39E-1 & $"$ & 4.997 & 97.5 & $80-120$ \\
\hline Nickel & $4.74 \mathrm{E} 0$ & 9.33E-2 & $"$ & 4.997 & 94.8 & $80-120$ \\
\hline Phosphorus & $<1.03 \mathrm{E} 0$ & $1.03 \mathrm{E} 0$ & $"$ & & & $80-120$ \\
\hline Lead & 4.75E0 & 4.32E-1 & $"$ & 4.997 & 95.1 & $80-120$ \\
\hline Selenium & $4.94 \mathrm{E} 0$ & $1.95 \mathrm{E} 0$ & $"$ & 4.997 & 98.9 & $80-120$ \\
\hline Strontium & $<5.22 \mathrm{E}-2$ & $5.22 \mathrm{E}-2$ & $"$ & & & $80-120$ \\
\hline Thallium & $4.78 \mathrm{E} 0$ & $1.03 \mathrm{E} 0$ & $"$ & 4.997 & 95.7 & $80-120$ \\
\hline Vanadium & 4.89E0 & 4.53E-2 & $"$ & 4.997 & 97.8 & $80-120$ \\
\hline Zinc & $4.60 \mathrm{E} 0$ & $9.27 \mathrm{E}-2$ & $"$ & 4.997 & 92.1 & $80-120$ \\
\hline Sodium & $5.34 \mathrm{E} 0$ & $6.69 \mathrm{E}-1$ & $"$ & 4.997 & 107 & $80-120$ \\
\hline Silicon & $2.85 \mathrm{E} 0$ & $1.50 \mathrm{E} 0$ & $"$ & 2.498 & 114 & $80-120$ \\
\hline Sulfur & $<3.08 \mathrm{E} 0$ & $3.08 \mathrm{E} 0$ & $"$ & & & $80-120$ \\
\hline Titanium & 4.87E0 & $8.76 \mathrm{E}-3$ & $"$ & 4.997 & 97.4 & $80-120$ \\
\hline Zirconium & $<1.50 \mathrm{E}-1$ & $1.50 \mathrm{E}-1$ & $"$ & & & $80-120$ \\
\hline Silver & 4.94E0 & $7.71 \mathrm{E}-2$ & $"$ & 4.997 & 99.0 & $80-120$ \\
\hline Rhenium & $<1.52 \mathrm{E}-1$ & $1.52 \mathrm{E}-1$ & $"$ & & & $80-120$ \\
\hline Antimony & 4.93E0 & 6.33E-1 & $"$ & 4.997 & 98.6 & $80-120$ \\
\hline
\end{tabular}


Total Metals by PNNL-AGG-ICP-AES/Water Extract - Quality Control

Environmental Science Laboratory

\begin{tabular}{|lrrrrrrrrrr}
\hline & & Reporting & & Spike & Source & & \%REC & & RPD & \\
Analyte & Result & Limit & Units & Level & Result & \%REC & Limits & RPD & Limit & Notes \\
\hline
\end{tabular}

Batch 8F30003 - 1:1 Water Extract (ICP/ICPMS)

\begin{tabular}{|c|c|c|c|c|c|c|}
\hline \multirow{2}{*}{$\begin{array}{l}\text { Duplicate (8F30003-DUP1) } \\
\text { Aluminum }\end{array}$} & \multicolumn{3}{|c|}{ Source: 0805020-11 } & \multirow{2}{*}{$\begin{array}{r}\text { Prepared: 06/25/08 } \\
2.57 \mathrm{E} 0\end{array}$} & \multicolumn{2}{|l|}{ Analyzed: 06/30/08 } \\
\hline & $1.87 \mathrm{E} 0$ & $9.00 \mathrm{E}-2$ & ug/g dry & & 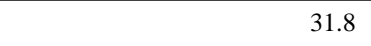 & 35 \\
\hline Arsenic & 8.91E-1 & $3.78 \mathrm{E}-1$ & $"$ & 7.7E-1 & 14.5 & 35 \\
\hline Boron & $<2.04 \mathrm{E} 0$ & 2.04E0 & $"$ & ND & & 35 \\
\hline Barium & $7.22 \mathrm{E}-2$ & $9.23 \mathrm{E}-3$ & $"$ & $1.07 \mathrm{E}-1$ & 38.7 & 35 \\
\hline Beryllium & $<2.98 \mathrm{E}-2$ & $2.98 \mathrm{E}-2$ & $"$ & ND & & 35 \\
\hline Bismuth & $<1.97 \mathrm{E}-1$ & $1.97 \mathrm{E}-1$ & $"$ & ND & & 35 \\
\hline Calcium & 1.17E1 & 4.06E-1 & $"$ & $1.77 \mathrm{E} 1$ & 40.6 & 35 \\
\hline Cadmium & $<2.82 \mathrm{E}-2$ & $2.82 \mathrm{E}-2$ & $"$ & ND & & 35 \\
\hline Cobalt & $<1.01 \mathrm{E}-1$ & $1.01 \mathrm{E}-1$ & $"$ & ND & & 35 \\
\hline Chromium & $<3.49 \mathrm{E}-2$ & $3.49 \mathrm{E}-2$ & $"$ & ND & & 35 \\
\hline Copper & $1.28 \mathrm{E}-1$ & $8.44 \mathrm{E}-2$ & $"$ & $1.24 \mathrm{E}-1$ & 3.74 & 35 \\
\hline Iron & 2.92E0 & $1.49 \mathrm{E}-1$ & $"$ & 3.9E0 & 28.6 & 35 \\
\hline Potassium & $4.89 \mathrm{E} 0$ & $2.44 \mathrm{E} 0$ & $"$ & 4.88E0 & 0.308 & 35 \\
\hline Lithium & $<5.67 \mathrm{E}-1$ & 5.67E-1 & $"$ & ND & & 35 \\
\hline Magnesium & $1.09 \mathrm{E} 0$ & 8.75E-2 & $"$ & $1.58 \mathrm{E} 0$ & 36.6 & 35 \\
\hline Manganese & $1.05 \mathrm{E}-1$ & $1.80 \mathrm{E}-2$ & $"$ & $1.65 \mathrm{E}-1$ & 44.4 & 35 \\
\hline Molybdenum & $<1.46 \mathrm{E}-1$ & $1.46 \mathrm{E}-1$ & $"$ & ND & & 35 \\
\hline Nickel & $<9.79 \mathrm{E}-2$ & $9.79 \mathrm{E}-2$ & $"$ & ND & & 35 \\
\hline Phosphorus & 5.49E0 & $1.08 \mathrm{E} 0$ & $"$ & $5.11 \mathrm{E} 0$ & 7.17 & 35 \\
\hline Lead & $<4.53 \mathrm{E}-1$ & 4.53E-1 & $"$ & ND & & 35 \\
\hline Selenium & $<2.05 \mathrm{E} 0$ & $2.05 \mathrm{E} 0$ & $"$ & ND & & 35 \\
\hline Strontium & $<5.48 \mathrm{E}-2$ & $5.48 \mathrm{E}-2$ & $"$ & ND & & 35 \\
\hline Thallium & $<1.08 \mathrm{E} 0$ & $1.08 \mathrm{E} 0$ & $"$ & ND & & 35 \\
\hline Vanadium & 3.97E-1 & 4.75E-2 & $"$ & $3.58 \mathrm{E}-1$ & 10.3 & 35 \\
\hline Zinc & $<9.73 \mathrm{E}-2$ & $9.73 \mathrm{E}-2$ & $"$ & ND & & 35 \\
\hline Sodium & $5.02 \mathrm{E} 2$ & 7.02E-1 & $"$ & 4.95E2 & 1.31 & 35 \\
\hline Silicon & $1.89 \mathrm{E} 1$ & $1.57 \mathrm{E} 0$ & $"$ & 2.03E1 & 7.00 & 35 \\
\hline Sulfur & $6.97 \mathrm{E} 0$ & 3.23E0 & $"$ & 7.02E0 & 0.729 & 35 \\
\hline Titanium & 1.66E-1 & $9.19 \mathrm{E}-3$ & $"$ & 3.05E-1 & 58.9 & 35 \\
\hline Zirconium & $<1.57 \mathrm{E}-1$ & $1.57 \mathrm{E}-1$ & $"$ & ND & & 35 \\
\hline Silver & $<8.09 \mathrm{E}-2$ & 8.09E-2 & $"$ & ND & & 35 \\
\hline Rhenium & $<1.59 \mathrm{E}-1$ & $1.59 \mathrm{E}-1$ & $"$ & ND & & 35 \\
\hline Antimony & $<6.64 \mathrm{E}-1$ & 6.64E-1 & $"$ & ND & & 35 \\
\hline
\end{tabular}


Total Metals by PNNL-AGG-ICP-AES/Water Extract - Quality Control

Environmental Science Laboratory

\begin{tabular}{|lrrrrrrrrrr}
\hline & & Reporting & & Spike & Source & & \%REC & & RPD & \\
Analyte & Result & Limit & Units & Level & Result & \%REC & Limits & RPD & Limit & Notes \\
\hline
\end{tabular}

Batch 8F30003 - 1:1 Water Extract (ICP/ICPMS)

\begin{tabular}{|c|c|c|c|c|c|c|}
\hline \multirow{2}{*}{$\begin{array}{l}\text { Duplicate (8F30003-DUP2) } \\
\text { Aluminum }\end{array}$} & \multicolumn{3}{|c|}{ Source: 0805020-27 } & \multirow{2}{*}{$\begin{array}{r}\text { Prepared: } 06 / 25 / 08 \\
\text { ND }\end{array}$} & \multicolumn{2}{|l|}{ Analyzed: 06/30/08 } \\
\hline & $<8.51 \mathrm{E}-2$ & $8.51 \mathrm{E}-2$ & ug/g dry & & & 35 \\
\hline Arsenic & $<3.57 \mathrm{E}-1$ & 3.57E-1 & $"$ & ND & & 35 \\
\hline Boron & $<1.92 \mathrm{E} 0$ & $1.92 \mathrm{E} 0$ & $"$ & ND & & 35 \\
\hline Barium & $2.62 \mathrm{E}-2$ & $8.71 \mathrm{E}-3$ & $"$ & $3.5 \mathrm{E}-2$ & 28.9 & 35 \\
\hline Beryllium & $<2.82 \mathrm{E}-2$ & $2.82 \mathrm{E}-2$ & $"$ & ND & & 35 \\
\hline Bismuth & $<1.86 \mathrm{E}-1$ & $1.86 \mathrm{E}-1$ & $"$ & ND & & 35 \\
\hline Calcium & $4.58 \mathrm{E} 1$ & $3.84 \mathrm{E}-1$ & $"$ & $4.11 \mathrm{E} 1$ & 10.8 & 35 \\
\hline Cadmium & $<2.66 \mathrm{E}-2$ & $2.66 \mathrm{E}-2$ & $"$ & ND & & 35 \\
\hline Cobalt & $<9.52 \mathrm{E}-2$ & $9.52 \mathrm{E}-2$ & $"$ & ND & & 35 \\
\hline Chromium & $<3.30 \mathrm{E}-2$ & $3.30 \mathrm{E}-2$ & $"$ & ND & & 35 \\
\hline Copper & $<7.97 \mathrm{E}-2$ & 7.97E-2 & $"$ & ND & & 35 \\
\hline Iron & $<1.41 \mathrm{E}-1$ & $1.41 \mathrm{E}-1$ & $"$ & ND & & 35 \\
\hline Potassium & $1.24 \mathrm{E} 1$ & 2.31E0 & $"$ & $1.23 \mathrm{E} 1$ & 0.0315 & 35 \\
\hline Lithium & $<5.35 \mathrm{E}-1$ & 5.35E-1 & $"$ & ND & & 35 \\
\hline Magnesium & $1.86 \mathrm{E} 1$ & 8.27E-2 & $"$ & $1.59 \mathrm{E} 1$ & 15.7 & 35 \\
\hline Manganese & $<1.70 \mathrm{E}-2$ & $1.70 \mathrm{E}-2$ & $"$ & ND & & 35 \\
\hline Molybdenum & $<1.38 \mathrm{E}-1$ & $1.38 \mathrm{E}-1$ & $"$ & ND & & 35 \\
\hline Nickel & $<9.25 \mathrm{E}-2$ & $9.25 \mathrm{E}-2$ & $"$ & ND & & 35 \\
\hline Phosphorus & $<1.02 \mathrm{E} 0$ & $1.02 \mathrm{E} 0$ & $"$ & ND & & 35 \\
\hline Lead & $<4.28 \mathrm{E}-1$ & 4.28E-1 & $"$ & ND & & 35 \\
\hline Selenium & $<1.93 \mathrm{E} 0$ & $1.93 \mathrm{E} 0$ & $"$ & ND & & 35 \\
\hline Strontium & 2.26E-1 & 5.17E-2 & $"$ & 1.93E-1 & 15.4 & 35 \\
\hline Thallium & $<1.02 \mathrm{E} 0$ & $1.02 \mathrm{E} 0$ & $"$ & ND & & 35 \\
\hline Vanadium & $<4.49 \mathrm{E}-2$ & 4.49E-2 & $"$ & ND & & 35 \\
\hline Zinc & $<9.19 \mathrm{E}-2$ & $9.19 \mathrm{E}-2$ & $"$ & ND & & 35 \\
\hline Sodium & $1.92 \mathrm{E} 1$ & 6.63E-1 & $"$ & $1.99 \mathrm{E} 1$ & 3.93 & 35 \\
\hline Silicon & 8.93Е0 & 1.49E0 & $"$ & 8.6Е0 & 3.85 & 35 \\
\hline Sulfur & $5.06 \mathrm{E} 1$ & 3.05E0 & $"$ & 4.03E1 & 22.6 & 35 \\
\hline Titanium & $<8.68 \mathrm{E}-3$ & $8.68 \mathrm{E}-3$ & $"$ & ND & & 35 \\
\hline Zirconium & $<1.49 \mathrm{E}-1$ & $1.49 \mathrm{E}-1$ & $"$ & ND & & 35 \\
\hline Silver & $<7.64 \mathrm{E}-2$ & 7.64E-2 & $"$ & ND & & 35 \\
\hline Rhenium & $<1.50 \mathrm{E}-1$ & $1.50 \mathrm{E}-1$ & $"$ & ND & & 35 \\
\hline Antimony & $<6.28 \mathrm{E}-1$ & $6.28 \mathrm{E}-1$ & $"$ & ND & & 35 \\
\hline
\end{tabular}


Total Metals by PNNL-AGG-ICP-AES/Water Extract - Quality Control

Environmental Science Laboratory

\begin{tabular}{|c|c|c|c|c|c|c|c|c|c|c|}
\hline & & porting & & Spike & Source & & \%REC & & RPD & \\
\hline Analyte & Result & Limit & Units & Level & Result & $\%$ REC & Limits & RPD & Limit & Notes \\
\hline
\end{tabular}

Batch 8F30003 - 1:1 Water Extract (ICP/ICPMS)

\begin{tabular}{|c|c|c|c|c|c|c|c|}
\hline \multirow{2}{*}{$\begin{array}{l}\text { Post Spike (8F30003-PS1) } \\
\text { Aluminum }\end{array}$} & \multicolumn{3}{|c|}{ Source: 0805020-11 } & \multicolumn{4}{|c|}{ Prepared \& Analyzed: 06/30/08 } \\
\hline & $1.41 \mathrm{E} 3$ & N/A & ug/L & 500 & 85.1E1 & 112 & $75-125$ \\
\hline Arsenic & 7.71E2 & N/A & $"$ & 500 & $25.5 \mathrm{E} 1$ & 103 & $75-125$ \\
\hline Boron & 5.53E2 & N/A & $"$ & 500 & $37.2 \mathrm{E} 0$ & 103 & $75-125$ \\
\hline Barium & 2.87E2 & N/A & $"$ & 250 & $35.4 \mathrm{E} 0$ & 101 & $75-125$ \\
\hline Beryllium & $2.5 \mathrm{E} 2$ & N/A & $"$ & 250 & $27.5 \mathrm{E}-2$ & 99.8 & $75-125$ \\
\hline Bismuth & 4.71E2 & N/A & $"$ & 500 & 11.3E-1 & 94 & $75-125$ \\
\hline Calcium & 6.63E3 & N/A & $"$ & 500 & 58.7E2 & 153 & $75-125$ \\
\hline Cadmium & 2.39E2 & N/A & $"$ & 250 & $12.2 \mathrm{E}-2$ & 95.7 & $75-125$ \\
\hline Cobalt & 2.35E2 & N/A & $"$ & 250 & 12.9E-1 & 93.4 & $75-125$ \\
\hline Chromium & $1.24 \mathrm{E} 2$ & N/A & $"$ & 125 & 11.9E-1 & 98.5 & $75-125$ \\
\hline Copper & 5.63E2 & N/A & $"$ & 500 & 40.9E0 & 104 & $75-125$ \\
\hline Iron & $1.8 \mathrm{E} 3$ & N/A & $"$ & 500 & 12.9E2 & 101 & $75-125$ \\
\hline Potassium & $2.96 \mathrm{E} 3$ & N/A & $"$ & 1250 & 16.2E2 & 108 & $75-125$ \\
\hline Lithium & $5.3 \mathrm{E} 2$ & N/A & $"$ & 500 & $12.7 \mathrm{E} 0$ & 103 & $75-125$ \\
\hline Magnesium & 9.78E2 & N/A & $"$ & 500 & $52.4 \mathrm{E} 1$ & 90.7 & $75-125$ \\
\hline Manganese & 2.99E2 & N/A & $"$ & 250 & 54.7E0 & 97.6 & $75-125$ \\
\hline Molybdenum & 5.27E2 & N/A & $"$ & 500 & 23.1E0 & 101 & $75-125$ \\
\hline Nickel & 4.77E2 & N/A & $"$ & 500 & $54.9 \mathrm{E}-1$ & 94.3 & $75-125$ \\
\hline Phosphorus & $2.99 \mathrm{E} 3$ & N/A & $"$ & 1250 & $16.9 \mathrm{E} 2$ & 104 & $75-125$ \\
\hline Lead & 4.71E2 & N/A & $"$ & 500 & ND & 96.1 & $75-125$ \\
\hline Selenium & 4.78E2 & N/A & $"$ & 500 & ND & 99.3 & $75-125$ \\
\hline Strontium & $5.48 \mathrm{E} 2$ & N/A & $"$ & 500 & 12.7E0 & 107 & $75-125$ \\
\hline Thallium & 4.34E2 & N/A & $"$ & 500 & ND & 98.9 & $75-125$ \\
\hline Vanadium & 3.71E2 & N/A & $"$ & 250 & 11.9E1 & 101 & $75-125$ \\
\hline Zinc & $2.64 \mathrm{E} 2$ & N/A & $"$ & 250 & 16.9E0 & 98.8 & $75-125$ \\
\hline Sodium & 1.69E5 & N/A & $"$ & 500 & 16.4E4 & 1000 & $75-125$ \\
\hline Silicon & 7.38E3 & N/A & $"$ & 500 & 67.1E2 & 134 & $75-125$ \\
\hline Sulfur & 3.3E3 & N/A & $"$ & 1000 & 23.2E2 & 97.3 & $75-125$ \\
\hline Titanium & 2.93E2 & N/A & " & 250 & 10.1E1 & 76.9 & $75-125$ \\
\hline Zirconium & $2.54 \mathrm{E} 2$ & N/A & $"$ & 250 & 11.E0 & 97.1 & $75-125$ \\
\hline Silver & $5.02 \mathrm{E} 2$ & N/A & $"$ & 500 & ND & 101 & $75-125$ \\
\hline Rhenium & 4.95E2 & N/A & $"$ & 500 & 39.3E-1 & 98.3 & $75-125$ \\
\hline Antimony & 5.E2 & N/A & $"$ & 500 & $66.4 \mathrm{E}-1$ & 98.7 & $75-125$ \\
\hline
\end{tabular}


Total Metals by PNNL-AGG-ICP-AES/Water Extract - Quality Control

Environmental Science Laboratory

\begin{tabular}{|c|c|c|c|c|c|c|c|c|c|c|}
\hline & & porting & & Spike & Source & & \%REC & & RPD & \\
\hline Analyte & Result & Limit & Units & Level & Result & $\%$ REC & Limits & RPD & Limit & Notes \\
\hline
\end{tabular}

Batch 8F30003 - 1:1 Water Extract (ICP/ICPMS)

\begin{tabular}{|c|c|c|c|c|c|c|c|}
\hline \multirow{2}{*}{$\begin{array}{l}\text { Post Spike (8F30003-PS2) } \\
\text { Aluminum }\end{array}$} & \multicolumn{3}{|c|}{ Source: 0805020-27 } & \multicolumn{4}{|c|}{ Prepared \& Analyzed: 06/30/08 } \\
\hline & 5.17E2 & N/A & $\mathrm{ug} / \mathrm{L}$ & 500 & 14.1E0 & 101 & $75-125$ \\
\hline Arsenic & $4.82 \mathrm{E} 2$ & N/A & $"$ & 500 & ND & 99.7 & $75-125$ \\
\hline Boron & 5.25E2 & N/A & $"$ & 500 & 14.1E0 & 102 & $75-125$ \\
\hline Barium & $2.58 \mathrm{E} 2$ & N/A & $"$ & 250 & 11.7E0 & 98.4 & $75-125$ \\
\hline Beryllium & 2.53E2 & N/A & $"$ & 250 & $10.8 \mathrm{E}-2$ & 101 & $75-125$ \\
\hline Bismuth & $5.1 \mathrm{E} 2$ & N/A & $"$ & 500 & 22.8E0 & 97.5 & $75-125$ \\
\hline Calcium & $1.42 \mathrm{E} 4$ & N/A & $"$ & 500 & 13.7E3 & 104 & $75-125$ \\
\hline Cadmium & 2.38E2 & N/A & $"$ & 250 & ND & 95.1 & $75-125$ \\
\hline Cobalt & 2.37E2 & N/A & $"$ & 250 & ND & 95.2 & $75-125$ \\
\hline Chromium & $1.22 \mathrm{E} 2$ & N/A & $"$ & 125 & ND & 99.6 & $75-125$ \\
\hline Copper & 5.23E2 & N/A & $"$ & 500 & ND & 105 & $75-125$ \\
\hline Iron & 5.01E2 & N/A & $"$ & 500 & 21.E-1 & 99.9 & $75-125$ \\
\hline Potassium & $5.46 \mathrm{E} 3$ & N/A & $"$ & 1250 & 41.1E2 & 108 & $75-125$ \\
\hline Lithium & 5.27E2 & N/A & $"$ & 500 & $10.9 \mathrm{E} 0$ & 103 & $75-125$ \\
\hline Magnesium & $5.82 \mathrm{E} 3$ & N/A & $"$ & 500 & 53.E2 & 105 & $75-125$ \\
\hline Manganese & $2.5 \mathrm{E} 2$ & N/A & $"$ & 250 & ND & 102 & $75-125$ \\
\hline Molybdenum & 5.33E2 & N/A & $"$ & 500 & 28.8E0 & 101 & $75-125$ \\
\hline Nickel & 4.82E2 & N/A & $"$ & 500 & ND & 96.5 & $75-125$ \\
\hline Phosphorus & $1.31 \mathrm{E} 3$ & N/A & $"$ & 1250 & 39.8E0 & 101 & $75-125$ \\
\hline Lead & 4.76E2 & N/A & $"$ & 500 & ND & 97.9 & $75-125$ \\
\hline Selenium & 4.51E2 & N/A & $"$ & 500 & ND & 103 & $75-125$ \\
\hline Strontium & 5.81E2 & N/A & $"$ & 500 & $64.4 \mathrm{E} 0$ & 103 & $75-125$ \\
\hline Thallium & 4.E2 & N/A & $"$ & 500 & ND & 98.4 & $75-125$ \\
\hline Vanadium & $2.44 \mathrm{E} 2$ & N/A & $"$ & 250 & ND & 99.7 & $75-125$ \\
\hline Zinc & 2.58E2 & N/A & $"$ & 250 & 12.7E0 & 98.3 & $75-125$ \\
\hline Sodium & 7.12E3 & N/A & $"$ & 500 & $66.4 \mathrm{E} 2$ & 96.2 & $75-125$ \\
\hline Silicon & $3.44 \mathrm{E} 3$ & N/A & $"$ & 500 & 28.6E2 & 116 & $75-125$ \\
\hline Sulfur & $1.45 \mathrm{E} 4$ & N/A & $"$ & 1000 & $13.4 \mathrm{E} 3$ & 111 & $75-125$ \\
\hline Titanium & 2.46E2 & N/A & " & 250 & ND & 98.7 & $75-125$ \\
\hline Zirconium & $2.52 \mathrm{E} 2$ & N/A & $"$ & 250 & $67.4 \mathrm{E}-1$ & 98.2 & $75-125$ \\
\hline Silver & 4.63E2 & N/A & $"$ & 500 & $14.6 \mathrm{E}-3$ & 92.5 & $75-125$ \\
\hline Rhenium & $4.96 \mathrm{E} 2$ & N/A & $"$ & 500 & 83.5E-1 & 97.5 & $75-125$ \\
\hline Antimony & 5.05E2 & N/A & $"$ & 500 & 28.6E-1 & 100 & $75-125$ \\
\hline
\end{tabular}


Total Metals by PNNL-AGG-ICP-AES/Acid Extract - Quality Control

Environmental Science Laboratory

\begin{tabular}{|c|c|c|c|c|c|c|c|c|c|c|}
\hline Analyte & Result & $\begin{array}{c}\text { orting } \\
\text { Limit }\end{array}$ & Units & $\begin{array}{l}\text { Spike } \\
\text { Level }\end{array}$ & $\begin{array}{l}\text { Source } \\
\text { Result }\end{array}$ & \%REC & $\begin{array}{l}\text { \%REC } \\
\text { Limits }\end{array}$ & RPD & $\begin{array}{l}\text { RPD } \\
\text { Limit }\end{array}$ & Notes \\
\hline
\end{tabular}

Batch 8G01001 - ASTM D 5198 (ICP/ICPMS)

\begin{tabular}{|c|c|c|c|}
\hline \multicolumn{4}{|c|}{ Blank (8G01001-BLK1) } \\
\hline Aluminum & $<9.88 \mathrm{E} 0$ & 9.88E0 & ug/g wet \\
\hline Arsenic & $<2.40 \mathrm{E} 1$ & $2.40 \mathrm{E} 1$ & $"$ \\
\hline Boron & $<2.09 \mathrm{E} 2$ & 2.09E2 & $"$ \\
\hline Barium & $<9.41 \mathrm{E}-1$ & $9.41 \mathrm{E}-1$ & $"$ \\
\hline Beryllium & $<3.73 \mathrm{E}-1$ & 3.73E-1 & $"$ \\
\hline Bismuth & $<4.99 \mathrm{E} 0$ & 4.99E0 & $"$ \\
\hline Calcium & $<3.67 \mathrm{E} 1$ & 3.67E1 & $"$ \\
\hline Cadmium & $<5.61 \mathrm{E}-1$ & $5.61 \mathrm{E}-1$ & $"$ \\
\hline Cobalt & $<2.05 \mathrm{E} 0$ & $2.05 \mathrm{E} 0$ & $"$ \\
\hline Chromium & $<8.21 \mathrm{E}-1$ & $8.21 \mathrm{E}-1$ & $"$ \\
\hline Copper & $<8.75 \mathrm{E} 0$ & 8.75E0 & $"$ \\
\hline Iron & $<2.51 \mathrm{E} 1$ & $2.51 \mathrm{E} 1$ & $"$ \\
\hline Potassium & $<2.33 \mathrm{E} 1$ & 2.33E1 & $"$ \\
\hline Lithium & $<3.56 \mathrm{E} 0$ & 3.56E0 & $"$ \\
\hline Magnesium & $<7.77 \mathrm{E} 0$ & 7.77E0 & $"$ \\
\hline Manganese & $<2.94 \mathrm{E}-1$ & $2.94 \mathrm{E}-1$ & $"$ \\
\hline Molybdenum & $<5.57 \mathrm{E} 0$ & 5.57E0 & $"$ \\
\hline Nickel & $<2.02 \mathrm{E} 0$ & 2.02E0 & $"$ \\
\hline Phosphorus & $<1.85 \mathrm{E} 1$ & $1.85 \mathrm{E} 1$ & $"$ \\
\hline Lead & $<7.81 \mathrm{E} 0$ & 7.81E0 & " \\
\hline Strontium & $<3.56 \mathrm{E}-1$ & $3.56 \mathrm{E}-1$ & $"$ \\
\hline Zinc & $<1.54 \mathrm{E} 0$ & $1.54 \mathrm{E} 0$ & $"$ \\
\hline Sodium & $<2.63 \mathrm{E} 2$ & 2.63E2 & $"$ \\
\hline Silicon & $<3.43 \mathrm{E} 2$ & 3.43E2 & $"$ \\
\hline Sulfur & $<5.82 \mathrm{E} 1$ & $5.82 \mathrm{E} 1$ & $"$ \\
\hline Titanium & $<7.75 \mathrm{E} 0$ & 7.75E0 & $"$ \\
\hline Zirconium & $<5.00 \mathrm{E} 0$ & $5.00 \mathrm{E} 0$ & $"$ \\
\hline Rhenium & $<4.33 \mathrm{E} 0$ & 4.33E0 & $"$ \\
\hline Antimony & $<2.75 \mathrm{E} 1$ & 2.75E1 & $"$ \\
\hline
\end{tabular}


Total Metals by PNNL-AGG-ICP-AES/Acid Extract - Quality Control

Environmental Science Laboratory

\begin{tabular}{|rrrrrrrrrrrr}
\hline & & Reporting & & Spike & Source & & \%REC & & RPD \\
Analyte & Result & Limit & Units & Level & Result & \%REC & Limits & RPD & Limit & Notes \\
\hline
\end{tabular}

Batch 8G01001 - ASTM D 5198 (ICP/ICPMS)

\begin{tabular}{|c|c|c|c|}
\hline \multicolumn{4}{|c|}{ Blank (8G01001-BLK2) } \\
\hline Aluminum & $<9.88 \mathrm{E} 0$ & $9.88 \mathrm{E} 0$ & ug/g wet \\
\hline Arsenic & $<2.40 \mathrm{E} 1$ & $2.40 \mathrm{E} 1$ & $"$ \\
\hline Boron & $<2.09 \mathrm{E} 2$ & 2.09E2 & $"$ \\
\hline Barium & $<9.41 \mathrm{E}-1$ & $9.41 \mathrm{E}-1$ & " \\
\hline Beryllium & $<3.73 \mathrm{E}-1$ & 3.73E-1 & $"$ \\
\hline Bismuth & $<4.99 \mathrm{E} 0$ & 4.99E0 & $"$ \\
\hline Calcium & $<3.67 \mathrm{E} 1$ & 3.67E1 & $"$ \\
\hline Cadmium & $<5.61 \mathrm{E}-1$ & 5.61E-1 & $"$ \\
\hline Cobalt & $<2.05 \mathrm{E} 0$ & 2.05E0 & $"$ \\
\hline Chromium & $<8.21 \mathrm{E}-1$ & $8.21 \mathrm{E}-1$ & $"$ \\
\hline Copper & $<8.75 \mathrm{E} 0$ & 8.75E0 & $"$ \\
\hline Iron & $<2.51 \mathrm{E} 1$ & $2.51 \mathrm{E} 1$ & $"$ \\
\hline Potassium & $<2.33 \mathrm{E} 1$ & 2.33E1 & $"$ \\
\hline Lithium & $<3.56 \mathrm{E} 0$ & $3.56 \mathrm{E} 0$ & $"$ \\
\hline Magnesium & $<7.77 \mathrm{E} 0$ & 7.77E0 & $"$ \\
\hline Manganese & $<2.94 \mathrm{E}-1$ & $2.94 \mathrm{E}-1$ & $"$ \\
\hline Molybdenum & $<5.57 \mathrm{E} 0$ & 5.57E0 & $"$ \\
\hline Nickel & $<2.02 \mathrm{E} 0$ & 2.02E0 & $"$ \\
\hline Phosphorus & $<1.85 \mathrm{E} 1$ & $1.85 \mathrm{E} 1$ & $"$ \\
\hline Lead & $<7.81 \mathrm{E} 0$ & 7.81E0 & $"$ \\
\hline Strontium & $<3.56 \mathrm{E}-1$ & $3.56 \mathrm{E}-1$ & $"$ \\
\hline Zinc & $<1.54 \mathrm{E} 0$ & $1.54 \mathrm{E} 0$ & $"$ \\
\hline Sodium & $<2.63 \mathrm{E} 2$ & 2.63E2 & $"$ \\
\hline Silicon & $<3.43 \mathrm{E} 2$ & 3.43E2 & $"$ \\
\hline Sulfur & $<5.82 \mathrm{E} 1$ & $5.82 \mathrm{E} 1$ & $"$ \\
\hline Titanium & $<7.75 \mathrm{E} 0$ & 7.75E0 & $"$ \\
\hline Zirconium & $<5.00 \mathrm{E} 0$ & $5.00 \mathrm{E} 0$ & $"$ \\
\hline Rhenium & $<4.33 \mathrm{E} 0$ & 4.33E0 & $"$ \\
\hline Antimony & $<2.75 \mathrm{E} 1$ & $2.75 \mathrm{E} 1$ & $"$ \\
\hline
\end{tabular}


Total Metals by PNNL-AGG-ICP-AES/Acid Extract - Quality Control

Environmental Science Laboratory

\begin{tabular}{|c|c|c|c|c|c|c|c|c|c|c|}
\hline & & porting & & Spike & Source & & \%REC & & RPD & \\
\hline Analyte & Result & Limit & Units & Level & Result & \%REC & Limits & RPD & Limit & Notes \\
\hline
\end{tabular}

Batch 8G01001 - ASTM D 5198 (ICP/ICPMS)

\begin{tabular}{|c|c|c|c|c|c|c|}
\hline LCS (8G01001-BS1) & & & & Prepared: 06/26/08 & Analyze & 7/01/08 \\
\hline Aluminum & $4.75 \mathrm{E} 0$ & 1.39E-1 & ug/g wet & 5.000 & 95.1 & $80-120$ \\
\hline Arsenic & $4.85 \mathrm{E} 0$ & 3.37E-1 & $"$ & 5.000 & 97.0 & $80-120$ \\
\hline Boron & 4.73E0 & 2.93E0 & $"$ & 5.000 & 94.6 & $80-120$ \\
\hline Barium & $5.18 \mathrm{E} 0$ & $1.32 \mathrm{E}-2$ & $"$ & 5.000 & 104 & $80-120$ \\
\hline Beryllium & 4.87E0 & $5.24 \mathrm{E}-3$ & $"$ & 5.000 & 97.4 & $80-120$ \\
\hline Bismuth & $<7.01 \mathrm{E}-2$ & 7.01E-2 & $"$ & & & $80-120$ \\
\hline Calcium & $5.04 \mathrm{E} 0$ & 5.15E-1 & $"$ & 5.000 & 101 & $80-120$ \\
\hline Cadmium & $4.91 \mathrm{E} 0$ & $7.88 \mathrm{E}-3$ & $"$ & 5.000 & 98.3 & $80-120$ \\
\hline Cobalt & $4.91 \mathrm{E} 0$ & $2.88 \mathrm{E}-2$ & $"$ & 5.000 & 98.3 & $80-120$ \\
\hline Chromium & $5.31 \mathrm{E} 0$ & $1.15 \mathrm{E}-2$ & $"$ & 5.000 & 106 & $80-120$ \\
\hline Copper & 5.17E0 & 1.23E-1 & $"$ & 5.000 & 103 & $80-120$ \\
\hline Iron & 5.13E0 & $3.52 \mathrm{E}-1$ & $"$ & 5.000 & 103 & $80-120$ \\
\hline Potassium & $5.13 \mathrm{E} 1$ & 3.27E-1 & $"$ & 50.00 & 103 & $80-120$ \\
\hline Lithium & $<5.00 \mathrm{E}-2$ & $5.00 \mathrm{E}-2$ & $"$ & & & $80-120$ \\
\hline Magnesium & 4.73E0 & 1.09E-1 & $"$ & 5.000 & 94.5 & $80-120$ \\
\hline Manganese & 5.08E0 & 4.13E-3 & $"$ & 5.000 & 102 & $80-120$ \\
\hline Molybdenum & $5.16 \mathrm{E} 0$ & 7.82E-2 & $"$ & 5.000 & 103 & $80-120$ \\
\hline Nickel & $5.01 \mathrm{E} 0$ & 2.84E-2 & $"$ & 5.000 & 100 & $80-120$ \\
\hline Phosphorus & $<2.60 \mathrm{E}-1$ & $2.60 \mathrm{E}-1$ & $"$ & & & $80-120$ \\
\hline Lead & $5.04 \mathrm{E} 0$ & $1.10 \mathrm{E}-1$ & $"$ & 5.000 & 101 & $80-120$ \\
\hline Strontium & $<5.00 \mathrm{E}-3$ & $5.00 \mathrm{E}-3$ & $"$ & & & $80-120$ \\
\hline Zinc & 4.91E0 & $2.16 \mathrm{E}-2$ & $"$ & 5.000 & 98.1 & $80-120$ \\
\hline Sodium & $5.75 \mathrm{E} 0$ & $3.69 \mathrm{E} 0$ & $"$ & 5.000 & 115 & $80-120$ \\
\hline Silicon & $<4.82 \mathrm{E} 0$ & $4.82 \mathrm{E} 0$ & $"$ & 2.500 & & $80-120$ \\
\hline Sulfur & $<8.17 \mathrm{E}-1$ & 8.17E-1 & $"$ & & & $80-120$ \\
\hline Titanium & $5.10 \mathrm{E} 0$ & 1.09E-1 & $"$ & 5.000 & 102 & $80-120$ \\
\hline Zirconium & 7.43E-2 & 7.02E-2 & $"$ & & & $80-120$ \\
\hline Rhenium & $<6.08 \mathrm{E}-2$ & $6.08 \mathrm{E}-2$ & $"$ & & & $80-120$ \\
\hline Antimony & $4.96 \mathrm{E} 0$ & 3.86E-1 & $"$ & 5.000 & 99.2 & $80-120$ \\
\hline
\end{tabular}


Total Metals by PNNL-AGG-ICP-AES/Acid Extract - Quality Control

Environmental Science Laboratory

\begin{tabular}{|c|c|c|c|c|c|c|c|c|c|c|}
\hline & & porting & & Spike & Source & & \%REC & & RPD & \\
\hline Analyte & Result & Limit & Units & Level & Result & \%REC & Limits & RPD & Limit & Notes \\
\hline
\end{tabular}

Batch 8G01001 - ASTM D 5198 (ICP/ICPMS)

\begin{tabular}{|c|c|c|c|c|c|c|}
\hline \multirow{2}{*}{$\begin{array}{l}\text { LCS (8G01001-BS2) } \\
\text { Aluminum }\end{array}$} & \multicolumn{6}{|c|}{ Prepared: 06/27/08 Analyzed: 07/01/08 } \\
\hline & $4.75 \mathrm{E} 0$ & $1.38 \mathrm{E}-1$ & ug/g wet & 5.000 & 95.0 & $80-120$ \\
\hline Arsenic & $4.90 \mathrm{E} 0$ & 3.35E-1 & $"$ & 5.000 & 98.1 & $80-120$ \\
\hline Boron & $4.78 \mathrm{E} 0$ & 2.92E0 & $"$ & 5.000 & 95.6 & $80-120$ \\
\hline Barium & $5.12 \mathrm{E} 0$ & $1.31 \mathrm{E}-2$ & $"$ & 5.000 & 102 & $80-120$ \\
\hline Beryllium & 4.93Е0 & $5.21 \mathrm{E}-3$ & $"$ & 5.000 & 98.6 & $80-120$ \\
\hline Bismuth & $<6.97 \mathrm{E}-2$ & $6.97 \mathrm{E}-2$ & $"$ & & & $80-120$ \\
\hline Calcium & 4.95E0 & $5.12 \mathrm{E}-1$ & $"$ & 5.000 & 99.0 & $80-120$ \\
\hline Cadmium & $4.94 \mathrm{E} 0$ & 7.83E-3 & $"$ & 5.000 & 98.8 & $80-120$ \\
\hline Cobalt & 4.93E0 & $2.86 \mathrm{E}-2$ & $"$ & 5.000 & 98.6 & $80-120$ \\
\hline Chromium & 5.31E0 & $1.15 \mathrm{E}-2$ & $"$ & 5.000 & 106 & $80-120$ \\
\hline Copper & 5.17E0 & $1.22 \mathrm{E}-1$ & " & 5.000 & 103 & $80-120$ \\
\hline Iron & $5.12 \mathrm{E} 0$ & $3.50 \mathrm{E}-1$ & " & 5.000 & 102 & $80-120$ \\
\hline Potassium & 5.07E1 & $3.25 \mathrm{E}-1$ & $"$ & 50.00 & 101 & $80-120$ \\
\hline Lithium & $<4.97 \mathrm{E}-2$ & 4.97E-2 & $"$ & & & $80-120$ \\
\hline Magnesium & $4.71 \mathrm{E} 0$ & $1.08 \mathrm{E}-1$ & " & 5.000 & 94.3 & $80-120$ \\
\hline Manganese & $5.08 \mathrm{E} 0$ & $4.11 \mathrm{E}-3$ & " & 5.000 & 102 & $80-120$ \\
\hline Molybdenum & 5.23E0 & $7.78 \mathrm{E}-2$ & $"$ & 5.000 & 105 & $80-120$ \\
\hline Nickel & $5.04 \mathrm{E} 0$ & $2.82 \mathrm{E}-2$ & " & 5.000 & 101 & $80-120$ \\
\hline Phosphorus & $<2.58 \mathrm{E}-1$ & $2.58 \mathrm{E}-1$ & $"$ & & & $80-120$ \\
\hline Lead & 5.07E0 & $1.09 \mathrm{E}-1$ & $"$ & 5.000 & 101 & $80-120$ \\
\hline Strontium & $<4.97 \mathrm{E}-3$ & 4.97E-3 & " & & & $80-120$ \\
\hline Zinc & $5.05 \mathrm{E} 0$ & $2.15 \mathrm{E}-2$ & $"$ & 5.000 & 101 & $80-120$ \\
\hline Sodium & 5.52E0 & 3.67E0 & " & 5.000 & 110 & $80-120$ \\
\hline Silicon & $<4.79 \mathrm{E} 0$ & 4.79E0 & $"$ & 2.500 & & $80-120$ \\
\hline Sulfur & $<8.13 \mathrm{E}-1$ & 8.13E-1 & " & & & $80-120$ \\
\hline Titanium & $5.20 \mathrm{E} 0$ & $1.08 \mathrm{E}-1$ & " & 5.000 & 104 & $80-120$ \\
\hline Zirconium & $<6.98 \mathrm{E}-2$ & $6.98 \mathrm{E}-2$ & $"$ & & & $80-120$ \\
\hline Rhenium & $<6.05 \mathrm{E}-2$ & $6.05 \mathrm{E}-2$ & $"$ & & & $80-120$ \\
\hline Antimony & 5.01E0 & $3.84 \mathrm{E}-1$ & $"$ & 5.000 & 100 & $80-120$ \\
\hline
\end{tabular}


Total Metals by PNNL-AGG-ICP-AES/Acid Extract - Quality Control

Environmental Science Laboratory

\begin{tabular}{|lrrrrrrrrrr}
\hline & & Reporting & & Spike & Source & & \%REC & & RPD & \\
Analyte & Result & Limit & Units & Level & Result & \%REC & Limits & RPD & Limit & Notes \\
\hline
\end{tabular}

Batch 8G01001 - ASTM D 5198 (ICP/ICPMS)

\begin{tabular}{|c|c|c|c|c|c|c|}
\hline \multirow{2}{*}{$\begin{array}{l}\text { Duplicate (8G01001-DUP1) } \\
\text { Aluminum }\end{array}$} & \multicolumn{3}{|c|}{ Source: 0805020-11 } & \multirow{2}{*}{$\begin{array}{r}\text { Prepared: 06/26/08 } \\
6.11 \mathrm{E} 3\end{array}$} & \multicolumn{2}{|l|}{ Analyzed: 07/01/08 } \\
\hline & $6.71 \mathrm{E} 3$ & $3.98 \mathrm{E} 1$ & ug/g dry & & 9.48 & 35 \\
\hline Arsenic & $<9.66 \mathrm{E} 1$ & $9.66 \mathrm{E} 1$ & $"$ & ND & & 35 \\
\hline Boron & $<8.41 \mathrm{E} 2$ & $8.41 \mathrm{E} 2$ & $"$ & ND & & 35 \\
\hline Barium & 8.55E1 & 3.79E0 & $"$ & $8.02 \mathrm{E} 1$ & 6.44 & 35 \\
\hline Beryllium & $<1.50 \mathrm{E} 0$ & $1.50 \mathrm{E} 0$ & $"$ & ND & & 35 \\
\hline Bismuth & $<2.01 \mathrm{E} 1$ & $2.01 \mathrm{E} 1$ & $"$ & ND & & 35 \\
\hline Calcium & $9.86 \mathrm{E} 3$ & $1.48 \mathrm{E} 2$ & $"$ & 8.77E3 & 11.7 & 35 \\
\hline Cadmium & $<2.26 \mathrm{E} 0$ & $2.26 \mathrm{E} 0$ & $"$ & ND & & 35 \\
\hline Cobalt & $<8.25 \mathrm{E} 0$ & $8.25 \mathrm{E} 0$ & $"$ & ND & & 35 \\
\hline Chromium & 5.59E0 & 3.30E0 & $"$ & $4.96 \mathrm{E} 0$ & 11.9 & 35 \\
\hline Copper & $<3.52 \mathrm{E} 1$ & 3.52E1 & $"$ & ND & & 35 \\
\hline Iron & $1.44 \mathrm{E} 4$ & $1.01 \mathrm{E} 2$ & $"$ & $1.33 \mathrm{E} 4$ & 7.91 & 35 \\
\hline Potassium & $1.42 \mathrm{E} 3$ & $9.38 \mathrm{E} 1$ & $"$ & $1.27 \mathrm{E} 3$ & 11.1 & 35 \\
\hline Lithium & $<1.43 \mathrm{E} 1$ & $1.43 \mathrm{E} 1$ & $"$ & ND & & 35 \\
\hline Magnesium & $4.50 \mathrm{E} 3$ & 3.13E1 & $"$ & $4.14 \mathrm{E} 3$ & 8.22 & 35 \\
\hline Manganese & 2.97E2 & $1.18 \mathrm{E} 0$ & $"$ & $2.82 \mathrm{E} 2$ & 5.25 & 35 \\
\hline Molybdenum & $<2.24 \mathrm{E} 1$ & 2.24E1 & $"$ & ND & & 35 \\
\hline Nickel & 8.37E0 & 8.13E0 & $"$ & ND & & 35 \\
\hline Phosphorus & 7.45E2 & 7.45E1 & $"$ & $6.73 \mathrm{E} 2$ & 10.2 & 35 \\
\hline Lead & $<3.14 \mathrm{E} 1$ & $3.14 \mathrm{E} 1$ & $"$ & ND & & 35 \\
\hline Strontium & $3.20 \mathrm{E} 1$ & 1.43E0 & $"$ & $2.91 \mathrm{E} 1$ & 9.45 & 35 \\
\hline Zinc & 3.73E1 & $6.20 \mathrm{E} 0$ & $"$ & $3.44 \mathrm{E} 1$ & 8.24 & 35 \\
\hline Sodium & $2.19 \mathrm{E} 3$ & $1.06 \mathrm{E} 3$ & $"$ & $2.01 \mathrm{E} 3$ & 8.77 & 35 \\
\hline Silicon & $<1.38 \mathrm{E} 3$ & $1.38 \mathrm{E} 3$ & $"$ & ND & & 35 \\
\hline Sulfur & $<2.34 \mathrm{E} 2$ & 2.34E2 & $"$ & ND & & 35 \\
\hline Titanium & 7.47E2 & 3.12E1 & $"$ & 6.63E2 & 11.8 & 35 \\
\hline Zirconium & $<2.01 \mathrm{E} 1$ & $2.01 \mathrm{E} 1$ & $"$ & ND & & 35 \\
\hline Rhenium & $<1.74 \mathrm{E} 1$ & $1.74 \mathrm{E} 1$ & $"$ & ND & & 35 \\
\hline Antimony & $<1.11 \mathrm{E} 2$ & $1.11 \mathrm{E} 2$ & $"$ & ND & & 35 \\
\hline
\end{tabular}


Total Metals by PNNL-AGG-ICP-AES/Acid Extract - Quality Control

Environmental Science Laboratory

\begin{tabular}{|lrrrrrrrrrr}
\hline & & Reporting & & Spike & Source & & \%REC & & RPD & \\
Analyte & Result & Limit & Units & Level & Result & \%REC & Limits & RPD & Limit & Notes \\
\hline
\end{tabular}

Batch 8G01001 - ASTM D 5198 (ICP/ICPMS)

\begin{tabular}{|c|c|c|c|c|c|c|}
\hline \multirow{2}{*}{$\begin{array}{l}\text { Duplicate (8G01001-DUP2) } \\
\text { Aluminum }\end{array}$} & \multicolumn{3}{|c|}{ Source: 0805020-27 } & \multirow{2}{*}{$\begin{array}{r}\text { Prepared: 06/27/08 } \\
4.61 \mathrm{E} 3\end{array}$} & \multicolumn{2}{|l|}{ Analyzed: 07/01/08 } \\
\hline & $4.83 \mathrm{E} 3$ & 3.63E1 & ug/g dry & & 4.55 & 35 \\
\hline Arsenic & $<8.81 \mathrm{E} 1$ & $8.81 \mathrm{E} 1$ & $"$ & ND & & 35 \\
\hline Boron & $<7.67 \mathrm{E} 2$ & 7.67E2 & $"$ & ND & & 35 \\
\hline Barium & $5.42 \mathrm{E} 1$ & $3.45 \mathrm{E} 0$ & $"$ & $5.14 \mathrm{E} 1$ & 5.44 & 35 \\
\hline Beryllium & $<1.37 \mathrm{E} 0$ & $1.37 \mathrm{E} 0$ & $"$ & $\mathrm{ND}$ & & 35 \\
\hline Bismuth & $<1.83 \mathrm{E} 1$ & $1.83 \mathrm{E} 1$ & $"$ & ND & & 35 \\
\hline Calcium & $8.60 \mathrm{E} 3$ & $1.35 \mathrm{E} 2$ & $"$ & $7.56 \mathrm{E} 3$ & 12.9 & 35 \\
\hline Cadmium & $<2.06 \mathrm{E} 0$ & $2.06 \mathrm{E} 0$ & $"$ & ND & & 35 \\
\hline Cobalt & $<7.53 \mathrm{E} 0$ & 7.53E0 & $"$ & ND & & 35 \\
\hline Chromium & $1.00 \mathrm{E} 1$ & $3.01 \mathrm{E} 0$ & $"$ & $9.9 \mathrm{E} 0$ & 1.02 & 35 \\
\hline Copper & $<3.21 \mathrm{E} 1$ & $3.21 \mathrm{E} 1$ & $"$ & ND & & 35 \\
\hline Iron & $8.84 \mathrm{E} 3$ & $9.21 \mathrm{E} 1$ & $"$ & $9.05 \mathrm{E} 3$ & 2.33 & 35 \\
\hline Potassium & $1.18 \mathrm{E} 3$ & $8.55 \mathrm{E} 1$ & $"$ & $1.12 \mathrm{E} 3$ & 4.95 & 35 \\
\hline Lithium & $<1.31 \mathrm{E} 1$ & $1.31 \mathrm{E} 1$ & $"$ & ND & & 35 \\
\hline Magnesium & 3.71E3 & $2.85 \mathrm{E} 1$ & $"$ & $3.51 \mathrm{E} 3$ & 5.54 & 35 \\
\hline Manganese & 2.17E2 & $1.08 \mathrm{E} 0$ & $"$ & $2.14 \mathrm{E} 2$ & 1.71 & 35 \\
\hline Molybdenum & $<2.04 \mathrm{E} 1$ & $2.04 \mathrm{E} 1$ & $"$ & ND & & 35 \\
\hline Nickel & 8.94E0 & 7.42E0 & $"$ & 8.89E0 & 0.601 & 35 \\
\hline Phosphorus & 3.52E2 & $6.79 \mathrm{E} 1$ & $"$ & $3.44 \mathrm{E} 2$ & 2.18 & 35 \\
\hline Lead & $<2.87 \mathrm{E} 1$ & 2.87E1 & $"$ & ND & & 35 \\
\hline Strontium & 2.99E1 & $1.31 \mathrm{E} 0$ & $"$ & $2.68 \mathrm{E} 1$ & 10.9 & 35 \\
\hline Zinc & 2.62E1 & $5.65 \mathrm{E} 0$ & $"$ & 2.43E1 & 7.63 & 35 \\
\hline Sodium & $<9.66 \mathrm{E} 2$ & $9.66 \mathrm{E} 2$ & $"$ & ND & & 35 \\
\hline Silicon & $<1.26 \mathrm{E} 3$ & $1.26 \mathrm{E} 3$ & $"$ & ND & & 35 \\
\hline Sulfur & $<2.14 \mathrm{E} 2$ & $2.14 \mathrm{E} 2$ & $"$ & ND & & 35 \\
\hline Titanium & $3.80 \mathrm{E} 2$ & $2.85 \mathrm{E} 1$ & $"$ & 3.39E2 & 11.5 & 35 \\
\hline Zirconium & $<1.84 \mathrm{E} 1$ & $1.84 \mathrm{E} 1$ & $"$ & ND & & 35 \\
\hline Rhenium & $<1.59 \mathrm{E} 1$ & $1.59 \mathrm{E} 1$ & $"$ & ND & & 35 \\
\hline Antimony & $<1.01 \mathrm{E} 2$ & $1.01 \mathrm{E} 2$ & $"$ & ND & & 35 \\
\hline
\end{tabular}


Total Metals by PNNL-AGG-ICP-AES/Acid Extract - Quality Control

Environmental Science Laboratory

\begin{tabular}{|c|c|c|c|c|c|c|c|c|c|c|}
\hline & & porting & & Spike & Source & & \%REC & & RPD & \\
\hline Analyte & Result & Limit & Units & Level & Result & $\%$ REC & Limits & RPD & Limit & Notes \\
\hline
\end{tabular}

Batch 8G01001 - ASTM D 5198 (ICP/ICPMS)

\begin{tabular}{|c|c|c|c|c|c|c|c|}
\hline \multirow{2}{*}{$\begin{array}{l}\text { Post Spike (8G01001-PS1) } \\
\text { Aluminum }\end{array}$} & \multicolumn{3}{|c|}{ Source: 0805020-11 } & \multicolumn{4}{|c|}{ Prepared \& Analyzed: 07/01/08 } \\
\hline & $1.57 \mathrm{E} 4$ & 39.3E2 & ug/g dry & 500 & $15.3 \mathrm{E} 3$ & 75.2 & $75-125$ \\
\hline Arsenic & 4.32E2 & $95.5 \mathrm{E} 2$ & $"$ & 500 & ND & 101 & $75-125$ \\
\hline Boron & 5.E2 & $83.2 \mathrm{E} 3$ & $"$ & 500 & 71.3E-1 & 98.6 & $75-125$ \\
\hline Barium & 4.69E2 & $37.4 \mathrm{E} 1$ & $"$ & 250 & $20.2 \mathrm{E} 1$ & 107 & $75-125$ \\
\hline Beryllium & $2.42 \mathrm{E} 2$ & $14.8 \mathrm{E} 1$ & $"$ & 250 & 40.7E-2 & 96.7 & $75-125$ \\
\hline Bismuth & $5.15 \mathrm{E} 2$ & 19.9E2 & $"$ & 500 & 34.3E0 & 96.1 & $75-125$ \\
\hline Calcium & 2.27E4 & $14.6 \mathrm{E} 3$ & $"$ & 500 & 22.E3 & 131 & $75-125$ \\
\hline Cadmium & 2.33E2 & 22.3E1 & $"$ & 250 & ND & 94 & $75-125$ \\
\hline Cobalt & 2.45E2 & 81.6E1 & " & 250 & $17.4 \mathrm{E} 0$ & 90.9 & $75-125$ \\
\hline Chromium & $1.34 \mathrm{E} 2$ & $32.7 \mathrm{E} 1$ & $"$ & 125 & $12.5 \mathrm{E} 0$ & 96.9 & $75-125$ \\
\hline Copper & 5.37E2 & $34.8 \mathrm{E} 2$ & $"$ & 500 & 21.5E0 & 103 & $75-125$ \\
\hline Iron & 3.39E4 & 99.9E2 & $"$ & 500 & $33.5 \mathrm{E} 3$ & 81.3 & $75-125$ \\
\hline Potassium & $4.46 \mathrm{E} 3$ & $92.7 \mathrm{E} 2$ & $"$ & 1250 & $31.8 \mathrm{E} 2$ & 103 & $75-125$ \\
\hline Lithium & 4.82E2 & $14.2 \mathrm{E} 2$ & $"$ & 500 & $30.9 \mathrm{E} 0$ & 90.3 & $75-125$ \\
\hline Magnesium & $1.09 \mathrm{E} 4$ & $30.9 \mathrm{E} 2$ & $"$ & 500 & $10.4 \mathrm{E} 3$ & 88.9 & $75-125$ \\
\hline Manganese & $9.55 \mathrm{E} 2$ & 11.7E1 & $"$ & 250 & $70.9 \mathrm{E} 1$ & 98.7 & $75-125$ \\
\hline Molybdenum & 4.91E2 & 22.2E2 & $"$ & 500 & $14.6 \mathrm{E} 0$ & 95.2 & $75-125$ \\
\hline Nickel & 4.83E2 & $80.4 \mathrm{E} 1$ & $"$ & 500 & 20.1E0 & 92.5 & $75-125$ \\
\hline Phosphorus & 2.9E3 & $73.6 \mathrm{E} 2$ & $"$ & 1250 & $16.9 \mathrm{E} 2$ & 96.4 & $75-125$ \\
\hline Lead & 4.73E2 & 31.1E2 & $"$ & 500 & ND & 94.6 & $75-125$ \\
\hline Strontium & $6.12 \mathrm{E} 2$ & $14.2 \mathrm{E} 1$ & $"$ & 500 & $73.2 \mathrm{E} 0$ & 108 & $75-125$ \\
\hline Zinc & $3.55 \mathrm{E} 2$ & 61.3E1 & $"$ & 250 & 86.4E0 & 108 & $75-125$ \\
\hline Sodium & 5.53E3 & $10.5 \mathrm{E} 4$ & $"$ & 500 & $50.4 \mathrm{E} 2$ & 97.4 & $75-125$ \\
\hline Silicon & 5.75E2 & 13.7E4 & $"$ & 500 & 57.9E0 & 103 & $75-125$ \\
\hline Sulfur & 7.91E2 & $23.2 \mathrm{E} 3$ & $"$ & 1000 & ND & 97.8 & $75-125$ \\
\hline Titanium & $1.91 \mathrm{E} 3$ & $30.8 \mathrm{E} 2$ & $"$ & 250 & 16.7E2 & 95.7 & $75-125$ \\
\hline Zirconium & $2.82 \mathrm{E} 2$ & 19.9E2 & " & 250 & 37.8E0 & 97.5 & $75-125$ \\
\hline Rhenium & 4.81E2 & $17.2 \mathrm{E} 2$ & $"$ & 500 & 16.4E-1 & 95.9 & $75-125$ \\
\hline Antimony & $4.86 \mathrm{E} 2$ & 10.9E3 & $"$ & 500 & 26.E-2 & 97.1 & $75-125$ \\
\hline
\end{tabular}


Total Metals by PNNL-AGG-ICP-AES/Acid Extract - Quality Control

Environmental Science Laboratory

\begin{tabular}{|c|c|c|c|c|c|c|c|c|c|c|}
\hline & & porting & & Spike & Source & & \%REC & & RPD & \\
\hline Analyte & Result & Limit & Units & Level & Result & $\%$ REC & Limits & RPD & Limit & Notes \\
\hline
\end{tabular}

Batch 8G01001 - ASTM D 5198 (ICP/ICPMS)

\begin{tabular}{|c|c|c|c|c|c|c|c|}
\hline \multirow{2}{*}{$\begin{array}{l}\text { Post Spike (8G01001-PS2) } \\
\text { Aluminum }\end{array}$} & \multicolumn{3}{|c|}{ Source: 0805020-27 } & \multicolumn{4}{|c|}{ Prepared \& Analyzed: 07/01/08 } \\
\hline & $1.3 \mathrm{E} 4$ & $36.8 \mathrm{E} 2$ & ug/g dry & 500 & $12.4 \mathrm{E} 3$ & 120 & $75-125$ \\
\hline Arsenic & $4.4 \mathrm{E} 2$ & 89.4E2 & $"$ & 500 & ND & 96.3 & $75-125$ \\
\hline Boron & $5.04 \mathrm{E} 2$ & 77.9E3 & $"$ & 500 & 51.9E-1 & 99.7 & $75-125$ \\
\hline Barium & 4.18E2 & $35.1 \mathrm{E} 1$ & $"$ & 250 & $13.8 \mathrm{E} 1$ & 112 & $75-125$ \\
\hline Beryllium & $2.44 \mathrm{E} 2$ & 13.9E1 & $"$ & 250 & $15.2 \mathrm{E}-2$ & 97.5 & $75-125$ \\
\hline Bismuth & 5.31E2 & 18.6E2 & $"$ & 500 & 39.8E0 & 98.1 & $75-125$ \\
\hline Calcium & 2.27E4 & 13.7E3 & $"$ & 500 & 20.3E3 & 484 & $75-125$ \\
\hline Cadmium & 2.34E2 & 20.9E1 & $"$ & 250 & ND & 94.4 & $75-125$ \\
\hline Cobalt & $2.4 \mathrm{E} 2$ & $76.4 \mathrm{E} 1$ & $"$ & 250 & $10.9 \mathrm{E} 0$ & 91.5 & $75-125$ \\
\hline Chromium & $1.49 \mathrm{E} 2$ & $30.6 \mathrm{E} 1$ & $"$ & 125 & 26.6E0 & 97.7 & $75-125$ \\
\hline Copper & $5.35 \mathrm{E} 2$ & $32.6 \mathrm{E} 2$ & $"$ & 500 & $12.2 \mathrm{E} 0$ & 105 & $75-125$ \\
\hline Iron & $2.34 \mathrm{E} 4$ & 93.5E2 & $"$ & 500 & 24.3E3 & NR & $75-125$ \\
\hline Potassium & 4.34E3 & 86.8E2 & $"$ & 1250 & 30.1E2 & 106 & $75-125$ \\
\hline Lithium & 5.17E2 & 13.3E2 & $"$ & 500 & 32.E0 & 97.1 & $75-125$ \\
\hline Magnesium & $1.02 \mathrm{E} 4$ & 28.9E2 & $"$ & 500 & $94.2 \mathrm{E} 2$ & 156 & $75-125$ \\
\hline Manganese & 8.28E2 & 11.0E1 & $"$ & 250 & 57.4E1 & 102 & $75-125$ \\
\hline Molybdenum & 4.91E2 & 20.7E2 & " & 500 & $15.4 \mathrm{E} 0$ & 95.2 & $75-125$ \\
\hline Nickel & 4.87E2 & $75.2 \mathrm{E} 1$ & $"$ & 500 & 23.9E0 & 92.6 & $75-125$ \\
\hline Phosphorus & 2.15E3 & 68.9E2 & $"$ & 1250 & 92.4E1 & 97.9 & $75-125$ \\
\hline Lead & 4.79E2 & 29.1E2 & " & 500 & ND & 97 & $75-125$ \\
\hline Strontium & $6.28 \mathrm{E} 2$ & 13.3E1 & $"$ & 500 & 72.1E0 & 111 & $75-125$ \\
\hline Zinc & 3.34E2 & 57.4E1 & $"$ & 250 & $65.2 \mathrm{E} 0$ & 107 & $75-125$ \\
\hline Sodium & 8.83E2 & $98.0 \mathrm{E} 3$ & $"$ & 500 & $34.6 \mathrm{E} 1$ & 107 & $75-125$ \\
\hline Silicon & 5.74E2 & $12.8 \mathrm{E} 4$ & $"$ & 500 & 50.9E0 & 105 & $75-125$ \\
\hline Sulfur & 8.38E2 & 21.7E3 & $"$ & 1000 & ND & 95.4 & $75-125$ \\
\hline Titanium & $1.22 \mathrm{E} 3$ & 28.9E2 & $"$ & 250 & 91.0E1 & 124 & $75-125$ \\
\hline Zirconium & 2.7E2 & 18.6E2 & $"$ & 250 & 22.5E0 & 99.2 & $75-125$ \\
\hline Rhenium & 4.85E2 & 16.1E2 & $"$ & 500 & $81.1 \mathrm{E}-2$ & 96.9 & $75-125$ \\
\hline Antimony & 5.05E2 & 10.2E3 & " & 500 & 10.9E0 & 98.8 & $75-125$ \\
\hline
\end{tabular}




\section{Radionuclides by ICP-MS/Acid Extract - Quality Control \\ Environmental Science Laboratory}

\begin{tabular}{|c|c|c|c|c|c|c|c|c|c|c|}
\hline Analyte & Result & $\begin{array}{c}\text { orting } \\
\text { Limit }\end{array}$ & Units & $\begin{array}{l}\text { Spike } \\
\text { Level }\end{array}$ & $\begin{array}{l}\text { Source } \\
\text { Result }\end{array}$ & \%REC & $\begin{array}{l}\text { \%REC } \\
\text { Limits }\end{array}$ & RPD & $\begin{array}{l}\text { RPD } \\
\text { Limit }\end{array}$ & Notes \\
\hline
\end{tabular}

Batch 8G02004 - ASTM D 5198 (ICP/ICPMS)

Blank (8G02004-BLK1)

Technetium-99

Uranium 238

Duplicate (8G02004-DUP1)

Technetium-99

Uranium 238

Post Spike (8G02004-PS1)

Technetium-99

Uranium 238

Batch 8G02006 - ASTM D 5198 (ICP/ICPMS)

Blank (8G02006-BLK1)

Technetium-99

Uranium 238

Duplicate (8G02006-DUP1)

Technetium-99

Uranium 238

Post Spike (8G02006-PS1)

Technetium-99

Uranium 238

Uraning 238
Prepared \& Analyzed: 07/02/08

$\begin{array}{ccc}<1.19 \mathrm{E}-3 & 1.19 \mathrm{E}-3 & \mathrm{ug} / \mathrm{g} \text { wet } \\ <8.64 \mathrm{E}-3 & 8.64 \mathrm{E}-3 & "\end{array}$

Source: 0805020-11

Prepared \& Analyzed: 07/02/08

$\begin{array}{cccccc}<4.79 \mathrm{E}-3 & 4.79 \mathrm{E}-3 & \text { ug/g dry } & \text { ND } & 35 \\ 5.47 \mathrm{E}-1 & 3.48 \mathrm{E}-2 & " & 5.27 \mathrm{E}-1 & 3.71 & 35\end{array}$

Source: 0805020-11

Prepared \& Analyzed: 07/02/08

$\begin{array}{rcccccc}4.84 \mathrm{E}-1 & \text { N/A } & \text { ug/L } & 0.5 & 27 . \mathrm{E}-4 & 96.2 & 75-125 \\ 1.8 \mathrm{E} 0 & \text { N/A } & " & 0.5 & 13.3 \mathrm{E}-1 & 94.1 & 75-125\end{array}$

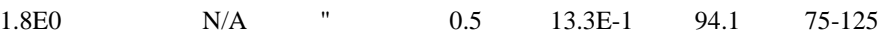

Batch 8G15009 - ASTM D 5198 (ICP/ICPMS)

Blank (8G15009-BLK1)

Neptunium-237

Plutonium-239

Americium-241
Prepared \& Analyzed: 07/02/08

$\begin{array}{llc}<1.19 \mathrm{E}-3 & 1.19 \mathrm{E}-3 & \mathrm{ug} / \mathrm{g} \text { wet } \\ <8.64 \mathrm{E}-3 & 8.64 \mathrm{E}-3 & "\end{array}$

Source: 0805020-27 Prepared \& Analyzed: 07/02/08

$\begin{array}{lllll}<4.37 \mathrm{E}-3 & 4.37 \mathrm{E}-3 & \text { ug/g dry } & \text { ND } & 35\end{array}$

3.43E-1 3.17E-2 "

$3.59 \mathrm{E}-1$

$4.64 \quad 35$

Source: 0805020-27 Prepared \& Analyzed: 07/02/08

\begin{tabular}{rcccccc}
\multicolumn{4}{r}{ Source: $0805020-27$} & \multicolumn{5}{c}{ Prepared \& Analyzed: $07 / 02 / 08$} \\
\hline N.45E-1 & N/A & ug/L & 0.5 & $26.4 \mathrm{E}-4$ & 88.4 & $75-125$ \\
$1.37 \mathrm{E} 0$ & N/A & $"$ & 0.5 & $96.5 \mathrm{E}-2$ & 81.3 & $75-125$
\end{tabular}




\section{Radionuclides by ICP-MS/Acid Extract - Quality Control \\ Environmental Science Laboratory}

\begin{tabular}{|c|c|c|c|c|c|c|c|c|c|c|}
\hline Analyte & Result & $\begin{array}{r}\text { porting } \\
\text { Limit }\end{array}$ & Units & $\begin{array}{l}\text { Spike } \\
\text { Level }\end{array}$ & $\begin{array}{l}\text { Source } \\
\text { Result }\end{array}$ & \%REC & $\begin{array}{l}\text { \%REC } \\
\text { Limits }\end{array}$ & RPD & $\begin{array}{l}\text { RPD } \\
\text { Limit }\end{array}$ & Notes \\
\hline
\end{tabular}

Batch 8G15009 - ASTM D 5198 (ICP/ICPMS)

Duplicate (8G15009-DUP1)

Neptunium-237

Plutonium-239

Americium-241

Post Spike (8G15009-PS1)

Neptunium-237

Plutonium-239

Americium-241

\begin{tabular}{|c|c|c|c|c|c|c|}
\hline \multicolumn{3}{|c|}{ Source: $0805020-11$} & \multicolumn{2}{|c|}{ Prepared: 07/15/08 } & \multicolumn{2}{|c|}{ Analyzed: 07/17/08 } \\
\hline$<3.70 \mathrm{E}-3$ & $3.70 \mathrm{E}-3$ & ug/g dry & & ND & & \\
\hline$<9.20 \mathrm{E}-3$ & $9.20 \mathrm{E}-3$ & $"$ & & ND & & \\
\hline$<7.96 \mathrm{E}-3$ & 7.96E-3 & $"$ & & ND & & \\
\hline \multicolumn{3}{|c|}{ Source: 0805020-11 } & Prepare & $7 / 15 / 08$ & Analyzec & $7 / 17 / 0$ \\
\hline $8.61 \mathrm{E}-2$ & N/A & ug/L & 0.1 & 29.4E-4 & 83.1 & $75-12$ \\
\hline 9.17E-2 & N/A & $"$ & 0.1 & 41.E-4 & 87.6 & $75-1$ \\
\hline 8.37E-2 & N/A & $"$ & 0.1 & $32.2 \mathrm{E}-4$ & 80.4 & $75-1$ \\
\hline
\end{tabular}

Batch 8G15010 - ASTM D 5198 (ICP/ICPMS)

Blank (8G15010-BLK1)

Neptunium-237

Plutonium-239

Americium-241

Duplicate (8G15010-DUP1)

Neptunium-237

Plutonium-239

Americium-241

Post Spike (8G15010-PS1)

Neptunium-237

Plutonium-239

Americium-241
Prepared: 07/15/08 Analyzed: 07/17/08

$\begin{array}{llc}<9.19 \mathrm{E}-4 & 9.19 \mathrm{E}-4 & \text { ug/g wet } \\ <2.28 \mathrm{E}-3 & 2.28 \mathrm{E}-3 & " \\ <1.98 \mathrm{E}-3 & 1.98 \mathrm{E}-3 & "\end{array}$

Source: 0805020-27 Prepared: 07/15/08 Analyzed: 07/17/08

$\begin{array}{ccccc}<3.37 \mathrm{E}-3 & 3.37 \mathrm{E}-3 & \text { ug/g dry } & \text { ND } & 35 \\ <8.39 \mathrm{E}-3 & 8.39 \mathrm{E}-3 & " & \text { ND } & 35 \\ <7.26 \mathrm{E}-3 & 7.26 \mathrm{E}-3 & " & \text { ND } & 35\end{array}$

Source: 0805020-27 Prepared: 07/15/08 Analyzed: 07/17/08

$\begin{array}{rcccccc}1.05 \mathrm{E}-1 & \text { N/A } & \text { ug/L } & 0.1 & 31 . \mathrm{E}-4 & 102 & 75-125 \\ 1.1 \mathrm{E}-1 & \text { N/A } & " & 0.1 & 38.9 \mathrm{E}-4 & 106 & 75-125 \\ 9.87 \mathrm{E}-2 & \text { N/A } & " & 0.1 & 35.5 \mathrm{E}-4 & 95.2 & 75-125\end{array}$




\section{Radionuclides by ICP-MS/Water Extract - Quality Control \\ Environmental Science Laboratory}

\begin{tabular}{|lrrrrrrrrrrr}
\hline & & Reporting & & Spike & Source & & & \%REC & & RPD & \\
Analyte & Result & Limit & Units & Level & Result & \%REC & Limits & RPD & Limit & Notes \\
\hline
\end{tabular}

Batch 8E29006 - 1:1 Water Extract (ICP/ICPMS)

Blank (8E29006-BLK1)

Technetium-99

Uranium 238

Blank (8E29006-BLK2)

Technetium-99

Uranium 238

Duplicate (8E29006-DUP1)

Technetium-99

Uranium 238

Batch 8F09006 - 1:1 Water Extract (ICP/ICPMS)

Blank (8F09006-BLK1)

Technetium-99

Uranium 238

Duplicate (8F09006-DUP1)

Technetium-99

Uranium 238

$\begin{array}{ccc}<2.30 \mathrm{E}-5 & 2.30 \mathrm{E}-5 & \text { ug/g wet } \\ <5.64 \mathrm{E}-4 & 5.64 \mathrm{E}-4 & "\end{array}$

Prepared: 05/29/08 Analyzed: 05/30/08

Prepared: 05/29/08 Analyzed: 05/30/08

$<2.30 \mathrm{E}-5 \quad 2.30 \mathrm{E}-5 \quad$ ug/g wet

$<5.64 \mathrm{E}-4 \quad 5.64 \mathrm{E}-4 \quad "$

Source: 0805020-25

Prepared: 05/29/08 Analyzed: 05/30/08

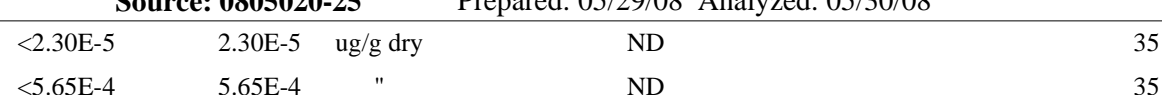

每

Prepared \& Analyzed: 06/09/08

$<2.30 \mathrm{E}-5 \quad 2.30 \mathrm{E}-5 \quad \mathrm{ug} / \mathrm{g}$ wet

$<5.64 \mathrm{E}-4 \quad 5.64 \mathrm{E}-4 \quad "$

Batch 8G01002 - 1:1 Water Extract (ICP/ICPMS)

Blank (8G01002-BLK1)

Technetium-99

Uranium 238

$\begin{array}{ccc}<2.30 \mathrm{E}-5 & 2.30 \mathrm{E}-5 & \text { ug/g wet } \\ <5.64 \mathrm{E}-4 & 5.64 \mathrm{E}-4 & "\end{array}$

Prepared \& Analyzed: 07/01/08

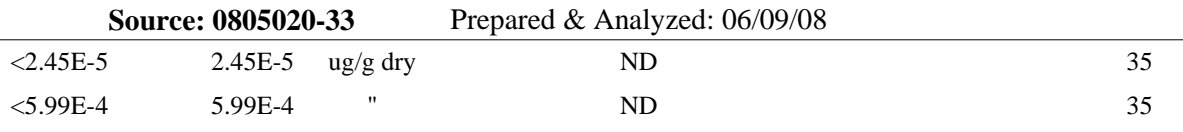




\section{Radionuclides by ICP-MS/Water Extract - Quality Control \\ Environmental Science Laboratory}

\begin{tabular}{|c|c|c|c|c|c|c|c|c|c|c|}
\hline & & orting & & Spike & Source & & \%REC & & RPD & \\
\hline Analyte & Result & Limit & Units & Level & Result & \%REC & Limits & RPD & Limit & Notes \\
\hline
\end{tabular}

Batch 8G01002 - 1:1 Water Extract (ICP/ICPMS)

Duplicate (8G01002-DUP1)

Technetium-99

Uranium 238

Post Spike (8G01002-PS1)

Technetium-99

Uranium 238

Batch 8G01003 - 1:1 Water Extract (ICP/ICPMS)

Blank (8G01003-BLK1)

Technetium-99

Uranium 238

Duplicate (8G01003-DUP1)

Technetium-99

Uranium 238

Post Spike (8G01003-PS1)

Technetium-99

Uranium 238

\begin{tabular}{|c|c|c|}
\hline \multicolumn{3}{|c|}{ Source: 0805020-11 } \\
\hline $6.21 \mathrm{E}-5$ & $2.41 \mathrm{E}-5$ & ug/g dry \\
\hline $3.26 \mathrm{E}-1$ & $5.91 \mathrm{E}-4$ & " \\
\hline
\end{tabular}

Prepared: 07/01/08 Analyzed: 07/22/08

4.93E-5

3.18E-1

Prepared: 07/01/08 Analyzed: 07/22/08

Source: 0805020-11

$\begin{array}{rcccccc}4.49 \mathrm{E}-1 & \text { N/A } & \text { ug/L } & 0.5 & 98 . \mathrm{E}-4 & 87.8 & 75-125 \\ 6.34 \mathrm{E} 1 & \text { N/A } & " & 0.5 & 63.2 \mathrm{E} 0 & 41.8 & 75-125\end{array}$

$\begin{array}{ll}22.9 & 35 \\ 2.39 & 35\end{array}$


RCRA Metals By PNNL-AGG-415/Water Extract - Quality Control

\begin{tabular}{|c|c|c|c|c|c|c|c|c|c|c|}
\hline Analyte & Result & $\begin{array}{c}\text { orting } \\
\text { Limit }\end{array}$ & Units & $\begin{array}{l}\text { Spike } \\
\text { Level }\end{array}$ & $\begin{array}{l}\text { Source } \\
\text { Result }\end{array}$ & \%REC & $\begin{array}{l}\text { \%REC } \\
\text { Limits }\end{array}$ & RPD & $\begin{array}{l}\text { RPD } \\
\text { Limit }\end{array}$ & Notes \\
\hline
\end{tabular}

Batch 8G01004 - 1:1 Water Extract (ICP/ICPMS)

Blank (8G01004-BLK1)

Chromium 52

Chromium 53

Copper 63

Copper 65

Selenium 82

Molybdenum 95

Molybdenum 97

Molybdenum 98

Ruthenium 101

Ruthenium 102

Ruthenium 104

Silver 107

Silver 109

Cadmium 111

Cadmium 114

Antimony 121

Lead 206

Lead 208
LCS (8G01004-BS1)

Chromium 52

Chromium 53

Copper 63

Copper 65

Selenium 82

Molybdenum 95

Molybdenum 97

Molybdenum 98

Ruthenium 101

Ruthenium 102

Ruthenium 104

Silver 107

Silver 109

Cadmium 111

Cadmium 114

Lead 206

Lead 208
Environmental Science Laboratory

Prepared \& Analyzed: 07/01/08

$\begin{array}{llc}<2.06 \mathrm{E}-3 & 2.06 \mathrm{E}-3 & \text { ug/g wet } \\ <6.40 \mathrm{E}-3 & 6.40 \mathrm{E}-3 & " \\ <3.48 \mathrm{E}-3 & 3.48 \mathrm{E}-3 & " \\ <4.84 \mathrm{E}-3 & 4.84 \mathrm{E}-3 & " \\ <1.10 \mathrm{E}-2 & 1.10 \mathrm{E}-2 & " \\ <1.60 \mathrm{E}-3 & 1.60 \mathrm{E}-3 & " \\ <2.04 \mathrm{E}-3 & 2.04 \mathrm{E}-3 & " \\ <1.60 \mathrm{E}-3 & 1.60 \mathrm{E}-3 & " \\ <8.20 \mathrm{E}-4 & 8.20 \mathrm{E}-4 & " \\ <5.65 \mathrm{E}-4 & 5.65 \mathrm{E}-4 & " \\ <3.65 \mathrm{E}-4 & 3.65 \mathrm{E}-4 & " \\ <9.25 \mathrm{E}-4 & 9.25 \mathrm{E}-4 & " \\ <1.07 \mathrm{E}-3 & 1.07 \mathrm{E}-3 & " \\ <2.95 \mathrm{E}-4 & 2.95 \mathrm{E}-4 & " \\ <6.25 \mathrm{E}-4 & 6.25 \mathrm{E}-4 & " \\ <5.40 \mathrm{E}-4 & 5.40 \mathrm{E}-4 & " \\ <7.20 \mathrm{E}-4 & 7.20 \mathrm{E}-4 & " \\ <5.60 \mathrm{E}-4 & 5.60 \mathrm{E}-4 & "\end{array}$

Prepared \& Analyzed: 07/01/08

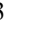

Result

\begin{abstract}
$5.60 \mathrm{E}-4$
\end{abstract}


RCRA Metals By PNNL-AGG-415/Water Extract - Quality Control

Environmental Science Laboratory

\begin{tabular}{|lrrrrrrrrrrr}
\hline & & Reporting & & Spike & Source & & & \%REC & & RPD & \\
Analyte & Result & Limit & Units & Level & Result & \%REC & Limits & RPD & Limit & Notes \\
\hline
\end{tabular}

Batch 8G01004 - 1:1 Water Extract (ICP/ICPMS)

Duplicate (8G01004-DUP1)

Chromium 52
Chromium 53
Copper 63
Copper 65
Selenium 82
Molybdenum 95
Molybdenum 97
Molybdenum 98
Ruthenium 101
Ruthenium 102
Ruthenium 104
Silver 107
Silver 109
Cadmium 111
Cadmium 114
Antimony 121
Lead 206
Lead 208

Source: 0805020-11

Prepared \& Analyzed: 07/01/08

$\begin{array}{rrc}1.68 \mathrm{E}-2 & 2.16 \mathrm{E}-3 & \text { ug/g dry } \\ 1.65 \mathrm{E}-2 & 6.72 \mathrm{E}-3 & " \\ 1.27 \mathrm{E}-1 & 3.65 \mathrm{E}-3 & " \\ 1.22 \mathrm{E}-1 & 5.08 \mathrm{E}-3 & " \\ <1.16 \mathrm{E}-2 & 1.16 \mathrm{E}-2 & " \\ 4.83 \mathrm{E}-2 & 1.68 \mathrm{E}-3 & " \\ 4.54 \mathrm{E}-2 & 2.15 \mathrm{E}-3 & " \\ 4.41 \mathrm{E}-2 & 1.68 \mathrm{E}-3 & " \\ 1.06 \mathrm{E}-3 & 8.61 \mathrm{E}-4 & " \\ <5.93 \mathrm{E}-4 & 5.93 \mathrm{E}-4 & " \\ <3.83 \mathrm{E}-4 & 3.83 \mathrm{E}-4 & " \\ 1.91 \mathrm{E}-3 & 9.71 \mathrm{E}-4 & " \\ <1.12 \mathrm{E}-3 & 1.12 \mathrm{E}-3 & " \\ 4.02 \mathrm{E}-4 & 3.10 \mathrm{E}-4 & " \\ <6.56 \mathrm{E}-4 & 6.56 \mathrm{E}-4 & " \\ 1.58 \mathrm{E}-3 & 5.67 \mathrm{E}-4 & " \\ 2.87 \mathrm{E}-3 & 7.56 \mathrm{E}-4 & " \\ 2.88 \mathrm{E}-3 & 5.88 \mathrm{E}-4 & "\end{array}$

$\begin{array}{lll}1.23 \mathrm{E}-2 & 30.8 & 35 \\ 1.22 \mathrm{E}-2 & 30.6 & 35 \\ 1.25 \mathrm{E}-1 & 1.47 & 35 \\ 1.19 \mathrm{E}-1 & 2.18 & 35 \\ \mathrm{ND} & & 35 \\ 6.55 \mathrm{E}-2 & 30.3 & 35 \\ 6.21 \mathrm{E}-2 & 31.0 & 35 \\ 6.14 \mathrm{E}-2 & 32.7 & 35 \\ 1.03 \mathrm{E}-3 & 2.73 & 35 \\ \mathrm{ND} & & 35 \\ \mathrm{ND} & & 35 \\ 1.77 \mathrm{E}-3 & & 35 \\ \mathrm{ND} & 7.58 & 35 \\ 4.68 \mathrm{E}-4 & & 35 \\ \mathrm{ND} & 15.0 & 35 \\ 1.76 \mathrm{E}-3 & & 35 \\ 3.85 \mathrm{E}-3 & 10.4 & 35 \\ 3.84 \mathrm{E}-3 & 29.1 & \end{array}$

Post Spike (8G01004-PS1)

Source: 0805020-11

Prepared \& Analyzed: 07/01/08

\begin{tabular}{lr}
\hline Chromium 52 & $7.77 \mathrm{E} 0$ \\
Chromium 53 & $7.57 \mathrm{E} 0$ \\
Copper 63 & $2.91 \mathrm{E} 1$ \\
Copper 65 & $2.8 \mathrm{E} 1$ \\
Selenium 82 & $7.26 \mathrm{E} 0$ \\
Molybdenum 95 & $1.8 \mathrm{E} 1$ \\
Molybdenum 97 & $1.75 \mathrm{E} 1$ \\
Molybdenum 98 & $1.72 \mathrm{E} 1$ \\
Ruthenium 101 & $5.31 \mathrm{E} 0$ \\
Ruthenium 102 & $5.14 \mathrm{E} 0$ \\
Ruthenium 104 & $4.3 \mathrm{E} 0$ \\
Silver 107 & $4.93 \mathrm{E} 0$ \\
Silver 109 & $4.66 \mathrm{E} 0$ \\
Cadmium 111 & $5.13 \mathrm{E} 0$ \\
Cadmium 114 & $5.07 \mathrm{E} 0$ \\
Antimony 121 & $5.47 \mathrm{E} 0$ \\
Lead 206 & $5.95 \mathrm{E} 0$ \\
Lead 208 & $5.9 \mathrm{E} 0$
\end{tabular}

$\begin{array}{lccccc}\text { N/A } & \text { ug/L } & 5 & 24.5 \mathrm{E}-1 & 106 & 75-125 \\ \text { N/A } & " & 5 & 24.2 \mathrm{E}-1 & 103 & 75-125 \\ \text { N/A } & " & 5 & 24.8 \mathrm{E} 0 & 84.9 & 75-125 \\ \text { N/A } & " & 5 & 23.7 \mathrm{E} 0 & 86.9 & 75-125 \\ \text { N/A } & " & 5 & 12.1 \mathrm{E}-1 & 121 & 75-125 \\ \text { N/A } & " & 5 & 13 . \mathrm{E} 0 & 100 & 75-125 \\ \text { N/A } & " & 5 & 12.3 \mathrm{E} 0 & 103 & 75-125 \\ \text { N/A } & " & 5 & 12.2 \mathrm{E} 0 & 100 & 75-125 \\ \text { N/A } & " & 5 & 20.5 \mathrm{E}-2 & 102 & 75-125 \\ \text { N/A } & " & 5 & 27.6 \mathrm{E}-3 & 102 & 75-125 \\ \text { N/A } & " & 5 & \text { ND } & 101 & 75-125 \\ \text { N/A } & " & 5 & 35.2 \mathrm{E}-2 & 91.6 & 75-125 \\ \text { N/A } & " & 5 & 76.8 \mathrm{E}-3 & 91.6 & 75-125 \\ \text { N/A } & " & 5 & 93 . \mathrm{E}-3 & 101 & 75-125 \\ \text { N/A } & " & 5 & 44.8 \mathrm{E}-3 & 101 & 75-125 \\ \text { N/A } & " & 5 & 34.9 \mathrm{E}-2 & 102 & 75-125 \\ \text { N/A } & " & 5 & 76.5 \mathrm{E}-2 & 104 & 75-125 \\ \text { N/A } & " & 5 & 76.3 \mathrm{E}-2 & 103 & 75-125\end{array}$


RCRA Metals By PNNL-AGG-415/Water Extract - Quality Control

\begin{tabular}{|c|c|c|c|c|c|c|c|c|c|c|}
\hline Analyte & Result & $\begin{array}{c}\text { orting } \\
\text { Limit }\end{array}$ & Units & $\begin{array}{l}\text { Spike } \\
\text { Level }\end{array}$ & $\begin{array}{l}\text { Source } \\
\text { Result }\end{array}$ & \%REC & $\begin{array}{l}\text { \%REC } \\
\text { Limits }\end{array}$ & RPD & $\begin{array}{l}\text { RPD } \\
\text { Limit }\end{array}$ & Notes \\
\hline
\end{tabular}

Batch 8G01005 - 1:1 Water Extract (ICP/ICPMS)

Blank (8G01005-BLK1)

Chromium 52

Chromium 53

Copper 63

Copper 65

Selenium 82

Molybdenum 95

Molybdenum 97

Molybdenum 98

Ruthenium 101

Ruthenium 102

Ruthenium 104

Silver 107

Silver 109

Cadmium 111

Cadmium 114

Antimony 121

Lead 206

Lead 208
LCS (8G01005-BS1)

Chromium 52

Chromium 53

Copper 63

Copper 65

Selenium 82

Molybdenum 95

Molybdenum 97

Molybdenum 98

Ruthenium 101

Ruthenium 102

Ruthenium 104

Silver 107

Silver 109

Cadmium 111

Cadmium 114

Lead 206

Lead 208

$4.87 \mathrm{E} 0 \quad 2.06 \mathrm{E}-1 \quad \mathrm{ug} / \mathrm{g}$ wet

\begin{tabular}{|c|c|c|c|c|c|}
\hline 4.87E0 & $2.06 \mathrm{E}-1$ & ug/g wet & 4.997 & 97.4 & $80-120$ \\
\hline $4.81 \mathrm{E} 0$ & $6.40 \mathrm{E}-1$ & $"$ & 4.997 & 96.3 & $80-120$ \\
\hline 4.64E0 & 3.48E-1 & $"$ & 4.997 & 92.8 & $80-120$ \\
\hline $4.58 \mathrm{E} 0$ & $4.84 \mathrm{E}-1$ & $"$ & 4.997 & 91.7 & $80-120$ \\
\hline $5.25 \mathrm{E} 0$ & 1.10E0 & $"$ & 4.997 & 105 & $80-120$ \\
\hline 4.64E0 & $1.60 \mathrm{E}-1$ & $"$ & 4.997 & 92.8 & $80-120$ \\
\hline 4.62E0 & $2.04 \mathrm{E}-1$ & $"$ & 4.997 & 92.4 & $80-120$ \\
\hline 4.65E0 & $1.60 \mathrm{E}-1$ & $"$ & 4.997 & 93.0 & $80-120$ \\
\hline$<8.20 \mathrm{E}-2$ & $8.20 \mathrm{E}-2$ & $"$ & & & $80-120$ \\
\hline$<5.65 \mathrm{E}-2$ & 5.65E-2 & $"$ & & & $80-120$ \\
\hline$<3.65 \mathrm{E}-2$ & $3.65 \mathrm{E}-2$ & $"$ & & & $80-120$ \\
\hline 4.55E0 & $9.25 \mathrm{E}-2$ & $"$ & 4.997 & 91.0 & $80-120$ \\
\hline 4.53E0 & $1.07 \mathrm{E}-1$ & $"$ & 4.997 & 90.7 & $80-120$ \\
\hline 4.79E0 & $2.95 \mathrm{E}-2$ & $"$ & 4.997 & 95.9 & $80-120$ \\
\hline 4.78E0 & $6.25 \mathrm{E}-2$ & $"$ & 4.997 & 95.7 & $80-120$ \\
\hline 4.53E0 & 7.20E-2 & $"$ & 4.997 & 90.6 & $80-120$ \\
\hline 4.48E0 & $5.60 \mathrm{E}-2$ & $"$ & 4.997 & 89.6 & $80-120$ \\
\hline
\end{tabular}

Environmental Science Laboratory

Prepared \& Analyzed: 07/01/08

$\begin{array}{cccc} & <2.06 \mathrm{E}-3 & 2.06 \mathrm{E}-3 & \mathrm{ug} / \mathrm{g} \text { wet } \\ & <6.40 \mathrm{E}-3 & 6.40 \mathrm{E}-3 & " \\ & <3.48 \mathrm{E}-3 & 3.48 \mathrm{E}-3 & " \\ & <4.84 \mathrm{E}-3 & 4.84 \mathrm{E}-3 & " \\ & <1.10 \mathrm{E}-2 & 1.10 \mathrm{E}-2 & " \\ & <1.60 \mathrm{E}-3 & 1.60 \mathrm{E}-3 & " \\ & <2.04 \mathrm{E}-3 & 2.04 \mathrm{E}-3 & " \\ & <1.60 \mathrm{E}-3 & 1.60 \mathrm{E}-3 & " \\ & <8.20 \mathrm{E}-4 & 8.20 \mathrm{E}-4 & " \\ & <5.65 \mathrm{E}-4 & 5.65 \mathrm{E}-4 & " \\ & <3.65 \mathrm{E}-4 & 3.65 \mathrm{E}-4 & " \\ & <9.25 \mathrm{E}-4 & 9.25 \mathrm{E}-4 & " \\ & <1.07 \mathrm{E}-3 & 1.07 \mathrm{E}-3 & " \\ & <2.95 \mathrm{E}-4 & 2.95 \mathrm{E}-4 & " \\ & <6.25 \mathrm{E}-4 & 6.25 \mathrm{E}-4 & " \\ & <5.40 \mathrm{E}-4 & 5.40 \mathrm{E}-4 & " \\ <7.20 \mathrm{E}-4 & 7.20 \mathrm{E}-4 & " \\ & <5.60 \mathrm{E}-4 & 5.60 \mathrm{E}-4 & \\ & & \\ & & \end{array}$

Prepared \& Analyzed: 07/01/08

3

Result 
RCRA Metals By PNNL-AGG-415/Water Extract - Quality Control

Environmental Science Laboratory

\begin{tabular}{|c|c|c|c|c|c|c|c|c|c|c|}
\hline Analyte & Result & $\begin{array}{c}\text { orting } \\
\text { Limit }\end{array}$ & Units & $\begin{array}{l}\text { Spike } \\
\text { Level }\end{array}$ & $\begin{array}{l}\text { Source } \\
\text { Result }\end{array}$ & \%REC & $\begin{array}{l}\text { \%REC } \\
\text { Limits }\end{array}$ & RPD & $\begin{array}{l}\text { RPD } \\
\text { Limit }\end{array}$ & Notes \\
\hline
\end{tabular}

Batch 8G01005 - 1:1 Water Extract (ICP/ICPMS)

Duplicate (8G01005-DUP1)

Chromium 52

Chromium 53

Copper 63

Copper 65

Selenium 82

Molybdenum 95

Molybdenum 97

Molybdenum 98

Ruthenium 101

Ruthenium 102

Ruthenium 104

Silver 107

Silver 109

Cadmium 111

Cadmium 114

Antimony 121

Lead 206

Lead 208
Source: 0805020-27 Prepared \& Analyzed: 07/01/08

$\begin{array}{rlc}<2.04 \mathrm{E}-3 & 2.04 \mathrm{E}-3 & \text { ug/g dry } \\ <6.34 \mathrm{E}-3 & 6.34 \mathrm{E}-3 & " \\ <3.45 \mathrm{E}-3 & 3.45 \mathrm{E}-3 & " \\ <4.80 \mathrm{E}-3 & 4.80 \mathrm{E}-3 & " \\ <1.10 \mathrm{E}-2 & 1.10 \mathrm{E}-2 & " \\ 5.85 \mathrm{E}-2 & 1.59 \mathrm{E}-3 & " \\ 5.81 \mathrm{E}-2 & 2.03 \mathrm{E}-3 & " \\ 5.82 \mathrm{E}-2 & 1.59 \mathrm{E}-3 & " \\ <8.13 \mathrm{E}-4 & 8.13 \mathrm{E}-4 & " \\ <5.60 \mathrm{E}-4 & 5.60 \mathrm{E}-4 & " \\ <3.62 \mathrm{E}-4 & 3.62 \mathrm{E}-4 & " \\ <9.17 \mathrm{E}-4 & 9.17 \mathrm{E}-4 & " \\ <1.06 \mathrm{E}-3 & 1.06 \mathrm{E}-3 & " \\ <2.92 \mathrm{E}-4 & 2.92 \mathrm{E}-4 & " \\ <6.20 \mathrm{E}-4 & 6.20 \mathrm{E}-4 & " \\ <5.35 \mathrm{E}-4 & 5.35 \mathrm{E}-4 & " \\ <7.14 \mathrm{E}-4 & 7.14 \mathrm{E}-4 & " \\ <5.55 \mathrm{E}-4 & 5.55 \mathrm{E}-4 & "\end{array}$

Source: 0805020-27

Prepared \& Analyzed: 07/01/08

Chromium 52

Chromium 53

Copper 63

Copper 65

Selenium 82

Molybdenum 95

Molybdenum 97

Molybdenum 98

Ruthenium 101

Ruthenium 102

Ruthenium 104

Silver 107

Silver 109

Cadmium 111

Cadmium 114

Antimony 121

Lead 206

Lead 208
$5.41 \mathrm{E} 0$

$5.43 \mathrm{E} 0$

$5.14 \mathrm{E} 0$

5.17E0

6.58E0

$1.69 \mathrm{E} 1$

$1.68 \mathrm{E} 1$

$1.7 \mathrm{E} 1$

$5.11 \mathrm{E} 0$

5.09E0

$5.14 \mathrm{E} 0$

$4.84 \mathrm{E} 0$

$4.83 \mathrm{E} 0$

5.33E0

$5.17 \mathrm{E} 0$

5.E0

$5.04 \mathrm{E} 0$

4.98E0

N/A

N/A ug/L

$$
\text { N/A }
$$

$$
\text { N/A }
$$

$$
\text { N/A }
$$$$
\text { N/A }
$$$$
\text { N/A }
$$$$
\text { N/A }
$$$$
\text { N/A }
$$$$
\text { N/A }
$$$$
\text { N/A }
$$$$
\text { N/A }
$$$$
\text { N/A }
$$$$
\text { N/A }
$$$$
\text { N/A }
$$$$
\text { N/A }
$$$$
\text { N/A }
$$$$
\text { N/A }
$$

\begin{tabular}{|c|c|}
\hline ND & \\
\hline ND & \\
\hline ND & \\
\hline ND & \\
\hline ND & \\
\hline $5.95 \mathrm{E}-2$ & 1.69 \\
\hline 5.93E-2 & 1.97 \\
\hline $5.94 \mathrm{E}-2$ & 2.06 \\
\hline ND & \\
\hline ND & \\
\hline ND & \\
\hline ND & \\
\hline ND & \\
\hline ND & \\
\hline ND & \\
\hline ND & \\
\hline ND & \\
\hline ND & \\
\hline
\end{tabular}$$
\text { N/A }
$$

ustis

$\begin{array}{cccc}5 & 33.4 \mathrm{E}-3 & 108 & 75-125 \\ 5 & 20.7 \mathrm{E}-2 & 104 & 75-125 \\ 5 & 36.9 \mathrm{E}-3 & 102 & 75-125 \\ 5 & 18.9 \mathrm{E}-2 & 99.7 & 75-125 \\ 5 & 52.6 \mathrm{E}-2 & 121 & 75-125 \\ 5 & 11.9 \mathrm{E} 0 & 101 & 75-125 \\ 5 & 11.8 \mathrm{E} 0 & 99.5 & 75-125 \\ 5 & 11.9 \mathrm{E} 0 & 103 & 75-125 \\ 5 & 21.4 \mathrm{E}-3 & 102 & 75-125 \\ 5 & 46.2 \mathrm{E}-4 & 102 & 75-125 \\ 5 & 60.6 \mathrm{E}-3 & 102 & 75-125 \\ 5 & 88.4 \mathrm{E}-4 & 96.6 & 75-125 \\ 5 & 95.1 \mathrm{E}-4 & 96.4 & 75-125 \\ 5 & 44.3 \mathrm{E}-3 & 106 & 75-125 \\ 5 & 11.3 \mathrm{E}-3 & 103 & 75-125 \\ 5 & 88.7 \mathrm{E}-3 & 98.3 & 75-125 \\ 5 & 11.3 \mathrm{E}-3 & 101 & 75-125 \\ 5 & 16.9 \mathrm{E}-3 & 99.2 & 75-125\end{array}$




\section{RCRA Metals By PNNL-AGG-415/Acid Extract - Quality Control}

Environmental Science Laboratory

\begin{tabular}{|c|c|c|c|c|c|c|c|c|c|c|}
\hline Analyte & Result & $\begin{array}{c}\text { orting } \\
\text { Limit }\end{array}$ & Units & $\begin{array}{l}\text { Spike } \\
\text { Level }\end{array}$ & $\begin{array}{l}\text { Source } \\
\text { Result }\end{array}$ & \%REC & $\begin{array}{l}\text { \%REC } \\
\text { Limits }\end{array}$ & RPD & $\begin{array}{l}\text { RPD } \\
\text { Limit }\end{array}$ & Notes \\
\hline
\end{tabular}

Batch 8G02005 - ASTM D 5198 (ICP/ICPMS)
Blank (8G02005-BLK1)

\section{Selenium 82}

Molybdenum 95

Molybdenum 97

Molybdenum 98

Ruthenium 101

Ruthenium 102

Ruthenium 104

Cadmium 111

Cadmium 114

Antimony 121

LCS (8G02005-BS1)

Selenium 82

Molybdenum 95

Molybdenum 97

Molybdenum 98

Ruthenium 101

Ruthenium 102

Ruthenium 104

Cadmium 111

Cadmium 114

Antimony 121

$\begin{array}{llc}<3.28 \mathrm{E}-1 & 3.28 \mathrm{E}-1 & \text { ug/g wet } \\ <6.72 \mathrm{E}-2 & 6.72 \mathrm{E}-2 & " \\ <6.16 \mathrm{E}-2 & 6.16 \mathrm{E}-2 & " \\ <2.55 \mathrm{E}-2 & 2.55 \mathrm{E}-2 & " \\ <1.29 \mathrm{E}-2 & 1.29 \mathrm{E}-2 & " \\ <7.80 \mathrm{E}-3 & 7.80 \mathrm{E}-3 & " \\ <2.18 \mathrm{E}-2 & 2.18 \mathrm{E}-2 & " \\ <1.41 \mathrm{E}-2 & 1.41 \mathrm{E}-2 & " \\ <3.20 \mathrm{E}-3 & 3.20 \mathrm{E}-3 & " \\ <2.28 \mathrm{E}-2 & 2.28 \mathrm{E}-2 & "\end{array}$

Prepared \& Analyzed: 07/02/08

Prepared: 07/02/08 Analyzed: 07/03/08

$\begin{array}{rrrrrr}5.18 \mathrm{E} 0 & 4.61 \mathrm{E}-1 & \text { ug/g wet } & 5.000 & 104 & 80-120 \\ 5.17 \mathrm{E} 0 & 9.44 \mathrm{E}-2 & " & 5.000 & 103 & 80-120 \\ 5.14 \mathrm{E} 0 & 8.65 \mathrm{E}-2 & " & 5.000 & 103 & 80-120 \\ 5.19 \mathrm{E} 0 & 3.58 \mathrm{E}-2 & " & 5.000 & 104 & 80-120 \\ <1.81 \mathrm{E}-2 & 1.81 \mathrm{E}-2 & " & & & 80-120 \\ <1.10 \mathrm{E}-2 & 1.10 \mathrm{E}-2 & " & & & 80-120 \\ <3.06 \mathrm{E}-2 & 3.06 \mathrm{E}-2 & " & & & 80-120 \\ 5.13 \mathrm{E} 0 & 1.98 \mathrm{E}-2 & " & 5.000 & 103 & 80-120 \\ 5.10 \mathrm{E} 0 & 4.49 \mathrm{E}-3 & " & 5.000 & 102 & 80-120 \\ 4.98 \mathrm{E} 0 & 3.20 \mathrm{E}-2 & " & 5.000 & 99.6 & 80-120\end{array}$

Duplicate (8G02005-DUP1)

Source: 0805020-11 Prepared \& Analyzed: 07/02/08

Selenium 82

$<1.32 \mathrm{E} 0 \quad 1.32 \mathrm{E} 0 \quad$ ug/g dry

$<2.71 \mathrm{E}-1$

$2.71 \mathrm{E}-1$

ND

3.03E-1

$2.91 \mathrm{E}-1$

2.78E-1

2.12E-1

2.48E-1

1.03E-1

ND

ND

ND

8.95E-2

6.85E-2

ND

$\begin{array}{ll}35 \\ 27.0 & 35 \\ & 35 \\ & 35 \\ & 35 \\ & 35 \\ 10.9 & 35 \\ 3.18 & 35 \\ & 35\end{array}$


RCRA Metals By PNNL-AGG-415/Acid Extract - Quality Control

Environmental Science Laboratory

\begin{tabular}{|c|c|c|c|c|c|c|c|c|c|c|}
\hline Analyte & Result & $\begin{array}{c}\text { orting } \\
\text { Limit }\end{array}$ & Units & $\begin{array}{l}\text { Spike } \\
\text { Level }\end{array}$ & $\begin{array}{l}\text { Source } \\
\text { Result }\end{array}$ & \%REC & $\begin{array}{l}\text { \%REC } \\
\text { Limits }\end{array}$ & RPD & $\begin{array}{l}\text { RPD } \\
\text { Limit }\end{array}$ & Notes \\
\hline
\end{tabular}

Batch 8G02005 - ASTM D 5198 (ICP/ICPMS)

\begin{tabular}{|c|c|c|c|c|c|c|c|}
\hline \multirow{2}{*}{$\begin{array}{l}\text { Post Spike (8G02005-PS1) } \\
\text { Selenium } 82\end{array}$} & \multicolumn{3}{|c|}{ Source: 0805020-11 } & \multicolumn{4}{|c|}{ Prepared \& Analyzed: 07/02/08 } \\
\hline & $5.96 \mathrm{E} 0$ & N/A & ug/L & 5 & 34.7E-2 & 112 & $75-125$ \\
\hline Molybdenum 95 & 5.93E0 & N/A & $"$ & 5 & 76.2E-2 & 103 & $75-125$ \\
\hline Molybdenum 97 & $5.86 \mathrm{E} 0$ & N/A & $"$ & 5 & 73.2E-2 & 103 & $75-125$ \\
\hline Molybdenum 98 & 5.95E0 & N/A & $"$ & 5 & 69.9E-2 & 105 & $75-125$ \\
\hline Ruthenium 101 & 4.88E0 & N/A & $"$ & 5 & $32.5 \mathrm{E}-4$ & 97.6 & $75-125$ \\
\hline Ruthenium 102 & $4.85 \mathrm{E} 0$ & N/A & $"$ & 5 & ND & 97.5 & $75-125$ \\
\hline Ruthenium 104 & 4.29E0 & N/A & $"$ & 5 & ND & 96.4 & $75-125$ \\
\hline Cadmium 111 & 5.09E0 & N/A & $"$ & 5 & $22.5 \mathrm{E}-2$ & 97.4 & $75-125$ \\
\hline Cadmium 114 & 5.07E0 & N/A & $"$ & 5 & $17.2 \mathrm{E}-2$ & 98 & $75-125$ \\
\hline Antimony 121 & 4.87E0 & N/A & $"$ & 5 & 31.E-3 & 96.7 & $75-125$ \\
\hline
\end{tabular}

Batch 8G02007 - ASTM D 5198 (ICP/ICPMS)

\begin{tabular}{|c|c|c|c|c|c|c|}
\hline Blank (8G02007-BLK1) & \multicolumn{6}{|c|}{ Prepared \& Analyzed: 07/02/08 } \\
\hline Selenium 82 & $<3.28 \mathrm{E}-1$ & $3.28 \mathrm{E}-1$ & ug/g wet & & & \\
\hline Molybdenum 95 & $<6.72 \mathrm{E}-2$ & $6.72 \mathrm{E}-2$ & $"$ & & & \\
\hline Molybdenum 97 & $<6.16 \mathrm{E}-2$ & $6.16 \mathrm{E}-2$ & $"$ & & & \\
\hline Molybdenum 98 & $<2.55 \mathrm{E}-2$ & $2.55 \mathrm{E}-2$ & $"$ & & & \\
\hline Ruthenium 101 & $<1.29 \mathrm{E}-2$ & $1.29 \mathrm{E}-2$ & $"$ & & & \\
\hline Ruthenium 102 & $<7.80 \mathrm{E}-3$ & $7.80 \mathrm{E}-3$ & $"$ & & & \\
\hline Ruthenium 104 & $<2.18 \mathrm{E}-2$ & $2.18 \mathrm{E}-2$ & $"$ & & & \\
\hline Cadmium 111 & $<1.41 \mathrm{E}-2$ & $1.41 \mathrm{E}-2$ & $"$ & & & \\
\hline Cadmium 114 & $<3.20 \mathrm{E}-3$ & $3.20 \mathrm{E}-3$ & $"$ & & & \\
\hline Antimony 121 & $<2.28 \mathrm{E}-2$ & $2.28 \mathrm{E}-2$ & $"$ & & & \\
\hline LCS (8G02007-BS1) & \multicolumn{6}{|c|}{ Prepared: 07/02/08 Analyzed: 07/03/08 } \\
\hline Selenium 82 & $5.20 \mathrm{E} 0$ & $4.58 \mathrm{E}-1$ & ug/g wet & 5.000 & 104 & $80-120$ \\
\hline Molybdenum 95 & $5.16 \mathrm{E} 0$ & $9.38 \mathrm{E}-2$ & $"$ & 5.000 & 103 & $80-120$ \\
\hline Molybdenum 97 & $5.22 \mathrm{E} 0$ & $8.60 \mathrm{E}-2$ & $"$ & 5.000 & 104 & $80-120$ \\
\hline Molybdenum 98 & $5.27 \mathrm{E} 0$ & $3.56 \mathrm{E}-2$ & $"$ & 5.000 & 105 & $80-120$ \\
\hline Ruthenium 101 & $<1.80 \mathrm{E}-2$ & $1.80 \mathrm{E}-2$ & $"$ & & & $80-120$ \\
\hline Ruthenium 102 & $<1.09 \mathrm{E}-2$ & $1.09 \mathrm{E}-2$ & $"$ & & & $80-120$ \\
\hline Ruthenium 104 & $<3.04 \mathrm{E}-2$ & $3.04 \mathrm{E}-2$ & $"$ & & & $80-120$ \\
\hline Cadmium 111 & $5.21 \mathrm{E} 0$ & 1.97E-2 & $"$ & 5.000 & 104 & $80-120$ \\
\hline Cadmium 114 & $5.20 \mathrm{E} 0$ & 4.47E-3 & $"$ & 5.000 & 104 & $80-120$ \\
\hline Antimony 121 & $5.05 \mathrm{E} 0$ & $3.18 \mathrm{E}-2$ & $"$ & 5.000 & 101 & $80-120$ \\
\hline
\end{tabular}


RCRA Metals By PNNL-AGG-415/Acid Extract - Quality Control

Environmental Science Laboratory

\begin{tabular}{|c|c|c|c|c|c|c|c|c|c|c|}
\hline Analyte & Result & $\begin{array}{c}\text { orting } \\
\text { Limit }\end{array}$ & Units & $\begin{array}{l}\text { Spike } \\
\text { Level }\end{array}$ & $\begin{array}{l}\text { Source } \\
\text { Result }\end{array}$ & \%REC & $\begin{array}{l}\text { \%REC } \\
\text { Limits }\end{array}$ & RPD & $\begin{array}{l}\text { RPD } \\
\text { Limit }\end{array}$ & Notes \\
\hline
\end{tabular}

Batch 8G02007 - ASTM D 5198 (ICP/ICPMS)

\begin{tabular}{|c|c|c|c|c|c|c|c|c|c|}
\hline \multirow{2}{*}{$\begin{array}{l}\text { Duplicate (8G02007-DUP1) } \\
\text { Selenium } 82\end{array}$} & \multicolumn{3}{|c|}{ Source: 0805020-27 } & \multicolumn{6}{|c|}{ Prepared \& Analyzed: 07/02/08 } \\
\hline & $<1.20 \mathrm{E} 0$ & $1.20 \mathrm{E} 0$ & ug/g dry & & ND & & & & 35 \\
\hline Molybdenum 95 & $<2.47 \mathrm{E}-1$ & 2.47E-1 & $"$ & & $2.66 \mathrm{E}-1$ & & & & 35 \\
\hline Molybdenum 97 & 2.35E-1 & $2.26 \mathrm{E}-1$ & $"$ & & 2.64E-1 & & & 11.5 & 35 \\
\hline Molybdenum 98 & 2.34E-1 & $9.36 \mathrm{E}-2$ & $"$ & & $2.56 \mathrm{E}-1$ & & & 8.67 & 35 \\
\hline Ruthenium 101 & $<4.74 \mathrm{E}-2$ & 4.74E-2 & $"$ & & ND & & & & 35 \\
\hline Ruthenium 102 & $<2.86 \mathrm{E}-2$ & $2.86 \mathrm{E}-2$ & $"$ & & ND & & & & 35 \\
\hline Ruthenium 104 & $<8.00 \mathrm{E}-2$ & 8.00E-2 & $"$ & & ND & & & & 35 \\
\hline Cadmium 111 & $5.30 \mathrm{E}-2$ & $5.18 \mathrm{E}-2$ & $"$ & & ND & & & & 35 \\
\hline Cadmium 114 & $3.84 \mathrm{E}-2$ & 1.17E-2 & $"$ & & 3.67E-2 & & & 4.63 & 35 \\
\hline Antimony 121 & $<8.37 \mathrm{E}-2$ & 8.37E-2 & $"$ & & ND & & & & 35 \\
\hline Post Spike (8G02007-PS1) & \multicolumn{3}{|c|}{ Source: 0805020-27 } & \multicolumn{4}{|c|}{ Prepared \& Analyzed: 07/02/08 } & & \\
\hline Selenium 82 & 5.79E0 & N/A & ug/L & 5 & 12.2E-3 & 116 & $75-125$ & & \\
\hline Molybdenum 95 & 5.93E0 & N/A & $"$ & 5 & $71.4 \mathrm{E}-2$ & 104 & $75-125$ & & \\
\hline Molybdenum 97 & 5.94E0 & N/A & $"$ & 5 & $70.8 \mathrm{E}-2$ & 105 & $75-125$ & & \\
\hline Molybdenum 98 & 5.93E0 & N/A & $"$ & 5 & $68.6 \mathrm{E}-2$ & 105 & $75-125$ & & \\
\hline Ruthenium 101 & 4.97E0 & N/A & $"$ & 5 & ND & 99.6 & $75-125$ & & \\
\hline Ruthenium 102 & 4.98E0 & N/A & $"$ & 5 & ND & 99.9 & $75-125$ & & \\
\hline Ruthenium 104 & 4.69E0 & N/A & $"$ & 5 & ND & 98.7 & $75-125$ & & \\
\hline Cadmium 111 & $5.26 \mathrm{E} 0$ & N/A & $"$ & 5 & $13.4 \mathrm{E}-2$ & 102 & $75-125$ & & \\
\hline Cadmium 114 & 5.18E0 & N/A & $"$ & 5 & 98.5E-3 & 102 & $75-125$ & & \\
\hline Antimony 121 & $5.1 \mathrm{E} 0$ & N/A & $"$ & 5 & 20.E-3 & 102 & $75-125$ & & \\
\hline
\end{tabular}


Total Alpha Total Beta/Acid Extract - Quality Control

Environmental Science Laboratory

\begin{tabular}{|lrrrrrrrrrr}
\hline & & Reporting & & Spike & Source & & \%REC & & RPD & \\
Analyte & Result & Limit & Units & Level & Result & \%REC & Limits & RPD & Limit & Notes \\
\hline
\end{tabular}

\section{Batch 8G02015 - ASTM D 5198 (RadChem)}

\begin{tabular}{|c|c|c|c|c|c|}
\hline \multirow{2}{*}{$\begin{array}{l}\text { Blank (8G02015-BLK1) } \\
\text { Gross Beta }\end{array}$} & \multicolumn{5}{|c|}{ Prepared \& Analyzed: 07/02/08 } \\
\hline & $<1.30 \mathrm{E} 1$ & 13.0E0 & $\mathrm{pCi} / \mathrm{g}$ wet & & \\
\hline Gross Alpha & $<5.25 \mathrm{E} 0$ & 52.5E-1 & " & & \\
\hline Blank (8G02015-BLK2) & \multicolumn{5}{|c|}{ Prepared \& Analyzed: 07/02/08 } \\
\hline Gross Beta & $<1.30 \mathrm{E} 1$ & 13.0E0 & $\mathrm{pCi} / \mathrm{g}$ wet & & \\
\hline Gross Alpha & $<5.25 \mathrm{E} 0$ & $52.5 \mathrm{E}-1$ & " & & \\
\hline Duplicate (8G02015-DUP1) & \multicolumn{3}{|c|}{ Source: 0805020-11 } & \multicolumn{2}{|l|}{ Prepared \& Analyzed: 07/02/08 } \\
\hline Gross Beta & $<1.30 \mathrm{E} 1$ & $13.0 \mathrm{E} 0$ & pCi/g dry & ND & 35 \\
\hline Gross Alpha & $<5.25 \mathrm{E} 0$ & $52.5 \mathrm{E}-1$ & " & ND & 35 \\
\hline Duplicate (8G02015-DUP2) & \multicolumn{3}{|c|}{ Source: 0805020-27 } & Prepared \& Analyzed: 07/02/08 & \\
\hline Gross Beta & $<1.30 \mathrm{E} 1$ & $13.0 \mathrm{E} 0$ & pCi/g dry & ND & 35 \\
\hline Gross Alpha & $<5.25 \mathrm{E} 0$ & $52.5 \mathrm{E}-1$ & " & ND & 35 \\
\hline
\end{tabular}




\section{Total Alpha Total Beta/Water Extract - Quality Control \\ Environmental Science Laboratory}

\begin{tabular}{|lrrrrrrrrrrr}
\hline & & Reporting & & Spike & Source & & & \%REC & & RPD & \\
Analyte & Result & Limit & Units & Level & Result & \%REC & Limits & RPD & Limit & Notes \\
\hline
\end{tabular}

Batch 8G02003 - 1:1 Water Extract (RadChem)

\begin{tabular}{|c|c|c|c|c|c|}
\hline \multirow{2}{*}{$\begin{array}{l}\text { Blank (8G02003-BLK1) } \\
\text { Gross Beta }\end{array}$} & \multicolumn{5}{|c|}{ Prepared \& Analyzed: 07/01/08 } \\
\hline & $<1.21 \mathrm{E} 1$ & $12.1 \mathrm{E} 0$ & $\mathrm{pCi} / \mathrm{g}$ wet & & \\
\hline Gross Alpha & $<5.56 \mathrm{E} 0$ & $55.6 \mathrm{E}-1$ & " & & \\
\hline Blank (8G02003-BLK2) & \multicolumn{5}{|c|}{ Prepared \& Analyzed: 07/01/08 } \\
\hline Gross Beta & $<1.21 \mathrm{E} 1$ & $12.1 \mathrm{E} 0$ & $\mathrm{pCi} / \mathrm{g}$ wet & & \\
\hline Gross Alpha & $<5.56 \mathrm{E} 0$ & $55.6 \mathrm{E}-1$ & " & & \\
\hline Duplicate (8G02003-DUP1) & \multicolumn{3}{|c|}{ Source: 0805020-11 } & \multicolumn{2}{|l|}{ Prepared \& Analyzed: 07/01/08 } \\
\hline Gross Beta & $<1.21 \mathrm{E} 1$ & $12.1 \mathrm{E} 0$ & pCi/g dry & ND & 35 \\
\hline Gross Alpha & $<5.56 \mathrm{E} 0$ & $55.6 \mathrm{E}-1$ & " & ND & 35 \\
\hline Duplicate (8G02003-DUP2) & \multicolumn{3}{|c|}{ Source: 0805020-27 } & Prepared \& Analyzed: 07/01/08 & \\
\hline Gross Beta & $<1.21 \mathrm{E} 1$ & $12.1 \mathrm{E} 0$ & pCi/g dry & ND & 35 \\
\hline Gross Alpha & $<5.56 \mathrm{E} 0$ & $55.6 \mathrm{E}-1$ & " & ND & 35 \\
\hline
\end{tabular}




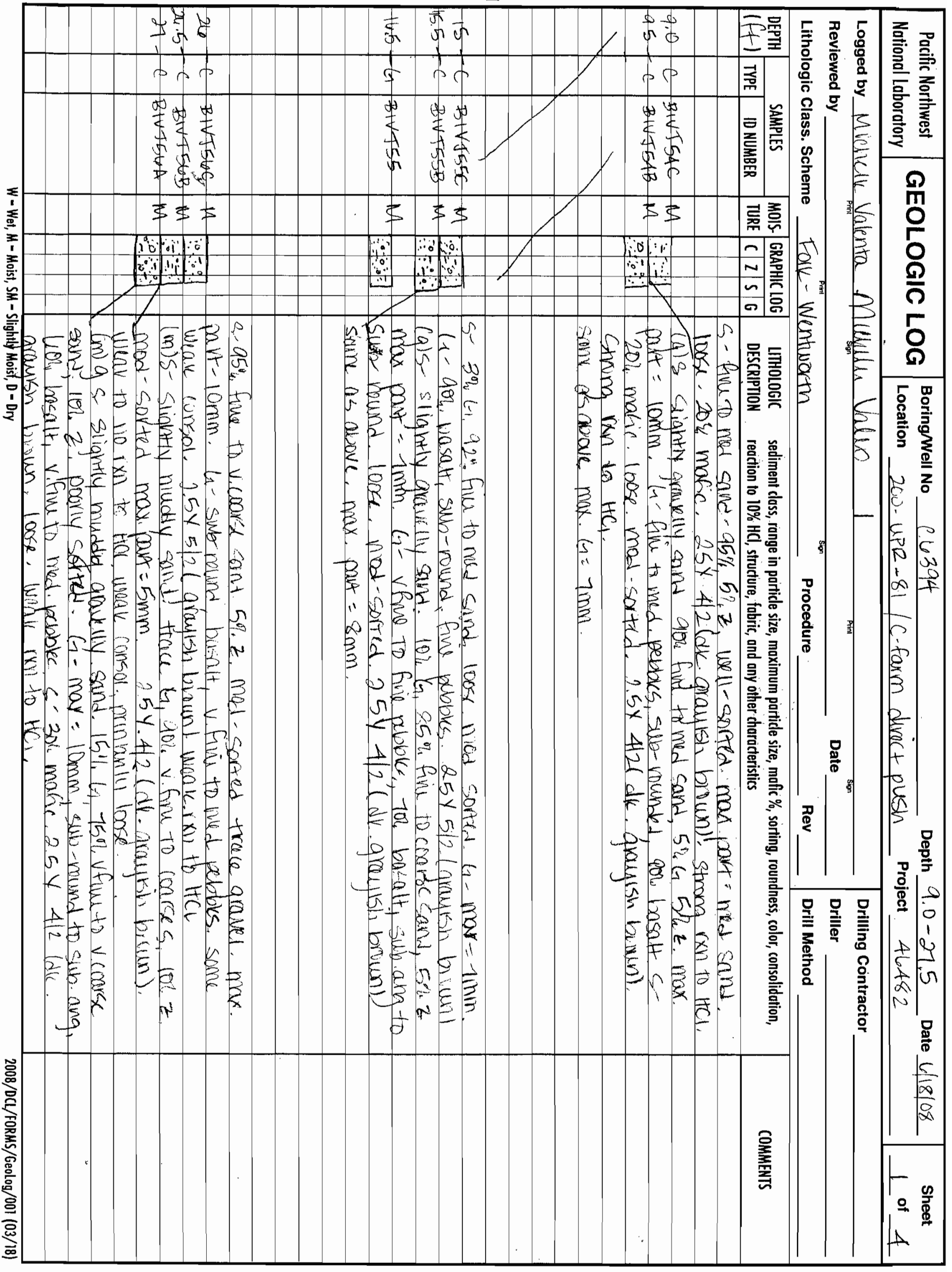




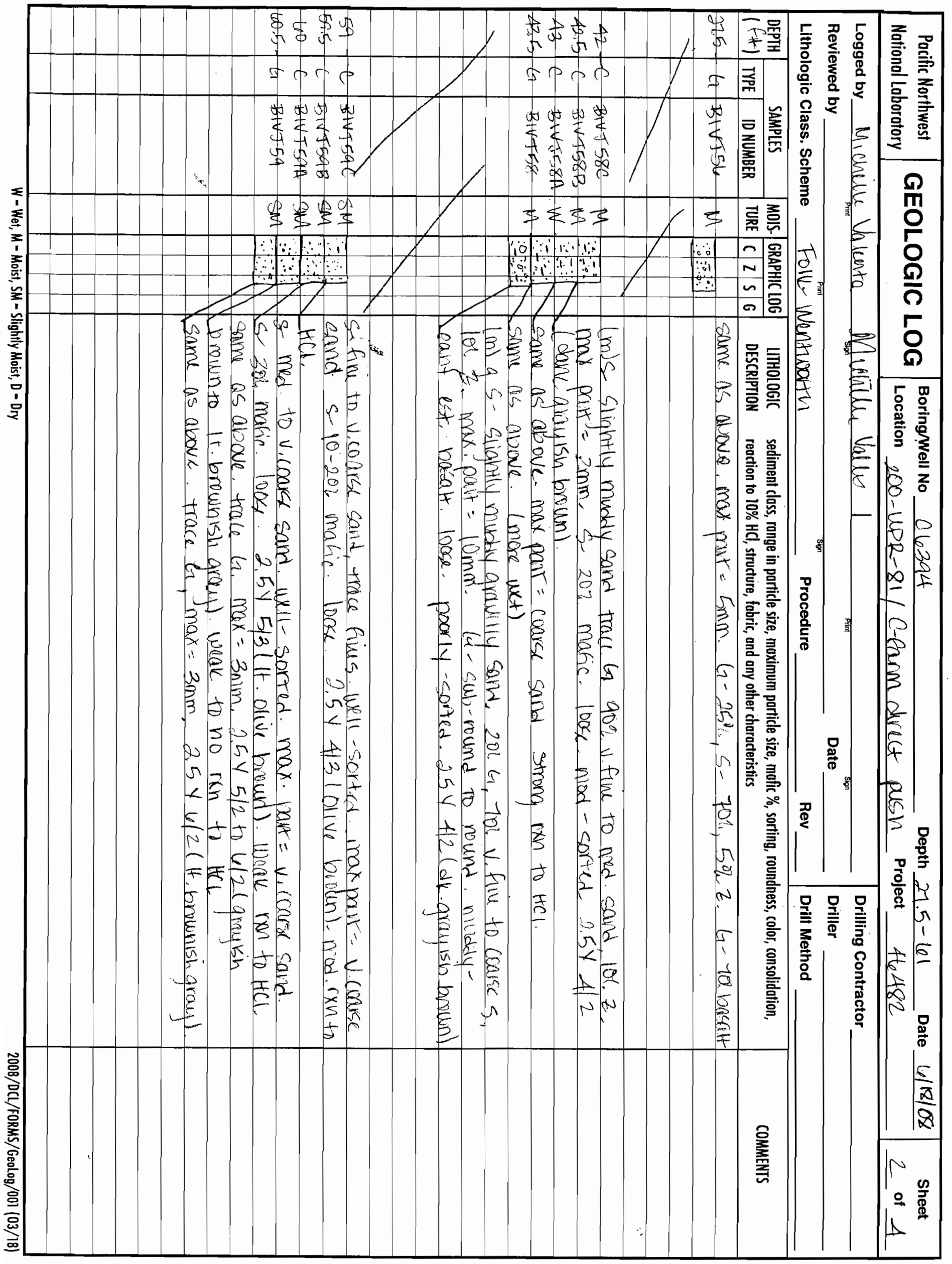




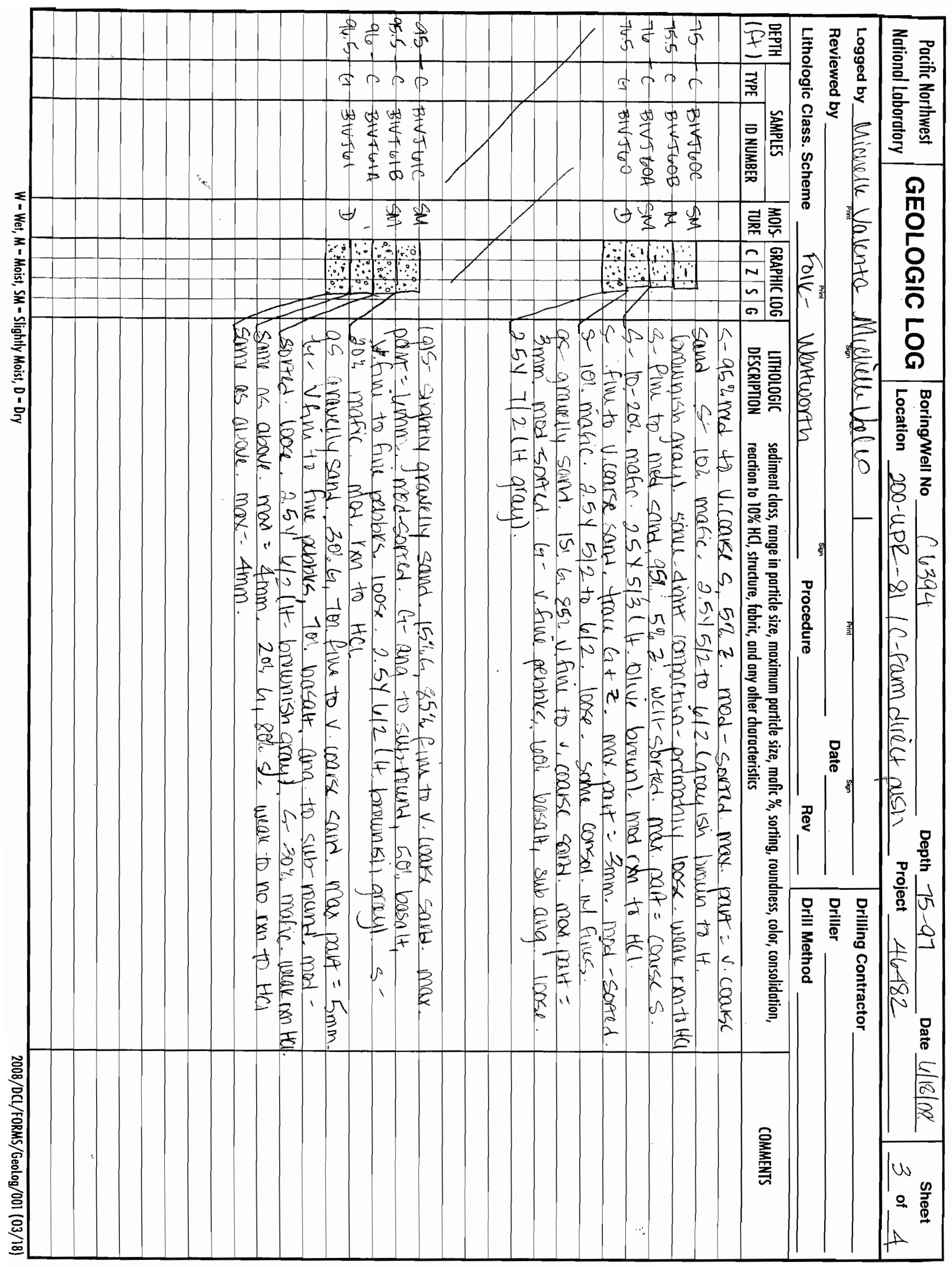




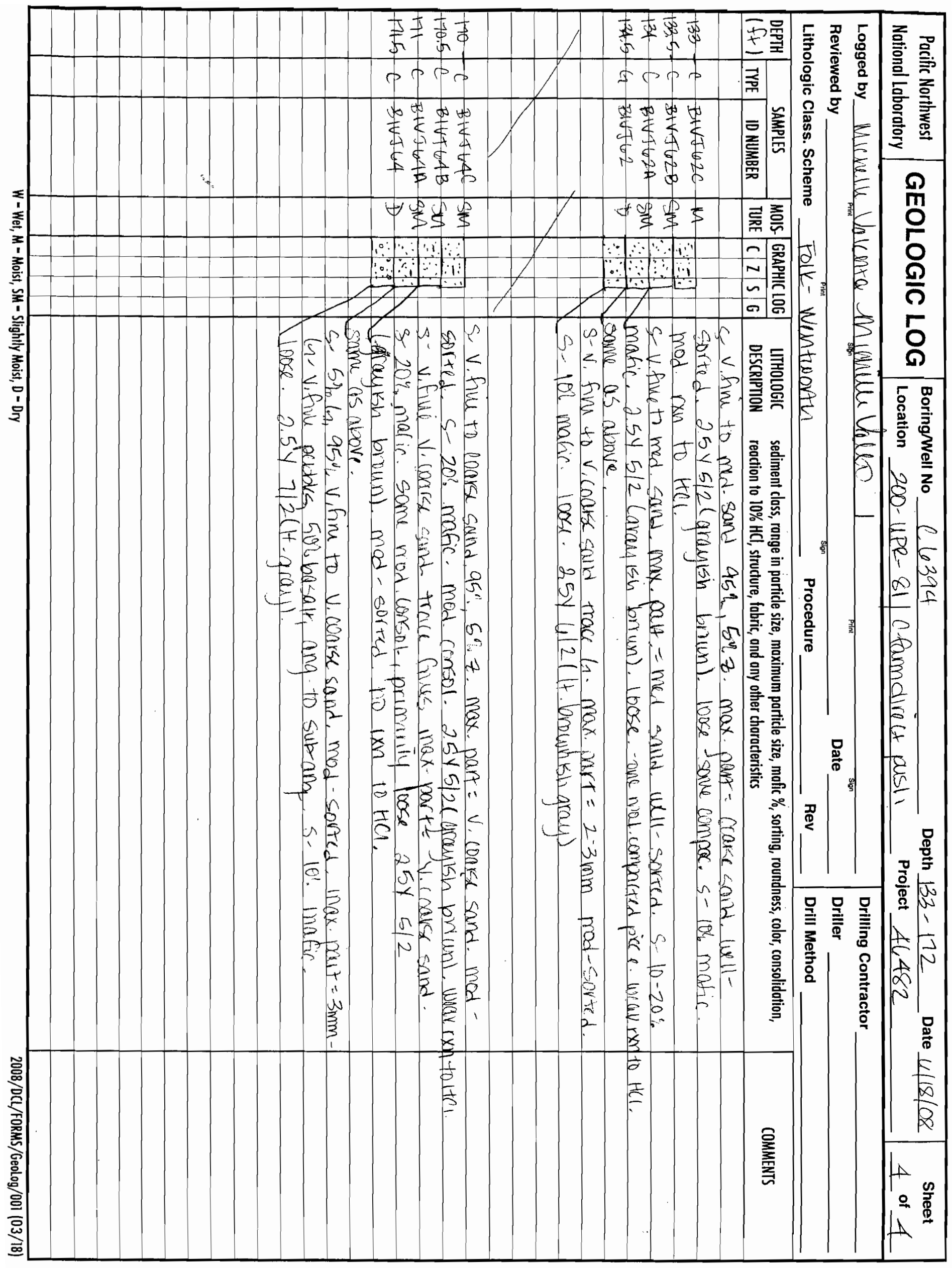




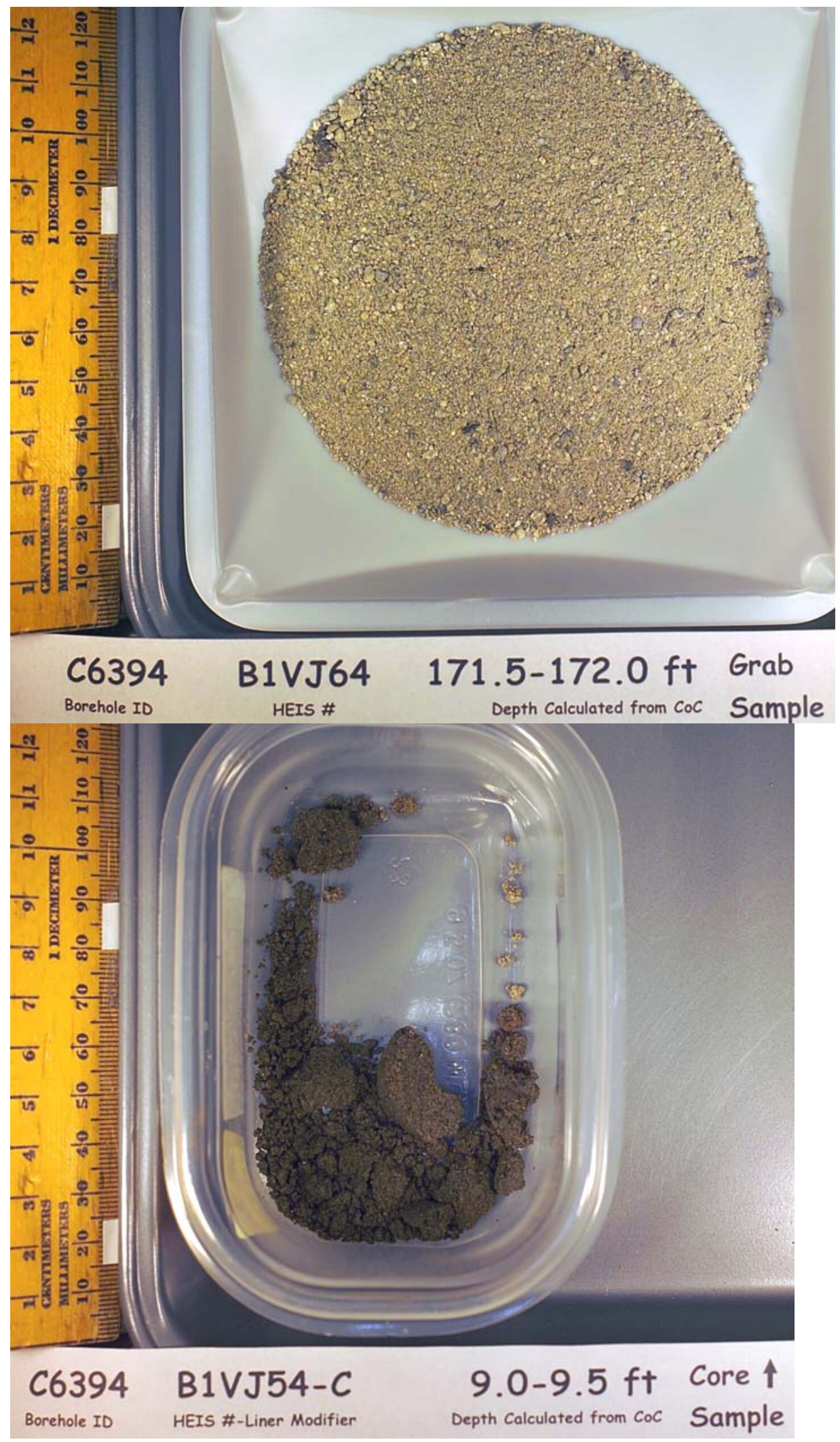




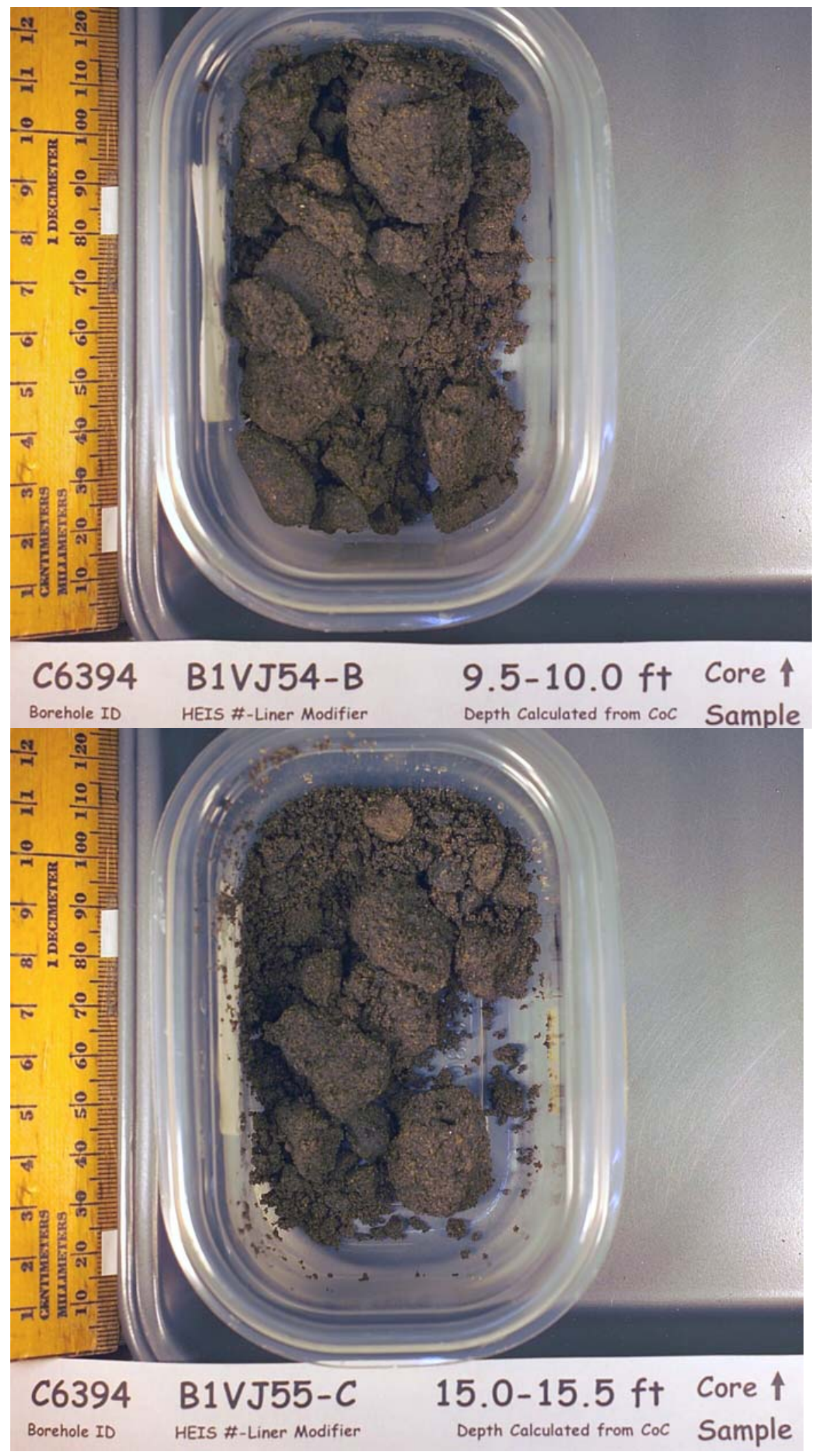




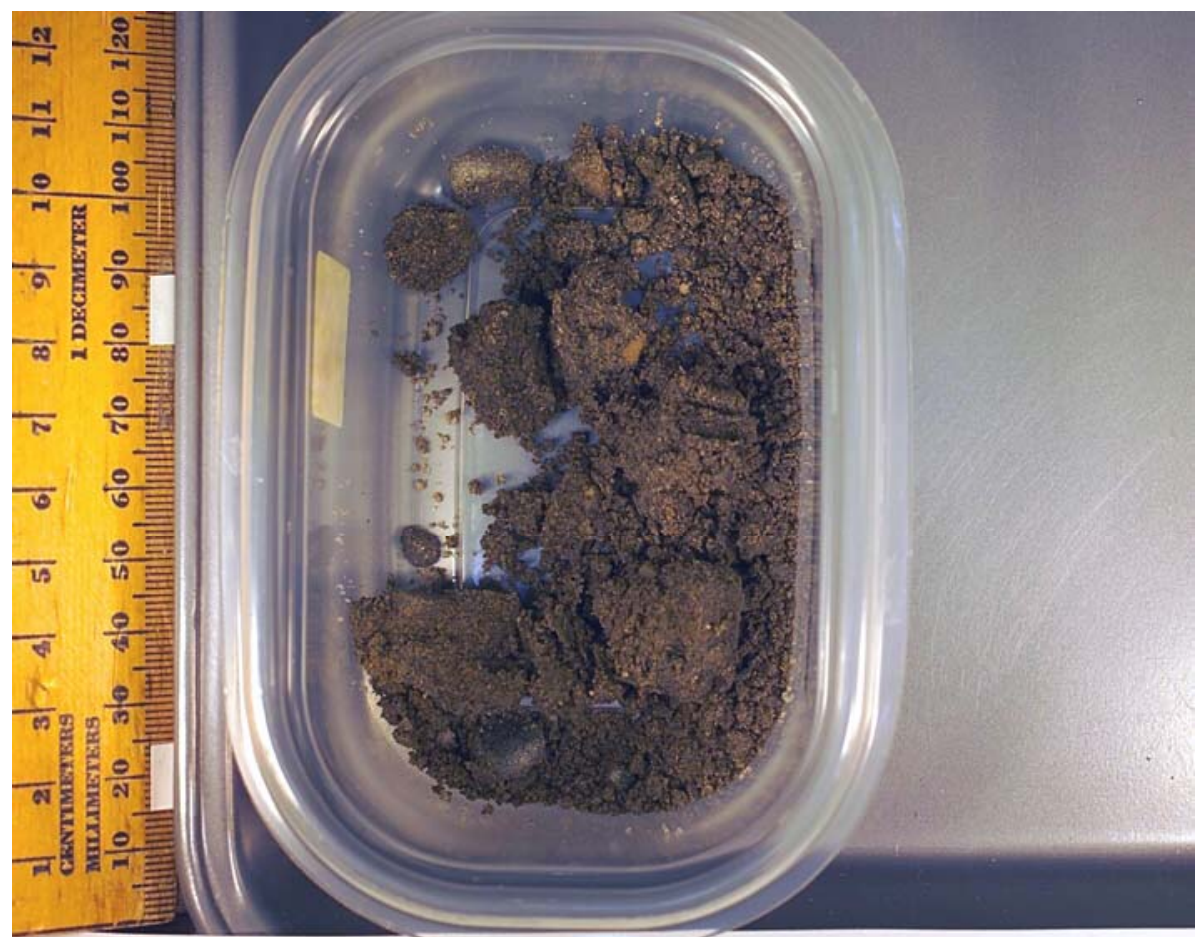
C6394
B1VJ55-B
15.5-16.0 ft Core $\uparrow$
Borehole ID
HEIS \#-Liner Modifier
Depth Calculated from $c 0 c$ Sample

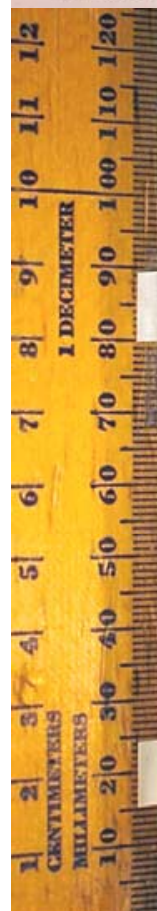

\section{C6394}

Borehole ID

B1VJ55

HEIS \#

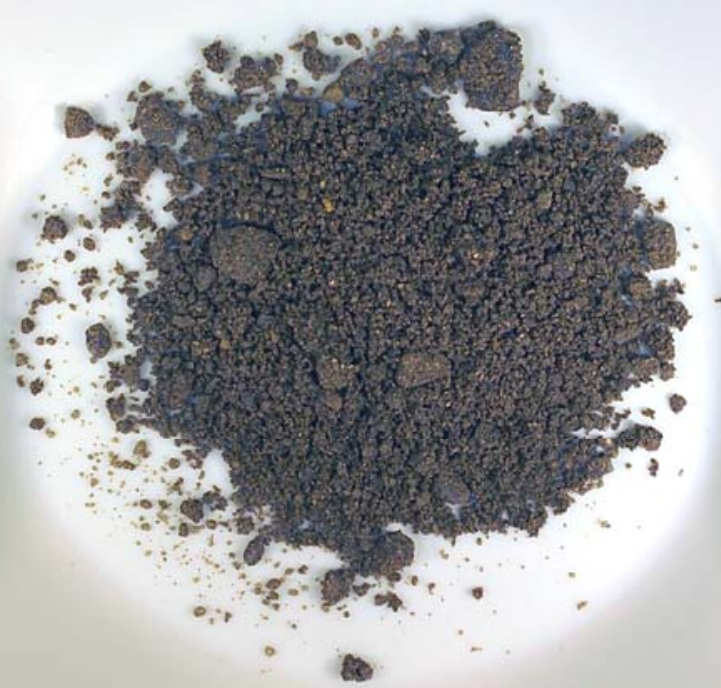

16.5-17.0 ft Grab Depth Calculated from coc Sample 


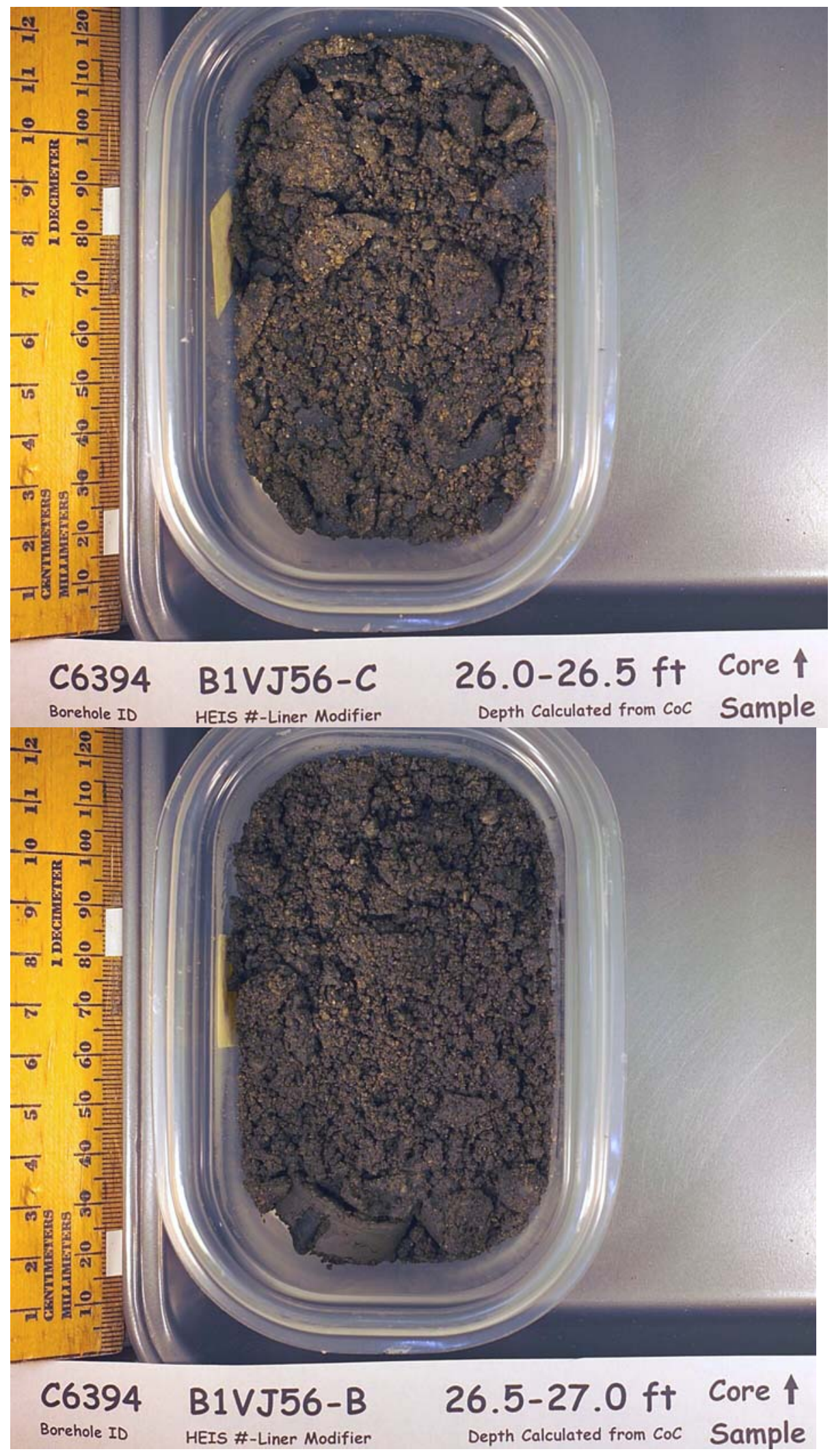




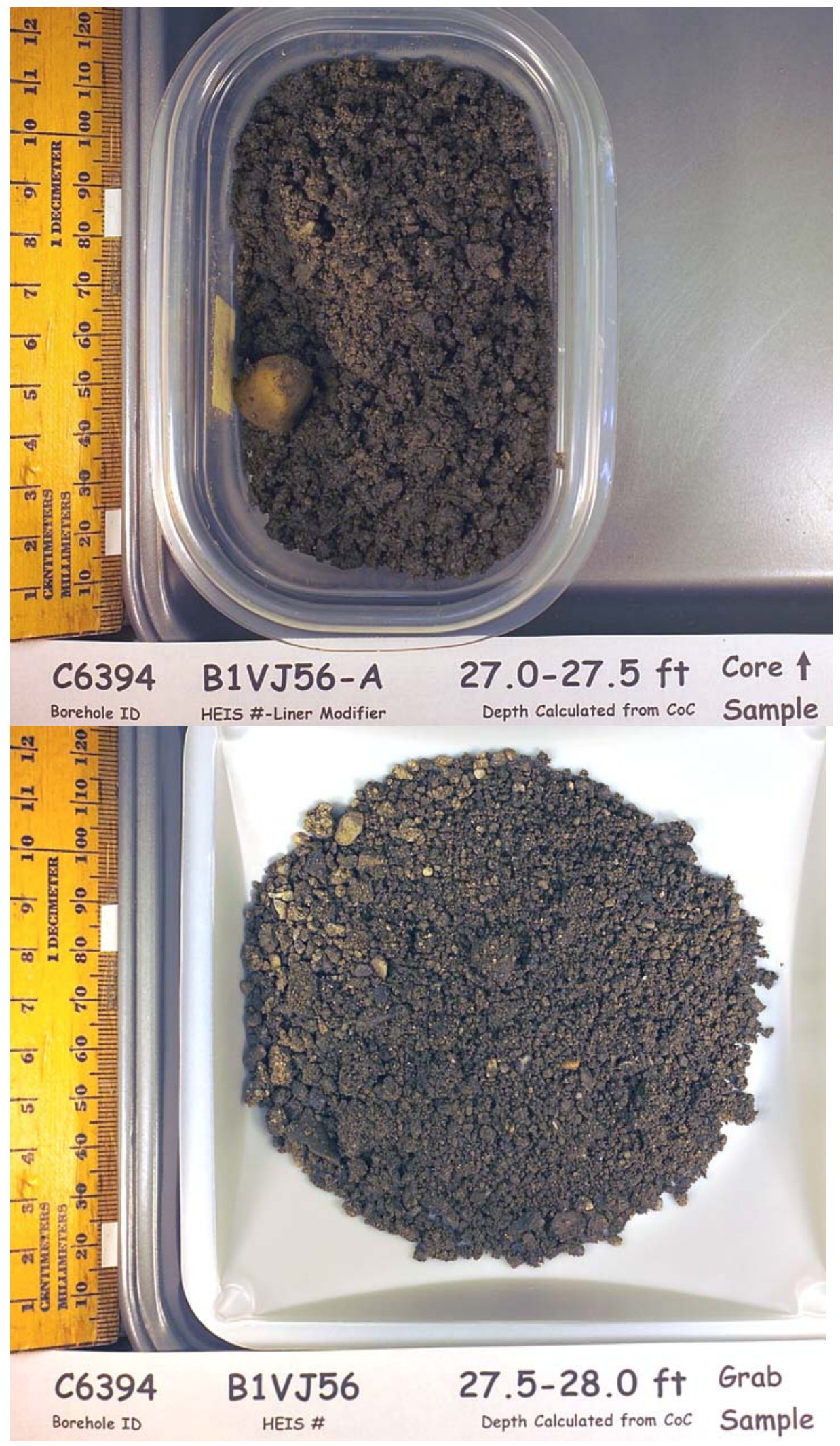




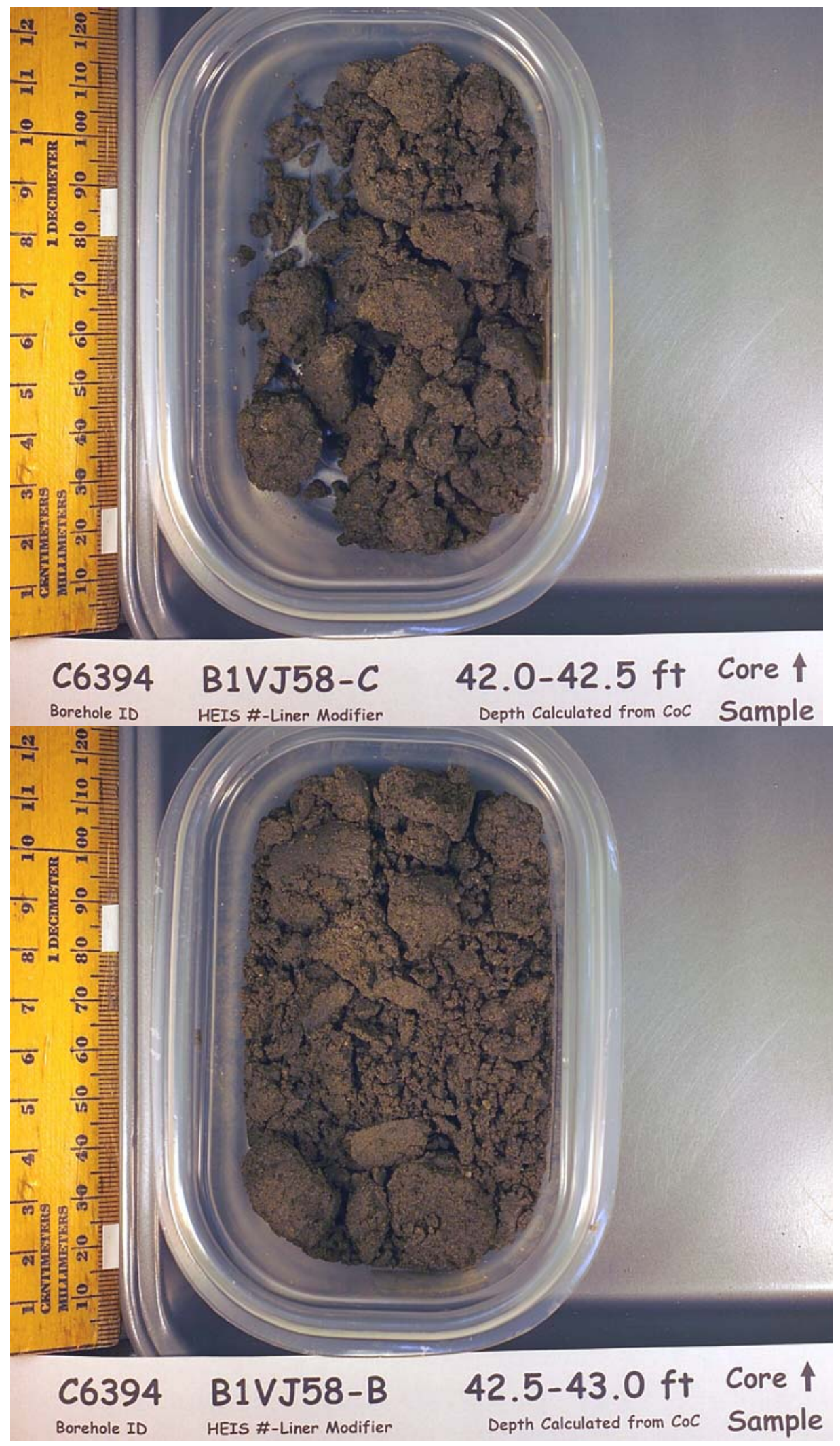




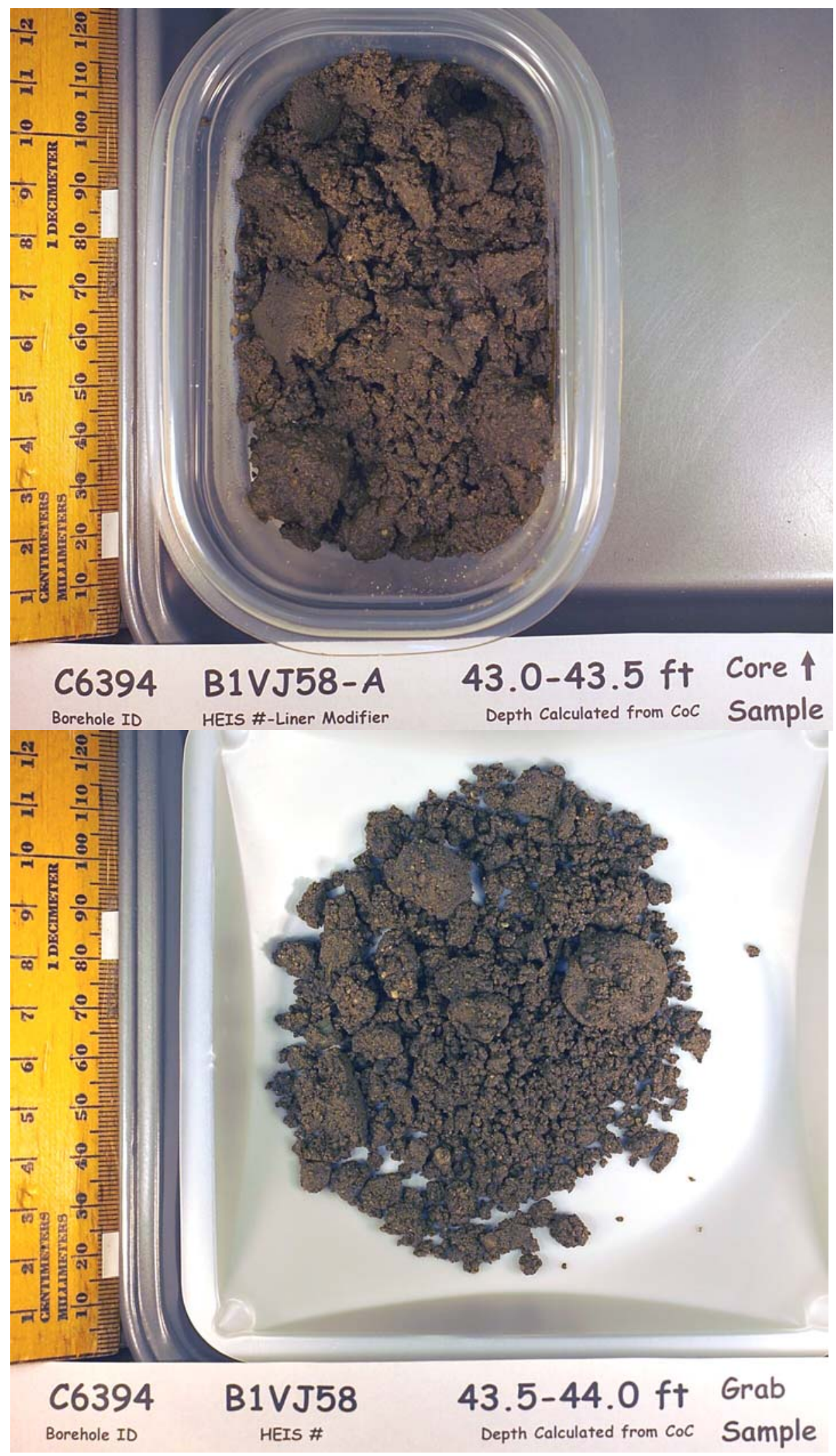




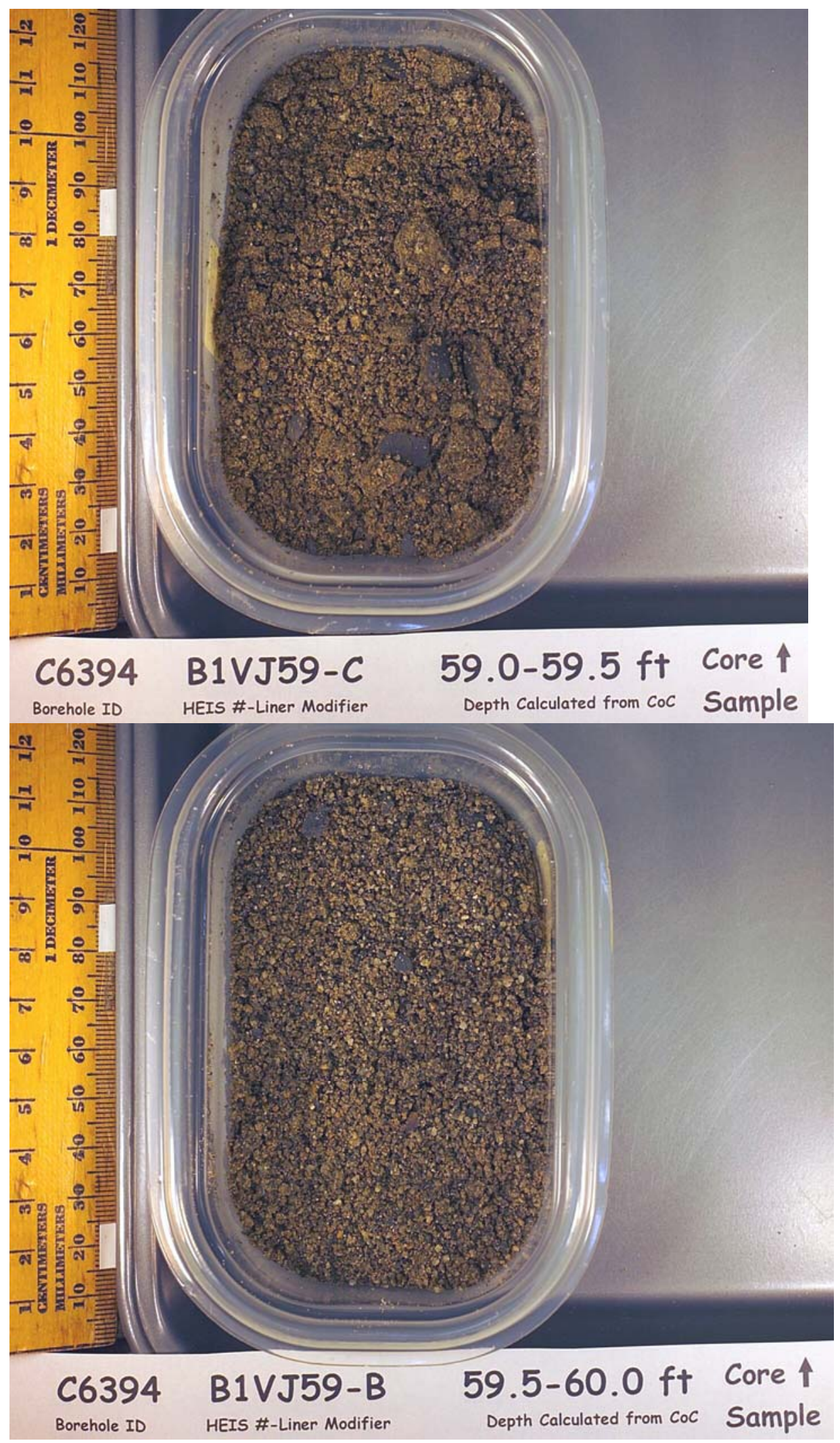




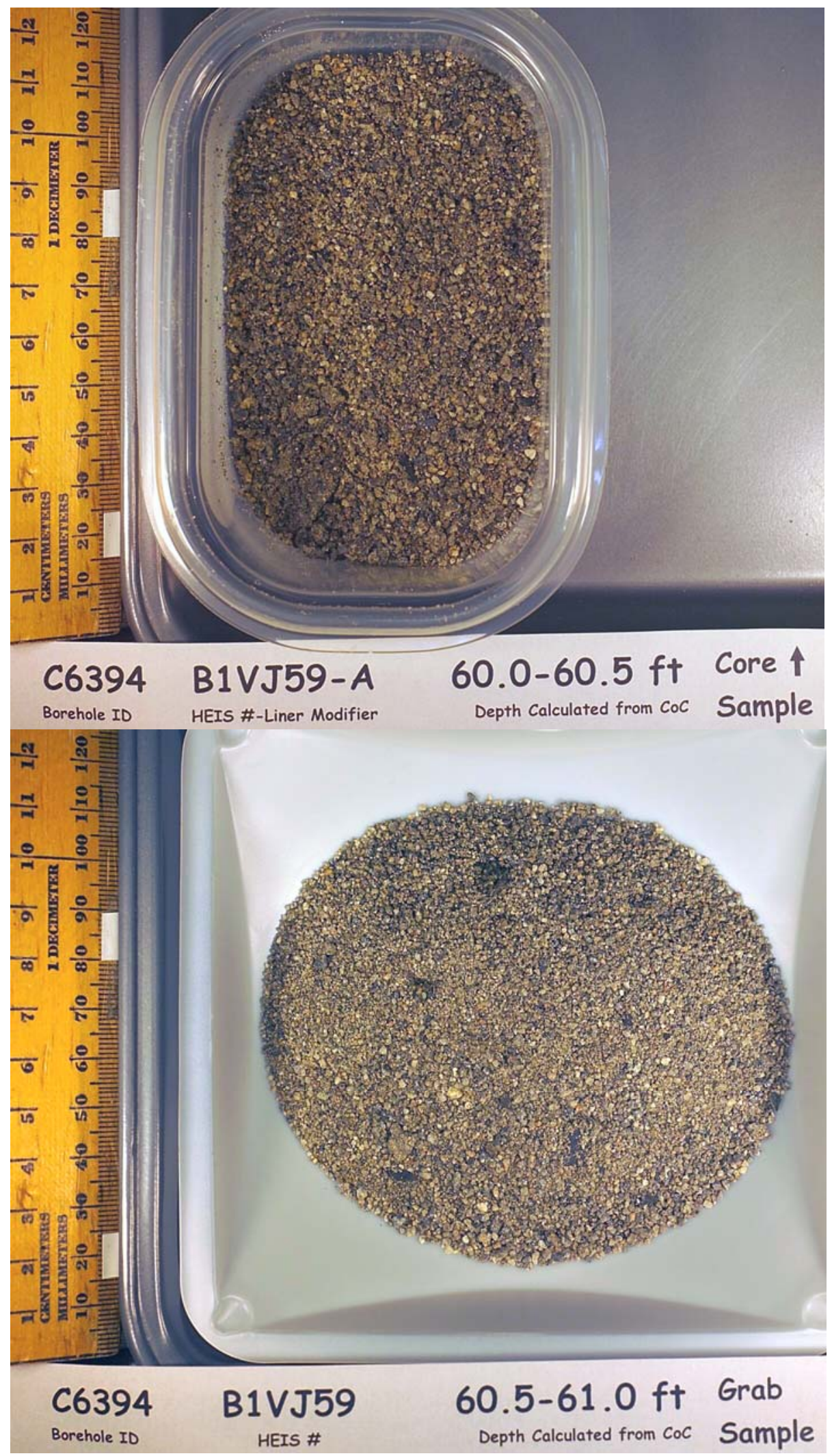




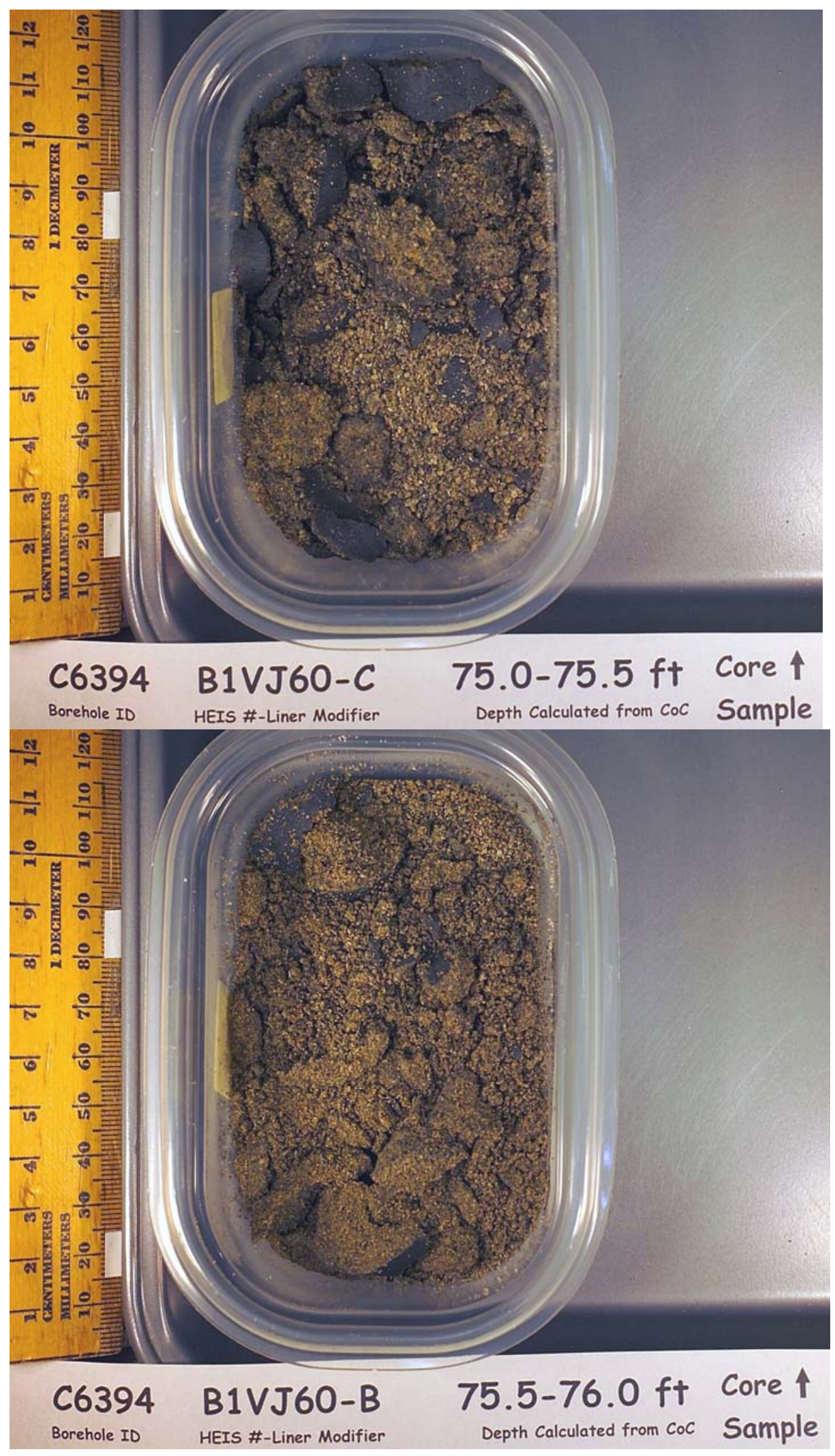




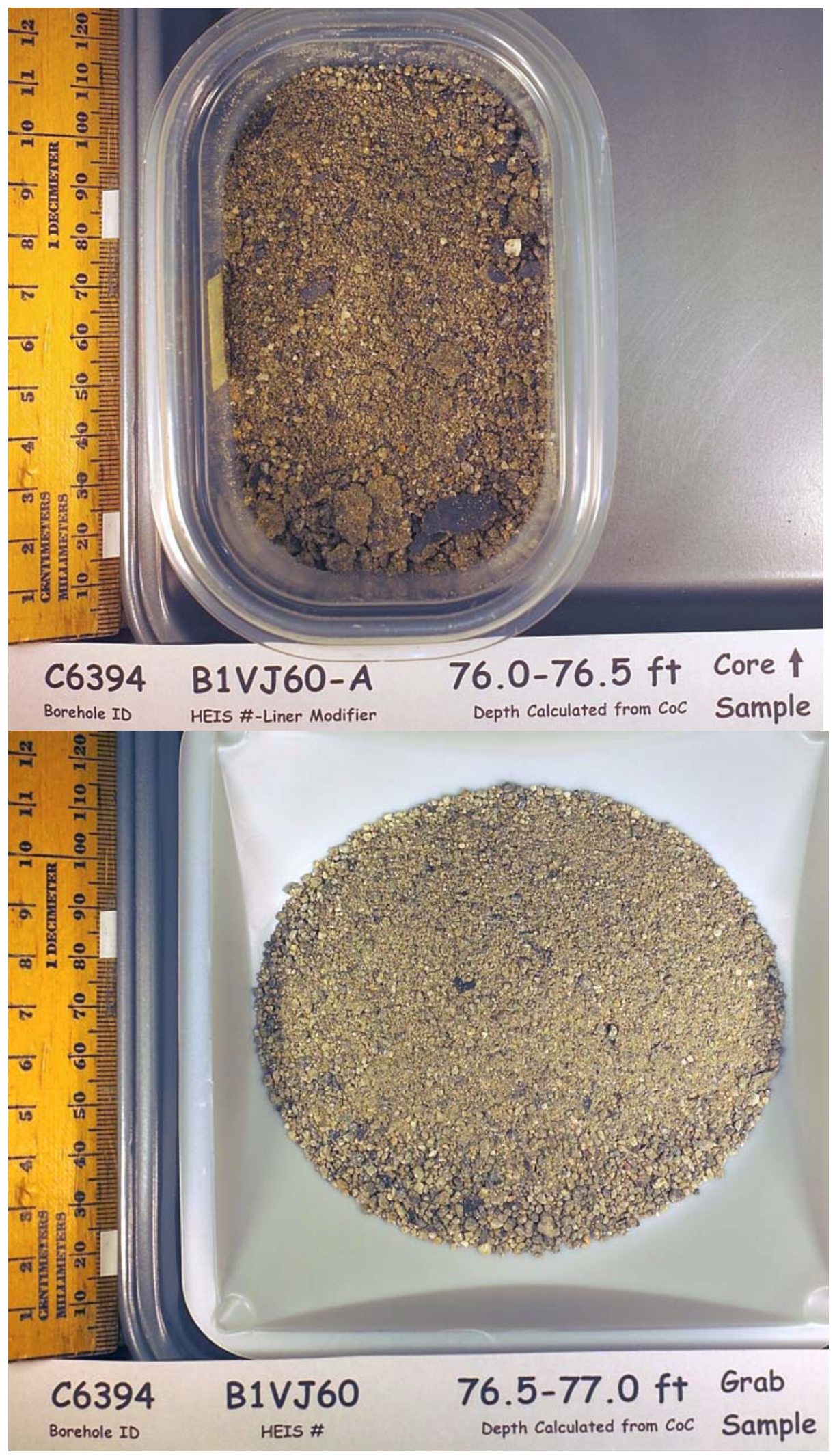




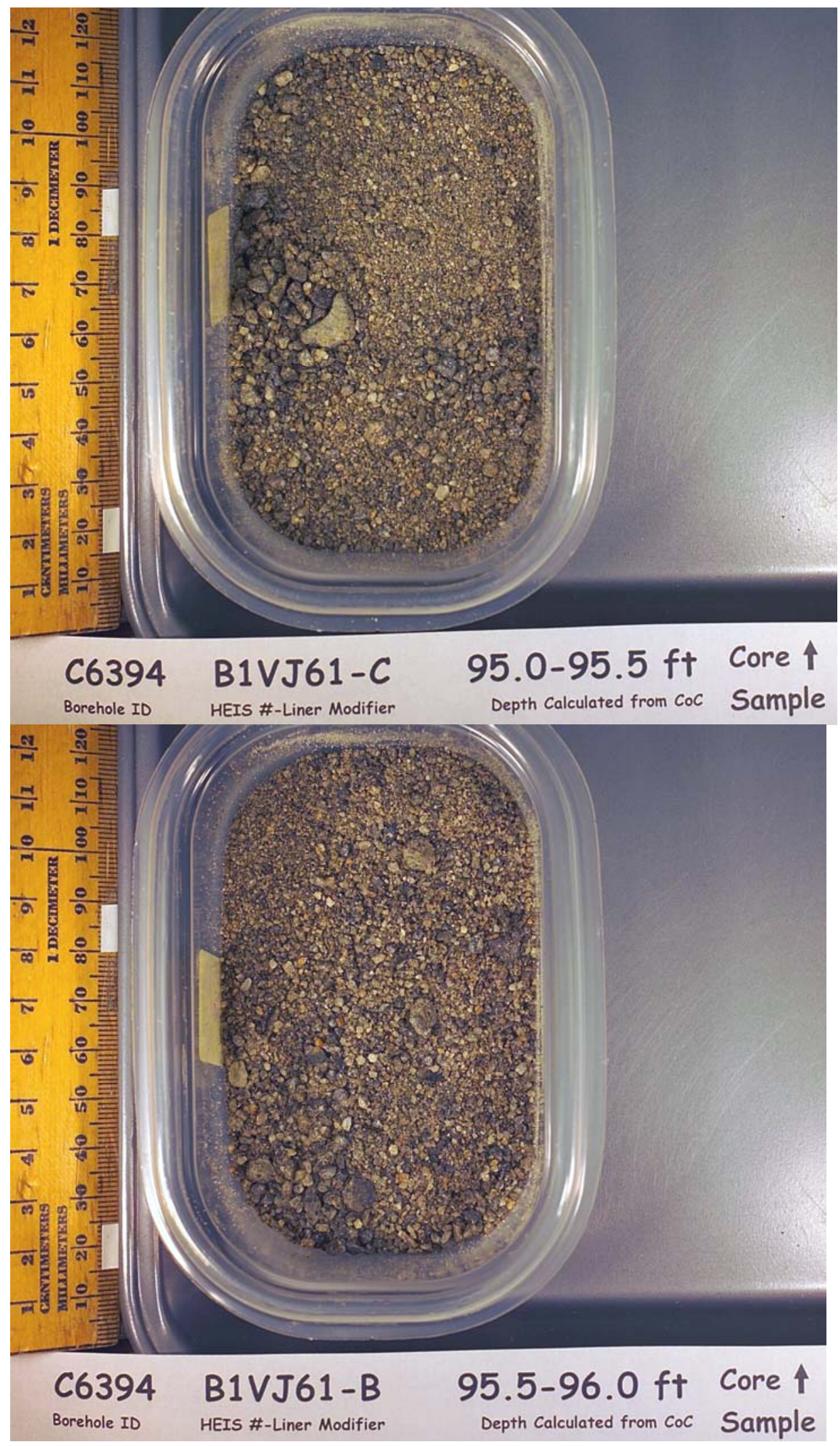




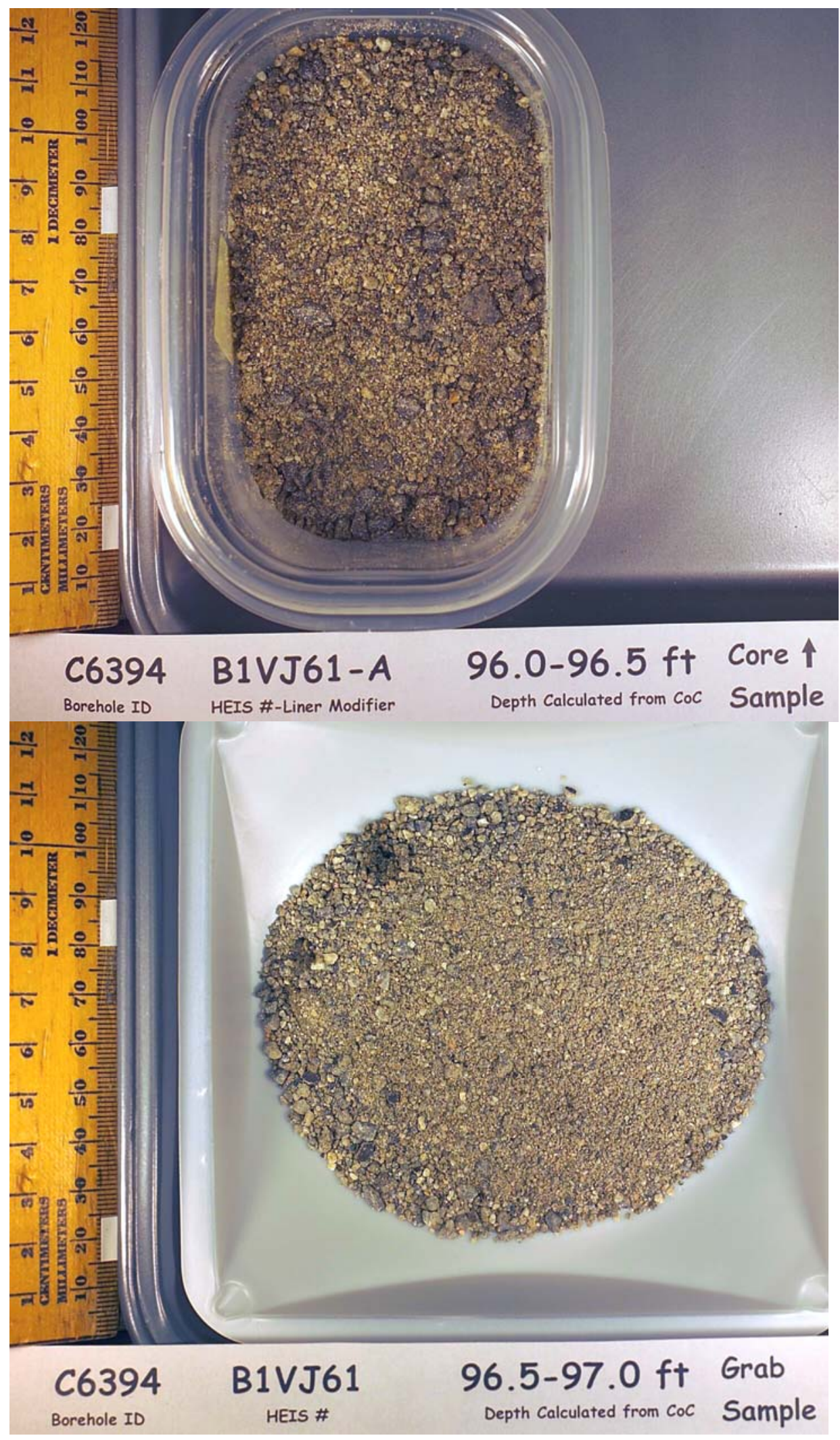



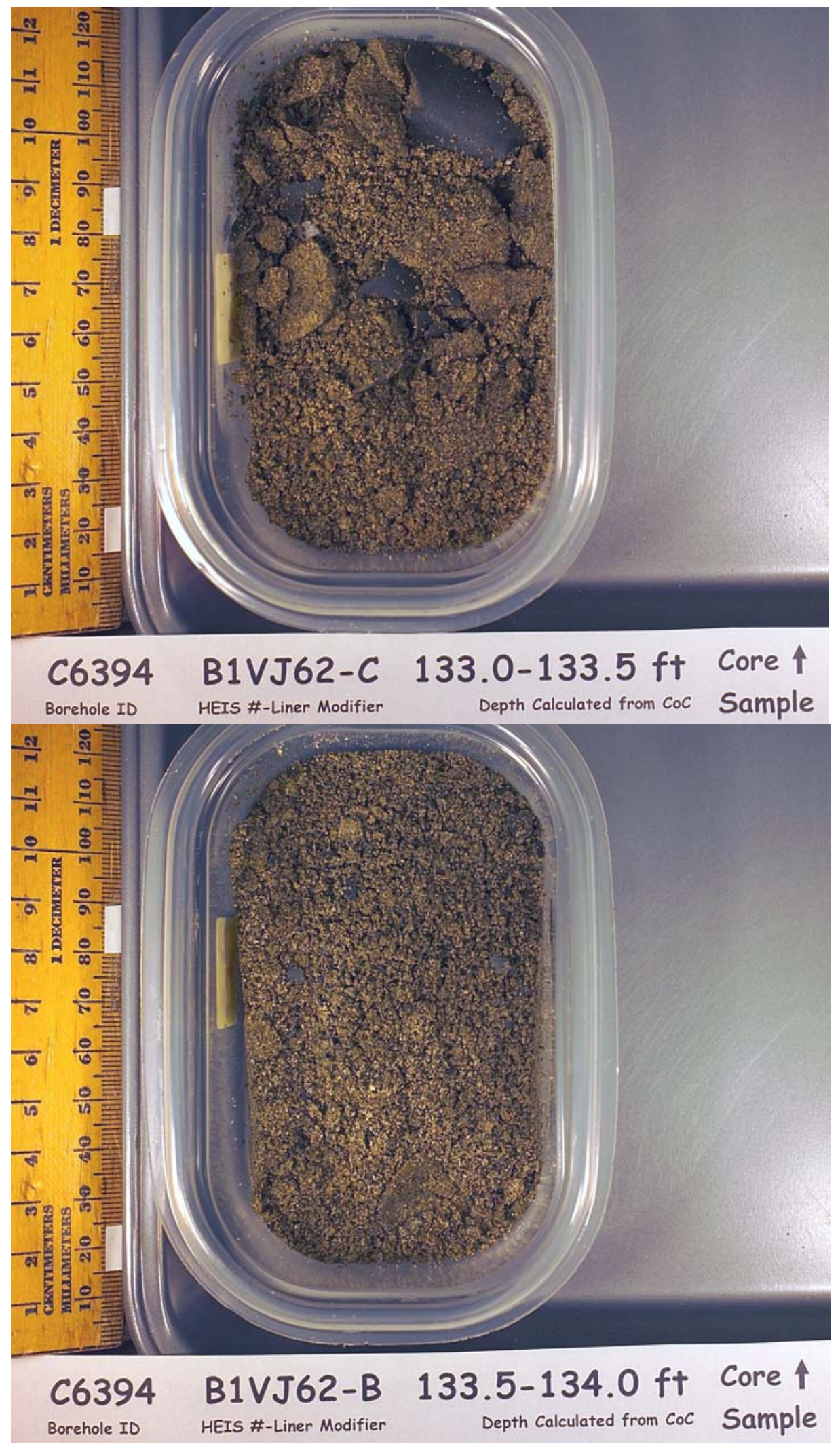

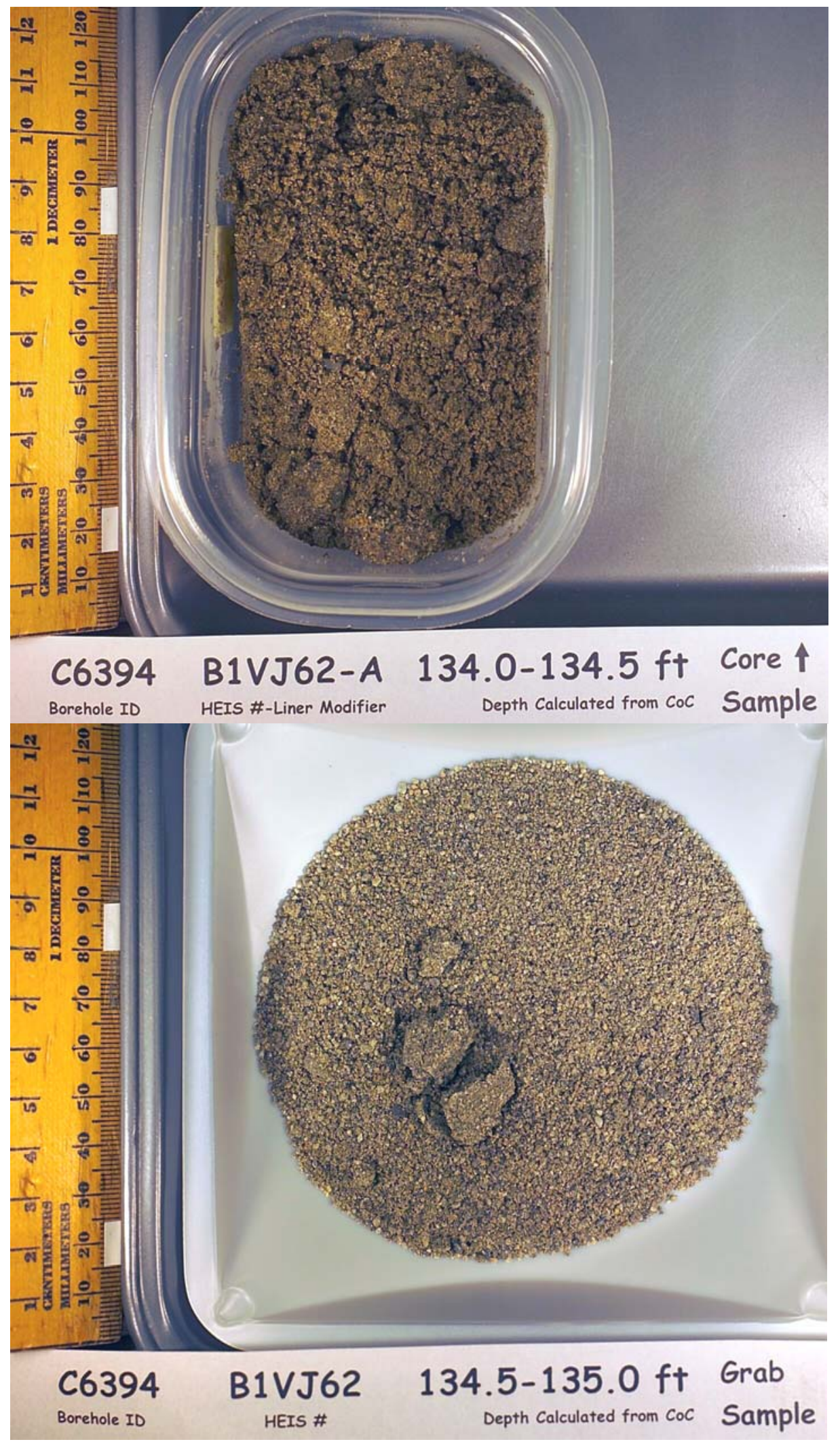

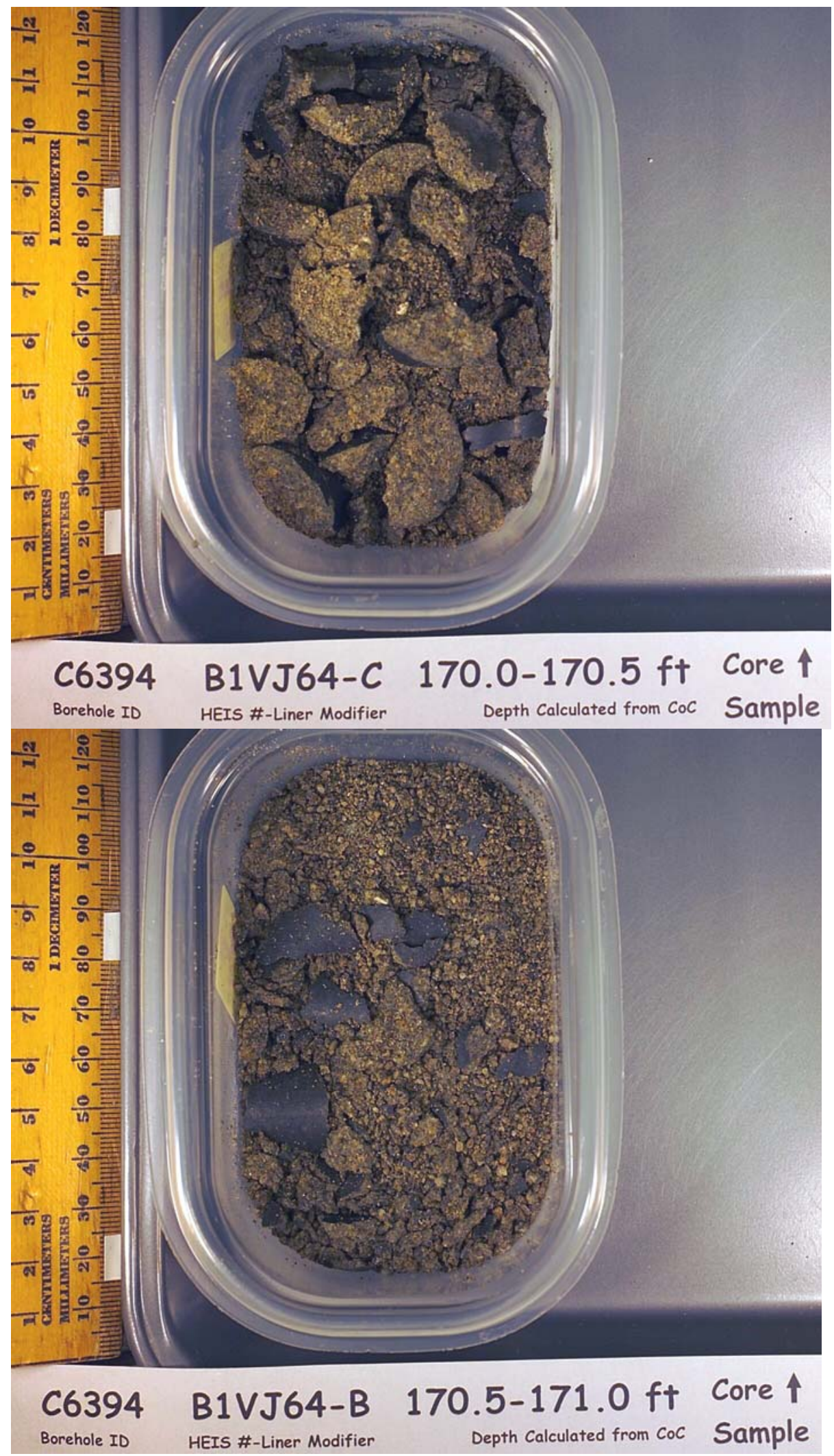


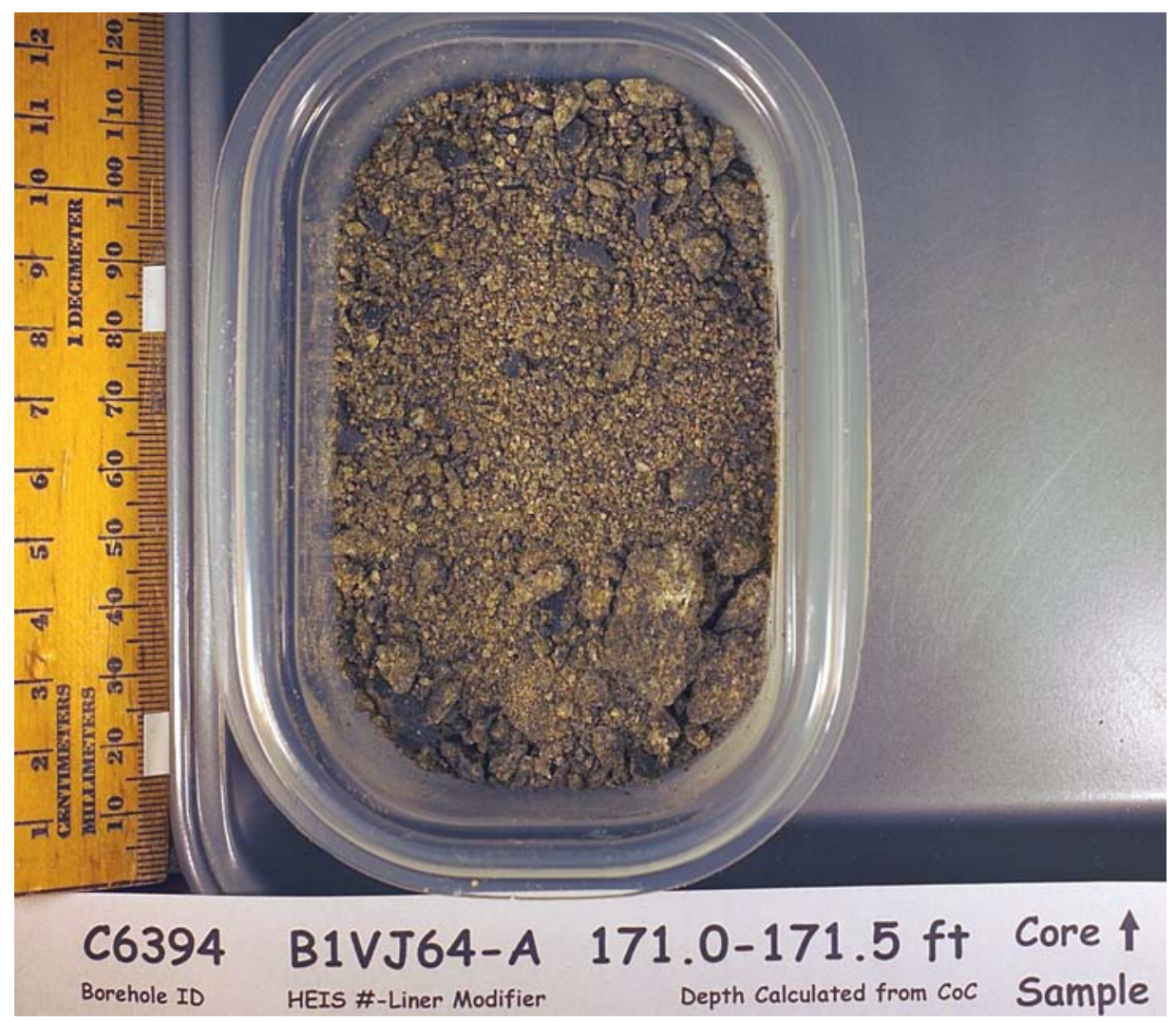




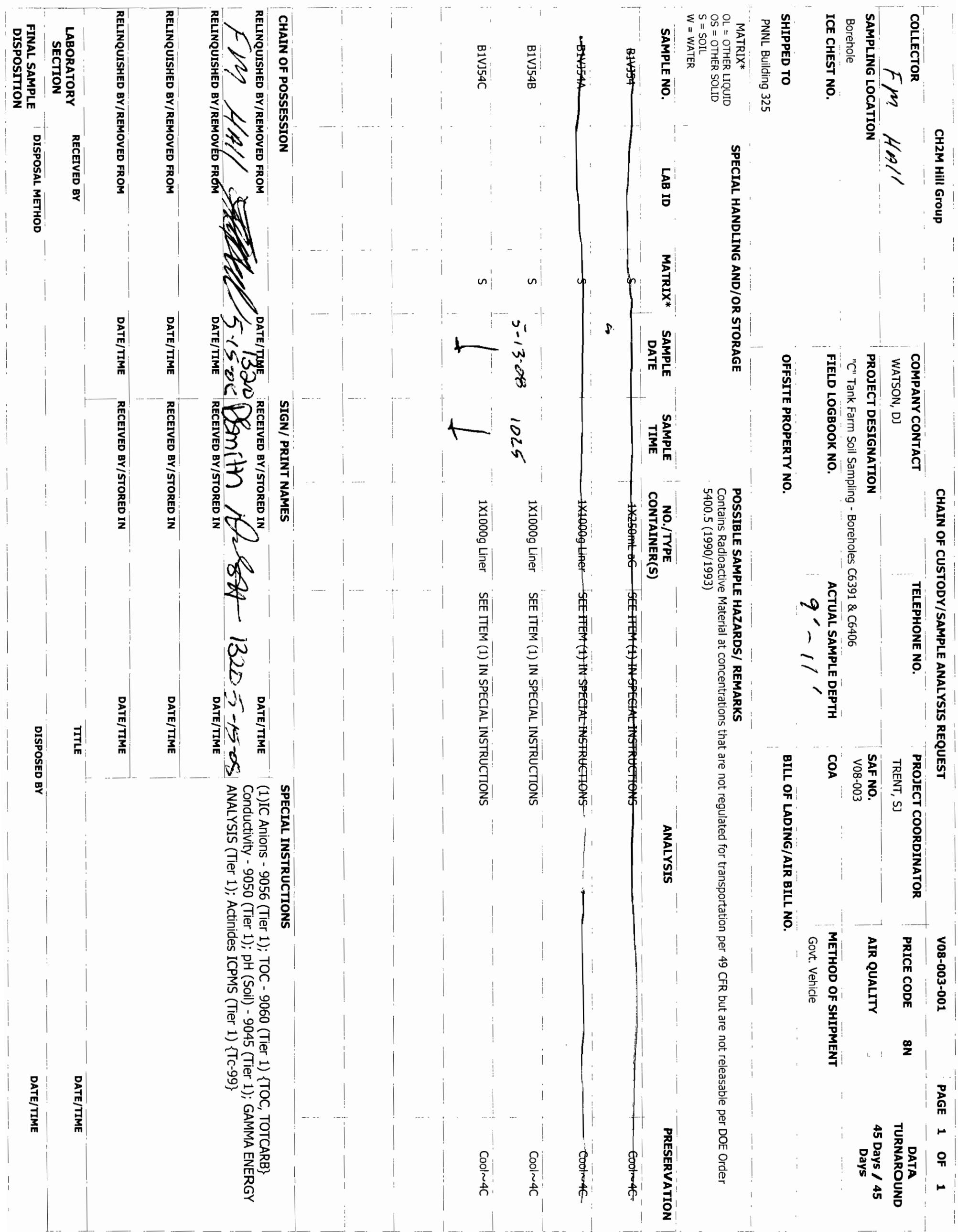




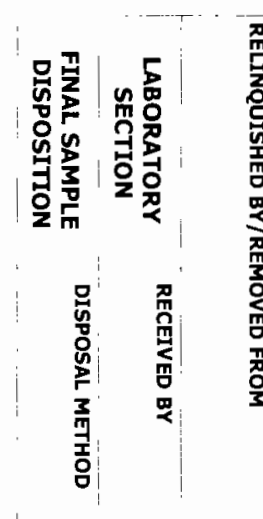

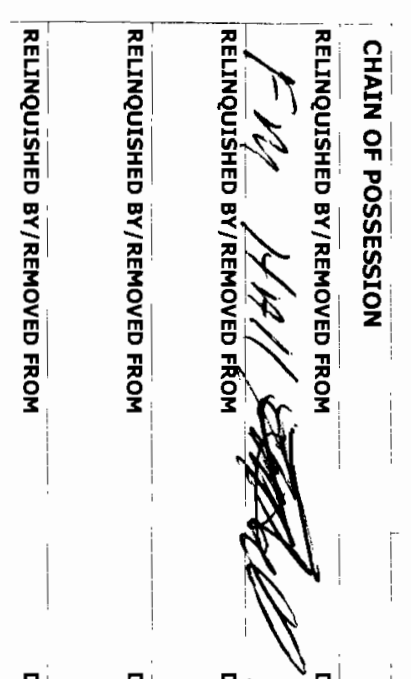

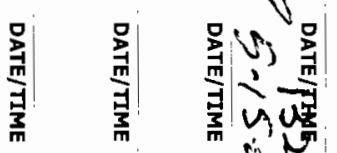

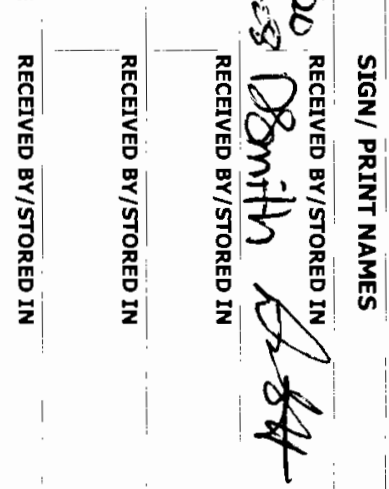

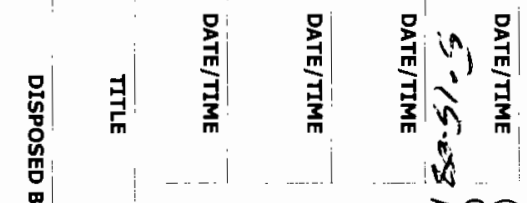

之导

氙寻

ज请

앵앵

곡 곻

융트

웅종ㅁㅇ

즐

氙点

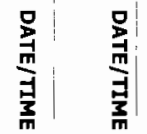

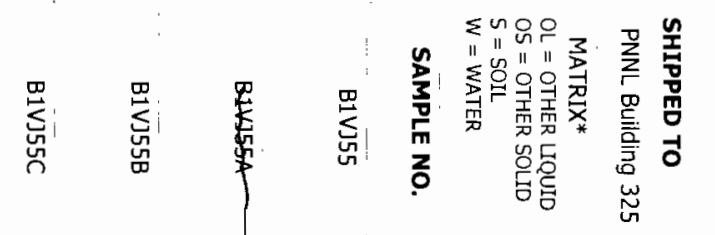

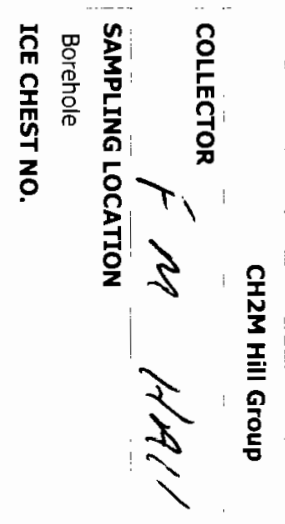

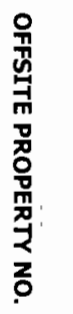

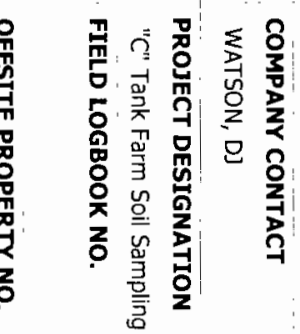

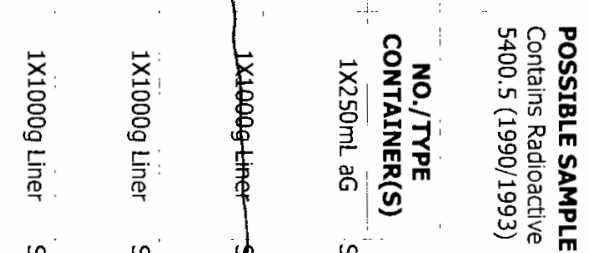

篅篎

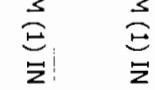

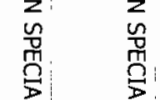

篮|

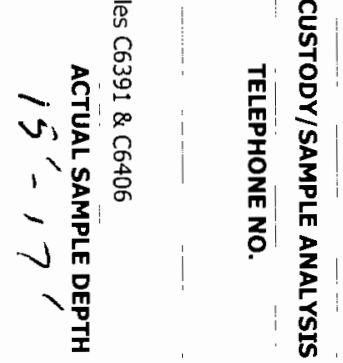

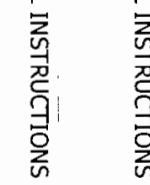

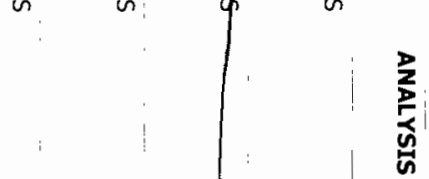

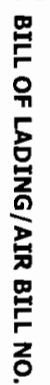

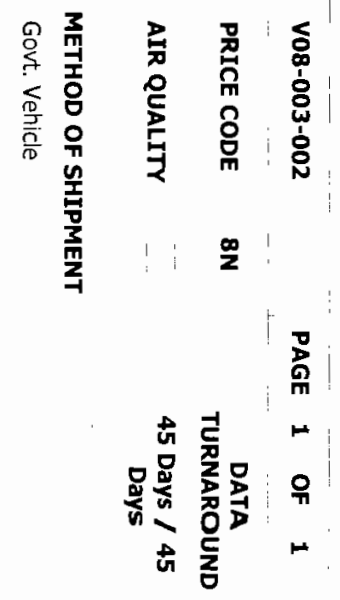




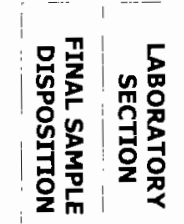
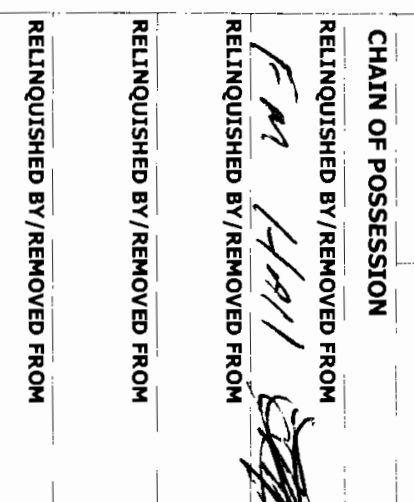

高高

1) 119

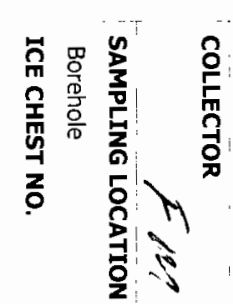

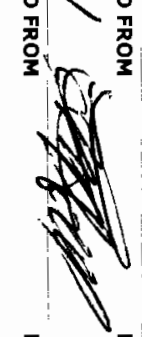

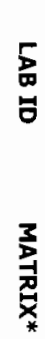
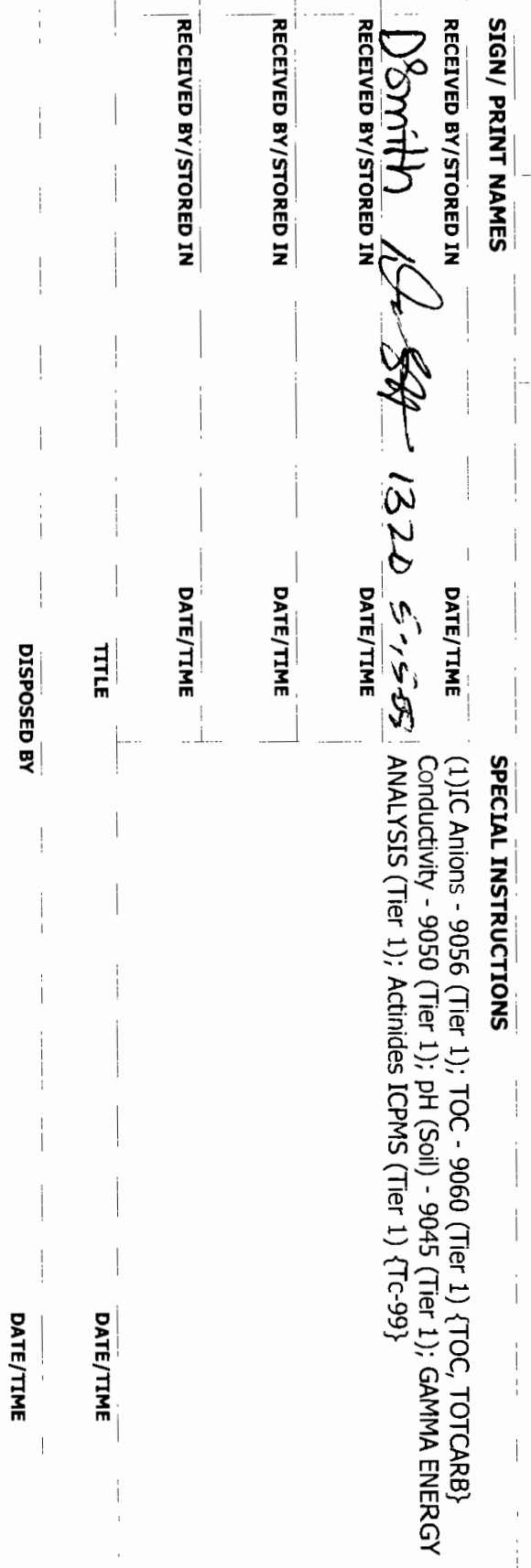

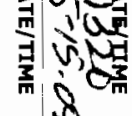
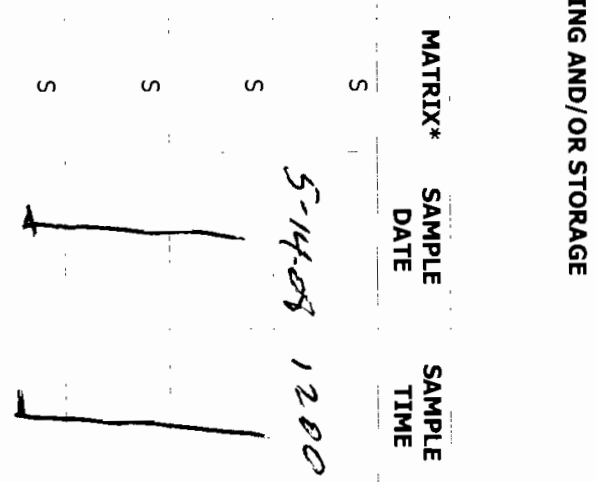

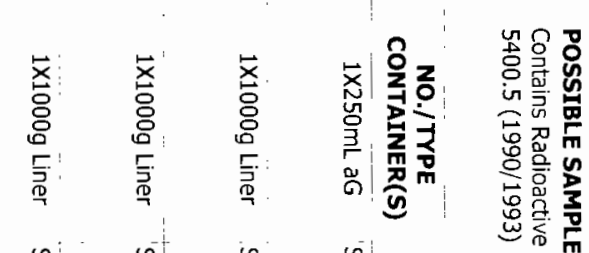

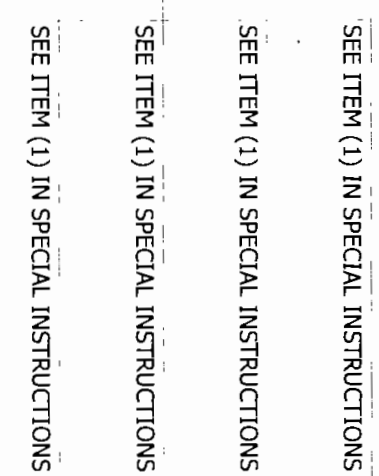

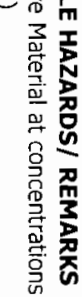
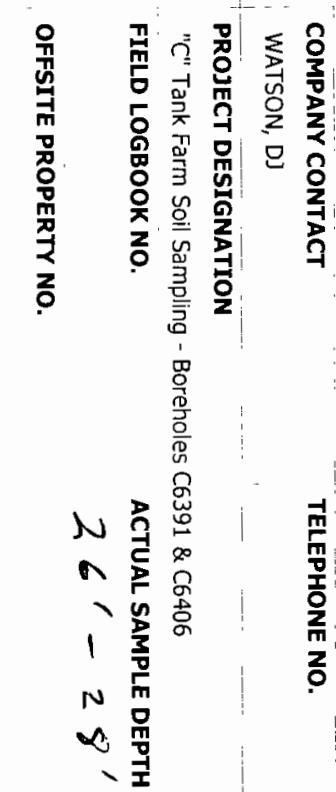

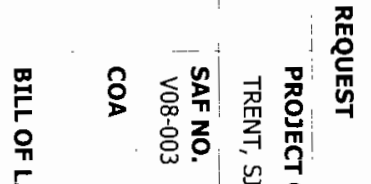

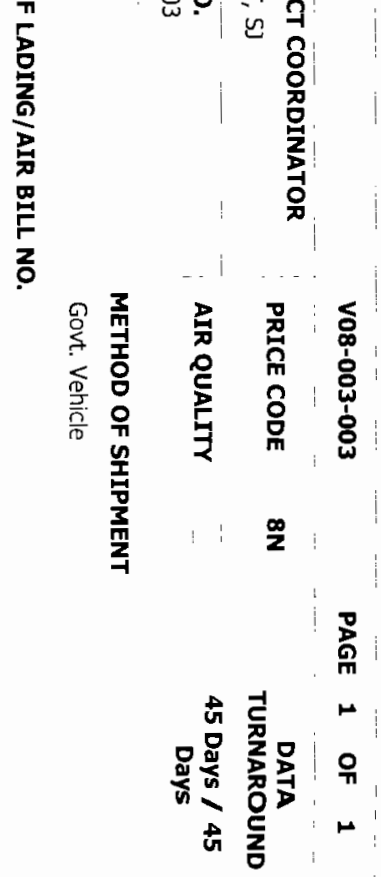




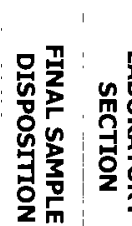

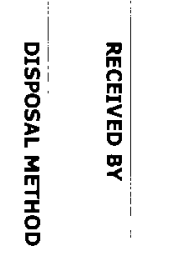

总咅

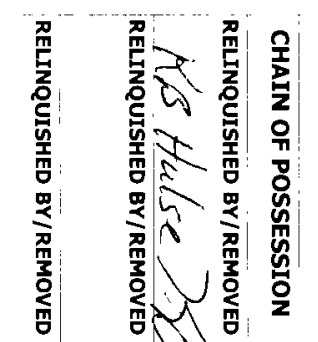

ग
$\mathbf{3}$
3

ôn

है

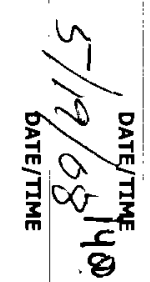

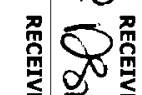

畜 学

哭

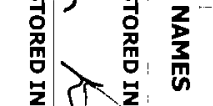

2

4

$v$

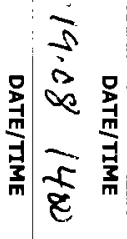

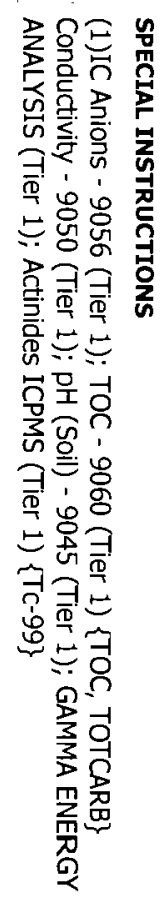

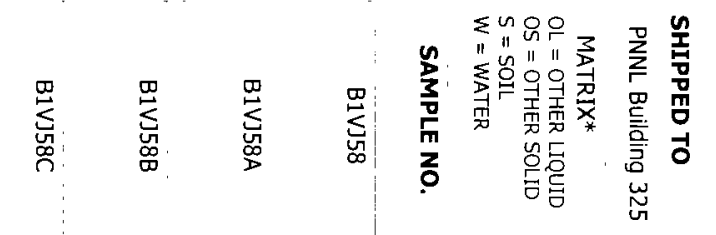

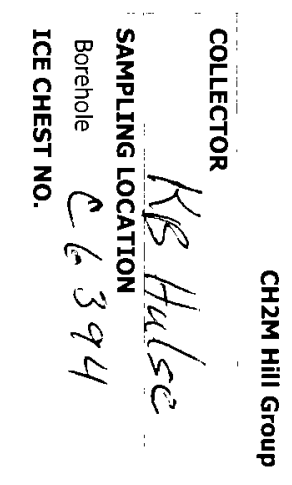

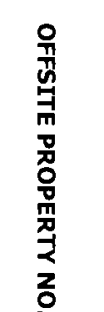

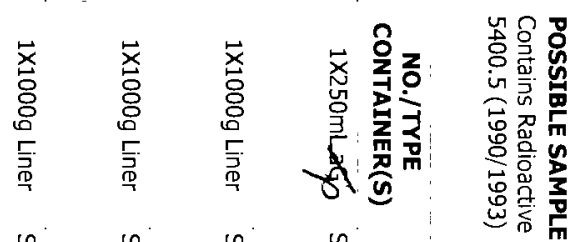

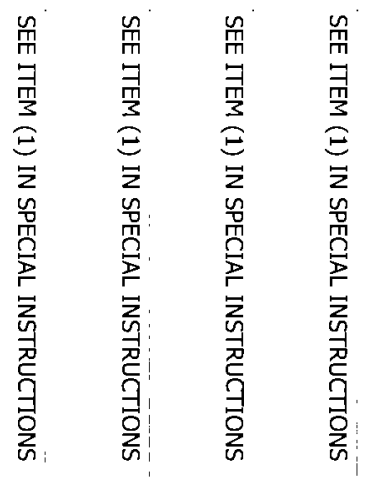

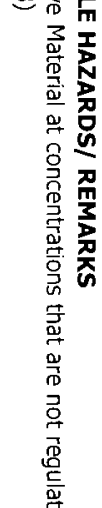

蛋

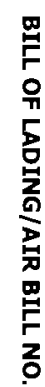

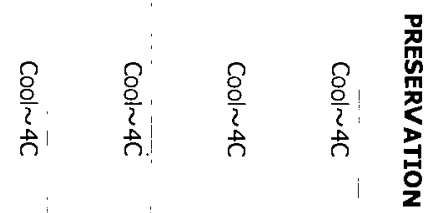

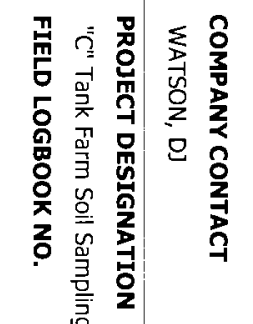

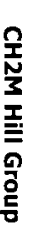
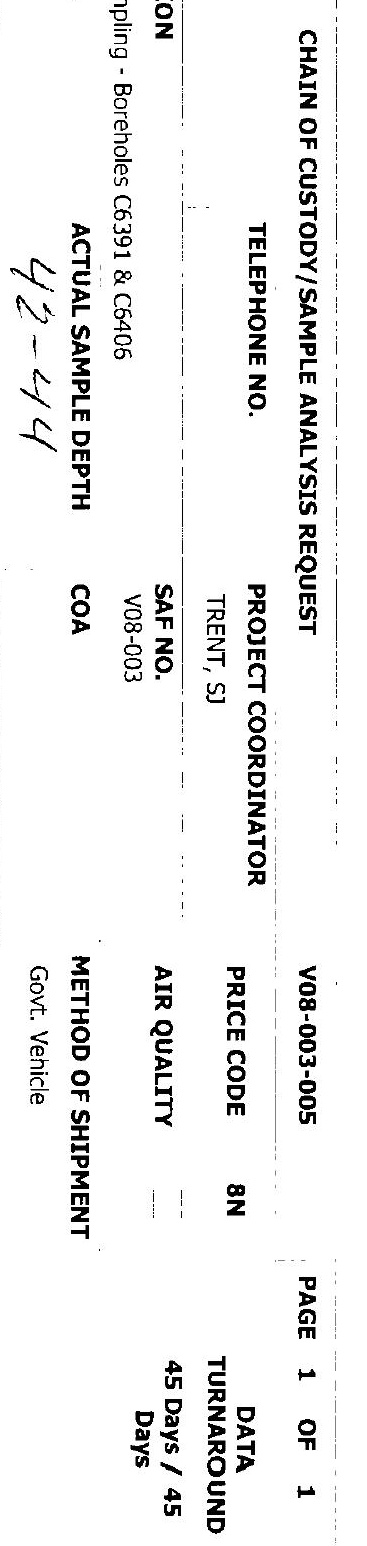


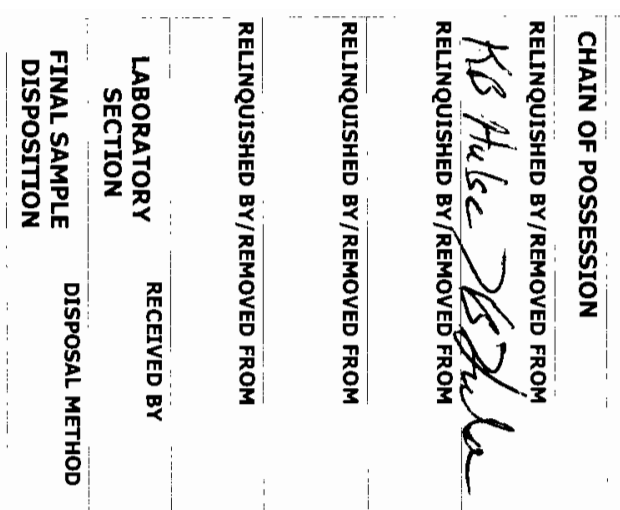

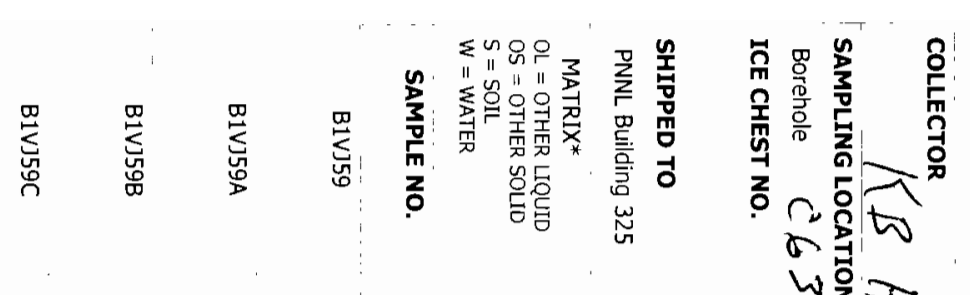

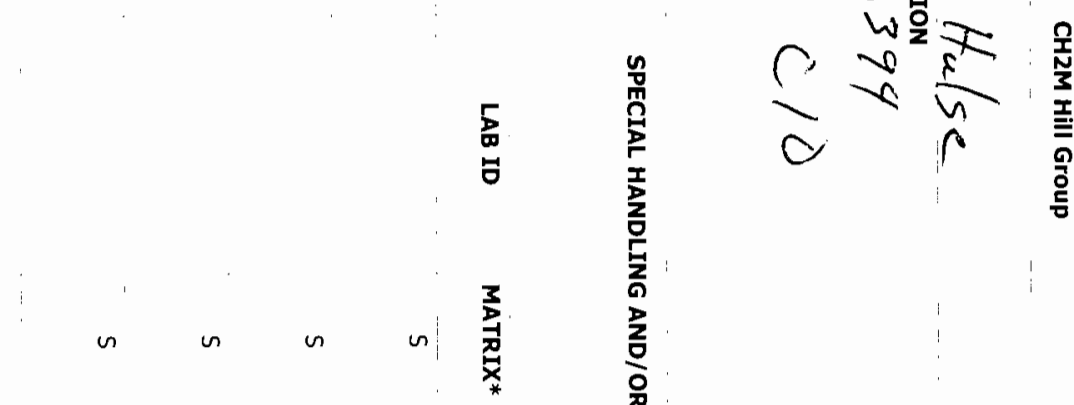

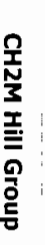
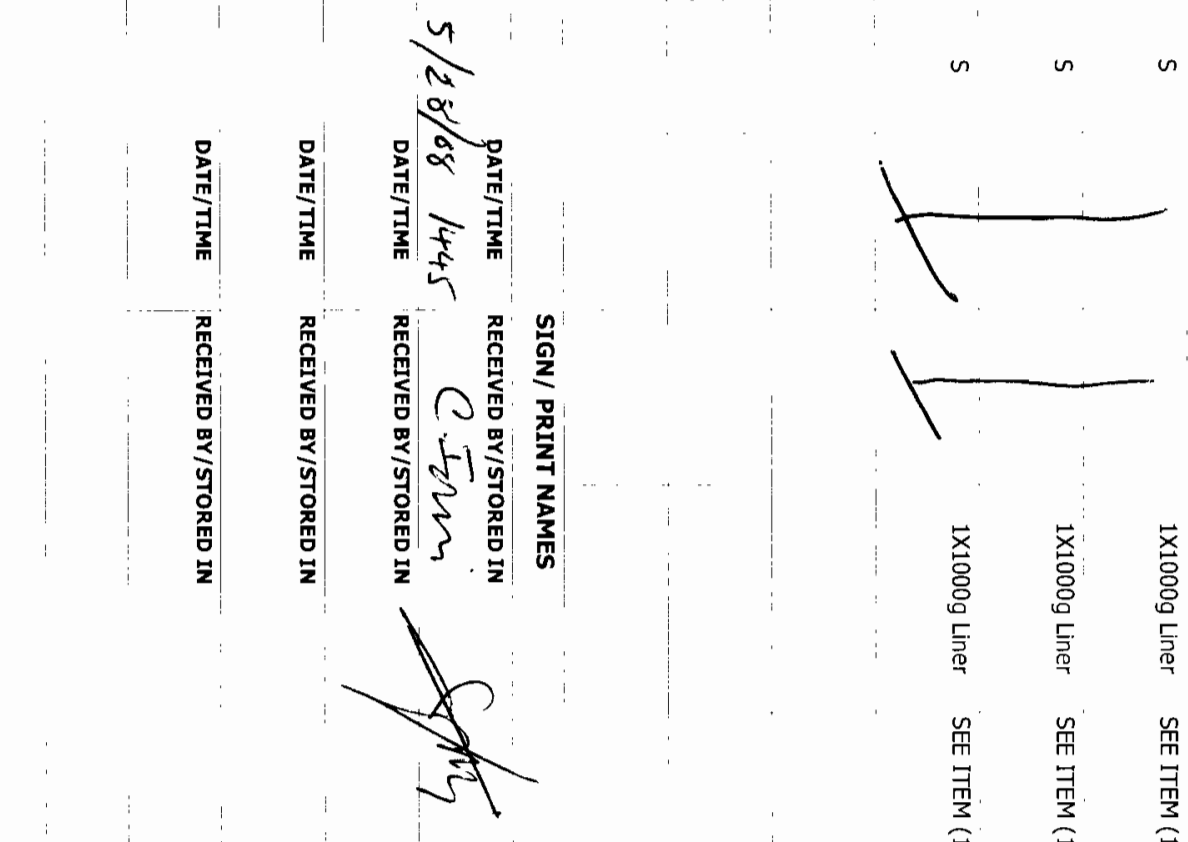

है
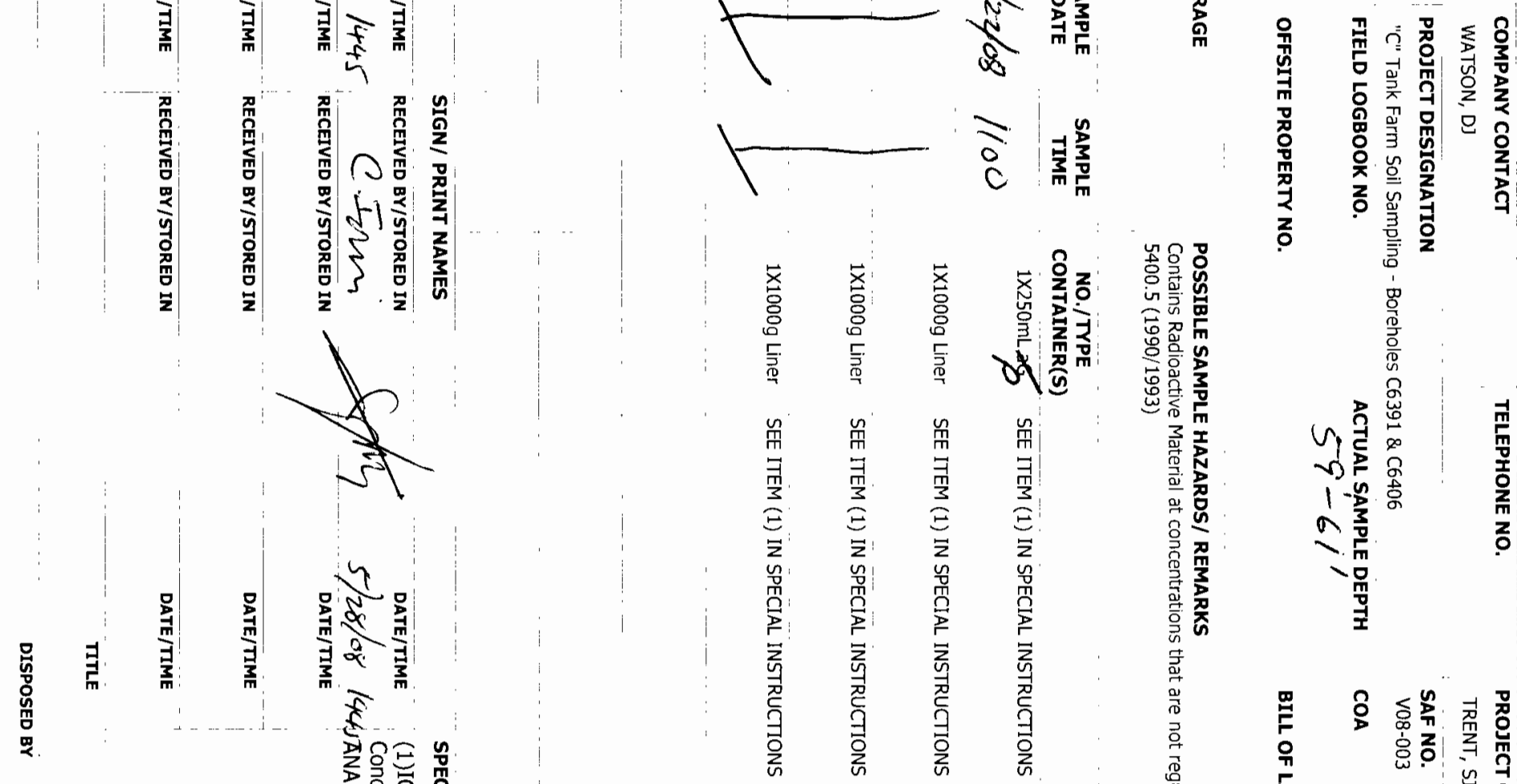

言

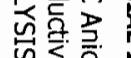

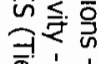

눈융

그ㅋㅡㅡ

产

员는

훅모용

ऊิ

홍ㅇㅇㅇ

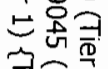

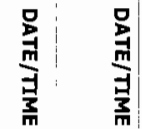

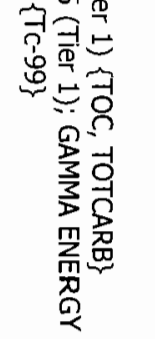




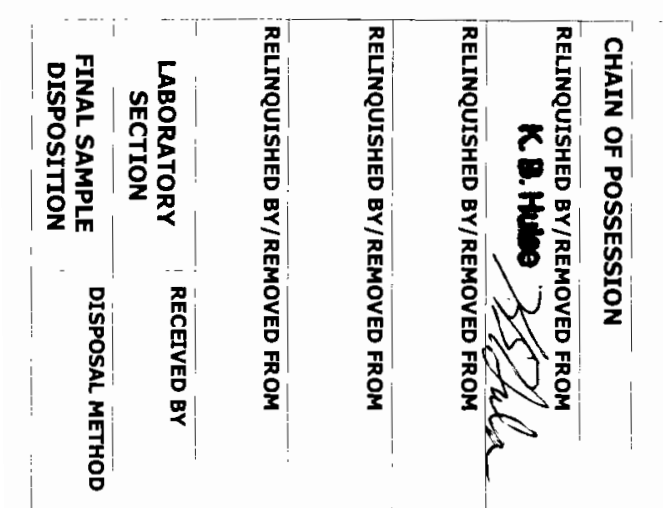

年1

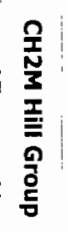
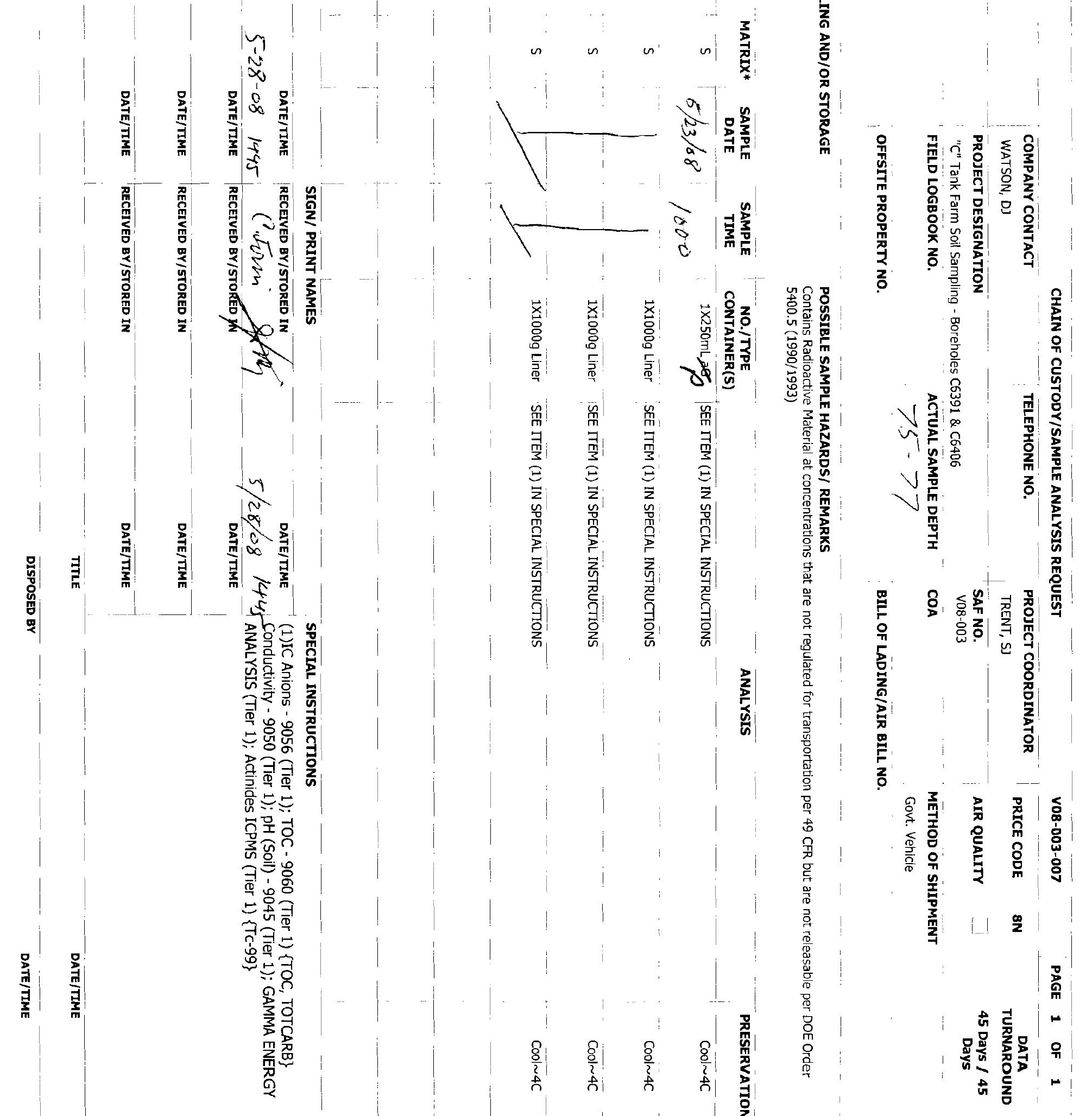


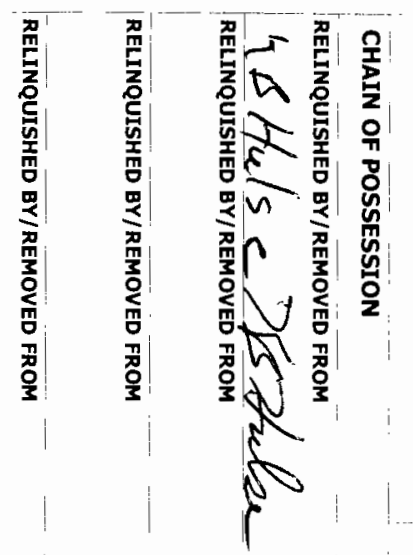

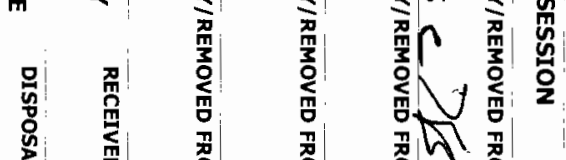

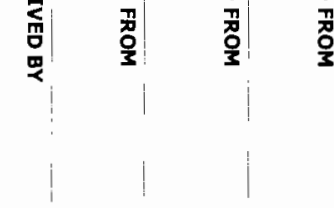
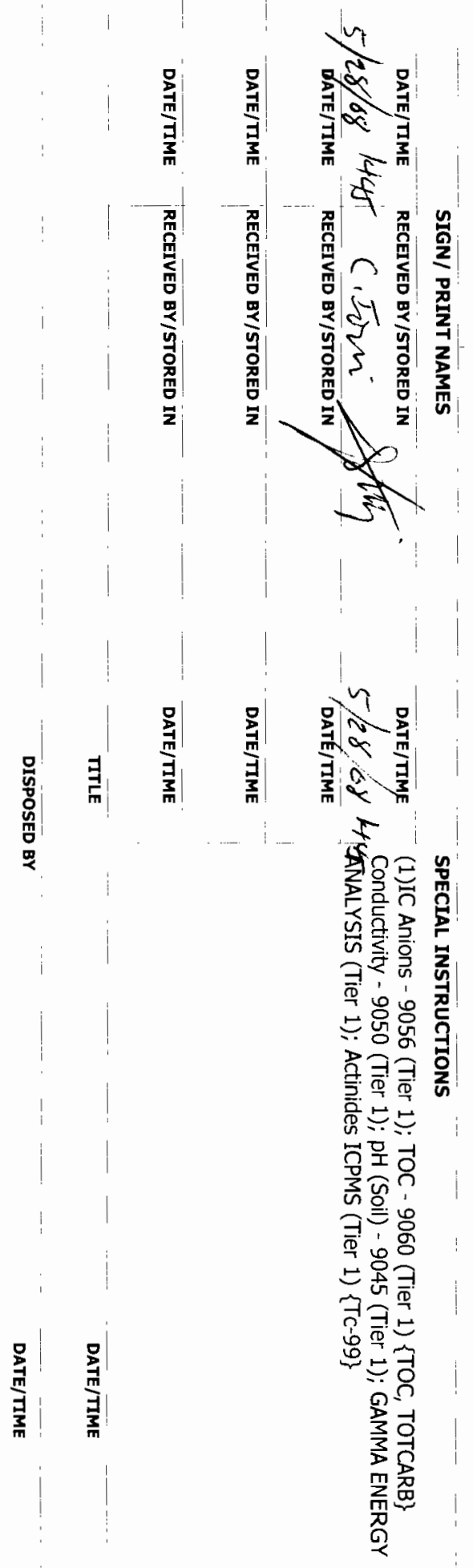

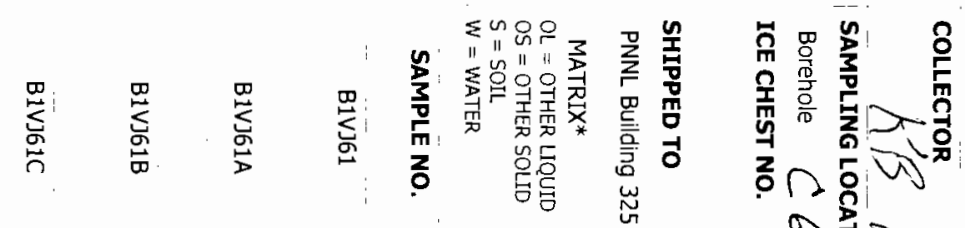
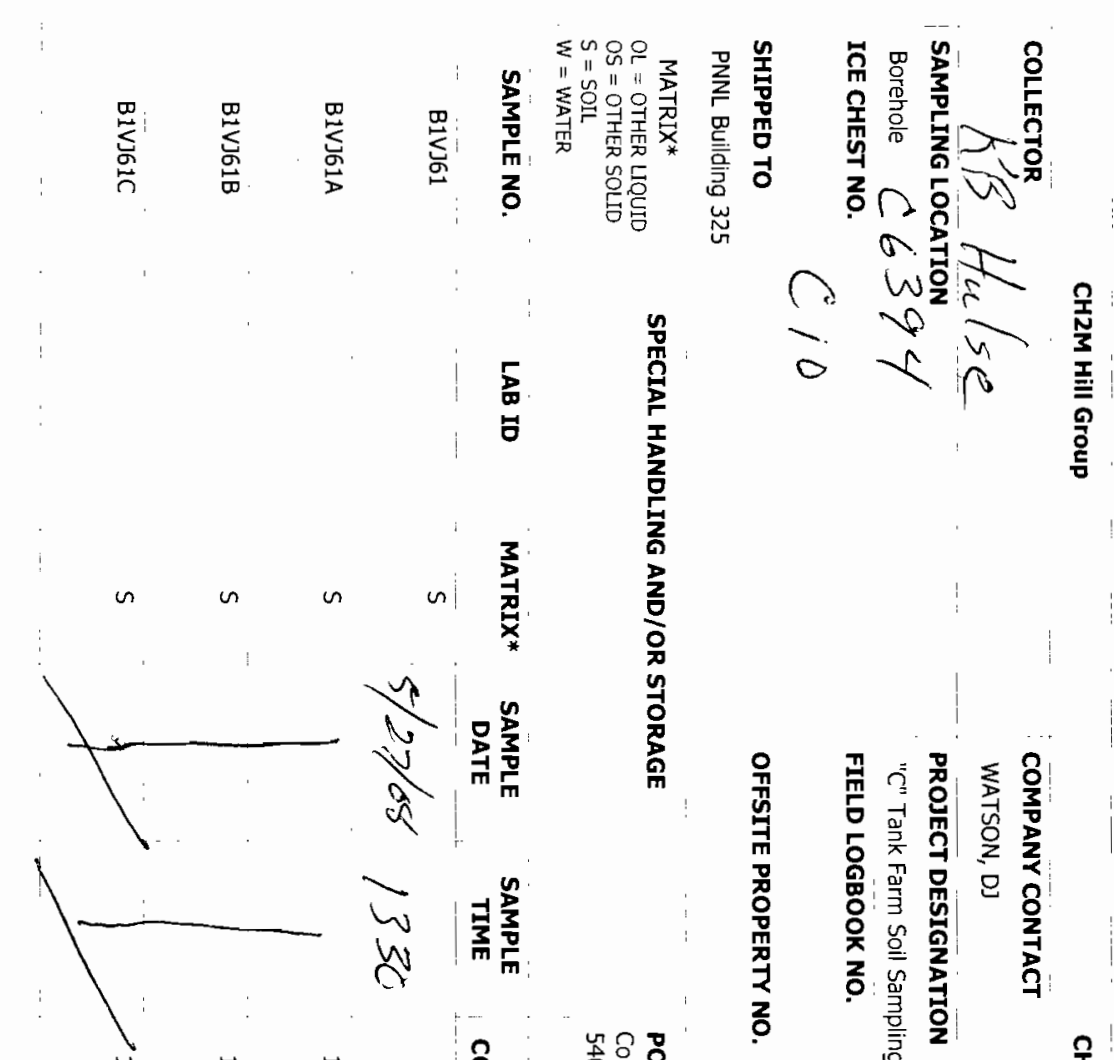

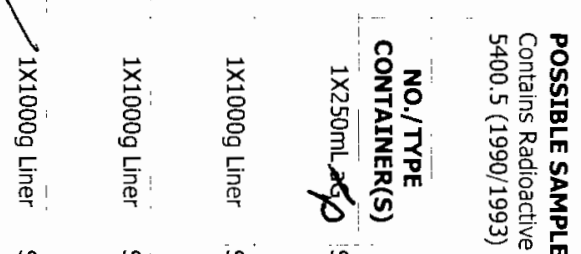

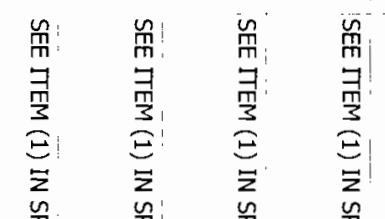

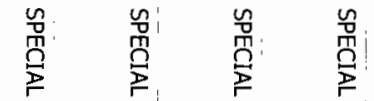

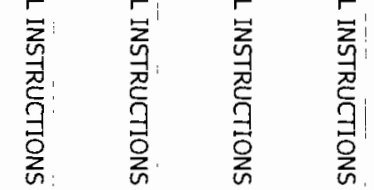

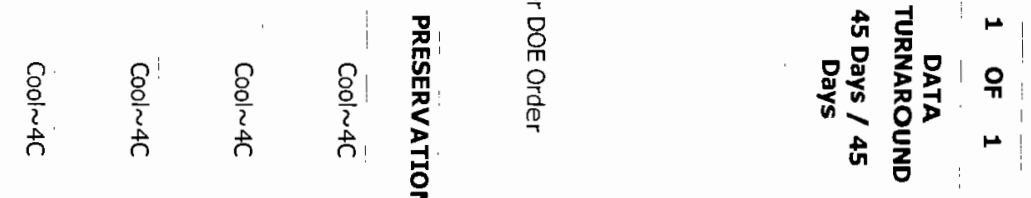




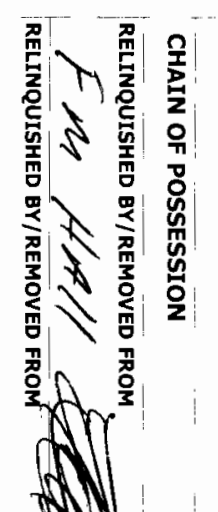

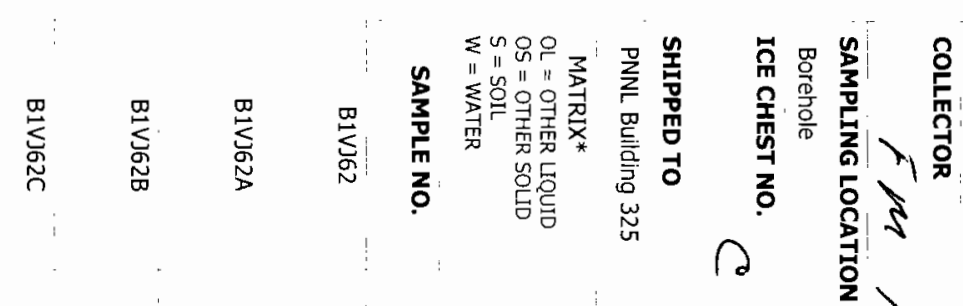

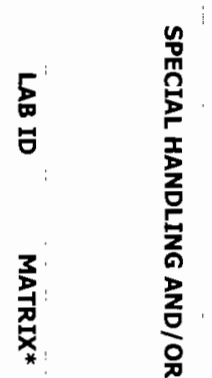
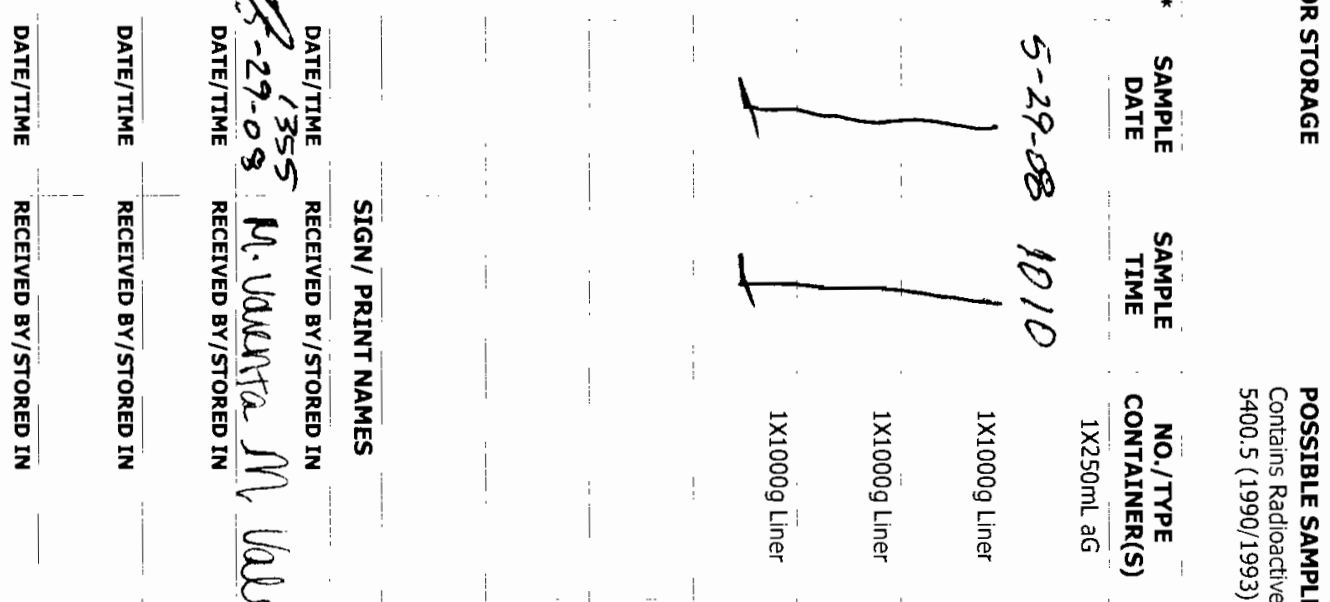

$\varepsilon$

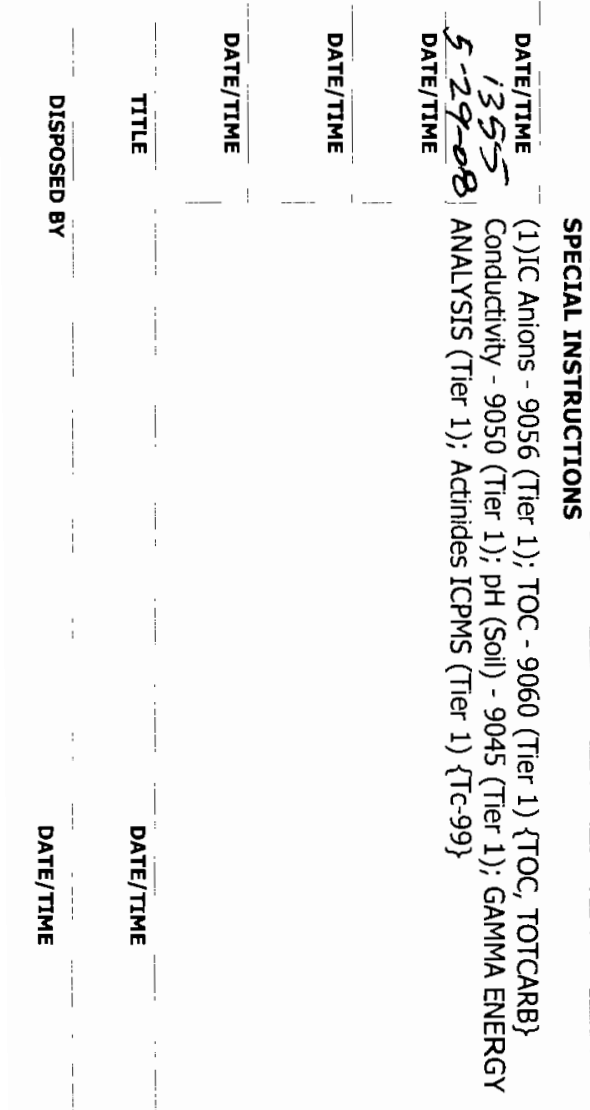

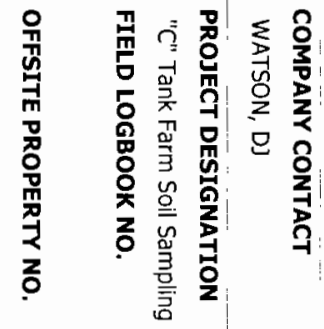
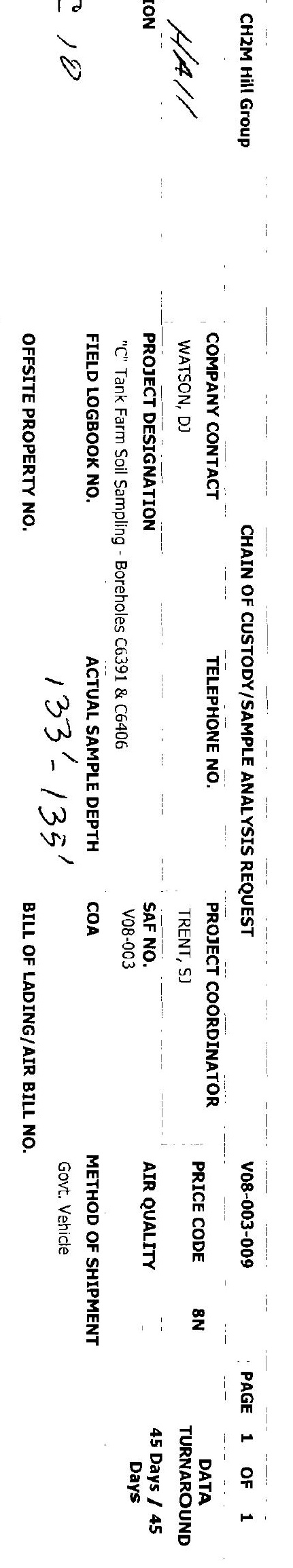


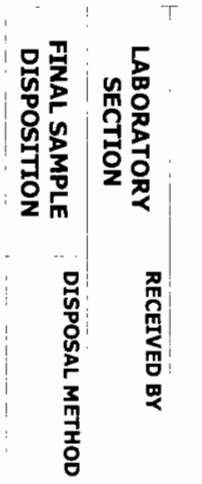
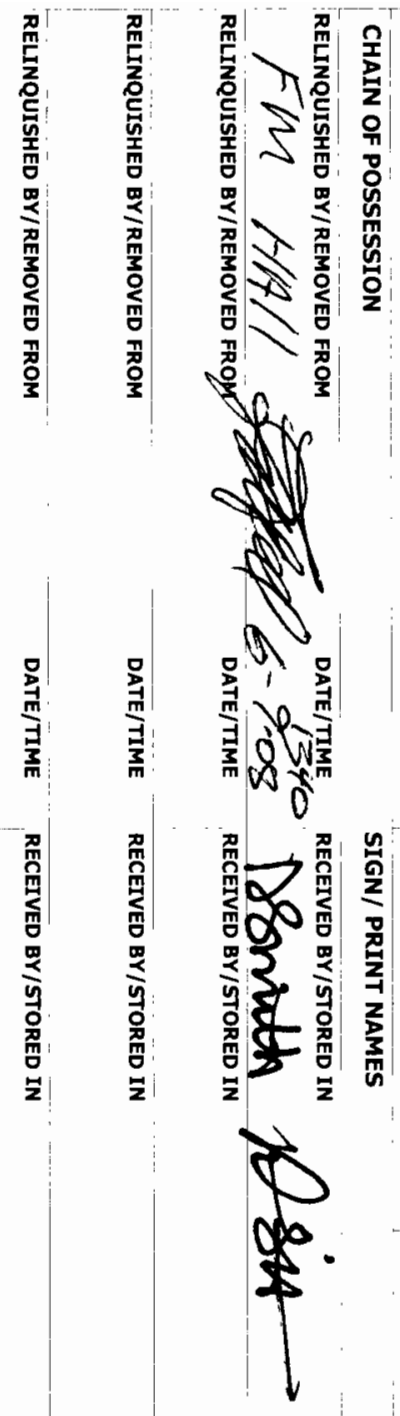

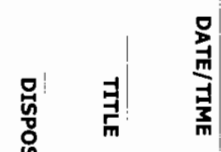

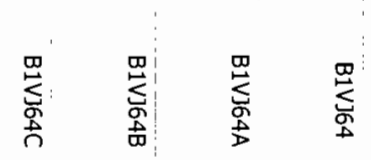
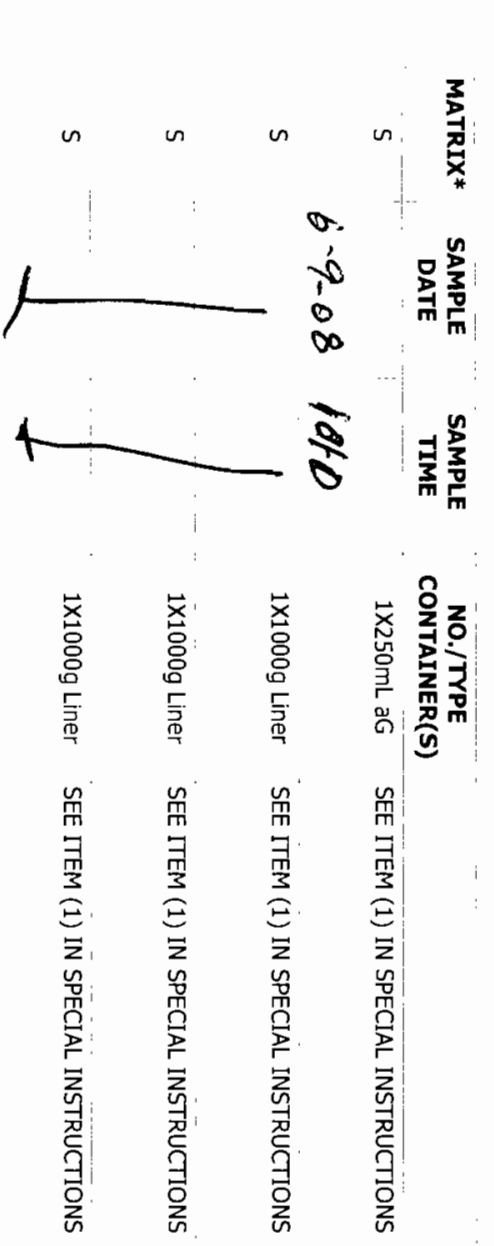

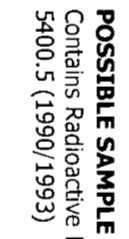
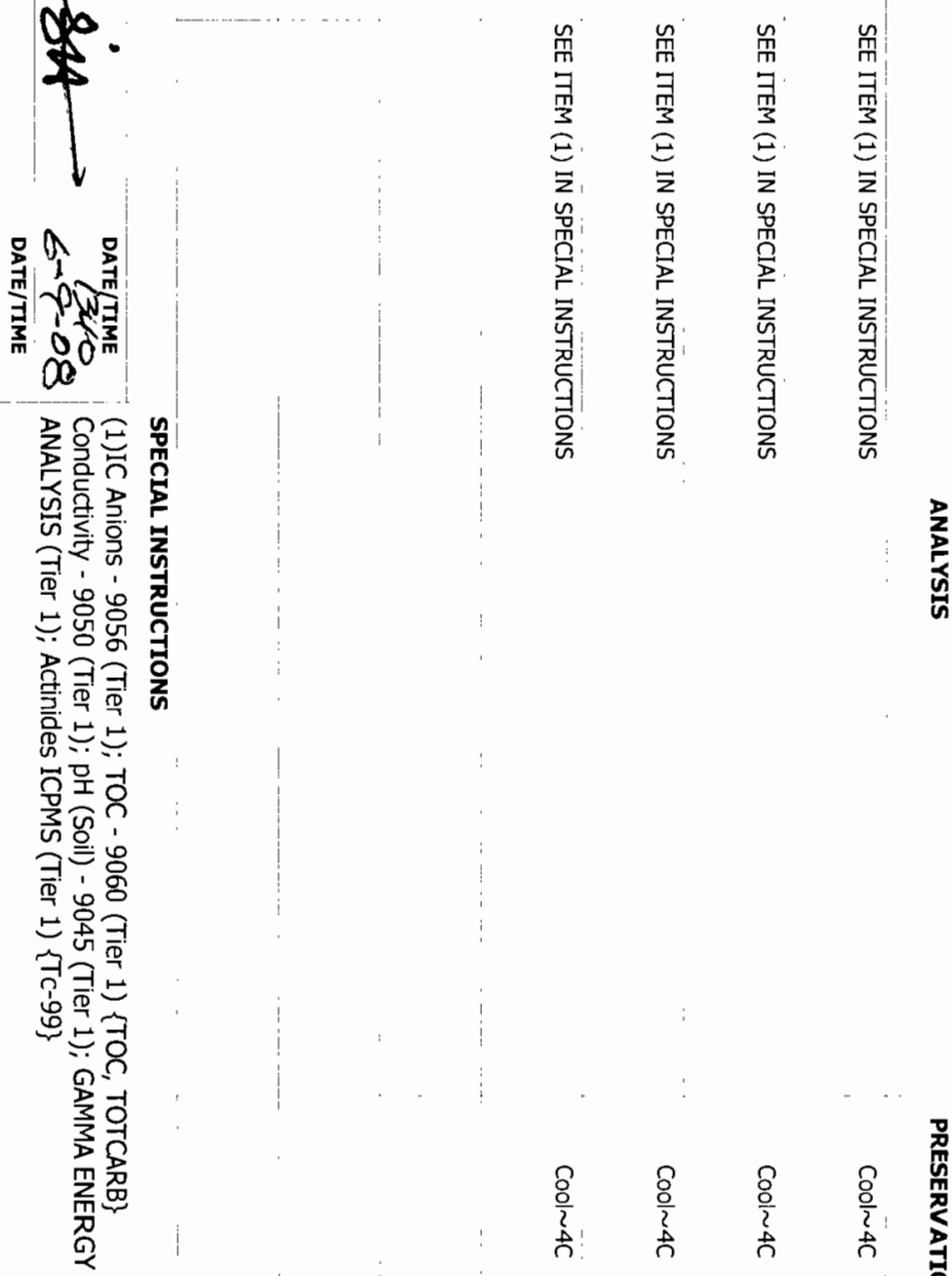

$\frac{5}{5}$

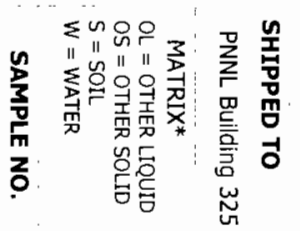

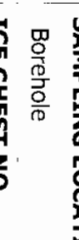

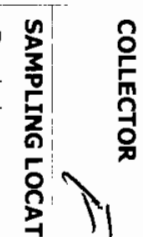
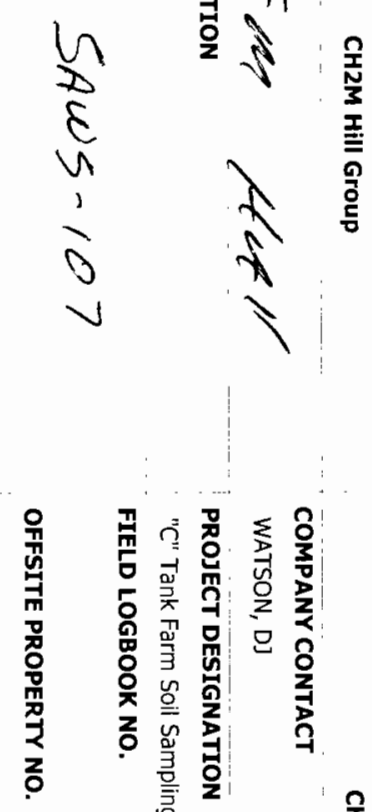

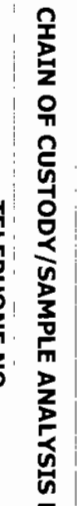
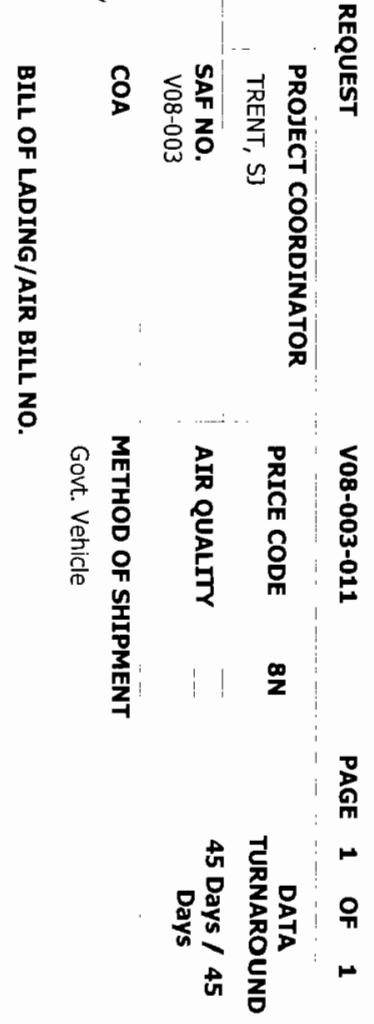
